\title{
Core Design and Operating Data for Cycles 1 and 2 of Peach Bottom 2
}

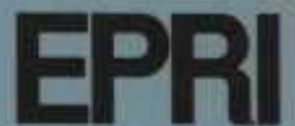

EPRI NP-563

Project 1020-1 Topical Report June 1978
BWR Core Design BWR Benchmark Data BWR Operating Data

Peach Bottom 2

Reactor Operating History 


\section{DISCLAIMER}

This report was prepared as an account of work sponsored by an agency of the United States Government. Neither the United States Government nor any agency Thereof, nor any of their employees, makes any warranty, express or implied, or assumes any legal liability or responsibility for the accuracy, completeness, or usefulness of any information, apparatus, product, or process disclosed, or represents that its use would not infringe privately owned rights. Reference herein to any specific commercial product, process, or service by trade name, trademark, manufacturer, or otherwise does not necessarily constitute or imply its endorsement, recommendation, or favoring by the United States Government or any agency thereof. The views and opinions of authors expressed herein do not necessarily state or reflect those of the United States Government or any agency thereof. 


\section{DISCLAIMER}

Portions of this document may be illegible in electronic image products. Images are produced from the best available original document. 


\title{
Core Design and Operating Data for Cycles 1 and 2 of Peach Bottom 2
}

\author{
NP-563 \\ Research Project 1020-1 \\ Topical Report, June 1978 \\ Prepared by \\ GENERAL ELECTRIC COMPANY \\ Nuclear Energy Engineerıng Divisıon \\ 175 Curtner Avenue \\ San Jose, California 95125 \\ Principal Investigator
}

N. H. Larsen

Prepared for

Electric Power Research Institute

3412 Hillview Avenue

Palo Alto, California 94304

EPRI Project Manager

Robert N. Whitesel

Nuclear Power Division 
LEGAL NOTICE

This report was prepared by General Electric (GE) as an account of work done in conjunction with Philadelphia Electric Company (PECO) and sponsored by both the Electric Power Research Institute, Inc (EPRI) and the General Electric Company Neither EPRI, members of EPRI, PECo, GE, nor any person acting on behalf of etther (a) Makes any warranty or representation, express or implied, with respect to the accuracy, completeness, or usefulness of the information contained in this report, or that the use of any information, apparatus, method, or process disclosed in this report may not infringe privately owned rights, or (b) Assumes any habilities with respect to the use of, or for damages resulting from the use of, any information, apparatus, method or process disclosed in this report 


\section{FOREWORD}

This report is a compilation of reactor design and operating data for Cycles 1 and 2 of the Peach Bottom Unit 2 BWR It has been prepared to provide reference quality data for use in the qualification of reactor core analysis methods

More specifically, these data have been collected to facilitate analyses of the pressure transient and stabılity tests performed at Peach Bottom 2 in Aprl 1977 just prior to the end of Cycle 2 A companion report, "Transient and Stability Tests at Peach Bottom Atomic Power Station Unit 2 at the End of Cycle 2," EPRI NP-564, contains the test data The project has been almed at measuring both the stability of a BWR core when subjected to small pressure oscillations and the response of the reactor to large pressure transients The data from such tests are important not only to the technical base of the licensing of BWR plants, but also as reference data for the qualification of reactor stability and transient computer codes

\section{BRUCE W CRAWFORD}

Program Manager

Nuclear Energy Engıneerıng Divisıon

General Electric Company
ROBERT N WHITESEL

Project Manager

Electric Power Research Institute 


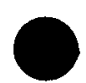




\begin{abstract}
This report contains the design and operating daia needed to define the fuel characteristics, vessel internal $c$ mponents, nuclear steam supply system components, and reactor operation characteristics for Cycles 1 and 2 of the Peach Bottom 2 reactor. The purpose is to provide reference quality data for use in the qualification of reactor core analysis methods and to provide the basis for the assessment of the irradiation environment during Cycles 1 and 2.

The design data includes fuel assembly description, core component arrangements, control rod descriptions, core loading patterns, reactor internals description, and major piping arrangements. Hydraulic characteristics of the assemblies and the inlet orifices are also provided. Operating data is compiled for 24 steadystate points during Cycle 1 and 13 during Cycle 2. Each state point includes core average exposure, thermal power, pressure, flux, inlet subcooling, control configuration and axial in-core detector readings.
\end{abstract}


0

. 
TABLE OF CONTENTS

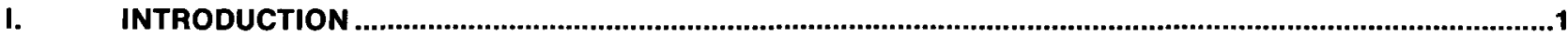

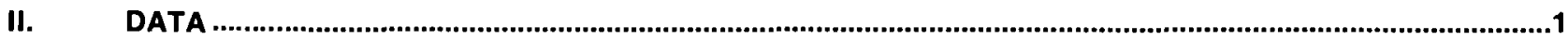

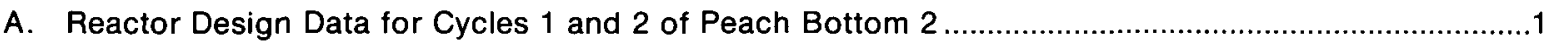

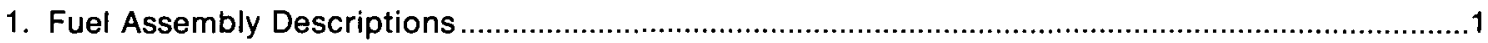

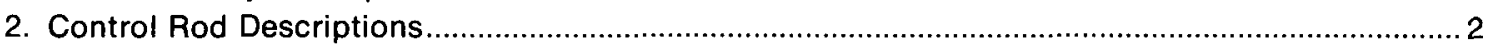

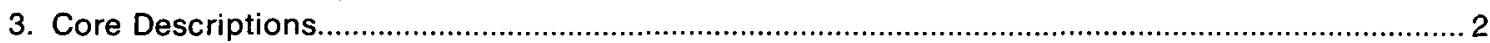

4. Vessel Internal Components, Elevation Drawings, etc........................................................ 2

5. Nuclear Steam Supply System Components Outside The Vessel ............................................ 2

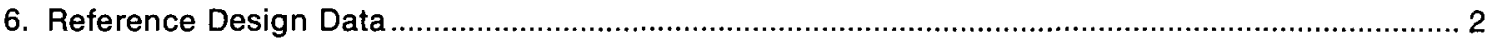

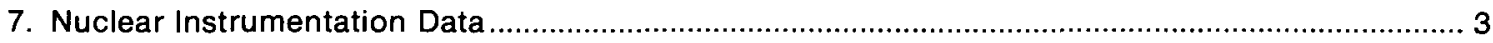

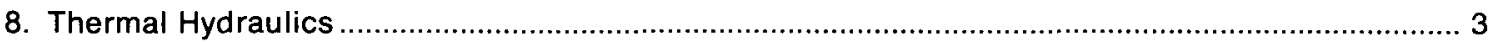

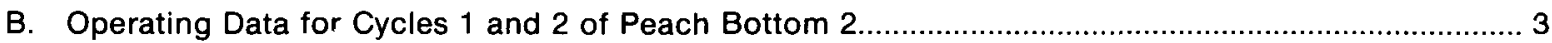

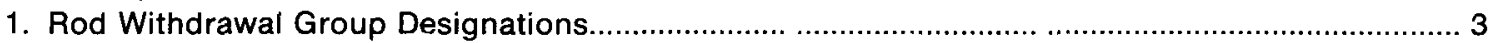

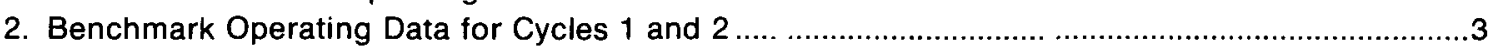

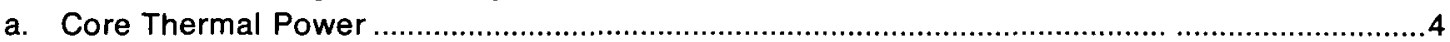

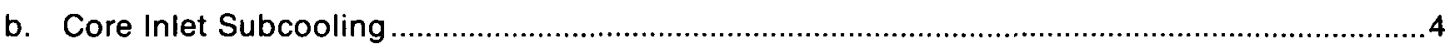

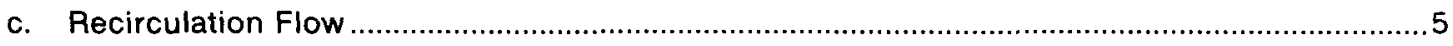

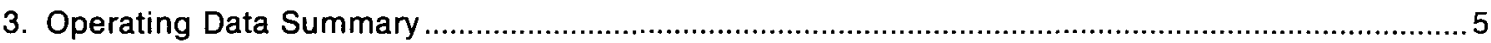


-

- 


\section{LIST OF TABLES}

Table

Title

Page

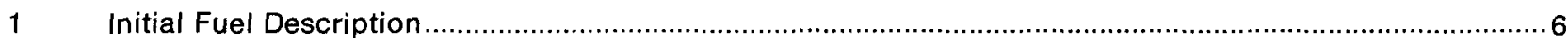

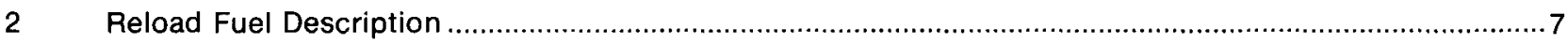

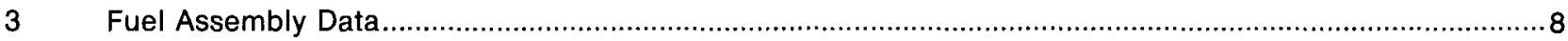

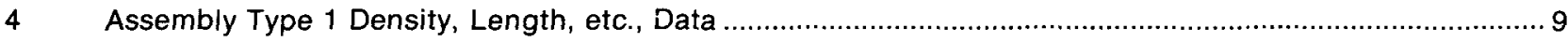

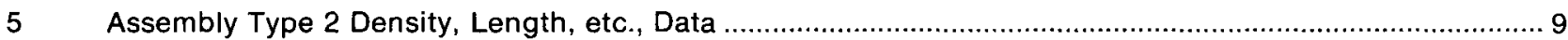

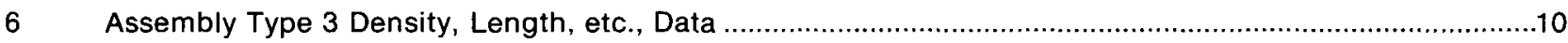

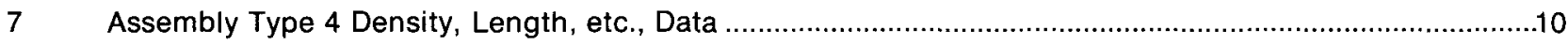

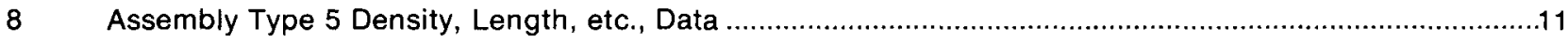

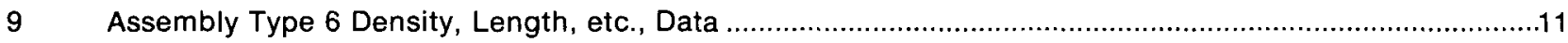

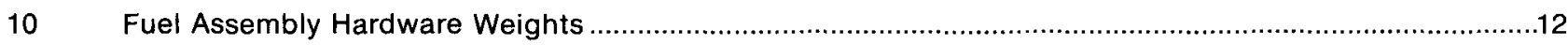

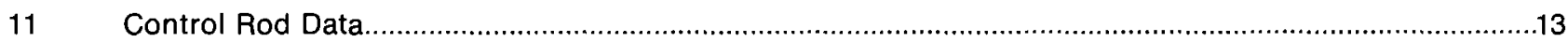

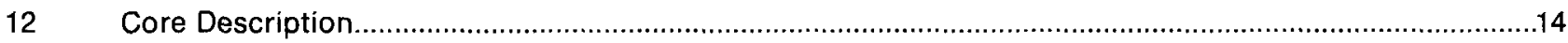

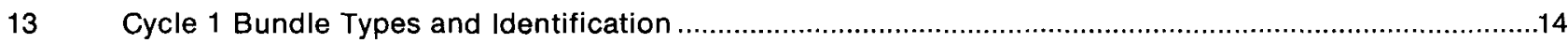

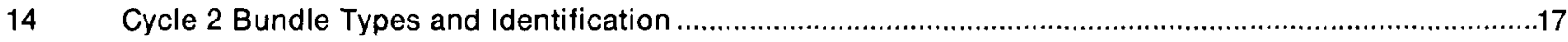

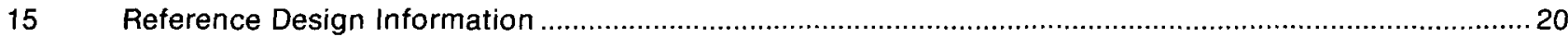

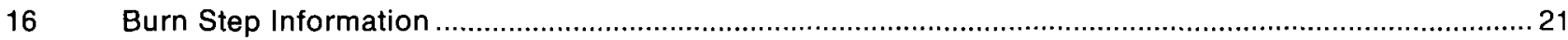


-$$
\text { . }
$$ 


\section{LIST OF ILLUSTRATIONS}

Figure

1 Bundle Design for Type 1 Initial Fuel.

2 Bundle Design for Type 2 Initial Fuel.

3 Spatial $\mathrm{Gd}_{2} \mathrm{O}_{3}$ Variation Initial Type 2 Fuel

Bundle Design for Type 3 Initial Fuel.

7 Bundle Design for Type $58 \times 8 \mathrm{UO}_{2}$ Reload,

8 Bundle Design for Type $68 \times 8 \mathrm{UO}_{2}$ Reload, LTA.

11 Reload Fuel Assembly Lattice for 100 mil Channels

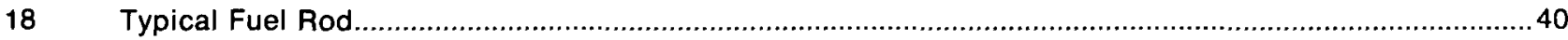

19 Spacer Positioning Rod for Type 3 Initial Fuel..............................................................................41

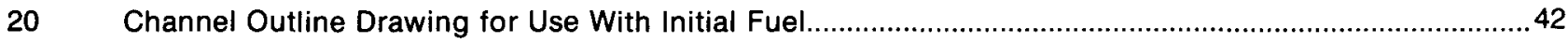

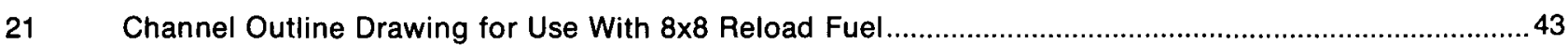

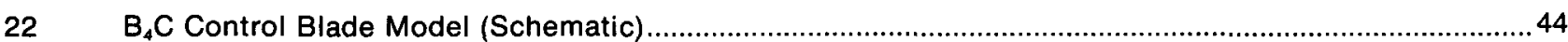

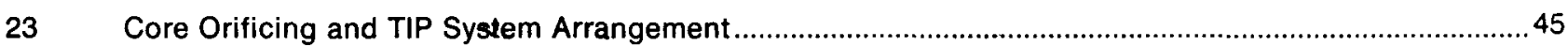

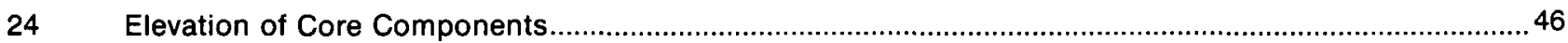

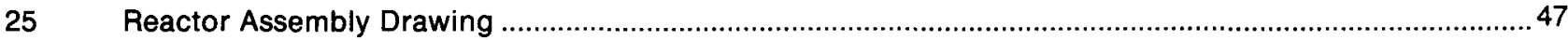




\section{LIST OF ILLUSTRATIONS (Continued)}

Figure

Title

Page

$26 \quad$ Shroud Drawing

27 Top Guide Drawing

28 Steam Separator Outlıne Drawing

$29 \quad$ Steam Dryer Drawing

30 Feedwater Sparger Outlıne

$31 \quad$ Orificed Fuel Support

32 Jet Pump Drawing

33 Recirculation Loop Piping

$34 \quad$ Prımary Steam Pıpıng

35 Prımary Steam Pipıng (Contınued)

37 Maın Steam and Bypass Lıne Pıpıng

38 Maın Steam and Bypass Lıne Pipıng (Contınued)

39 Reactor Primary System Weights and Volumes

40 Reactor Prımary System Weights and Volumes (Contınued)

41 Recirculation Pump Characterıstics at Rated Pump Speed

42 Recırculation Pump Characteristıcs at Varıous Pump Speeds

43 TIP/LPRM In-Core Assembly Cross Section

481469 in Orifice Diameter, 20 Btu/lb Subcooling 


\section{LIST OF ILLUSTRATIONS (Continued)}

Figure

Title

Page

$51 \quad 2.211$ in Orifice Diameter, $30 \mathrm{Btu} / \mathrm{lb}$ Subcooling

2.211 in Orifice Diameter With Orificed Lower Tie Plate, 20 Btu/lb Subcooling. .74

Core Bypass Flow for Cycle 2.

Peach Bottom 2 Control Rod A Sequence Groups $1-8$ .78 


\section{LIST OF ILLUSTRATIONS (Continued)}

Figure

76 Data Summaries, February 1975

77 Data Summarıes, March 1975

78 Data Summarıes, Aprıl 1975

79 Data Summaries, May 1975

80 Data Summarıes, June 1975

81 Data Summaries, July 1975

82 Data Summaries, August 1975

83 Data Summaries, September 1975

84 Data Summaries, October 1975

85 Data Summarıes, November 1975

86 Data Summarıes, December 1975

87 Data Summaries, January 1976

88 Data Summarıes, February 1976

89 Data Summaries, March 1976

90 Data Summaries, June 1976

$91 \quad$ Data Summarıes, July 1976

92 Data Summarıes, August 1976

93 Data Summarıes, September 1976

94 Data Summaries, October 1976

95 Data Summarıes, November 1976

96 Data Summarıes, December 1976

97 Data Summaries, January 1977

98 Data Summaries, February 1977

99 Data Summaries, March 1977

100 Data Summarıes, Aprıl 1977
Title

Page

98

99

100

101

102

103

104

105

106

107

108

109

110

111

112

113

114

115

116

117

118

119

120

121

122 


\section{LIST OF DATA SETS}

Page

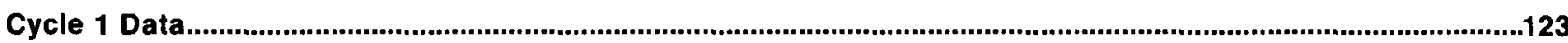

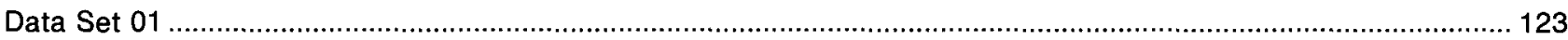

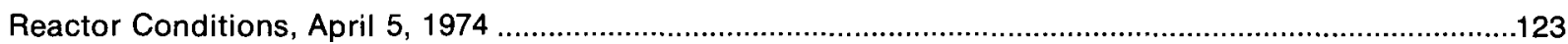

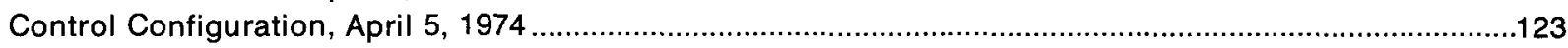

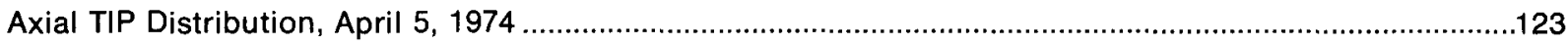

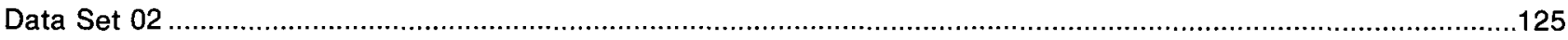

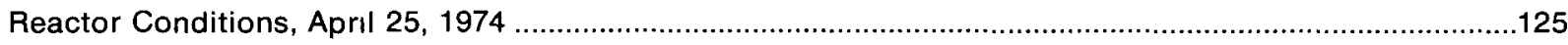

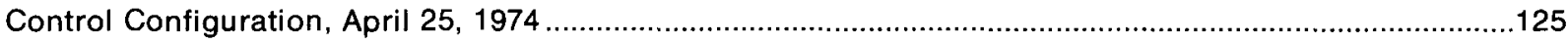

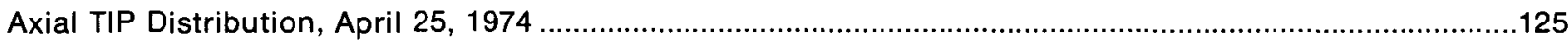

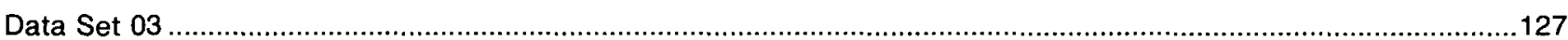

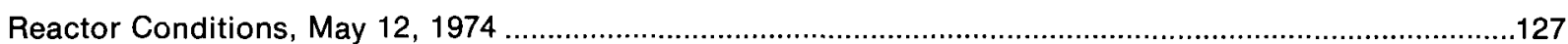

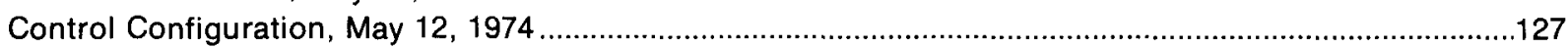

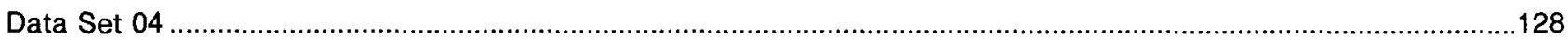

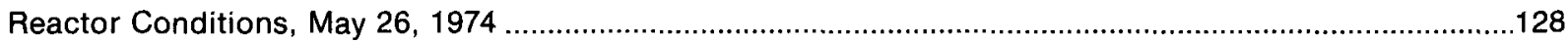

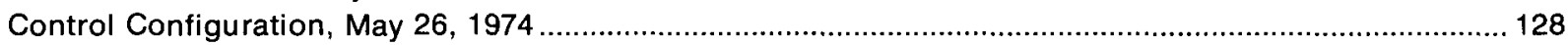

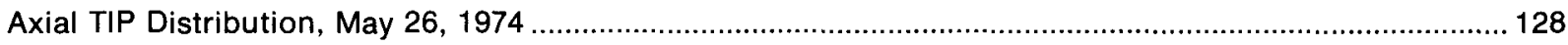

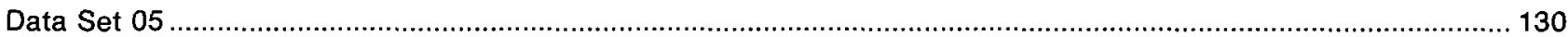

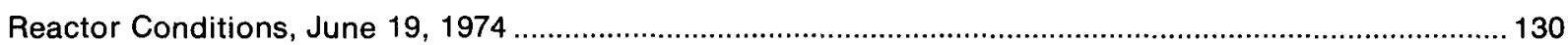

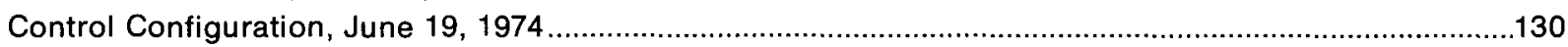

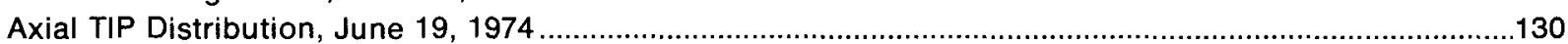

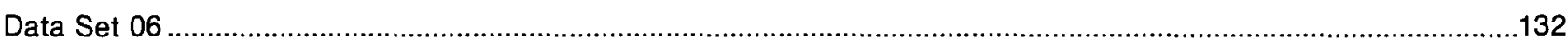

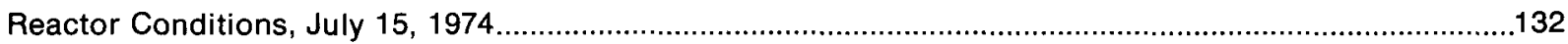

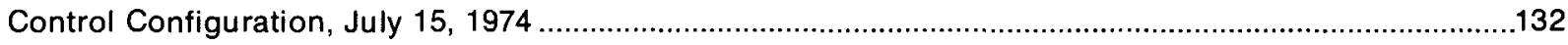

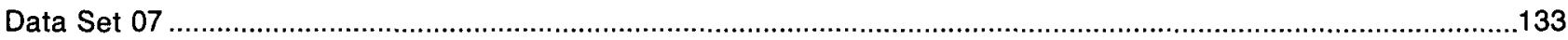

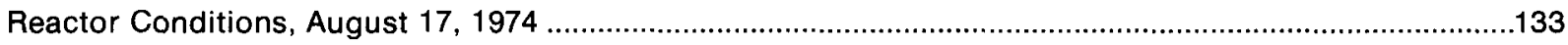

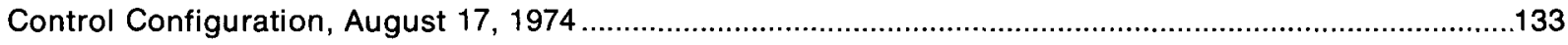

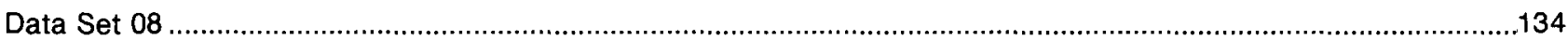

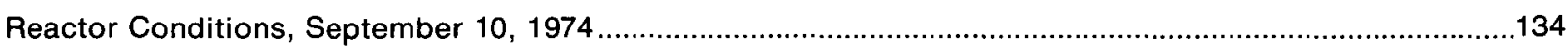

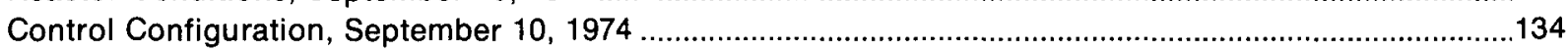

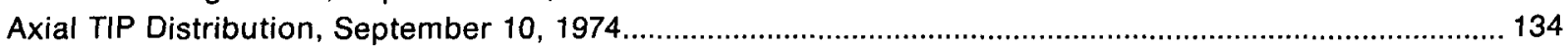

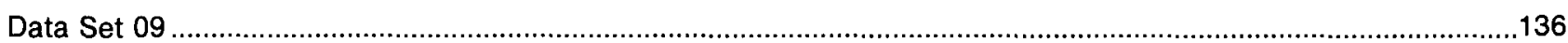

Reactor Conditions, October 4, 1974

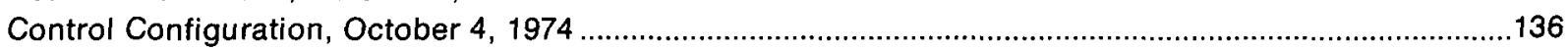

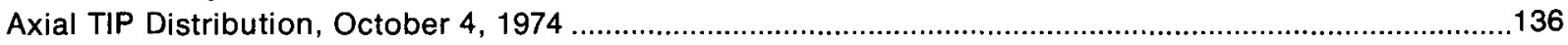

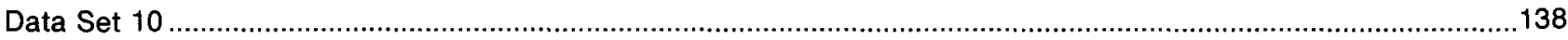

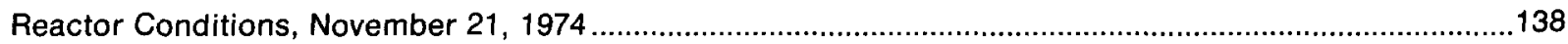

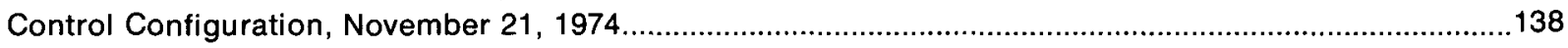

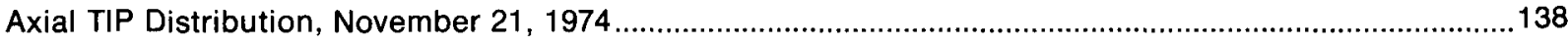




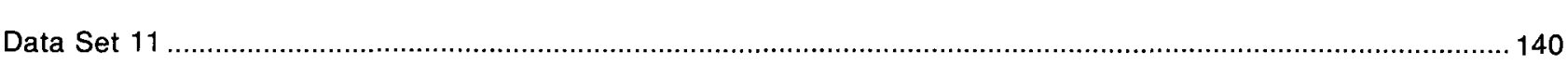

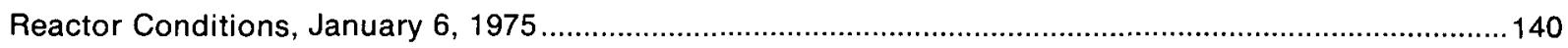

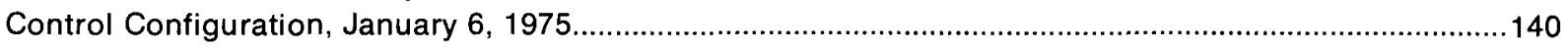

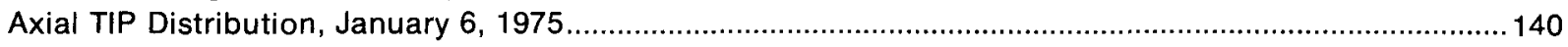

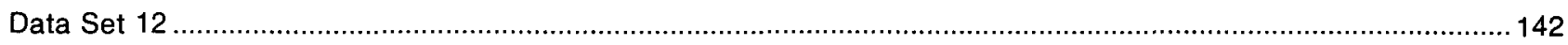

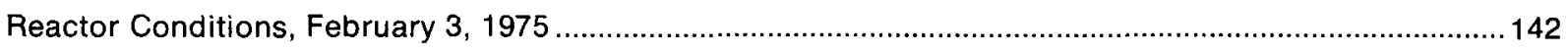

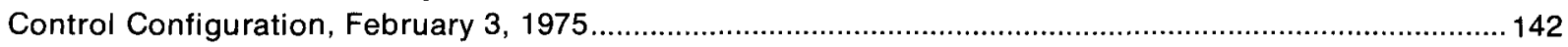

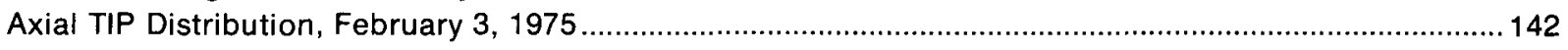

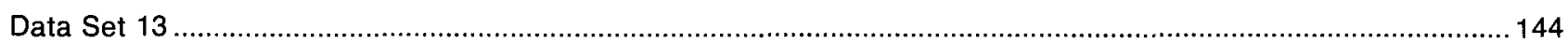

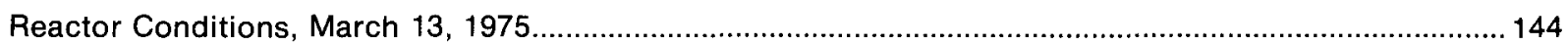

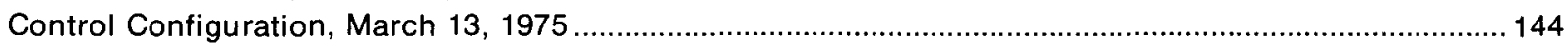

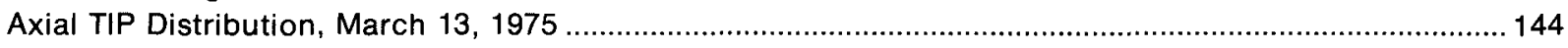

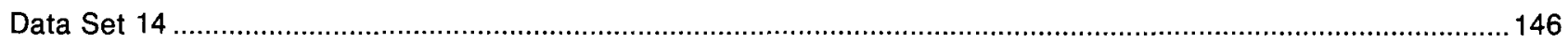

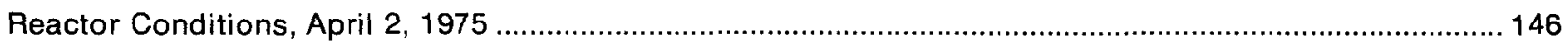

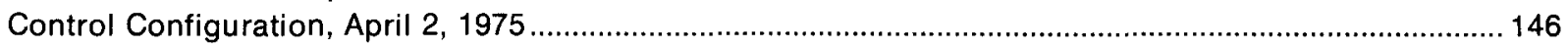

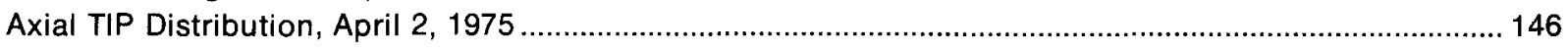

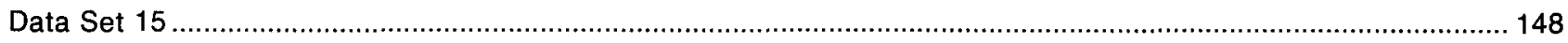

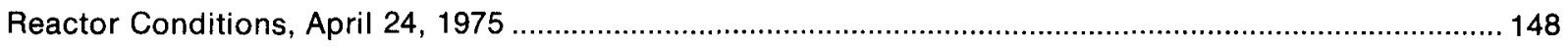

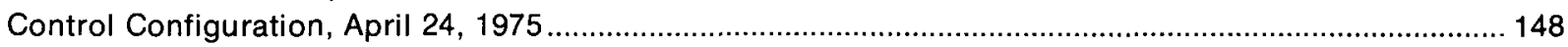

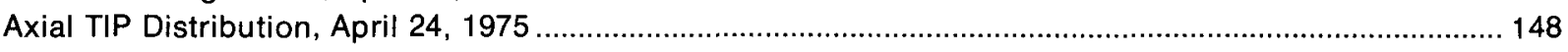

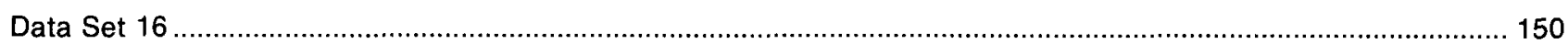

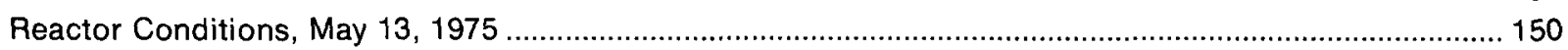

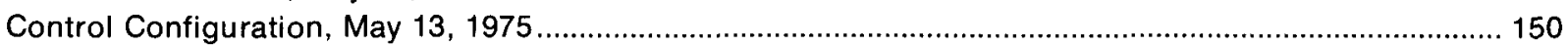

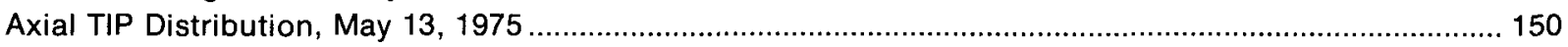

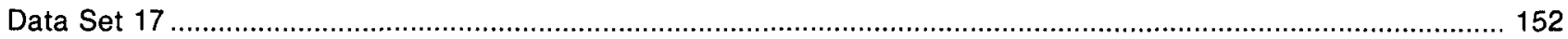

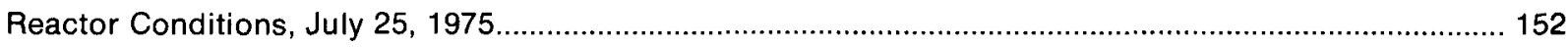

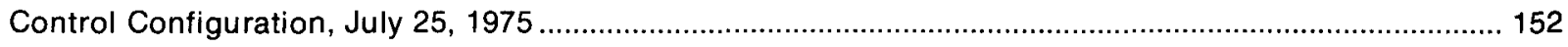

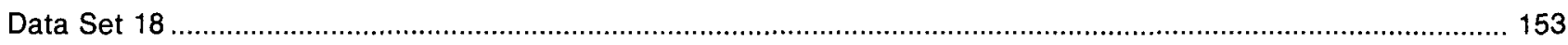

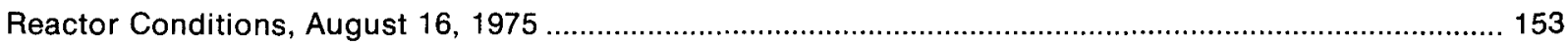

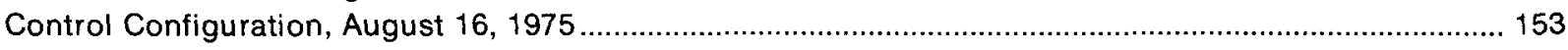

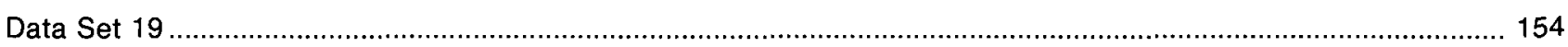

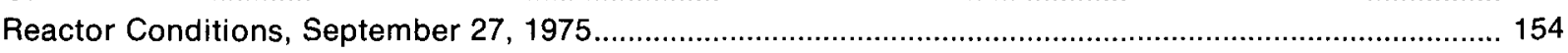

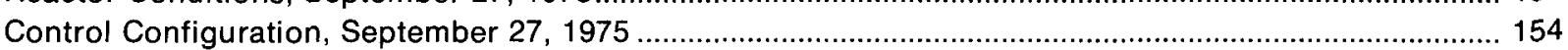

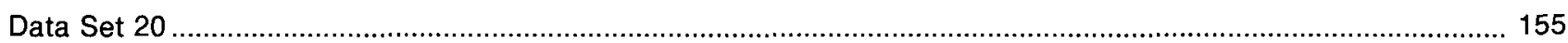

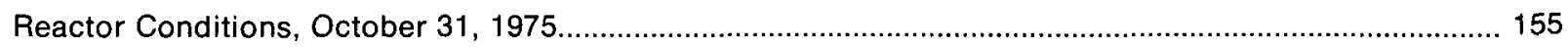

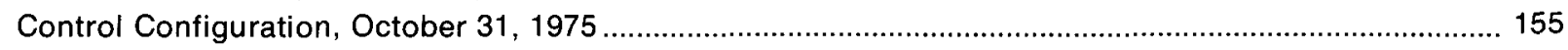

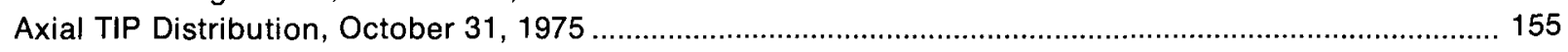

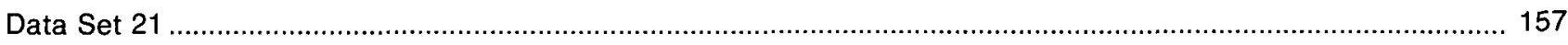

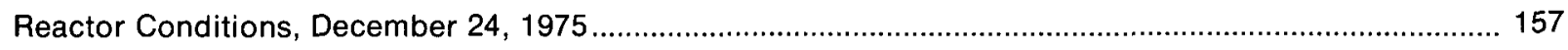

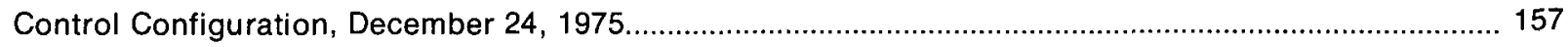




\section{LIST OF DATA SETS (Contınued)}

Data Set 22

Reactor Conditions, January 15, 1976

Control Configuration, January 15,1976 158

Axial TIP Distribution, January 15, 1976

Data Set 23

Reactor Conditions, February 14, $1976 \quad 160$

Control Configuration, February 14, 1976

Axial TIP Distribution, February 14, 1976

Data Set 24

Reactor Conditions, March 26, 1976

Control Configuration, March 26, 1976

Axial TIP Distribution, March 26, 1976

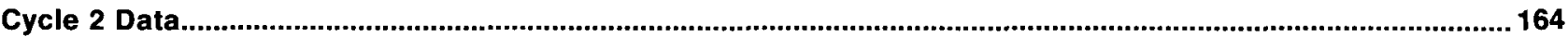

Data Set 25

Reactor Conditıons, June 28, 1976

Control Configuration, June 28, $1976 \quad 164$

Axial TIP Distribution, June 28, 1976

Data Set 26

Reactor Conditıons, July 14,1976

Control Confıguration, July 14, 1976

Axial TIP Distribution, July 14, 1976

Data Set 27

Reactor Conditions, September 1, 1976

Control Configuration, September 1, 1976

Axial TIP Distribution, September 1, 1976

Data Set 28

Reactor Conditıons, October 8, 1976

Control Configuration, October 8, 1976

Axıal TIP Distribution, October 8, 1976

Data Set 29

Reactor Conditions, October 28, 1976

Control Confıguration, October 28, 1976

Axıal TIP Distribution, October 28, 1976

Data Set 30

Reactor Conditions, December 16, 1976

Control Configuration, December 16,1976 174

Axıal TIP Distribution, December 16, 1976

Data Set $31 \quad 176$

Reactor Conditions, December 28, 1976

Control Configuration, December 28, 1976

Axıal TIP Distribution, December 28, 1976 


\section{LIST OF DATA SETS (Continued)}

Data Set 32 178

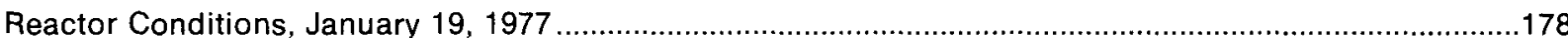

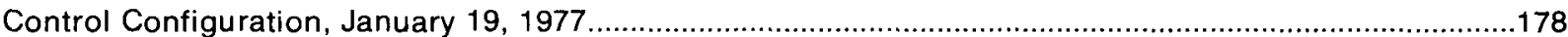

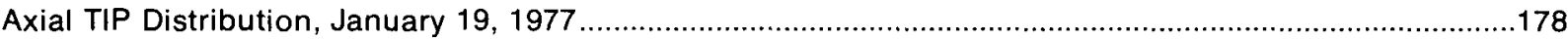

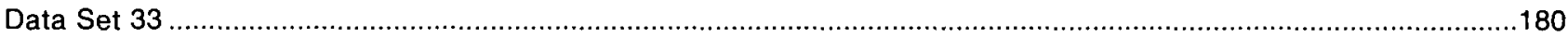

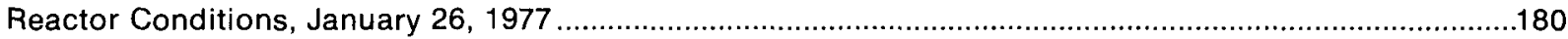

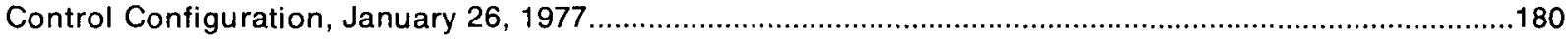

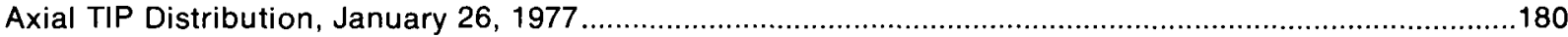

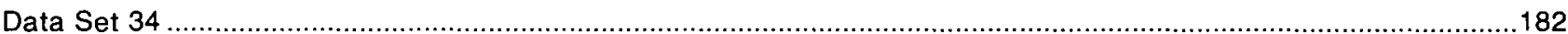

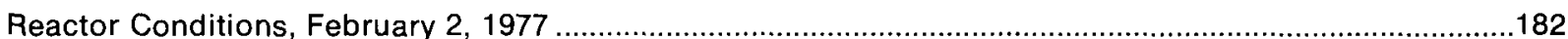

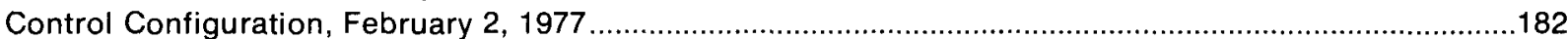

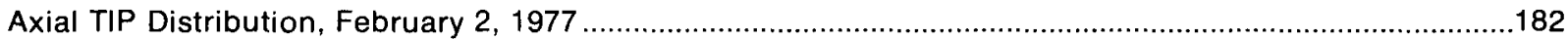

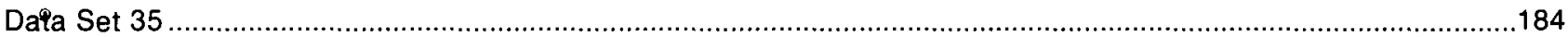

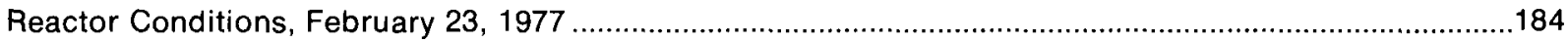

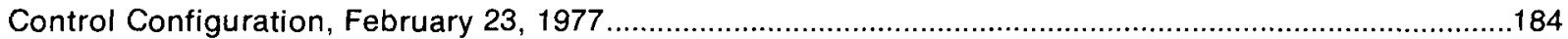

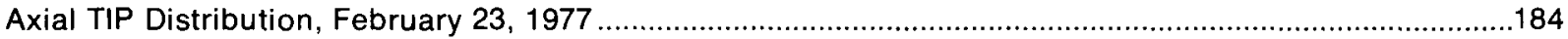

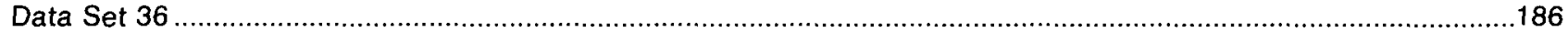

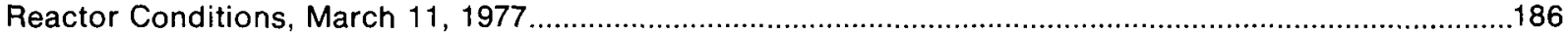

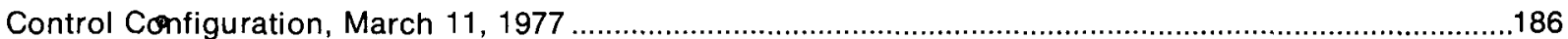

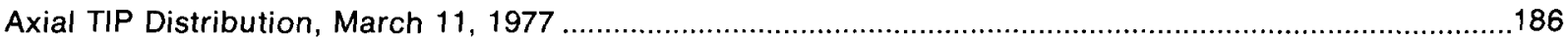

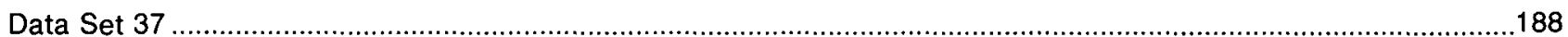

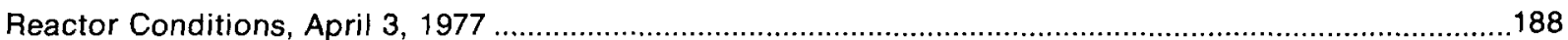

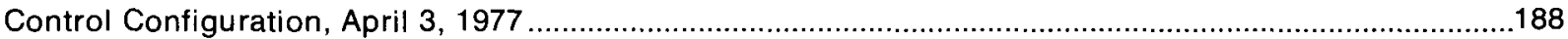

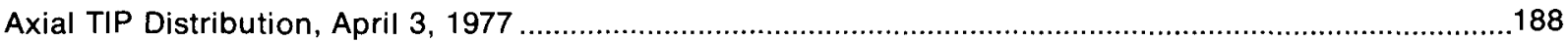




\section{SUMMARY}

This report contains the design and operating data needed to define the fuel characteristics, vessel internal components, nuclear steam supply system components, and reactor operation characteristics for Cycles 1 and 2 of the Peach Bottom 2 reactor The purpose is to provide reference quality data for use in the qualification of reactor core analysis methods and to provide the basis for the assessment of the irradiation environment during Cycles 1 and 2

The design data includes fuel assembly description, core component arrangements, control rod descriptions, core loading patterns, reactor internals description, and major pipıng arrangements. Hydraulic characteristıcs of the assemblies and the inlet orifices are also provided Operating data is compiled for 24 steady-state points during Cycle 1 and 13 during Cycle 2 Each state point includes core average exposure, thermal power, pressure, flux, inlet subcooling, control configuration and axial in-core detector readings 


\section{INTRODUCTION}

The design and operatıng data needed to define the fuel characterıstics and reactor operatıng characterıstics for Cycles 1 and 2 of the Peach Bottom 2 reactor are contained in this report The program was a joint effort of the General Electric Company, the Philadelphia Electric Company, and the Electric Power Research Institute

The fuel and core design data were extracted from appropriate reports and drawings Almost all of the operating data provided were obtained directly from process computer output edits of the reactor operation through Cycles 1 and 2

\section{DATA}

\section{A. REACTOR DESIGN DATA FOR CYCLES 1 AND 2 OF PEACH BOTTOM 2}

\section{Fuel Assembly Descriptions}

a Bundle desıgn data for Type 1 ınıtıal fuel, Type 2 ınıtıal fuel, Type 3 ınıtıal fuel, spatıal $\mathrm{Gd}_{2} \mathrm{O}_{3}$ varıatıon ın ınıtıal fuel, $8 \times 8$ reload fuel, and lead test fuel assemblies are included as Figures 1 to 9

b Fuel assembly lattıce drawings, including detalled dimensıons, for inıtıal fuel, reload fuel with 100 and $120 \mathrm{mil}$ channels, and the lead test assemblies are included as Figures 10 through 13 The numbers 100 and 120 refer to the wall thickness of the channel

Tables 1 and 2 summarize fuel rod arrays, fuel rod pitch, rod-to-channel spacing, gap thicknesses, control augmentation characteristics, $\mathrm{U}$ weights, channel characterıstıcs, and water/ $\mathrm{UO}_{2}$ volume ratıos for the ınıtıal $7 \times 7$ assemblies and the reload assemblies

Table 3 provides core loadıng, assembly pitch, fuel pın pitch, spacer data, average fuel compositıons, and fuel weights for all fuel assemblies during Cycles 1 and 2

Tables 4 to 9 include pellet and stack densities, $\mathrm{Gd}_{2} \mathrm{O}_{3}$ and $\mathrm{UO}_{2}$ weights, pellet lengths, pellet $\mathrm{Od}$, cladding o d cladding thıckness, and gas plenum lengths for all fuel used durıng Cycles 1 and 2 All fuel rods were backfilled with helıum at 0 to $10 \mathrm{psig}$ at $70^{\circ} \mathrm{F}$

Table 10 includes spacer weights, end plug weights, upper and lower tie plate weights, fission gas plenum material weights, the alloy compositıons recommended for nuclear analyses, and spacer placement identification

Figures 14 to 17 are assembly detail drawings for Initıal $7 \times 7$ fuel, reload $8 \times 8$ fuel, and the lead test assembly (LTA) fuel

A typical fuel rod is shown in Figure 18 A fueled spacer positıonıng rod for Type 3 initial fuel is shown in Figure 19 The purpose of the spacer positioning rod is to provide a lockıng tab which fixes the fuel rod spacers in their desıgned axial position This is accomplished by an end plug connector that contains a fork desıgn which catches a tab on the spacer The fuel rod is thus segmented into eight segments for the seven spacers Fission gas may travel from segment to segment by means of a hole in the center of the connector plugs For the $8 \times 8$ assemblies, the water rod is also the spacer positioning rod in this case, the positioning mechanism is simply welded to the tube, which is the same as the cladding for the fuel rods Holes are provided at the bottom and top of the water rod to provide water flow and ittle or no boiling inside the tube

Figures 20 and 21 are channel outline drawings for 80 and $100 \mathrm{mll}$ channels The $120 \mathrm{mll}$ thick development channels are sımılar to those shown 


\section{Control Rod Descriptions}

a. Table 11 contains physical data for the control rods including shape, pitch, stroke, control material, etc.

b. Figure 22 is a schematic drawing of a cross section of a control blade.

\section{Core Descriptions}

a. Table 12 identifies the total number of fuel assemblies, number of fuel assembly types, heat transfer surface area, total weight of $U$ in the core, etc., for Cycles 1 and 2.

b. Table 13 presents the bundle type and identification core loading array for Cycle 1.

c. Figure 23 is a core plan view showing the core orificing and TIP system arrangement.

d. Table 14 presents the bundle type and identification core loading array for Cycle 2.

4. Vessel Internal Components, Elevation Drawings, etc.

a. Figure 24 shows the elevation of the core instrumentation and control blade poison with respect to the active fuel.

b. An elevation view of the reactor internal components is presented in Figure 25 . Figures 26 through 30 are outlines of the various internal components; core shroud, top guide, steam separator, steam dryer, and feedwater sparger.

c. Figure 31 is an outline of the orificed fuel support piece.

d. Figure 32 is an outline drawing of the jet pump.

5. Nuclear Steam Supply System Components Outside The Vessel

a. Figure 33 shows the recirculation loop piping.

b. Figures 34 to 38 show the primary steam piping and the steam bypass lines.

c. Portions of the feedwater lines appear on several drawings made by Bechtel, San Francisco, under Job Number 6280 . These drawings are identified by Bechtel as $M-x x x$, where $M$ designates mechanical and $x x x$ is a particular drawing number, beginning with 1 . These drawings are not included in this report, but may be obtained from Bechtel.

d. Other characteristics, such as Main Turbine Stop and Bypass Valve Flow-Pressure relationship for the downstream bypass pressure reducers and the transfer functions representing the speed-torque response of the Feedpump Turbine Drives, are necessary information for analysis of the transient experiments. These data are proprietary to the General Electric Company.

\section{Reference Design Data}

Table 15 provides reference design information. Figures 39 and 40 are compilations of various weights and volumes of the reactor primary system. Figures 41 and 42 are recirculation pump characteristics for various conditions. 


\section{Nuclear Instrumentation Data}

Peach Bottom 2 is equipped with a system of Travelling In-Core Probe (TIP) detectors and fixed Local Power Range Monitor (LPRM) detectors desıgned to provide an accurate representation of the spatial distribution of the neutron flux The TIP detectors travel through a set of 43 vertical tubes which are distributed uniformly throughout the core with the planar density of one detector per four square feet Figure 23 shows the core location and coordinate identification of the TIP strings A cross section of a TIP/LPRM assembly is given in Figure 43

The TIP measures the axial neutron flux distribution in the water gap by use of a 1-In long U-235 fission chamber attached to a cable and motor which allows the chamber to be positioned at any point along the axial length of up to 10 core positions for each TIP machıne There are five TIP machınes in the Peach Bottom 2 reactor The TIP values reported in the data sets for 6-in intervals represent the weighted average value of seven measurements made at $1-$ in intervals (five interior measurements which are given twice the weightıng as the two end points) A total of 145 measurements is made for each core position resulting in 24 values of 6 in each

The TIP data are normalized to the common position The common position normalization is determined experimentally by traveling the common position with each TIP machine The normalization is determined so that all the TIP machınes produce the same readings when operated in the common position The axially averaged TIP reading for the common position is usually defined to be 100 The TIP data given in the data sets were obtained directly from the process computer For editing purposes, all values of some data sets have been multiplied by a constant such that all values are less than 1000 No other adjustments have been made

\section{Thermal Hydraulics}

The hydraulic characteristics of $7 \times 7$ and $8 \times 8$ fuel assemblies are presented in Figures 44 to 47 as functions of active coolant flow, active coolant power and subcooling These data may be applied over a pressure range of $1035 \pm 100$ psia Bundle pressure drop is somewhat insensitive to axial power distribution The data are based on a distribution peaked at the middle with a peak-to-average value of 15 With a bundle flow of 130x $10^{3} \mathrm{lb} / \mathrm{hr}$, bottom-peaked axial ( $3 / 8$ point of active fuel length) will yield a pressure drop about $066 \mathrm{psi}$ larger A top-peaked axıal yields essentially the same pressure drop as the middle peaked axıal

b The pressure drop characteristics of the central and peripheral region orifices are presented as functions of active coolant flow on Figures 48 through 53 The location of the orifice zones is given in Figure 23 it should be noted that all the $8 \times 8$ reload fuel had holes drilled in the lower tie plate for bypass flow augmentation, whereas there are no holes in the $7 \times 7$ lower tie plates

The total core bypass flow rates for Cycles 1 and 2 are presented in Figures 54 and 55, respectively It should be noted that the origınal core desıgn had holes drilled in the core support for bypass flow augmentation At $8100 \mathrm{MWd} / \mathrm{t}$ core average exposure into Cycle 1 (November, 1975) these holes were plugged, reducıng the bypass flow

\section{B. OPERATING DATA FOR CYCLES 1 AND 2 OF PEACH BOTTOM 2}

\section{Rod Withdrawal Group Designation}

Figures 56 to 61 present the control rod group desıgnatıons for Cycles 1 and 2

\section{Benchmark Operating Data for Cycles 1 and 2}

Data Sets 01 to 24 contaın the reactor data for 24 selected operatıng states durıng Cycle 1 and Data Sets 25 to 37 contaın the reactor data for 13 selected operatıng states durıng Cycle 2 Most data sets contaın the following data date, core average exposure, core thermal power, dome pressure, core flow, inlet subcoolıng, control configuration, and complete axial TIP distribution data for all 43 LPRM string locations The TIP data are the 
commonly normalized TIP readıngs at 6-inch intervals up the length of the assembly. The TIP data read from the bottom to the top of core; 1 e., the first entry is for the bottom 6-in. node. Exposure can be accumulated by using the calculated core power distribution for each of the data sets provided to advance to the next operatıng state When a control rod sequence change is encountered between data sets, the exposure may be advanced to the sequence exchange date, and the data set after the exchange used to advance the exposure to the date of the data set ımmediately following the exchange date. Experience has shown that takıng exposure steps finer than $700 \mathrm{MWd} / \mathrm{t}$ does not significantly add to the trackıng accuracy (see Table 16).

All of these data were taken during steady-state operation. The reactor had been operatıng for at least 48 hours with essentially constant power, flow, and rod pattern before the data were accumulated.

Core thermal power, inlet subcoolıng, and recirculatıon flow rate are important to the reactor data evaluatıon. The values for these items were taken directly from process computer PI output. The PI output does not contain the detailed data used to calculate the output values and the detaıled data are normally not available from the plant data (I.e., special edits must be requested or special readings taken). Therefore, the detailed data cannot be provided. However, the method used by the process computer to compute the values is given here.

\section{a. Core Thermal Power}

The core thermal power is obtained from the process computer which performs an energy balance on a system composed of the reactor vessel, recirculation loop pıpıng, and cleanup demıneralızer pıpıng. Flows enterıng the system are the reactor feedwater flow, which is assumed to enter in three branches, and the control rod drive system flow. The only flow assumed to be leaving the system is the primary steam flow. Nonflow power inputs are the fission power (core thermal power) and recirculation pumpıng power; nonflow power losses are the radiative power loss and the net power transferred across the boundary of the cleanup demıneralızer loop. Analytıcally, the energy balance is:

Core Power, MWt $=\frac{W_{f w}\left(h_{s}-h_{f w}\right)+W_{c r}\left(h_{s}-h_{c r}\right)}{C_{1}}+Q_{c u}+Q_{r}-Q_{o}$

where: $\quad W_{f w}=$ feedwater flow rate entering reactor at top of downcomer, Mlb/hr

$\mathrm{h}_{\mathrm{s}} \quad=$ enthalpy of steam leaving the reactor vessel, Btu/lb

$h_{f w}=$ feedwater enthalpy, Btu/lb

$\mathrm{W}_{\mathrm{cr}}=$ control rod drive system flow, Mlb/hr

$\mathrm{h}_{c r}=$ enthalpy of control rod drive system flow, Btu/lb

$Q_{p} \quad=$ power added to downcomer fluid by recırculation pumps, $\mathrm{MW}$

$\mathrm{Q}_{r} \quad=$ radiative power loss, $\mathrm{MW}$

$\mathrm{Q}_{c u}=$ power removed from downcomer fluid by cleanup demineralızer system, $\mathrm{MW}$

$\mathrm{C}_{1}=$ conversion constant $=3.413 \mathrm{MBtu} / \mathrm{MWh}$

\section{b. Core Inlet Subcooling}

The core inlet subcooling is obtained from the process computer by performıng an energy balance on the core downcomer (the volume between the core shroud and the vessel wall, and includıng the external recirculatıon and cleanup loops) yielding:

$$
W_{T} h_{o}=W_{r l} h_{f}+W_{r s} h_{g}+W_{f w} h_{f w}+W_{c r} h_{c r}+\left(Q_{p}-Q_{c u}\right) C_{l}
$$


where:

$$
\begin{aligned}
& \mathrm{W}_{T}=\text { flow rate enterıng core inlet plenum, } \mathrm{Mlb} / \mathrm{hr} \\
& h_{0} \quad=\text { core Inlet enthalpy (enthalpy of } W_{T} \text { ), BTu/lb } \\
& \mathrm{W}_{r l}=\text { flow rate of saturated lıquid entering downcomer, } \mathrm{Mlb} / \mathrm{hr} \\
& h_{f} \quad=\text { saturated lıquid enthalpy, Btu/lb } \\
& \mathrm{W}_{r \mathrm{~s}}=\text { flow rate of saturated steam enterıng downcomer (1.e., "carryunder'), MIb/hr } \\
& h_{g} \quad=\text { saturated steam enthalpy, Btu/lb }
\end{aligned}
$$

and other terms are defıned as above

The total flow entering the inlet plenum is:

$$
W_{T}=W_{r i}+W_{r s}+W_{f w}+W c r
$$

\section{c. Recirculation Flow}

The reactor core flow rate is monitored by the process computer by direct measurement of differential pressure across the jet pump diffusers. For illustratıve purposes, the 20 jet pumps can be divided into four groups of five each A typical group is shown in Figure 62. In each group, one jet pump contains a diffuser with two statıc pressure taps The remaınıng four units contaın only one pressure tap The "double tapped units" are calibrated by test prior to installation to determıne the relatıonshıp between flow and differentıal pressure over the range of expected operatıng flow rates. This information is used to perform in-reactor calıbration of the "top tap-to-lower plenum" pressure difference of all 20 jet pumps. After this calibration procedure has been completed, the total core flow is measured by electrically analyzing the signals from the single tap-to-lower plenum pressure transducers on all 20 jet pumps. The resulting total core flow rate output signal is displayed on the reactor control board. In addition, the 20 single tap and 4 double tap $\Delta P$ signals described above are avallable in the control room.

\section{OPERATING DATA SUMMARY}

Figures 63 to 100 present operatıng data summaries for each month during Cycles 1 and 2 . The data presented include daıly values of power level, flow, subcoolıng, and rod notch inventory (rod notches inserted) 
Table 1

INITIAL FUEL DESCRIPTION

Type 1

Type 2

Type 3

\section{Fuel Assembly}

Number of Fuel Assemblies per Batch .. 168

263

333

Fuel Rod Array

$7 \times 7$

Fuel Rod Pitch, in

0.738

$7 \times 7$

$7 \times 7$

0.738

0.738

Peripheral-Rod-to-Channel Spacing, in .

0.1435

$1 / 2$ Width of Wide Water Gap, in

0.375

0.188

$1 / 2$ Width of Narrow Water Gap, in ........

160

0.1435

0.1435

0.375

0.375

0.188

Cladding Length, in

0.188

160

Bundle Average Enrichment

(wt \% U-235 in Total U).

1.10

2.50

2.50

Control Augmentation

Type

NONE

Fuel Rods

Containing $\mathrm{Gd}_{2} \mathrm{O}_{3}$

4

144(3), 60(1)

Control Length, in

$3.0 \mathrm{wt} \% \mathrm{Gd}_{2} \mathrm{O}_{3}$

Control Material.

Locations

In Fuel Lattice

Fuel Rods

Containing $\mathrm{Gd}_{2} \mathrm{O}_{3}$

5

144(3), 108(1), 36(1)

3.0 wt $\% \mathrm{Gd}_{2} \mathrm{O}_{3}(3)$

$4.0 \mathrm{wt} \% \mathrm{Gd}_{2} \mathrm{O}_{3}(2)$

In Fuel Lattice

Weight of $U$ per Fuel Assembly

Ib. .

432.3

412.4

412.1

196.1

187.1

186.9

Channel

Outside Dimensions, in

$5.438 \times 5.438$

$5.438 \times 5.438$

$5.438 \times 5.438$

0.080

0.080

0.080

0.38

0.38

$\mathrm{Zr}-4$

$\mathrm{Zr}-4$

Zr-4

2.43

2.53

2.53 
Table 2

RELOAD FUEL DESCRIPTION

\section{Fuel Assembly}

Number of Fuel Assemblies per Batch .....

Fuel Rod Array

Fuel Rod Pitch, in

Peripheral-Rod-to-Channel Spacing, in.

$1 / 2$ Width of Wide Water Gap, in

$1 / 2$ Width of Narrow Water Gap, in........

Cladding Length, in

Bundle Average Enrichment

(wt \% U-235 in total U)

Control Augmentation

Type

Number

Control Length, in

Control Material

Locations

Weight of $U$ per Fuel Assembly

Ib

$\mathrm{kg}$

\section{Channel}

Outside Dimensions, in

Thickness, in

Inside Corner Radius, in

Material.

Water $/ \mathrm{UO}_{2}$ Volume Ratio (cold)
Type 4

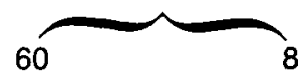

$8 \times 8$

0.640

0.153

0.355

0.167

160

2.74

2.74

2.74

2.60

Fuel Rods Containing $\mathrm{Gd}_{2} \mathrm{O}_{3}$

5

5

5

5

In Fuel Lattice

403.8

403.8

404.2

402.6

183.2

183.2

183.3

182.6

$5.478 \times 5.478$

0.100

$5.518 \times 5.518$

$5.478 \times 5.478$

$5.478 \times 5.478$

0.38

0.120

0.100

0.100

Zr-4

$\mathrm{Zr}-4$

0.38

0.38

$\mathrm{Zr}-4$

$\mathrm{Zr}-4$

2.56

2.51

2.56

2.75 
Table 3

FUEL ASSEMBLY DATA

\section{Initial Load}

Assembly Type.

No. of Assemblies, Initial Core...

No. of Assemblies, Cycle 2...

Geometry

Assembly Pitch, in

Fuel Rod Pitch

Fuel Rods per Assembly

Instrument Rods per Assembly.

Water Rods per Assembly

Burnable Poison Positions.

No. of Spacer Grids

Inconel per Grid, Ib

Zr-4 per Grid, Ib

Spacer Width, in

Assembly Average Fuel Composition

$\mathrm{Gd}_{2} \mathrm{O}_{3}, \mathrm{gm}$.

$\mathrm{UO}_{2}, \mathrm{~kg}$.....

Total Fuel, kg.

1
168
0
$7 \times 7$
6.0
0.738
49
0
0
0
7
0.102
0.537
1.625

\section{2}

263

261

$7 \times 7$

6.0

0.738

49

0

0

4

7

0.102

0.537

1.625

$\begin{array}{lll}0 & 441 & 547\end{array}$

$222.44 \quad 212.21$

222.44

\section{3}

333

315

$7 \times 7$

6.0

0.738

49

0

0

5

7

0.102

0.537

1.625

212.06

212.61
Reload

4
0

$68^{*}$

$8 \times 8$

6.0

0.640

63

0

0

5

7

0.102

0.614

1.625

490

207.78

208.27
Reload

\section{5}

0

116

$8 \times 8$

6.0

0.640

63

0

1

5

7

0.102

0.614

1.625

328

208.00

208.33
LTA Special

\section{6}

0

4

$8 \times 8$

6.0

0.640

62

0

2

5

7

0.102

0.614

1.625

313

207.14

207.45

*60 Assemblies channeled with 0.100 inch thick channels, 8 with 0.120 inch thick channels. 
Table 4

ASSEMBLY TYPE 1 DENSITY, LENGTH, etc., DATA

\begin{tabular}{|c|c|c|c|c|c|c|c|}
\hline \multicolumn{8}{|c|}{ ASSEMBLY TYPE 1} \\
\hline \multirow[b]{2}{*}{$\begin{array}{l}\text { Rod } \\
\text { Type }\end{array}$} & \multirow[b]{2}{*}{$\begin{array}{c}\text { Number of } \\
\text { Rods }\end{array}$} & \multicolumn{2}{|c|}{ Pellet Density } & \multirow{2}{*}{$\begin{array}{c}\text { Stack } \\
\text { Density } \\
(\mathrm{gm} / \mathrm{cc})\end{array}$} & \multirow[b]{2}{*}{$\begin{array}{c}\mathrm{Gd}_{2} \mathrm{O}_{3} \\
(\mathbf{g m})\end{array}$} & \multirow[b]{2}{*}{$\begin{array}{l}\mathrm{UO}_{2} \\
(\mathrm{gm})\end{array}$} & \multirow{2}{*}{$\begin{array}{c}\text { Stack } \\
\text { Lengtr } \\
\text { (in.) }\end{array}$} \\
\hline & & $\begin{array}{c}\mathrm{UO}_{2} \\
(\mathrm{gm} / \mathrm{cc})\end{array}$ & $\begin{array}{c}\mathrm{UO}_{2}+\mathrm{Gd}_{2} \mathrm{O}_{3} \\
(\mathrm{gm} / \mathrm{cc})\end{array}$ & & & & \\
\hline 1 & 31 & 10.42 & - & 10.34 & 0 & 4548 & 144 \\
\hline 2 & 17 & 10.42 & - & 10.34 & 0 & 4548 & 144 \\
\hline $2 S$ & 1 & 10.42 & - & 10.34 & 0 & 4140 & 130 \\
\hline
\end{tabular}

\footnotetext{
Pellet o.d. $=0.487$ inch all rods

Cladding $=$ Zircaloy-2, 0.563 -inch $0 . d . \times 0.032$-inch wall, all rods Gas Plenum Length $=16.0$ inches
}

Table 5

ASSEMBLY TYPE 2 DENSITY, LENGTH, etc., DATA

\begin{tabular}{|c|c|c|c|c|c|c|c|}
\hline & & & ASSEMBLY T & & & & \\
\hline & & & Density & Stack & & & Stack \\
\hline $\begin{array}{l}\text { Rod } \\
\text { Type }\end{array}$ & $\begin{array}{c}\text { Number of } \\
\text { Rods }\end{array}$ & $\begin{array}{c}\mathrm{UO}_{2} \\
(\mathrm{gm} / \mathrm{cc})\end{array}$ & $\begin{array}{c}\mathrm{UO}_{2}+\mathrm{Gd}_{2} \mathrm{O}_{3} \\
(\mathrm{gm} / \mathrm{cc})\end{array}$ & $\begin{array}{l}\text { Density } \\
\text { (gm/cc) }\end{array}$ & $\begin{array}{c}\mathrm{Gd}_{2} \mathrm{O}_{3} \\
(\mathrm{gm})\end{array}$ & $\begin{array}{c}\mathrm{UO}_{2} \\
(\mathrm{gm})\end{array}$ & $\begin{array}{l}\text { Length } \\
\text { (in.) }\end{array}$ \\
\hline 1 & 25 & 10.42 & - & 10.32 & 0 & 4352 & 144 \\
\hline 1s & 1 & 10.42 & - & 10.32 & 0 & 3935 & 130 \\
\hline 2 & 12 & 10.42 & - & 10.32 & 0 & 4352 & 144 \\
\hline 3 & 6 & 10.42 & - & 10.32 & 0 & 4352 & 144 \\
\hline 4 & 1 & 10.42 & - & 10.32 & 0 & 4352 & 144 \\
\hline $5 \mathrm{~A}$ & 3 & - & 10.29 & 10.19 & 129 & 4171 & 144 \\
\hline $6 \mathrm{~B}^{*}$ & 1 & 10.42 & 10.29 & 10.27 & 54 & 4277 & 144 \\
\hline
\end{tabular}

\footnotetext{
Pellet o.d. $=0.477$ inch all rods

Cladding $=$ Zircaloy-2, 0.563-inch 0. d. $\times 0.037$-inch wall, all rods Gas Plenum Length $=15.8$ inches
}

${ }^{\star}$ Contains two pellet types, see Figures 2 and 3 
Table 6

ASSEMBLY TYPE 3 DENSITY, LENGTH, etc., DATA

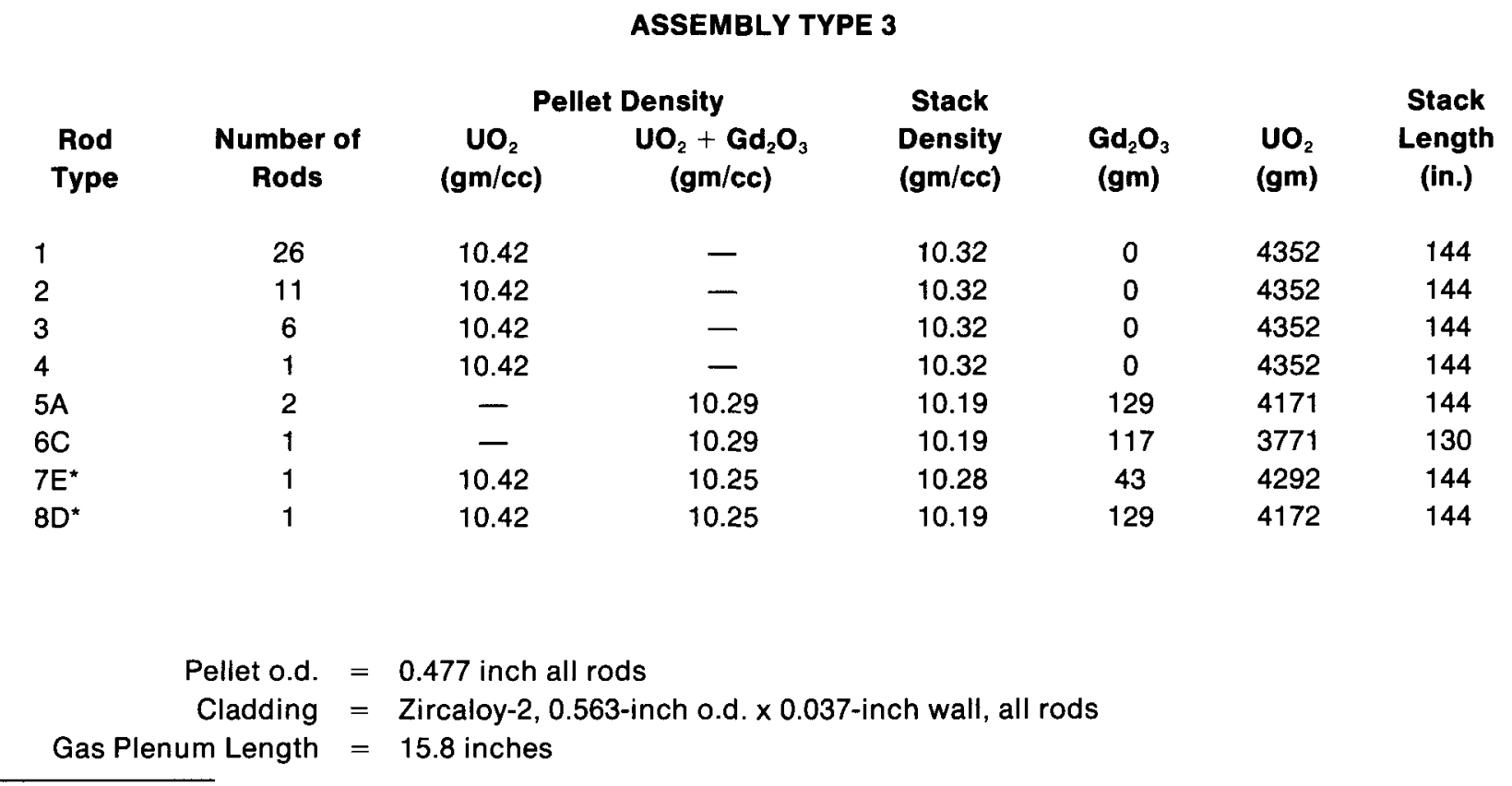

Table 7

ASSEMBLY TYPE 4 DENSITY, LENGTH, etc., DATA

\begin{tabular}{|c|c|c|c|c|c|c|c|}
\hline \multirow[b]{3}{*}{$\begin{array}{l}\text { Rod } \\
\text { Type }\end{array}$} & \multicolumn{6}{|c|}{ ASSEMBLY TYPE 4} & \multirow{3}{*}{$\begin{array}{c}\text { Stack } \\
\text { Length } \\
\text { (in.) }\end{array}$} \\
\hline & \multicolumn{3}{|c|}{ Pellet Density } & \multirow{2}{*}{$\begin{array}{c}\text { Stack } \\
\text { Density } \\
(\mathrm{gm} / \mathrm{cc})\end{array}$} & \multirow[b]{2}{*}{$\begin{array}{c}\mathrm{Gd}_{2} \mathrm{O}_{3} \\
(\mathrm{gm})\end{array}$} & \multirow[b]{2}{*}{$\begin{array}{c}\mathrm{UO}_{2} \\
\text { (gm) }\end{array}$} & \\
\hline & $\begin{array}{c}\text { Number of } \\
\text { Rods }\end{array}$ & $\begin{array}{c}\mathrm{UO}_{2} \\
(\mathrm{gm} / \mathrm{cc})\end{array}$ & $\begin{array}{c}\mathrm{UO}_{2}+\mathrm{Gd}_{2} \mathrm{O}_{3} \\
(\mathrm{gm} / \mathrm{cc})\end{array}$ & & & & \\
\hline 1 & 39 & 10.42 & - & 10.32 & 0 & 3309 & 144 \\
\hline 2 & 14 & 10.42 & - & 10.32 & 0 & 3309 & 144 \\
\hline 3 & 4 & 10.42 & - & 10.32 & 0 & 3309 & 144 \\
\hline 4 & 1 & 10.42 & - & 10.32 & 0 & 3309 & 144 \\
\hline 5 & 5 & - & 10.29 & 10.19 & 98 & 3172 & 144 \\
\hline ws & 1 & - & - & - & 0 & 0 & - \\
\hline
\end{tabular}

\footnotetext{
Pellet o.d. $=0.416$ inch all rods

Cladding = Zircaloy-2, 0.493-inch o.d. $\times 0.034$-inch wall, all rods

Gas Plenum Length $=16.0$ inches except water rod
}

$\mathrm{Gd}_{2} \mathrm{O}_{3}$ in rod type 5 runs full 144 inches

Water rod has holes drilled top and bottom to provide water flow and little or no boiling Water rod is also spacer positioning rod 


\section{ASSEMBLY TYPE 5}

\begin{tabular}{|c|c|c|c|c|c|c|c|}
\hline \multirow[b]{2}{*}{$\begin{array}{l}\text { Rod } \\
\text { Type }\end{array}$} & \multirow[b]{2}{*}{$\begin{array}{c}\text { Number of } \\
\text { Rods }\end{array}$} & \multicolumn{2}{|c|}{ Pellet Density } & \multirow{2}{*}{$\begin{array}{c}\text { Stack } \\
\text { Density } \\
\text { (gm/cc) }\end{array}$} & \multirow[b]{2}{*}{$\begin{array}{c}\mathrm{Gd}_{2} \mathrm{O}_{3} \\
(\mathrm{gm})\end{array}$} & \multirow[b]{2}{*}{$\begin{array}{l}\mathrm{UO}_{2} \\
(\mathrm{gm})\end{array}$} & \multirow{2}{*}{$\begin{array}{c}\text { Stack } \\
\text { Length } \\
\text { (in.) }\end{array}$} \\
\hline & & $\begin{array}{c}\mathrm{UO}_{2} \\
\text { (gm/cc) }\end{array}$ & $\begin{array}{c}\mathrm{UO}_{2}+\mathrm{Gd}_{2} \mathrm{O}_{3} \\
(\mathrm{gm} / \mathrm{cc})\end{array}$ & & & & \\
\hline 1 & 39 & 1042 & - & 1032 & 0 & 3309 & 144 \\
\hline 2 & 14 & 1042 & - & 1032 & 0 & 3309 & 144 \\
\hline 3 & 4 & 1042 & - & 1032 & 0 & 3309 & 144 \\
\hline 4 & 1 & 1042 & - & 1032 & 0 & 3309 & 144 \\
\hline 5 & 5 & - & 1033 & 1023 & 66 & 3216 & 144 \\
\hline WS & 1 & - & - & - & 0 & 0 & - \\
\hline
\end{tabular}

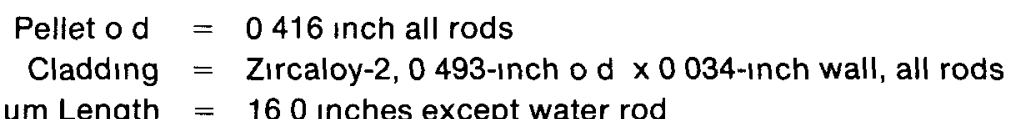

Gas Plenum Length $=160$ inches except water rod

$\mathrm{Gd}_{2} \mathrm{O}_{3}$ In rod type 5 runs full 144 inches

Water rod has holes drilled top and bottom to provide water flow and little or no boiling Water rod is also spacer positioning rod

Table 9

ASSEMBLY TYPE 6 DENSITY, LENGTH, etc., DATA

\begin{tabular}{|c|c|c|c|c|c|c|c|}
\hline \multirow[b]{3}{*}{$\begin{array}{l}\text { Rod } \\
\text { Type }\end{array}$} & \multicolumn{6}{|c|}{ ASSEMBLY TYPE 6* } & \multirow{3}{*}{$\begin{array}{c}\text { Stack } \\
\text { Length } \\
\text { (in.) }\end{array}$} \\
\hline & \multirow[b]{2}{*}{$\begin{array}{l}\text { Number of } \\
\text { Rods }\end{array}$} & \multicolumn{2}{|c|}{ Pellet Density } & \multirow{2}{*}{$\begin{array}{c}\text { Stack } \\
\text { Density } \\
\text { (gm/cc) }\end{array}$} & \multirow[b]{2}{*}{$\begin{array}{l}\mathrm{Gd}_{2} \mathrm{O}_{3} \\
(\mathbf{g m})\end{array}$} & \multirow[b]{2}{*}{$\begin{array}{l}\mathrm{UO}_{2} \\
(\mathrm{gm})\end{array}$} & \\
\hline & & $\begin{array}{c}\mathrm{UO}_{2} \\
(\mathrm{gm} / \mathrm{cc})\end{array}$ & $\begin{array}{c}\mathrm{UO}_{2}+\mathrm{Gd}_{2} \mathrm{O}_{3} \\
(\mathrm{gm} / \mathrm{cc})\end{array}$ & & & & \\
\hline 1 & 38 & 1042 & - & 1032 & 0 & 3125 & 140 \\
\hline 2 & 14 & 1042 & - & 1032 & 0 & 3125 & 140 \\
\hline 3 & 4 & 1042 & - & 1032 & 0 & 3125 & 140 \\
\hline 4 & 1 & 1042 & - & 1032 & 0 & 3125 & 140 \\
\hline 5 & 5 & - & 1033 & 1023 & 63 & 3037 & 140 \\
\hline WR, WS & 2 & - & - & - & 0 & 0 & - \\
\hline ENDS & 62 & 1042 & - & 1032 & 0 & 223 & 10 \\
\hline
\end{tabular}

Pellet o d $=0410$ inch all rods

Cladding $=$ Zircaloy-2, $0483-$ inch od $\times 0.032-$ inch wall, all fueled rods Zırcaloy-2, 0 591-ınch o d $\times 0$ 030-ınch wall, water rods

Gas Plenum Length $=948$ inches

$\mathrm{Gd}_{2} \mathrm{O}_{3}$ in rod type 5 runs full 140 inches

Water rods have holes drilled top and bottom to provide water flow and little or no boilıng WS rod is also spacer positionıng rod

*Each fuel rod contains three axial zones see Figures 8 and 9 
Table 10

FUEL ASSEMBLY HARDWARE WEIGHTS

$7 \times 7$ Initial

Assemblies

Spacers

Zircaloy-4

Inconel

End Plugs

Zircaloy-2

\section{Lower Tie Plate}

Type-304 Stainless Steel Inconel Finger Springs

Upper Tie Plate Assembly with Hardware

Type-304 Stainless Steel

1

4.222

9.612

0.106

3.757

0.717

3.565

128

$8 \times 8$

Reload Assemblies

LTA

Reload Assemblies

Quantity

Pounds

Quantity

Pounds

\section{Fission Gas Plenum}

Spring, Type-302 Stainless Steel

$49 \quad 4.073$

49

0.972

4.299

0.717

112

7

4.299

112

0.717

Getter, Zirconium Alloy
Wt \% Alloy Compositions for Nuclear Analyses
Metal

$\mathrm{Zr}$

$\mathrm{Fe}$

Sn

$\mathrm{Ni}$

$\mathrm{Cr}$

Ti.

$\mathrm{Mn}$

C...

Si.

S................

P................

Al
Zircaloy-4

98.24

0.21

1.45

0.10
Type-304

10.545

$4 \quad 0.106$

1

10.545

Inconel-X

4.151

62

1.360

62

1.338
Stainless Steel

\section{Placement}

There are seven spacers in the initial and reload fuel assemblies. Their center positions above the bottom of the active fuel in inches are 18.9, 39.0, 59.2, 79.3, 99.5, 119.6, and 139.7. Each spacer is 1.625 inches long. 


\section{Movable Control Rods}

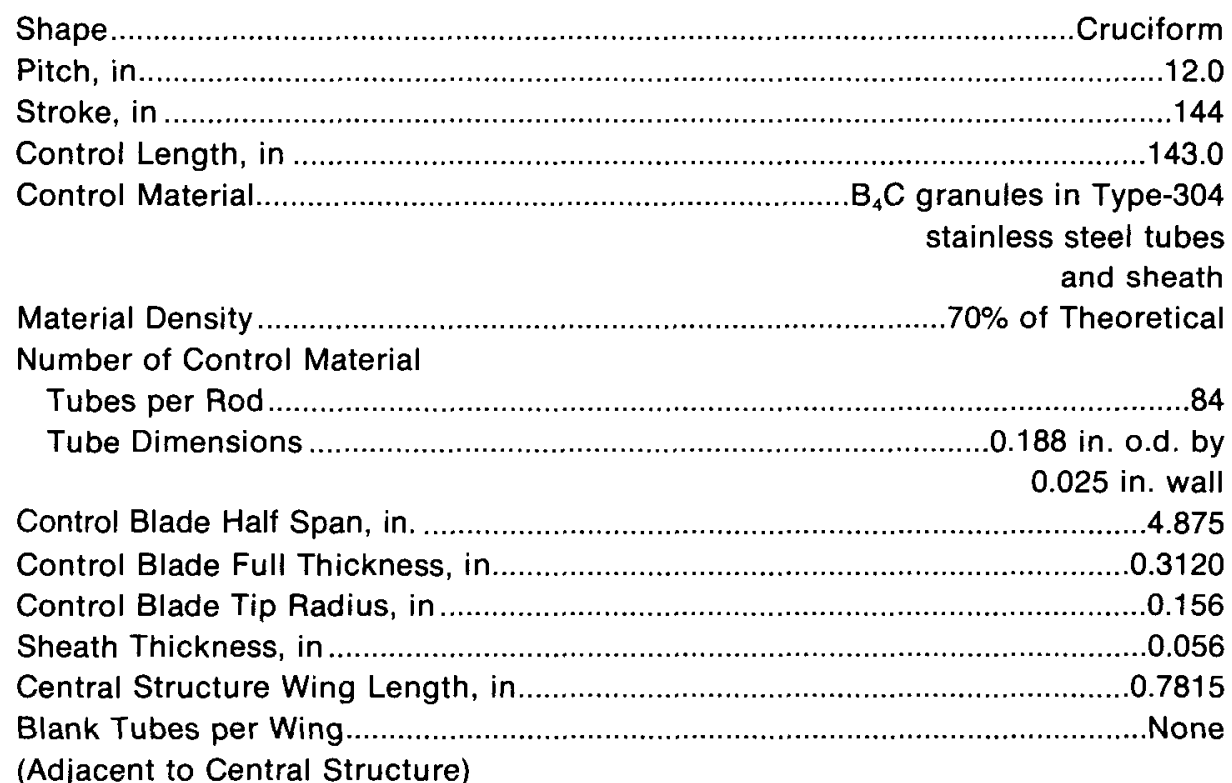

\section{Control Blade Position}

Control blade insertion is calibrated in notches, where one notch equals 3 inches. Position of the control blade is described by the number of notches withdrawn. Thus, 0 notch implies full insertion and 48 notches implies full withdrawal. Total travel of the control blade is 144 inches, the same as the length of the active fuel. At full blade insertion ( 0 notch), the top of the control material is 1 inch below the top of the active fuel. At full blade withdrawal, the top of the control material is 1 inch below the bottom of the active fuel. Since the physical notches in the control rod drive are 6 inches apart, the control blade notch position is always even. For the control patterns shown, the numbers shown indicate notches withdrawn and no notch number implies a fully withdrawn blade or a notch position of 48 for power operating patterns and a fully inserted blade or a notch position of 00 for cold critical patterns. 
Table 12

CORE DESCRIPTION

Cycle 1

Total Number of Fuel Assemblies

Number of Fuel Assembly Types

Number of Fuel Assemblies of Each Type

Total Number of Control Elements

Number of Control Element Types.

Number of Control Elements of Each Type.....

Total Number of In-core Flux Monitors.

Heat Transfer Surface Area, $\mathrm{ft}^{2}$

Average Linear Heat Rate, kw/ft.

Total Weight of $\mathrm{U}$ in Core, short tons.

Core

Core Lattice Pitch, in

Water/ $/ \mathrm{OO}_{2}$ Volume Ratio (cold)
764

3

See Table 3

185

1

185

43

66,214

7.037

159.2

12.0

2.51
Cycle 2

$$
764
$$

6

See Table 3

185

1

185

43

68,266

6.574

156.7

12.0

2.54

Table 13

CYCLE 1 BUNDLE TYPES AND IDENTIFICATION

PH 001 to $\mathrm{PH} 168$

$\mathrm{PH} 169$ to $\mathrm{PH} 431$

PH 432 to $\mathrm{PH} 764$

$\begin{array}{lll}7 \times 7 & \mathrm{UO}_{2} & 1.10 w t \% \\ 7 \times 7 & \mathrm{UO}_{2} & 2.50 w t \% \\ 7 \times 7 & \mathrm{UO}_{2} & 2.50 w t \%\end{array}$

Type 1 Fuel Without $\mathrm{Gd}_{2} \mathrm{O}_{3}$ Type 2 With $\mathrm{Gd}_{2} \mathrm{O}_{3}$ in 4 Rods Type 3 With $\mathrm{Gd}_{2} \mathrm{O}_{3}$ in 5 Rods 
CYCLE 1 BUNDLE TYPES AND IDENTIFICATION (Continued)

BUNDLE

IDENTIFICATION

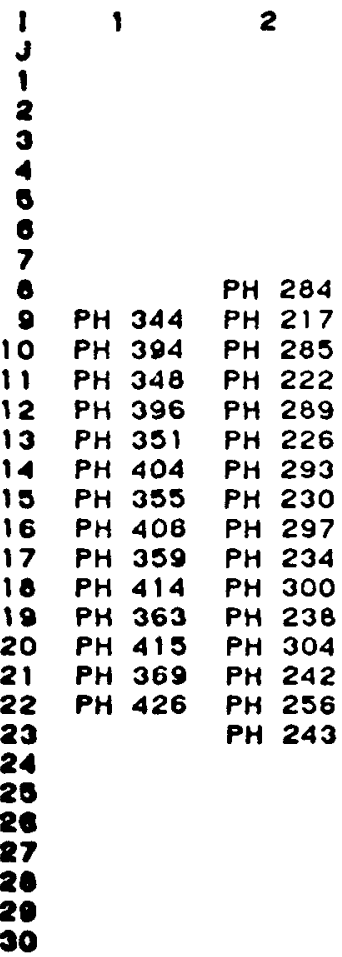

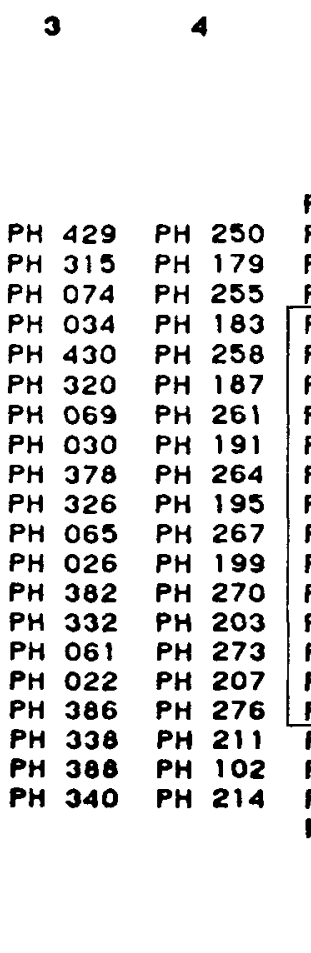

$\mathbf{5}$

6

7

PH 309 PH

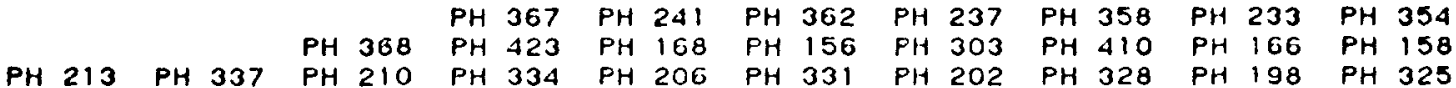

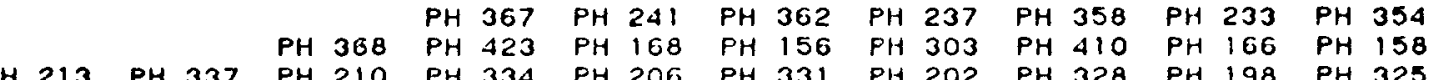

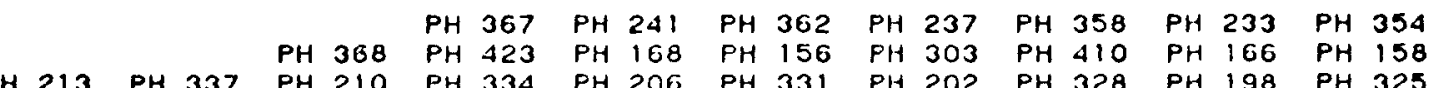

11

12

13

14

15

$\begin{array}{llllllllllllll} & & \end{array}$

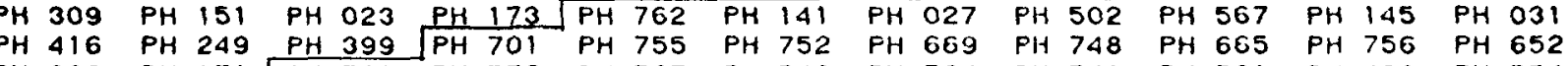

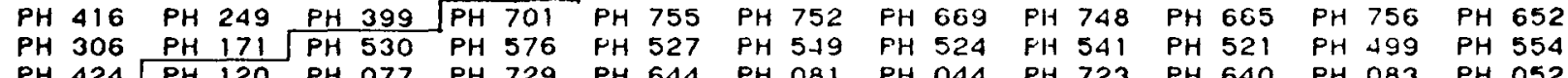

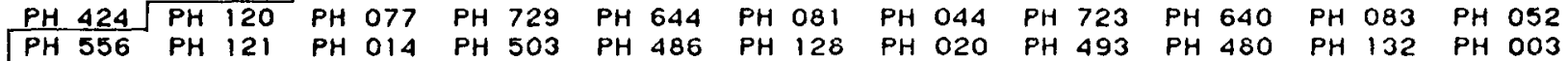

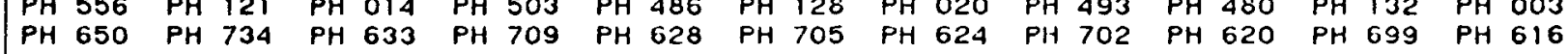

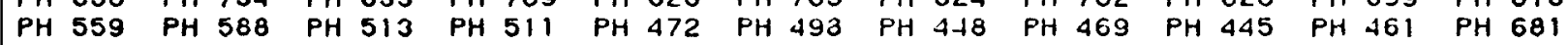

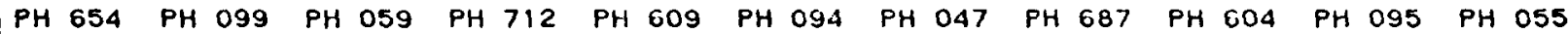

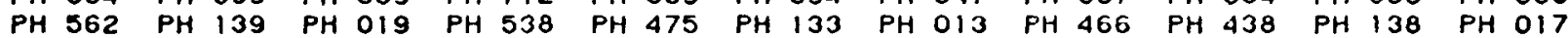

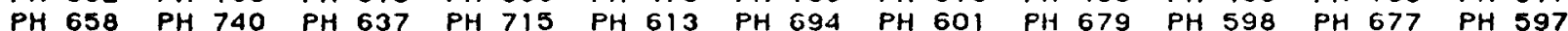

$\mathrm{PH} 565 \mathrm{PH} 577 \quad \mathrm{PH} 519 \mathrm{PH} 546 \quad \mathrm{PH} 478 \quad \mathrm{PH} 506 \quad \mathrm{PH} 443 \quad \mathrm{PH} 455$ PH $433 \quad \mathrm{PH} 454 \quad \mathrm{PH} 432$

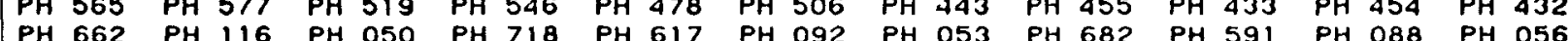

$\mathrm{PH} 568 \mathrm{PH} 144 \mathrm{PH} 006$ PH $535 \mathrm{PH} 481 \mathrm{PH} 129$ PH 009 PH 463 PH 434 PH $122 \quad \mathrm{PH} 033$

$\mathrm{PH} 666 \mathrm{PH} 746$ PH $641 \mathrm{PH} 721 \mathrm{PH} 621$ PH 700 PH 605 PH 685 PH 592 PH 672 PH 593

PH 571 PH 585 PH 525 PH 543 PH 484 PH 495 PH 449 PH 471 PH 440 PH 458 PH 444

$\mathrm{PH} 670$ PH 114 PH 042 PH 724 PH 625 $\mathrm{PH} 090$ PH 045 PH 688 PH 602 PH 098 PH 051

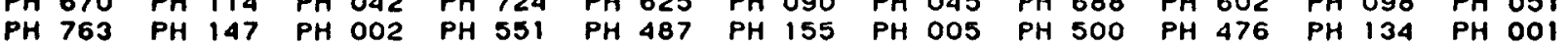

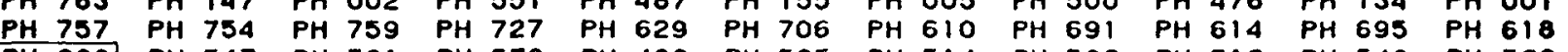

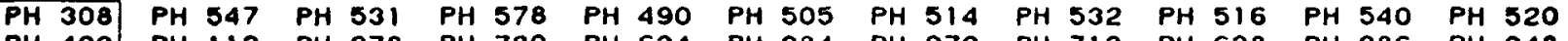

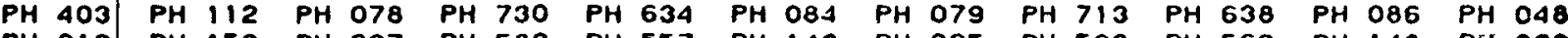

$\begin{array}{llllllllllllll} & & & \end{array}$

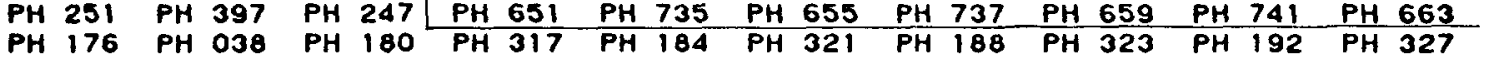

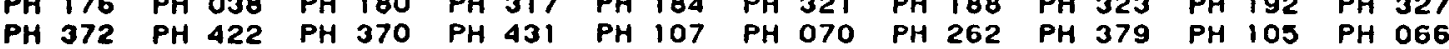

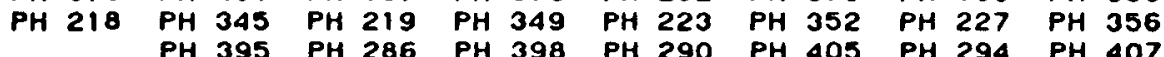

TYPE 1 AND 2 IN OUTER REGION

TYPE 1 AND 3 IN INNER REGION

SEE FIGURE 22 FOR TYPE 1 LOCATIONS 
Table 13

CYCLE 1 BUNDLE TYPES AND IDENTIFICATION (Continued)

BUNDLE

IDENTIFICATION

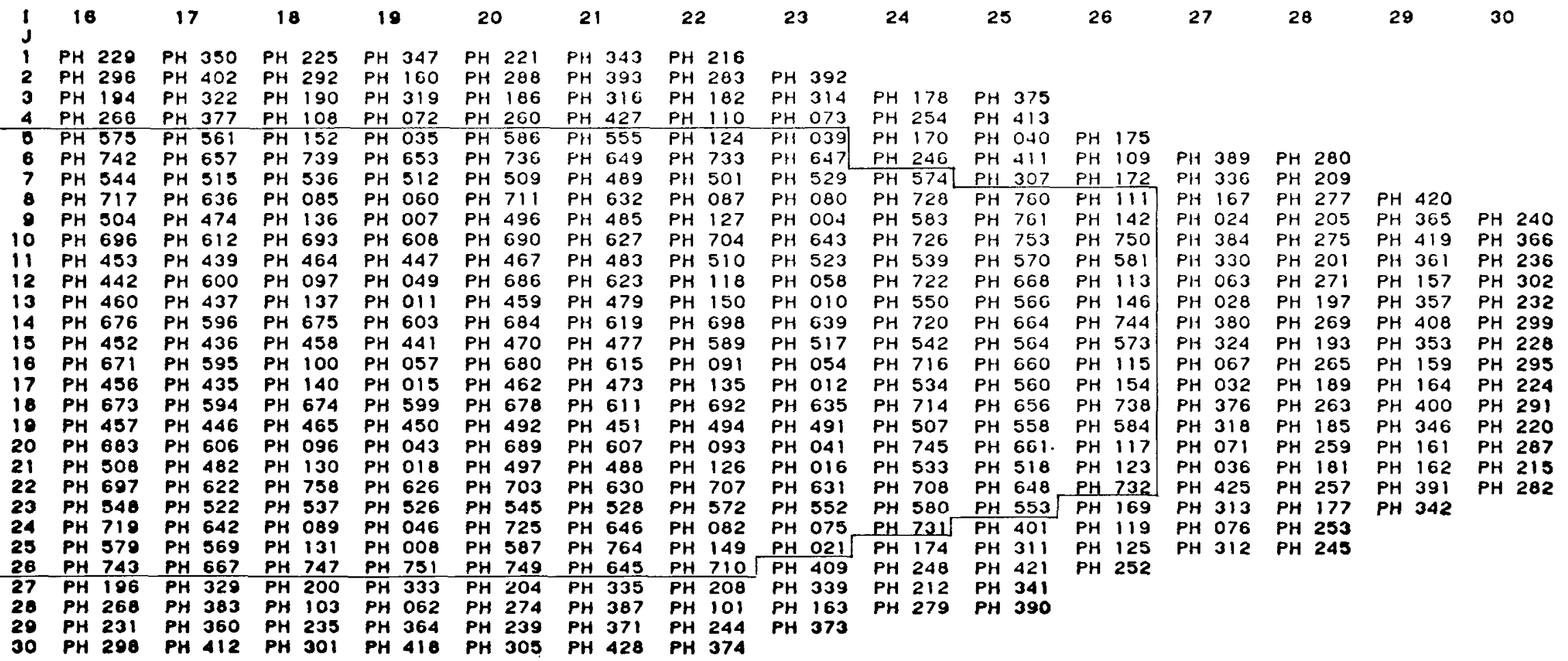

TYPE 1 AND 2 IN OUTER REGION

TYPE 1 AND 3 IN INNER REGION

SEE FIGURE 22 FOR TYPE 1 LOCATIONS 


\section{Table 14 \\ CYCLE 2 BUNDLE TYPES AND IDENTIFICATION}

PH 169 to $\mathrm{PH} 431$

$\mathrm{PH} 432$ to $\mathrm{PH} 764$

LJ3213 to LJ3280

LJ3098 to LJ3212, LJ3454

LJLTA1 to LJLTA4 $\begin{array}{lll}7 \times 7 & \mathrm{UO}_{2} & 2.50 \mathrm{wt} \%\end{array}$

$7 \times 7 \quad \mathrm{UO}_{2} \quad 2.50 \mathrm{wt} \%$

$8 \times 8 \quad \mathrm{UO}_{2} \quad 2.74 \mathrm{wt} \%$

$\begin{array}{lll}8 \times 8 & \mathrm{UO}_{2} & 2.74 w \mathrm{wt} \%\end{array}$

$\begin{cases}8 \times 8 & \mathrm{UO}_{2} \\ 8 \times 8 & \mathrm{UO}_{2}\end{cases}$
2.73 wt $\%$

0.71 wt $\%$
Type 2 With $\mathrm{Gd}_{2} \mathrm{O}_{3}$ in 4 Rods

Type 3 With $\mathrm{Gd}_{2} \mathrm{O}_{3}$ in 5 Rods

Type 4 With $3 \% \mathrm{Gd}_{2} \mathrm{O}_{3}$ in 5 Rods

Type 5 With $2 \% \mathrm{Gd}_{2} \mathrm{O}_{3}$ in 5 Rods

Type 6 With $2 \% \mathrm{Gd}_{2} \mathrm{O}_{3}$ in 5 Rods

in center, and $\mathrm{No} \mathrm{Gd}_{2} \mathrm{O}_{3}$ in ends

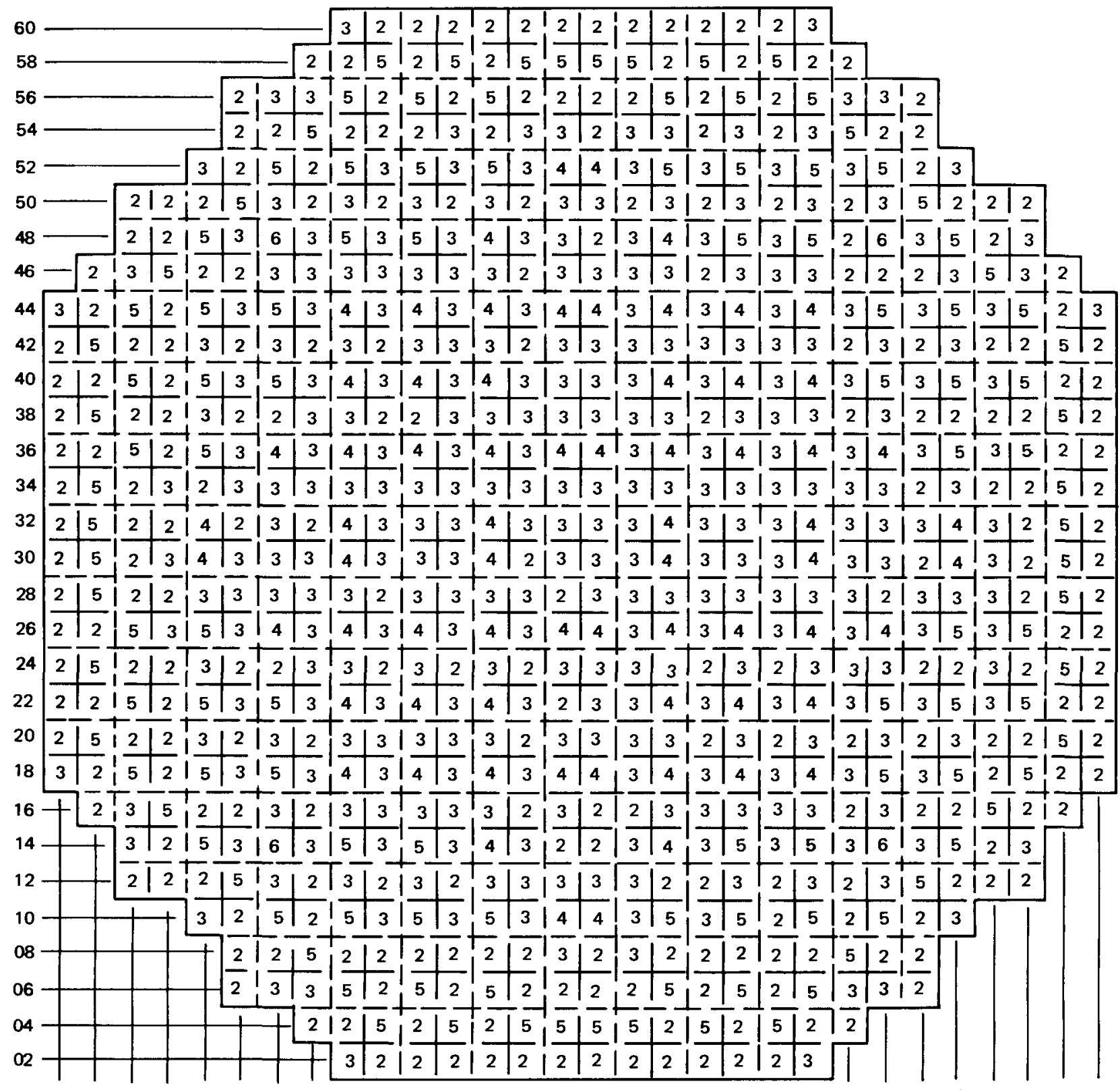

$\begin{array}{llllllllllllllllllllllllllllll}01 & 03 & 05 & 07 & 09 & 11 & 13 & 15 & 17 & 19 & 21 & 23 & 25 & 27 & 29 & 31 & 33 & 35 & 37 & 39 & 41 & 43 & 45 & 47 & 49 & 51 & 53 & 55 & 57 & 59\end{array}$ 
Table 14

CYCLE 2 BUNDLE TYPES AND IDENTIFICATION (Continued)

\section{BUNOLE IDENTIFICATION}

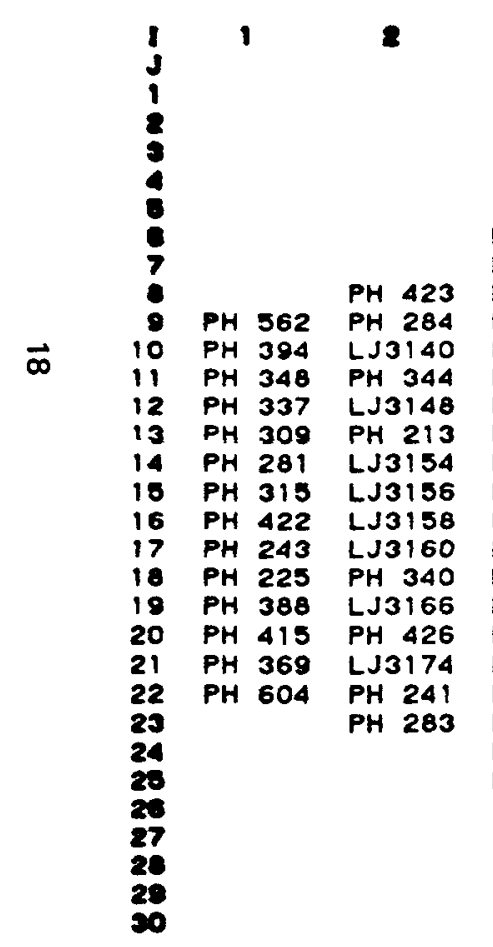

\section{BUNOLE IOENTIFICATION}

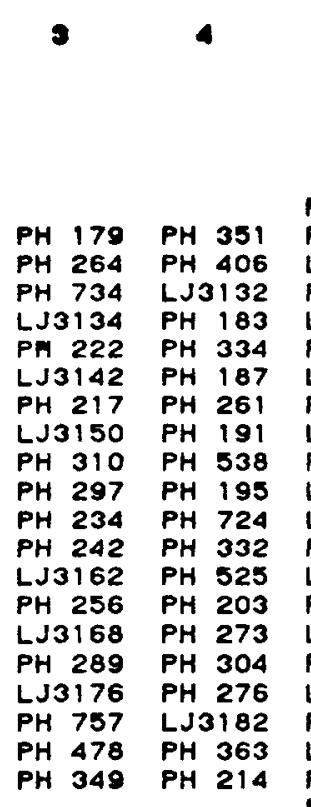

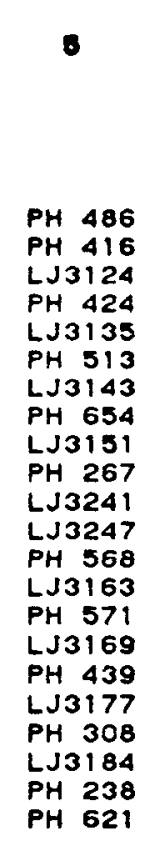

PH 404

PH 752

PH 202 LJ3114 PH 173 LJ3115

PH 226 PH 3136 PH 503 LJ3217

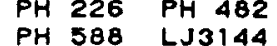

PH 293 PH 378 PH 712 PH 609

PH 662 L 3231 PH 457 PH 13232

$\mathrm{PH} 687 \mathrm{PH} 565$

PH 207 PH 519

PH 715 PH 613

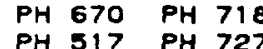

PH 199 LJ 585242

PH 746

LJ3253

PH 535 PH 481

PH $300 \quad \mathrm{PH} 382$ PH 543 PH 484

PH 389 LJ3170

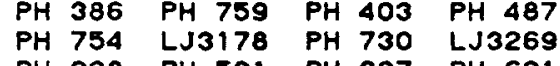

PH 641 LJLTA3

LJ3192

PH 706

$\mathrm{PH} 176 \mathrm{PH} 405$

$\begin{array}{ll}\mathrm{PH} & 514 \\ \mathrm{PH} & 370\end{array}$

PH 370 PH 55

LJ3202 $\mathrm{PH} 317$

$\begin{array}{lll}\mathrm{PH} & 667 & \mathrm{LH} \\ \mathrm{PH} & 414 & \mathrm{PH} 342 \\ \end{array}$

PH 371

PH 651

10

11

12

13

14

18

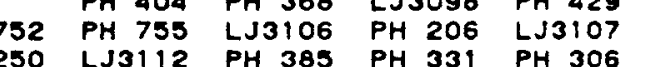

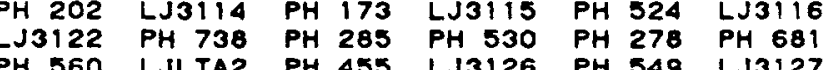

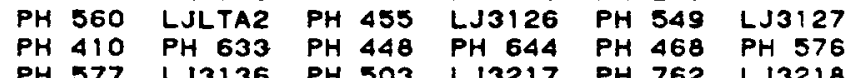

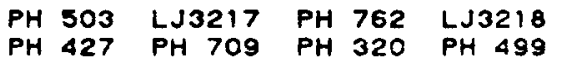

PH 511 LJ3225 PH 498 LJ3226

PH 457 L 3232 PH 740 L 2538

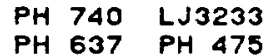

PH 506 PH 443

$\mathrm{PH} 682$ PH 469

PH 326 PH 559

PH 617 LJ3255

PH $700 \mathrm{PH} 180$

PH 625 LJ3264 $\mathrm{PH} 602$

PH 432 PH 582 PH 500

\begin{tabular}{l} 
PH 666 LJ3270 PH 578 LJ3271 \\
\hline PH
\end{tabular}

$\mathrm{PH} 713$ L 33187 PH 532 PJ3277

PH 75 LH

PH 352 PH 444 PH 431 PH 563

$\mathrm{PH} 563$
$\mathrm{LJ} 3197$

$\mathrm{PH} 737$

PH 188 PH 323

LJ3211 PH 372

$\begin{array}{llllll}\text { PH } 342 & \text { LJ3210 } & \text { PH } 395 & \text { LJ } 3211 & \text { PH } 372 \\ \text { PH } 286 & \text { PH } 398 & \text { PH } 232 & \text { PH } 417\end{array}$

PH 251 PH 227

LJ3212 LJ3125

PH 290 
Table 14

CYCLE 2 BUNDLE TYPES AND IDENTIFICATION (Continued)

DUNDLE IDENTIFICATION

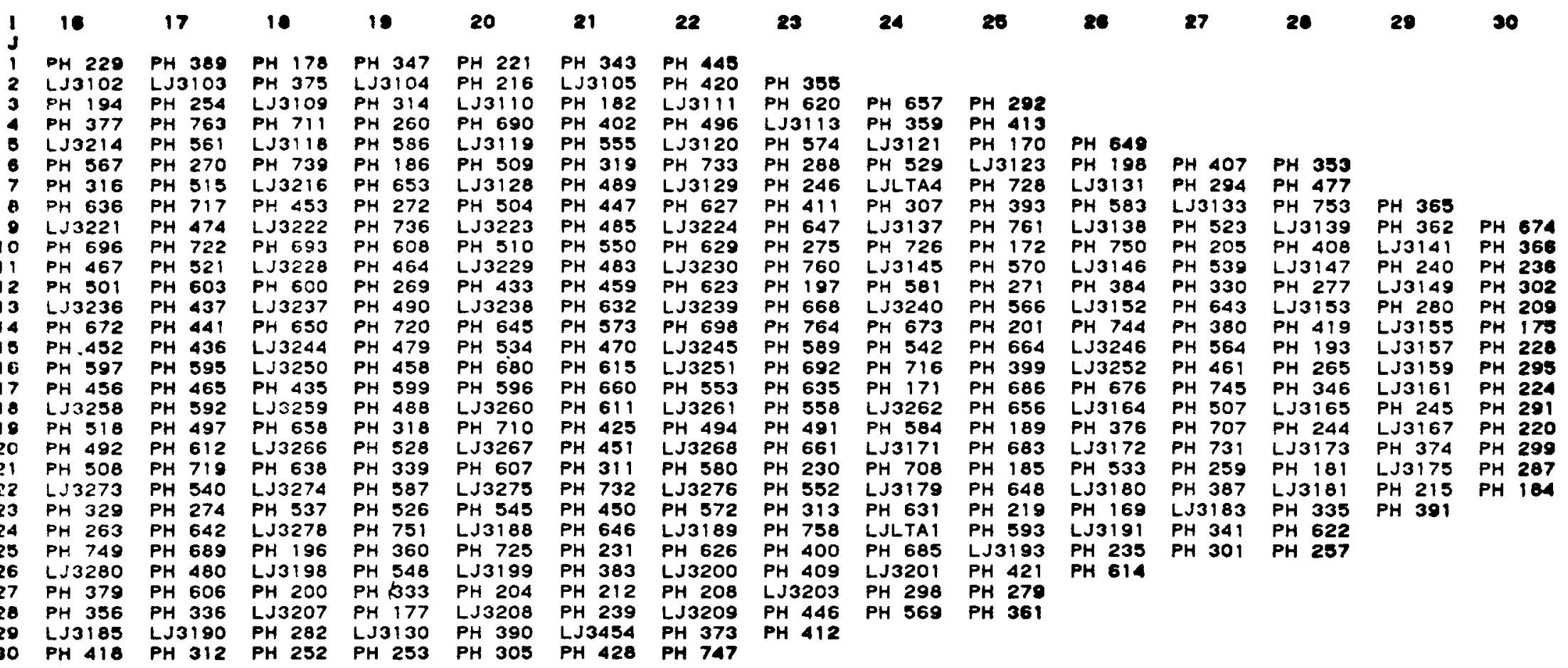


Rated Core Thermal Power, MW

Rated Core Total Flow Rate, Mlb/hr

Bypass Flow Rate, Fraction of Total Core Flow

Fraction of Core Thermal Power Passing Through Fuel Cladding

Approximate Bypass Coolant Total Power Fraction

Approximate Active Coolant Total Power Fraction

Approximate Channel Wall Direct Heating Fraction

Design Minimum Critical Power Ratio for 7x7 Assemblies (Cycle 2)

Design Minimum Critical Power Ratio for 8x8 Assemblies (Cycle 2)

Design Overpower for Turbine-Generator System

Turbine Inlet Pressure, psia

Rated Reactor Dome Pressure, psia

Rated Steam Flow Rate, Mlb/hr

Steam Moisture Content, Fraction

Rated Steam Dryer and Separator Pressure Drop, psi

Rated Core Pressure, psia

Core Pressure Drop at Rated Conditions, psi

Approximate Core Inlet Pressure, psia

Core Inlet Enthalpy, Btu/lb

Enthalpy Rise Across Core, Btu/lb (Average)

Core Support Plate Pressure Drop, psi

Core Orifice and Lower Tie Plate Pressure Drop

Fuel Bundle Pressure Drop

Reactor Average Exit Quality At Rated Conditions

Design Hot Channel Active Coolant Exit Quality

Design Bypass Coolant Exit Quality

Total Feedwater Flow Rate, MIb/hr

Feedwater Temperature, ${ }^{\circ} \mathrm{F}$

Control Rod Drive Flow Rate, Ib/hr

Control Rod Drive Flow Temperature, ${ }^{\circ} \mathrm{F}$

Cleanup Demineralizer Flow Rate, Ib/hr

Cleanup Demineralizer Inlet Temperature, ${ }^{\circ} \mathrm{F}$

Cleanup Demineralizer Outlet Temperature, ${ }^{\circ} \mathrm{F}$

Location of Demineralized Water Return

Jet Pump Design M Ratio

Jet Pump Design N Ratio

Number of Recirculation Loops

Recirculation Pump Type

Recirculation Pump Rated Flow, MIb/Hr

Total Developed Pump Head, $\mathrm{ft}$

Recirculation Pump Efficiency, Percent

Head Loss From Vessel Recirculation Outlet to Vessel Inlet, $\mathrm{ft}$

Head Loss From Vessel Recirculation Inlet to Jet Pump $180^{\circ}$ Bend Entrance, ft
3293

102.5

Figures 54 to 55

0.96

0.02

0.02

0.0075

$\geqslant 1.28$

$\geqslant 1.31$

$105 \%$ Rated Steam Flow

965

1020

13.381

0.001

15

1035

22

1050

521.3

109.6

18

Figures 48 to 53

Figures 44 to 47

0.129

0.25

0.0

13.331

376.1

50000

80

133300

528

431

Feedwater Line

1.96

0.16

2

Centrifugal

17.1

710

87

59

11 
Table 16

BURN STEP INFORMATION

\section{Exposure Interval (MWd/t)}

0 to 230

230 to 390

390 to 439

439 to 648

648 to 741

741 to 1010

1010 to 1251

1251 to 1585

1585 to 2080

2080 to 2555

2555 to 2630

2630 to 2920

2920 to 3120

3120 to 3542

3542 to 3724

3724 to 4364

4364 to 4525

4525 to 4697

4697 to 4880

4880 to 5262

5262 to 5352

5352 to 5640

5640 to 6106

6106 to 6470

6470 to 6530

6530 to 7000

7000 to 7300

7300 to 7712

7712 to 8100

8100 to 8430

8430 to 8766

8766 to 9190

9190 to 9295

9295 to 9520

9520 to 10100

7974 to 8025

8025 to 8042

8042 to 8264

8264 to 8706

8706 to 9035

9035 to 9500

9500 to 9730

9730 to 10050

10050 to 10395

\section{Control Rod \\ Sequence \\ Cycle 1}

\section{Reactor Data From \\ Data Set Number}

$\begin{array}{ll}\text { A } & 1 \\ \text { A } & 2 \\ \text { A } & 2 \\ \text { B } & 2 \\ \text { A } & 3 \\ \text { A } & 4 \\ \text { A } & 5 \\ \text { A } & 5 \\ \text { B } & 6 \\ \text { B } & 6 \\ \text { B } & 7 \\ \text { A } & 8 \\ \text { A } & 8 \\ \text { A } & 9 \\ \text { A } & 9 \\ \text { B } & 9 \\ \text { B } & 10 \\ \text { A } & 10 \\ \text { A } & 11 \\ \text { A } & 11 \\ \text { A } & 12 \\ \text { B } & 12 \\ \text { B } & 13 \\ \text { B } & 13 \\ \text { B } & 13 \\ \text { A } & 14 \\ \text { A } & 15 \\ \text { A } & 16 \\ \text { B } & 16 \\ \text { B } & 16 \\ \text { B } & 17 \\ \text { A } & 18 \\ \text { A } & 19 \\ \text { A } & 20 \\ & 21 \\ & 22 \\ & 22 \\ & 23 \\ & 23 \\ & 24\end{array}$

\section{Cycle 2}

$\begin{array}{ll}\text { A } & 25 \\ \text { A } & 25 \\ \text { B } & 26 \\ \text { B } & 26 \\ \text { A } & 27 \\ \text { A } & 27 \\ \text { A } & 27 \\ \text { B } & 28 \\ \text { B } & 29\end{array}$


Table 16

BURN STEP INFORMATION (Continued)

10395 to 10730

10730 to 11030

11030 to 11092

11092 to 11260

11260 to 11420

11420 to 11570

11570 to 11630

11630 to 11910

11910 to 12070

12070 to 12190

12190 to 12530

12530 to 12800

$\begin{array}{ll}\text { A } & 30 \\ \text { A } & 30 \\ \text { A } & 31 \\ \text { B } & 32 \\ \text { B } & 32 \\ \text { B } & 33 \\ \text { B } & 34 \\ \text { B } & 35 \\ \text { B } & 35 \\ \text { A } & 36 \\ \text { A } & 37 \\ \text { A } & 37\end{array}$

30

30

31

32

32

33

34

35

35

36

37

37 


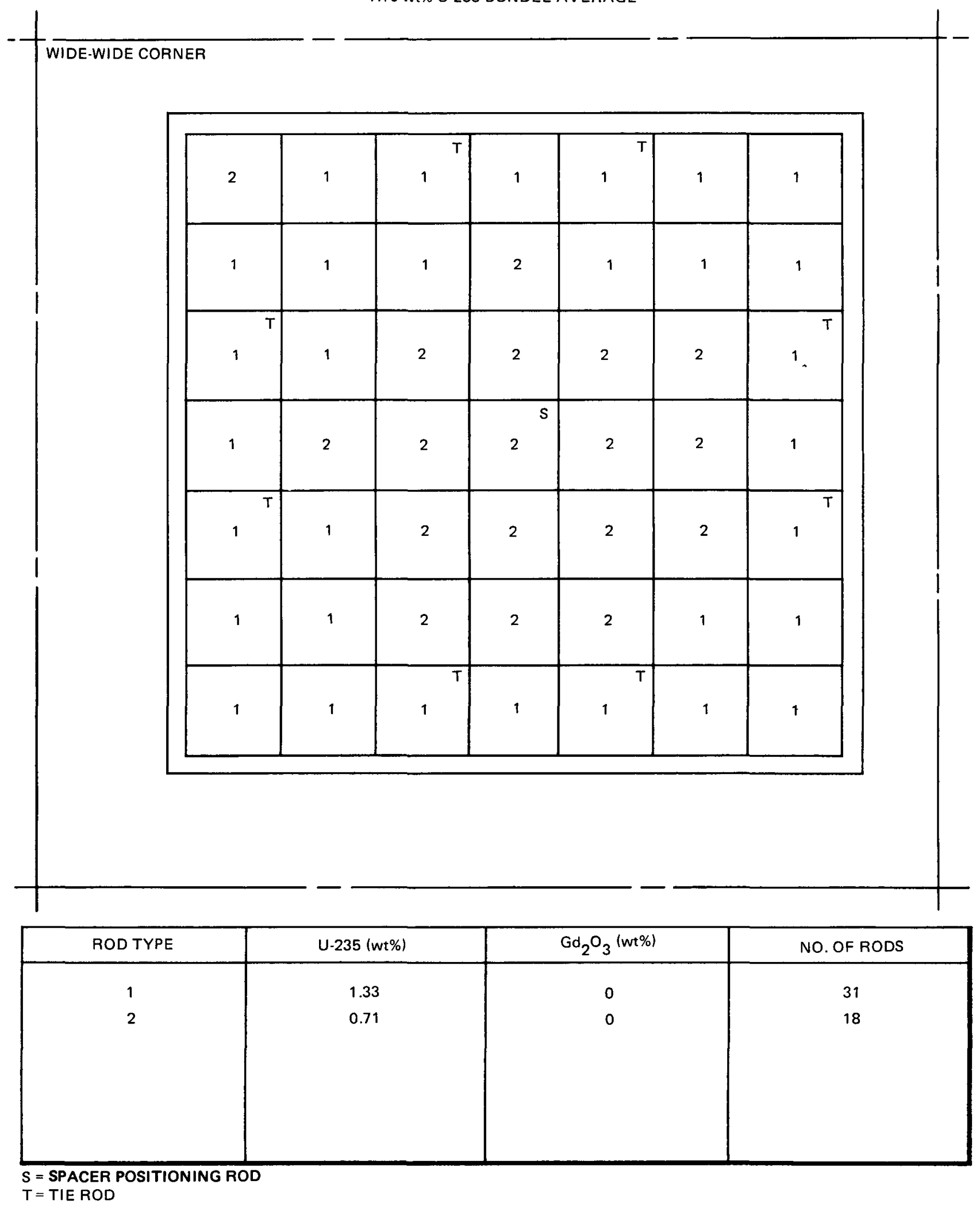

Figure 1. Bundle Design for Type 1 Initial Fuel 


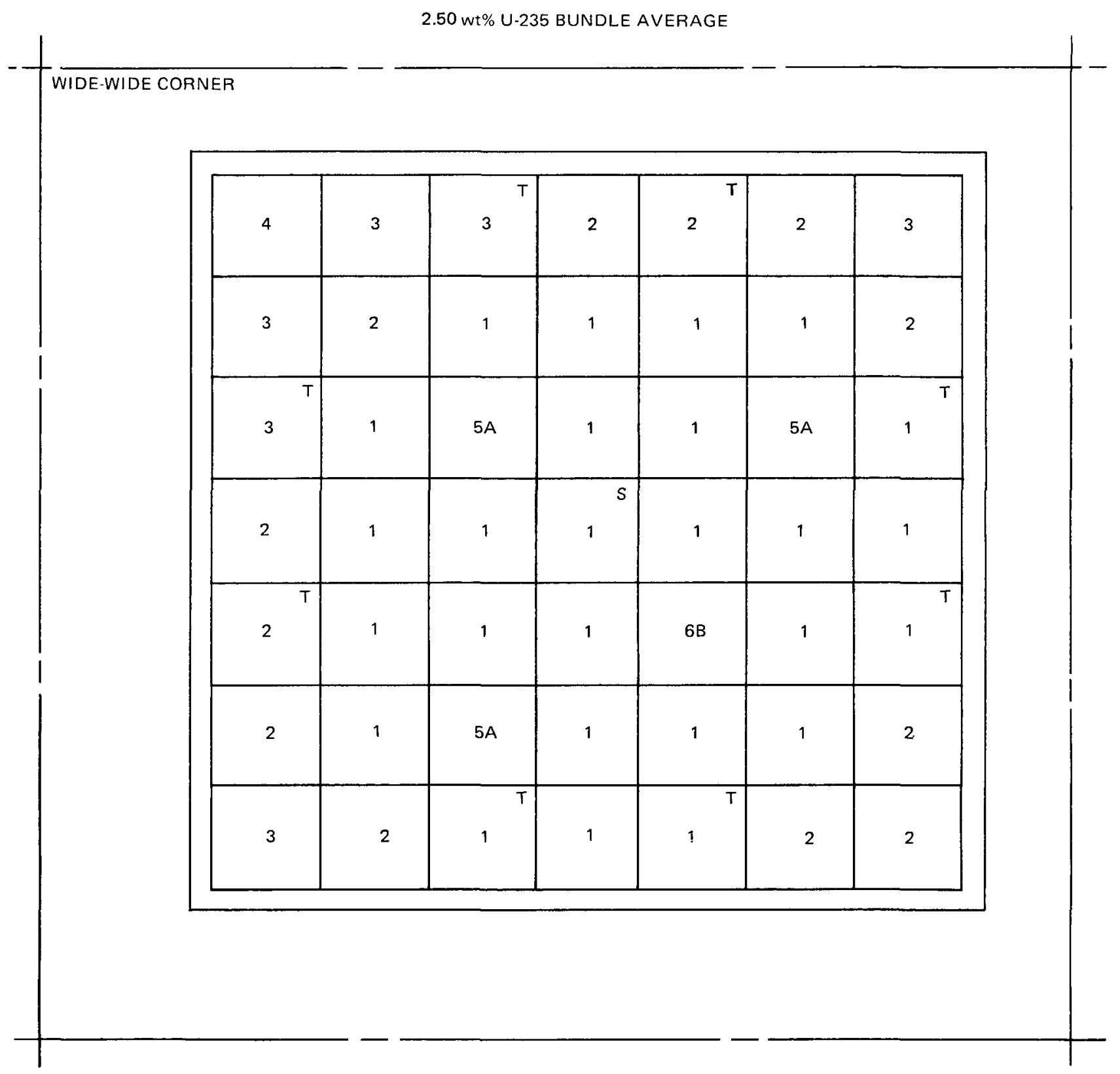

\begin{tabular}{|c|c|c|c|}
\hline ROD TYPE & U-235 $(w t \%)$ & $\mathrm{Gd}_{2} \mathrm{O}_{3}(\mathrm{wt} \%)$ & NO. OF RODS \\
\hline 1 & 2.93 & 0 & 26 \\
2 & 1.94 & 0 & 12 \\
3 & 1.69 & 0 & 6 \\
4 & 1.33 & 0 & 1 \\
$5 \mathrm{~A}$ & 2.93 & 3.0 & 3 \\
$6 \mathrm{~B}$ & 2.93 & 3.0 & 1 \\
& & & \\
S SPACER POSITIONING ROD &
\end{tabular}

Figure 2. Bundle Design for Type 2 Initial Fuel 

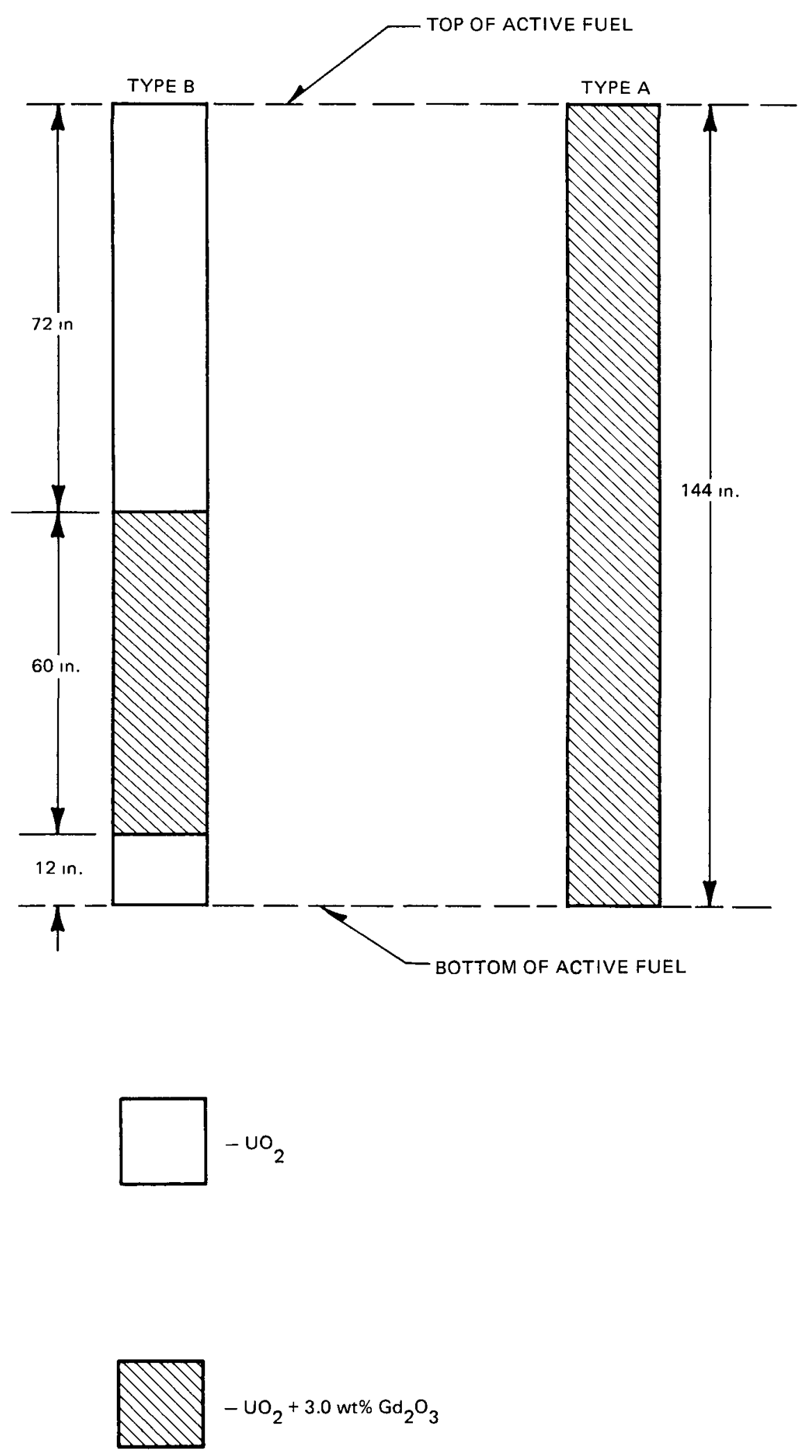

Figure 3. Spatial $\mathrm{Gd}_{2} \mathrm{O}_{3}$ Varration Initial Type 2 Fuel Rods 


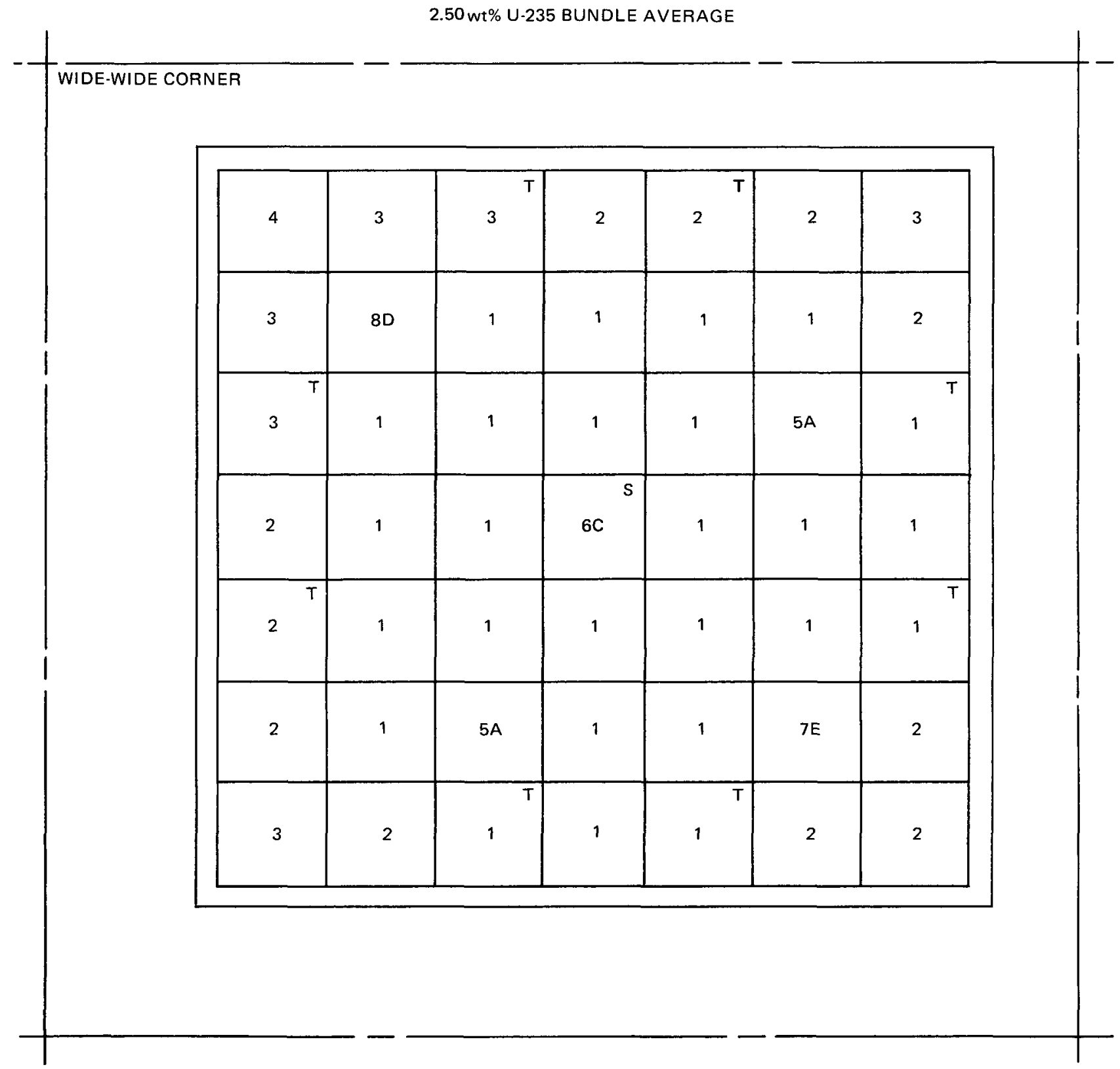

\begin{tabular}{|c|c|c|c|}
\hline ROD TYPE & U-235 (wt \%) & $\mathrm{Gd}_{2} \mathrm{O}_{3}(w \mathrm{t} \%)$ & NO. OF RODS \\
\hline 1 & 2.93 & 0 & 26 \\
3 & 1.94 & 0 & 11 \\
4 & 1.69 & 0 & 6 \\
5A & 1.33 & 0 & 1 \\
6C & 2.93 & 3.0 & 2 \\
7E & 2.93 & 3.0 & 1 \\
8D & 2.93 & 4.0 & 1 \\
\hline
\end{tabular}

Figure 4. Bundle Design for Type 3 Initial Fuel 


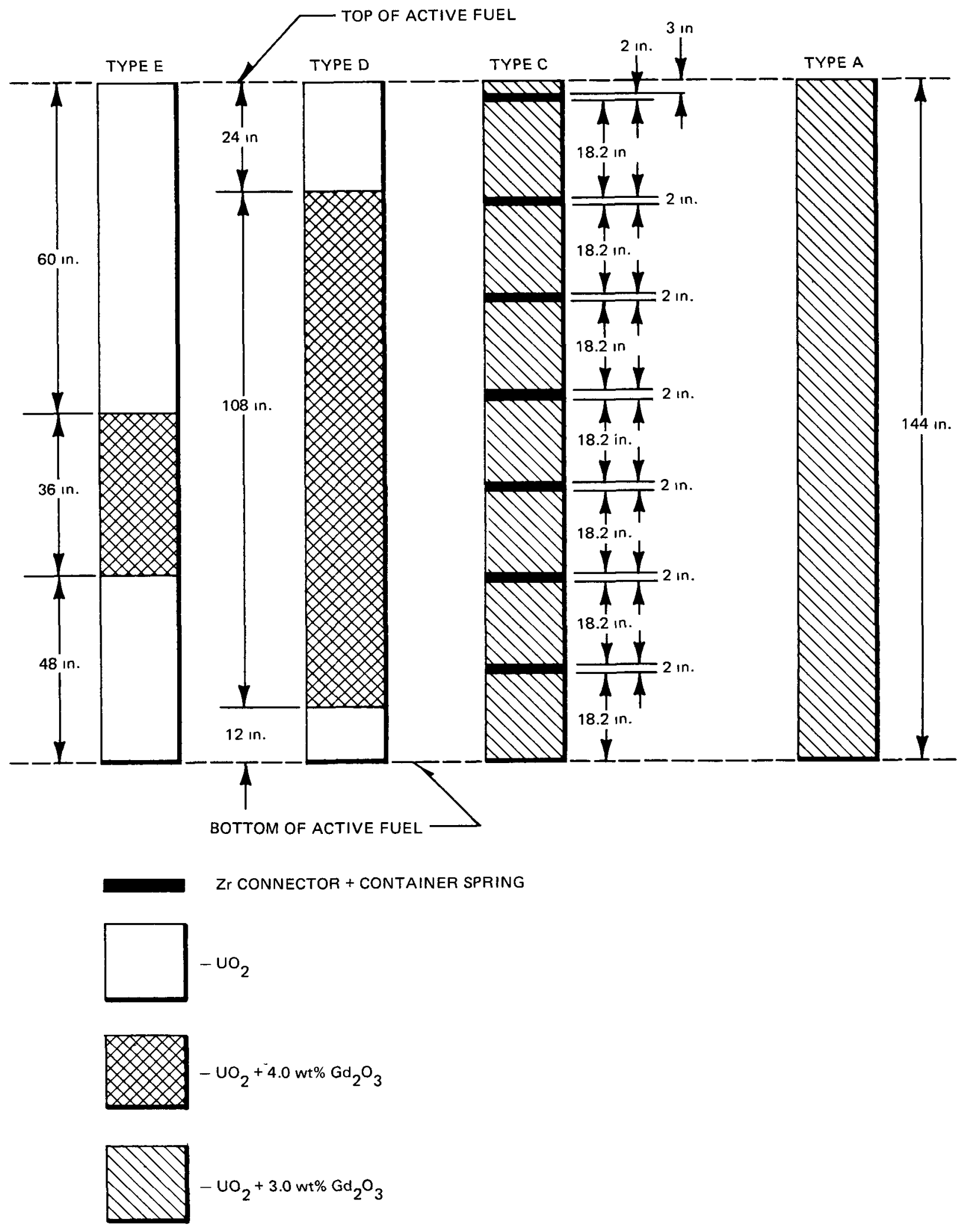

Figure 5. Spatial $\mathrm{Gd}_{2} \mathrm{O}_{3}$ Variation Initial Type 3 Fuel Rods 


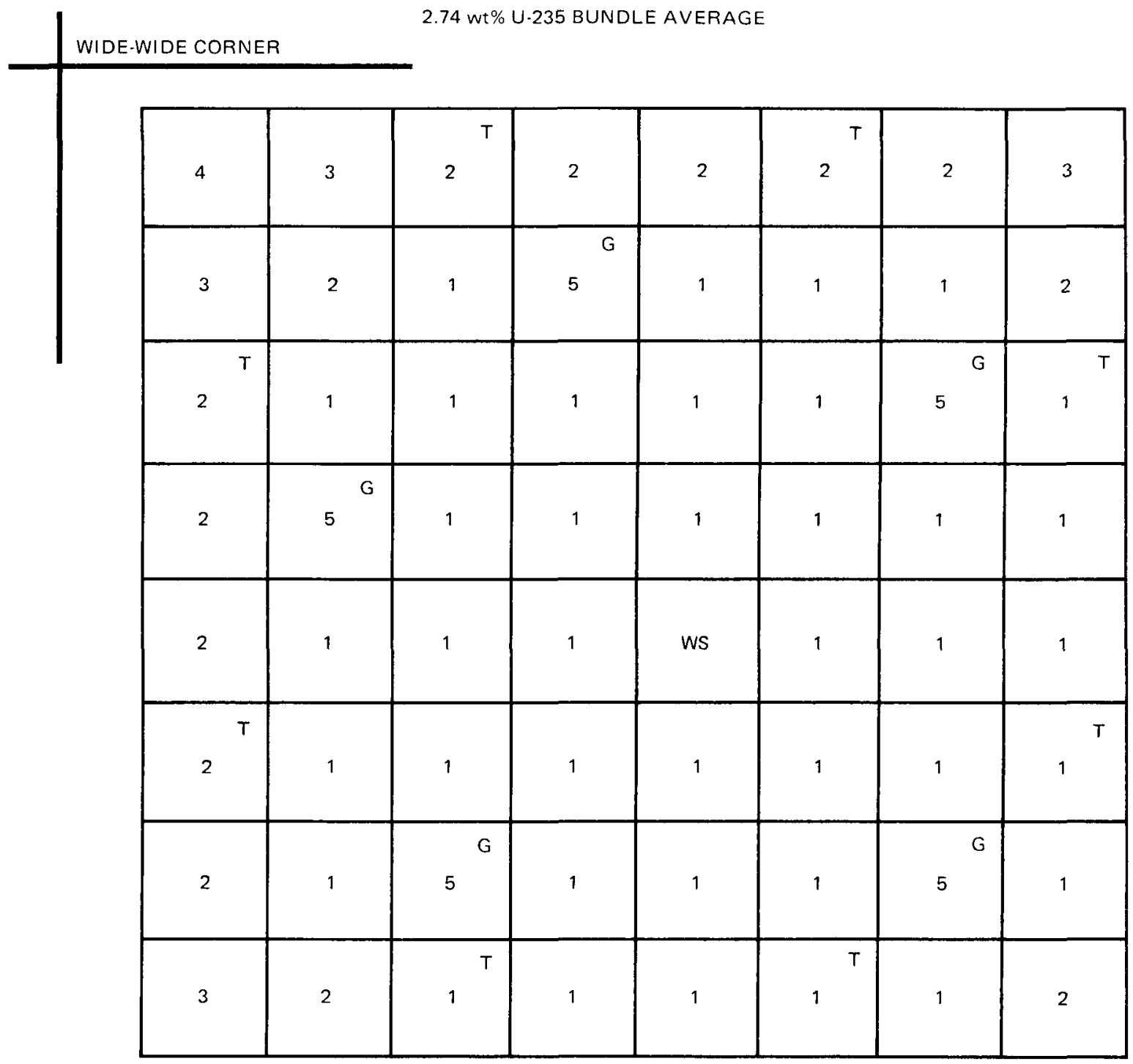

\begin{tabular}{|c|c|c|c|}
\hline ROD TYPE & $\begin{array}{c}\text { ENRICHMENT } \\
\text { wt } \% \text { U-235 }\end{array}$ & $\mathrm{Gd}_{2} \mathrm{O}_{3} w t \%$ & $\begin{array}{c}\text { NUMBER } \\
\text { OF RODS }\end{array}$ \\
\hline 1 & 3.01 & 0 & 39 \\
\hline 2 & 2.22 & 0 & 14 \\
\hline 3 & 1.87 & 0 & 4 \\
\hline 4 & 1.45 & 0 & 1 \\
\hline 5 & 3.01 & 3.0 & 5 \\
\hline WS & - & 0 & 1 \\
\hline
\end{tabular}

WS - SPACER POSITIONING WATER ROD

$T$ - TIE RODS

G - GADOLINIUM RODS

Figure 6. Bundle Design for Type $48 \times 8 \cup \mathrm{UO}_{2}$ Reload 


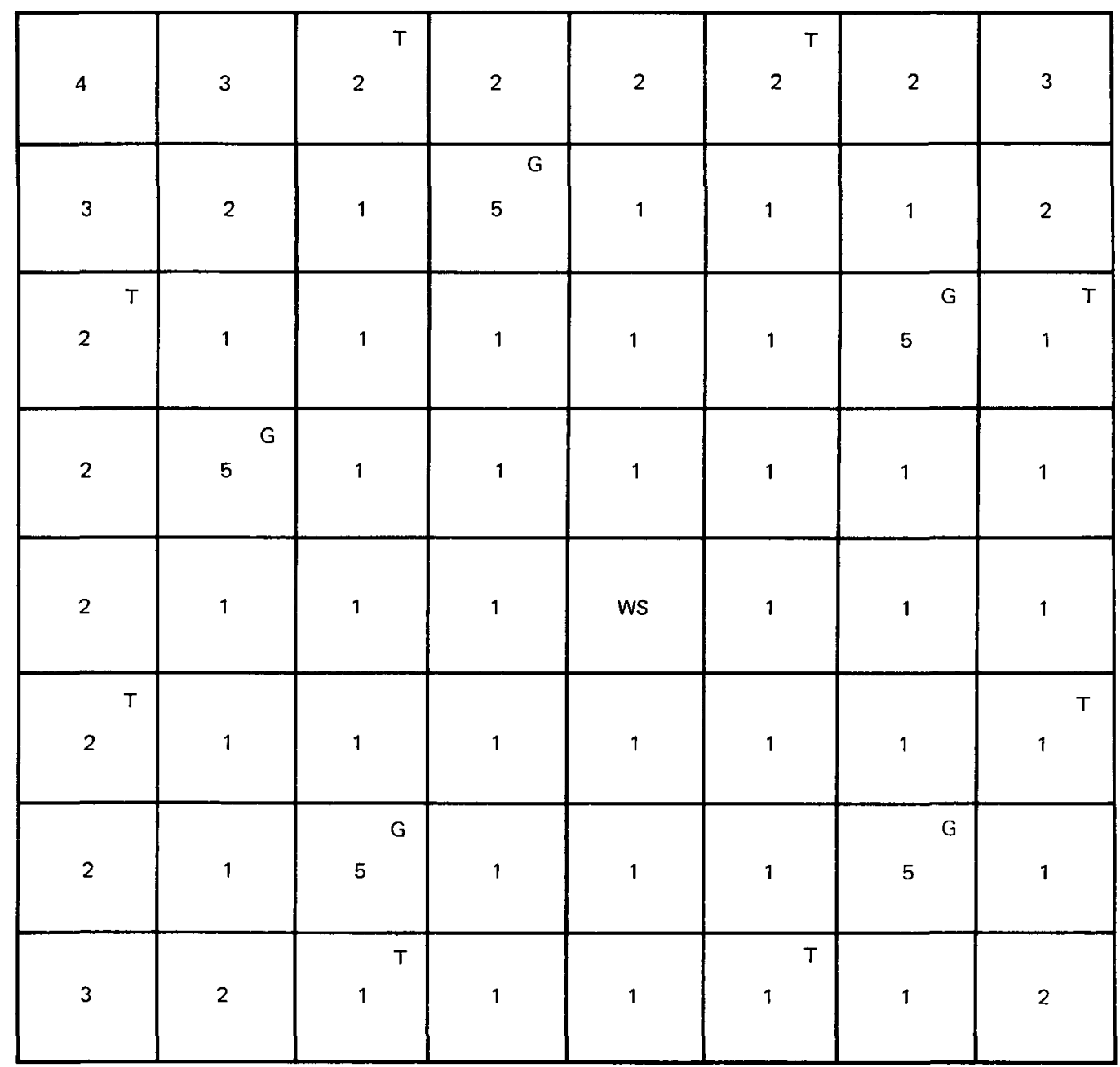

\begin{tabular}{|c|c|c|c|}
\hline ROD TYPE & $\begin{array}{c}\text { ENRICHMENT } \\
w t \% \cup 235\end{array}$ & $\mathrm{Gd}_{2} \mathrm{O}_{3} w t \%$ & $\begin{array}{c}\text { NUMBER } \\
\text { OF RODS }\end{array}$ \\
\hline 1 & 301 & 0 & 39 \\
\hline 2 & 222 & 0 & 14 \\
\hline 3 & 187 & 0 & 4 \\
\hline 4 & 145 & 0 & 1 \\
\hline 5 & 301 & 20 & 5 \\
\hline WS & - & 0 & 1 \\
\hline
\end{tabular}

WS - SPACER POSITIONING WATER ROD

$T$ - TIE RODS

G - GADOLINIUM RODS

Figure 7 Bundle Design for Type $58 \times 8 \cup_{2}$ Reload 


\begin{tabular}{|c|c|c|c|c|c|c|c|}
\hline 4 & 3 & $2^{T}$ & 2 & 2 & $2^{\top}$ & 2 & 3 \\
\hline 3 & 2 & 1 & 5 & 1 & 1 & 1 & 2 \\
\hline $2^{T}$ & 1 & 1 & 1 & 1 & 1 & $5^{G}$ & $\begin{array}{ll} & \\
& \end{array}$ \\
\hline 2 & $5^{G}$ & 1 & 1 & WR & 1 & 1 & 1 \\
\hline 2 & 1 & 1 & ws & 1 & 1 & 1 & 1 \\
\hline $2^{T}$ & 1 & 1 & 1 & 1 & 1 & 1 & ${ }_{1}{ }^{T}$ \\
\hline 2 & 1 & $5^{G}$ & 1 & 1 & 1 & $5^{G}$ & 1 \\
\hline 3 & 2 & 1 & 1 & 1 & $\begin{array}{ll} & \mathrm{T} \\
1 & \end{array}$ & 1 & 2 \\
\hline
\end{tabular}

"NATURAL UO 2 LOCATED IN END ZONES, 62 RODS. SEE FIGURE 9.

\begin{tabular}{|c|c|c|c|}
\hline ROD TYPE & $\begin{array}{c}\text { ENRICHMENT } \\
\text { Wt\% U-235 }\end{array}$ & $\mathrm{Gd}_{2} \mathrm{O}_{3} w t \%$ & $\begin{array}{c}\text { NUMBER } \\
\text { OF RODS }\end{array}$ \\
\hline 1 & 3.01 & 0 & 38 \\
\hline 2 & 2.22 & 0 & 14 \\
\hline 3 & 1.87 & 0 & 4 \\
\hline 4 & 1.45 & 0 & 1 \\
\hline 5 & 3.01 & 2.0 & 5 \\
\hline WS & - & 0 & 1 \\
\hline WR & - & 0 & 1 \\
\hline
\end{tabular}

WS - SPACER POSITIONING WATER ROD

WR - WATER ROD

$T$ - TIE RODS

G - GADOLINIUM RODS

Figure 8. Bundle Design for Type $68 \times 8 \cup_{2} \mathrm{O}_{2}$ Reload, LTA 


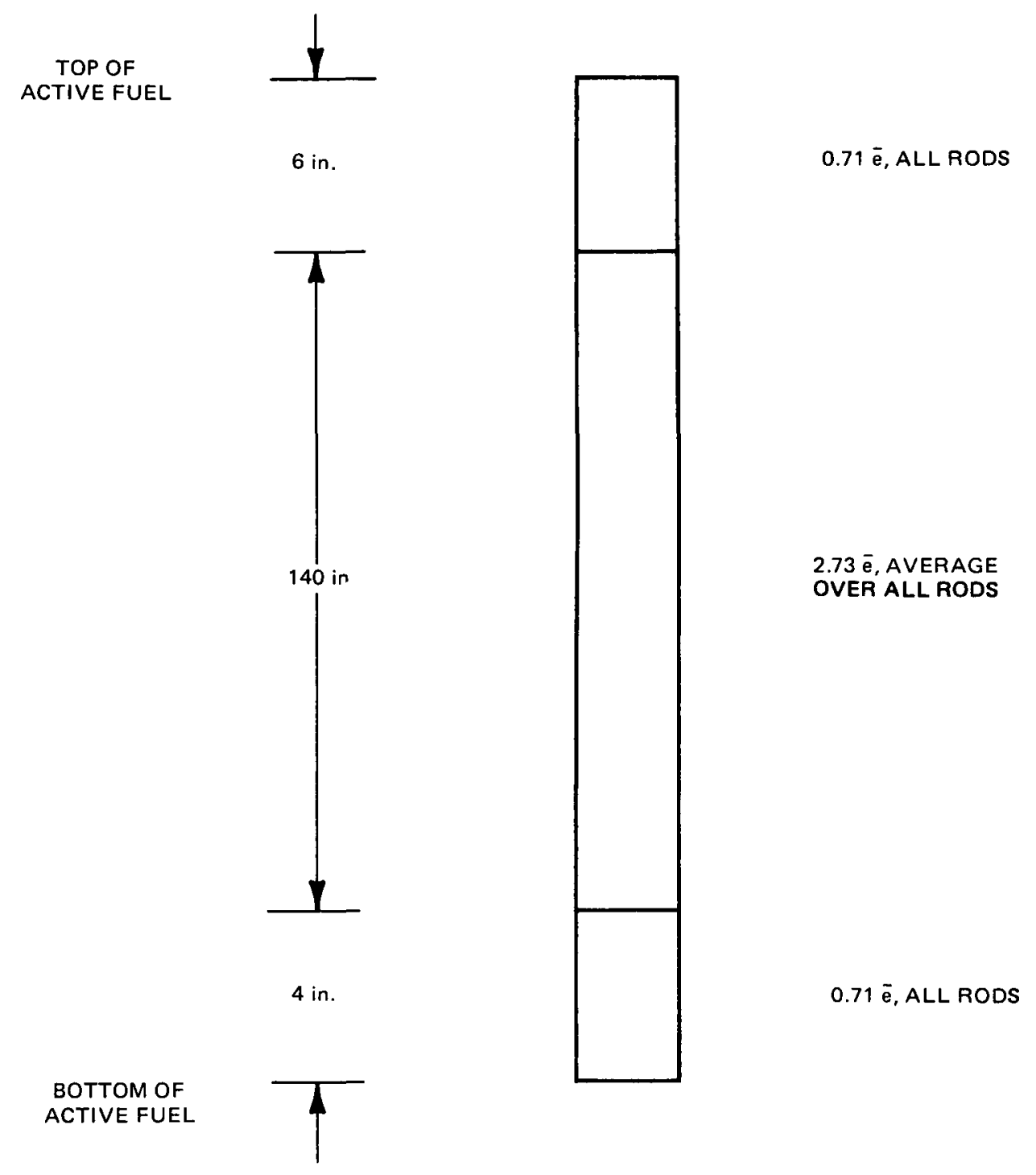

Figure 9. Lead Test Assembly U-235 Enrichment Axial Profile 


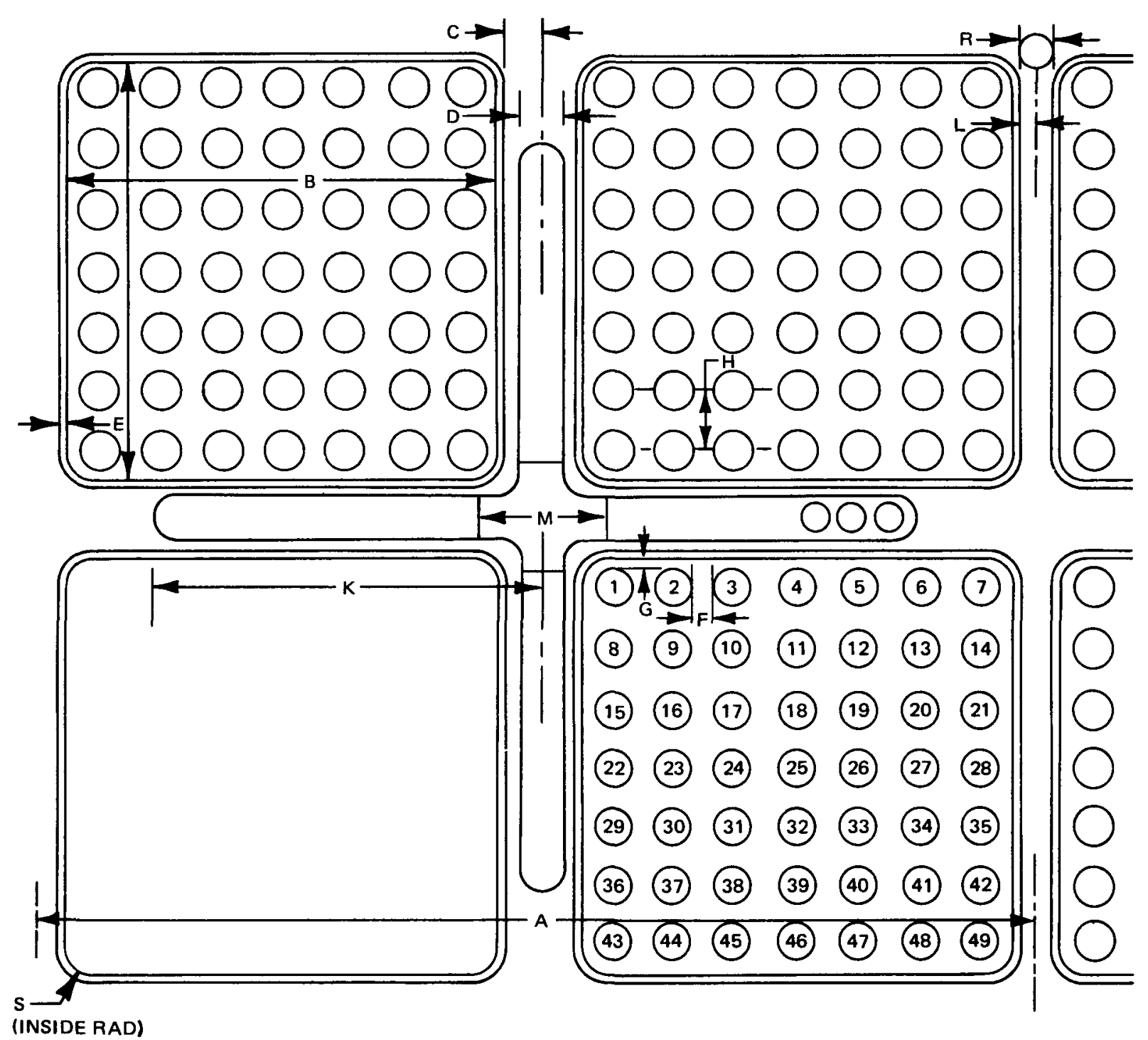

\begin{tabular}{|l|c|c|c|c|c|c|c|c|c|c|}
\hline DIM. IDENTIFICATION & A & B & C & D & E & F & G & H & I & J \\
\hline DIM. INCHES & 12.0 & 5.278 & 0.375 & & 0.080 & 0.175 & 0.1435 & 0.738 & & \\
\hline & & & & & & & & & & \\
\hline
\end{tabular}

Figure 10. Initial Fuel Assembly Lattice 


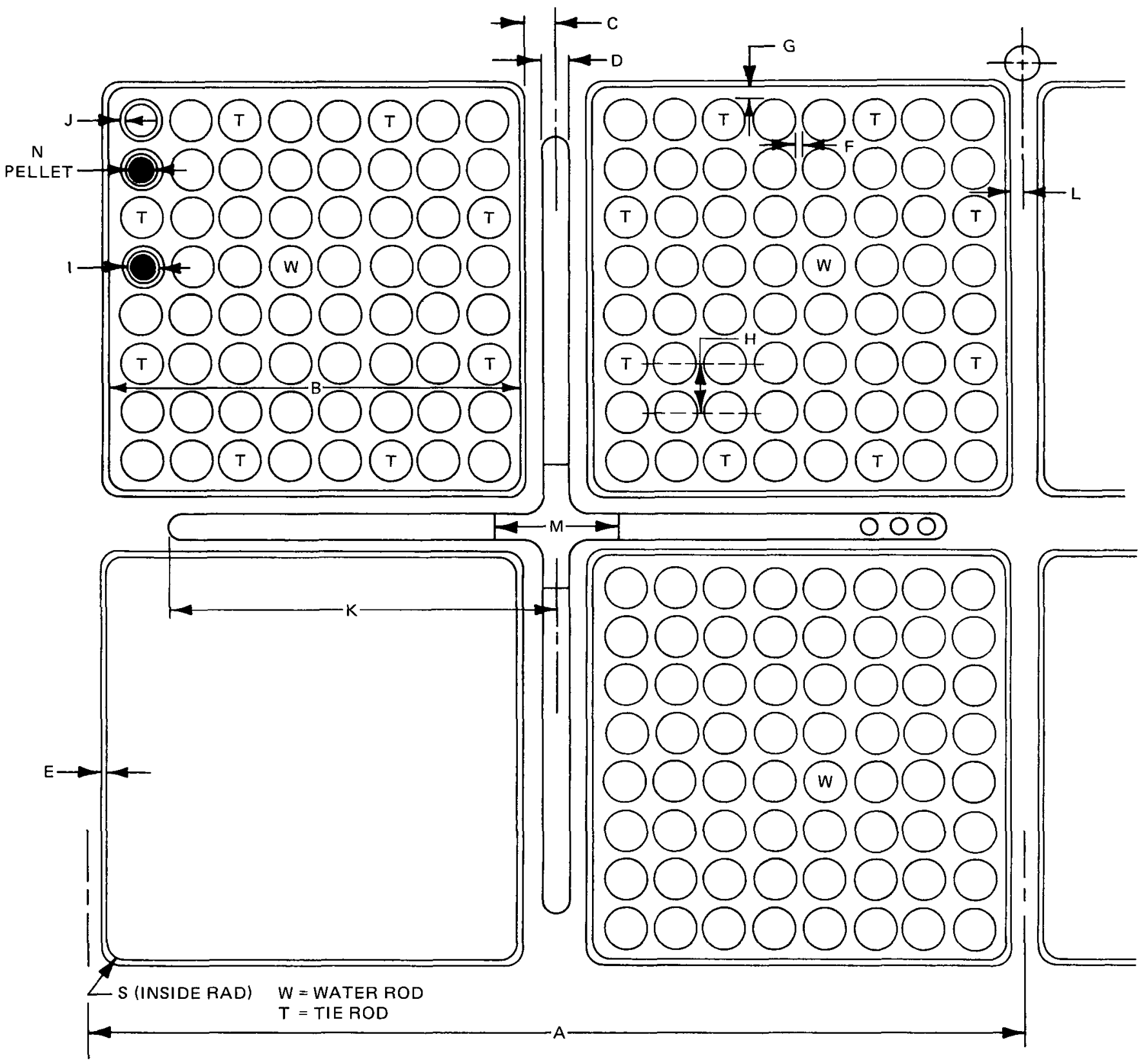

\begin{tabular}{|l|c|c|c|c|c|c|c|c|c|c|}
\hline DIM. IDENTIFICATION & A & B & C & D & E & F & G & H & I & J \\
\hline DIM. INCHES & 12.0 & 5.278 & 0.355 & & 0.100 & 0.147 & 0.153 & 0.640 & & \\
\hline & & & & & & & & & & \\
\hline
\end{tabular}

Figure 11. Reload Fuel Assembly Lattice for 100 mil Channels 


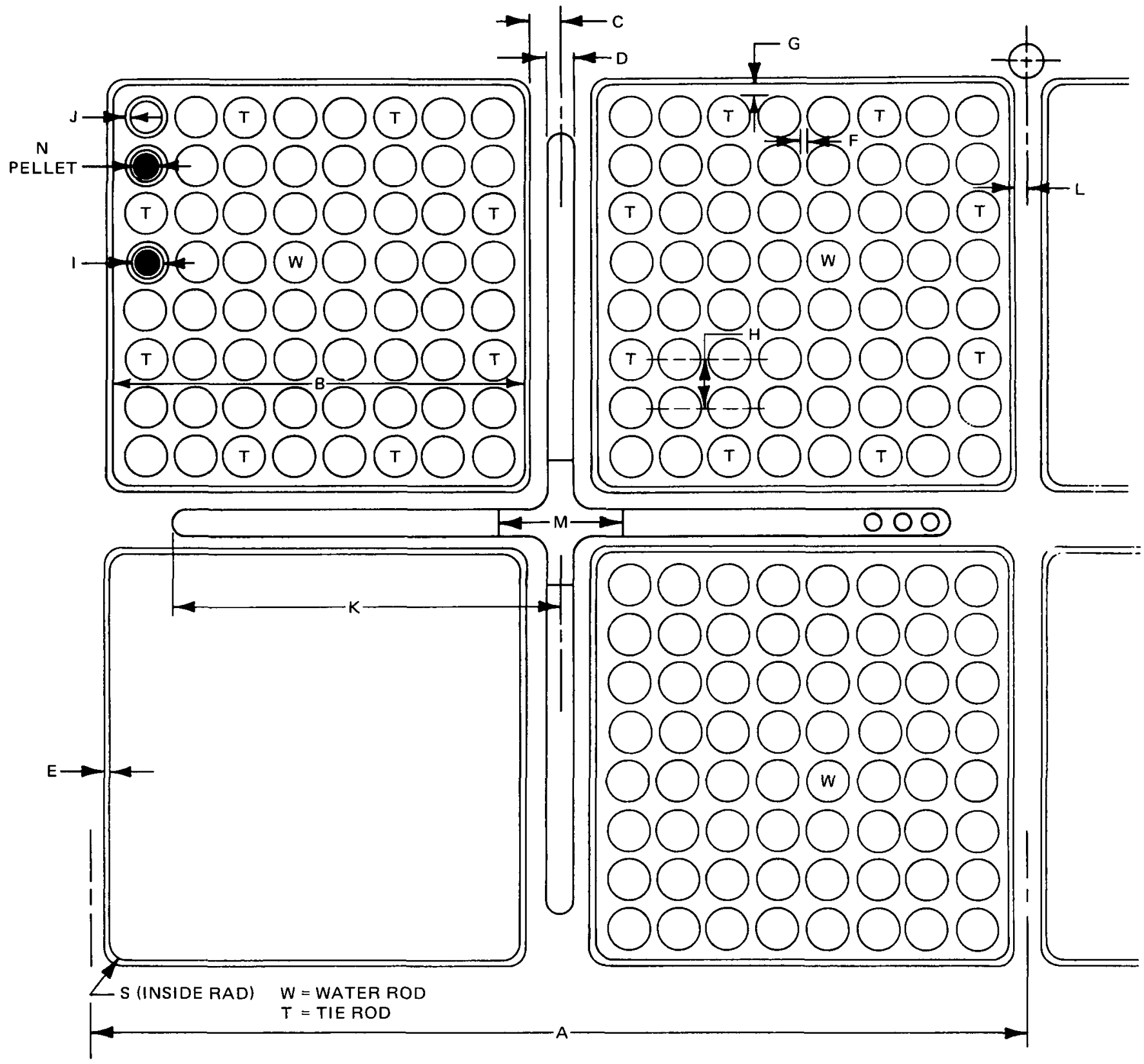

\begin{tabular}{|l|c|c|c|c|c|c|c|c|c|c|c|}
\hline DIM. IDENTIFICATION & A & B & C & D & E & F & G & H & I & J \\
\hline DIM. INCHES & 12.0 & 5.278 & 0.335 & & 0.120 & 0.147 & 0.153 & 0.640 & & \\
\hline & & & & & & & & & & \\
\hline
\end{tabular}

Figure 12. Reload Fuel Assembly Lattice for 120 mil Channels 


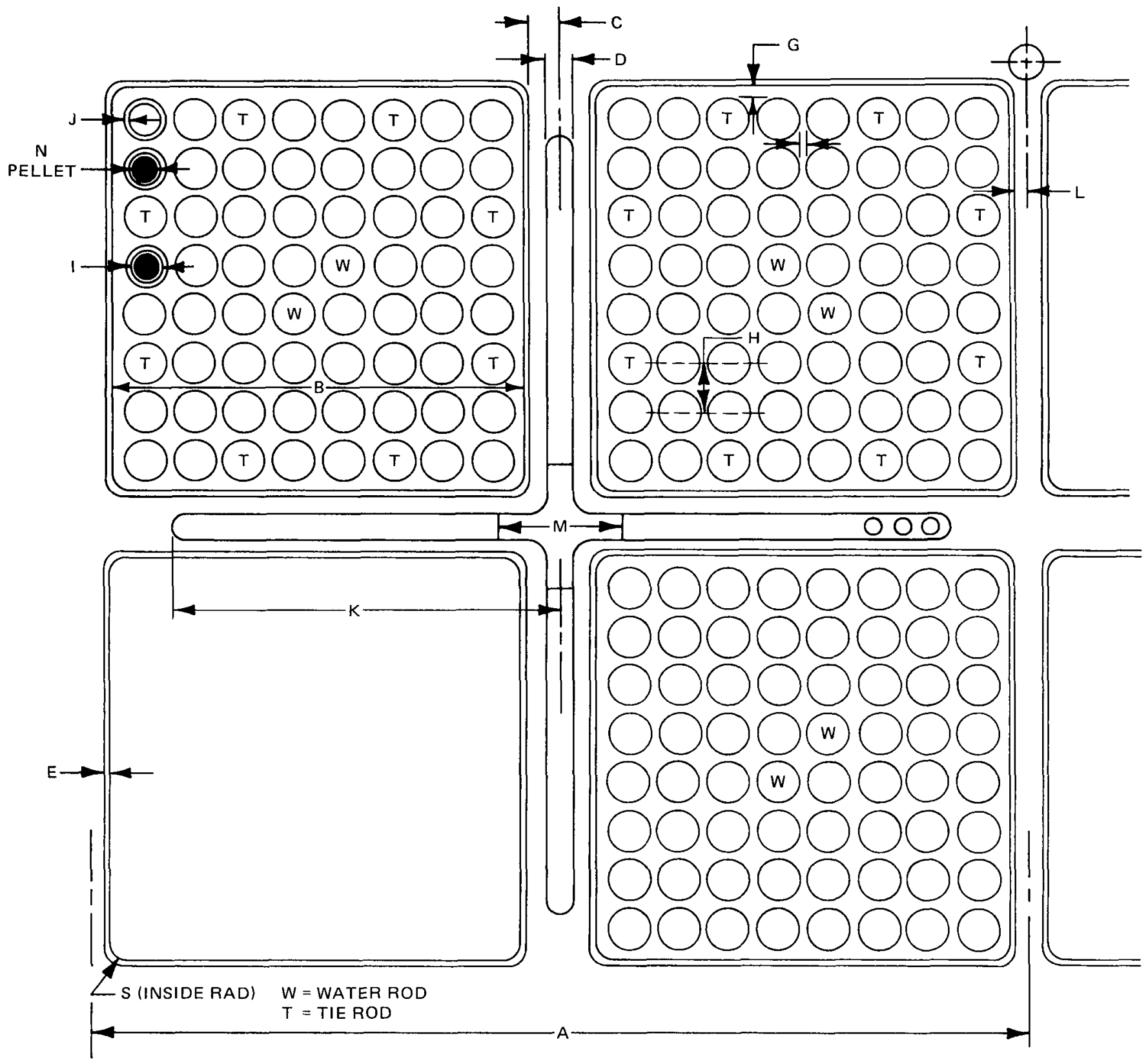

\begin{tabular}{|l|c|c|c|c|c|c|c|c|c|c|}
\hline DIM. IDENTIFICATION & A & B & C & D & E & F & G & H & I & J \\
\hline DIM. INCHES & 12.0 & 5.278 & 0.355 & & 0.100 & 0.157 & 0.158 & 0.640 & & \\
\hline & & & & & & & & & & \\
\hline
\end{tabular}

Figure 13. Reload Fuel Assembly Lattice for LTA Assemblies 


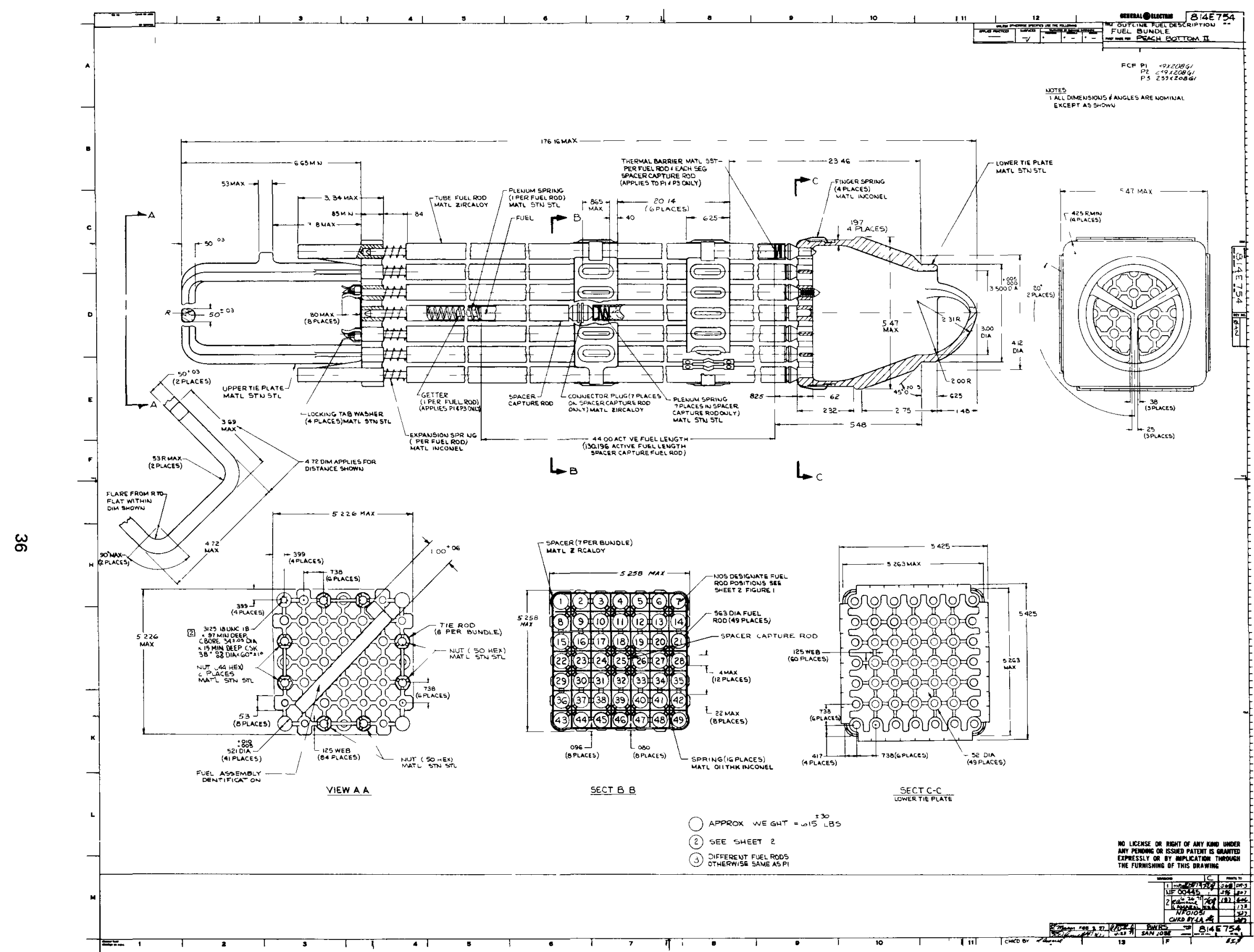

Figure 14 Fuel Assembly Drawing for Initial Core Fuel 


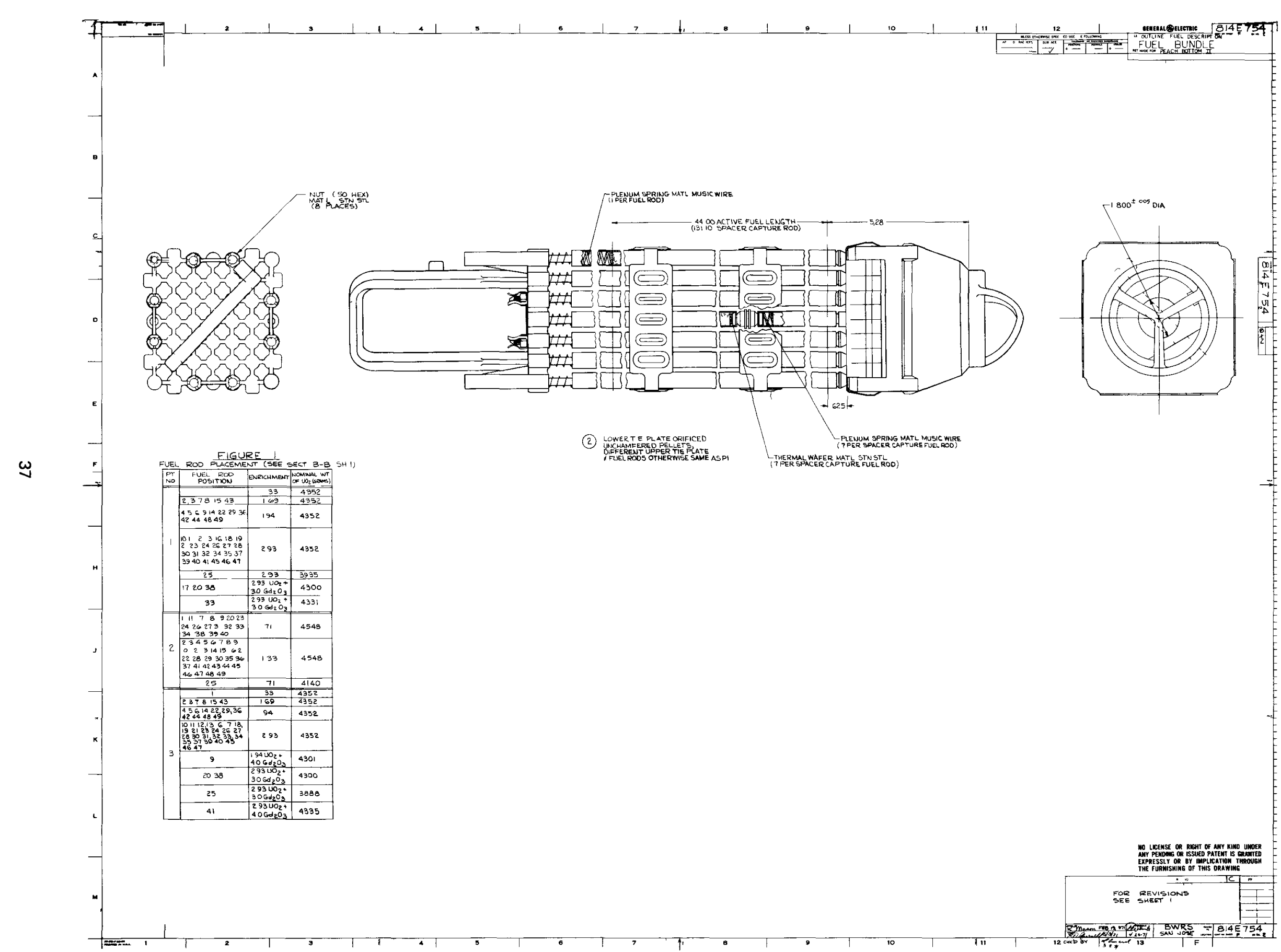




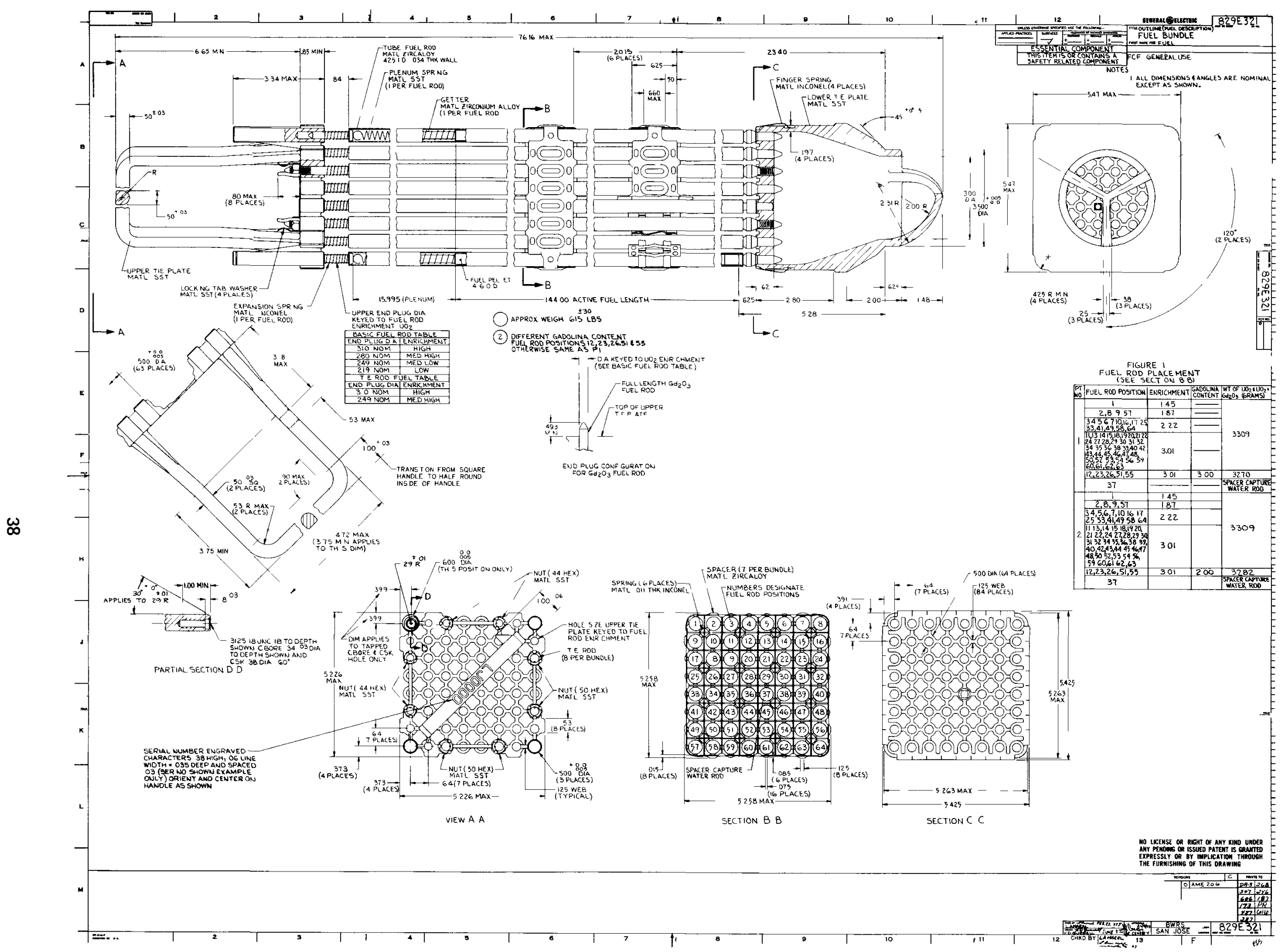




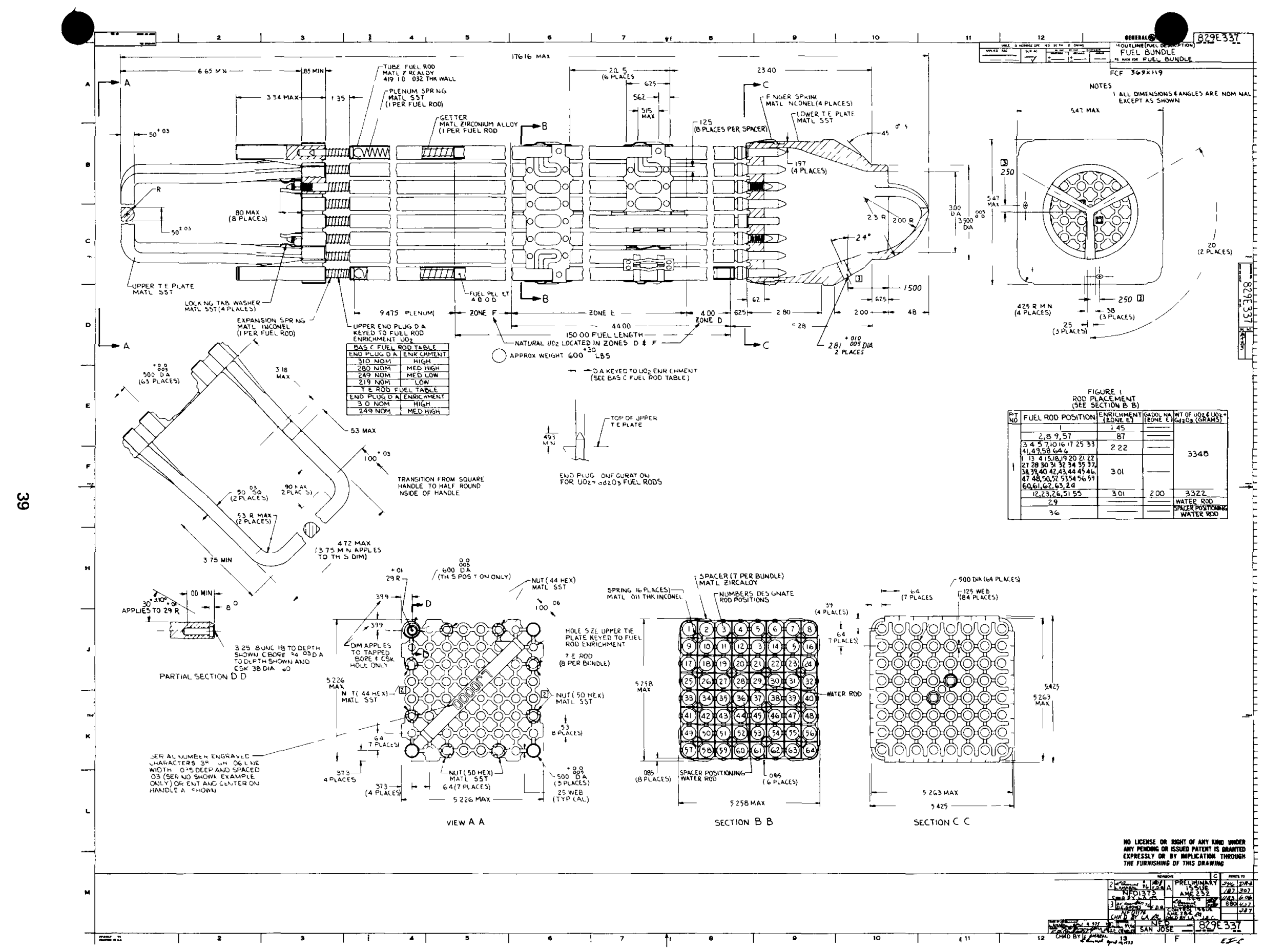




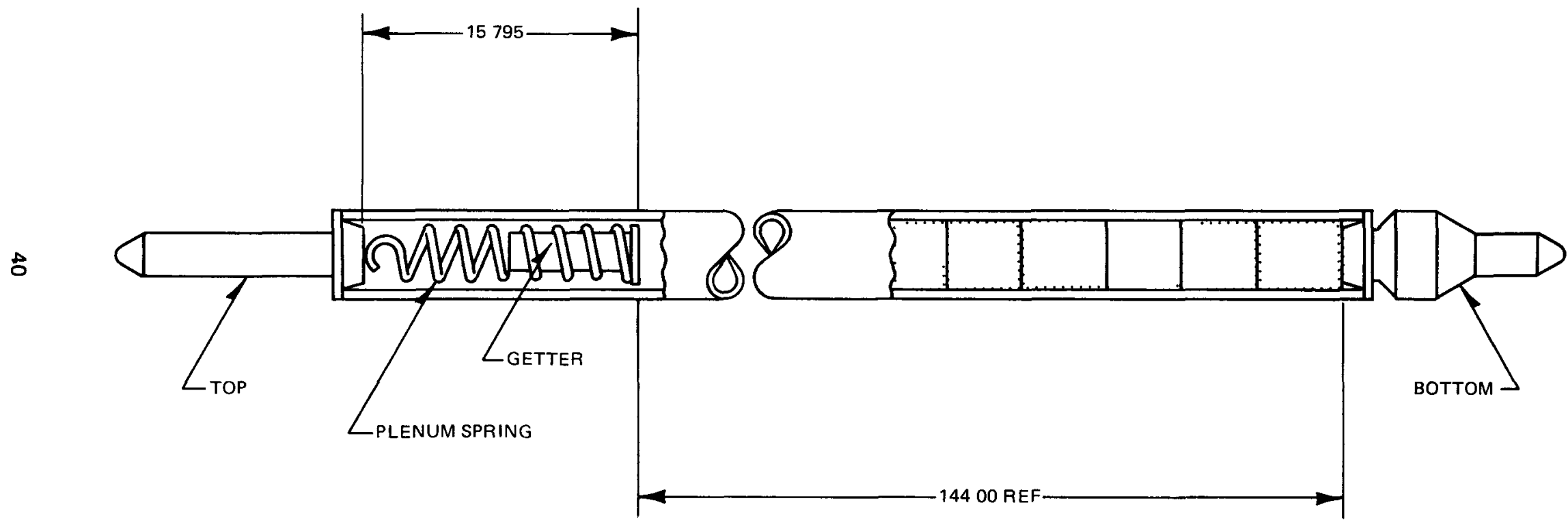

Figure 18 Typical Fuel Rod 


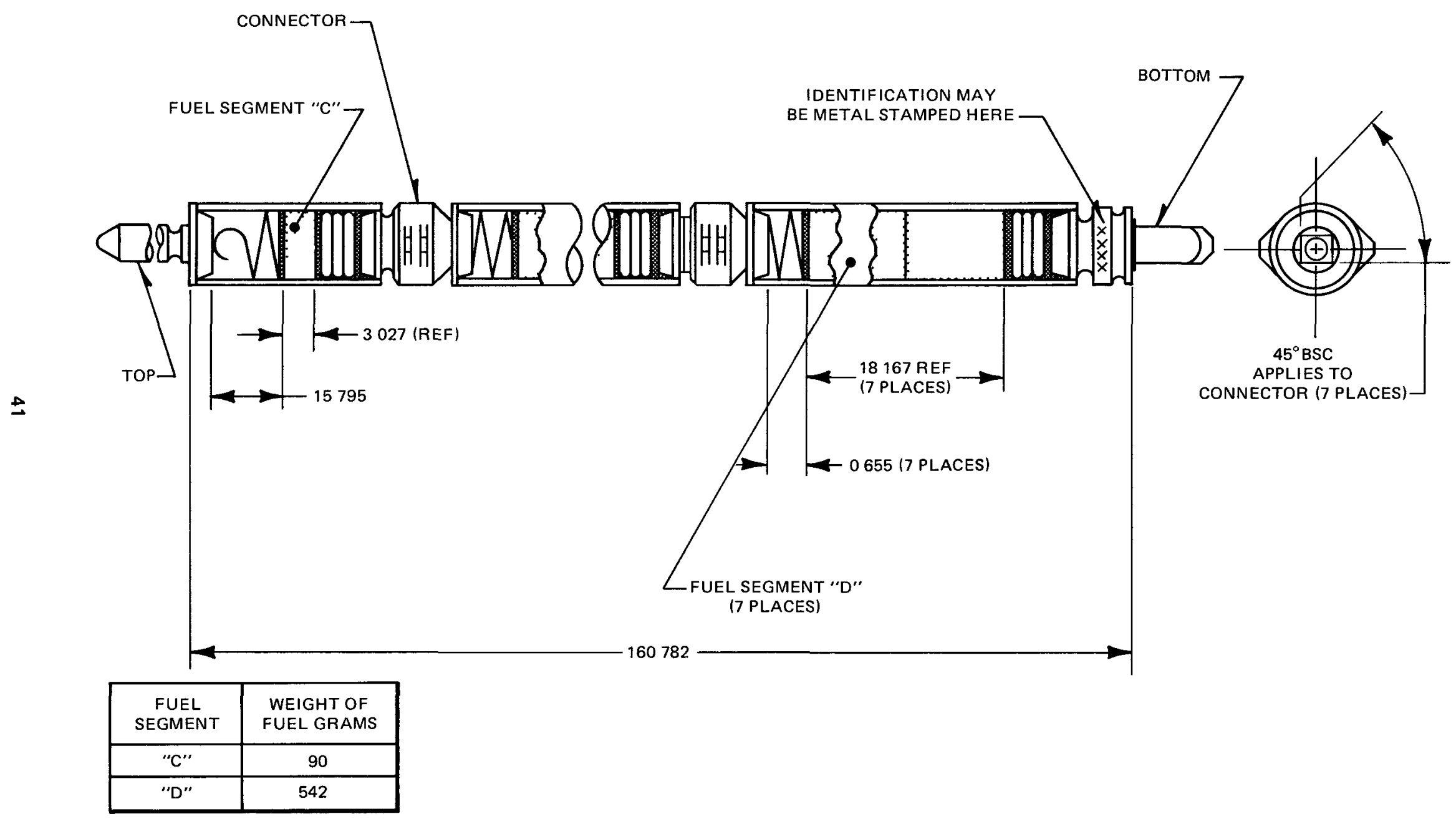

Figure 19 Spacer Positioning Rod for Type 3 Initial Fuel 


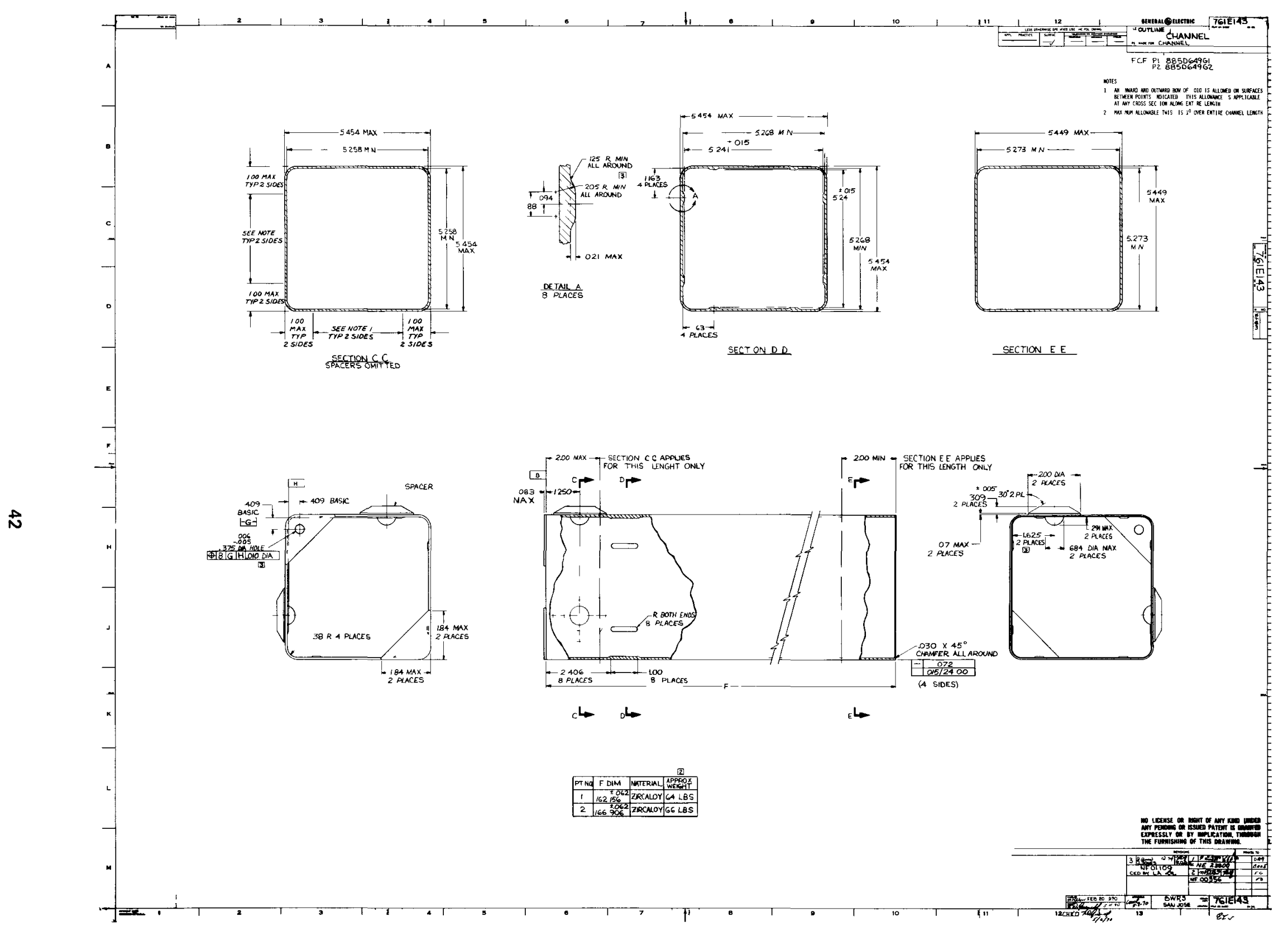

Figure 20 Channel Outline Drawing for Use With Initial Fuel 


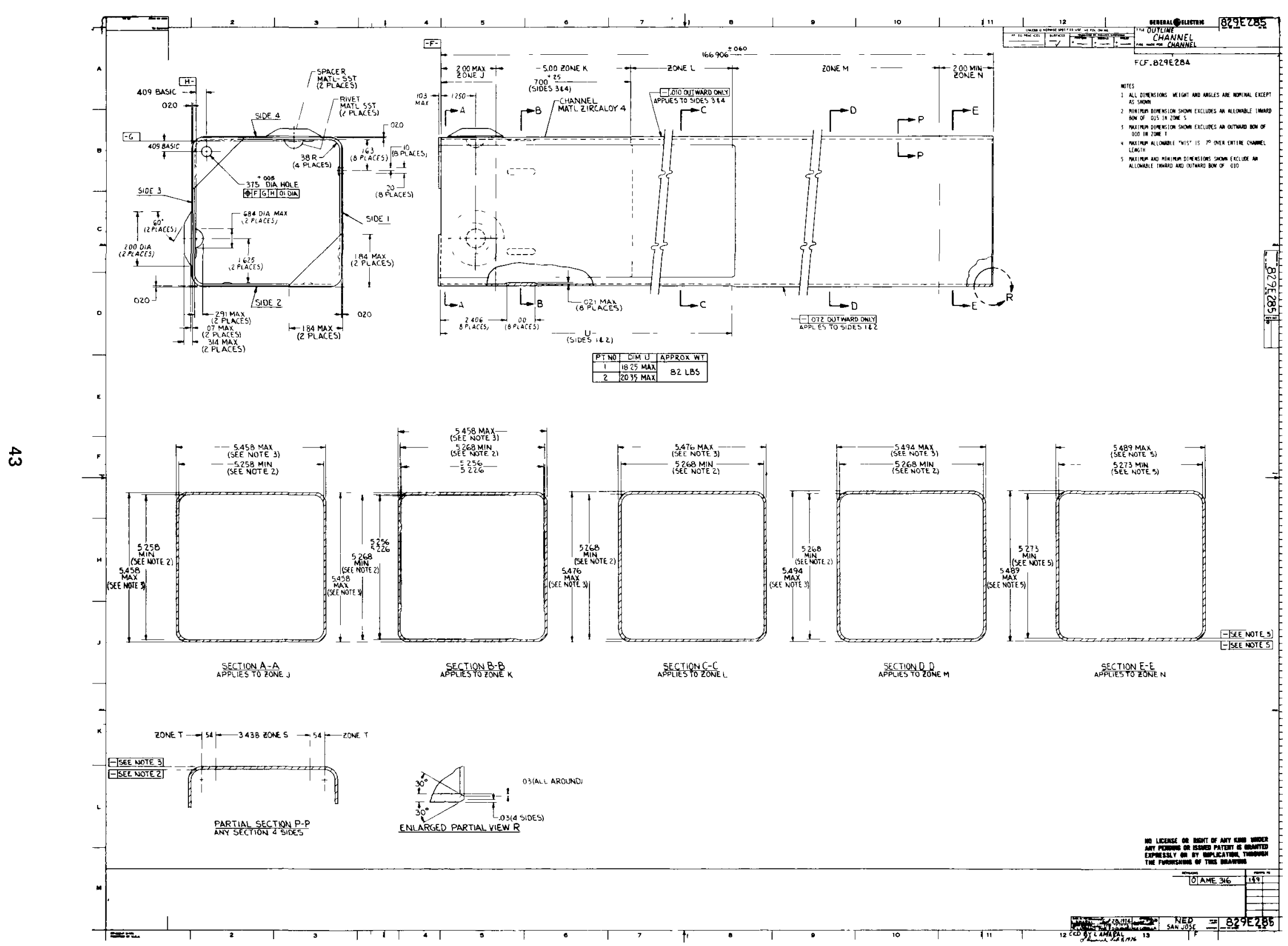

Figure 21. Channel Outline Drawing for Use With $8 \times 8$ Reload Fuel 


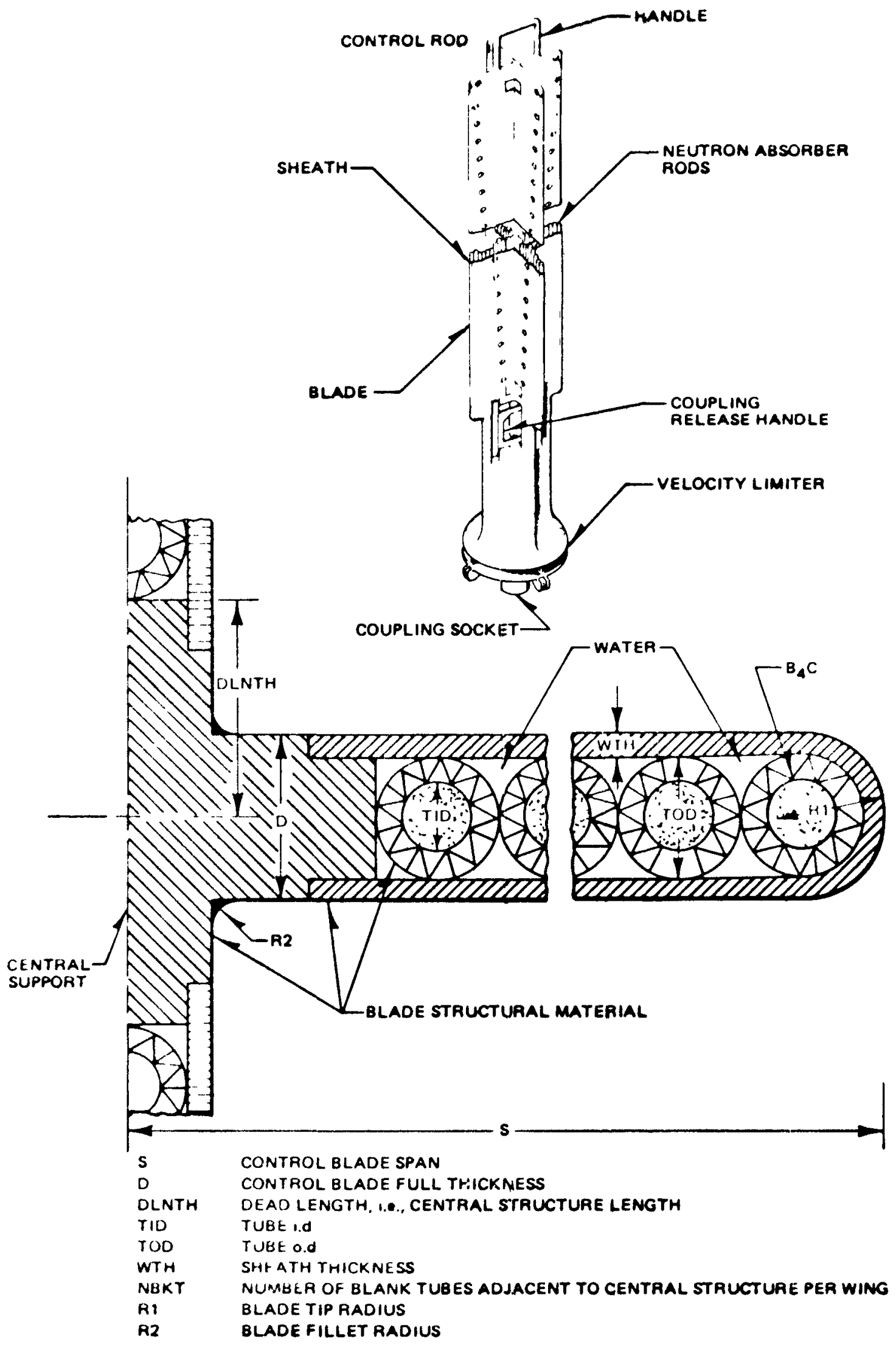

Figure 22. $B_{4} C$ Control Blade Model (Schematic) 


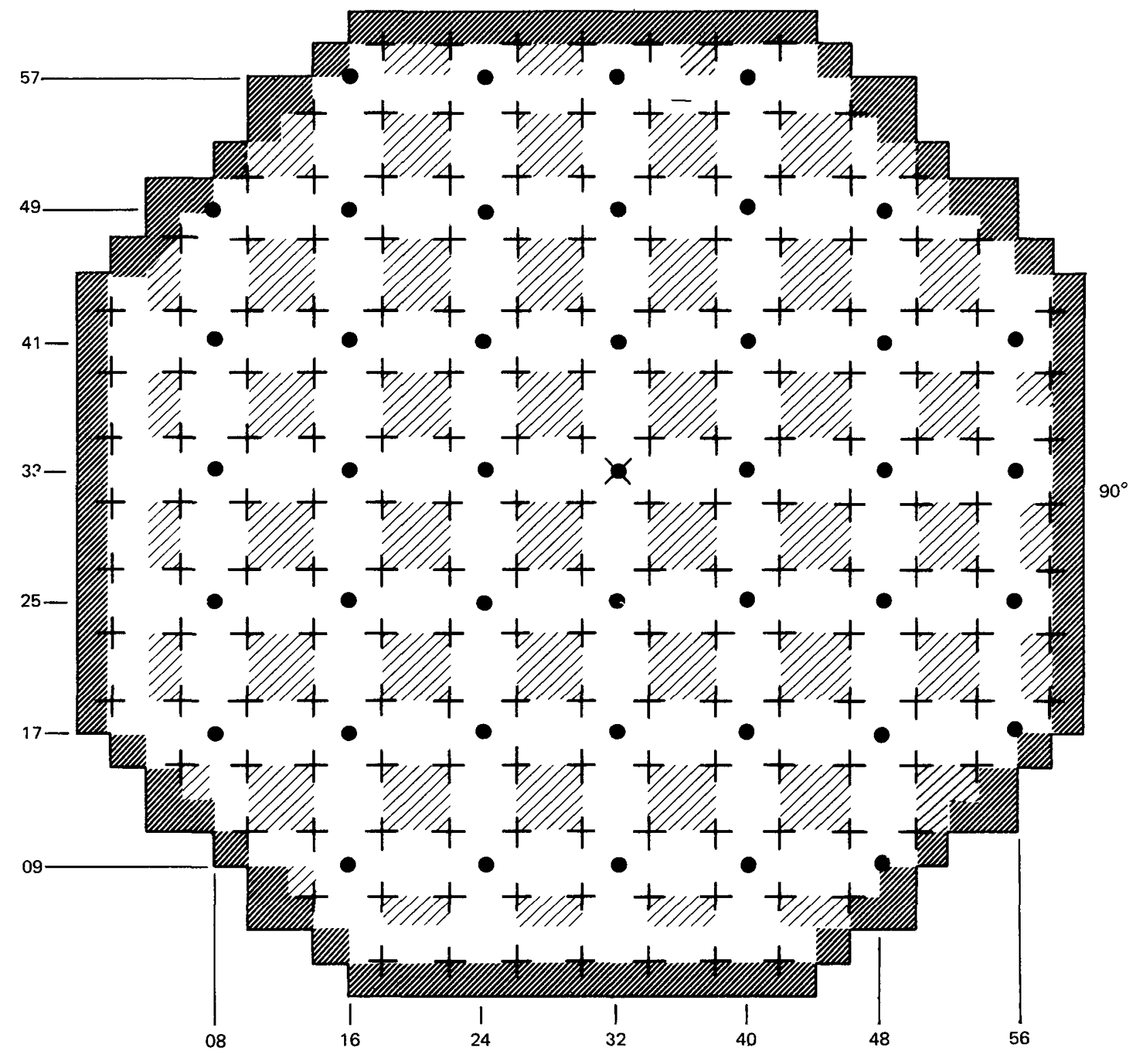

NUMBER OF FUEL ASSEMBLIES - 764

+ NUMBER OF CONTROL RODS - 185

X NUMBER OF TIP INSTRUMENT ASSEMBLIES -43

COMMON POSITION FOR ALL TIP MACHINES
FUEL BUNDLES WITH $2.211 \mathrm{in.}$

ORIFICE DIAMETER - 672

YIT FUEL BUNDLES WITH 1.469 in.

ORIFICE DIAMETER - 92

D TYPE 1 ASSEMBLIES WITH $2.211 \mathrm{in.}$ ORIFICE AND RESTRICTED LOWER TIE PLATE - 168 (CYCLE 1 ONLY)

Figure 23. Core Orificing and TrP System Arrangement 


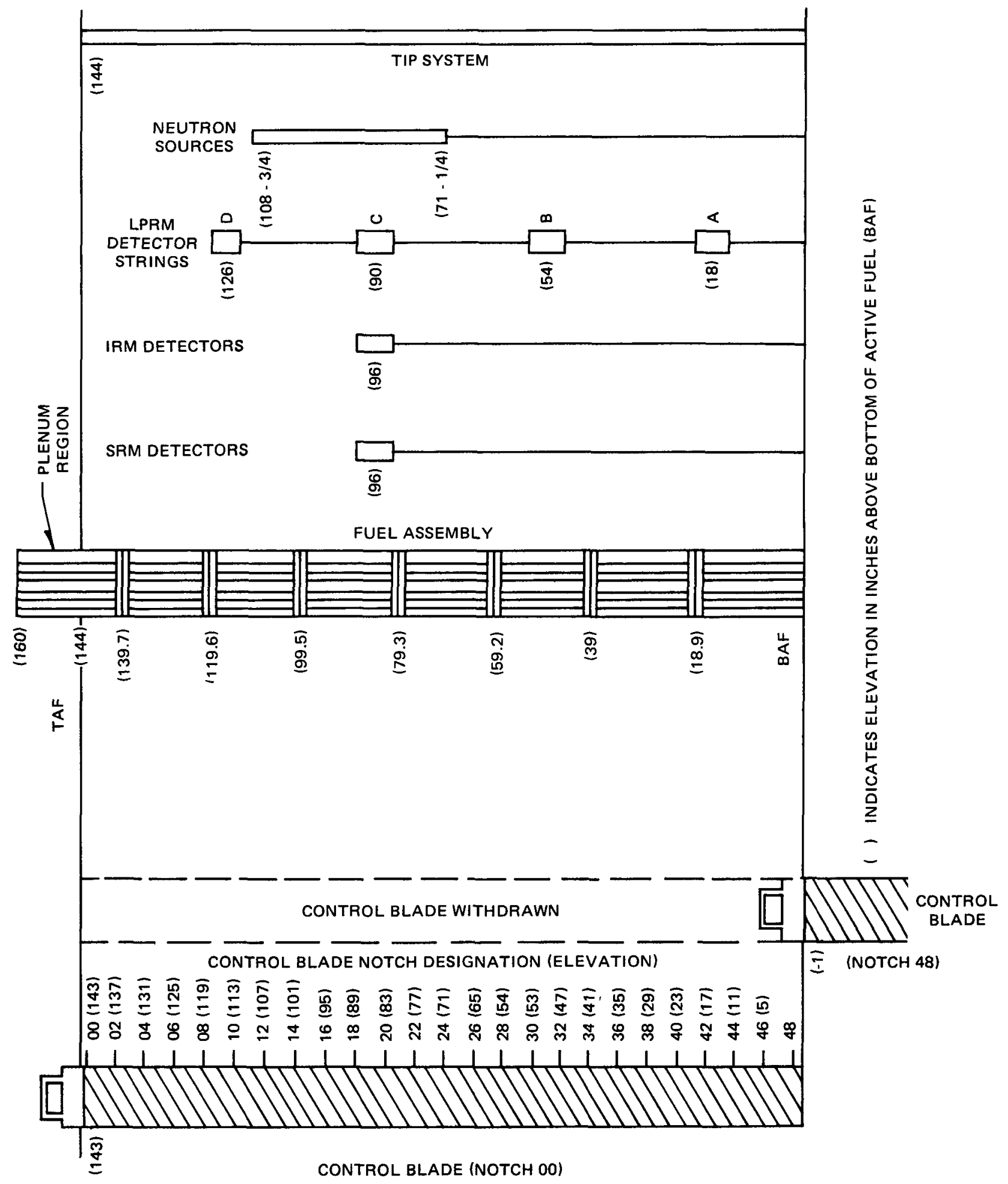

Figure 24. Elevation of Core Components 

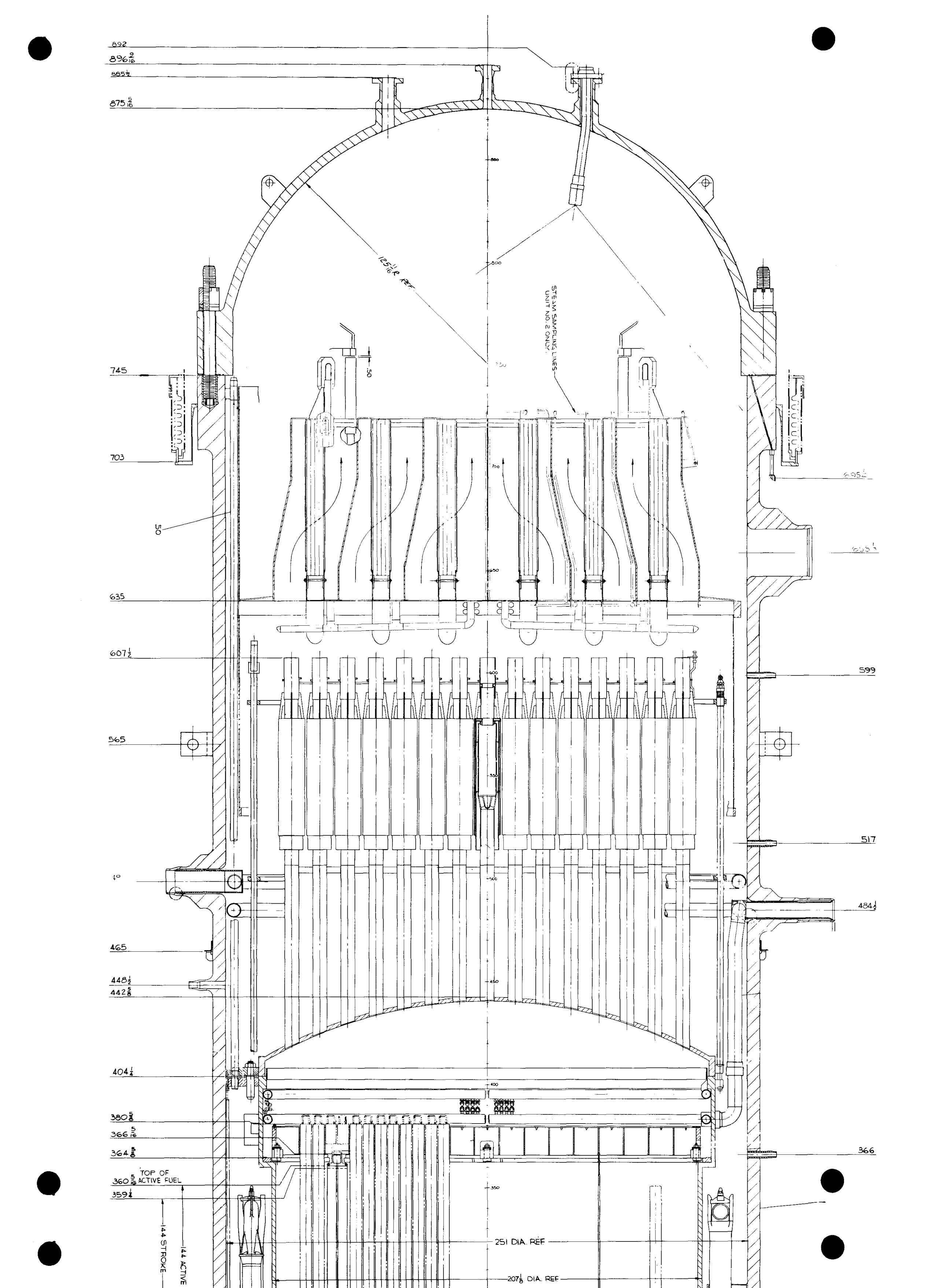

M०
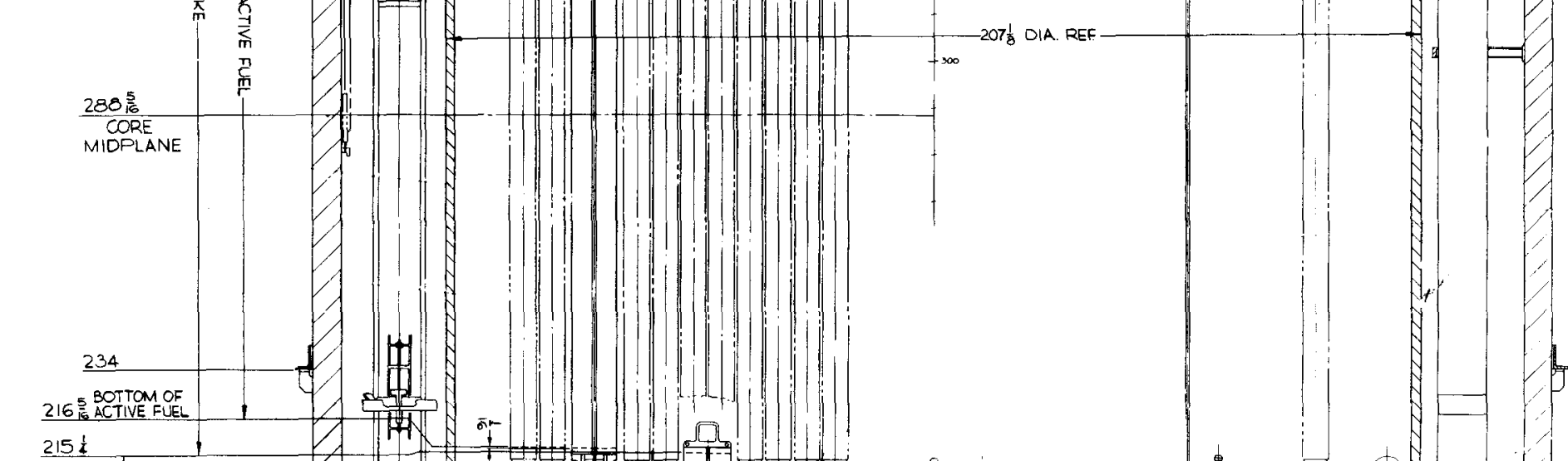

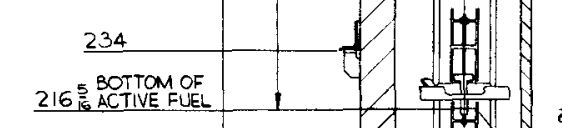
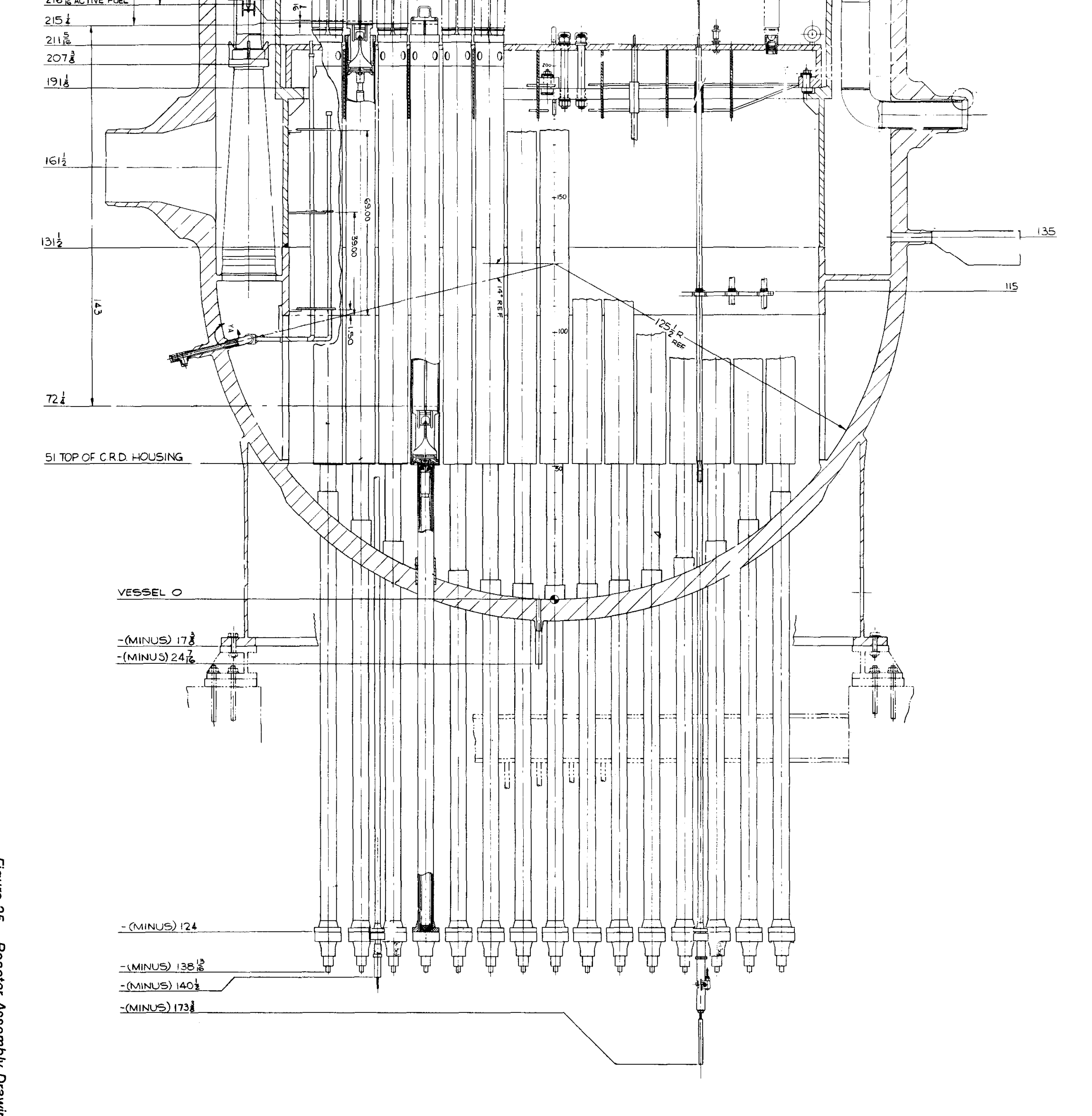

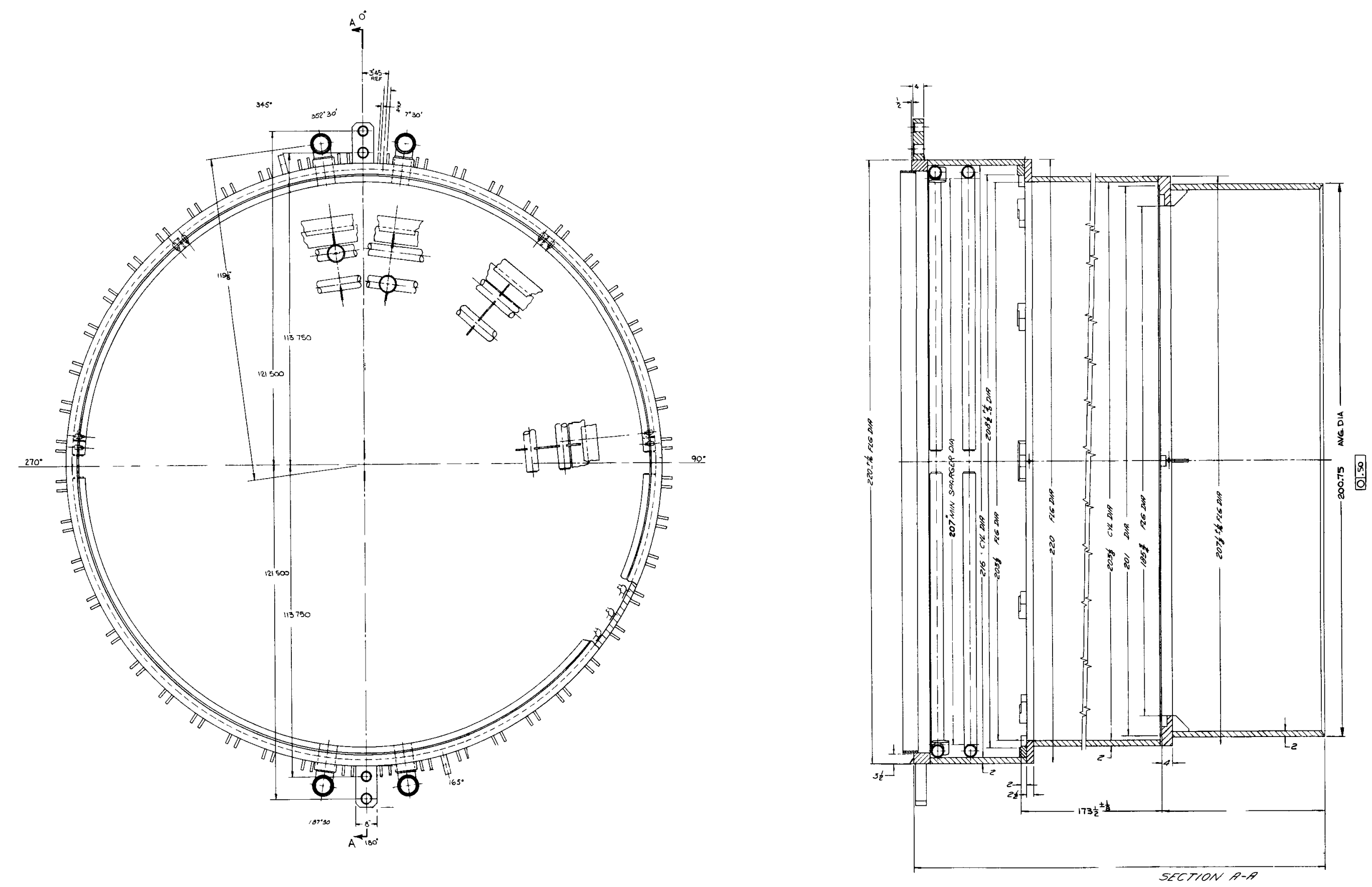

Figure 26. Shroud Drawing 


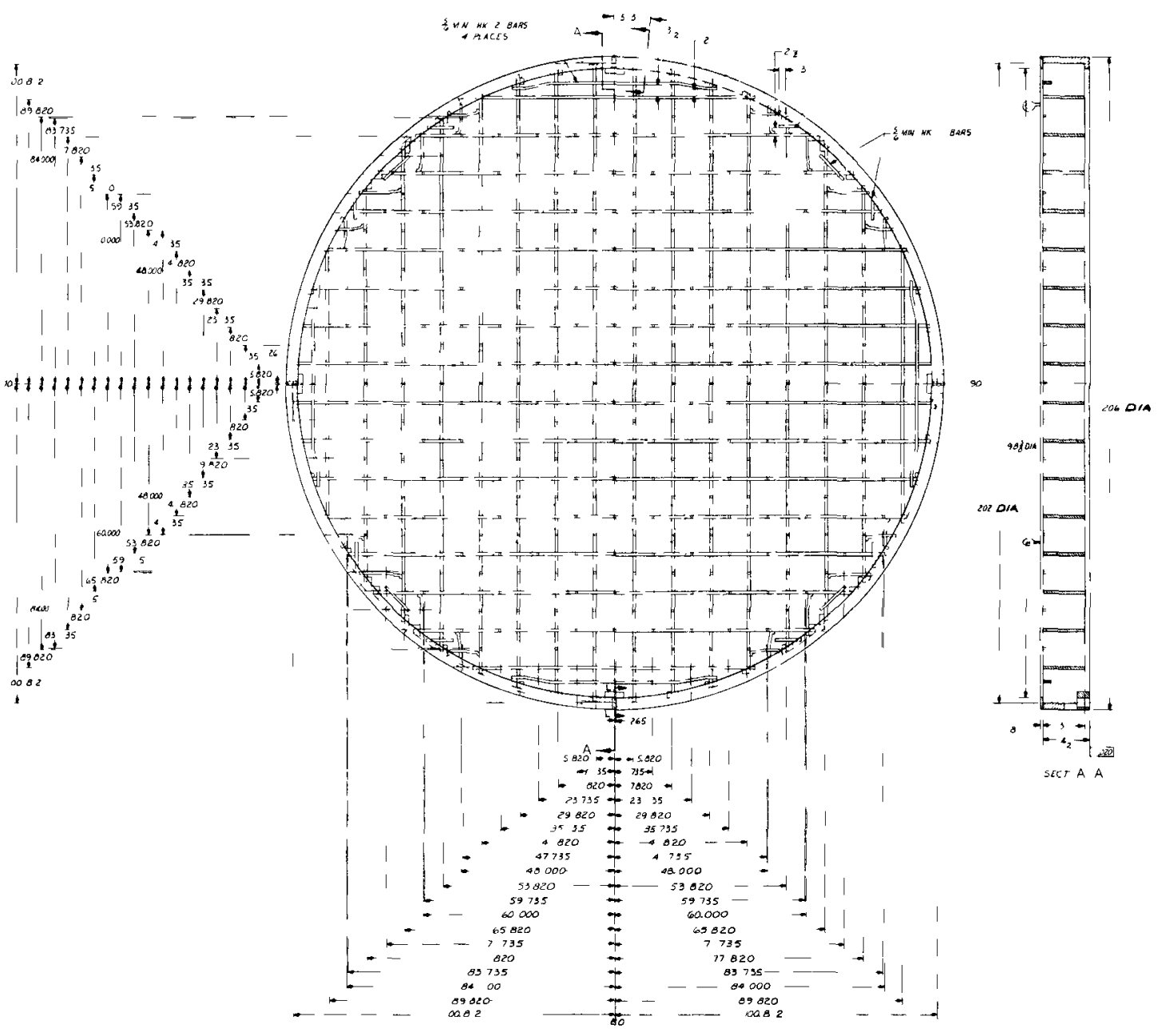




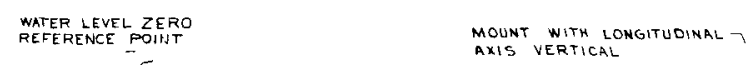
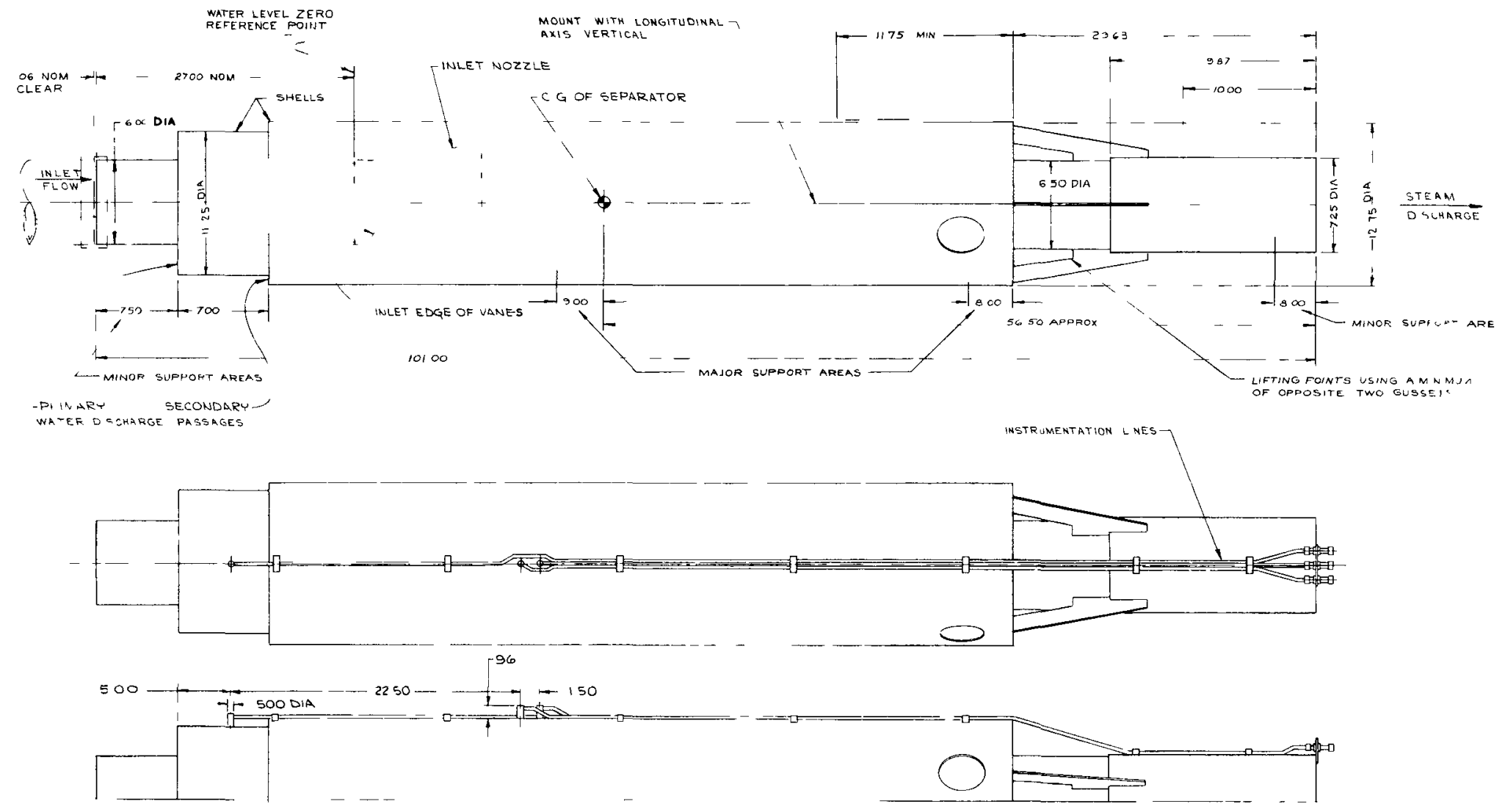

Figure 28. Steam Separator Outline Drawing 

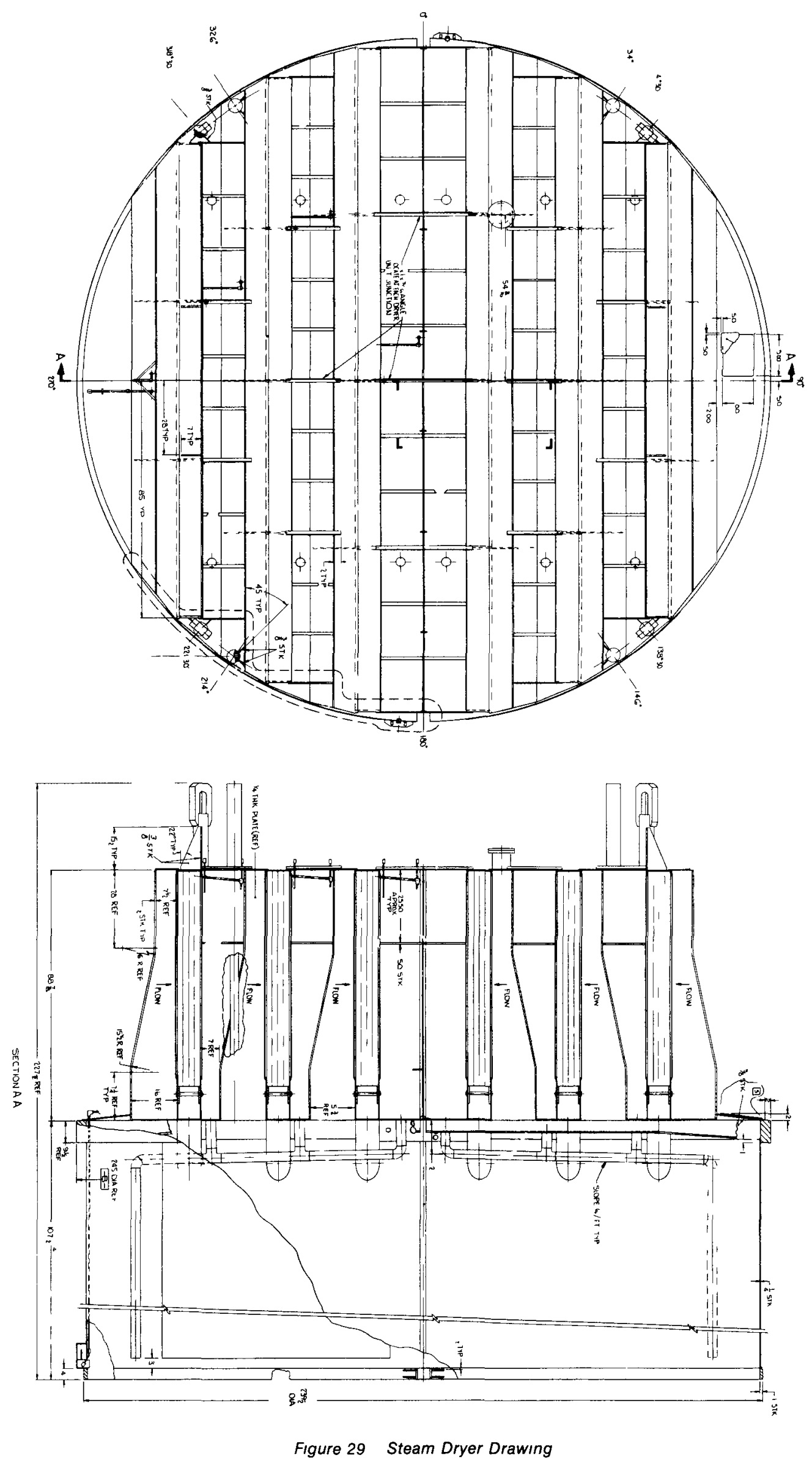

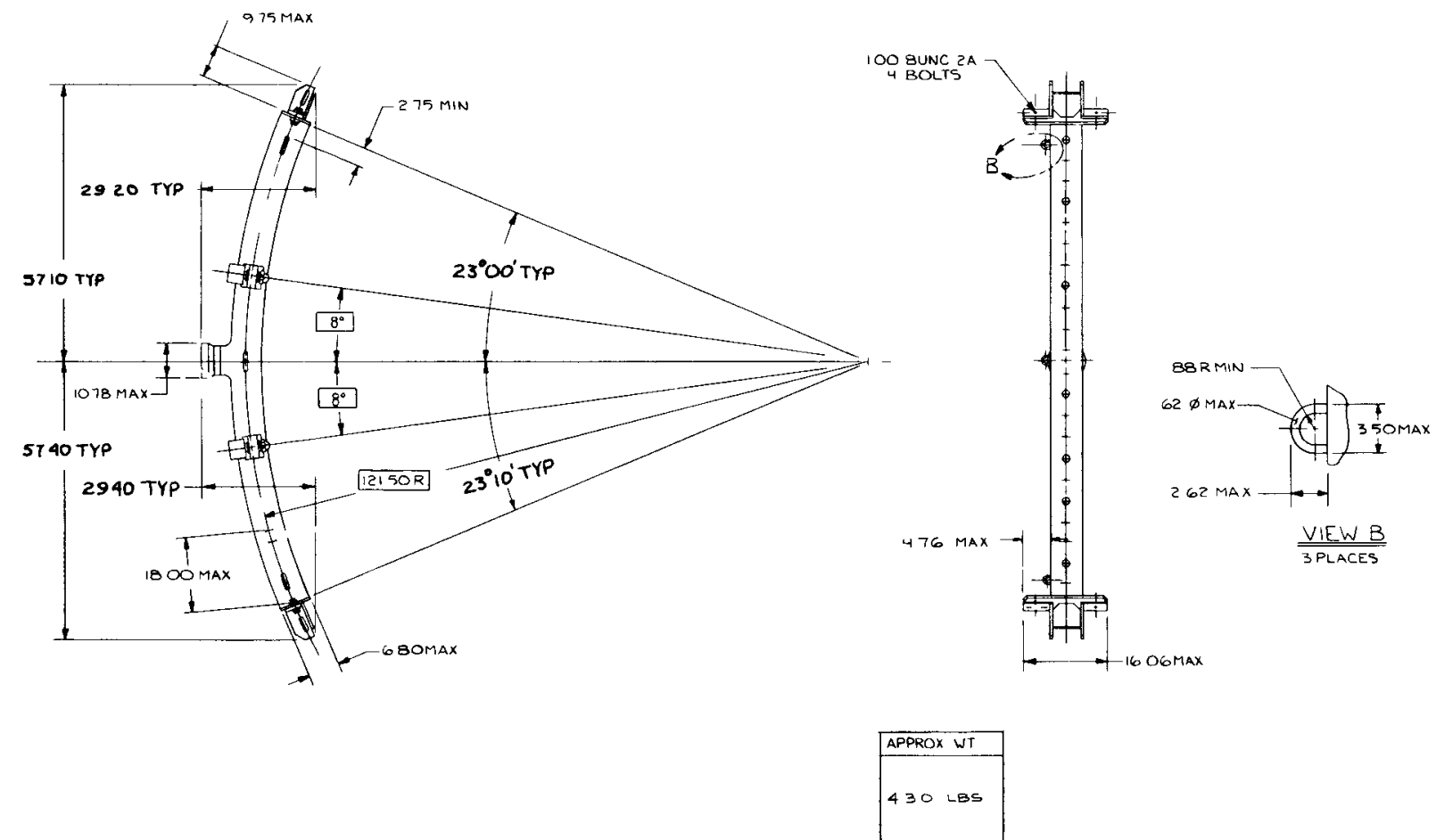

Figure 30. Feedwater Sparger Outline 


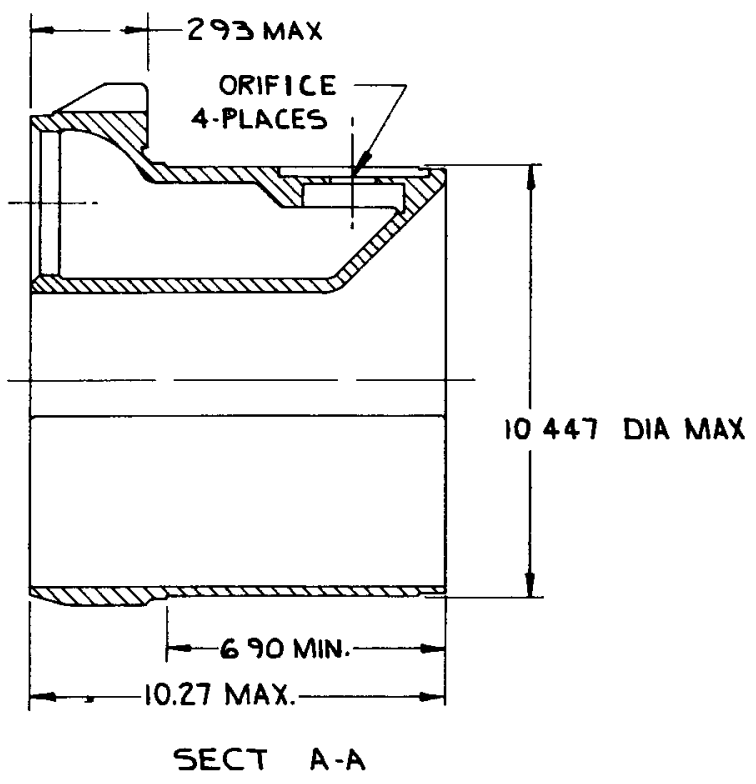

Figure 31 Orificed Fuel Support 


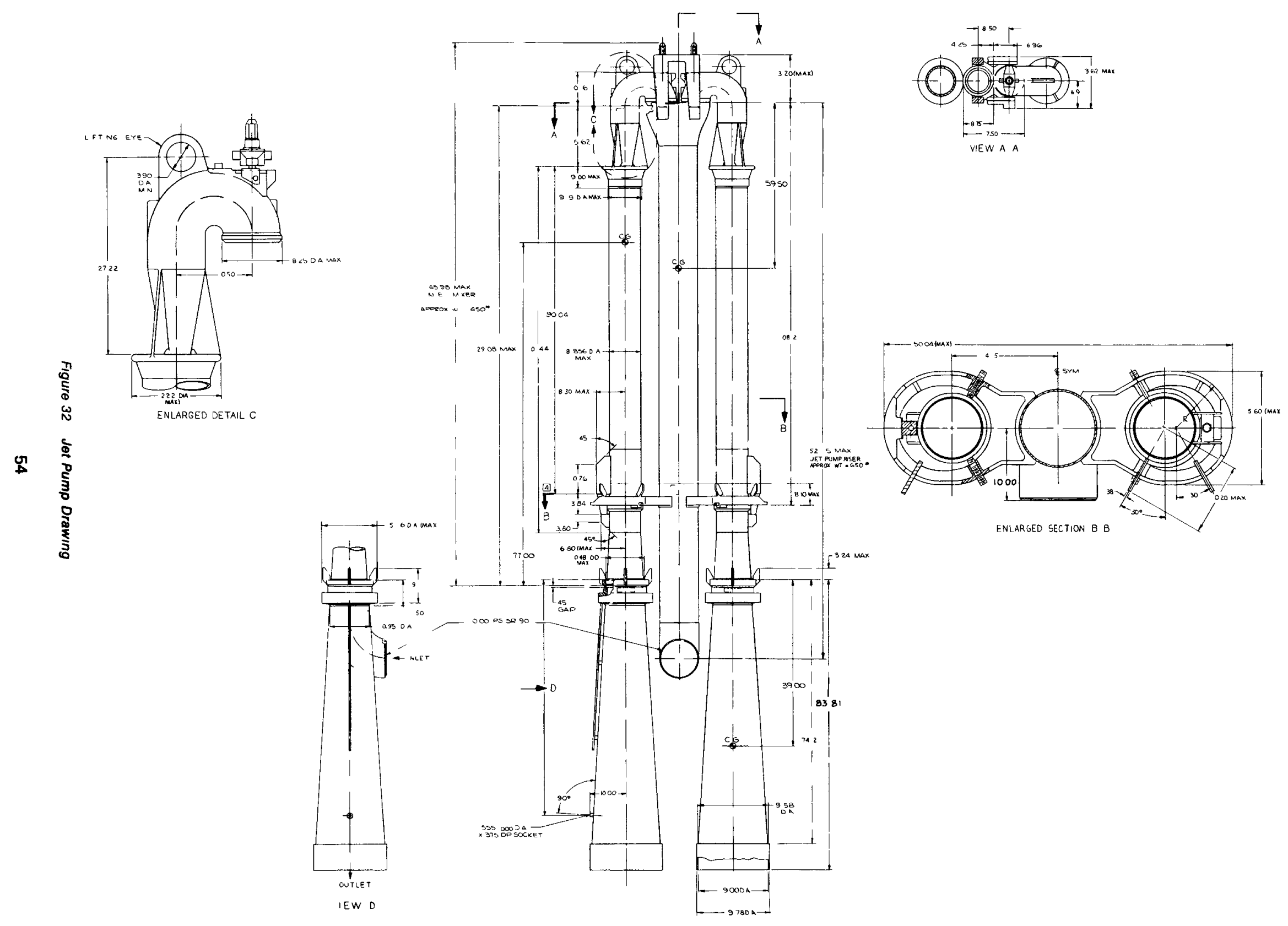

0 


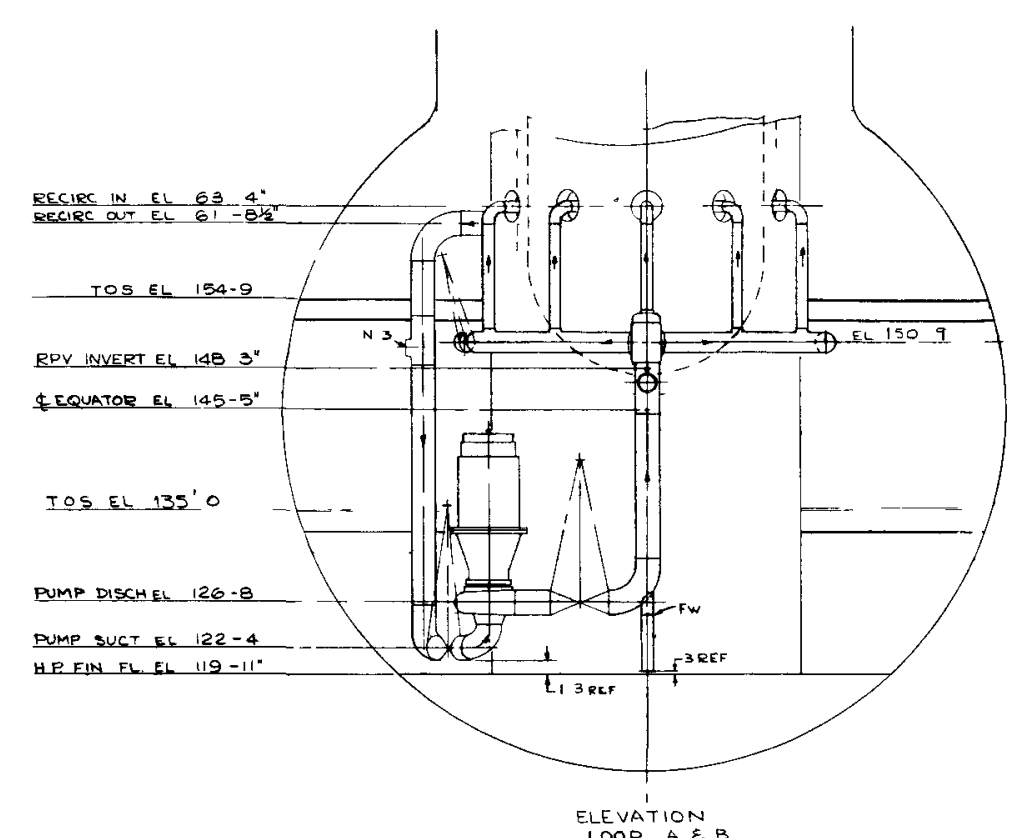

EeEvation
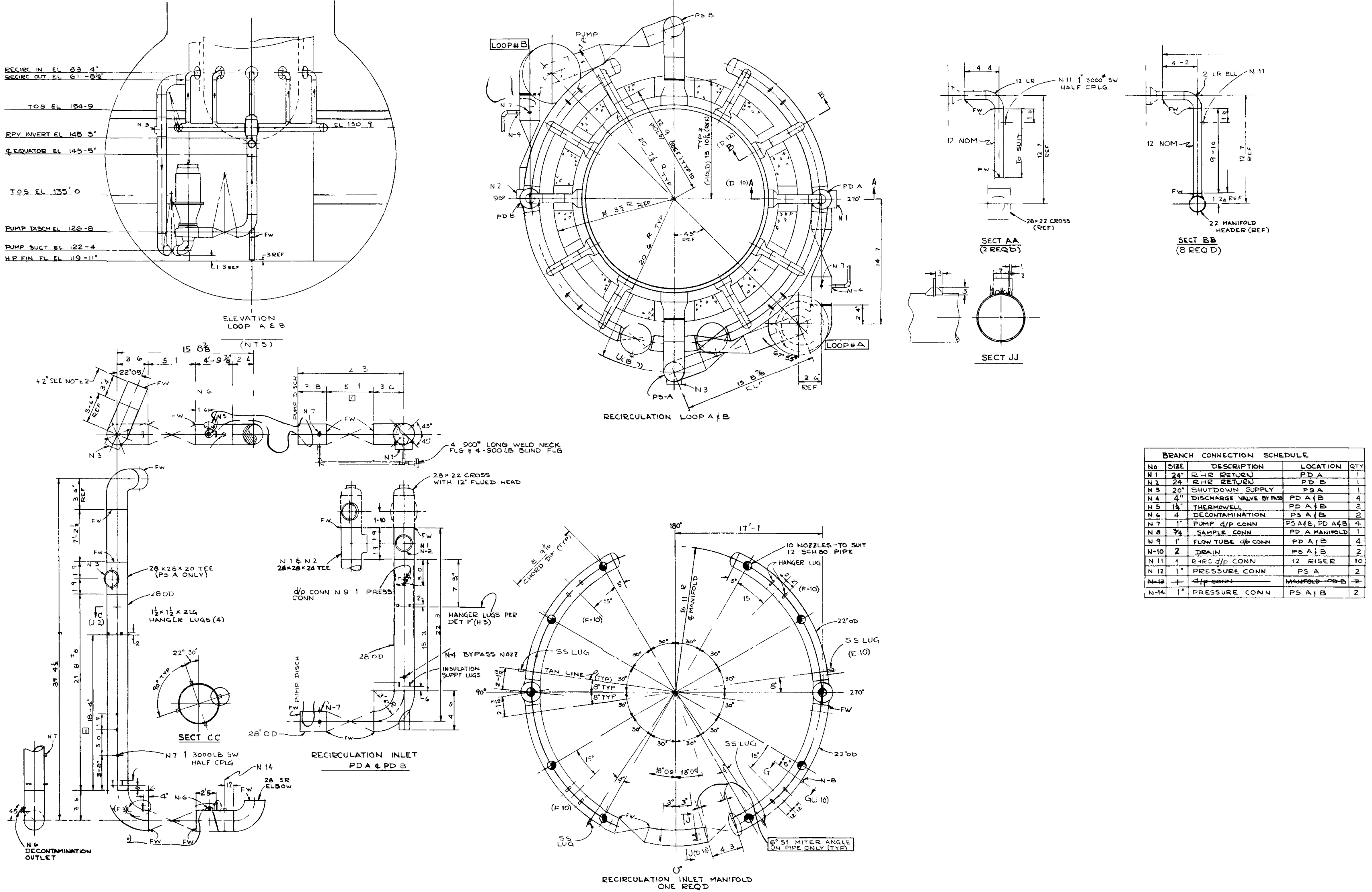

Figure 33. Recirculation Loop Piping 


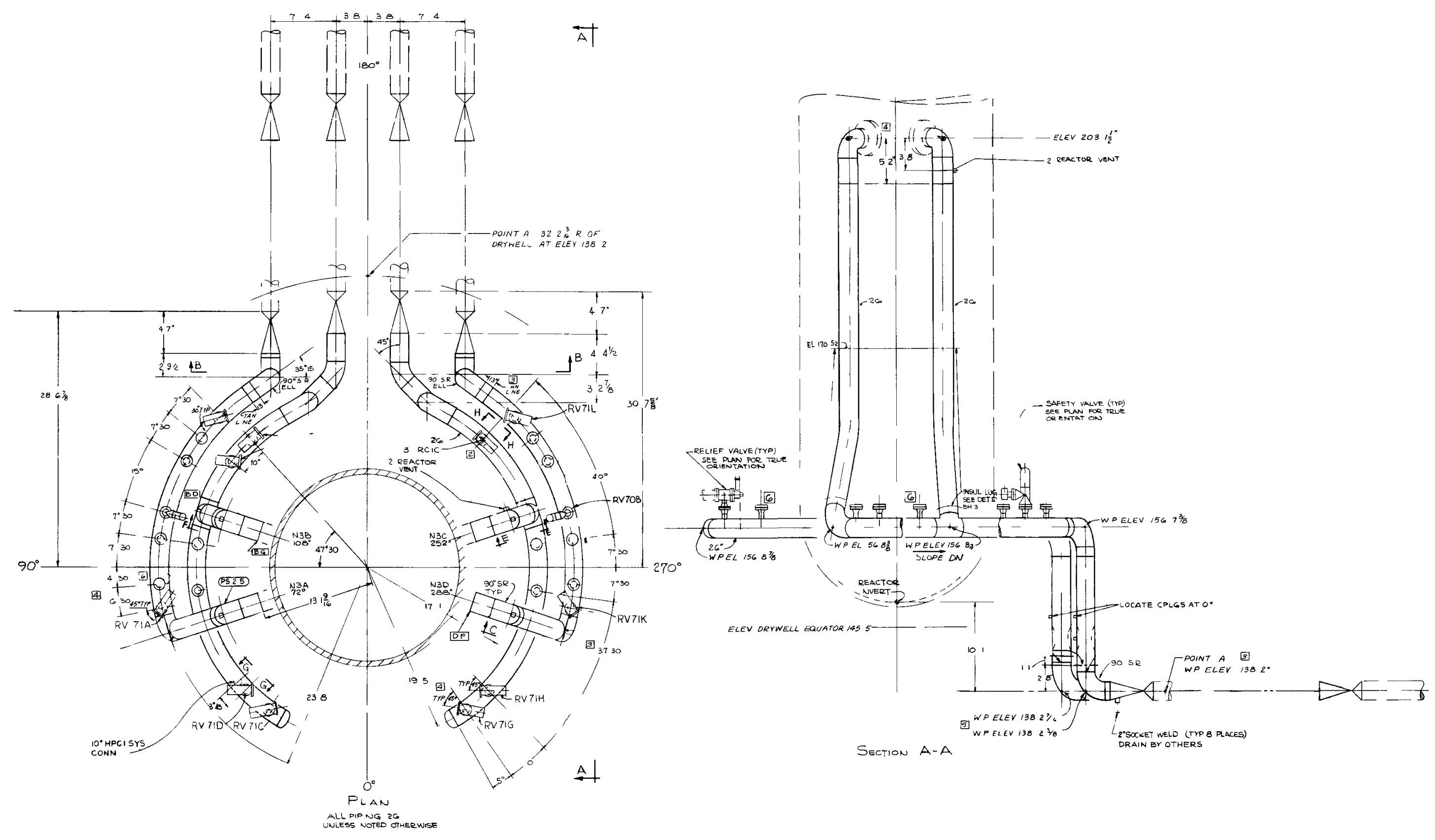

Figure 34. Primary Steam Pipıng 


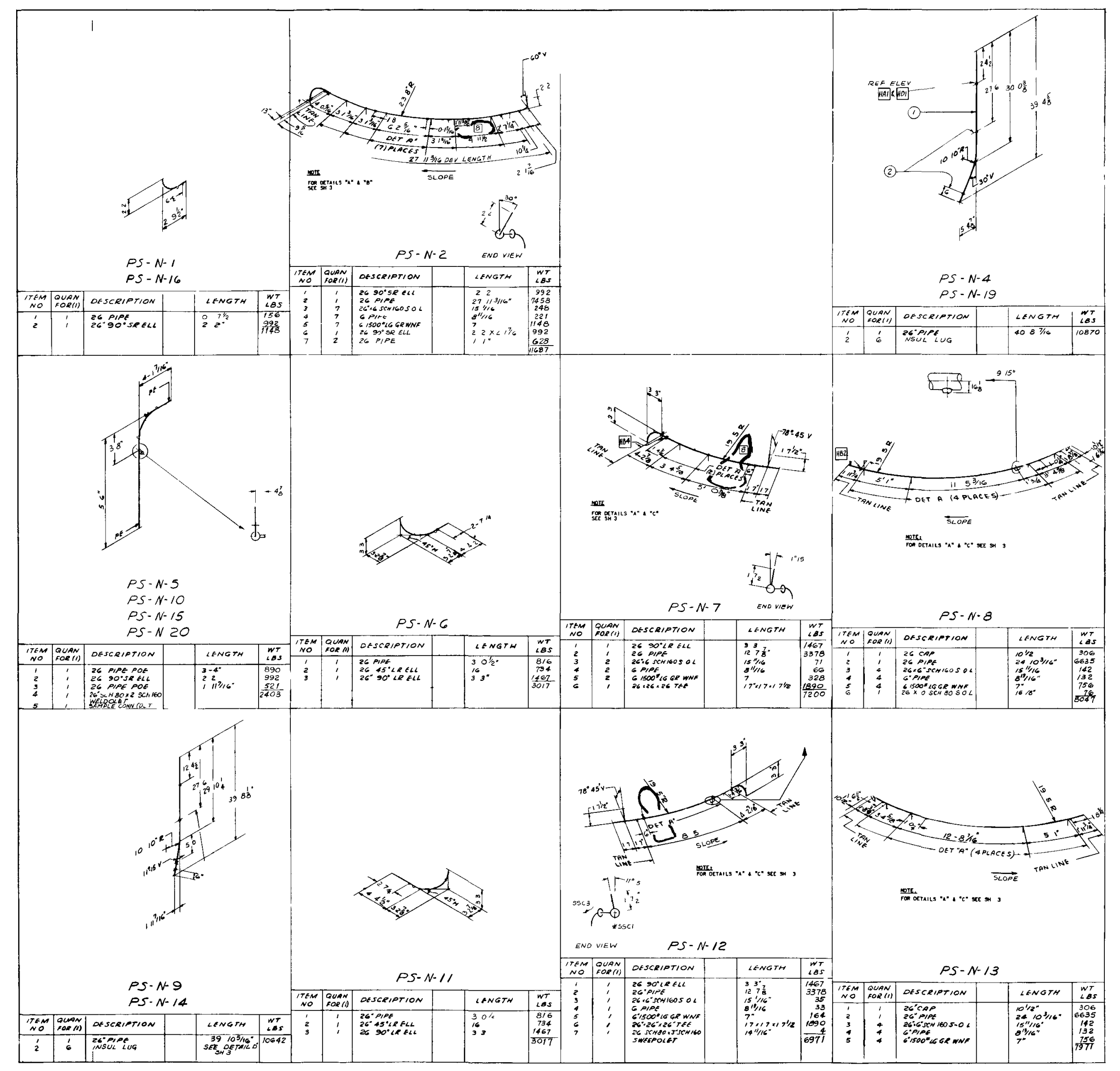

Figure 35. Primary Steam Piplng (Continued) 


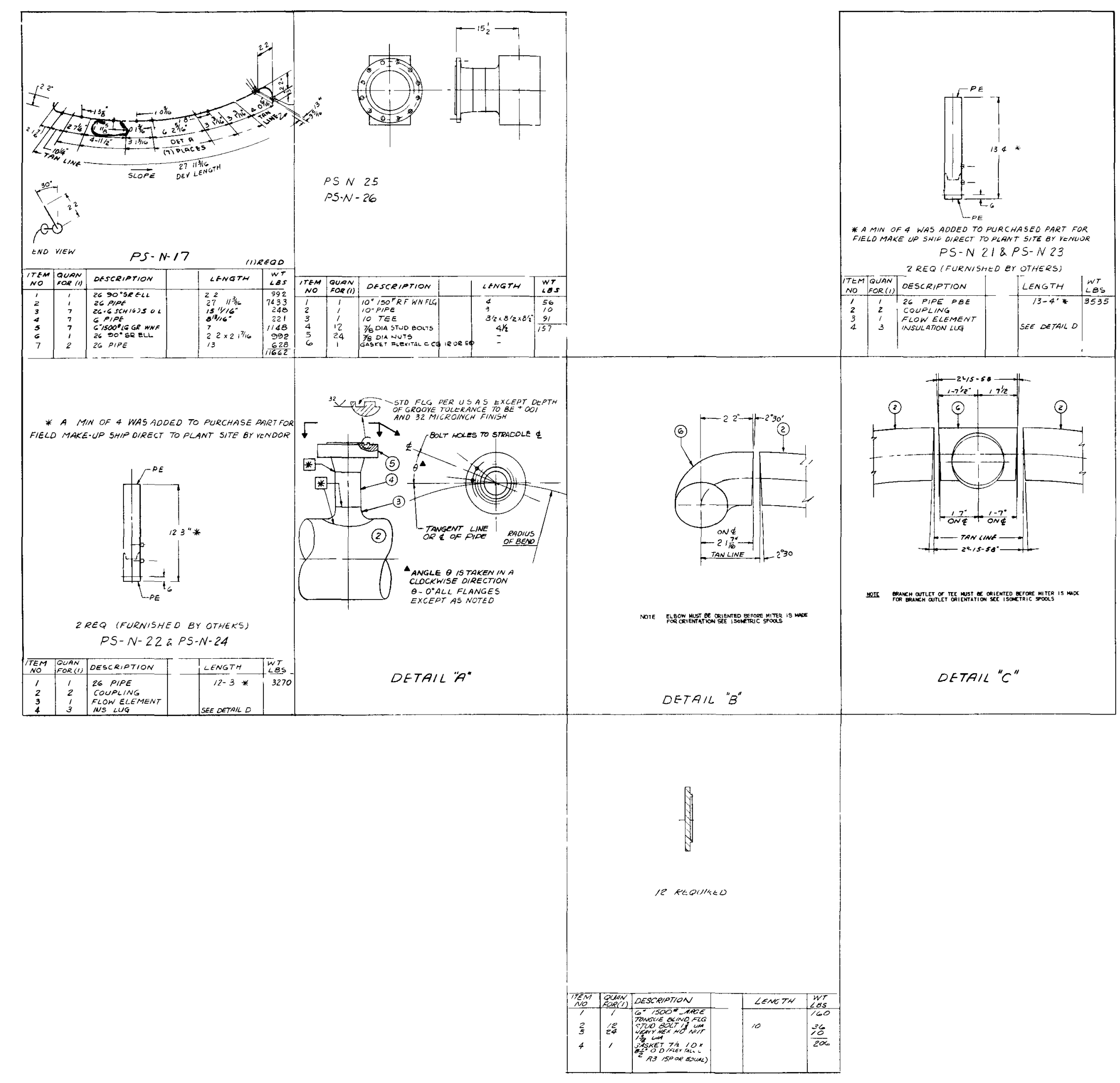

Figure 36. Primary Steam Piping (Continued) 


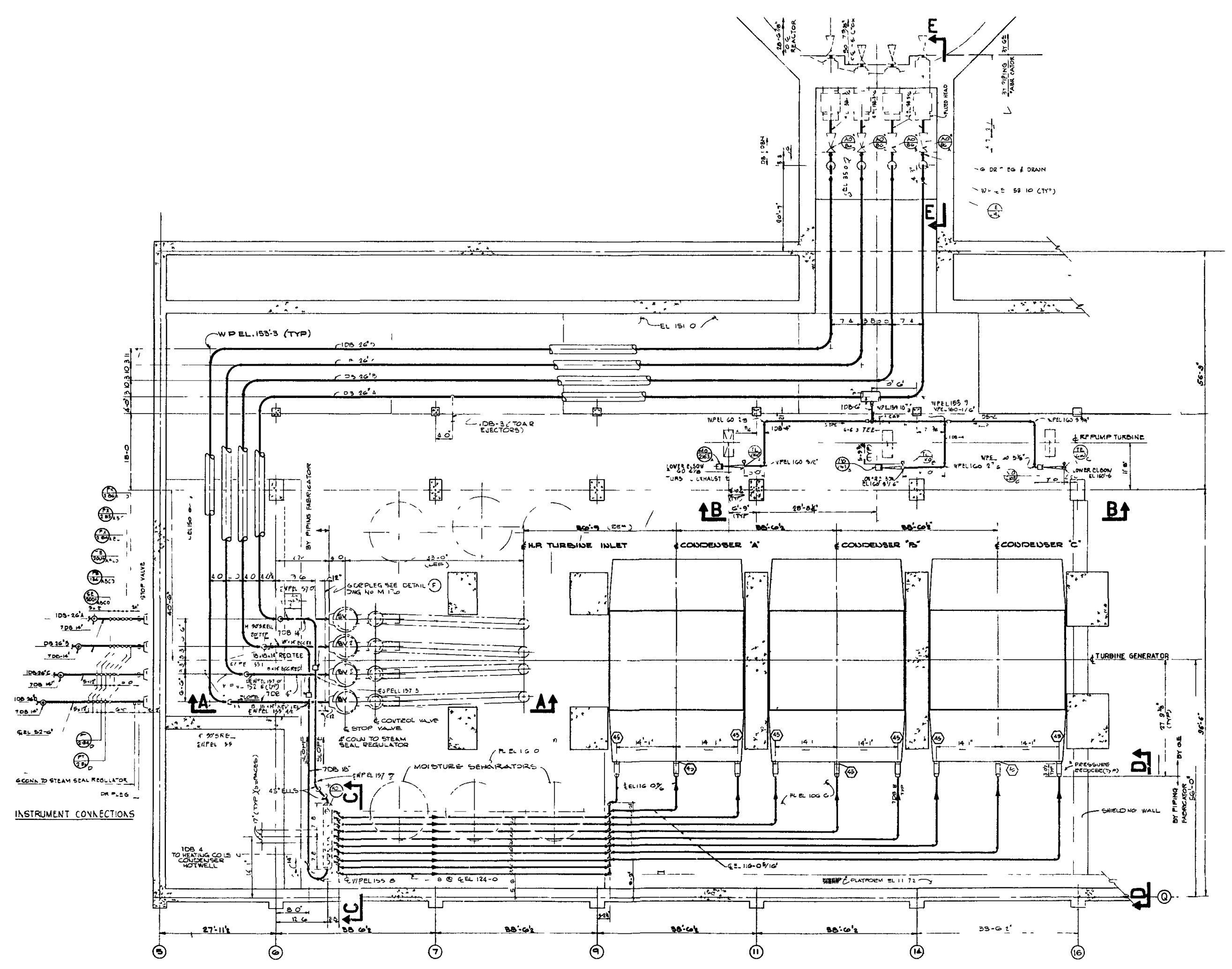

(N) $\rightarrow$

Figure 37. Main Steam and Bypass Line Piping 

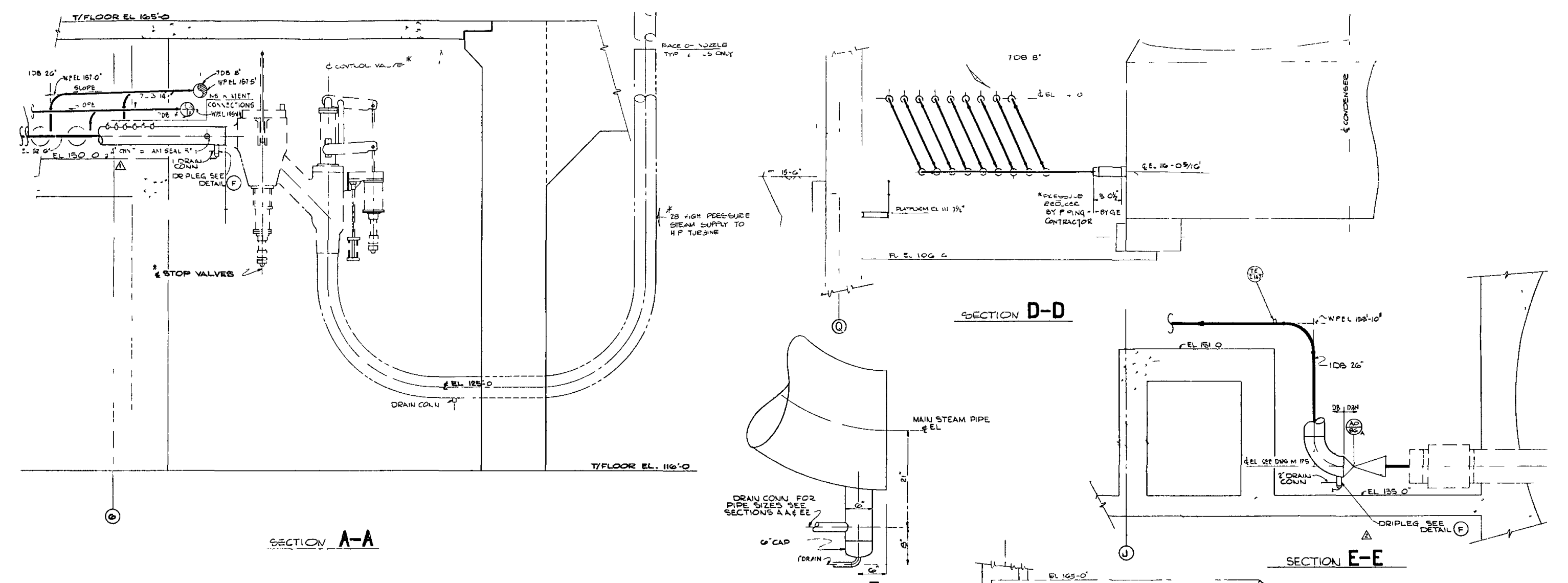

DRIPLEG DETAIL F

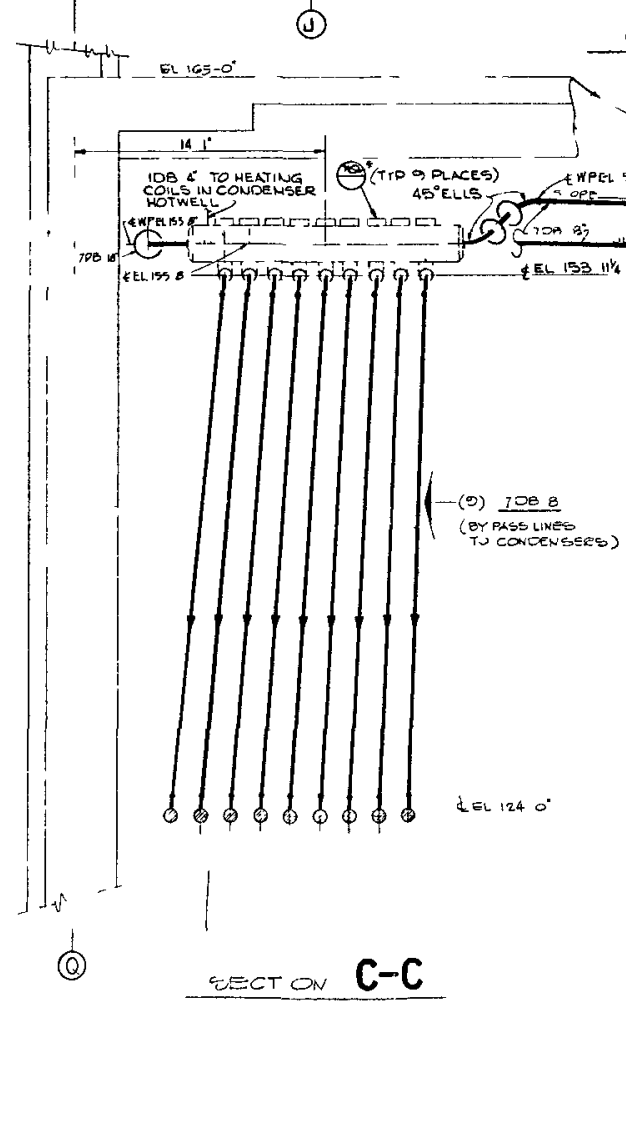

Figure 38. Main Steam and Bypass Line Piping (Continued) 


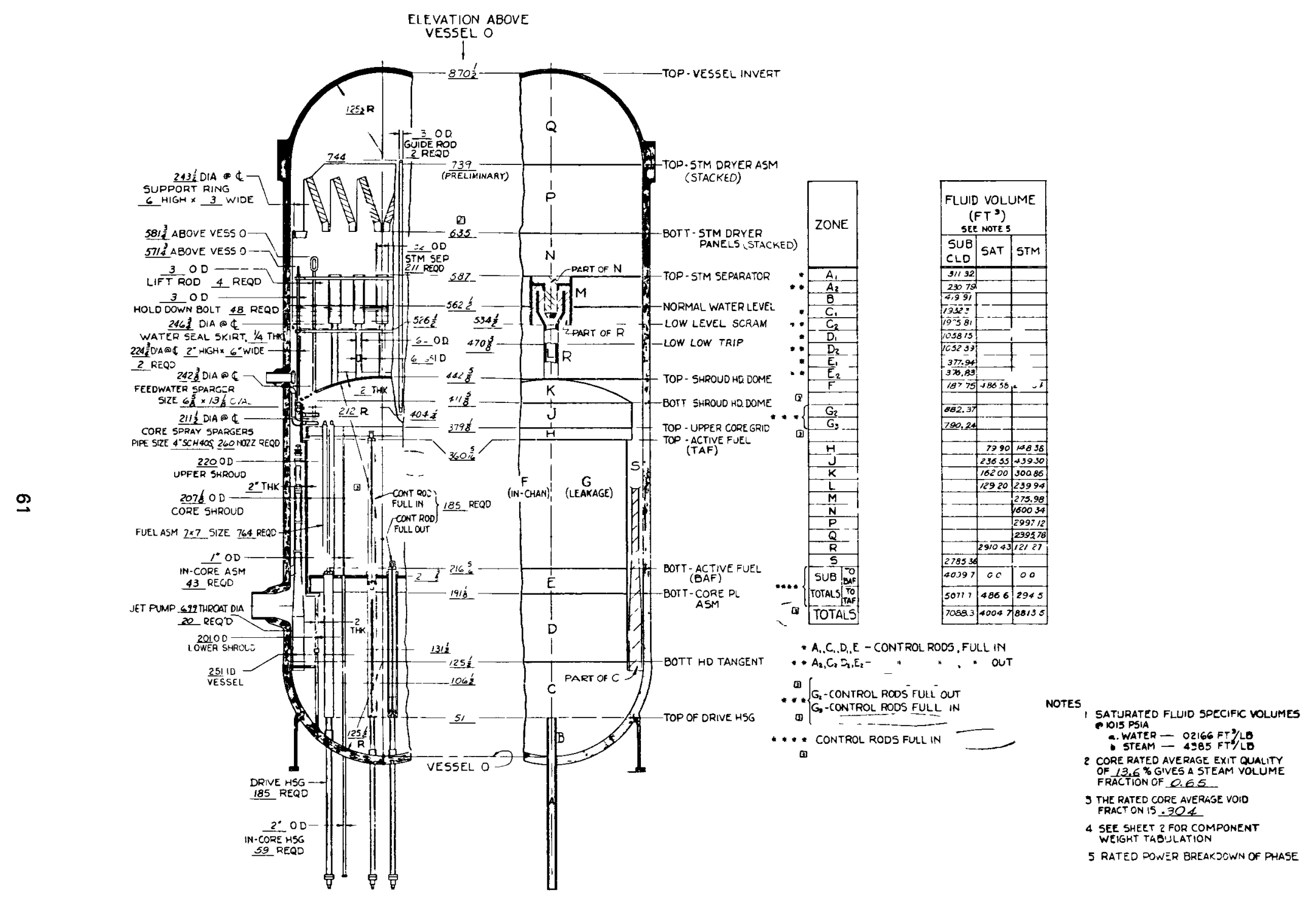

Figure 39. Reactor Primary System Weights and Volumes 


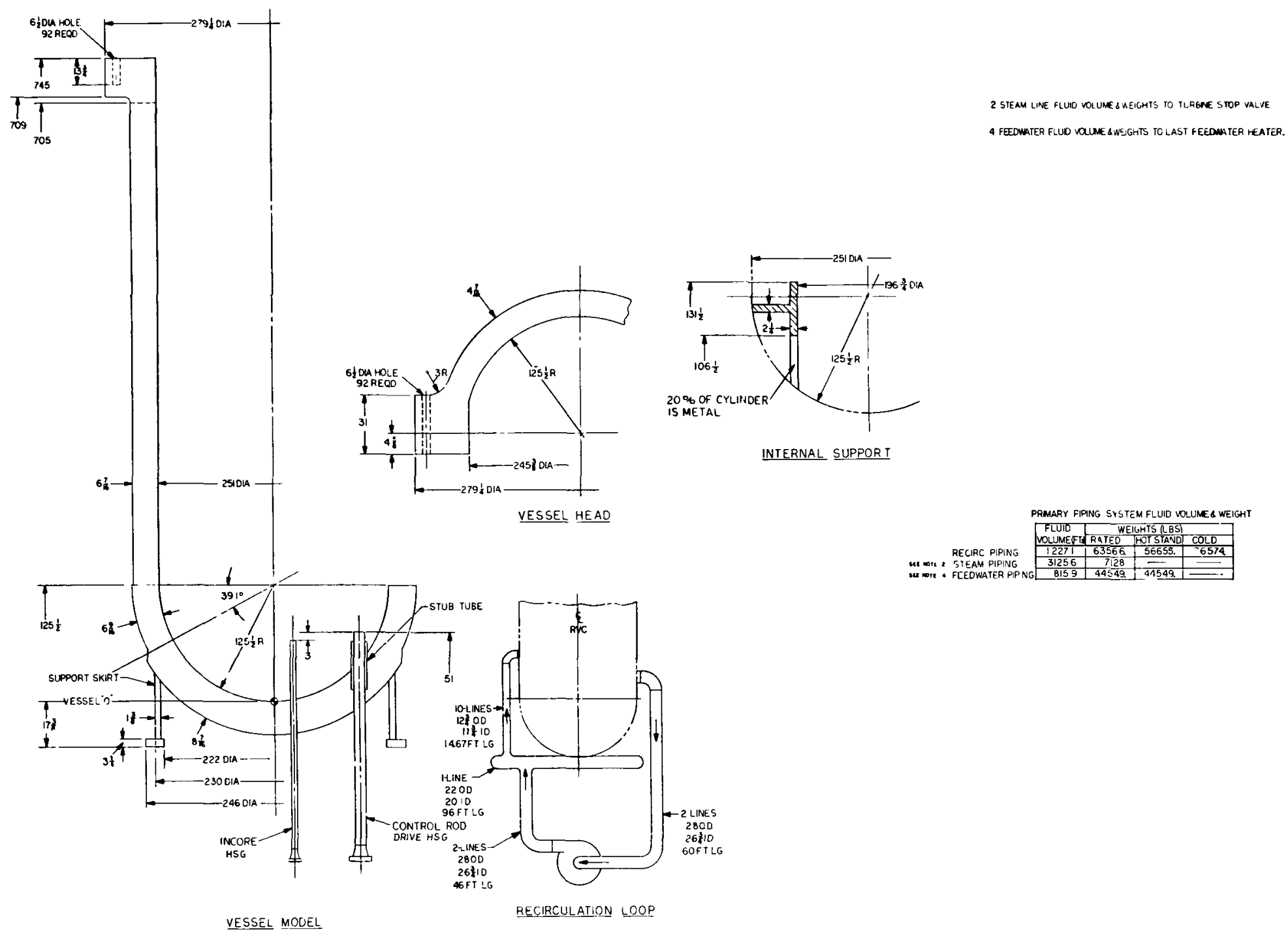

Figure 40. Reactor Primary System Weights and Volumes (Continued) 


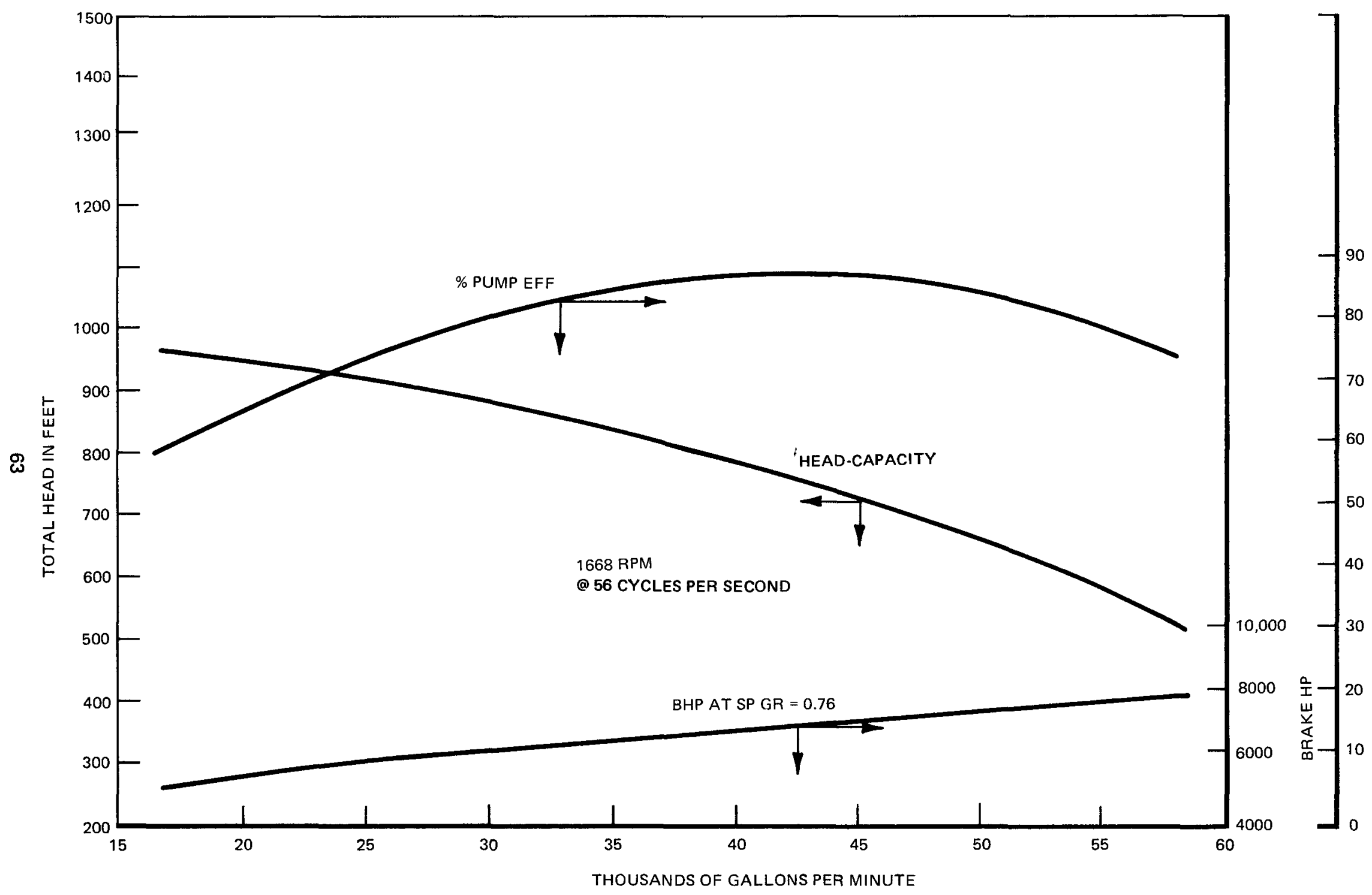

Figure 41. Recirculation Pump Characteristics at Rated Pump Speed 


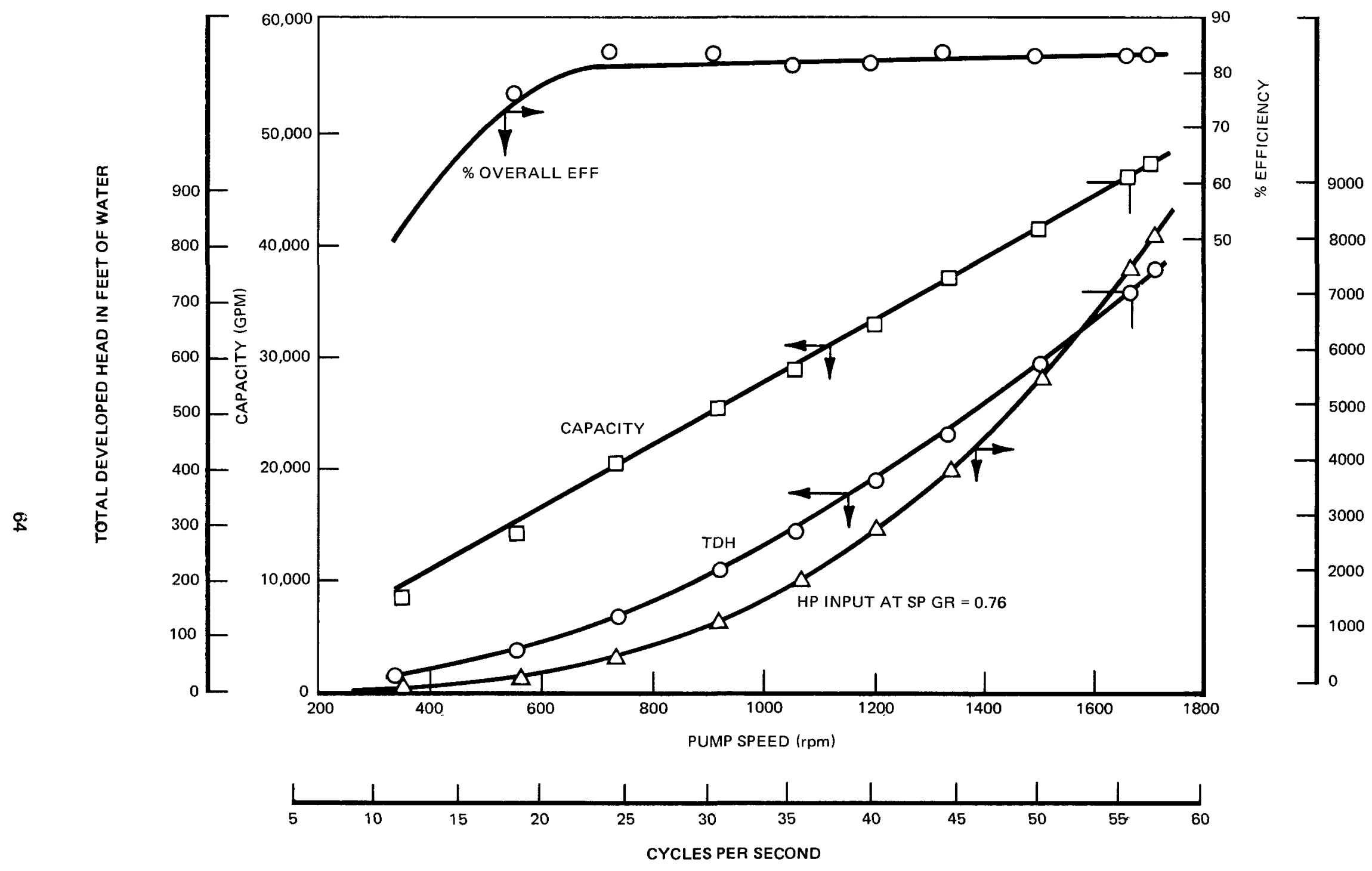

Figure 42. Recirculation Pump Characteristics at Various Pump Speeds 


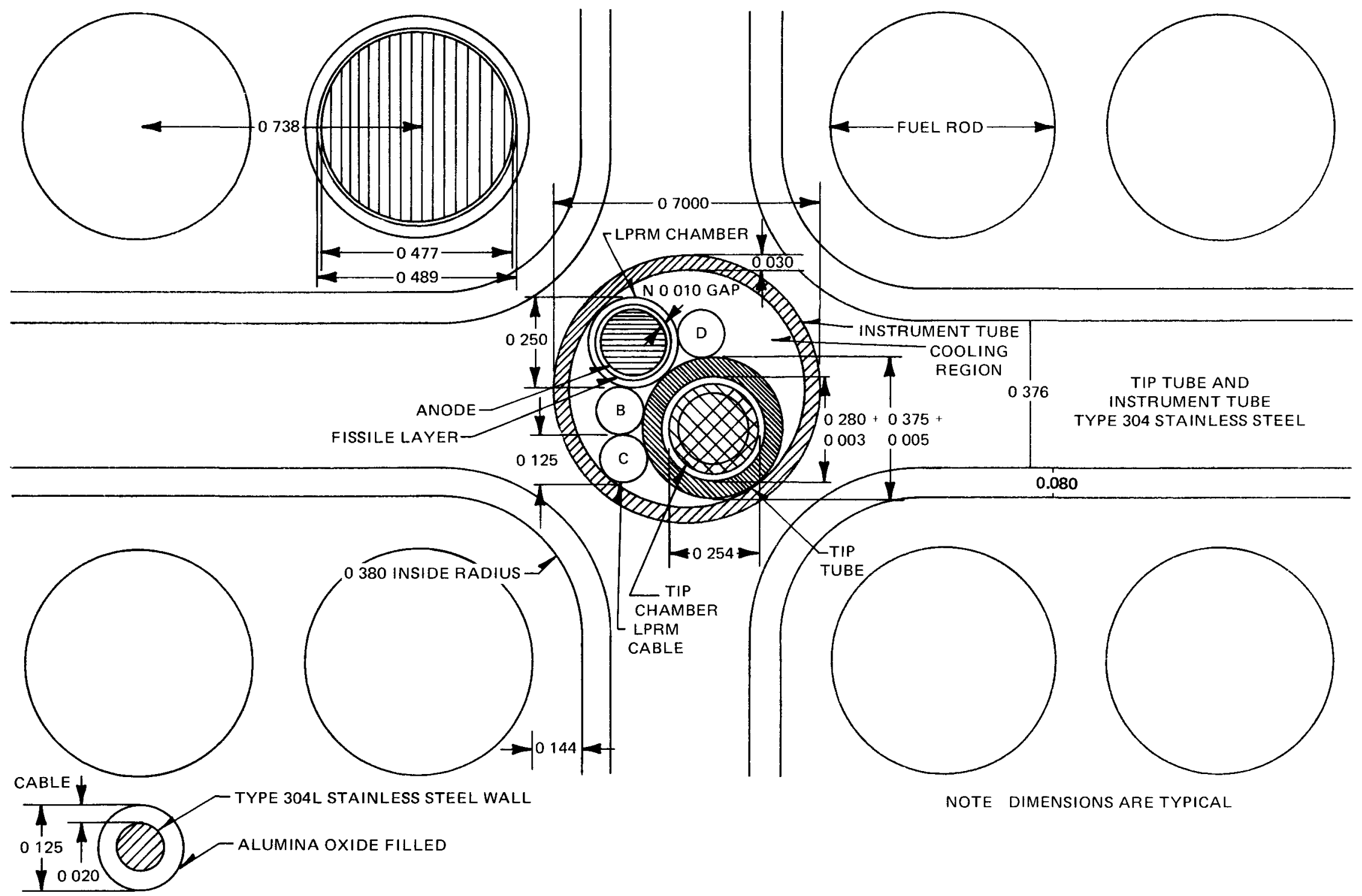

FIgure 43. TIPILPRM In-Core Assembly Cross Section 


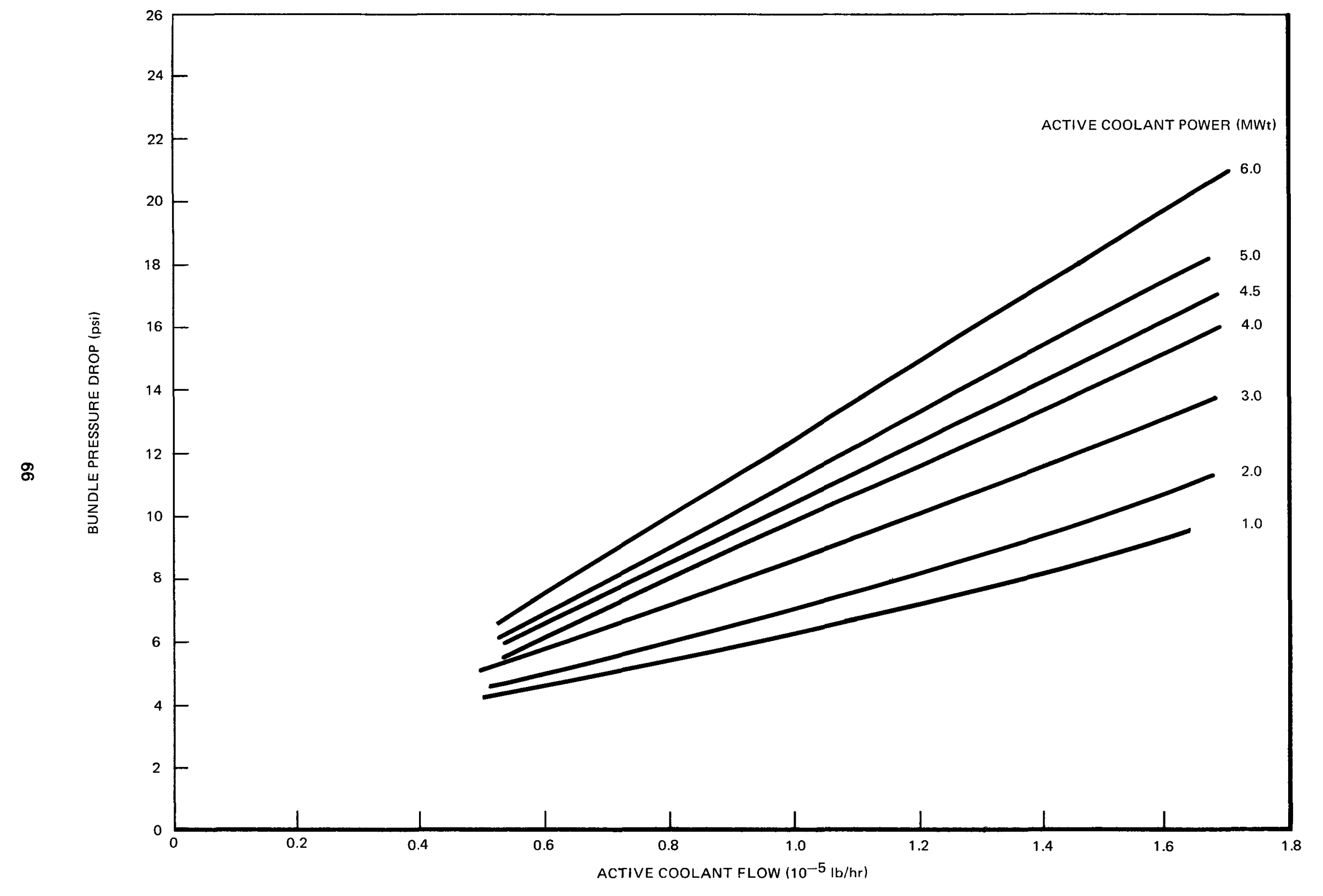

Figure 44. Flow Characteristics $7 \times 7$ Fuel Assemblies, 20 Btullb Subcooling 

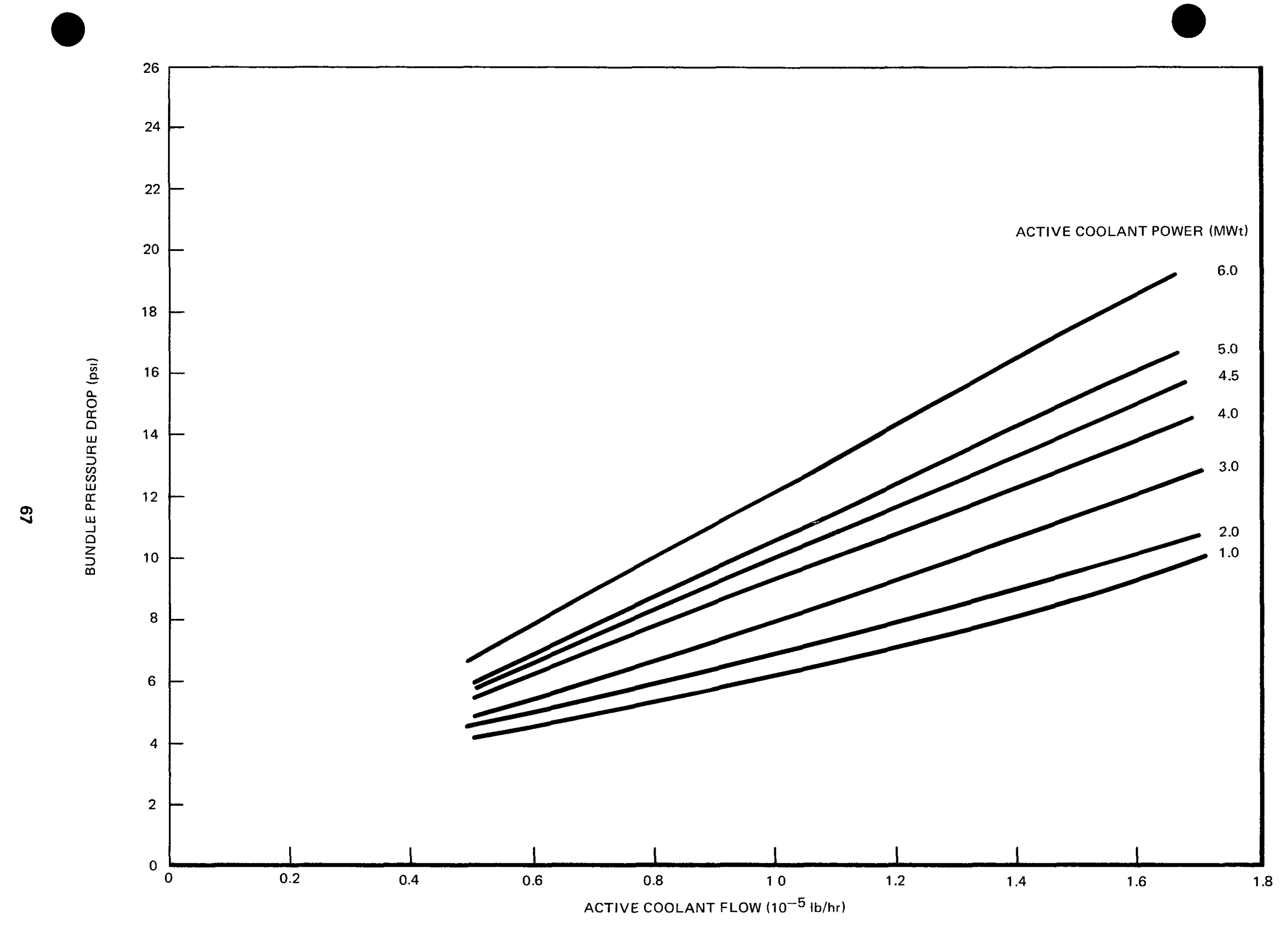

Figure 45. Flow Characteristics $7 \times 7$ Fuel Assemblies, 30 Btullb Subcooling 


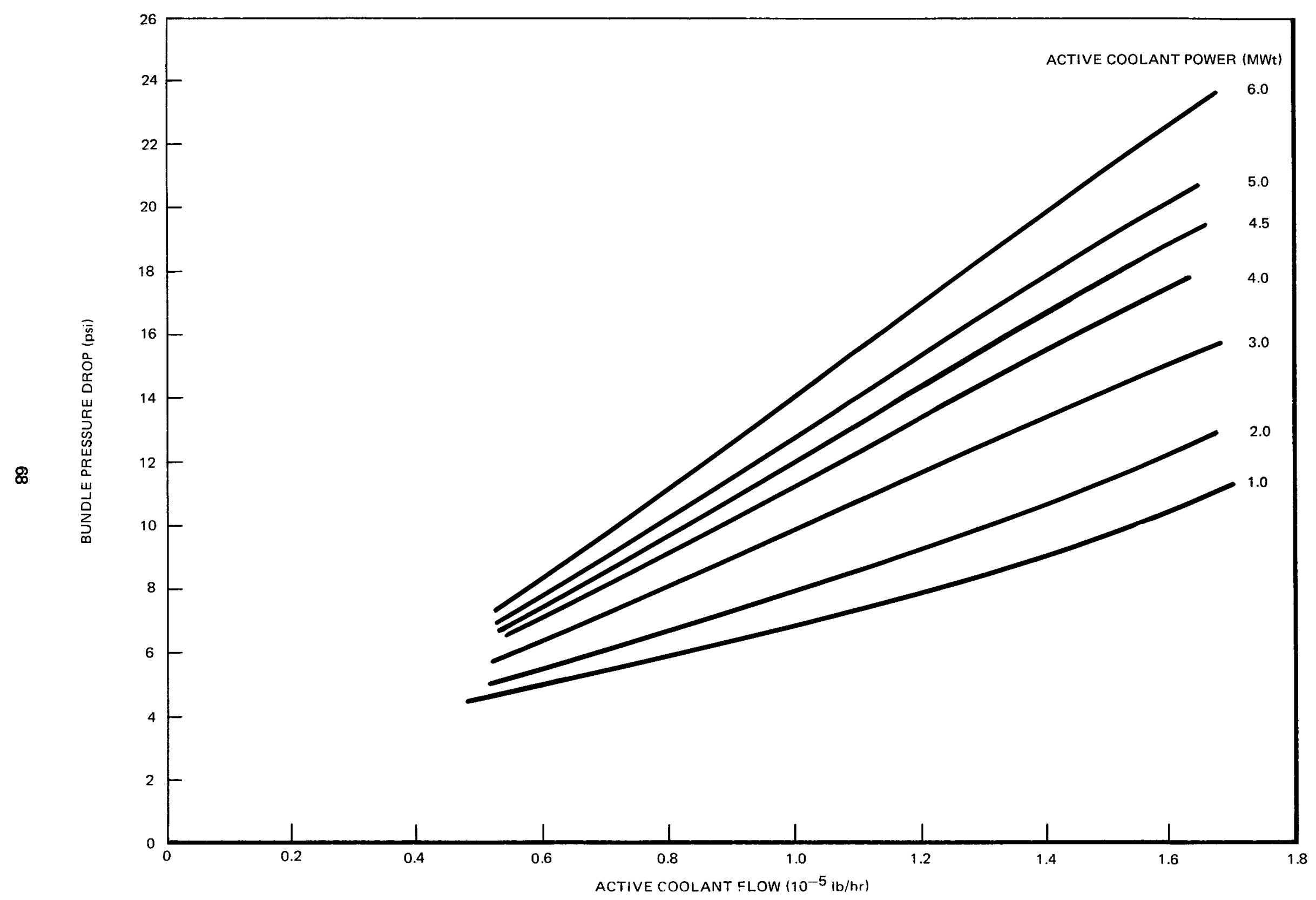

Figure 46. Flow Characteristics $8 \times 8$ Fuel Assemblies, 20 Btu/lb Subcooling 


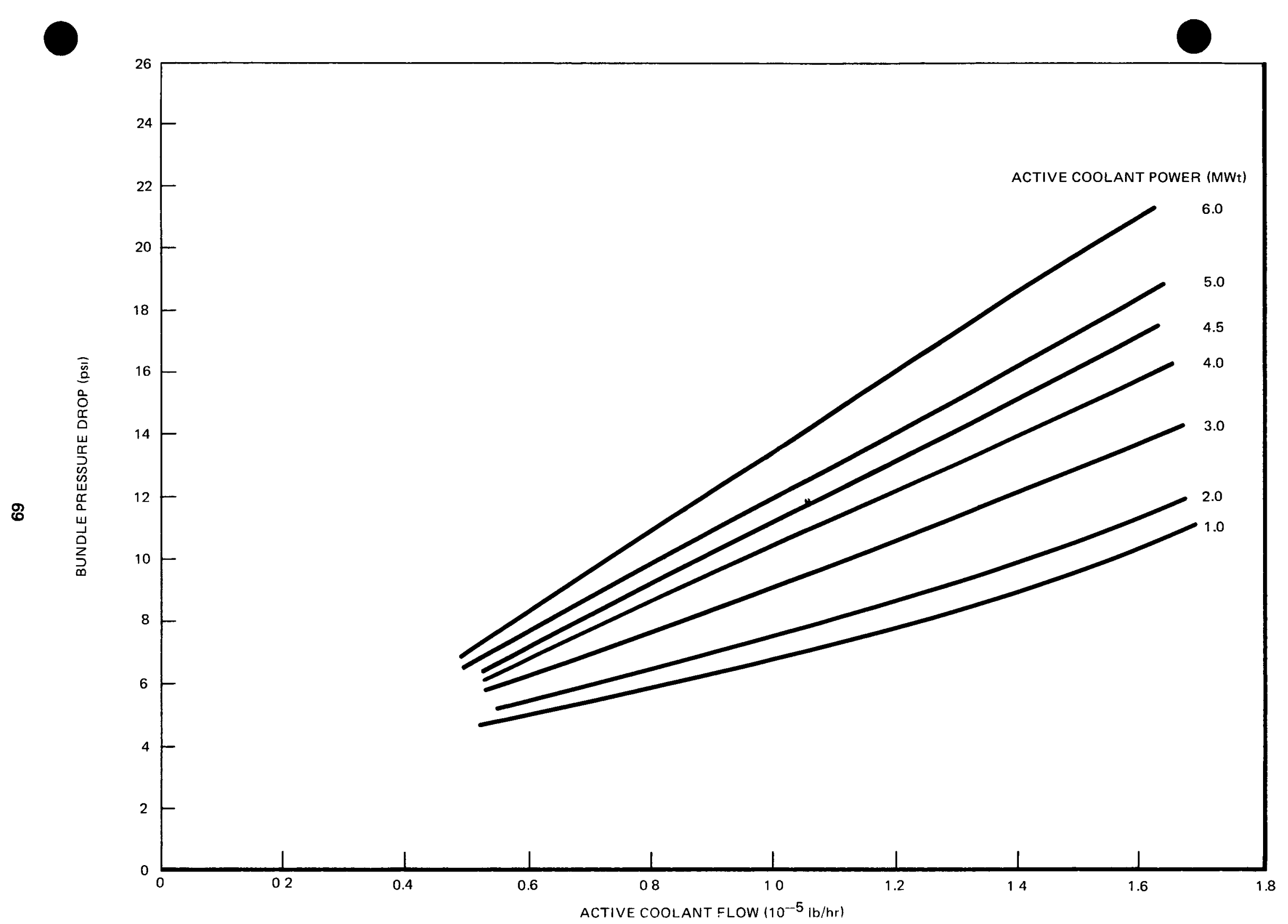

Figure 47. Flow Characteristics $8 \times 8$ Fuel Assemblies, 30 Btu/lb Subcooling 


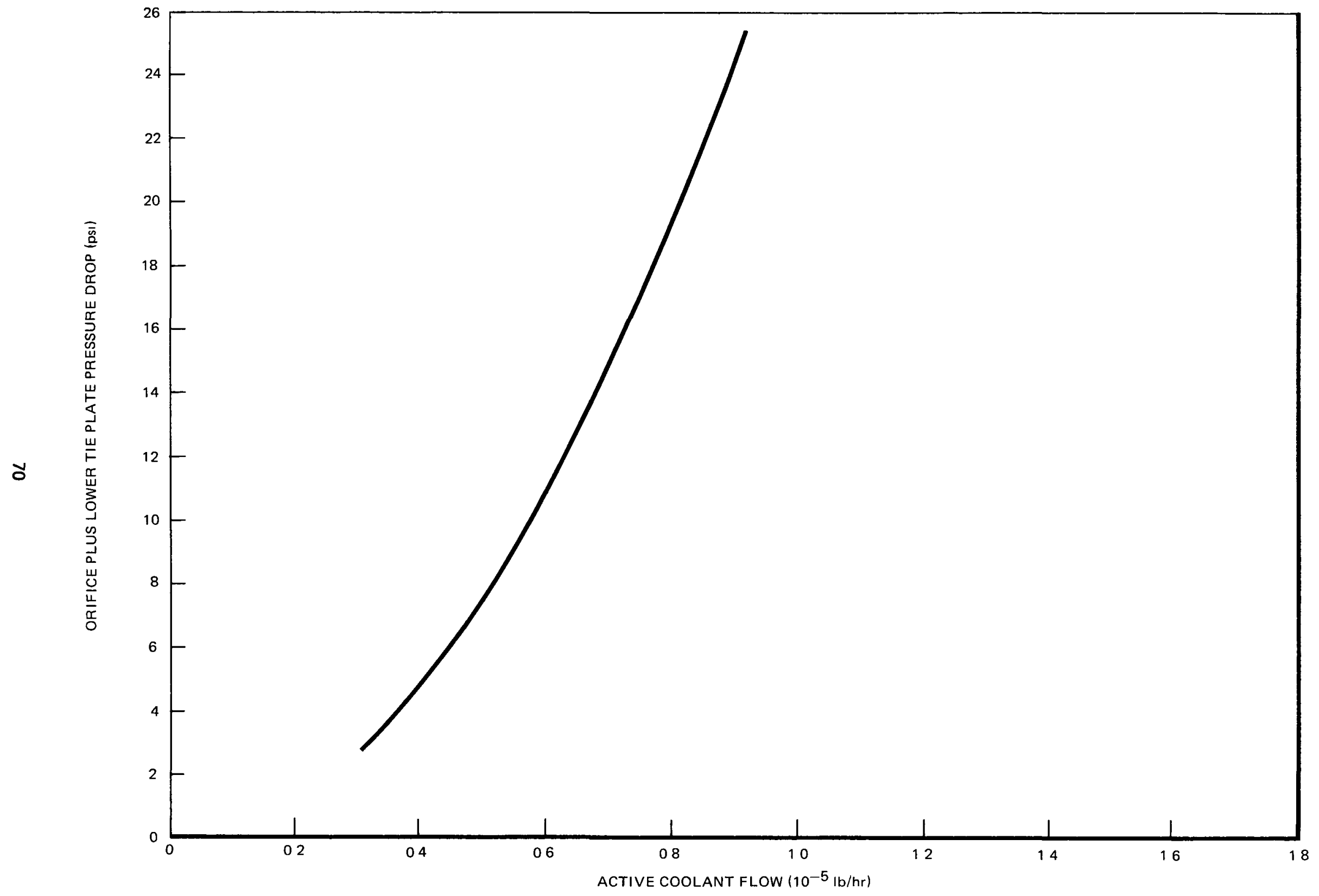

Figure 481469 in Orifice Diameter, 20 Btu/lb Subcoolıng 


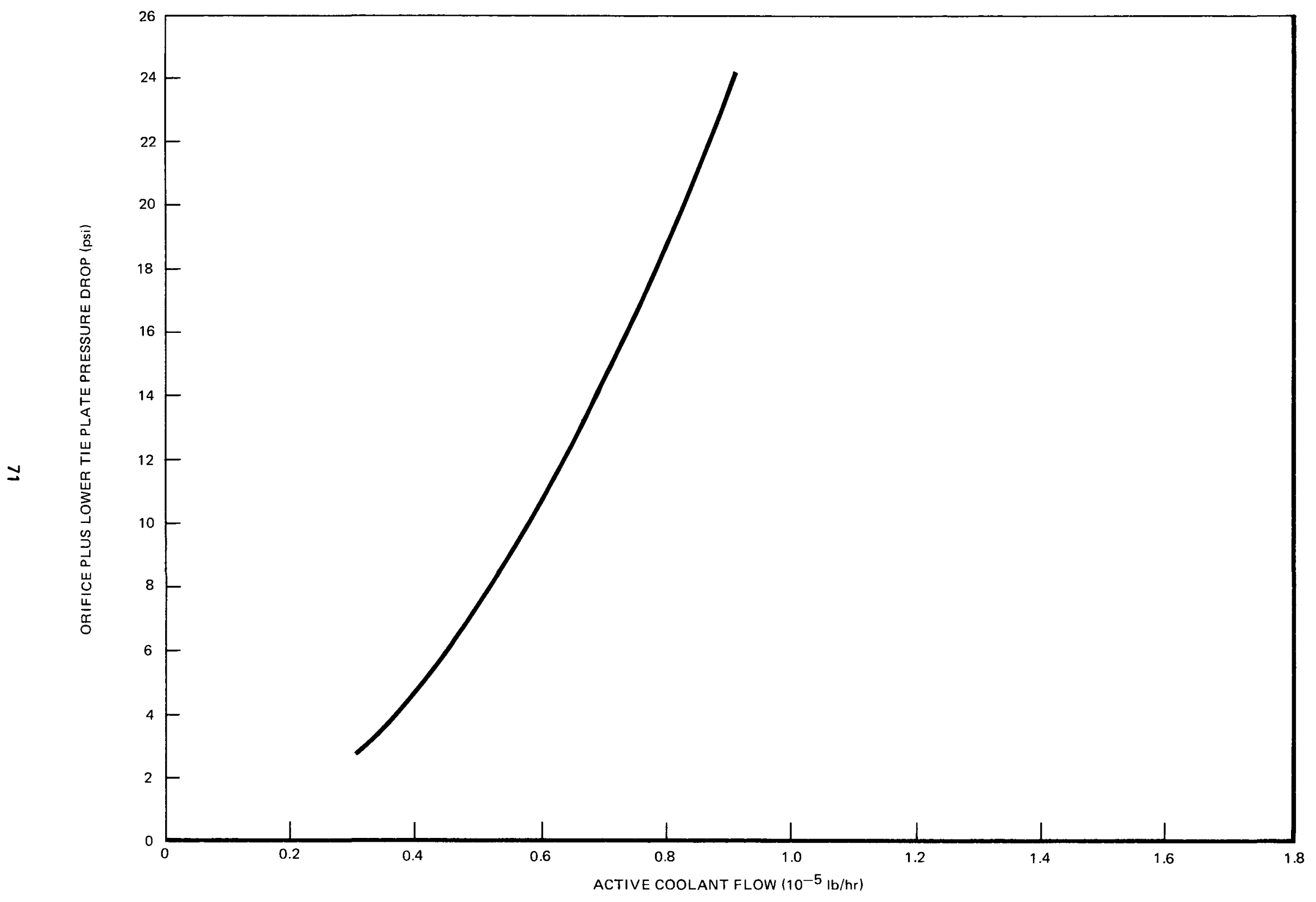

Figure 49. 1.469 in Orifice Diameter, 30 Btu/lb Subcooling 


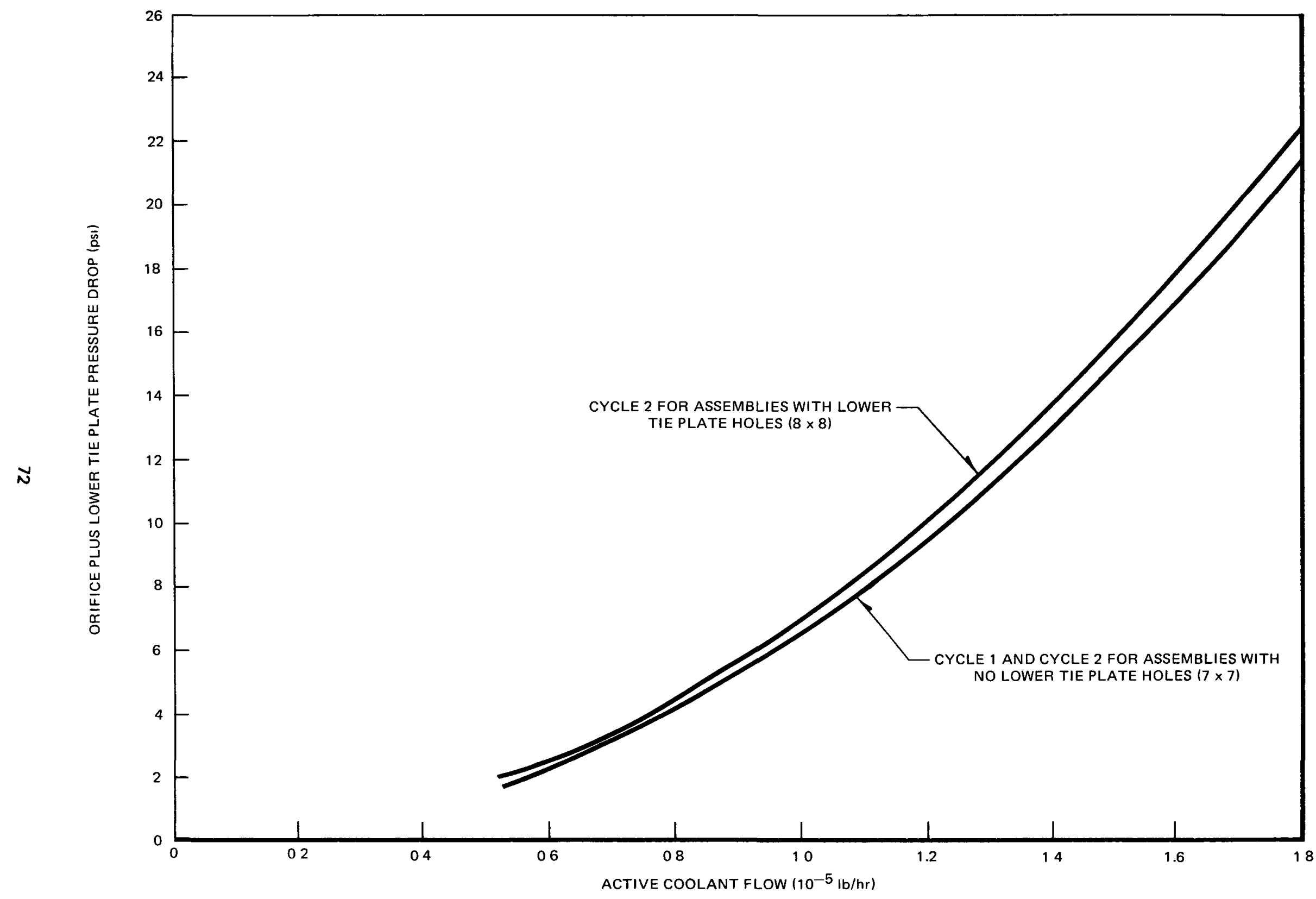

Figure 50. 2.211 in Orifice Diameter, 20 Btu/lb Subcoolıng 


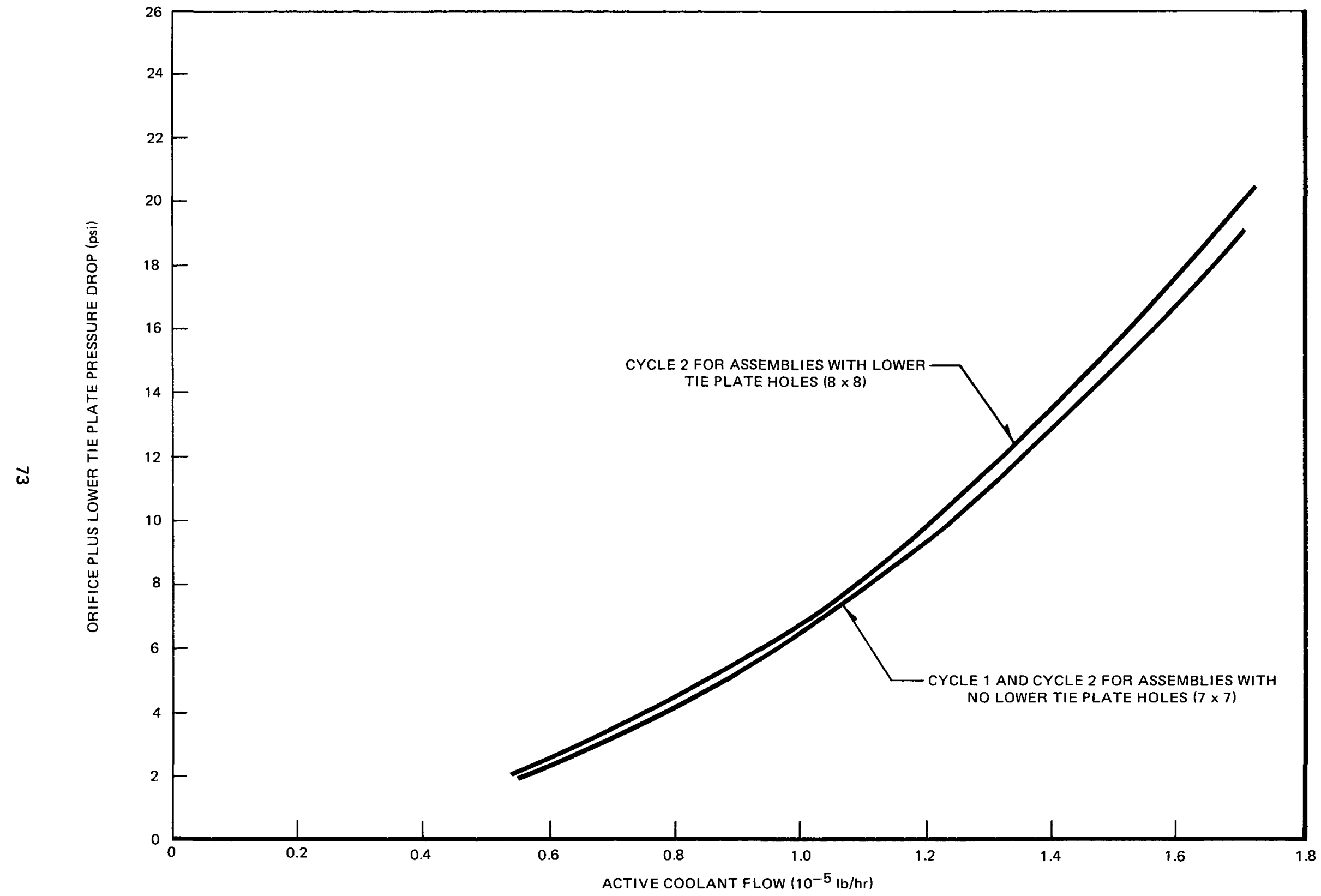

Figure 51. 2.211 in Orifice Diameter, 30 Btu/lb Subcooling 


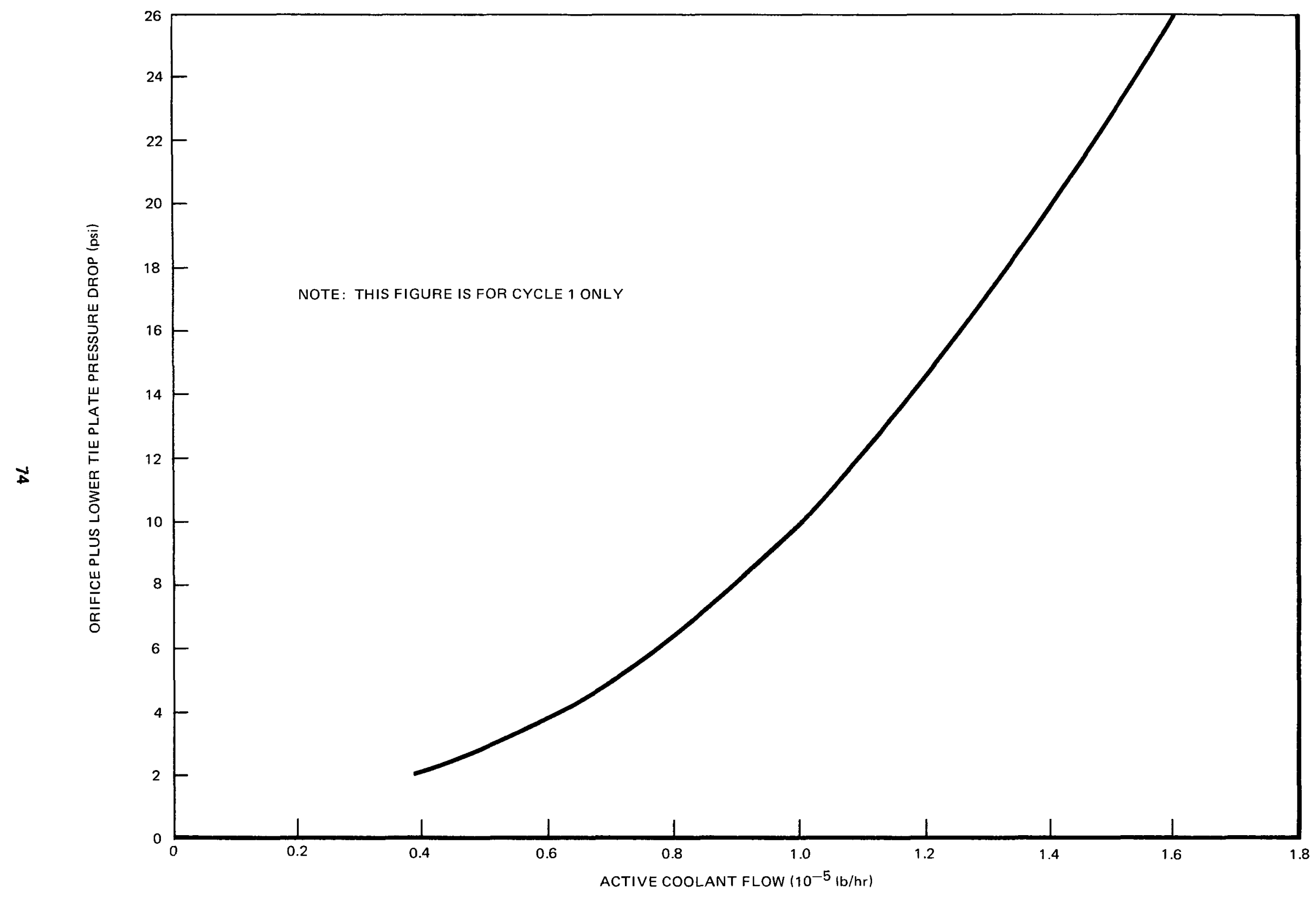

Figure 52. 2.211 in Orifice Diameter With Orificed Lower Tie Plate, 20 Btu/lb Subcooling 


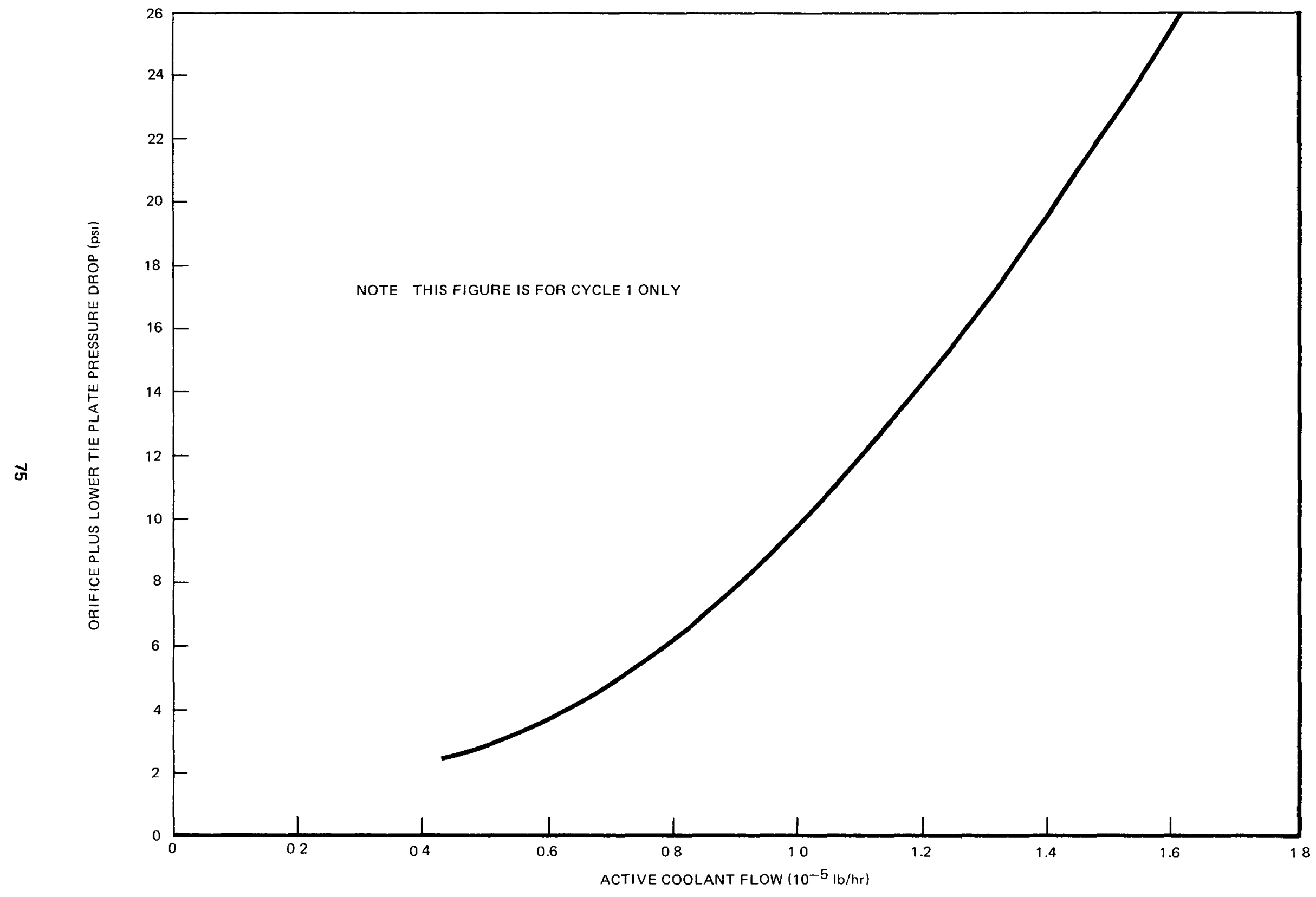

Figure 53. 2.211 in Orifice Diameter With Orificed Lower Tie Plate, 30 Btullb Subcoolıng 


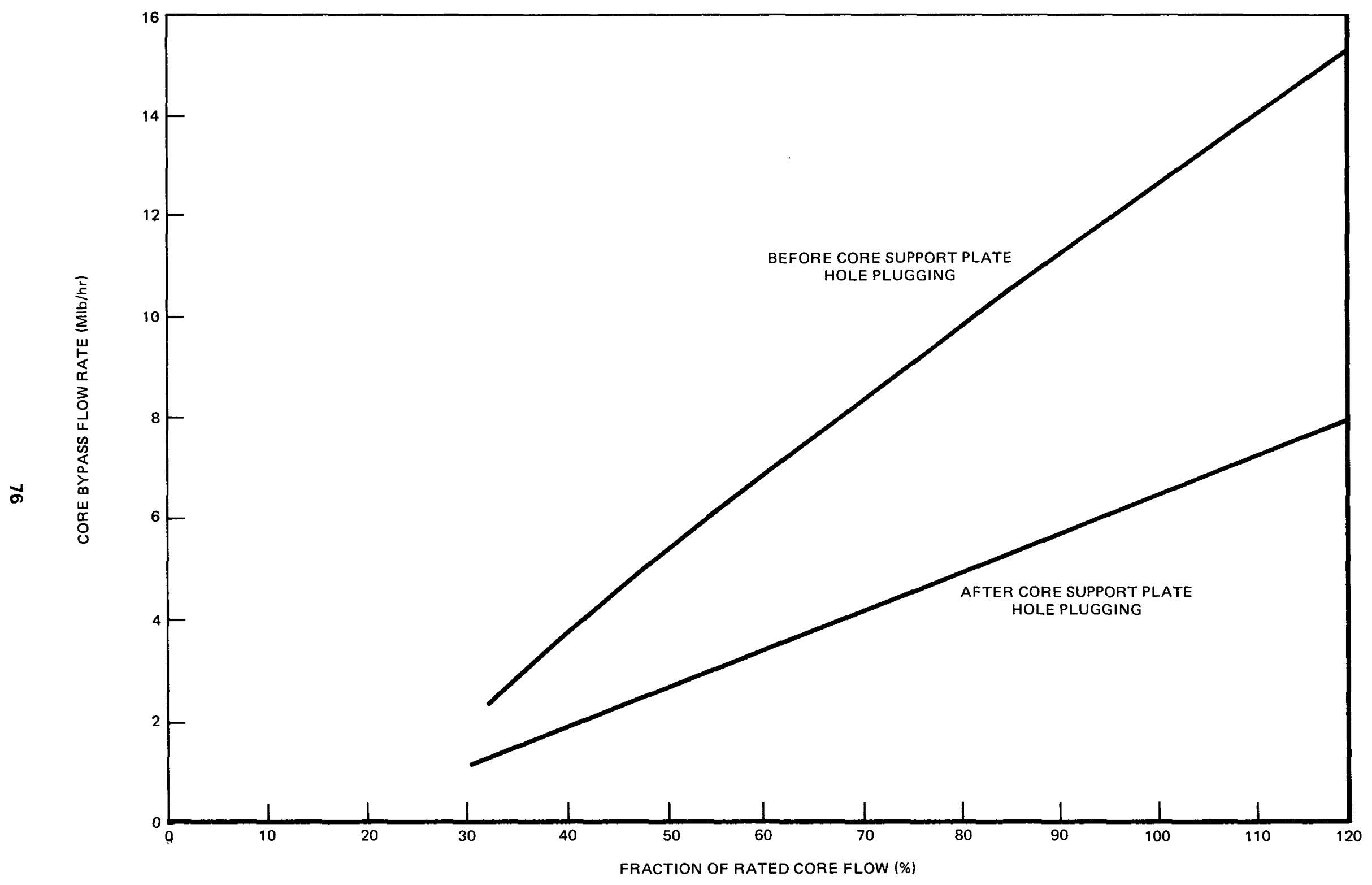

Figure 54. Core Bypass Flow for Cycle 1 


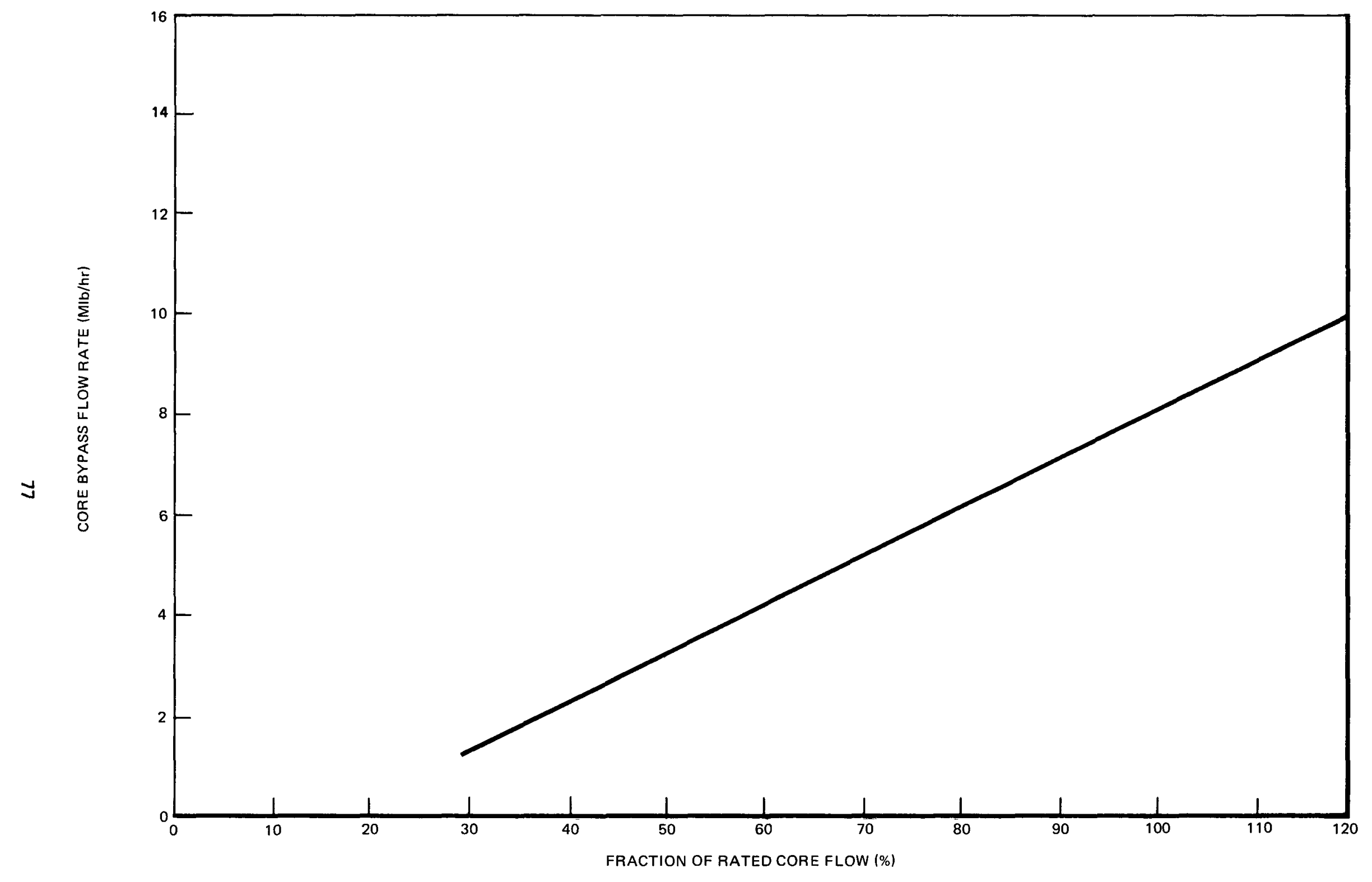

Figure 55. Core Bypass Flow for Cycle 2 


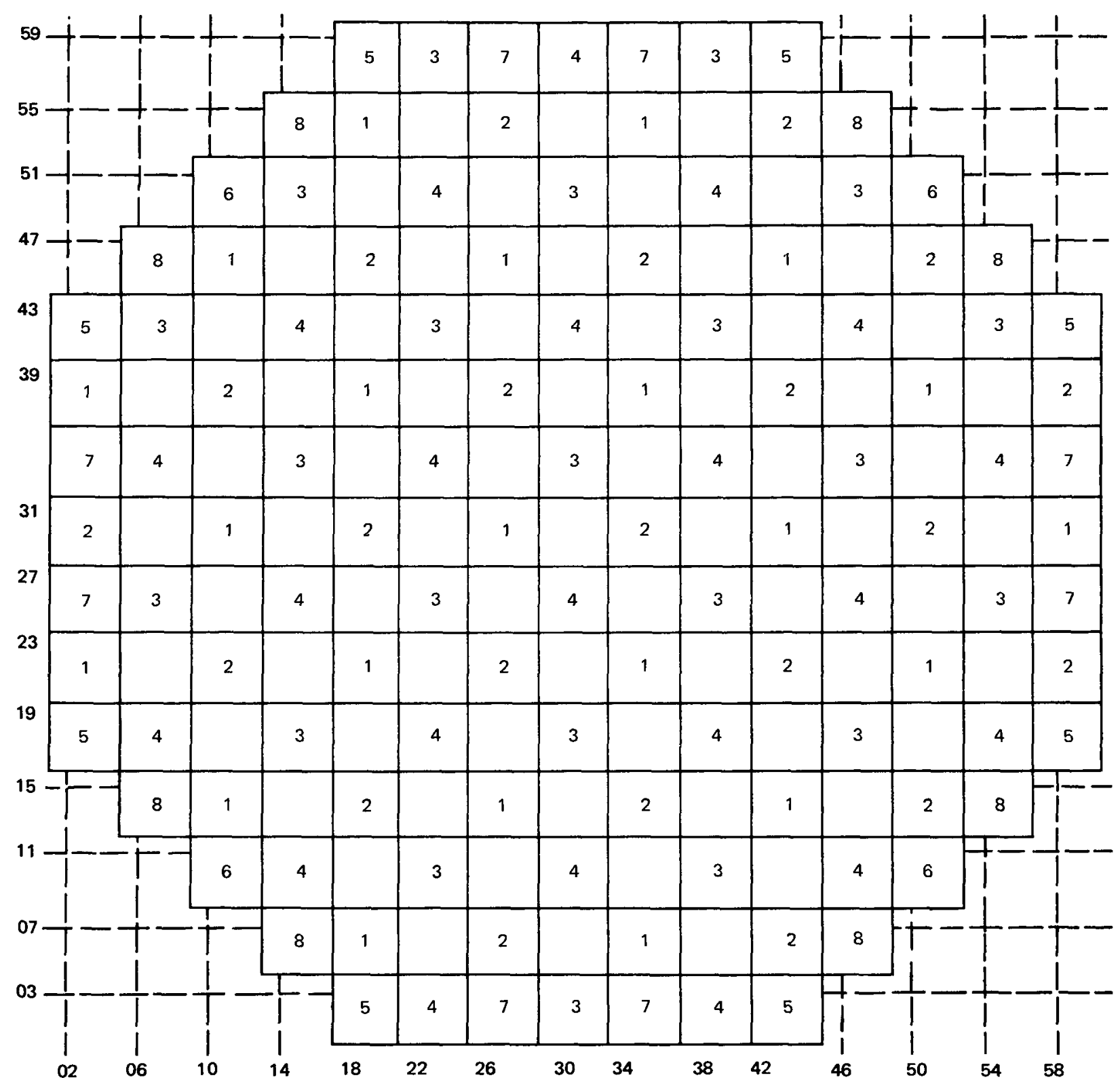

Figure 56. Peach Bottom 2 Control Rod A Sequence Groups 1-8 


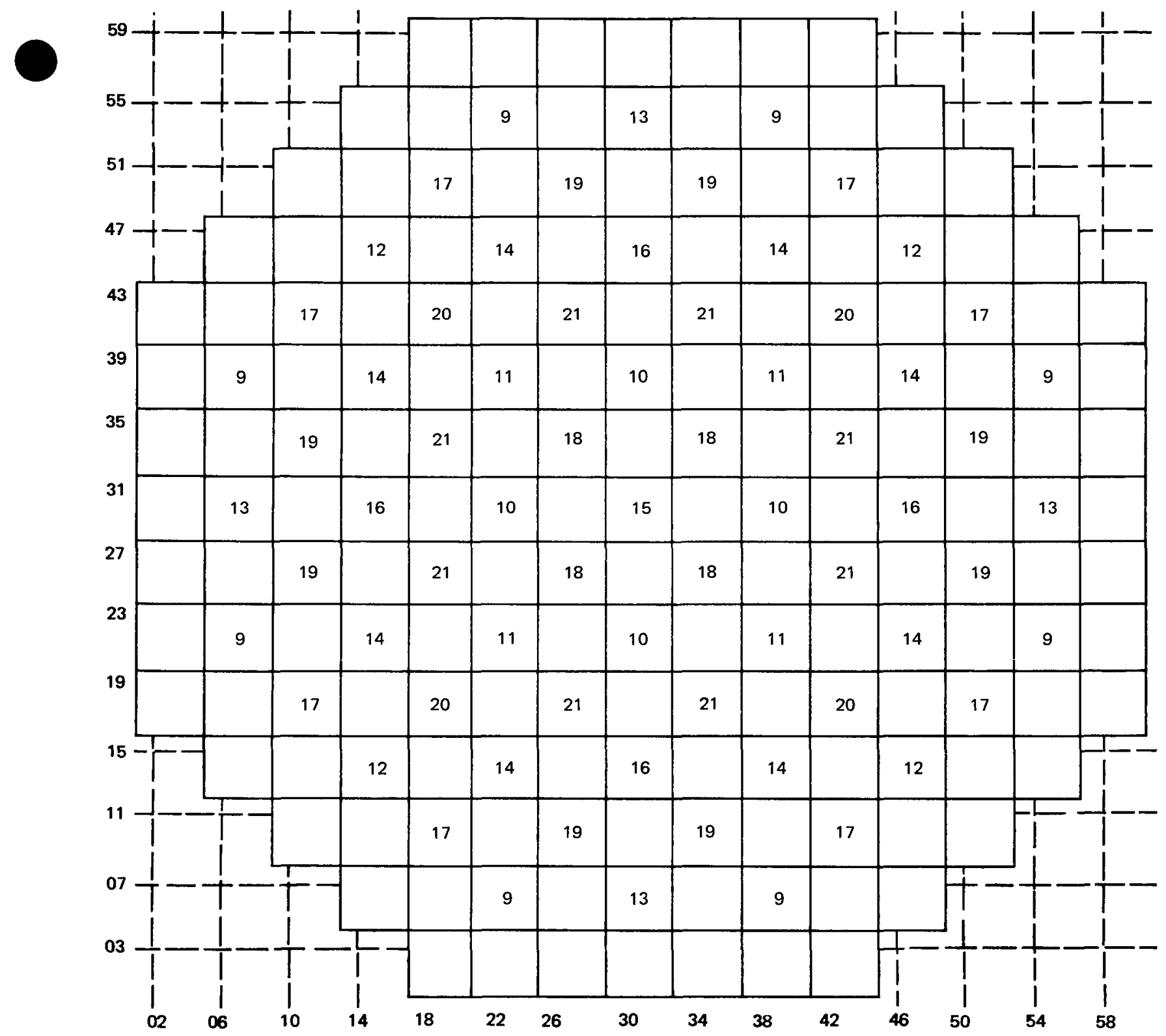

Figure 57. Peach Bottom 2 Control Rod A1 Sequence Groups 9-21 


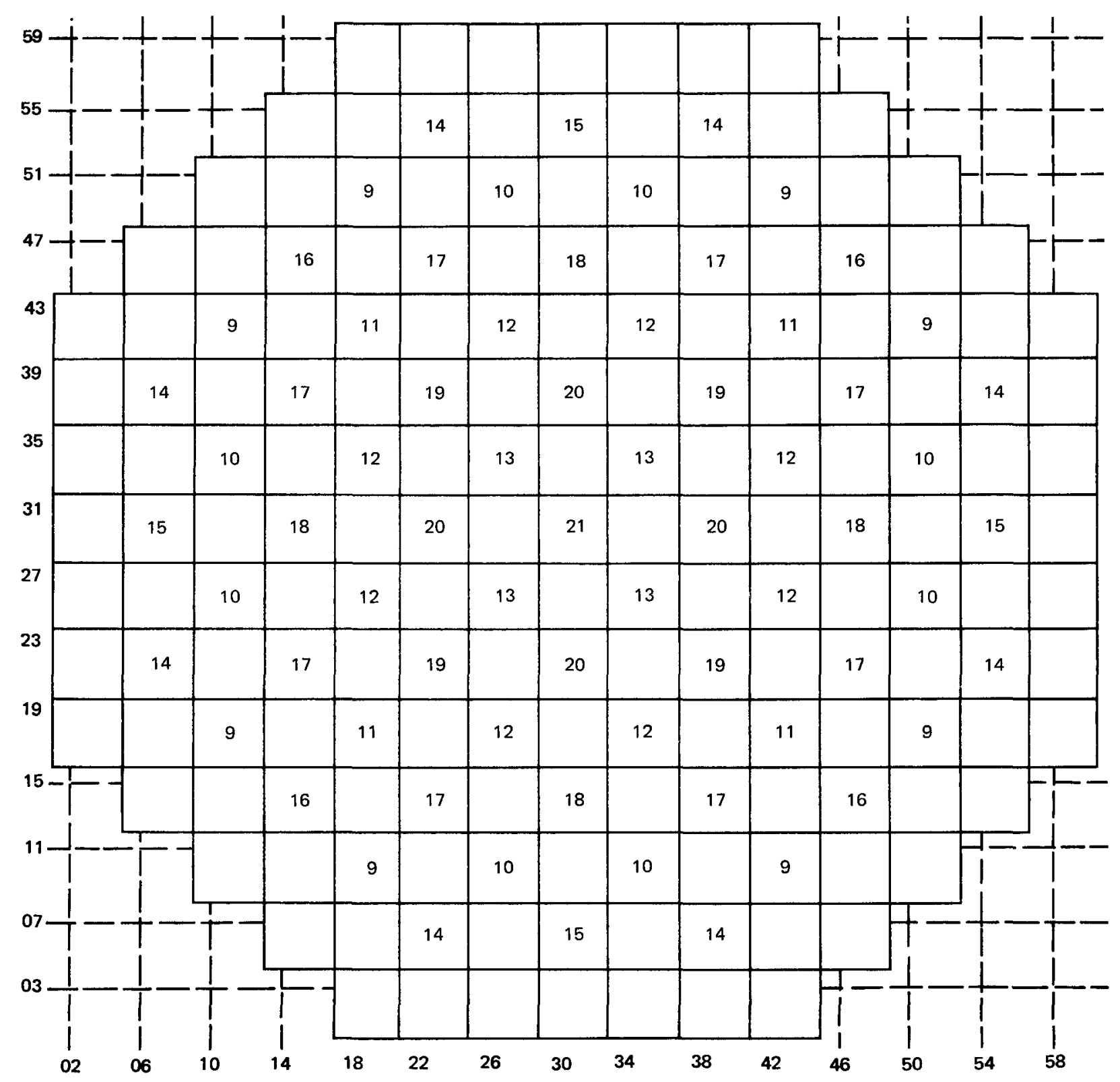

Figure 58. Peach Bottom 2 Control Rod A2 Sequence Groups 9-21 


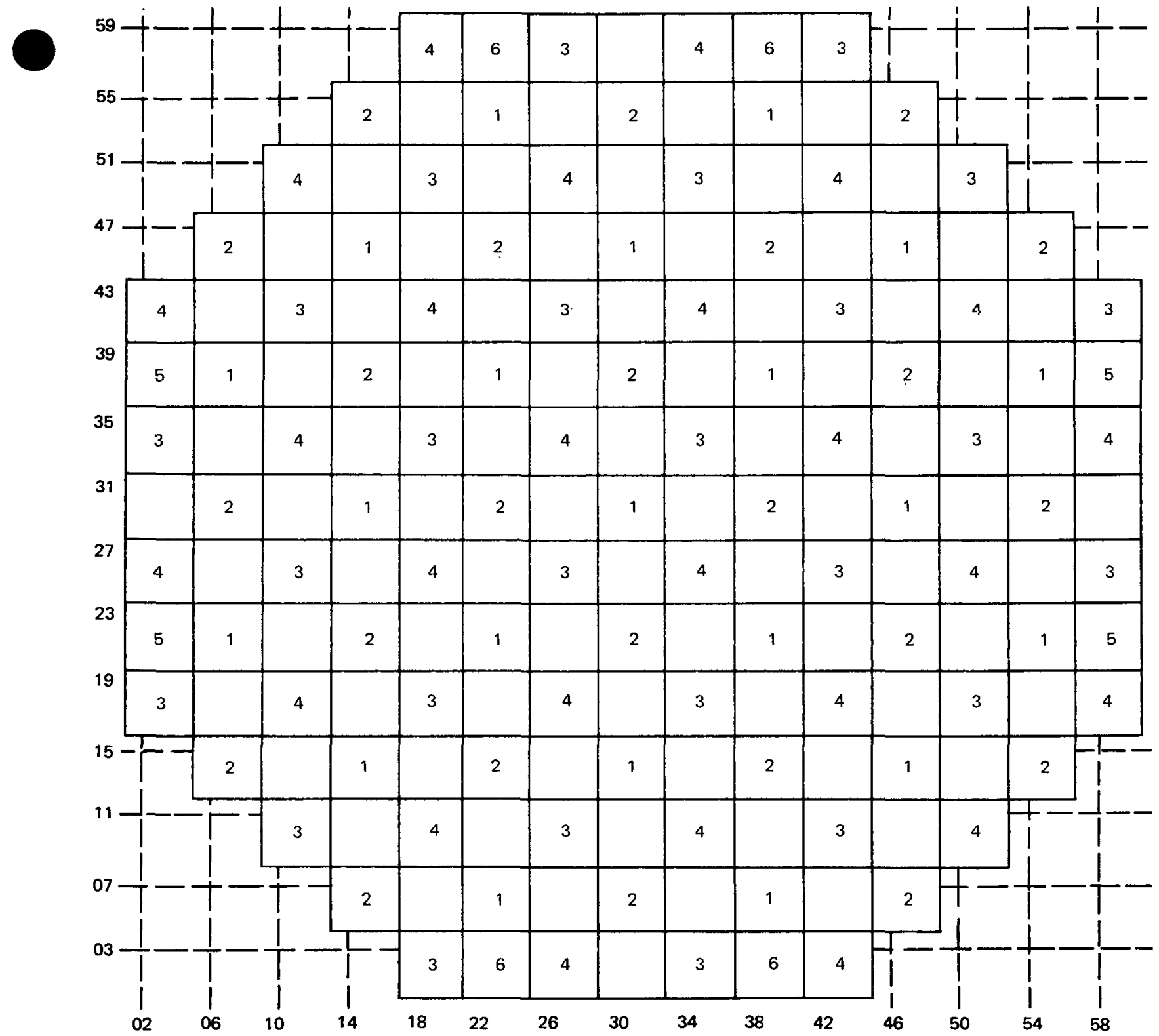

Figure 59. Peach Bottom 2 Control Rod B Sequence Groups 1-6 


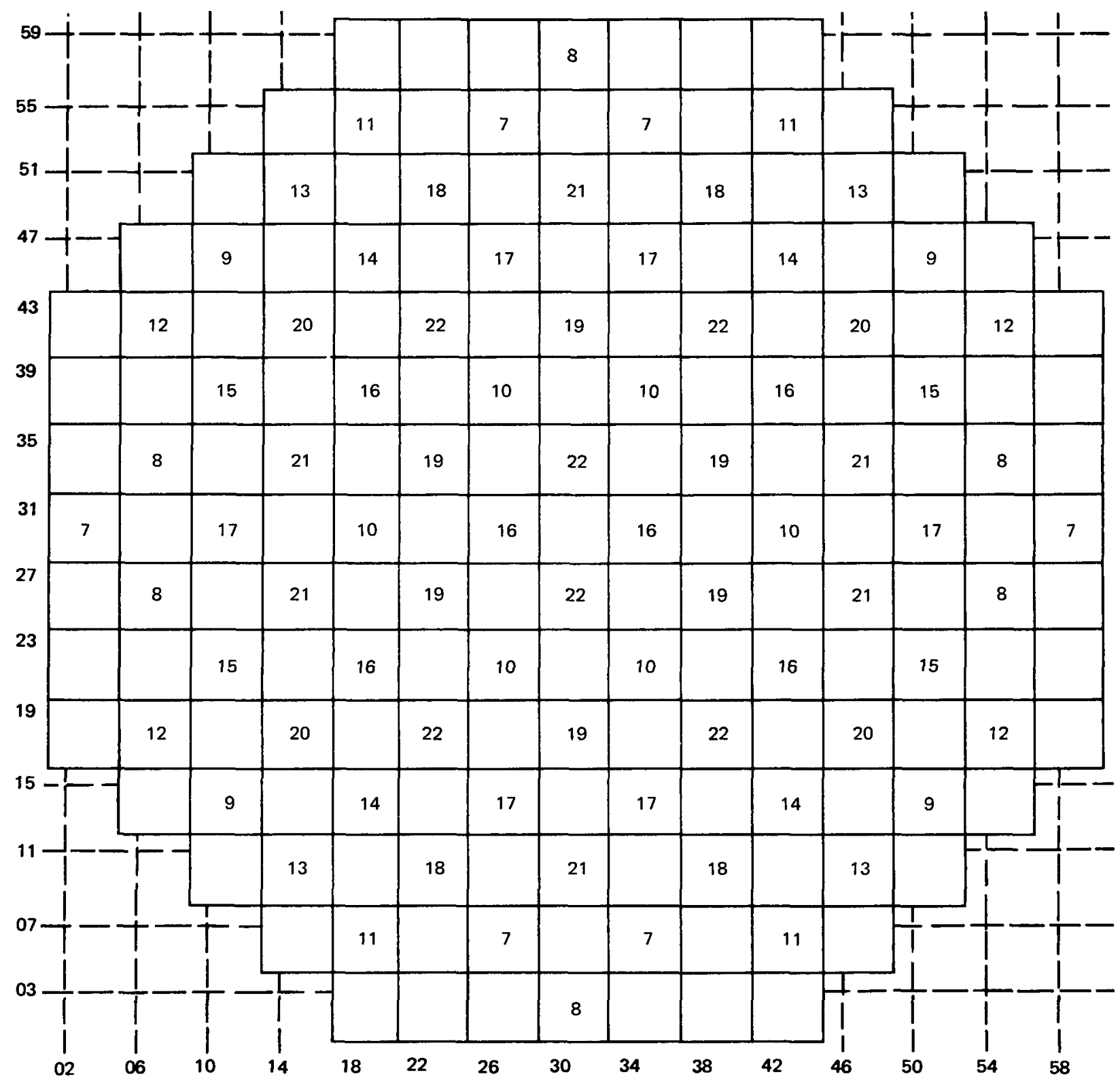

Figure 60. Peach Bottom 2 Control Rod B1 Sequence Groups 7-21 


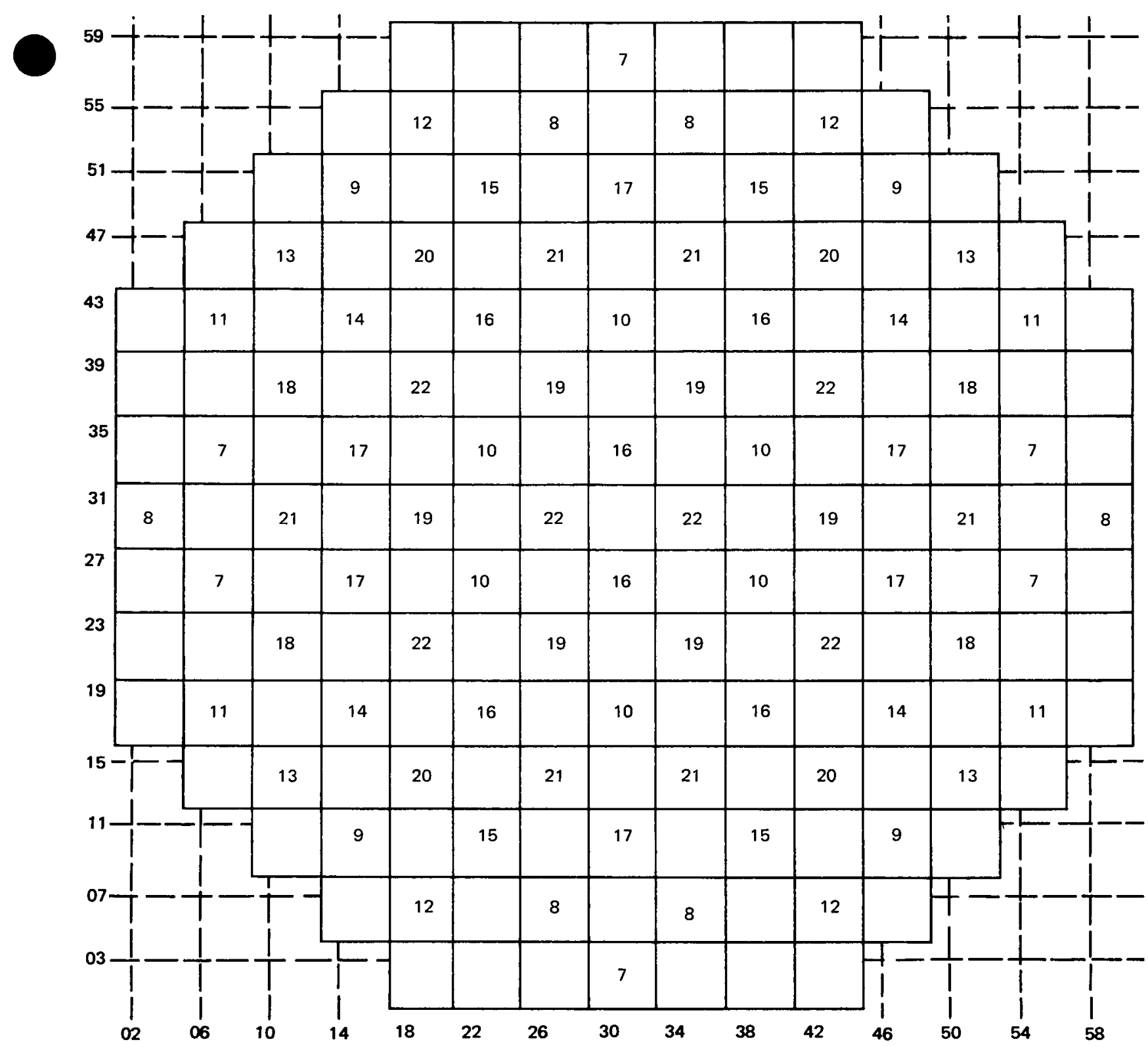

Figure 61. Peach Bottom 2 Control Rod B2 Sequence Groups 7-22 


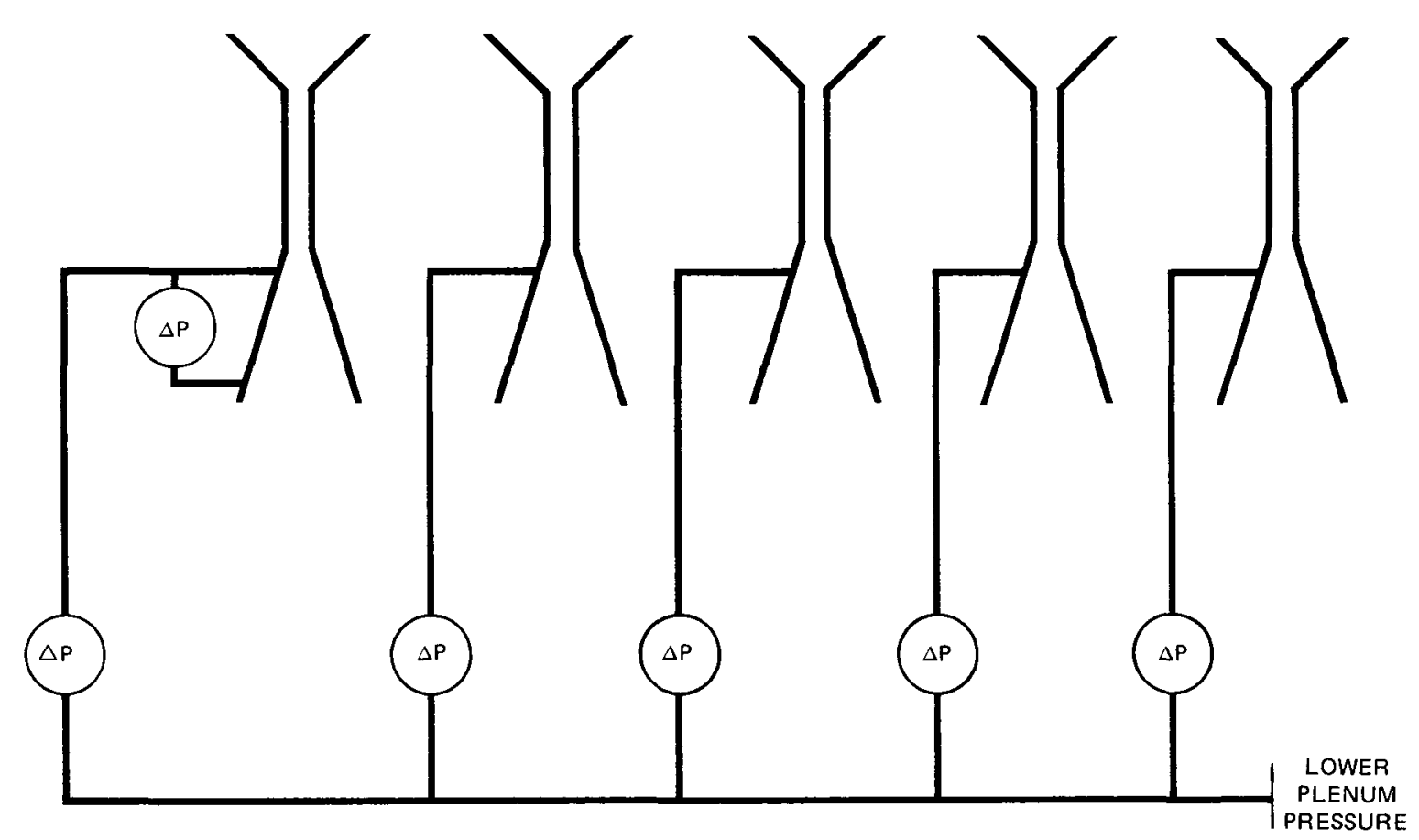

Figure 62. Core Flow Measurement System Schematic Showing One of Four Groups of Jet Pumps 


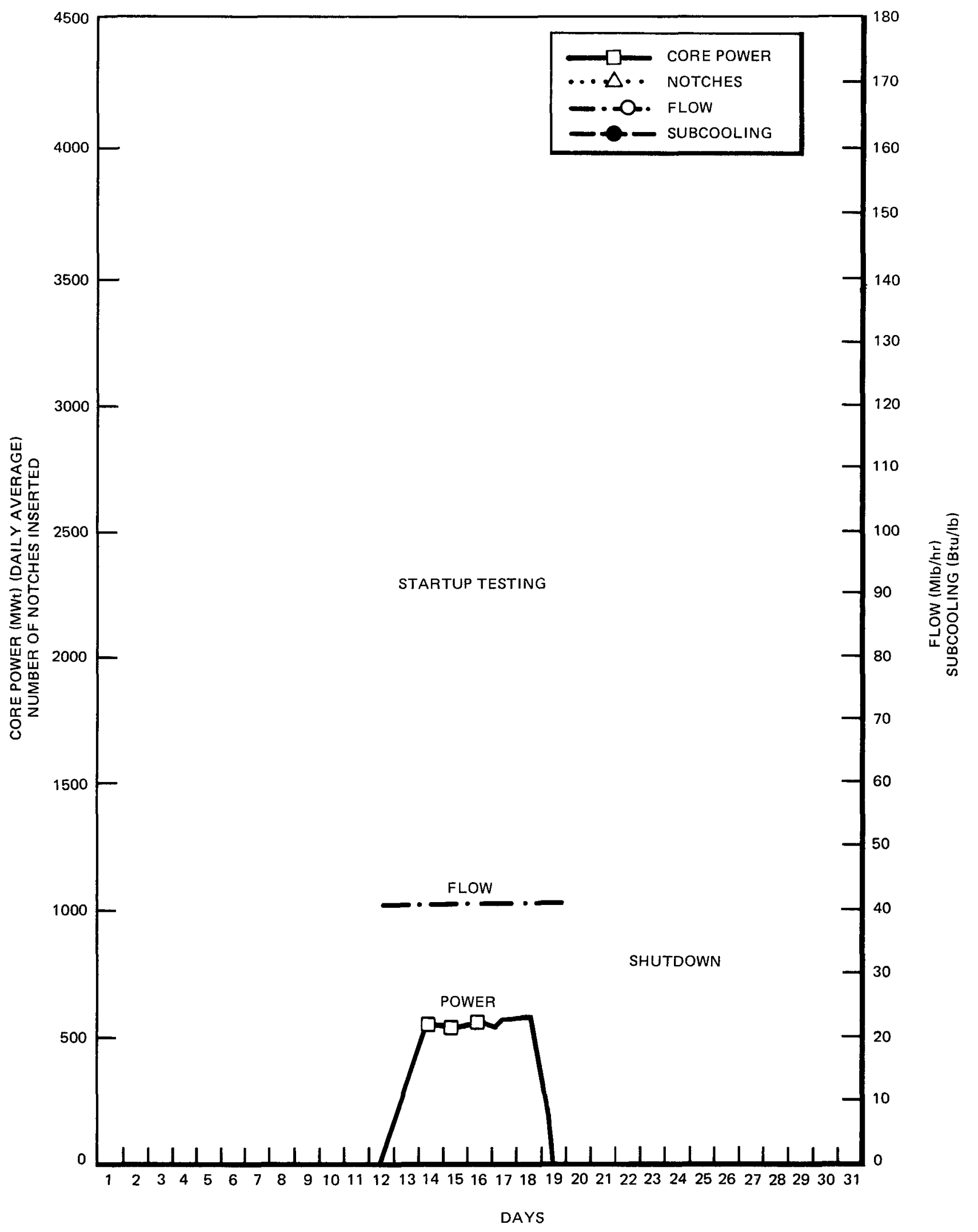

Figure 63. Data Summaries, January 1974 


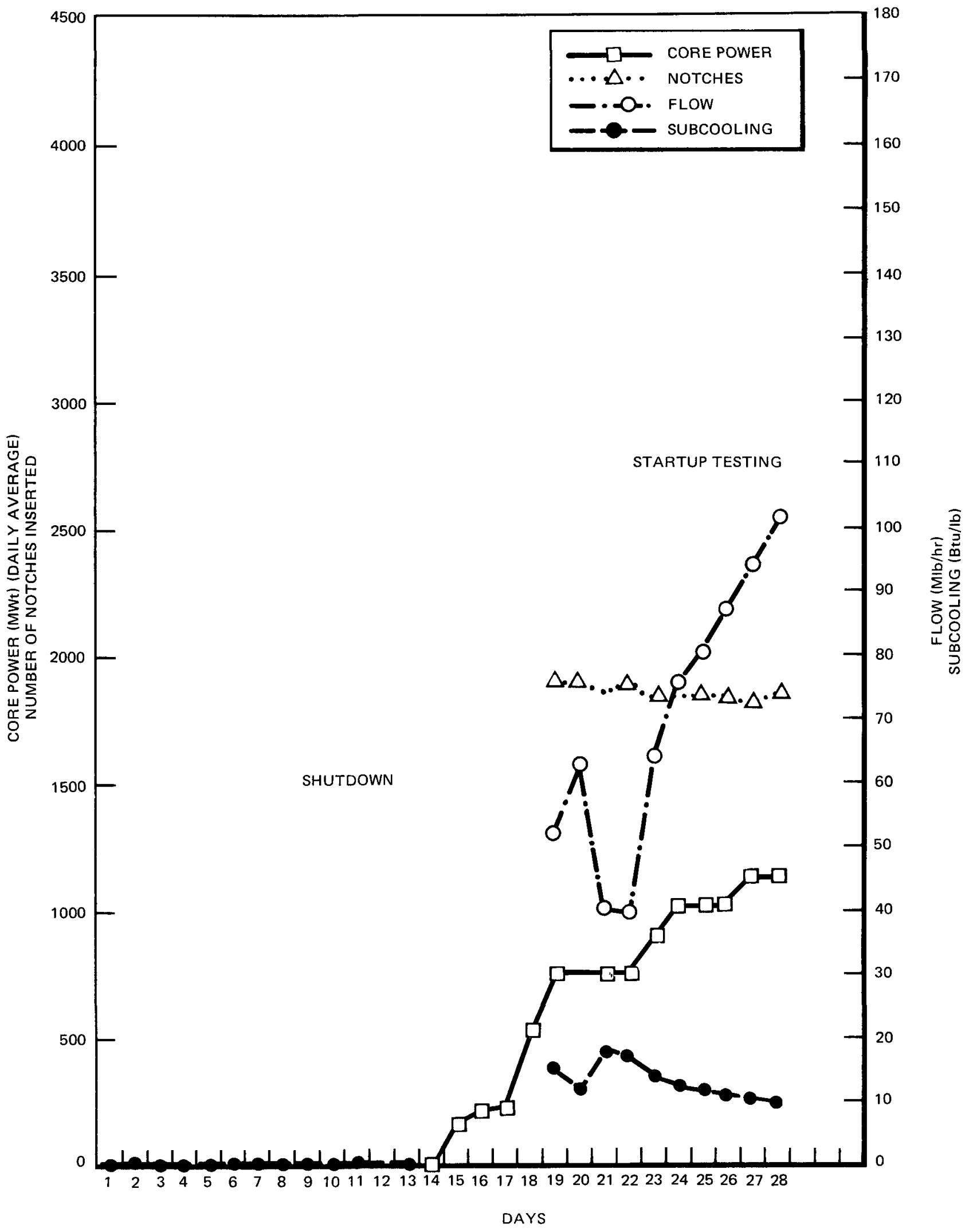

Figure 64. Data Summaries, February 1974 


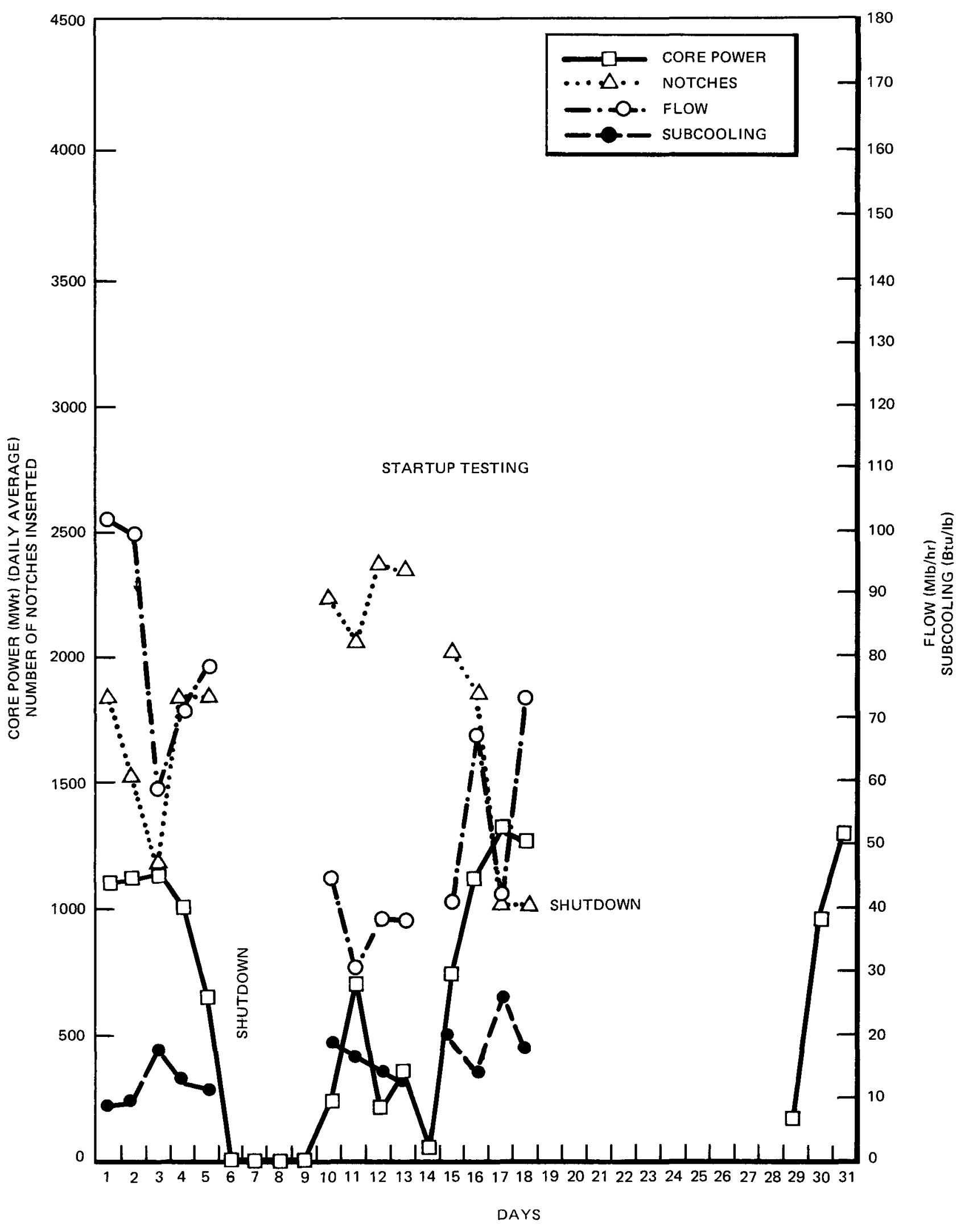

Figure 65. Data Summarıes, March 1974 


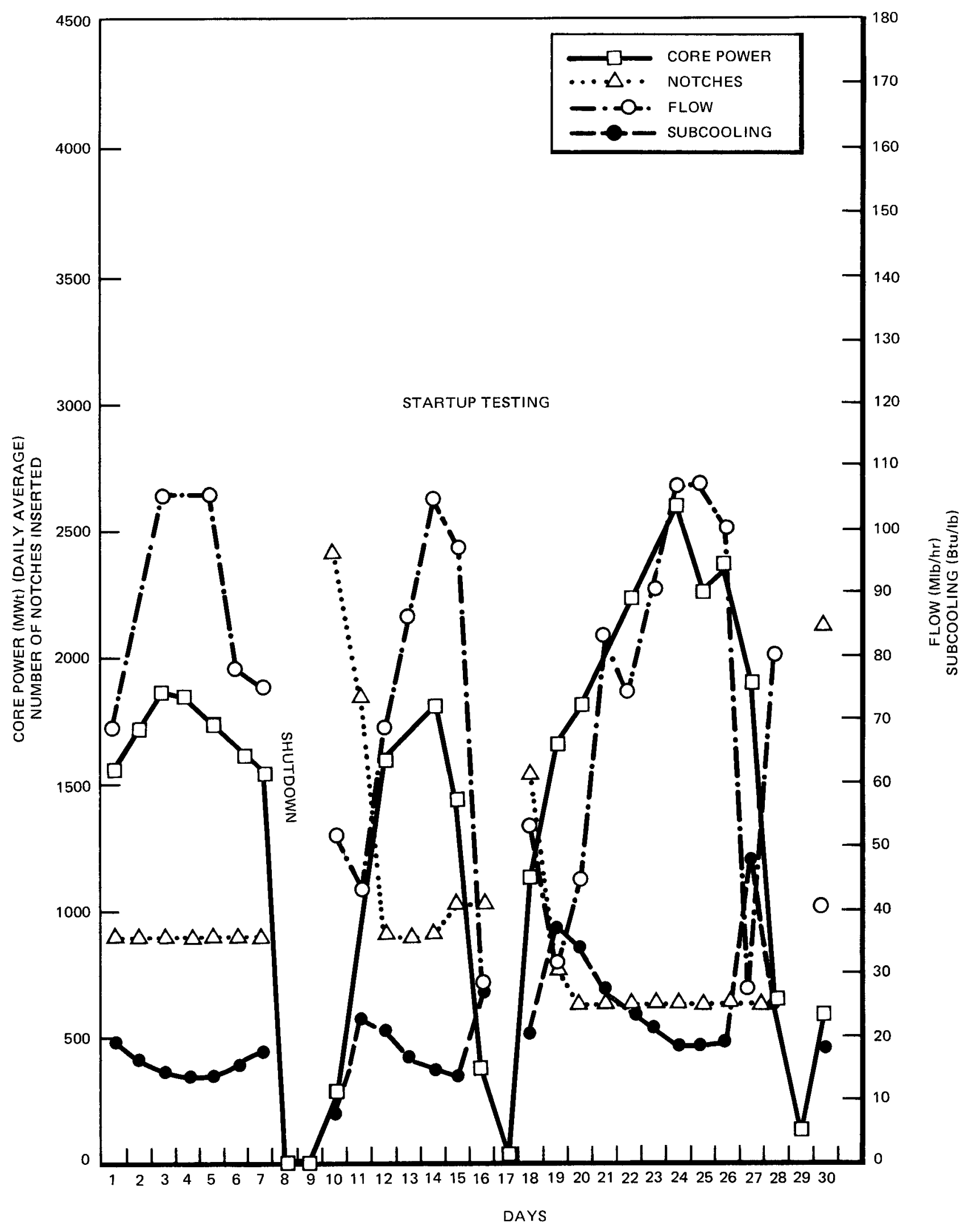

Figure 66. Data Summaries, April 1974 


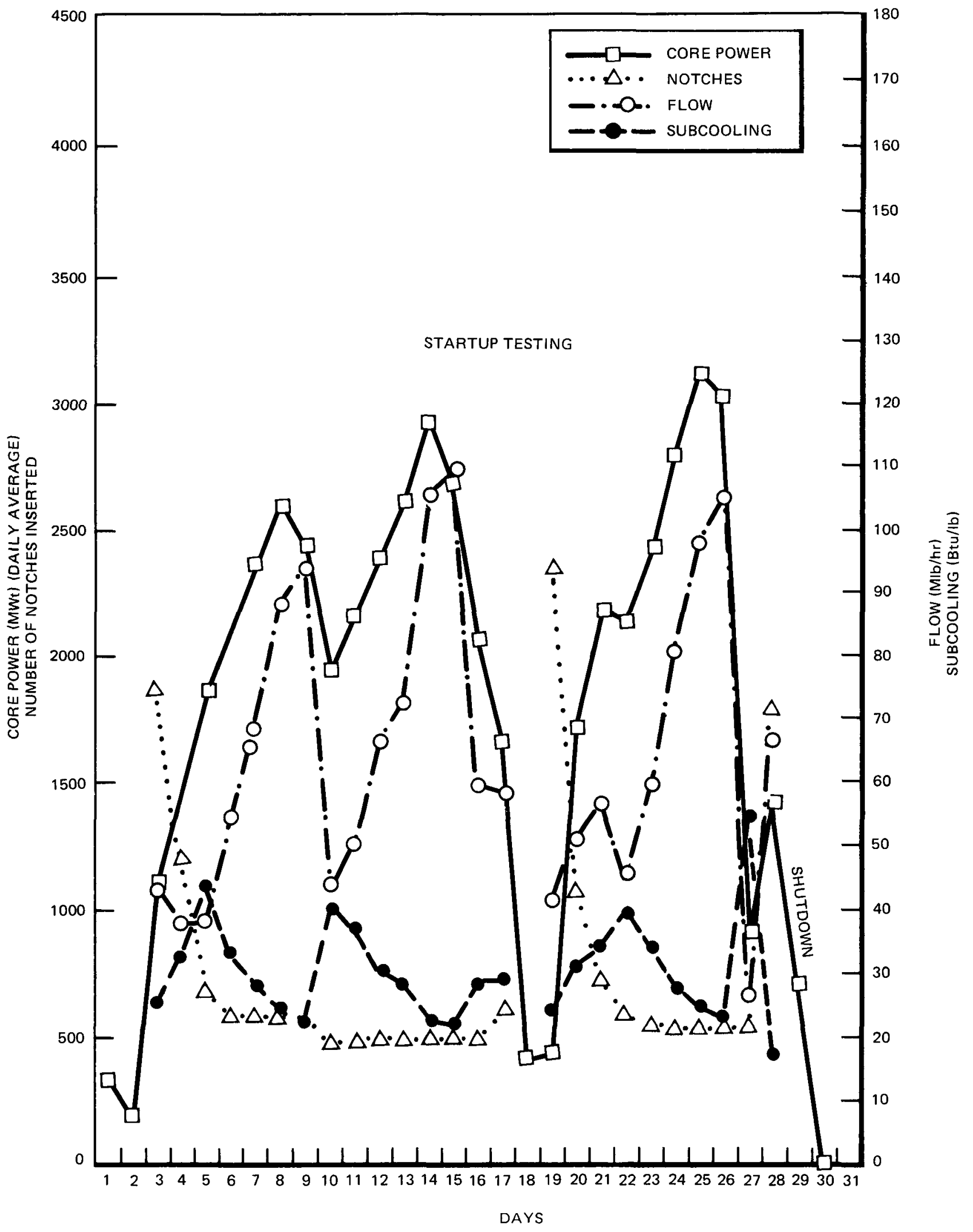

Figure 67. Data Summaries, May 1974 


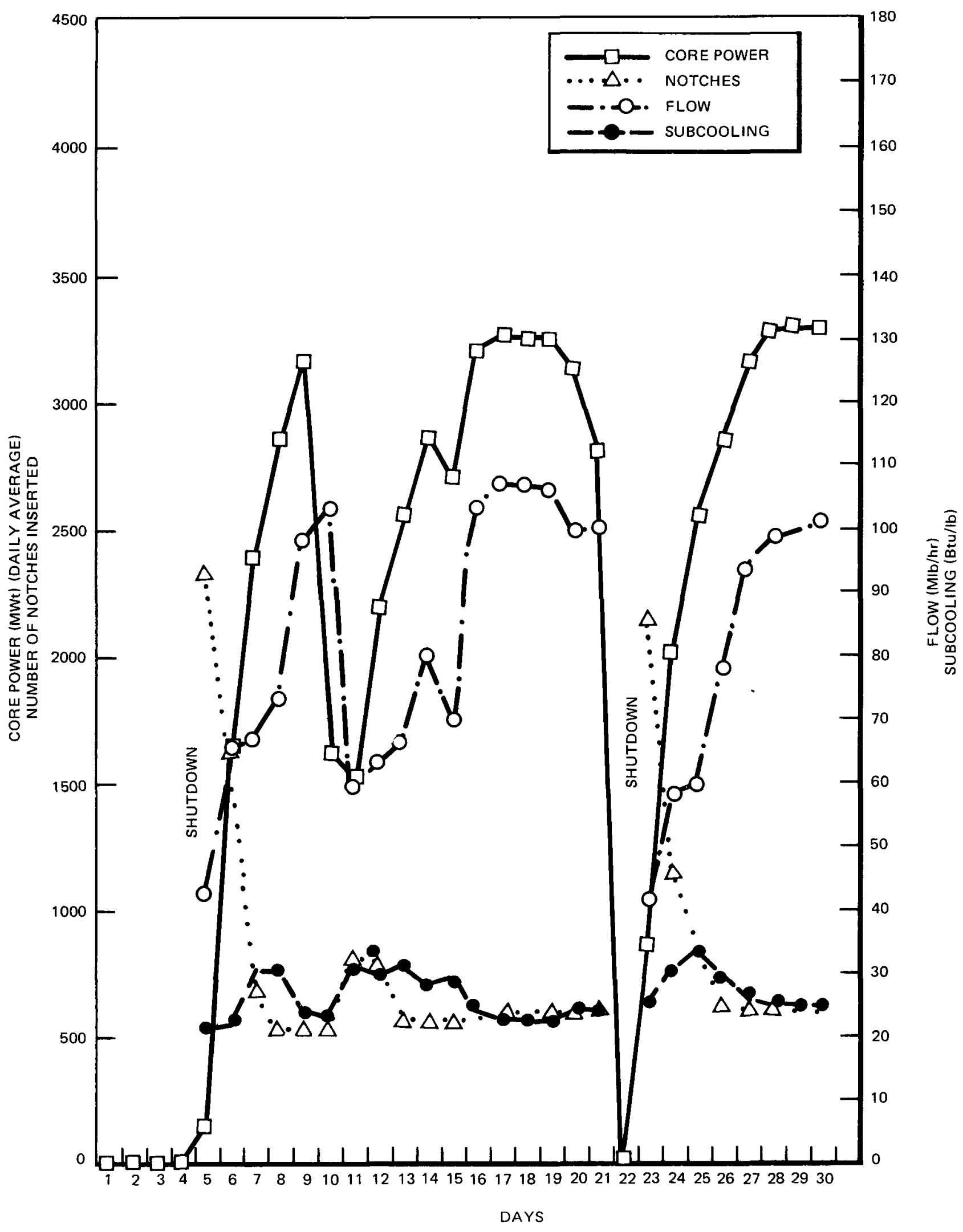

Figure 68. Data Summaries, June 1974 


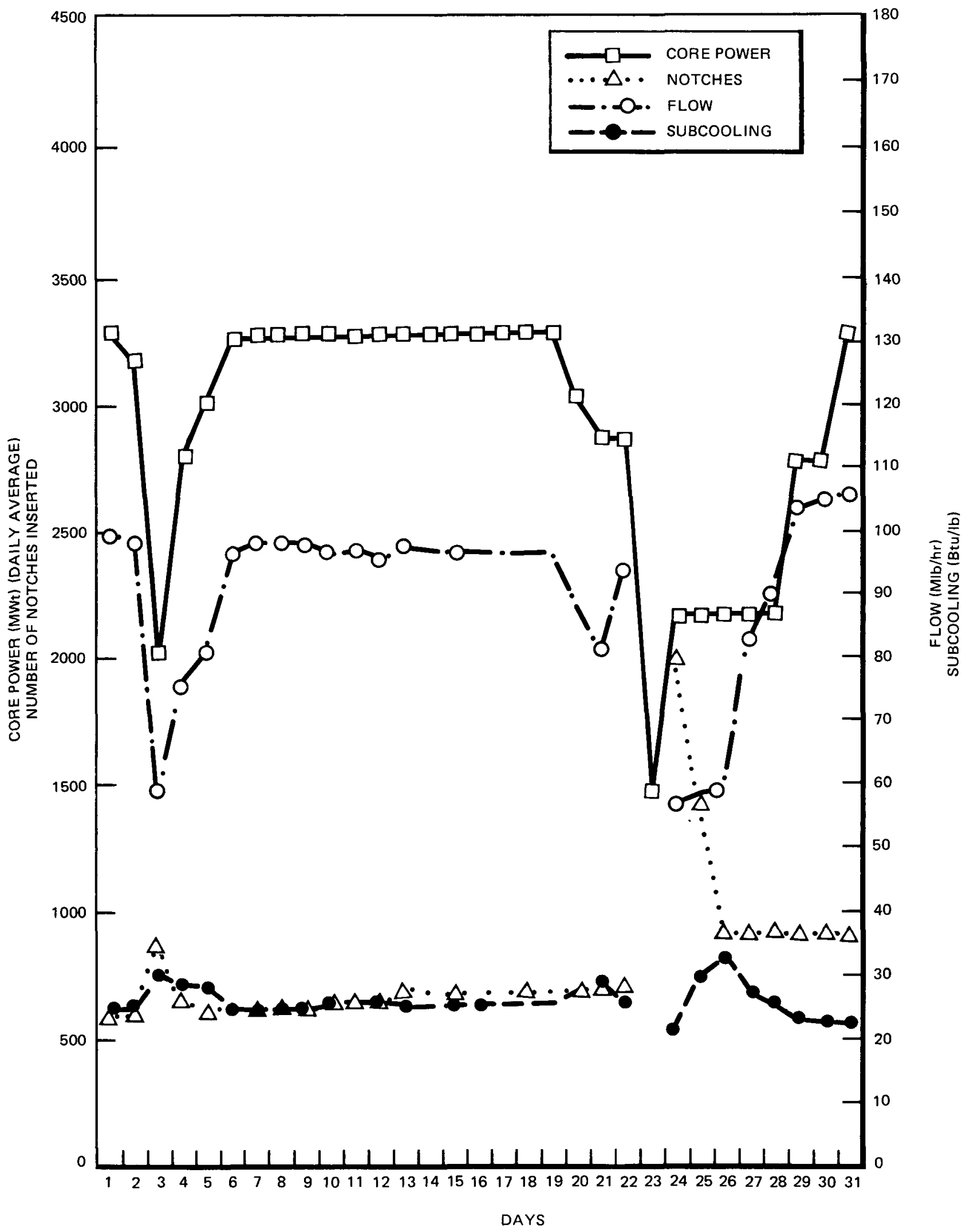

Figure 69. Data Summaries, July 1974 


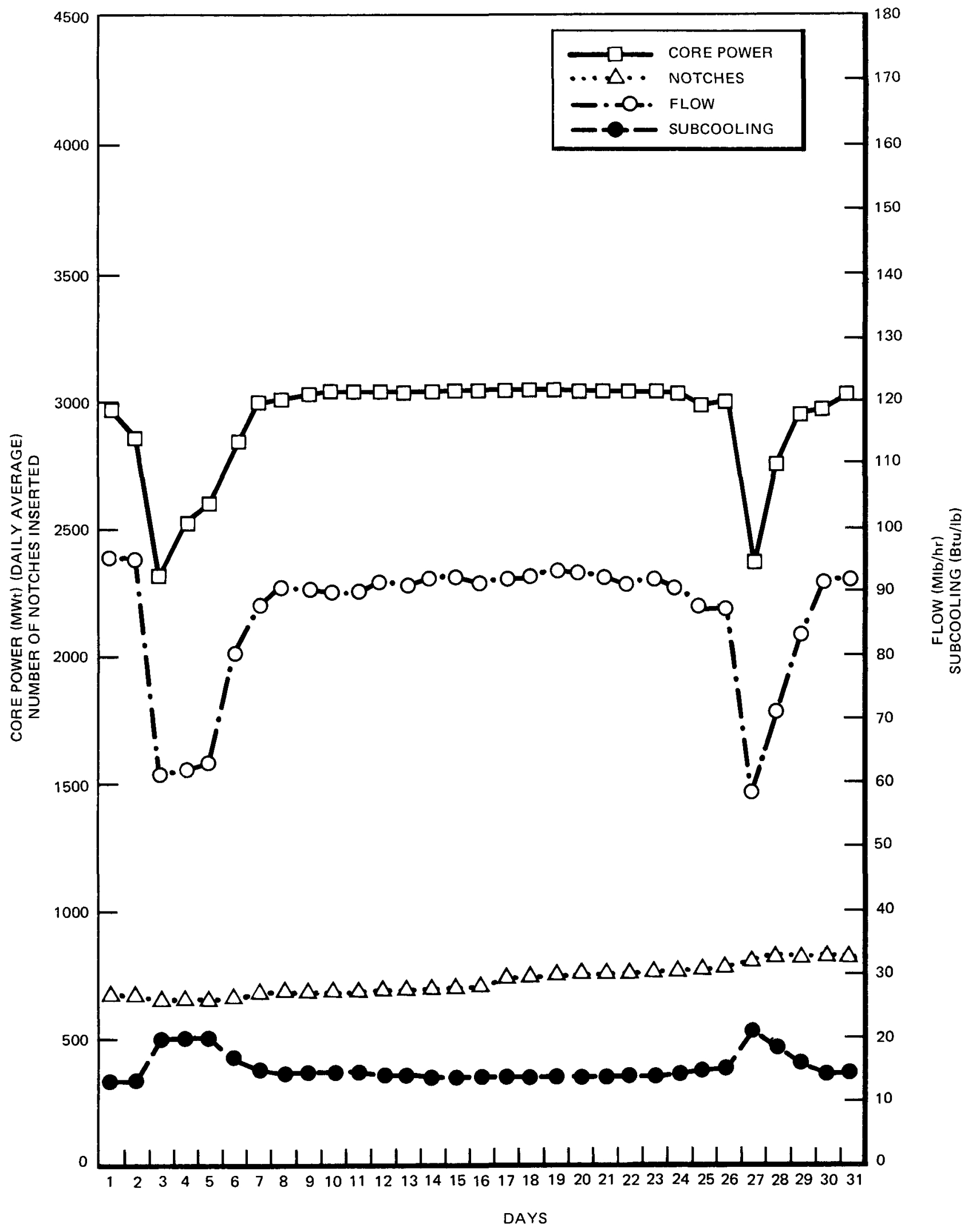

Figure 70. Data Summaries, August 1974 


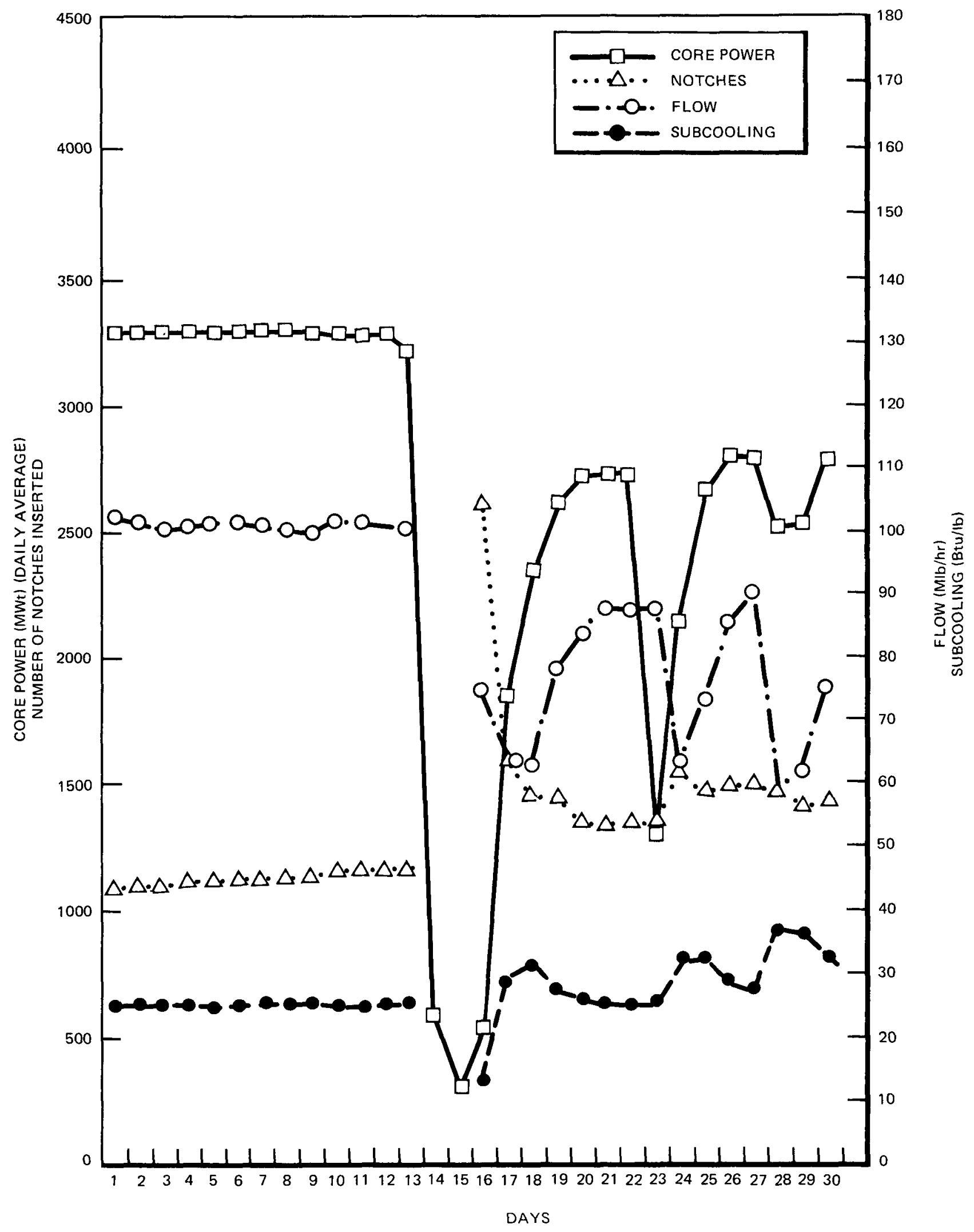

Figure 71. Data Summaries, September 1974 


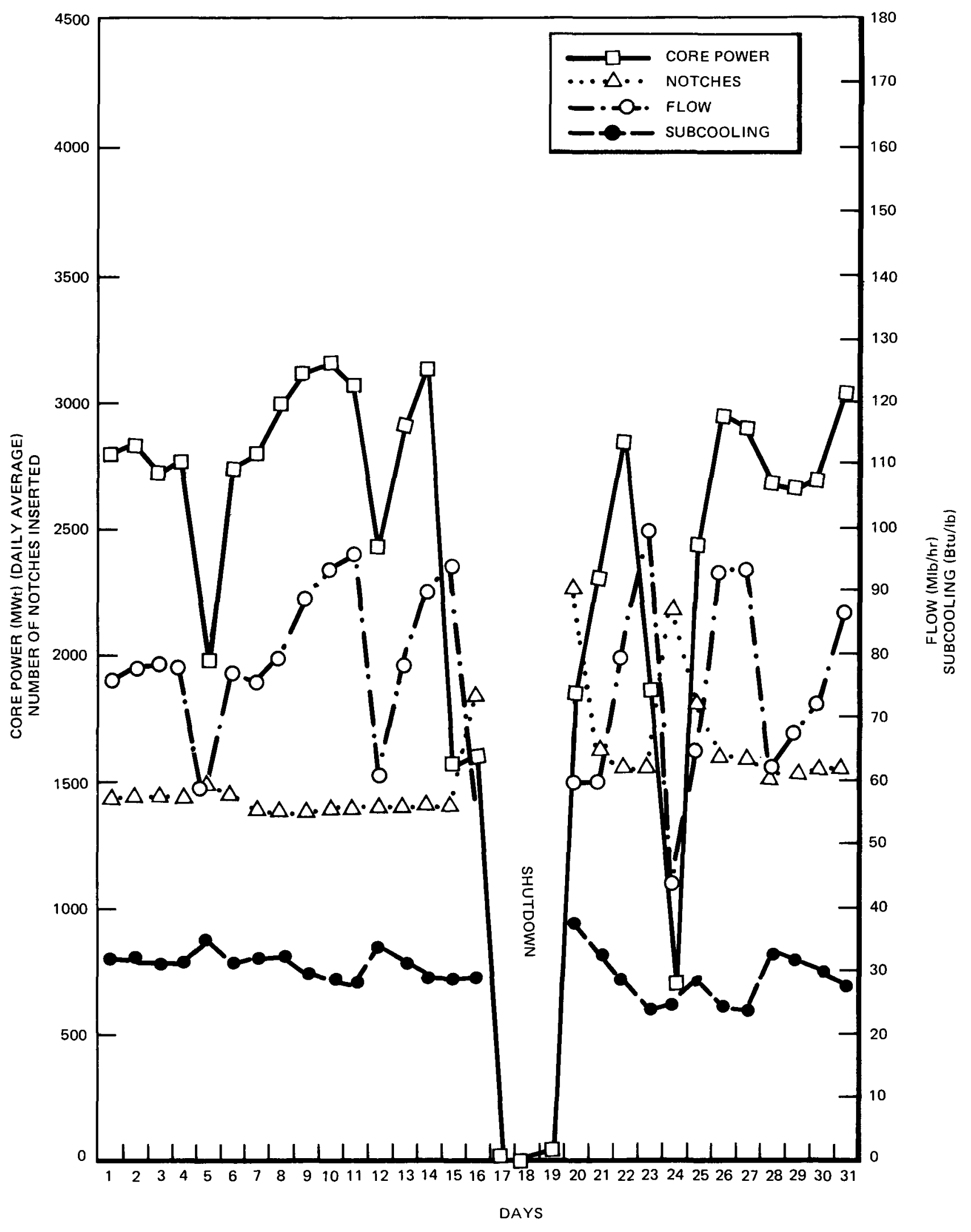

Figure 72. Data Summaries, October 1974 


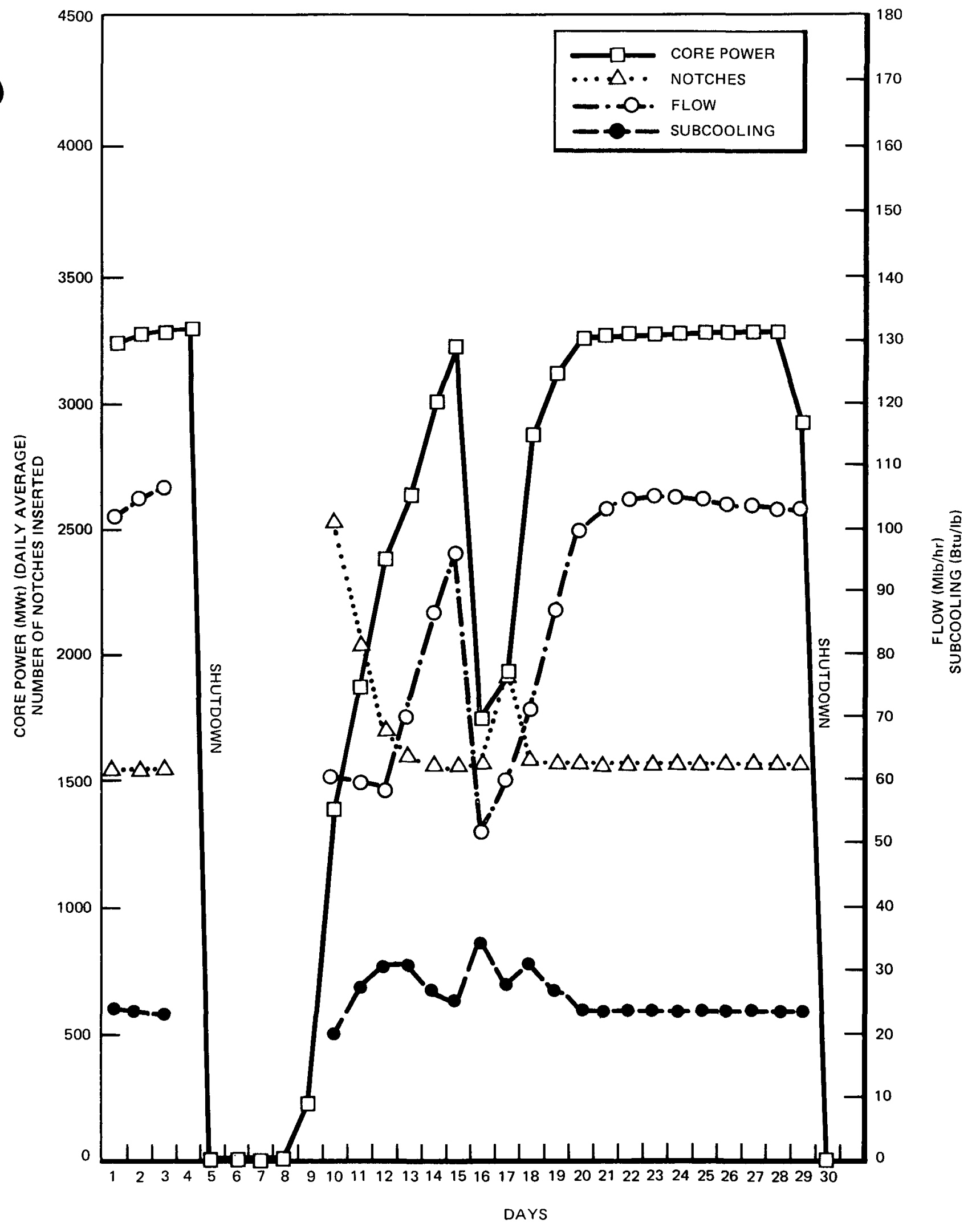

Figure 73. Data Summaries, November 1974 


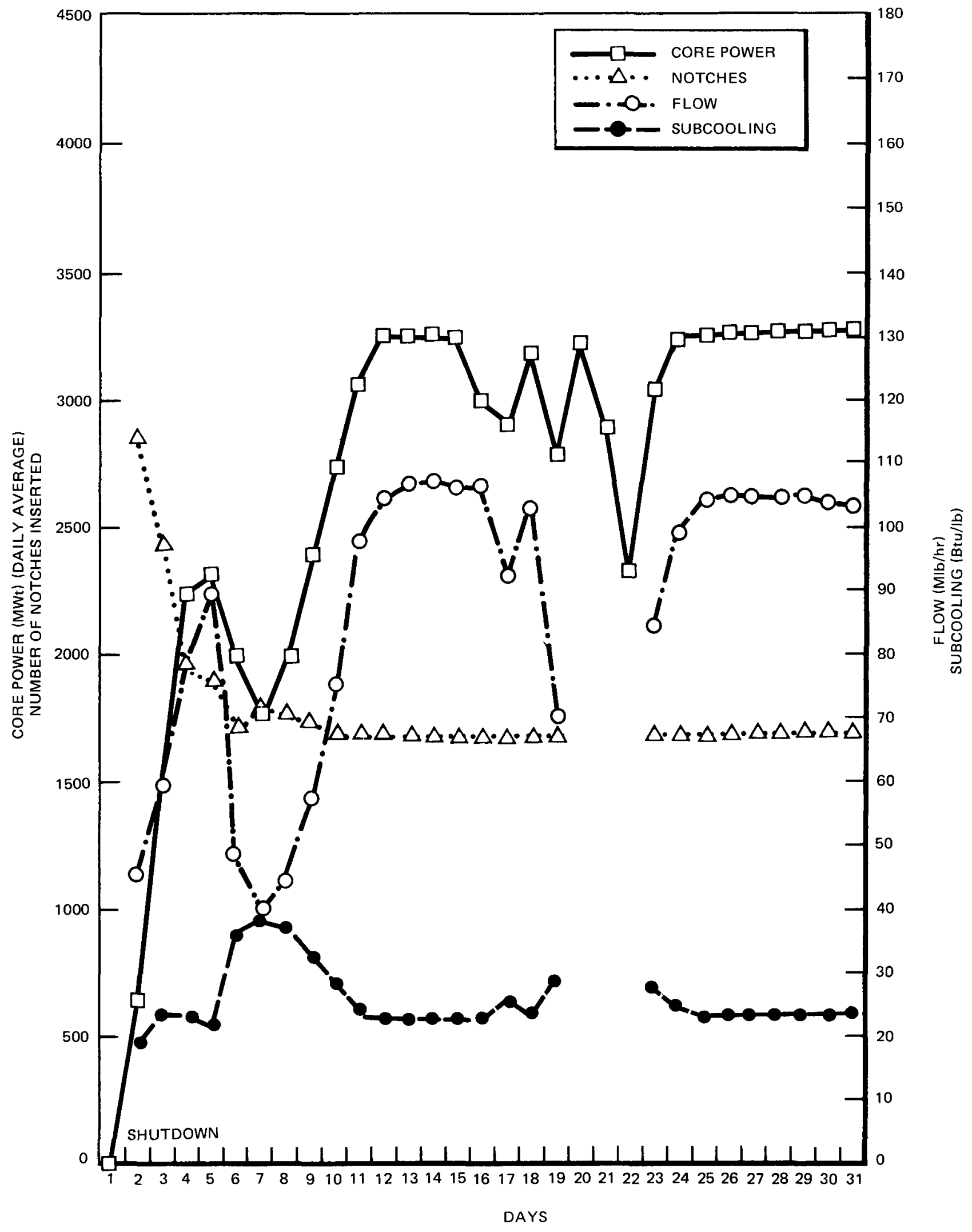

Figure 74. Data Summaries, December 1974 


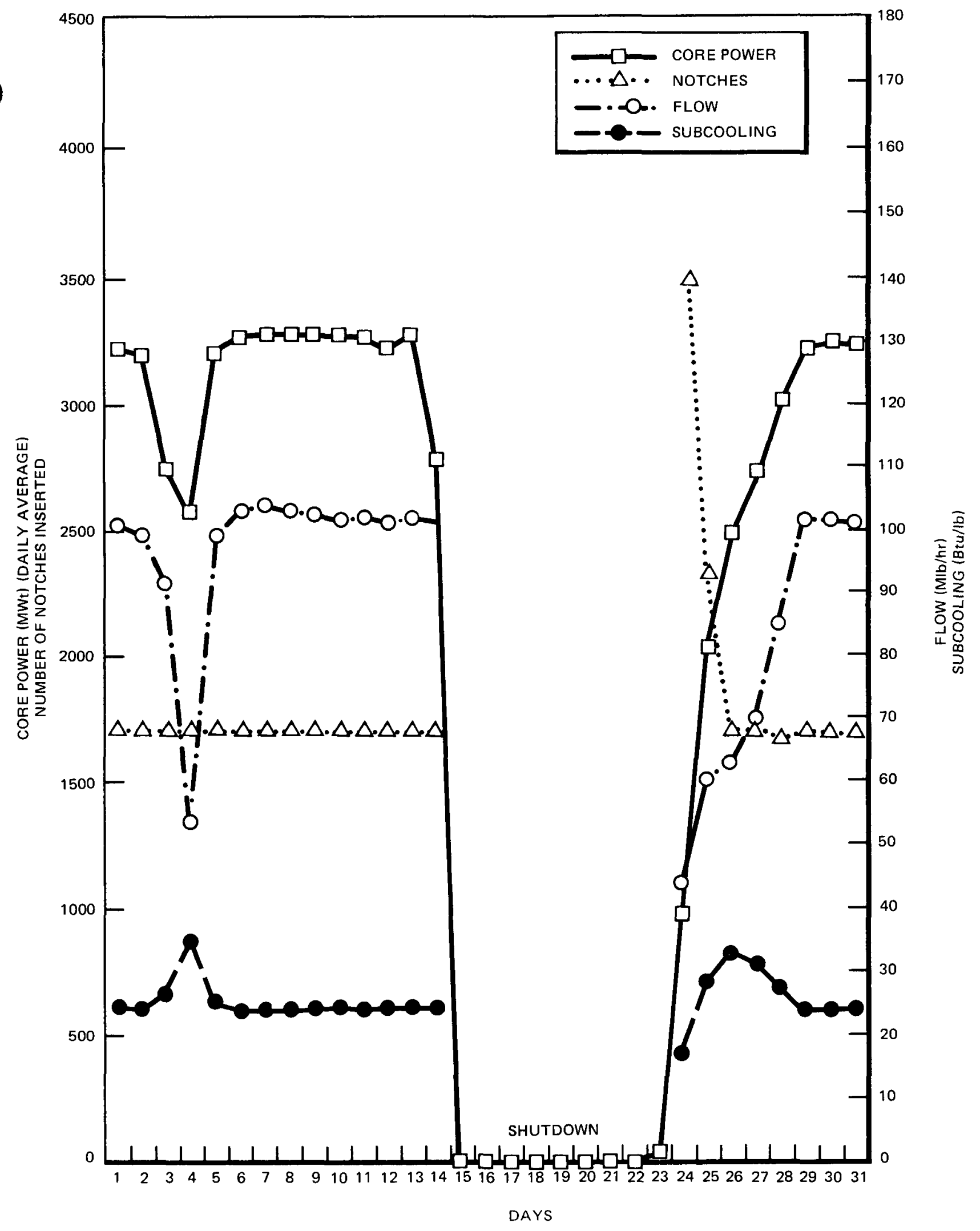

Figure 75. Data Summaries, January 1975 


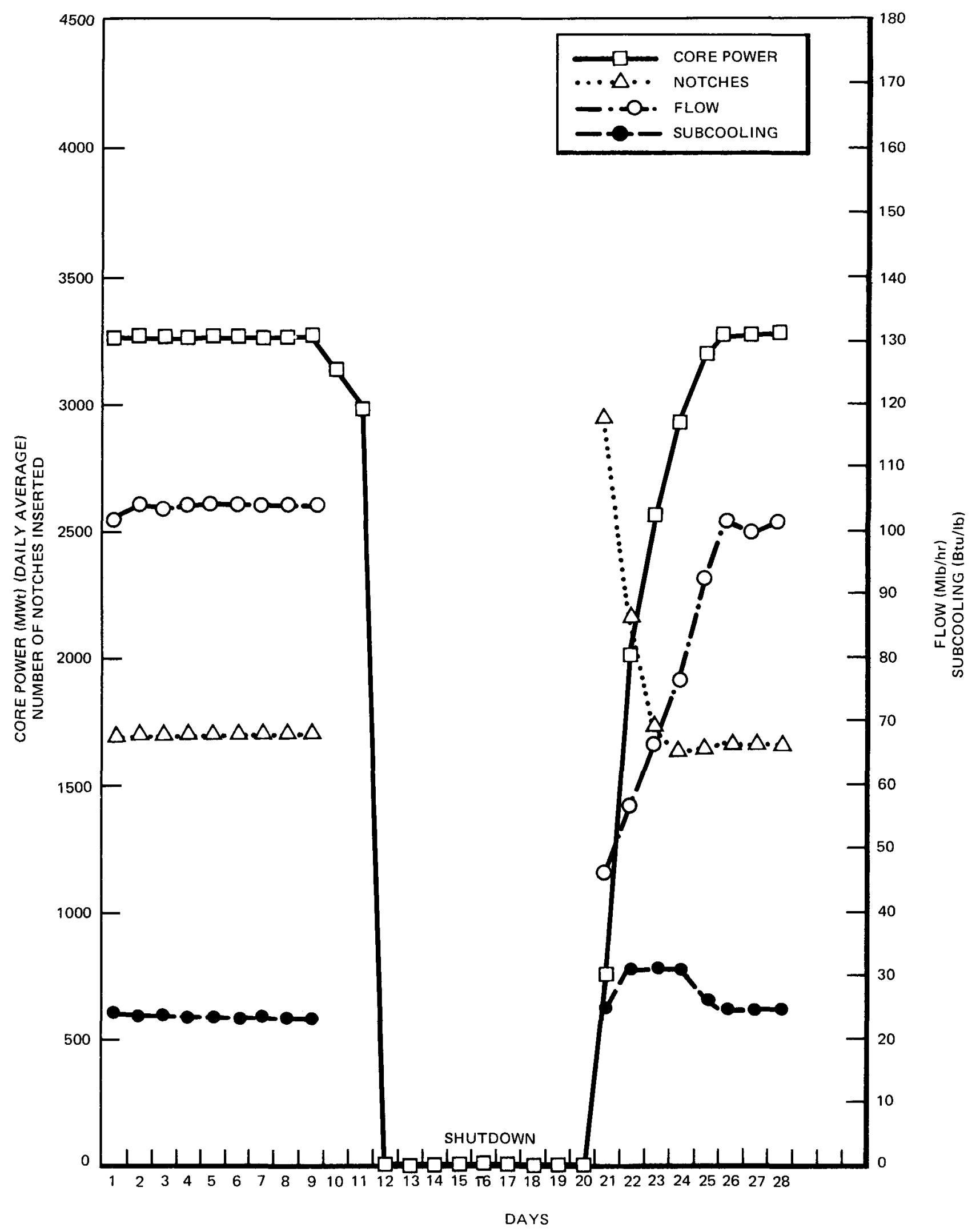

Figure 76. Data Summaries, February 1975 


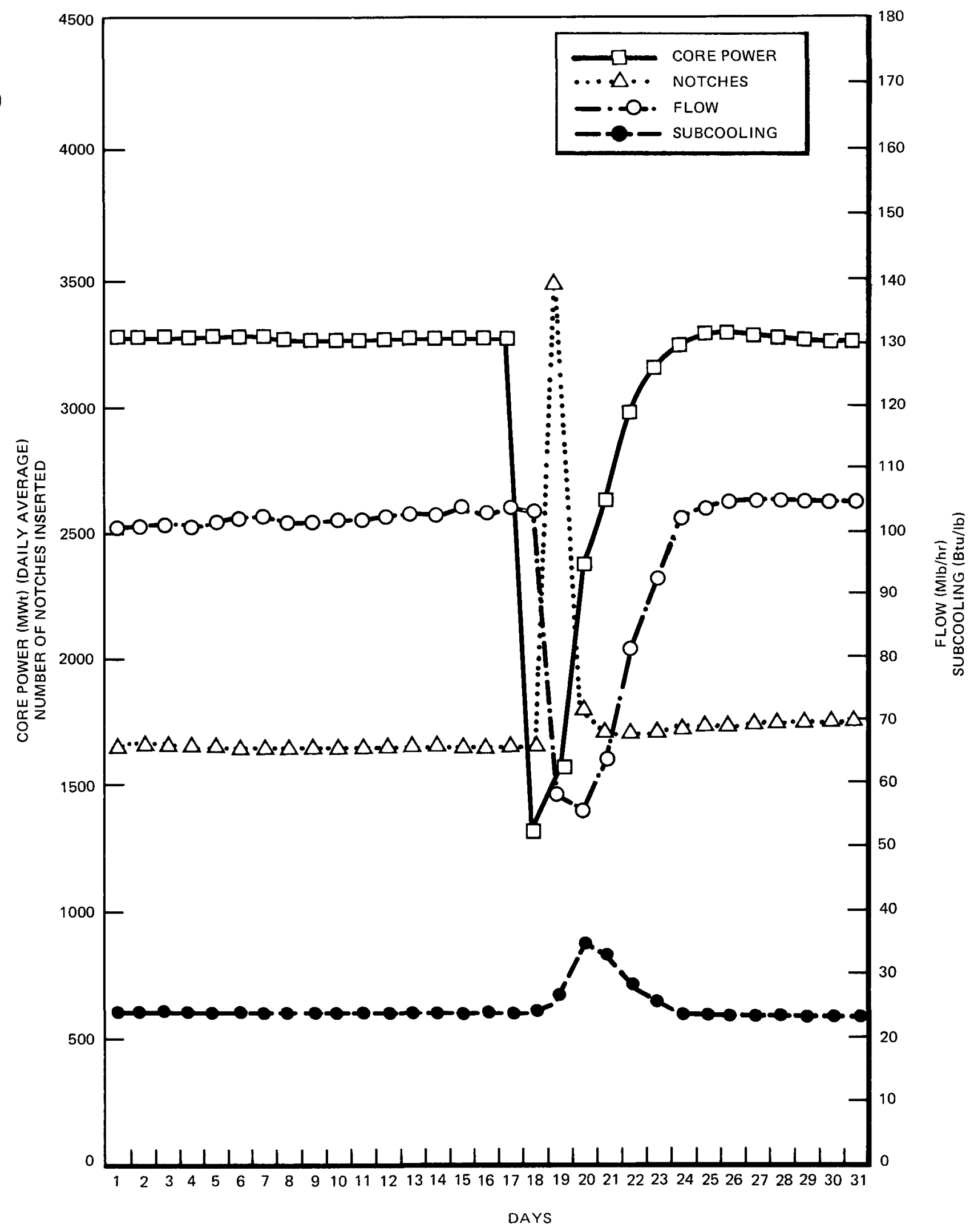

Figure 77. Data Summaries, March 1975 


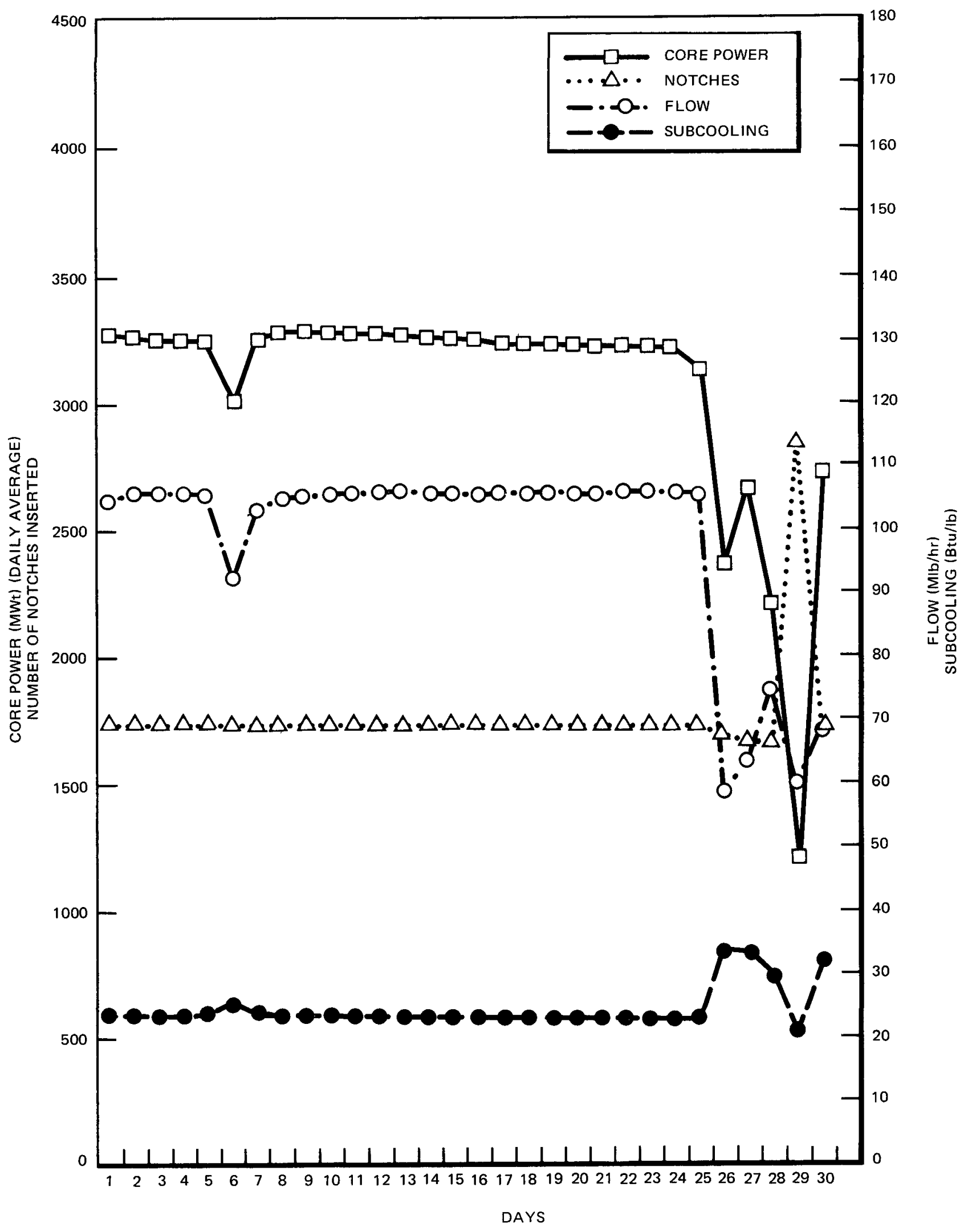

Figure 78. Data Summaries, April 1975 


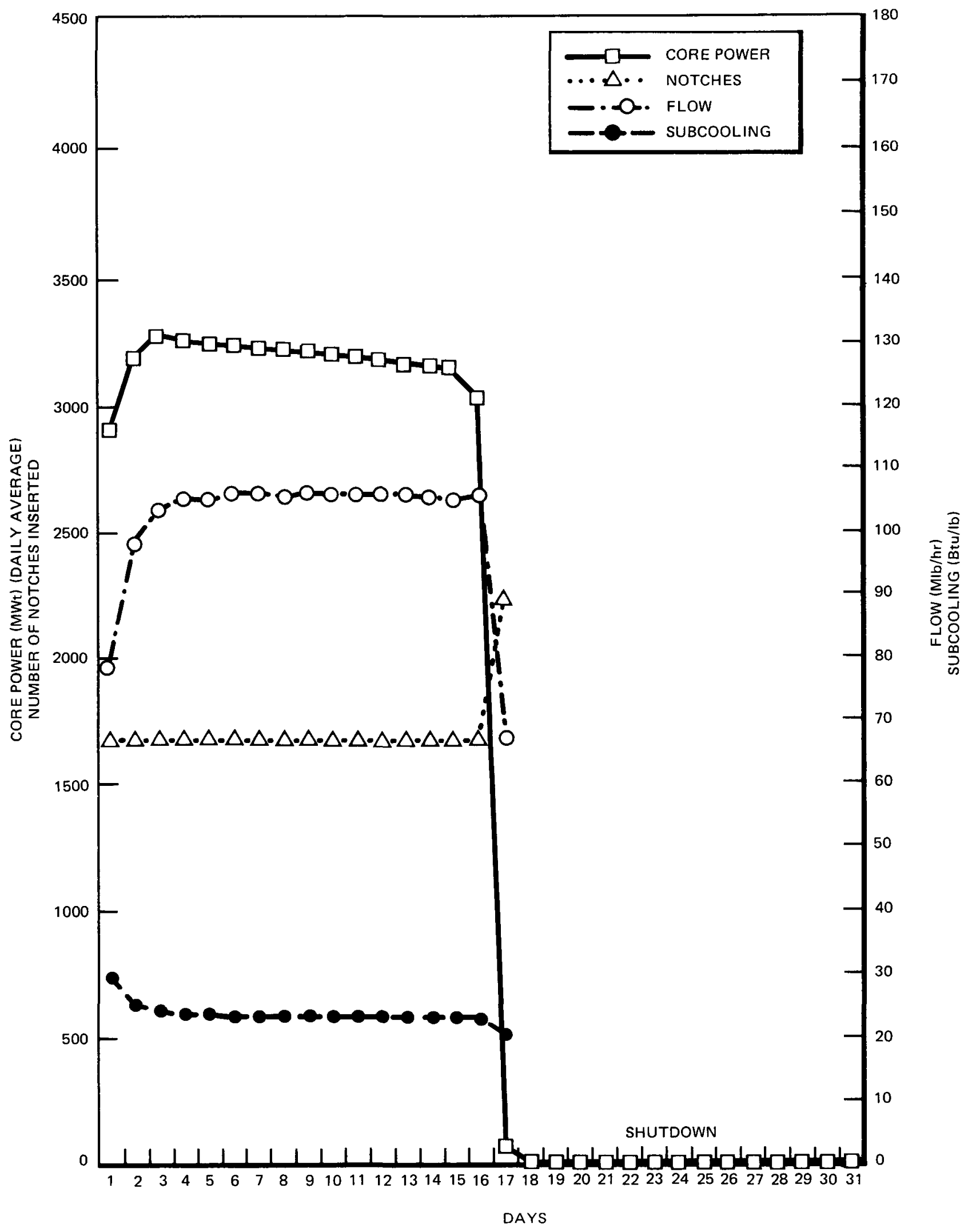

Figure 79. Data Summaries, May 1975 


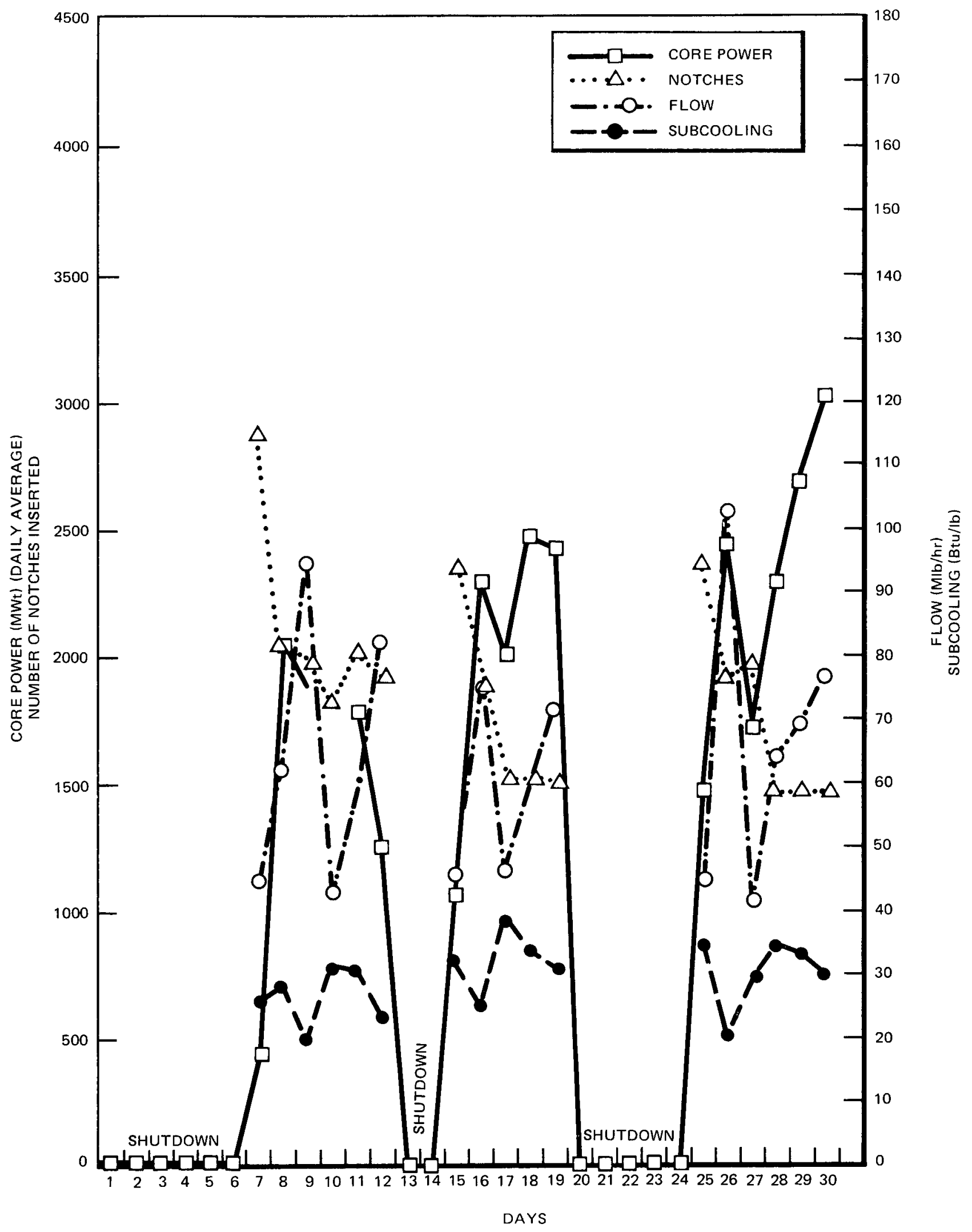

Figure 80. Data Summaries, June 1975 


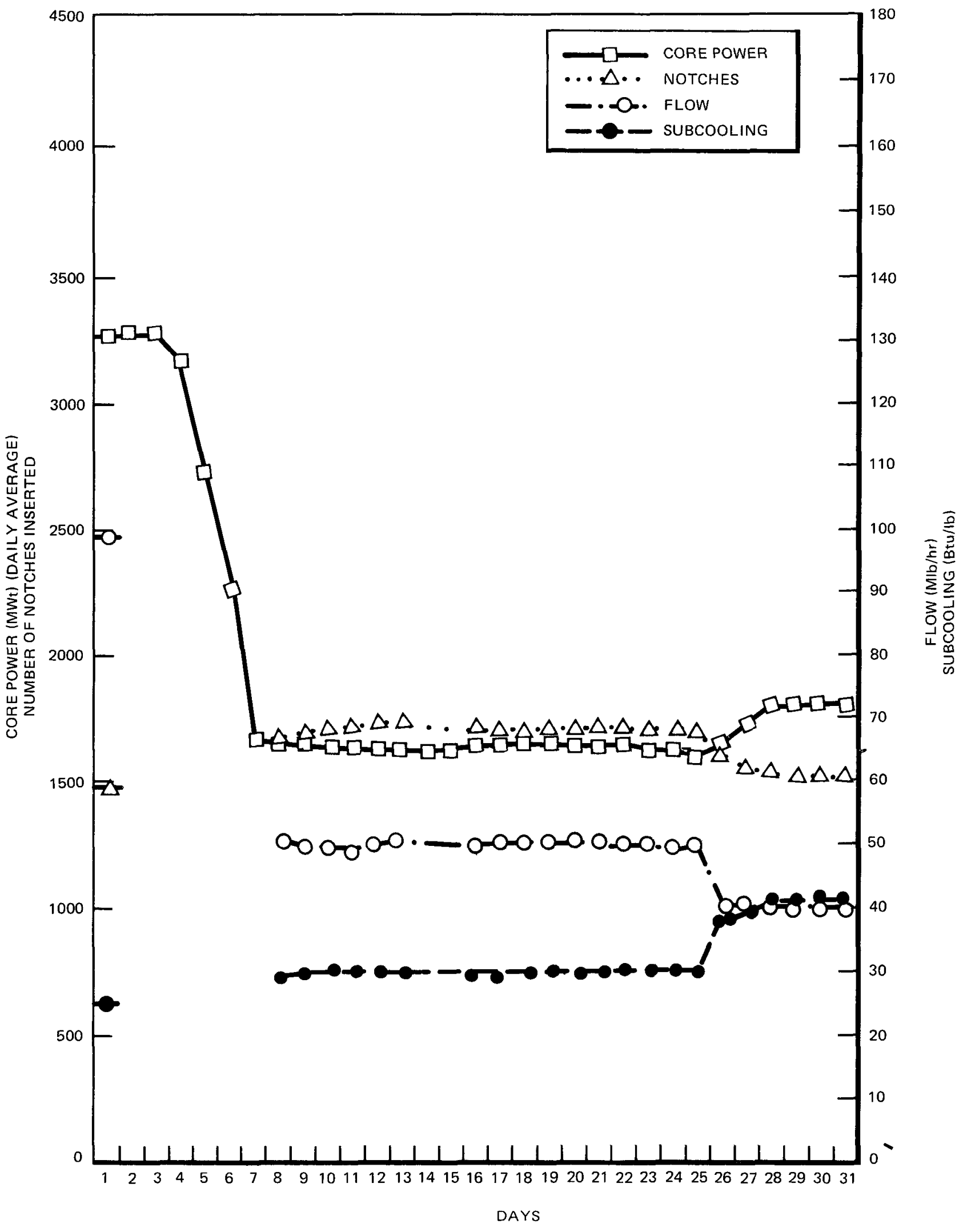

Figure 81. Data Summaries, July 1975 


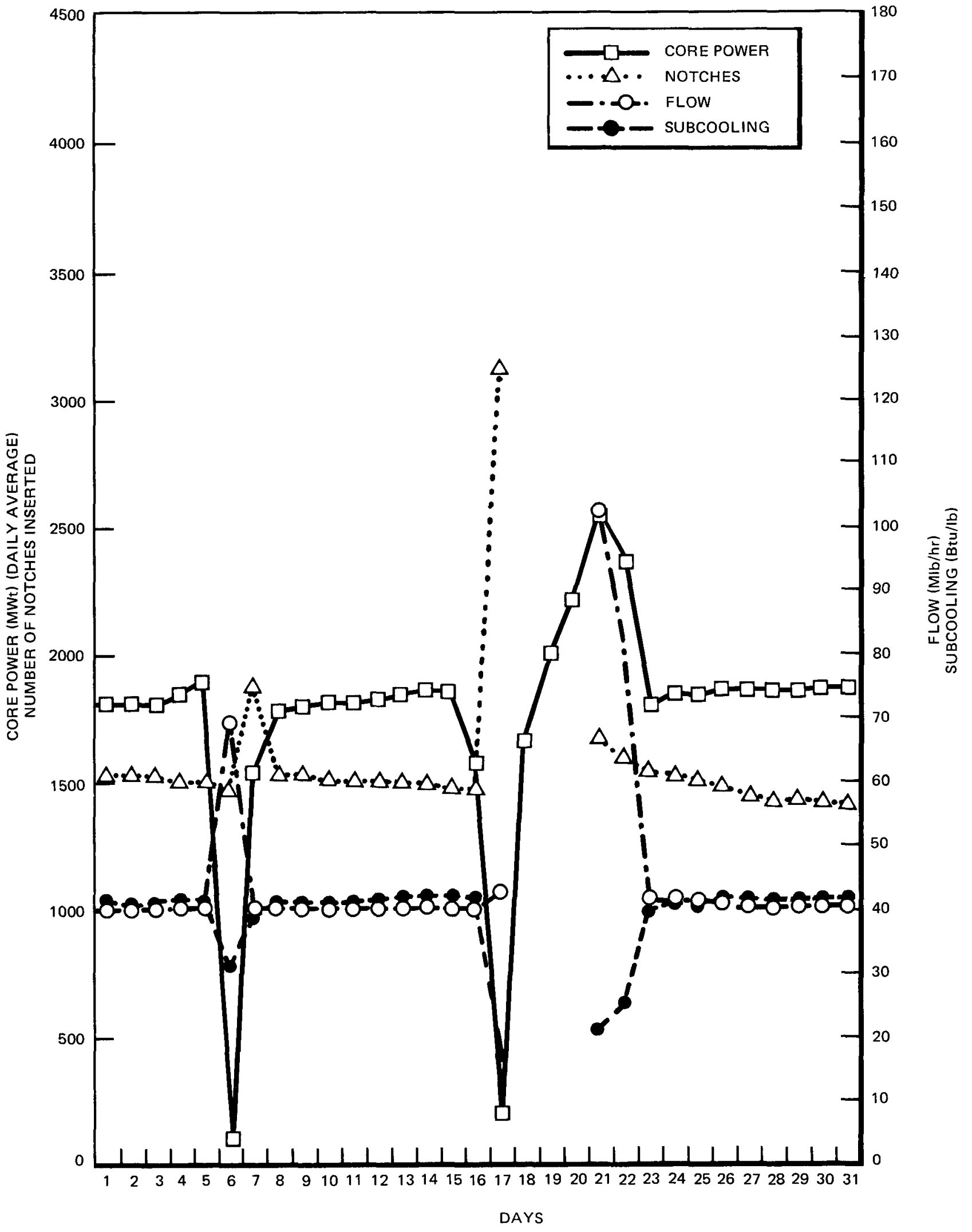

Figure 82. Data Summaries, August 1975 


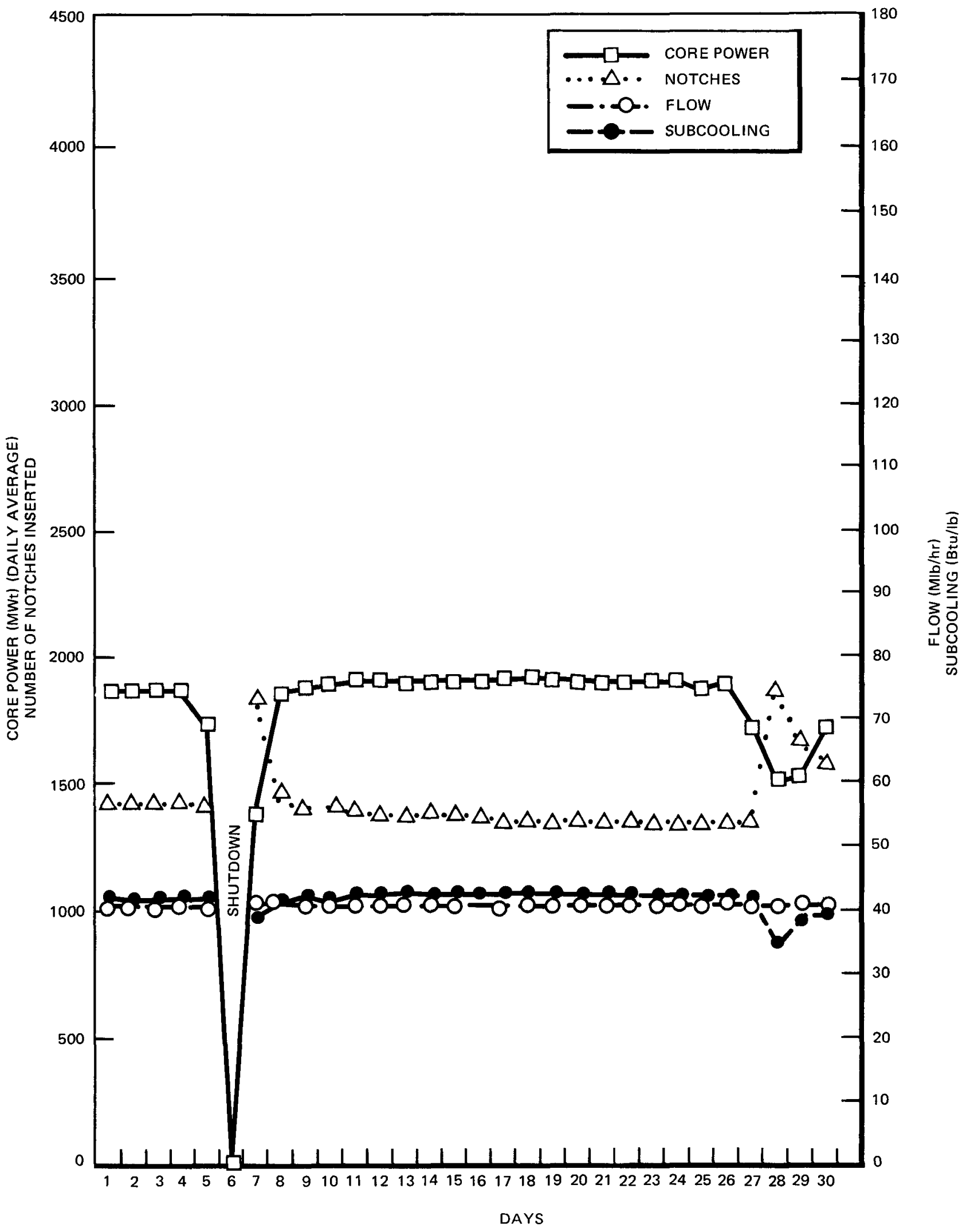

Figure 83. Data Summaries, September 1975 


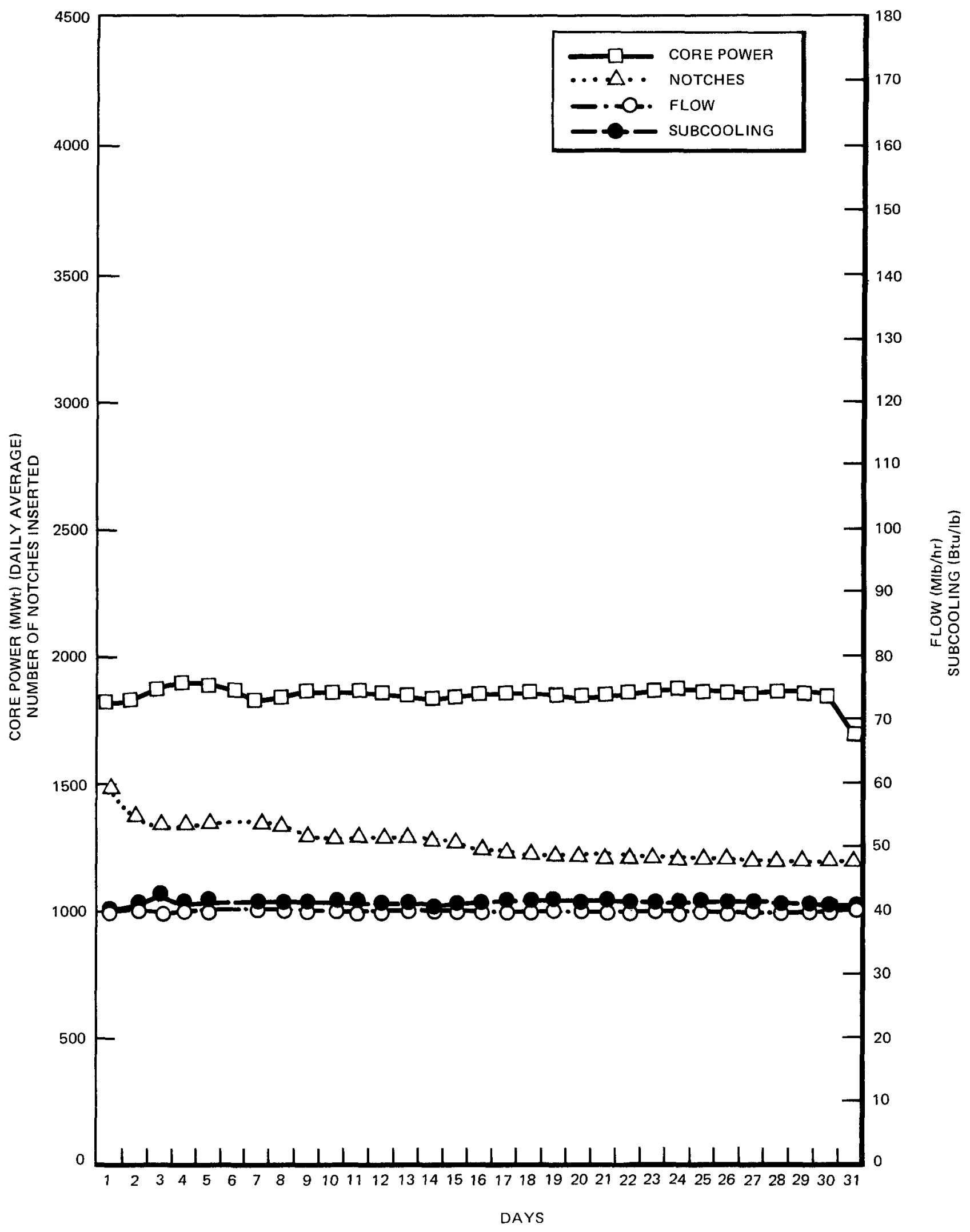

Figure 84. Data Summaries, October 1975 


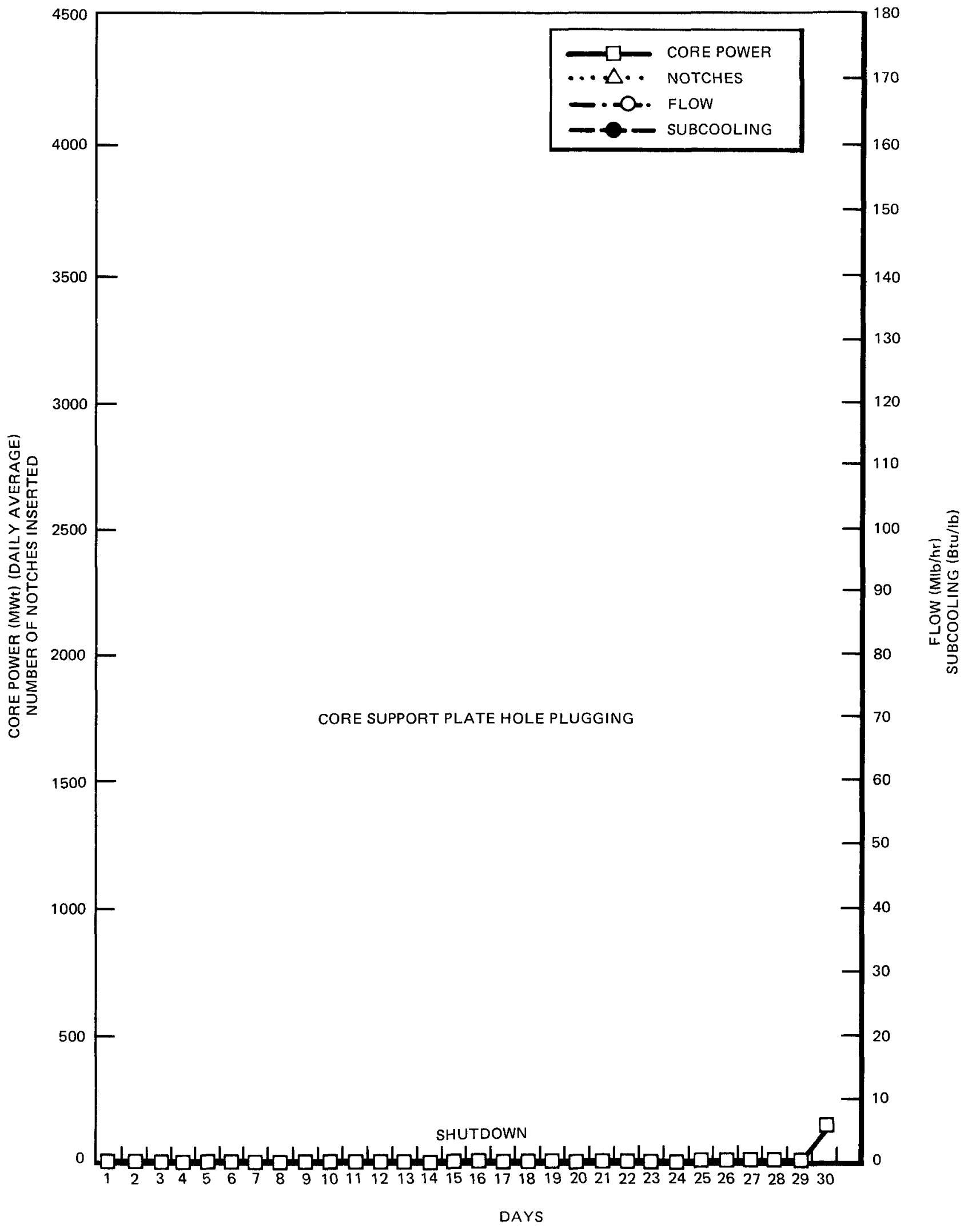

Figure 85. Data Summaries, November 1975 


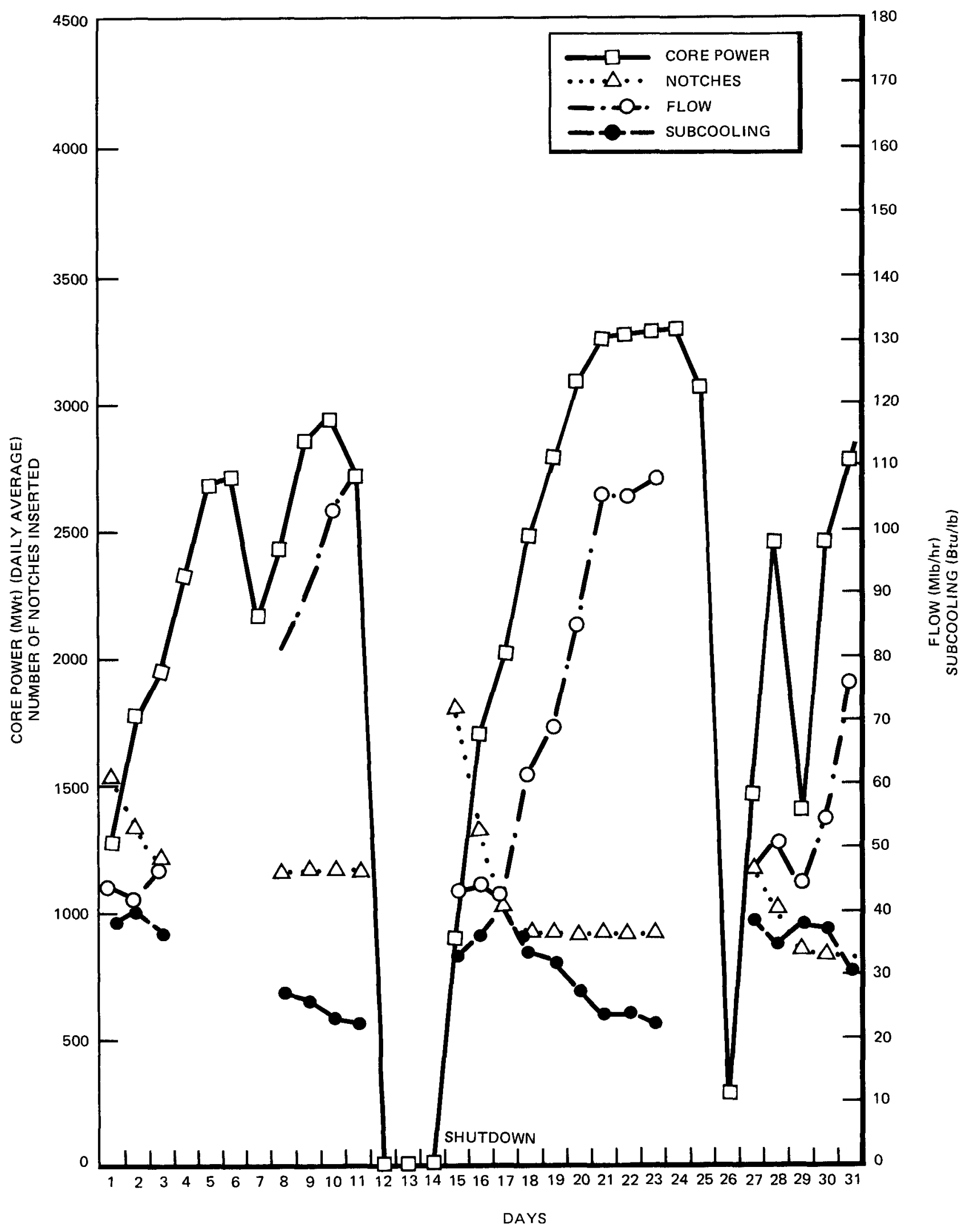

Figure 86. Data Summaries, December 1975 


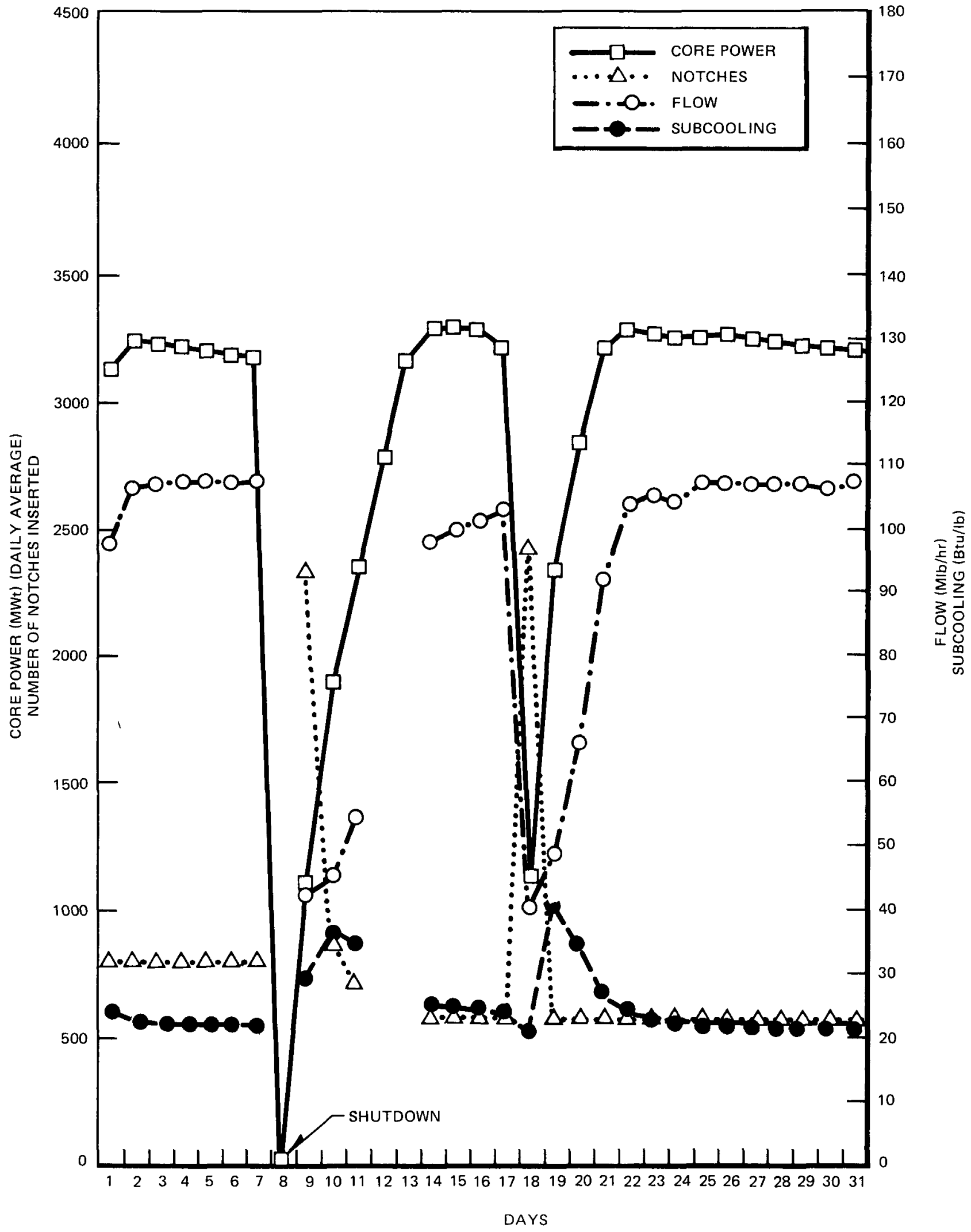

Figure 87. Data Summaries, January 1976 


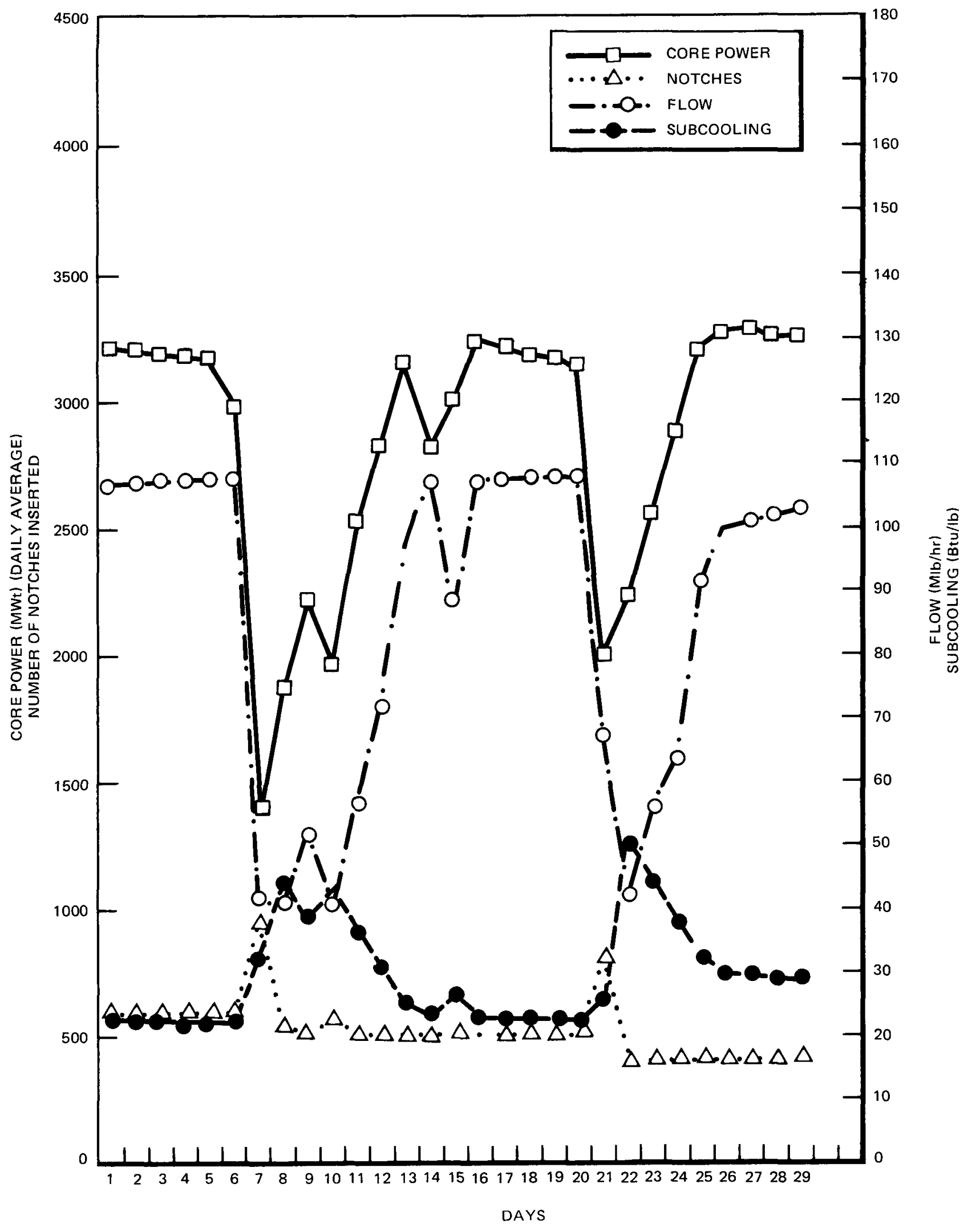

Figure 88. Data Summaries, February 1976 


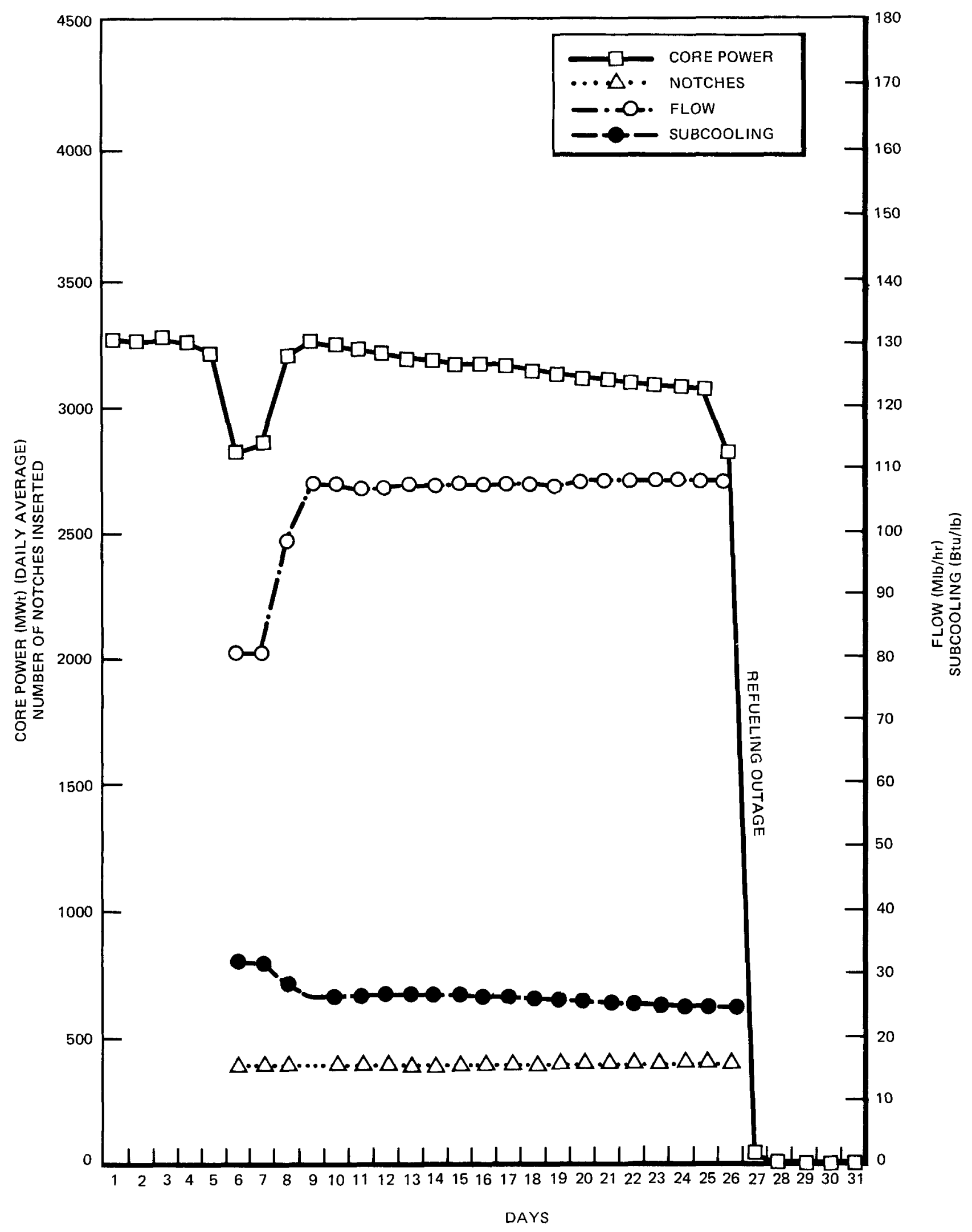

Figure 89. Data Summaries, March 1976 


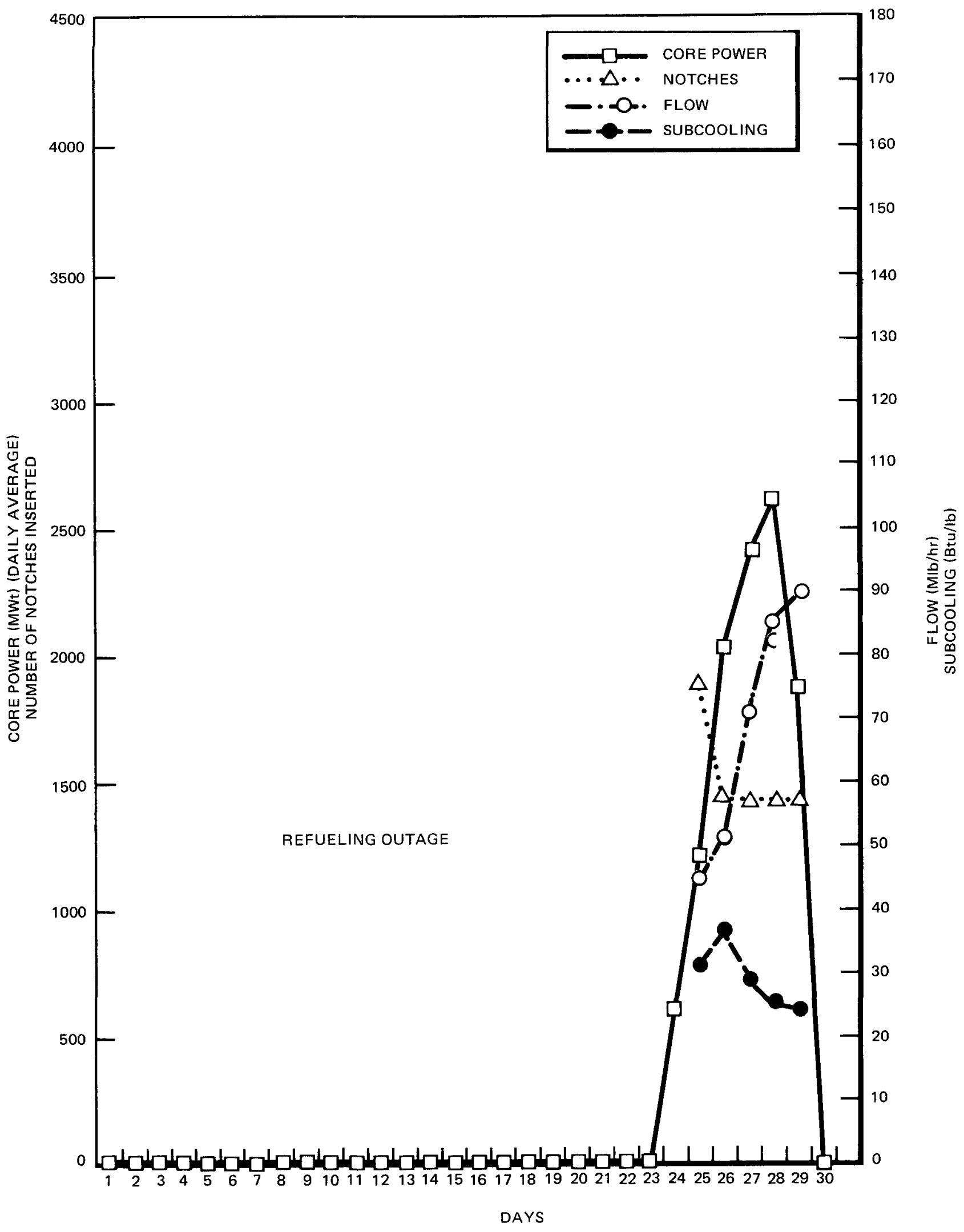

Figure 90. Data Summaries, June 1976 


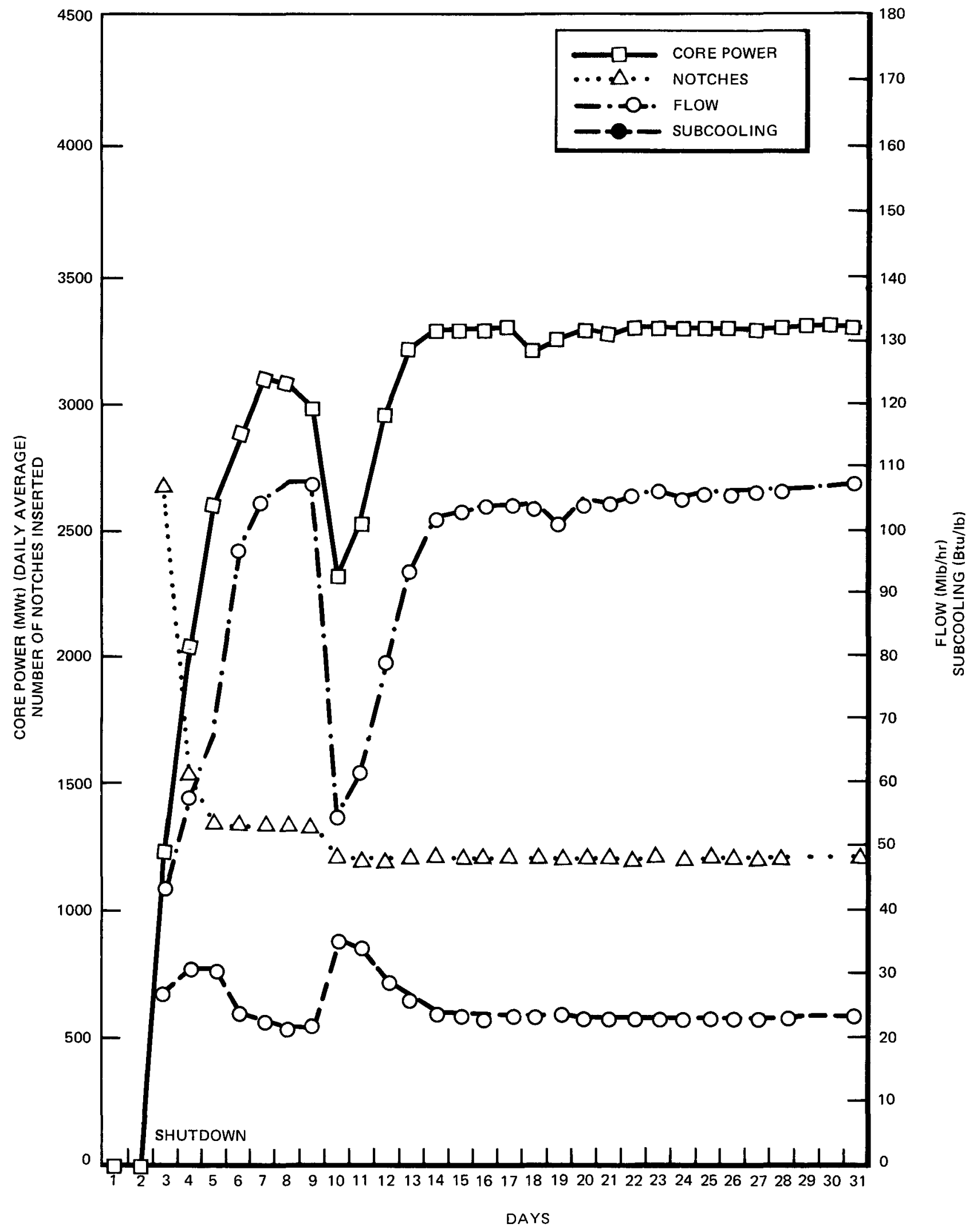

Figure 91. Data Summaries, July 1976 


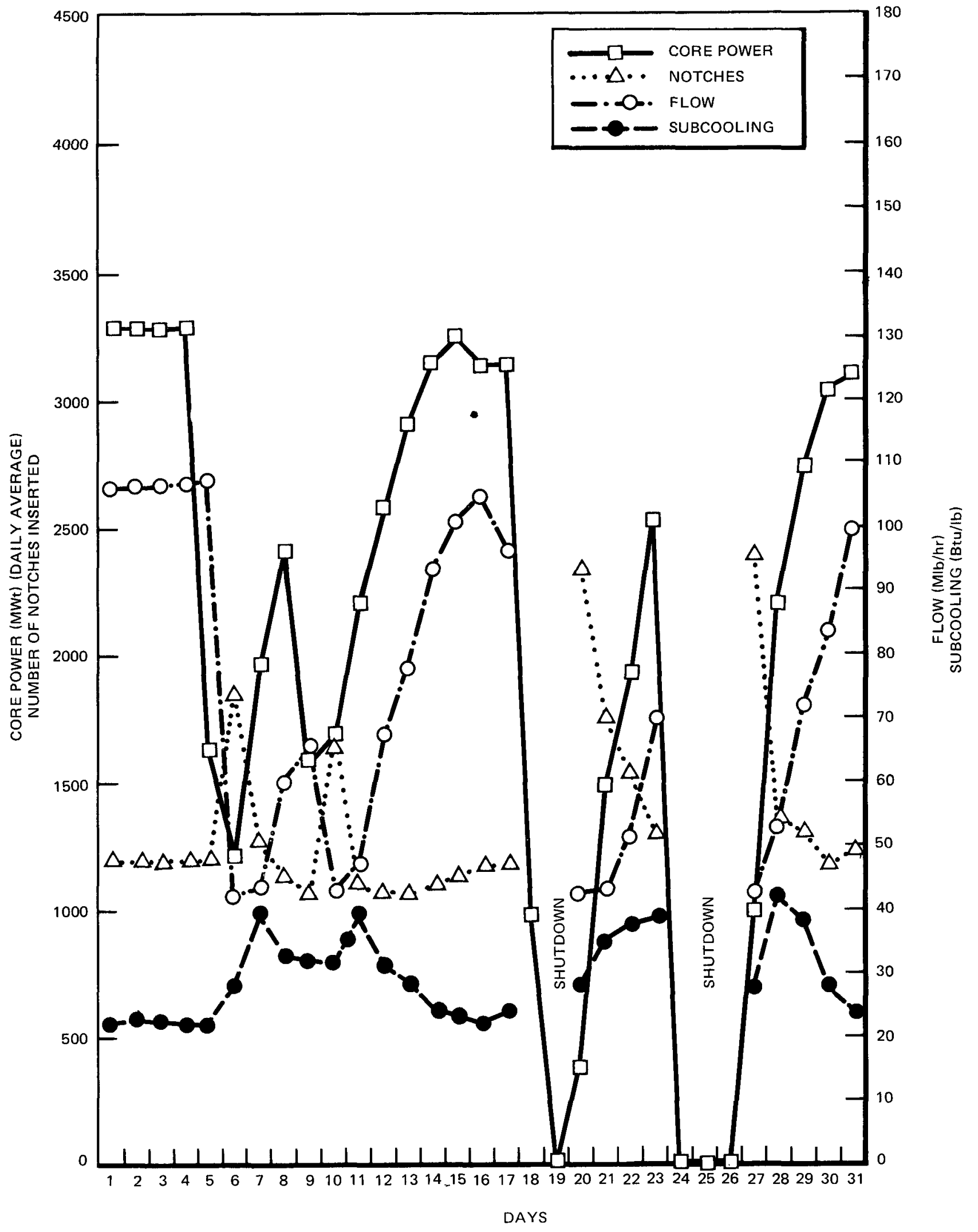

Figure 92. Data Summaries, August 1976 


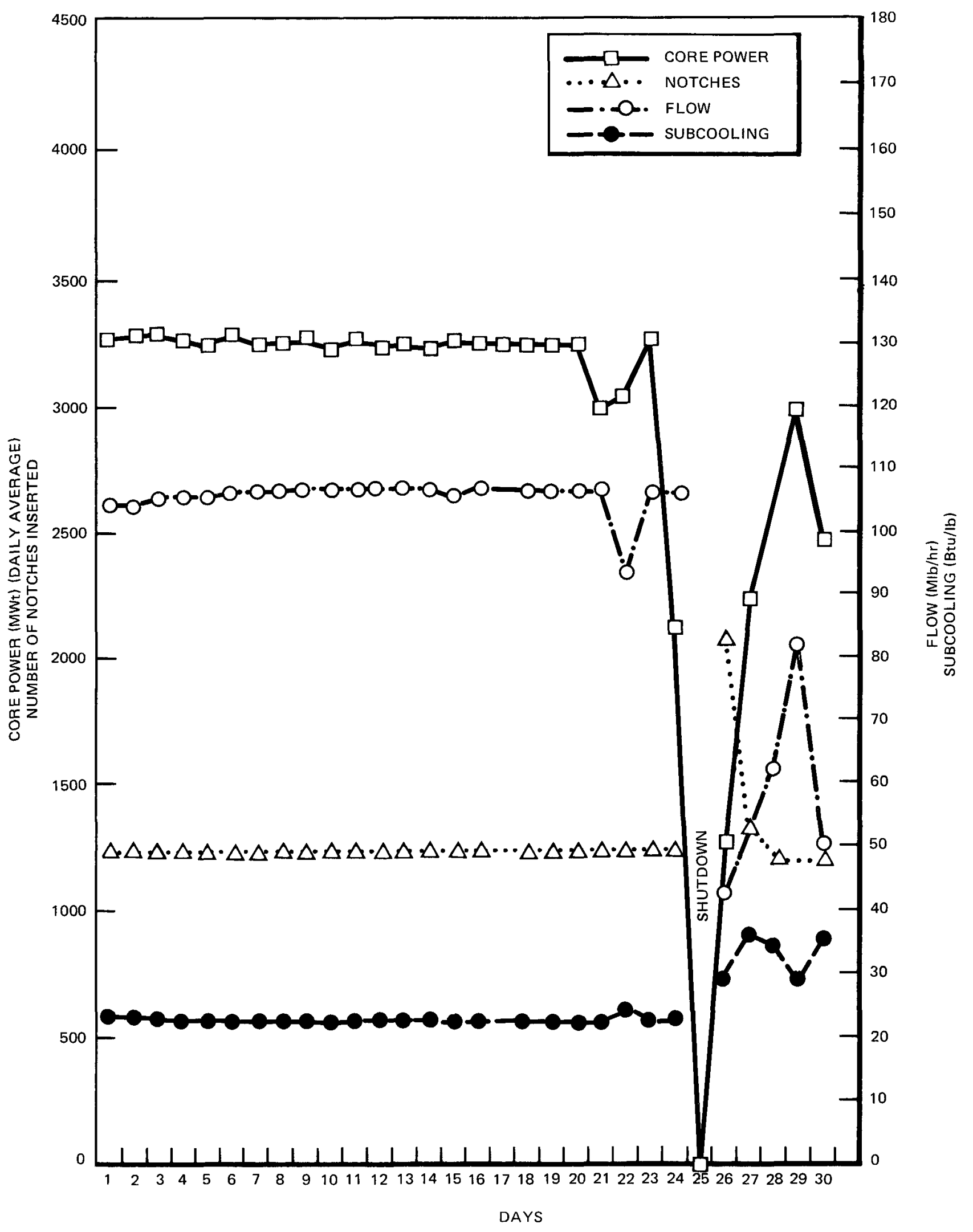

Figure 93. Data Summaries, September 1976 


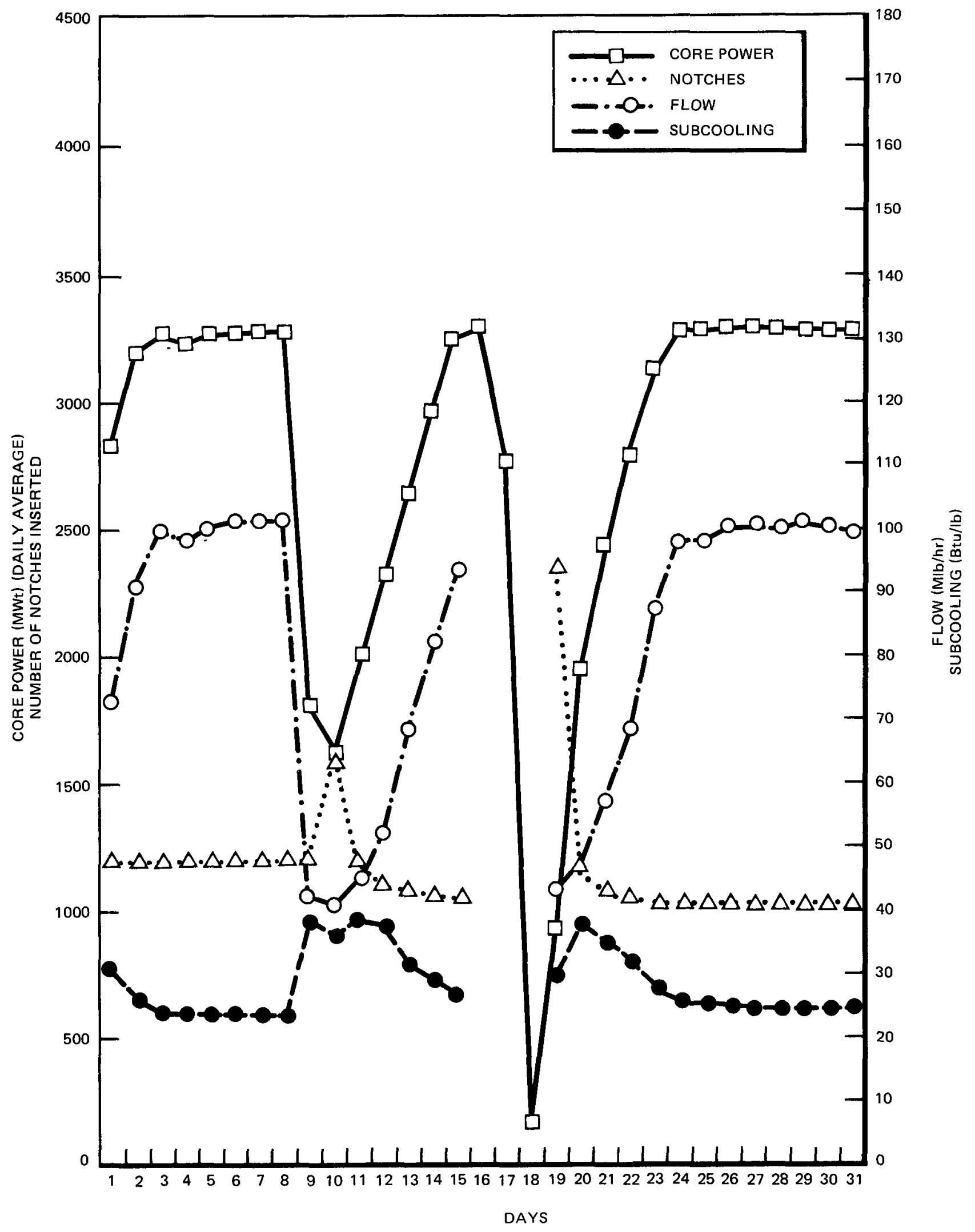

Figure 94. Data Summaries, October 1976 


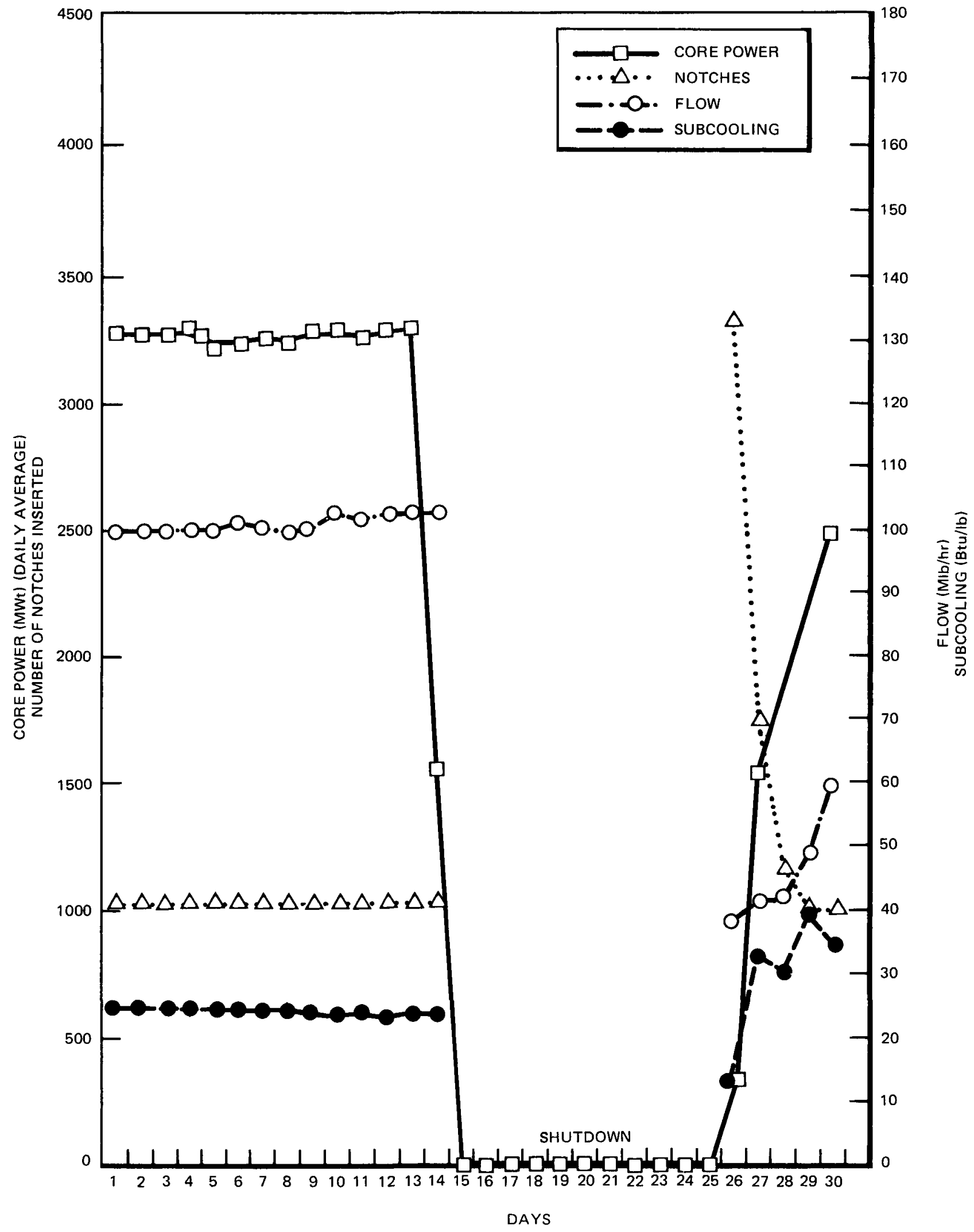

Figure 95. Data Summaries, November 1976 


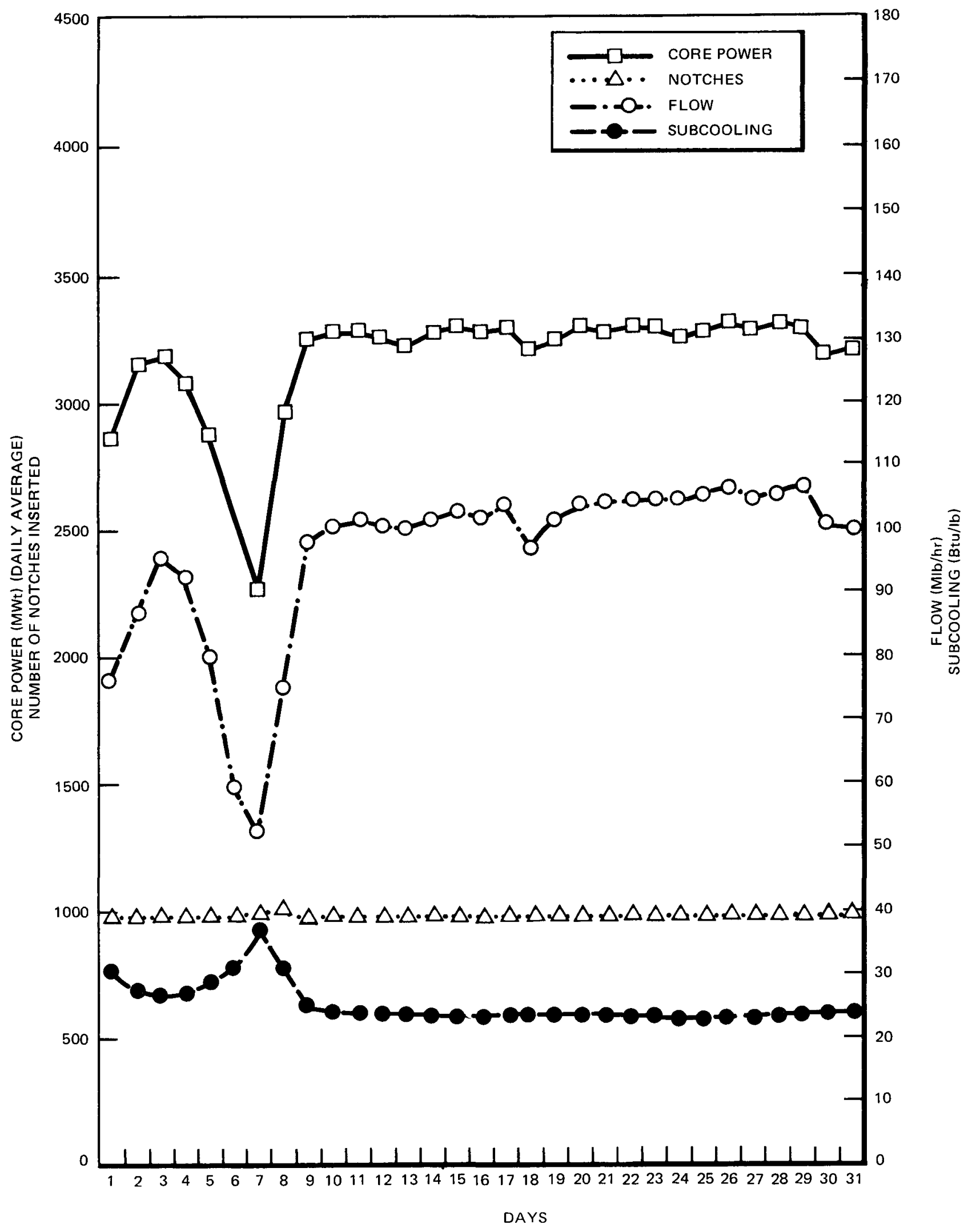

Figure 96. Data Summaries, December 1976 


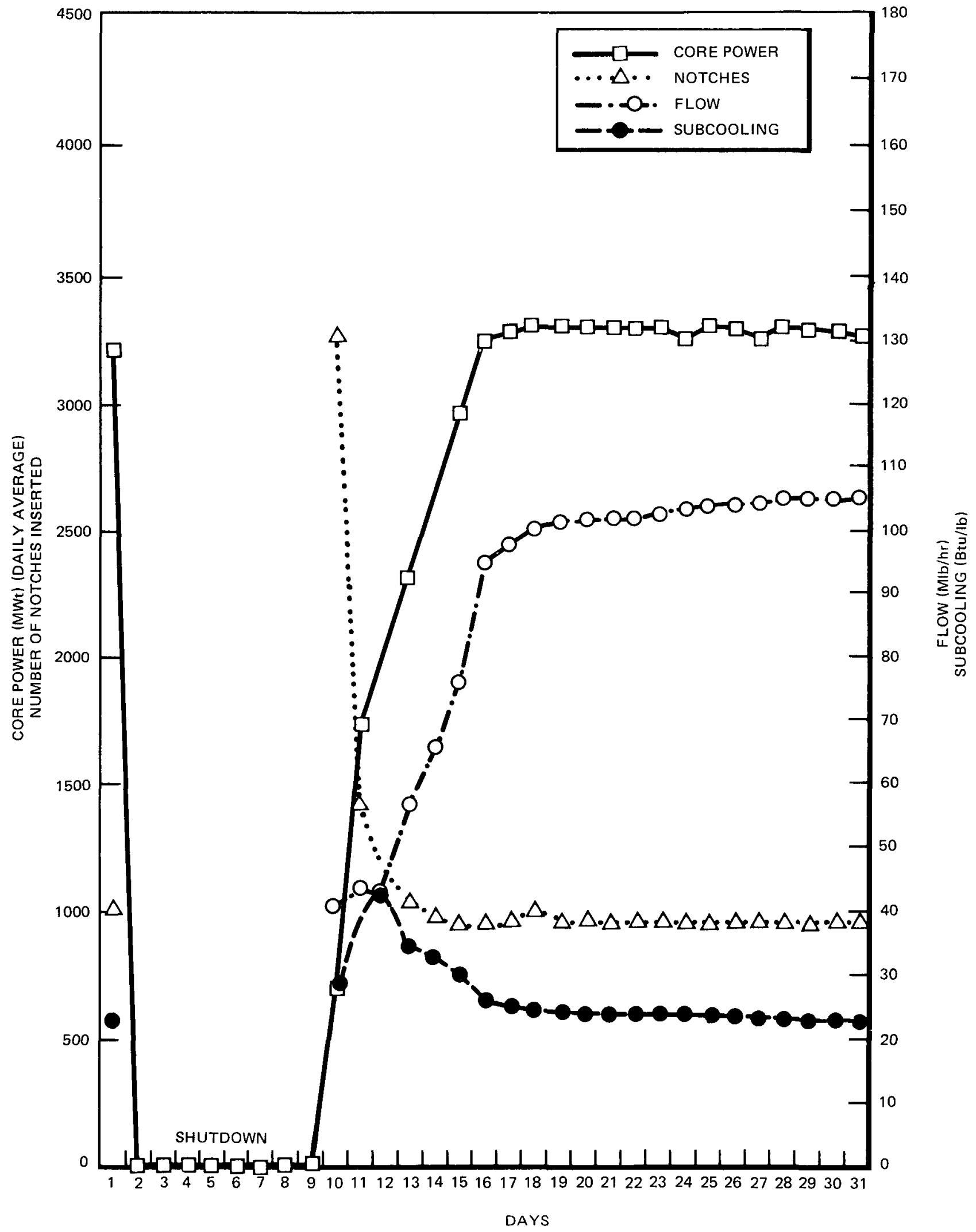

Figure 97. Data Summaries, January 1977 


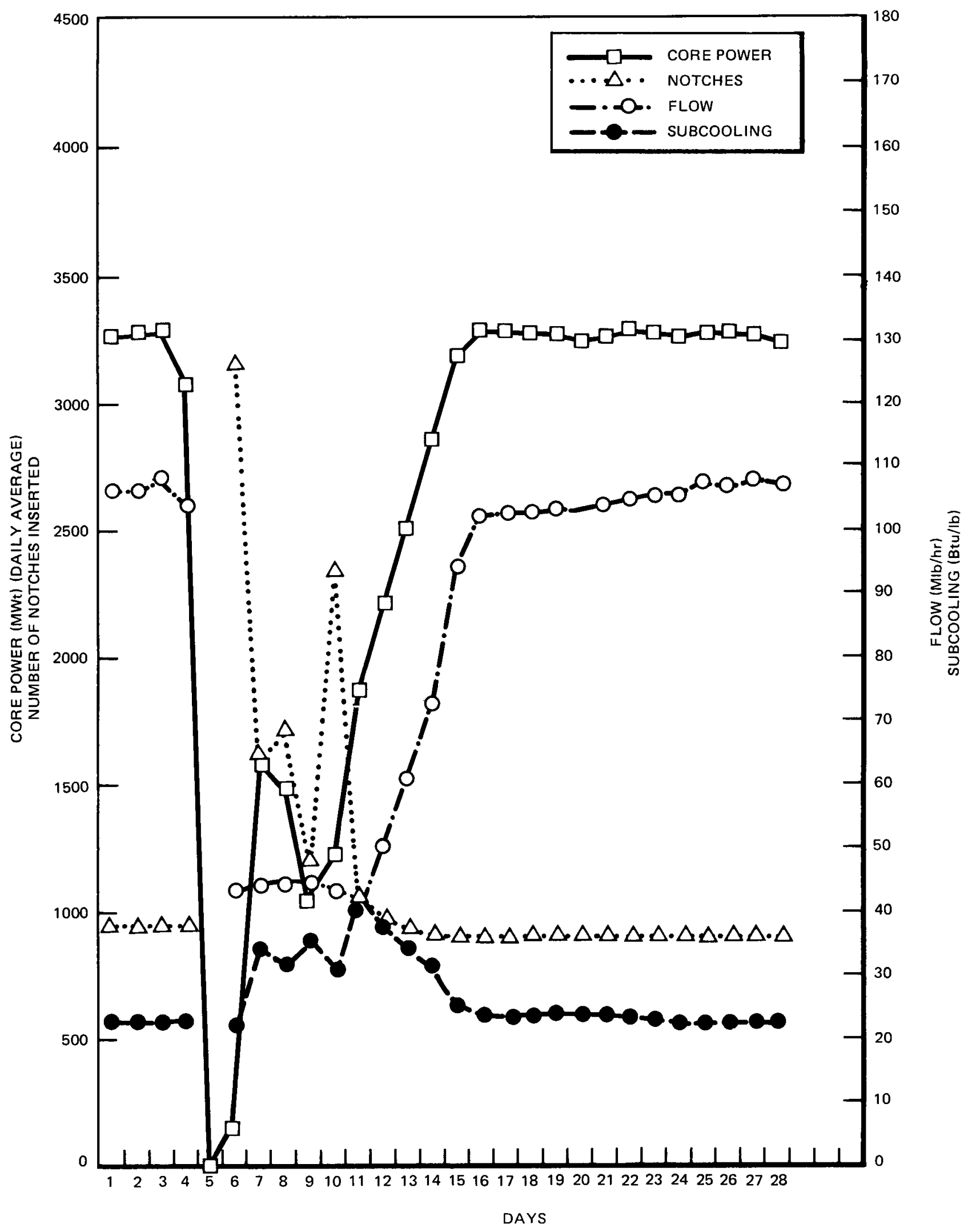

Figure 98. Data Summaries, February 1977 


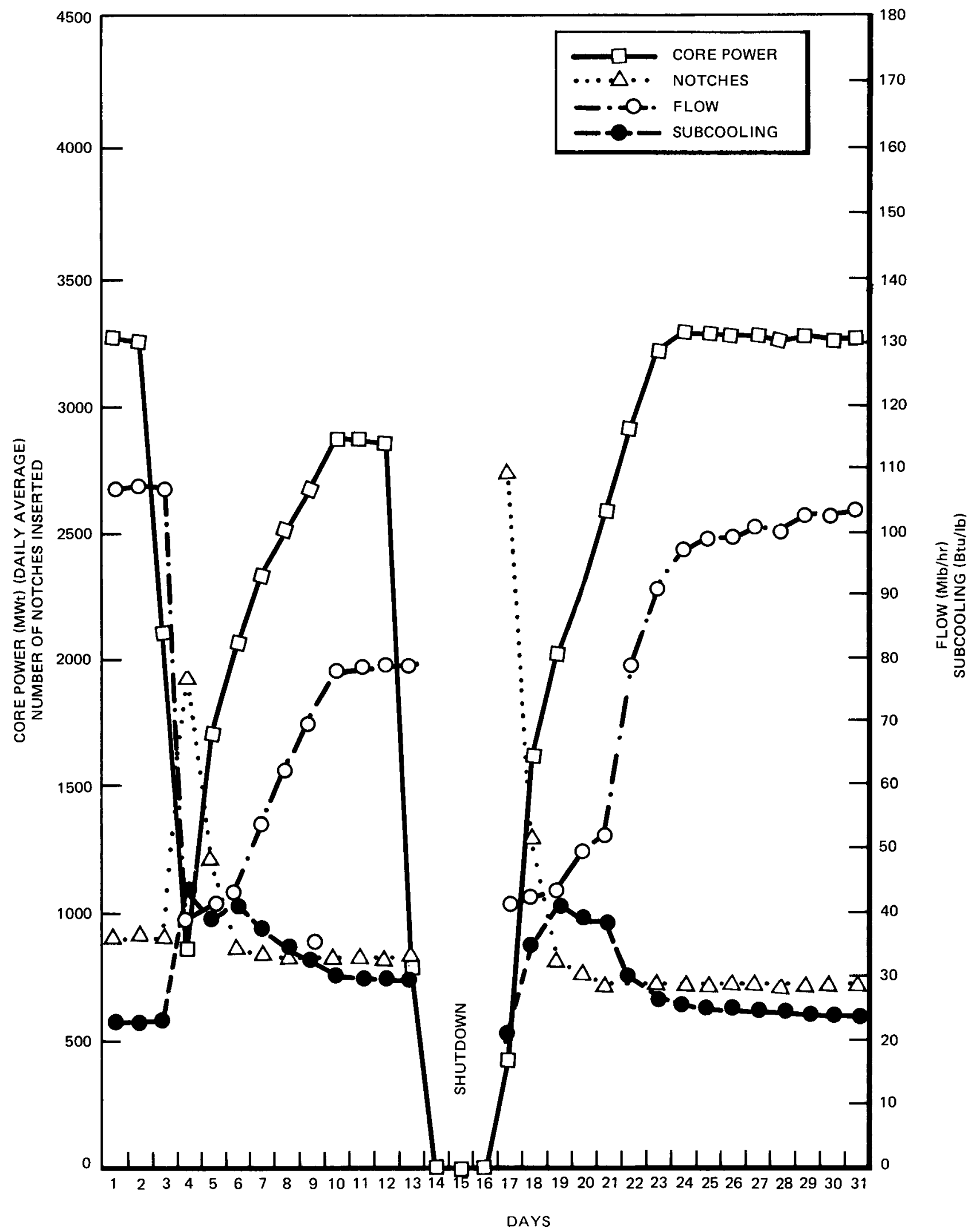

Figure 99. Data Summaries, March 1977 


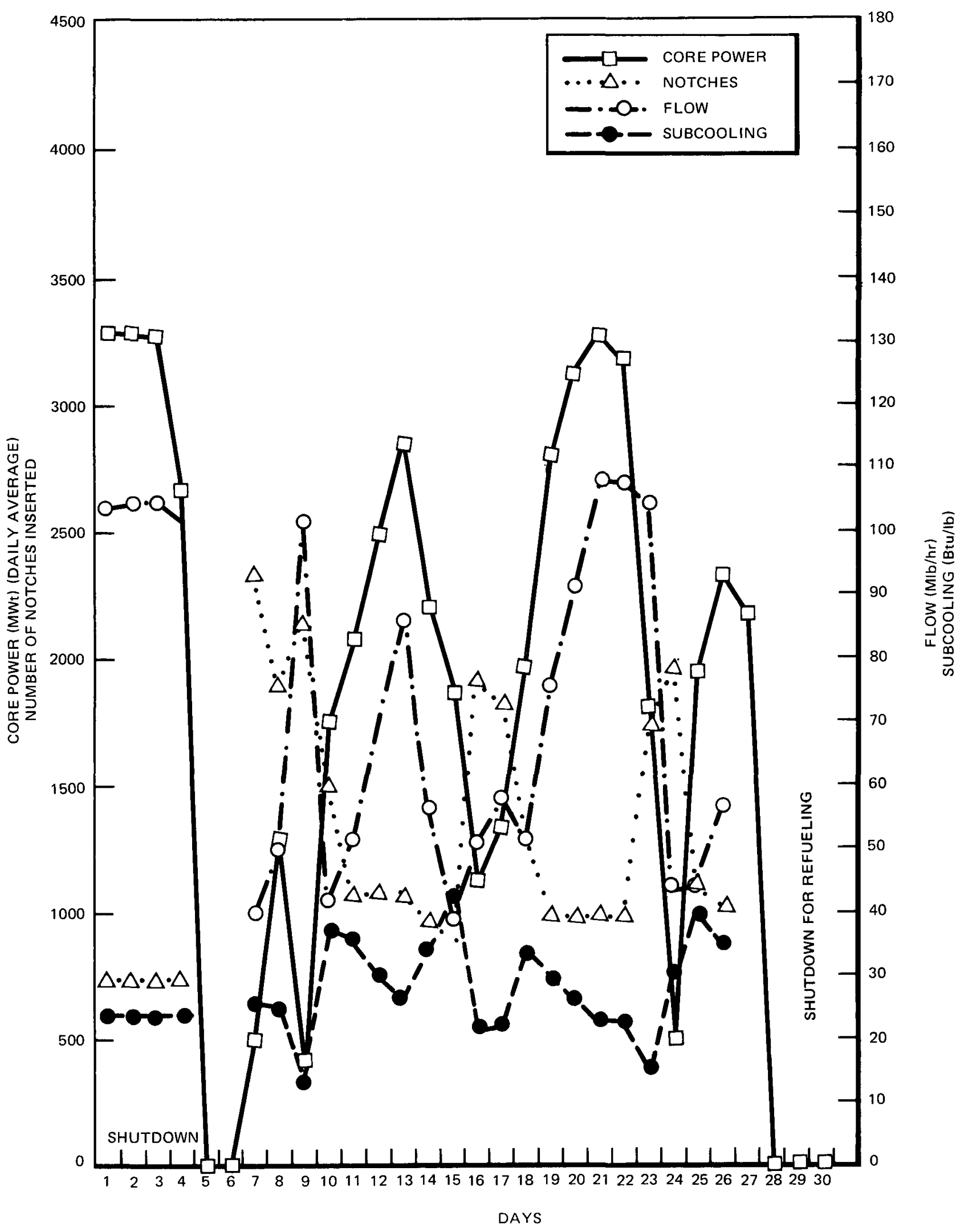

Figure 100. Data Summaries, April 1977 


\section{CYCLE 1 DATA}

\section{DATASET 01, APRIL 5, 1974}

\section{Reactor Conditions}

Core Average Exposure, $230 \mathrm{MWd} / \mathrm{t}$

Core Thermal Power, 1835 MWT

Dome Pressure, $\mathrm{P}, 973$ psia

Core Flow, $105.9 \mathrm{Mlb} / \mathrm{hr}$

Inlet Subcooling at P, 14.2 Btu/lb

Control Configuration

Legend: 48, Full Out; O, Full In.

\begin{tabular}{|c|c|c|c|c|c|c|c|c|c|c|c|c|c|c|}
\hline & $\begin{array}{r}48 \\
48 \\
48 \\
48 \\
48 \\
8 \\
48 \\
38 \\
48 \\
8 \\
88 \\
48\end{array}$ & $\begin{array}{l}48 \\
48\end{array}$ & $\begin{array}{r}48 \\
38 \\
48 \\
38 \\
48 \\
4 \\
48 \\
38 \\
48 \\
38 \\
48 \\
48 \\
48\end{array}$ & $\begin{array}{l}48 \\
48 \\
48 \\
48 \\
48 \\
48 \\
48 \\
48 \\
48 \\
48 \\
48 \\
48\end{array}$ & $\begin{array}{r}8 \\
48 \\
38 \\
48 \\
4 \\
48 \\
48 \\
38 \\
48 \\
4 \\
48\end{array}$ & $\begin{array}{l}48 . \\
48 .\end{array}$ & $\begin{array}{r}48 . \\
38 . \\
48 . \\
4 .\end{array}$ & & 48 & 48. & $\begin{array}{r}48 . \\
48 . \\
48 . \\
38 . \\
48 . \\
38 . \\
48 . \\
4 . \\
48 . \\
38 .\end{array}$ & $\begin{array}{l}4 \\
4 \\
4 \\
4\end{array}$ & $\begin{array}{r}48 . \\
8 . \\
88 .\end{array}$ & \\
\hline
\end{tabular}

Axial TIP Distribution, Bottom to Top of Core

See Figure 22

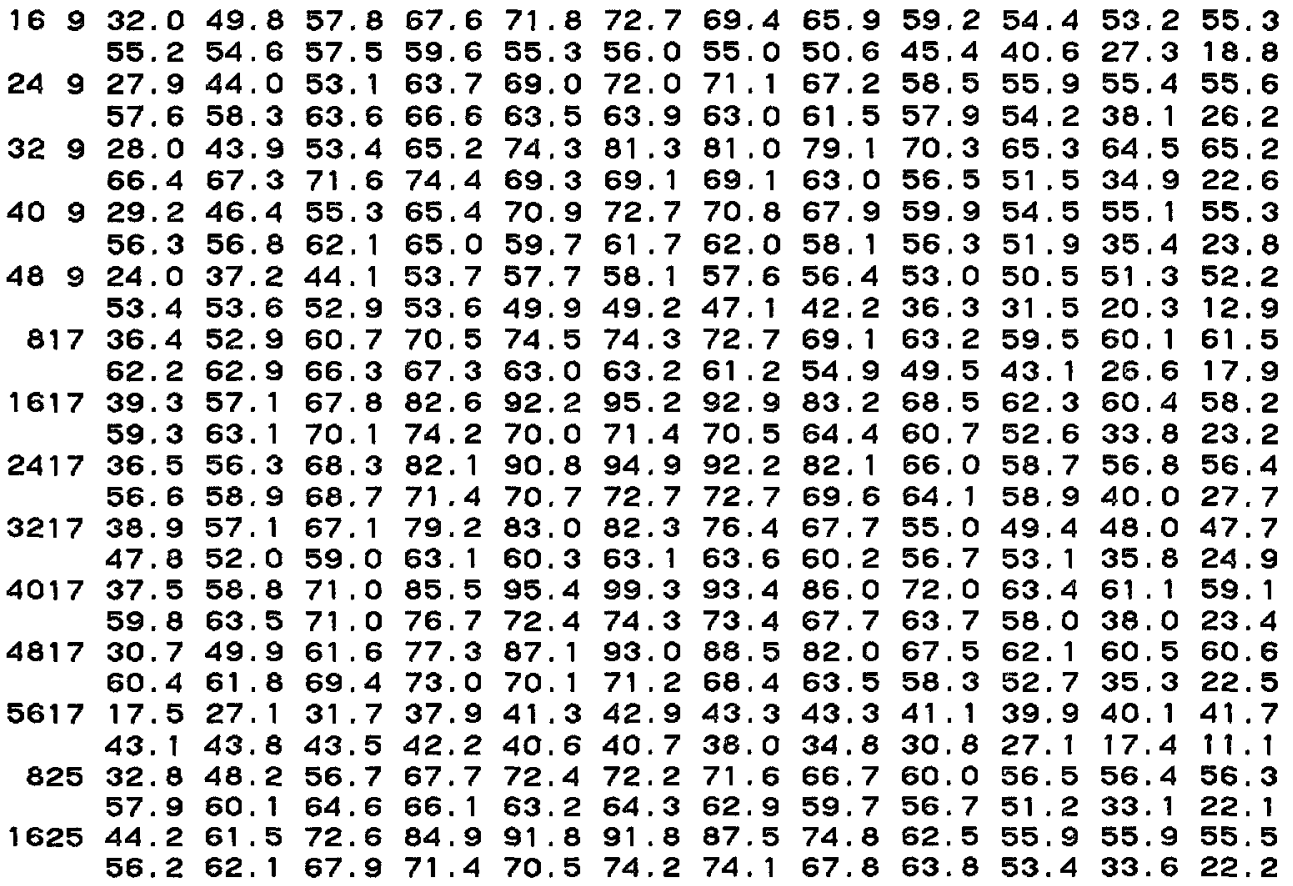


$242539.7 \quad 61.273 .0 \quad 85.0 \quad 90.1 \quad 89.6 \quad 81.6 \quad 72.0 \quad 57.2 \quad 51.1 \quad 49.950 .0$ $\begin{array}{lllllllllllll}51.4 & 56.5 & 66.8 & 73.9 & 73.1 & 77.3 & 79.8 & 77.4 & 74.9 & 70.5 & 48.7 & 33.7\end{array}$

$322540.0 \quad 60.972 .4 \quad 87.0 \quad 94.4 \quad 96.8 \quad 89.8 \quad 79.4 \quad 64.1 \quad 56.954 .6 \quad 54.2$ $\begin{array}{llllllllllll}54.4 & 58.4 & 68.1 & 72.8 & 70.4 & 73.8 & 76.4 & 73.3 & 69.2 & 63.7 & 43.2 & 28.9\end{array}$

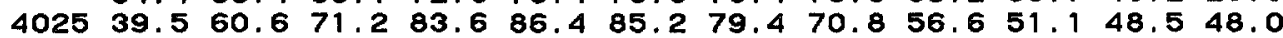

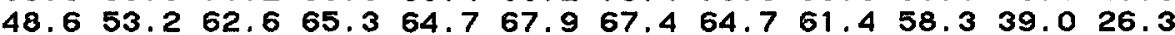

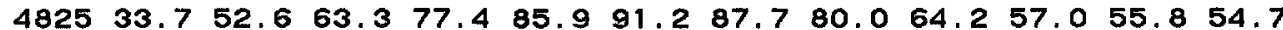
$55.760 .267 .7 \quad 70.768 .0 \quad 72.3 \quad 70.066 .462 .2 \quad 56.4 \quad 37.5 \quad 23.5$

$\begin{array}{lllllllllllllll}5625 & 16.1 & 26.1 & 31.3 & 39.1 & 44.1 & 47.6 & 48.1 & 49.0 & 46.6 & 46.7 & 47.9 & 48.1\end{array}$ $\begin{array}{lllllllllllll}50.1 & 51.1 & 51.5 & 52.1 & 50.1 & 51.2 & 51.3 & 49.3 & 47.3 & 44.1 & 28.8 & 17.7\end{array}$

$\begin{array}{llllllllllllll}833 & 35.8 & 50.4 & 59.7 & 72.1 & 80.6 & 84.0 & 84.2 & 76.9 & 69.0 & 64.3 & 65.3 & 67.8\end{array}$ $68.071 .474 .674 .870 .3 \quad 70.6 \quad 68.5 \quad 61.0 \quad 56.1 \quad 46.8 \quad 29.0 \quad 18.7$

$\begin{array}{llllllllllllll}1633 & 41.9 & 58.3 & 68.8 & 78.6 & 81.5 & 77.9 & 73.9 & 63.1 & 51.5 & 47.1 & 46.7 & 47.6\end{array}$ $47.554 .6 \quad 61.062 .961 .763 .7 \quad 64.1 \quad 60.4 \quad 56.9 \quad 47.5 \quad 30.821 .6$

$243341.6 \quad 60.4 \quad 70.6 \quad 84.1 \quad 91.1 \quad 91.6 \quad 87.0 \quad 75.3 \quad 62.1 \quad 55.3 \quad 53.7 \quad 53.4$ $\begin{array}{lllllllllllll}52.6 & 60.0 & 67.9 & 73.3 & 71.9 & 76.3 & 77.5 & 73.6 & 70.1 & 60.4 & 39.1 & 26.9\end{array}$

$\begin{array}{lllllllllllllll}3233 & 45.5 & 64.2 & 75.0 & 85.7 & 89.1 & 85.6 & 78.9 & 67.4 & 54.5 & 49.1 & 48.1 & 47.6\end{array}$ $\begin{array}{lllllllllllll}48.1 & 53.4 & 62.1 & 66.9 & 67.0 & 72.8 & 80.1 & 77.5 & 75.9 & 65.0 & 40.6 & 28.7\end{array}$

$403335.6 \quad 56.268 .4 \quad 83.2 \quad 90.9 \quad 92.3 \quad 89.1 \quad 80.1 \quad 65.4 \quad 58.254 .953 .4$ $\begin{array}{lllllllllllll}53.8 & 55.8 & 65.2 & 72.2 & 70.7 & 72.1 & 74.5 & 70.4 & 67.2 & 62.1 & 42.6 & 28.3\end{array}$

$\begin{array}{llllllllllllllll}4833 & 34.0 & 52.2 & 62.4 & 73.5 & 79.0 & 79.5 & 75.0 & 67.2 & 55.0 & 49.9 & 49.8 & 49.7\end{array}$ $50.654 .5 \quad 60.964 .1 \quad 62.6 \quad 63.963 .459 .7 \quad 56.852 .0 \quad 35.2 \quad 21.9$

$\begin{array}{llllllllllllll}5633 & 19.5 & 28.3 & 33.9 & 43.4 & 52.7 & 58.6 & 62.5 & 63.9 & 60.6 & 59.4 & 61.5 & 64.8\end{array}$ $\begin{array}{llllllllllll}63.8 & 64.4 & 64.2 & 63.4 & 58.1 & 57.1 & 54.9 & 49.0 & 43.4 & 36.7 & 22.4 & 15.2\end{array}$

$84135.5 \quad 50.8 \quad 59.1 \quad 69.1 \quad 73.6 \quad 74.5 \quad 72.3 \quad 66.6 \quad 59.3 \quad 56.3 \quad 56.0 \quad 57.0$

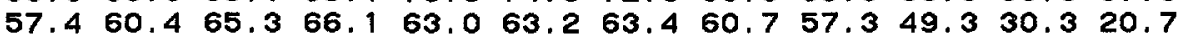

$\begin{array}{lllllllllllll}1641 & 35.9 & 56.1 & 68.2 & 84.3 & 93.4 & 99.4 & 94.5 & 88.0 & 71.4 & 64.2 & 61.7 & 61.6\end{array}$ $\begin{array}{llllllllllll}61.2 & 64.4 & 73.1 & 76.3 & 72.7 & 74.9 & 75.0 & 70.0 & 64.1 & 58.1 & 38.2 & 25.5\end{array}$

$244139.3 \quad 57.1 \quad 67.7 \quad 77.2 \quad 81.6 \quad 79.7 \quad 74.8 \quad 65.0 \quad 53.4 \quad 48.146 .945 .7$ $\begin{array}{lllllllllllll}46.5 & 51.6 & 59.2 & 63.1 & 60.0 & 63.3 & 62.7 & 59.7 & 57.1 & 50.5 & 34.6 & 23.8\end{array}$

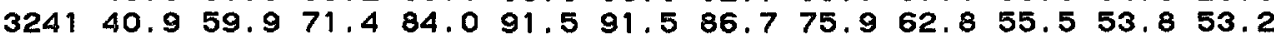

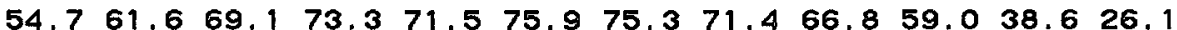

$404139.5 \quad 60.9 \quad 72.0 \quad 84.7$ 90.1 $88.8 \quad 82.4 \quad 73.4 \quad 59.3 \quad 53.752 .3 \quad 52.0$ $\begin{array}{llllllllllll}51.6 & 58.8 & 65.5 & 71.0 & 67.7 & 70.2 & 69.0 & 65.6 & 62.2 & 57.6 & 38.5 & 26.3\end{array}$

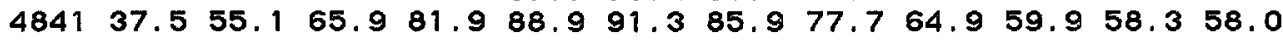
$\begin{array}{lllllllllllll}57.6 & 63.5 & 69.1 & 72.4 & 69.3 & 71.2 & 69.9 & 63.2 & 59.6 & 52.9 & 33.4 & 23.7\end{array}$

$\begin{array}{lllllllllllllll}5641 & 20.9 & 29.6 & 33.9 & 40.1 & 43.7 & 44.8 & 46.0 & 45.7 & 44.5 & 43.4 & 44.2 & 45.6\end{array}$ $\begin{array}{llllllllllllll}46.2 & 47.8 & 46.9 & 46.6 & 44.1 & 44.3 & 44.0 & 41.7 & 40.8 & 35.2 & 22.0 & 15.3\end{array}$

$84923.7 \quad 36.8 \quad 43.8 \quad 51.956 .7 \quad 59.1 \quad 58.2 \quad 57.6 \quad 53.1 \quad 49.0 \quad 48.848 .5$ $\begin{array}{lllllllllllll}50.3 & 50.1 & 50.3 & 51.0 & 48.2 & 48.3 & 46.0 & 39.8 & 35.2 & 30.3 & 19.5 & 12.4\end{array}$

$164934.7 \quad 52.6 \quad 63.7 \quad 78.3 \quad 88.1 \quad 91.9 \quad 89.8 \quad 80.367 .161 .260 .561 .5$ $\begin{array}{llllllllllllll}60.7 & 64.6 & 72.6 & 74.1 & 70.7 & 72.8 & 69.9 & 63.7 & 57.8 & 50.9 & 31.5 & 20.1\end{array}$

$244933.451 .261 .5 \quad 75.1 \quad 83.4 \quad 87.6 \quad 83.7 \quad 75.0 \quad 61.3 \quad 55.254 .5 \quad 54.7$

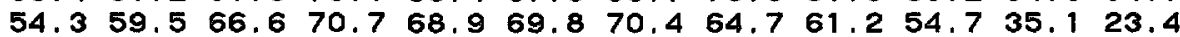

$324937.254 .963 .975 .7 \quad 80.0 \quad 80.1 \quad 75.267 .0 \quad 55.8 \quad 52.451 .250 .9$ $\begin{array}{llllllllllll}50.7 & 58.1 & 63.7 & 65.5 & 61.4 & 65.8 & 63.7 & 60.2 & 57.5 & 52.6 & 33.9 & 24.8\end{array}$

$\begin{array}{lllllllllllllll}4049 & 34.7 & 53.5 & 65.6 & 78.9 & 88.4 & 94.0 & 90.4 & 81.7 & 67.5 & 61.1 & 60.5 & 60.7\end{array}$ $61.6 \quad 65.173 .878 .8 \quad 77.479 .7 \quad 79.2 \quad 75.7 \quad 71.463 .141 .6 \quad 27.3$

$484928.944 .1 \quad 53.4 \quad 65.9 \quad 75.1 \quad 80.5 \quad 80.1 \quad 75.3 \quad 68.262 .562 .3 \quad 62.6$ $\begin{array}{lllllllllllll}63.6 & 64.4 & 66.0 & 68.0 & 62.9 & 63.6 & 60.4 & 53.6 & 48.8 & 42.7 & 26.2 & 17.9\end{array}$

$165717.2 \quad 26.1 \quad 31.0 \quad 37.0 \quad 40.0 \quad 41.4 \quad 41.4 \quad 41.7 \quad 39.7 \quad 38.9 \quad 39.841 .5$ $\begin{array}{llllllllllllll}42.1 & 42.6 & 42.4 & 42.8 & 40.5 & 40.3 & 39.1 & 34.8 & 31.2 & 26.4 & 16.5 & 10.5\end{array}$

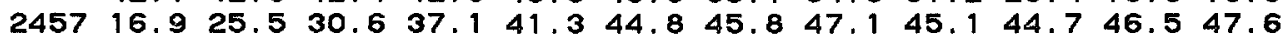
$\begin{array}{lllllllllllll}49.0 & 50.4 & 49.6 & 50.6 & 46.8 & 47.2 & 46.2 & 43.4 & 41.3 & 37.4 & 23.6 & 15.9\end{array}$

$325718.2 \quad 27.8 \quad 34.0 \quad 43.1 \quad 52.4 \quad 59.2 \quad 63.4 \quad 65.2 \quad 62.7 \quad 60.461 .3 \quad 63.8$ $\begin{array}{llllllllllllll}65.5 & 65.1 & 53.6 & 63.5 & 59.1 & 59.5 & 55.8 & 49.3 & 43.6 & 37.2 & 23.1 & 14.7\end{array}$

$\begin{array}{llllllllllllll}4057 & 18.5 & 27.9 & 32.7 & 39.2 & 42.9 & 44.6 & 45.2 & 45.5 & 44.4 & 43.0 & 44.9 & 45.8\end{array}$ $\begin{array}{llllllllllll}47.3 & 48.5 & 48.2 & 47.4 & 45.1 & 46.1 & 45.5 & 43.8 & 41.9 & 36.7 & 23.4 & 14.9\end{array}$ 


\section{CYCLE 1 DATA}

DATASET 02, APRIL 25, 1974

\section{Reactor Conditions}

Core Average Exposure, $390 \mathrm{MWd} / \mathrm{t}$

Core Thermal Power, 2603 MWT

Dome Pressure, P, 991 psia

Core Flow, 107.9 Mlb/hr

Inlet Subcooling at P, 18.7 Btu/lb

Control Configuration

Legend: 48, Full Out; O, Full In.

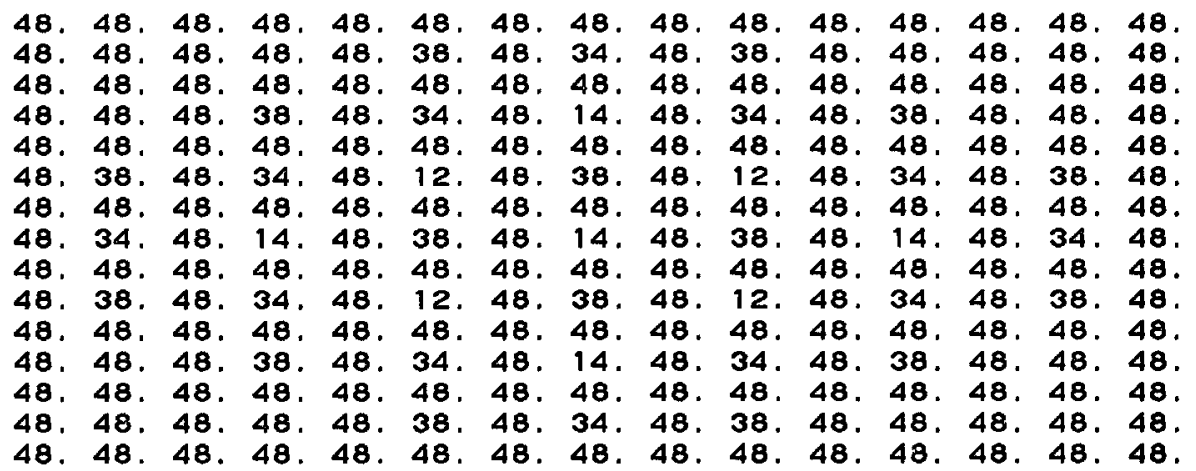

Axial TIP Distribution, Bottom to Top of Core

See Figure 22

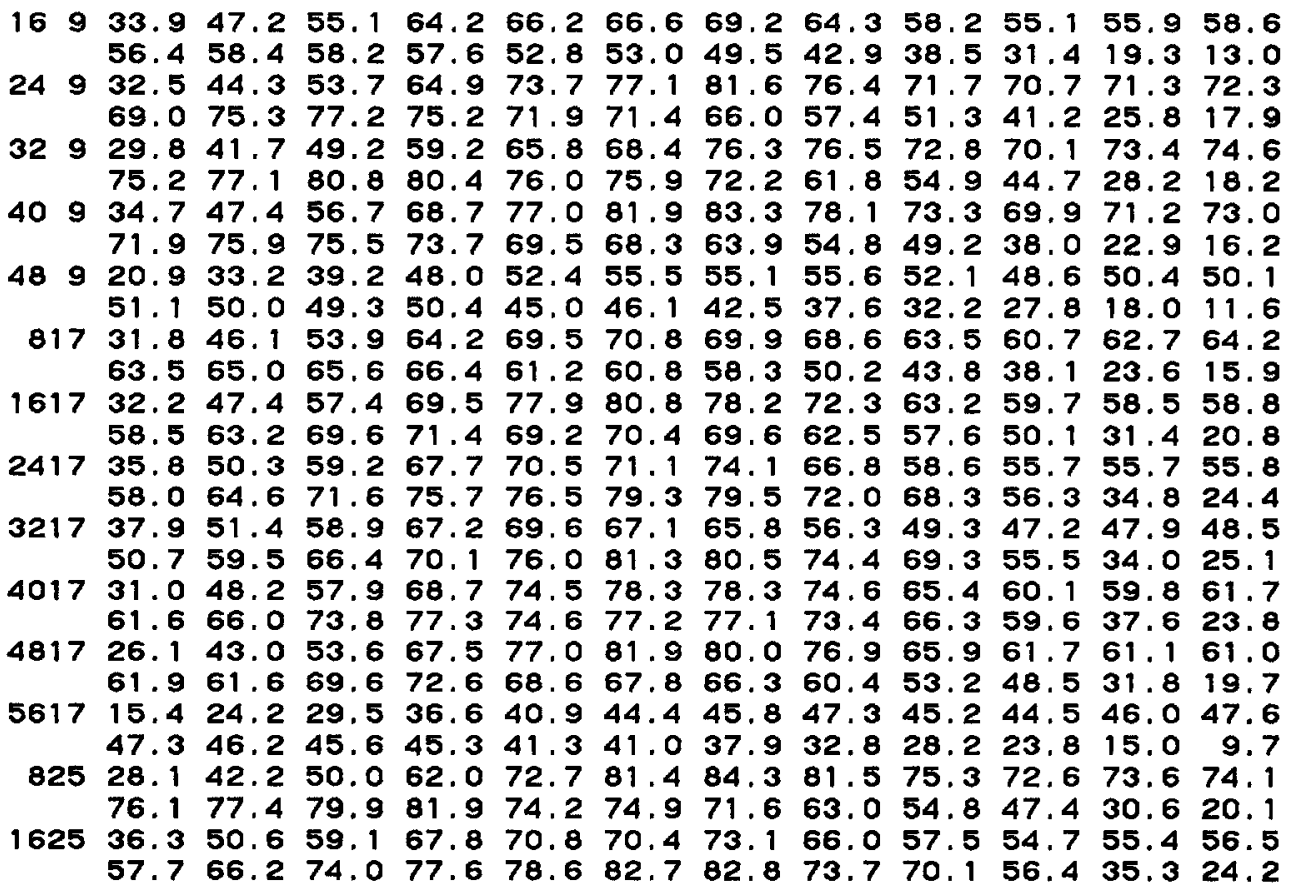


$242540.1 \quad 55.2 \quad 65.1 \quad 74.7 \quad 76.6 \quad 74.2 \quad 68.8 \quad 58.2 \quad 50.3 \quad 46.0 \quad 46.949 .8$

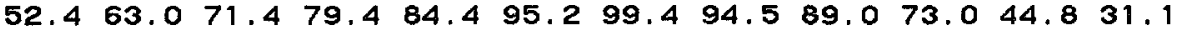

$322540.6 \quad 55.9 \quad 66.5 \quad 77.5 \quad 83.3 \quad 80.5 \quad 77.7 \quad 64.4 \quad 54.8 \quad 50.1 \quad 50.5 \quad 51.0$ $\begin{array}{lllllllllllllllll}53.0 & 63.3 & 72.1 & 76.7 & 78.5 & 82.8 & 84.9 & 78.4 & 73.5 & 60.7 & 37.7 & 27.0\end{array}$

$402533.0 \quad 49.6 \quad 58.4 \quad 69.5 \quad 72.5 \quad 71.7 \quad 67.8 \quad 60.6 \quad 50.2 \quad 46.2 \quad 46.5 \quad 46.9$ $\begin{array}{llllllllllllll}48.2 & 53.8 & 63.2 & 69.8 & 72.1 & 79.8 & 84.9 & 81.3 & 76.8 & 69.6 & 44.0 & 27.5\end{array}$

$\begin{array}{lllllllllllll}4825 & 28.1 & 43.1 & 52.1 & 64.3 & 70.2 & 73.2 & 75.0 & 74.2 & 63.5 & 59.7 & 60.4 & 60.3\end{array}$ $\begin{array}{llllllllllllll}61.1 & 65.5 & 72.6 & 77.7 & 76.0 & 75.7 & 75.2 & 70.2 & 63.7 & 57.3 & 37.2 & 23.8\end{array}$

$\begin{array}{llllllllllllll}5625 & 14.2 & 23.1 & 28.8 & 38.3 & 47.7 & 57.3 & 63.3 & 69.2 & 69.1 & 66.2 & 66.7 & 66.2\end{array}$

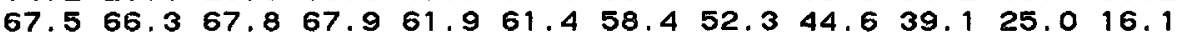

$\begin{array}{lllllllllllllll}833 & 29.5 & 42.0 & 49.9 & 60.7 & 66.7 & 70.9 & 76.5 & 77.0 & 74.4 & 71.7 & 73.4 & 75.1\end{array}$

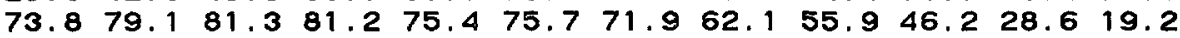

$\begin{array}{lllllllllllllll}1633 & 33.9 & 46.6 & 54.9 & 64.1 & 67.3 & 66.7 & 64.9 & 55.6 & 48.1 & 44.9 & 45.4 & 47.8\end{array}$ $\begin{array}{llllllllllll}48.4 & 57.9 & 65.5 & 70.8 & 74.2 & 80.7 & 81.2 & 76.6 & 70.6 & 56.2 & 34.3 & 23.3\end{array}$

$\begin{array}{lllllllllllllllll}2433 & 34.6 & 50.5 & 60.3 & 71.9 & 78.8 & 79.8 & 74.7 & 65.7 & 55.0 & 49.7 & 49.7 & 50.6\end{array}$ $\begin{array}{llllllllllllll}52.2 & 60.7 & 69.7 & 76.0 & 77.0 & 83.3 & 86.4 & 81.3 & 75.4 & 64.7 & 41.2 & 26.7\end{array}$

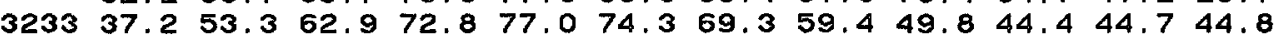
$\begin{array}{llllllllllll}47.0 & 55.2 & 63.6 & 73.0 & 76.1 & 85.8 & 88.6 & 83.4 & 78.3 & 67.9 & 42.7 & 29.8\end{array}$

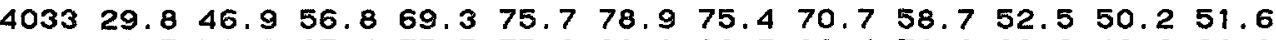

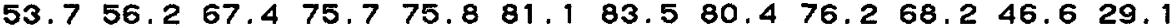

$\begin{array}{llllllllllllll}4833 & 28.2 & 43.5 & 51.8 & 60.9 & 66.3 & 68.8 & 68.4 & 63.2 & 55.0 & 52.4 & 53.2 & 54.3\end{array}$ $\begin{array}{llllllllllllll}55.2 & 59.0 & 66.9 & 73.7 & 75.8 & 80.5 & 80.7 & 75.4 & 69.1 & 61.3 & 40.3 & 24.7\end{array}$

$\begin{array}{lllllllllllllll}5633 & 16.7 & 24.5 & 30.0 & 38.3 & 44.2 & 50.6 & 59.1 & 65.4 & 67.7 & 67.9 & 69.5 & 72.7\end{array}$ $\begin{array}{lllllllllllll}71.4 & 70.6 & 69.9 & 68.1 & 60.4 & 61.2 & 56.8 & 48.4 & 41.1 & 34.4 & 21.0 & 14.7\end{array}$

$84130.944 .5 \quad 53.4 \quad 66.1 \quad 75.8 \quad 80.3 \quad 82.2 \quad 78.2 \quad 72.5 \quad 68.3 \quad 70.8 \quad 71.4$ $71.074 .076 .676 .7 \quad 71.3 \quad 70.5 \quad 66.0 \quad 57.4 \quad 51.3 \quad 42.2 \quad 25.5 \quad 16.6$

$\begin{array}{lllllllllllllll}1641 & 29.3 & 45.6 & 55.6 & 67.2 & 72.1 & 75.7 & 76.8 & 75.7 & 66.3 & 62.0 & 61.0 & 61.4\end{array}$ $61.967 .073 .978 .975 .277 .578 .273 .167 .960 .1 \quad 38.224 .9$

$\begin{array}{llllllllllllllll}2441 & 32.0 & 47.1 & 56.0 & 65.0 & 67.6 & 67.7 & 64.2 & 56.9 & 48.0 & 44.6 & 44.9 & 45.1\end{array}$ $46.052 .461 .065 .966 .7 \quad 74.179 .4 \quad 75.4 \quad 71.259 .6 \quad 39.227 .0$

$\begin{array}{lllllllllllll}3241 & 34.1 & 49.3 & 58.3 & 70.4 & 75.6 & 77.7 & 75.0 & 66.1 & 56.1 & 50.9 & 50.3 & 52.1\end{array}$ $\begin{array}{llllllllllll}53.4 & 62.0 & 70.7 & 77.4 & 78.7 & 84.3 & 85.8 & 79.2 & 73.7 & 63.7 & 41.2 & 27.1\end{array}$

$404132.9 \quad 49.8 \quad 58.968 .7 \quad 72.3 \quad 73.1 \quad 70.3 \quad 63.3 \quad 55.1 \quad 50.9 \quad 50.951 .3$ $\begin{array}{llllllllllllll}52.3 & 58.6 & 67.9 & 73.4 & 74.4 & 80.8 & 84.5 & 81.1 & 74.9 & 66.0 & 42.7 & 29.1\end{array}$

$\begin{array}{lllllllllllllll}4841 & 31.7 & 46.7 & 56.2 & 67.4 & 74.4 & 77.8 & 79.1 & 73.4 & 65.3 & 61.3 & 62.0 & 64.1\end{array}$

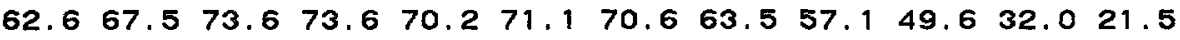

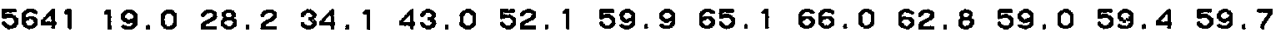
$\begin{array}{lllllllllllll}60.3 & 59.3 & 56.9 & 56.9 & 51.7 & 50.2 & 45.5 & 39.4 & 35.1 & 29.7 & 17.8 & 12.4\end{array}$

$\begin{array}{llllllllllllllll}849 & 20.7 & 31.9 & 38.3 & 46.7 & 51.6 & 53.9 & 54.7 & 52.9 & 49.4 & 47.2 & 46.4 & 48.1\end{array}$ $\begin{array}{lllllllllllllll}48.7 & 46.9 & 47.9 & 48.0 & 45.2 & 44.5 & 41.6 & 36.2 & 31.4 & 26.9 & 17.0 & 10.8\end{array}$

$164928.945 .0 \quad 55.368 .076 .7 \quad 81.5 \quad 78.7 \quad 74.164 .460 .661 .262 .5$ $\begin{array}{lllllllllllll}62.9 & 66.0 & 72.7 & 74.9 & 68.5 & 69.9 & 68.4 & 61.4 & 54.9 & 46.3 & 29.1 & 18.9\end{array}$

$244928.2 \quad 43.5 \quad 51.6 \quad 62.2 \quad 67.6 \quad 71.4 \quad 72.8 \quad 71.1 \quad 61.7 \quad 59.1 \quad 60.0 \quad 60.1$

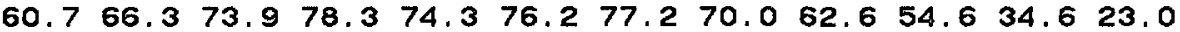

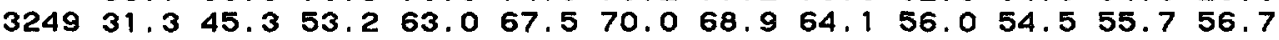
$\begin{array}{llllllllllllll}56.4 & 61.4 & 69.5 & 75.0 & 74.7 & 81.6 & 80.5 & 74.0 & 68.6 & 60.9 & 38.1 & 26.6\end{array}$

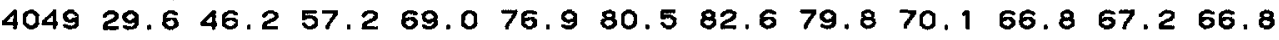
$\begin{array}{lllllllllllll}68.0 & 72.1 & 81.5 & 86.4 & 83.3 & 82.7 & 80.5 & 75.6 & 68.3 & 59.4 & 38.5 & 25.2\end{array}$

$\begin{array}{llllllllllllllll}4849 & 24.8 & 38.1 & 46.5 & 59.3 & 68.3 & 72.3 & 73.1 & 71.7 & 64.9 & 61.8 & 62.0 & 62.2\end{array}$ $\begin{array}{lllllllllllll}60.2 & 62.0 & 63.3 & 64.6 & 59.1 & 57.9 & 55.9 & 49.2 & 43.2 & 37.5 & 23.8 & 16.2\end{array}$

$\begin{array}{llllllllllllll}1657 & 15.5 & 23.7 & 28.4 & 35.4 & 40.4 & 43.4 & 44.6 & 46.4 & 45.5 & 45.0 & 45.8 & 46.1\end{array}$ $\begin{array}{llllllllllllll}46.1 & 44.8 & 45.3 & 46.1 & 41.8 & 41.5 & 38.9 & 33.4 & 28.4 & 24.1 & 14.8 & 9.4\end{array}$

$\begin{array}{lllllllllllllllll}2457 & 15.3 & 23.6 & 28.8 & 37.7 & 47.3 & 56.5 & 63.0 & 66.7 & 65.2 & 63.1 & 65.9 & 67.4\end{array}$ $\begin{array}{llllllllllll}66.2 & 65.3 & 65.8 & 63.8 & 57.8 & 56.6 & 52.6 & 45.3 & 38.2 & 32.4 & 19.9 & 12.5\end{array}$

$\begin{array}{llllllllllllllll}3257 & 16.0 & 24.7 & 30.1 & 38.7 & 45.5 & 51.8 & 60.5 & 66.8 & 68.5 & 68.0 & 70.5 & 73.5\end{array}$ $\begin{array}{llllllllllll}73.1 & 73.3 & 71.2 & 70.5 & 62.7 & 62.4 & 58.1 & 48.6 & 42.4 & 34.9 & 21.1 & 13.6\end{array}$

$4057 \quad 17.5 \quad 26.6 \quad 32.3 \quad 42.1 \quad 52.261 .265 .969 .2 \quad 65.8 \quad 63.5 \quad 64.3 \quad 65.0$ $\begin{array}{lllllllllllll}63.5 & 62.8 & 63.4 & 62.5 & 55.8 & 55.6 & 52.2 & 44.3 & 38.5 & 31.4 & 19.5 & 12.1\end{array}$ 


\section{CYCLE 1 DATA}

DATASET 03, MAY 12, 1974

\section{Reactor Conditions}

Core Average Exposure, $648 \mathrm{MWd} / \mathrm{t}$

Core Thermal Power, 2513 MWT

Dome Pressure, P, 1007 psia

Core Flow, 72.1 Mlb/hr

Inlet Subcooling at P, $28.4 \mathrm{Btu} / \mathrm{lb}$

Control Configuration

$\begin{array}{lllllllllllllll}48 & 48 & 48 & 48 & 48 & 48 & 48 & 48 & 48 & 48 & 48 & 48 & 48 & 48 & 48 \\ 48 & 48 & 48 & 48 & 42 & 48 & 40 & 48 & 40 & 48 & 42 & 48 & 48 & 48 & 48 \\ 48 & 48 & 48 & 48 & 48 & 48 & 48 & 48 & 48 & 48 & 48 & 48 & 48 & 48 & 48 \\ 48 & 48 & 48 & 48 & 38 & 48 & 32 & 48 & 32 & 48 & 38 & 48 & 48 & 48 & 48 \\ 48 & 48 & 48 & 48 & 48 & 48 & 48 & 48 & 48 & 48 & 48 & 48 & 48 & 48 & 48 \\ 48 & 48 & 38 & 48 & 14 & 48 & 40 & 48 & 40 & 48 & 14 & 48 & 38 & 48 & 48 \\ 48 & 48 & 48 & 48 & 48 & 48 & 48 & 48 & 48 & 48 & 48 & 48 & 48 & 48 & 48 \\ 48 & 48 & 30 & 48 & 38 & 48 & 16 & 48 & 16 & 48 & 38 & 48 & 30 & 48 & 48 \\ 48 & 48 & 48 & 48 & 48 & 48 & 48 & 48 & 48 & 48 & 48 & 48 & 48 & 48 & 48 \\ 48 & 48 & 38 & 48 & 14 & 48 & 40 & 48 & 40 & 48 & 14 & 48 & 38 & 48 & 48 \\ 48 & 48 & 48 & 48 & 48 & 48 & 48 & 48 & 48 & 48 & 48 & 48 & 48 & 48 & 48 \\ 48 & 48 & 48 & 48 & 38 & 48 & 32 & 48 & 32 & 48 & 38 & 48 & 48 & 48 & 48 \\ 48 & 48 & 48 & 48 & 48 & 48 & 48 & 48 & 48 & 48 & 48 & 48 & 48 & 48 & 48 \\ 48 & 48 & 48 & 48 & 42 & 48 & 40 & 48 & 40 & 48 & 42 & 48 & 48 & 48 & 48 \\ 48 & 48 & 48 & 48 & 48 & 48 & 48 & 48 & 48 & 48 & 48 & 48 & 48 & 48 & 48\end{array}$

Axial TIP Distribution,

No TIP data taken for this Data Set. 


\section{CYCLE 1 DATA}

DATASET 04, MAY 26, 1974

\section{Reactor Conditions}

Core Average Exposure, $741 \mathrm{MWd} / \mathrm{t}$

Core Thermal Power, 3164 MWT

Dome Pressure, P, 1009 psia

Core Flow, 107.7 Mlb/hr

Inlet Subcooling at P, 22.1 Btu/lb

Control Configuration

Legend: 48, Full Out; O, Full In.

\begin{tabular}{|c|c|c|c|c|c|c|c|c|c|c|c|c|c|c|}
\hline $\begin{array}{l}8 . \\
8 . \\
8 .\end{array}$ & $\begin{array}{l}48 \\
48 \\
48 \\
48\end{array}$ & $\begin{array}{l}8 . \\
8 . \\
8 . \\
8 .\end{array}$ & $\begin{array}{l}48 \\
48 \\
40\end{array}$ & $\begin{array}{l}48 \\
48 \\
48 \\
48\end{array}$ & $\begin{array}{l}30 \\
48 \\
38\end{array}$ & $\begin{array}{l}48 \\
48 \\
48\end{array}$ & 8. & 3. & 3. & $\begin{array}{l}48 . \\
48 .\end{array}$ & 48 . & 48 . & 8. & \\
\hline
\end{tabular}

Axial TIP Distribution, Bottom to Top of Core

See Figure 22

\begin{tabular}{|c|c|c|c|c|c|c|c|c|c|c|c|c|}
\hline $\mathbf{g}$ & 38. & & 5 & & & & $\begin{array}{l}66.9 \\
44.4\end{array}$ & $\begin{array}{l}63.1 \\
38.8\end{array}$ & 33 & $\begin{array}{l}53.4 \\
27.7\end{array}$ & $\begin{array}{l}54.7 \\
17.0\end{array}$ & 4 \\
\hline 249 & $\begin{array}{l}39.5 \\
73.4\end{array}$ & $\begin{array}{l}53.2 \\
74.0\end{array}$ & $\begin{array}{l}3.4 \\
4.6\end{array}$ & 3.8 & $\begin{array}{l}74.4 \\
67.5\end{array}$ & $\begin{array}{l}75.3 \\
65.7\end{array}$ & 76.4 & 70.7 & 69.6 & 67.9 & $\begin{array}{l}72.9 \\
21.4\end{array}$ & $\begin{array}{l}6.8 \\
5.7\end{array}$ \\
\hline$y$ & 37.5 & 54.7 & $\begin{array}{l}67.0 \\
67.2\end{array}$ & & 92.9 & 92.9 & 90.9 & 85.4 & $\begin{array}{l}44.1 \\
77.5\end{array}$ & 74.1 & 75.5 & 74.8 \\
\hline & 74.1 & 74.2 & 77.4 & 2 & 69.4 & 67 & 63.5 & 54.4 & 46.7 & 8.2 & 4.2 & 5 \\
\hline 9 & & & $\begin{array}{l}63.7 \\
74.6\end{array}$ & & 75.2 & $\begin{array}{l}72.4 \\
63.1\end{array}$ & 74.0 & 70.9 & 69.6 & 9.2 & $\begin{array}{l}71.9 \\
20.0\end{array}$ & $\begin{array}{l}2.0 \\
1.5\end{array}$ \\
\hline 48 & 24.2 & 38.2 & 46.3 & 56.7 & 9.8 & 58.8 & 57.2 & . 1 & 1.7 & 48.3 & 47.6 & 49.0 \\
\hline & 47.9 & 46.6 & 46.9 & .5 & 42.4 & 41.0 & 38.5 & 35 & .3 & 24.3 & 15.5 & 9.7 \\
\hline 817 & 36.1 & 52.2 & 60.4 & 72.1 & 74.6 & 73.5 & 70.6 & 67.8 & 62.5 & 58.2 & 60.7 & 62.7 \\
\hline & 61 & 61.2 & 61.8 & .8 & 55.6 & 54.9 & 51.7 & 9 & .4 & 2.7 & 19.8 & 14.1 \\
\hline & & 8 & 7 & 89.4 & .6 & 95.5 & 89.3 & 81.9 &.$<$ & 3.0 & 60.2 & $58 \cdot 2$ \\
\hline & & & 64.9 & & 62.0 & 62.5 & & & & 42.6 & & .4 \\
\hline 2417 & 46.1 & 65.5 & 78.4 & 92.0 & 5 & 97.8 & 92.9 & & & 62.4 & 9 & .7 \\
\hline & & 6 & 78 & 6 & 70 & 71.2 & .2 & 1 & .8 & 46.8 & 1 & .4 \\
\hline 7 & 47.7 & 65.4 & 78.4 & .9 & 7 & .0 & 8 & 3 & .3 & 62.1 & .4 & 3.1 \\
\hline & 60.7 & 6 & 71.7 & 2 & 5 & 70.1 & .9 & 0.3 & .8 & 44.0 & 7.0 & 20.1 \\
\hline 4 & 38.8 & 60.9 & 72.5 & .7 & 3 & 99.4 & 93.3 & 8 & 72.0 & 66.2 & 62.9 & 63.2 \\
\hline & 6 & 6 & 68 & 72.4 & 6 & 7 & 8 & 62.2 & 55.9 & 0.4 & 31.9 & .3 \\
\hline & .2 & $\begin{array}{l}7 \\
5\end{array}$ & 66.4 & 84.0 & .3 & 93.2 & .6 & 0. 1 & 1 & .5 & 60 & 14 \\
\hline & & & 4 & .8 & .4 & 6 & 58.6 & & 46. & 40.6 & & 9 \\
\hline & & & 8 & & & 4 & .9 & & 43 & .9 & 45.0 & .7 \\
\hline & & & 44.3 & & & 38 & 0 & & 25.1 & .4 & 6 & .5 \\
\hline & $\begin{array}{l}34.1 \\
75.6\end{array}$ & $\begin{array}{l}51.0 \\
74.8\end{array}$ & $\begin{array}{l}61.6 \\
77.1\end{array}$ & $\begin{array}{l}74.7 \\
76.2\end{array}$ & $\begin{array}{l}77.6 \\
70.4\end{array}$ & $\begin{array}{l}77.6 \\
68.0\end{array}$ & $\begin{array}{l}75.3 \\
62.7\end{array}$ & $\begin{array}{l}73.3 \\
54.8\end{array}$ & $\begin{array}{l}72.8 \\
47.4\end{array}$ & $\begin{array}{l}71.4 \\
40.5\end{array}$ & $\begin{array}{l}75.3 \\
25.6\end{array}$ & $\begin{array}{l}76.8 \\
16.7\end{array}$ \\
\hline 1625 & 44.9 & .6 & 76.5 & 88.9 & 95.0 & 92.1 & 89.0 & .5 & 67.7 & 61.4 & 61.1 & 61.1 \\
\hline & & & & & & 72.1 & 71.0 & & .0 & 47.7 & 29.0 & 3 \\
\hline & 0 & 2 & $\begin{array}{l}78.1 \\
71.3\end{array}$ & $\begin{array}{l}88.5 \\
78.5\end{array}$ & $\begin{array}{l}89.5 \\
79.6\end{array}$ & $\begin{array}{l}86.0 \\
86.0\end{array}$ & $\begin{array}{l}80.6 \\
84.9\end{array}$ & $\begin{array}{l}69.1 \\
78.5\end{array}$ & $\begin{array}{l}58.6 \\
71.0\end{array}$ & $\begin{array}{l}54.8 \\
57.7\end{array}$ & $\begin{array}{l}54.8 \\
35.8\end{array}$ & 25.0 \\
\hline
\end{tabular}


$\begin{array}{lllllllllllllll}3225 & 49.5 & 68.4 & 80.1 & 92.3 & 95.0 & 93.1 & 87.2 & 73.5 & 63.0 & 57.1 & 55.0 & 55.2\end{array}$

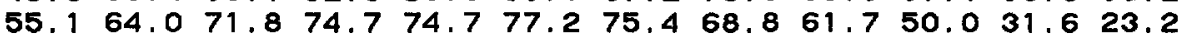

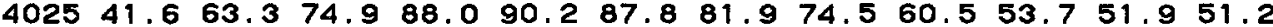
$\begin{array}{llllllllllll}50.4 & 54.4 & 63.2 & 67.9 & 69.4 & 74.8 & 76.5 & 71.3 & 65.2 & 57.1 & 36.4 & 24.3\end{array}$

$\begin{array}{lllllllllllllll}4825 & 35.7 & 55.6 & 67.4 & 84.9 & 90.6 & 98.2 & 92.6 & 84.9 & 73.6 & 67.8 & 65.1 & 64.6\end{array}$ $\begin{array}{lllllllllllll}62.3 & 64.9 & 70.8 & 74.3 & 70.0 & 67.6 & 68.1 & 59.9 & 53.3 & 47.0 & 30.3 & 20.7\end{array}$

$\begin{array}{llllllllllllllll}5625 & 16.8 & 27.3 & 33.7 & 42.5 & 48.2 & 52.6 & 53.6 & 58.0 & 59.4 & 62.3 & 67.4 & 68.0\end{array}$ $\begin{array}{llllllllllllll}68.9 & 66.6 & 66.4 & 66.7 & 59.9 & 58.1 & 54.1 & 48.1 & 40.0 & 35.1 & 22.1 & 14.1\end{array}$

$833 \quad 37.4 \quad 54.967 .7 \quad 84.6 \quad 90.8 \quad 92.6 \quad 88.4 \quad 84.6 \quad 77.7 \quad 72.6 \quad 74.475 .0$ $\begin{array}{llllllllllllll}73.7 & 74.9 & 76.5 & 76.3 & 68.4 & 68.3 & 62.7 & 54.3 & 46.5 & 38.3 & 23.6 & 16.5\end{array}$

$\begin{array}{lllllllllllll}1633 & 42.7 & 60.5 & 71.8 & 85.3 & 92.3 & 93.9 & 89.4 & 79.3 & 66.3 & 61.7 & 61.5 & 60.4\end{array}$ $\begin{array}{lllllllllllll}59.4 & 65.6 & 71.2 & 73.1 & 70.3 & 70.2 & 68.7 & 61.3 & 55.0 & 43.2 & 26.9 & 18.9\end{array}$

$243341.959 .9 \quad 71.9 \quad 84.7 \quad 89.2 \quad 89.4 \quad 82.0 \quad 72.8 \quad 62.3 \quad 54.0 \quad 53.8 \quad 54.1$ $\begin{array}{llllllllllll}53.7 & 59.3 & 67.2 & 73.9 & 72.9 & 76.2 & 76.1 & 69.6 & 64.1 & 54.9 & 34.5 & 22.4\end{array}$

$\begin{array}{lllllllllllll}3233 & 44.5 & 64.2 & 75.2 & 85.8 & 86.4 & 83.0 & 76.4 & 65.9 & 55.4 & 48.6 & 47.6 & 47.5\end{array}$ $\begin{array}{llllllllllll}48.6 & 56.4 & 64.6 & 72.4 & 73.7 & 79.9 & 81.0 & 74.0 & 67.5 & 57.3 & 36.2 & 26.1\end{array}$

$4033 \quad 37.0 \quad 58.8 \quad 71.4 \quad 86.4 \quad 93.8 \quad 94.8 \quad 90.0 \quad 83.4 \quad 70.7 \quad 63.5 \quad 59.7 \quad 58.4$ $\begin{array}{llllllllllllll}57.3 & 59.0 & 66.3 & 73.7 & 73.1 & 74.8 & 74.9 & 69.6 & 63.3 & 56.4 & 38.7 & 25.8\end{array}$

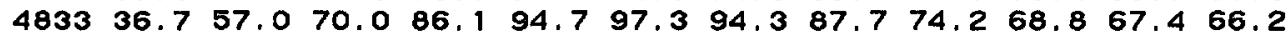

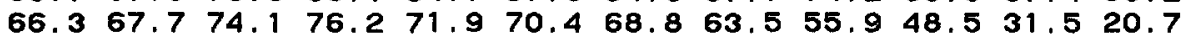

$\begin{array}{llllllllllllll}5633 & 21.1 & 31.6 & 40.9 & 54.5 & 64.2 & 67.7 & 69.6 & 70.2 & 68.4 & 66.3 & 66.5 & 67.2\end{array}$ $\begin{array}{llllllllllllll}66.3 & 64.5 & 63.9 & 62.2 & 53.9 & 53.5 & 49.4 & 41.7 & 34.7 & 28.8 & 17.9 & 12.0\end{array}$

$84135.251 .0 \quad 60.6 \quad 71.3 \quad 74.7 \quad 75.3 \quad 72.5 \quad 71.3 \quad 67.8 \quad 67.8 \quad 69.8 \quad 73.3$ $\begin{array}{lllllllllllll}71.3 & 72.1 & 73.9 & 73.3 & 66.7 & 66.1 & 61.2 & 53.2 & 46.8 & 38.6 & 23.3 & 16.0\end{array}$

$164138.4 \quad 58.9 \quad 70.9 \quad 86.1 \quad 95.1 \quad 98.3 \quad 93.5 \quad 85.4 \quad 72.3 \quad 65.8 \quad 63.162 .5$ $\begin{array}{lllllllllllll}61.7 & 63.0 & 69.2 & 72.7 & 68.3 & 70.8 & 68.9 & 63.0 & 56.0 & 49.6 & 31.3 & 21.9\end{array}$

$244141.359 .2 \quad 69.3 \quad 82.3 \quad 83.6$ 80.9 $74.5 \quad 66.0 \quad 56.2 \quad 50.949 .849 .8$ $\begin{array}{llllllllllll}47.5 & 54.1 & 59.2 & 63.1 & 65.2 & 70.4 & 71.1 & 64.9 & 60.0 & 49.6 & 32.4 & 22.3\end{array}$

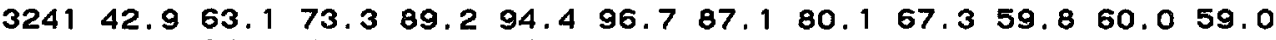
$\begin{array}{lllllllllllll}58.7 & 64.3 & 71.5 & 77.3 & 74.2 & 76.2 & 75.6 & 69.1 & 62.3 & 53.5 & 34.5 & 24.0\end{array}$

$404141.063 .075 .188 .5 \quad 91.7 \quad 89.0 \quad 81.7 \quad 73.0 \quad 60.8 \quad 54.452 .953 .3$ $\begin{array}{lllllllllllll}53.4 & 57.4 & 65.9 & 70.1 & 70.6 & 74.5 & 75.6 & 70.8 & 64.7 & 55.8 & 36.1 & 25.1\end{array}$

$\begin{array}{llllllllllllll}4841 & 39.2 & 58.3 & 69.8 & 85.4 & 92.2 & 91.9 & 88.0 & 79.9 & 70.4 & 64.5 & 64.2 & 64.1\end{array}$ $61.564 .969 .8 \quad 71.5 \quad 65.3 \quad 66.1 \quad 62.3 \quad 55.2 \quad 48.841 .1 \quad 26.1 \quad 17.4$

$\begin{array}{llllllllllllll}5641 & 21.9 & 31.0 & 35.9 & 43.2 & 47.5 & 49.1 & 52.0 & 54.2 & 56.2 & 59.9 & 61.7 & 62.8\end{array}$ $\begin{array}{llllllllllll}60.4 & 59.0 & 58.0 & 56.0 & 49.7 & 48.3 & 42.9 & 35.9 & 31.4 & 26.3 & 15.8 & 10.4\end{array}$

$\begin{array}{llllllllllllllll}849 & 24.8 & 37.7 & 45.4 & 53.7 & 56.8 & 56.7 & 55.5 & 53.5 & 49.4 & 45.8 & 45.0 & 44.3\end{array}$ $\begin{array}{lllllllllllll}44.0 & 44.1 & 43.6 & 42.6 & 39.9 & 39.1 & 37.0 & 31.7 & 27.1 & 23.0 & 14.3 & 9.9\end{array}$

$\begin{array}{llllllllllllll}1649 & 37.2 & 55.1 & 67.9 & 84.6 & 90.1 & 90.1 & 83.7 & 75.4 & 65.6 & 61.0 & 60.2 & 61.5\end{array}$ $\begin{array}{llllllllllll}59.8 & 63.1 & 67.1 & 68.8 & 63.0 & 62.9 & 58.8 & 53.2 & 46.1 & 38.1 & 23.6 & 16.2\end{array}$

$244937.0 \quad 55.267 .3 \quad 80.8 \quad 91.1 \quad 94.5 \quad 88.3 \quad 81.5 \quad 69.0 \quad 64.0 \quad 63.7 \quad 64.3$ $\begin{array}{lllllllllllllll}62.5 & 65.3 & 71.7 & 74.0 & 68.5 & 69.0 & 65.3 & 58.5 & 52.1 & 44.6 & 28.8 & 19.1\end{array}$

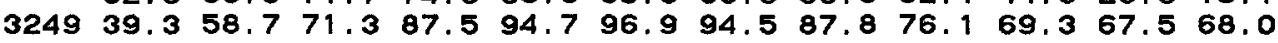
$\begin{array}{llllllllllll}65.7 & 69.4 & 74.4 & 74.8 & 69.0 & 70.6 & 68.4 & 62.2 & 54.6 & 46.6 & 30.1 & 21.2\end{array}$

$404937.1 \quad 57.4 \quad 70.3 \quad 84.993 .2 \quad 95.4 \quad 91.7 \quad 84.8 \quad 73.6 \quad 69.7 \quad 68.0 \quad 68.6$

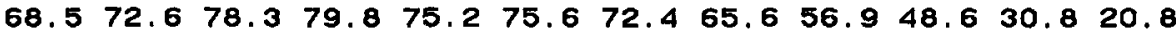

$484930.5 \quad 47.1 \quad 58.4 \quad 74.2 \quad 81.7 \quad 82.3 \quad 78.5 \quad 74.1 \quad 66.259 .6 \quad 59.0 \quad 57.8$ $\begin{array}{lllllllllllll}57.0 & 56.6 & 59.1 & 58.1 & 52.9 & 52.5 & 49.5 & 42.4 & 37.4 & 32.3 & 20.0 & 13.7\end{array}$

$\begin{array}{lllllllllllllll}1657 & 17.8 & 26.5 & 31.1 & 37.4 & 40.0 & 41.3 & 42.5 & 43.8 & 42.3 & 40.8 & 42.6 & 43.5\end{array}$ $\begin{array}{llllllllllll}43.8 & 44.9 & 43.4 & 42.7 & 38.0 & 37.6 & 34.6 & 29.2 & 25.3 & 20.6 & 12.5 & 8.2\end{array}$

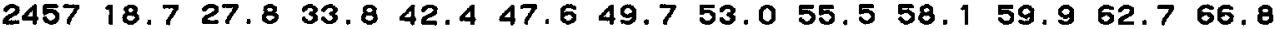
$\begin{array}{llllllllllllll}64.9 & 63.5 & 62.5 & 59.8 & 53.5 & 51.3 & 47.3 & 39.0 & 33.5 & 27.3 & 16.7 & 10.8\end{array}$

$325720.931 .7 \quad 40.354 .6 \quad 65.4 \quad 68.2 \quad 70.571 .8 \quad 67.6 \quad 66.0 \quad 67.1 \quad 68.3$ $\begin{array}{llllllllllllll}66.9 & 66.5 & 64.1 & 63.0 & 56.0 & 54.2 & 49.7 & 41.4 & 35.0 & 28.8 & 17.3 & 11.2\end{array}$

$\begin{array}{lllllllllllll}4057 & 19.5 & 29.1 & 33.6 & 40.7 & 45.1 & 47.9 & 50.5 & 54.1 & 56.4 & 59.2 & 63.0 & 63.4\end{array}$ $\begin{array}{lllllllllllll}61.9 & 62.4 & 59.6 & 59.4 & 53.7 & 50.7 & 46.0 & 39.5 & 32.8 & 26.7 & 16.3 & 10.4\end{array}$ 


\section{CYCLE 1 DATA}

DATASET 05, JUNE 19, 1974

\section{Reactor Conditions}

Core Average Exposure, $1010 \mathrm{MWd} / \mathrm{t}$

Core Thermal Power, 3261 MWT

Dome Pressure, P, 1017 psia

Core Flow, $105.6 \mathrm{MIb} / \mathrm{hr}$

Inlet Subcooling at $\mathrm{P}, 22.8 \mathrm{Btu} / \mathrm{lb}$

Control Configuration

Legend: 48, Full Out; O, Full In.

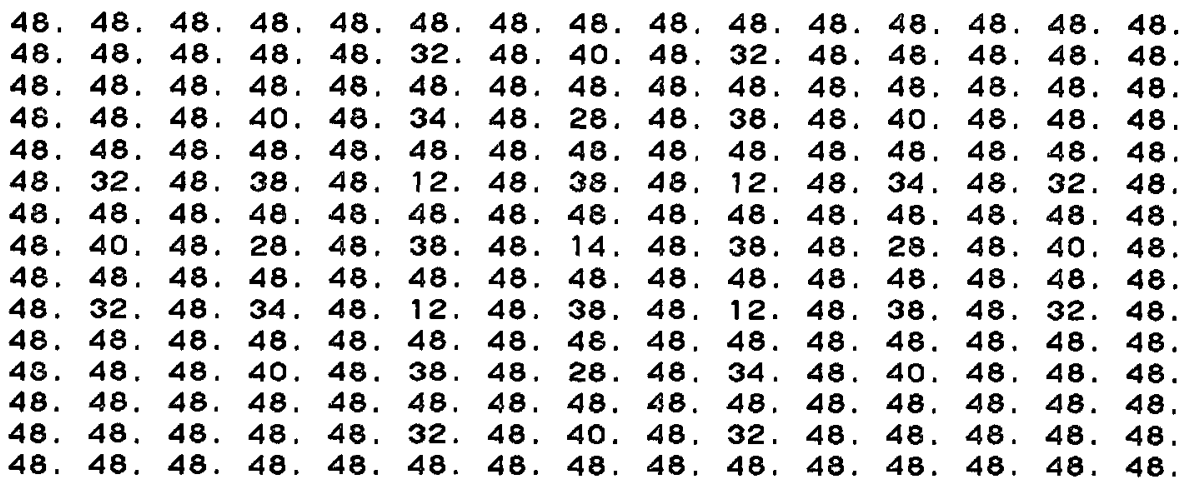

Axial TIP Distribution, Bottom to Top of Core

See Figure 22

$\begin{array}{lllllllllllllll}16 & 9 & 41.5 & 56.5 & 64.8 & 74.9 & 72.8 & 70.1 & 67.7 & 65.4 & 60.5 & 55.3 & 55.2 & 54.9\end{array}$ $\begin{array}{llllllllllllll}53.1 & 52.6 & 53.7 & 52.4 & 46.5 & 45.5 & 42.8 & 36.6 & 31.9 & 26.1 & 15.9 & 10.8\end{array}$ $24939.8 \quad 54.964 .674 .478 .074 .4 \quad 76.5 \quad 75.5 \quad 73.069 .972 .273 .5$ $\begin{array}{llllllllllllll}70.0 & 73.6 & 73.3 & 71.7 & 65.7 & 64.0 & 58.3 & 49.6 & 43.2 & 33.4 & 21.1 & 14.9\end{array}$

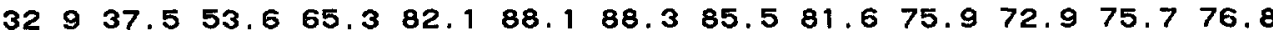

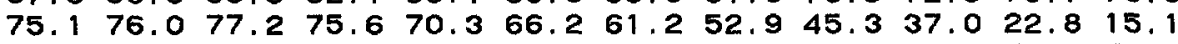

$\begin{array}{llllllllllllll}40 & 9 & 40.4 & 54.6 & 64.0 & 72.8 & 76.3 & 73.4 & 76.2 & 75.6 & 75.0 & 71.6 & 75.5 & 73.3\end{array}$ $71.073 .974 .5 \quad 70.5 \quad 64.3 \quad 61.957 .3 \quad 48.5 \quad 42.0 \quad 31.8 \quad 18.8 \quad 14.8$

$\begin{array}{llllllllllllllll}48 & 9 & 25.5 & 40.4 & 47.8 & 56.7 & 61.1 & 61.3 & 57.7 & 57.4 & 51.4 & 48.3 & 47.2 & 48.1\end{array}$ $\begin{array}{lllllllllllll}46.8 & 44.9 & 44.9 & 44.3 & 40.0 & 38.5 & 36.3 & 31.4 & 26.7 & 22.9 & 15.1 & 9.6\end{array}$

$81738.8 \quad 55.5 \quad 65.5 \quad 75.7 \quad 77.4 \quad 75.3 \quad 73.071 .0 \quad 63.962 .161 .963 .5$ $\begin{array}{llllllllllllll}61.7 & 60.6 & 61.8 & 60.3 & 55.3 & 53.3 & 49.1 & 42.7 & 36.7 & 30.3 & 18.8 & 12.0\end{array}$

$161742.463 .3 \quad 77.3 \quad 92.1 \quad 99.0 \quad 96.6 \quad 89.2 \quad 82.0 \quad 70.362 .6 \quad 62.060 .4$ $\begin{array}{lllllllllllll}59.6 & 59.9 & 65.1 & 67.2 & 62.6 & 60.9 & 58.6 & 53.3 & 47.5 & 40.9 & 25.9 & 17.8\end{array}$

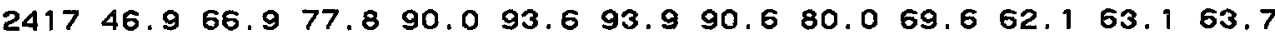

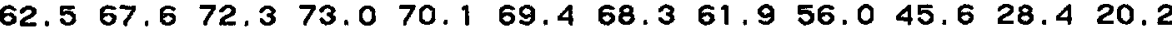

$\begin{array}{lllllllllllll}3217 & 47.4 & 64.9 & 73.7 & 80.6 & 82.0 & 78.7 & 74.6 & 64.9 & 60.5 & 60.1 & 63.5 & 65.1\end{array}$ $\begin{array}{llllllllllll}64.1 & 71.9 & 76.2 & 75.9 & 71.3 & 72.4 & 68.8 & 60.8 & 55.2 & 44.3 & 27.5 & 20.6\end{array}$

$401739.361 .3 \quad 71.8 \quad 85.9 \quad 88.5 \quad 89.0 \quad 85.1 \quad 83.0 \quad 72.967 .865 .4 \quad 64.7$

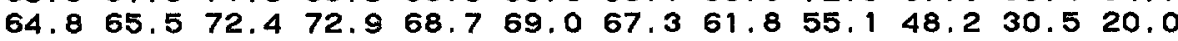

$\begin{array}{llllllllllllll}4817 & 33.4 & 55.8 & 70.3 & 86.1 & 95.0 & 96.1 & 89.4 & 83.5 & 70.3 & 64.1 & 61.3 & 60.2\end{array}$ $\begin{array}{llllllllllll}59.2 & 58.3 & 64.4 & 64.7 & 61.9 & 58.6 & 56.7 & 50.7 & 44.3 & 38.5 & 26.5 & 16.8\end{array}$

$\begin{array}{lllllllllllllll}5617 & 18.7 & 29.5 & 35.5 & 42.5 & 46.1 & 47.6 & 47.2 & 48.3 & 46.8 & 45.0 & 46.6 & 45.4\end{array}$ $\begin{array}{llllllllllllll}45.4 & 44.2 & 42.9 & 42.4 & 37.5 & 35.8 & 33.3 & 28.4 & 23.8 & 20.0 & 12.6 & 7.9\end{array}$

82535.251 .461 .373 .076 .477 .876 .478 .977 .174 .976 .578 .9 $\begin{array}{llllllllllll}76.1 & 76.6 & 76.6 & 77.2 & 70.4 & 66.6 & 62.7 & 54.3 & 46.6 & 39.6 & 24.9 & 16.2\end{array}$

$162546.463 .4 \quad 70.960 .5 \quad 80.5 \quad 79.0 \quad 79.1 \quad 73.6 \quad 66.6 \quad 62.462 .264 .5$

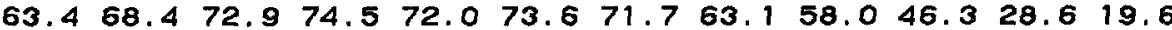


$242550.0 \quad 69.2 \quad 79.5 \quad 88.4 \quad 88.7 \quad 82.977 .5 \quad 66.6 \quad 58.1 \quad 53.6 \quad 53.955 .2$ $\begin{array}{lllllllllllll}55.0 & 62.2 & 68.8 & 72.5 & 73.3 & 78.7 & 79.4 & 72.8 & 65.0 & 52.7 & 33.6 & 23.4\end{array}$

$\begin{array}{llllllllllllll}3225 & 51.1 & 70.2 & 82.7 & 93.1 & 95.7 & 92.8 & 87.9 & 74.3 & 64.5 & 57.1 & 56.2 & 56.5\end{array}$ $\begin{array}{lllllllllllll}56.6 & 63.4 & 69.4 & 73.6 & 72.3 & 75.0 & 72.8 & 66.3 & 61.1 & 50.1 & 30.9 & 22.7\end{array}$

$\begin{array}{lllllllllllll}4025 & 42.5 & 63.8 & 74.9 & 87.9 & 88.4 & 86.2 & 79.3 & 70.9 & 59.0 & 53.8 & 52.3 & 52.2\end{array}$ $\begin{array}{llllllllllll}52.2 & 54.8 & 62.7 & 67.1 & 65.5 & 69.2 & 71.8 & 69.2 & 63.9 & 56.6 & 35.7 & 24.4\end{array}$

$\begin{array}{lllllllllllllll}4825 & 36.4 & 56.9 & 69.1 & 85.5 & 94.5 & 94.6 & 88.5 & 82.5 & 71.7 & 67.4 & 65.2 & 65.8\end{array}$ $\begin{array}{llllllllllll}65.0 & 65.7 & 71.7 & 75.7 & 69.9 & 69.3 & 66.3 & 60.5 & 51.5 & 46.2 & 29.5 & 19.7\end{array}$

$\begin{array}{llllllllllllll}5625 & 17.7 & 28.7 & 35.3 & 43.9 & 50.2 & 55.0 & 57.5 & 64.4 & 66.9 & 66.8 & 70.2 & 69.2\end{array}$ $\begin{array}{lllllllllllll}69.0 & 66.8 & 66.2 & 64.3 & 59.2 & 56.4 & 53.6 & 46.7 & 38.8 & 32.9 & 21.1 & 13.0\end{array}$

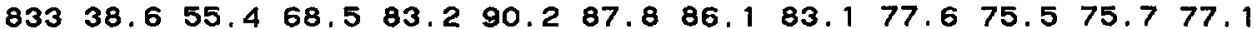
$\begin{array}{lllllllllllll}75.2 & 76.1 & 78.4 & 76.1 & 70.6 & 67.5 & 61.9 & 53.9 & 45.0 & 37.4 & 23.2 & 15.8\end{array}$

$\begin{array}{llllllllllllll}1633 & 43.1 & 60.7 & 71.3 & 79.1 & 80.3 & 78.0 & 73.7 & 65.7 & 58.2 & 57.5 & 61.9 & 64.3\end{array}$ $\begin{array}{lllllllllllll}63.5 & 69.2 & 74.8 & 76.8 & 72.9 & 72.8 & 70.5 & 61.6 & 55.4 & 44.3 & 27.8 & 19.2\end{array}$

$243344.463 .6 \quad 74.2 \quad 86.9 \quad 30.4 \quad 89.2 \quad 83.0 \quad 74.2 \quad 62.2 \quad 56.1 \quad 55.0 \quad 55.4$ $\begin{array}{llllllllllllll}54.7 & 60.5 & 66.9 & 72.1 & 70.6 & 74.3 & 74.1 & 68.1 & 61.8 & 52.7 & 33.3 & 22.8\end{array}$

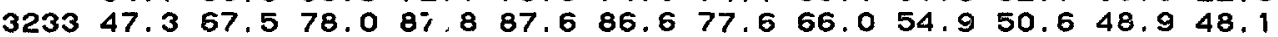
$\begin{array}{llllllllllll}48.9 & 54.9 & 61.6 & 67.5 & 69.6 & 75.6 & 77.0 & 71.9 & 65.5 & 53.8 & 34.1 & 24.2\end{array}$

$\begin{array}{lllllllllllll}4033 & 37.4 & 59.7 & 71.5 & 85.6 & 92.0 & 91.7 & 85.8 & 80.1 & 67.8 & 62.4 & 59.8 & 59.3\end{array}$ $\begin{array}{lllllllllllll}60.0 & 61.3 & 68.0 & 74.4 & 72.6 & 73.1 & 74.3 & 68.1 & 61.4 & 55.7 & 36.5 & 24.3\end{array}$

$\begin{array}{lllllllllllllll}4833 & 35.7 & 54.8 & 65.6 & 77.7 & 80.2 & 79.3 & 74.9 & 71.7 & 64.8 & 64.5 & 66.8 & 70.5\end{array}$

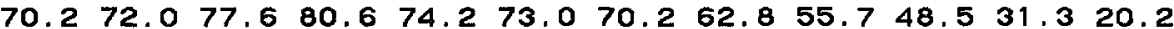

$\begin{array}{lllllllllllllll}5633 & 21.4 & 32.3 & 41.4 & 54.4 & 64.4 & 68.3 & 70.3 & 71.5 & 67.2 & 64.4 & 68.1 & 68.8\end{array}$ $\begin{array}{lllllllllllllll}66.6 & 65.3 & 63.2 & 60.6 & 54.6 & 52.9 & 47.5 & 40.8 & 34.8 & 27.9 & 17.1 & 11.5\end{array}$

$84137.0 \quad 53.1 \quad 62.7 \quad 74.978 .077 .4 \quad 76.175 .974 .671 .072 .472 .9$

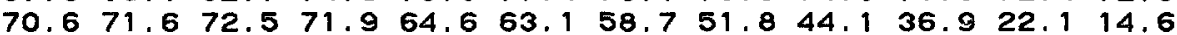

$\begin{array}{lllllllllllllllll}1641 & 40.4 & 61.1 & 73.9 & 89.1 & 96.1 & 99.4 & 93.6 & 86.0 & 72.5 & 67.0 & 64.2 & 65.1\end{array}$ $64.165 .8 \quad 71.6 \quad 72.8 \quad 67.969 .367 .5 \quad 60.8 \quad 54.5 \quad 47.0 \quad 30.0 \quad 20.3$

$\begin{array}{llllllllllllll}2441 & 40.7 & 59.4 & 68.5 & 77.8 & 79.3 & 76.0 & 70.7 & 63.6 & 54.8 & 49.8 & 50.1 & 49.7\end{array}$ $\begin{array}{lllllllllllll}49.8 & 53.9 & 57.8 & 63.0 & 61.1 & 66.1 & 66.8 & 63.0 & 58.1 & 47.4 & 31.0 & 21.8\end{array}$

$\begin{array}{llllllllllllll}3241 & 43.3 & 62.7 & 72.4 & 85.2 & 89.7 & 88.3 & 81.6 & 74.1 & 64.5 & 58.2 & 58.4 & 58.8\end{array}$ $\begin{array}{lllllllllllllll}58.9 & 64.4 & 71.1 & 73.6 & 70.6 & 74.6 & 73.6 & 67.1 & 59.1 & 50.9 & 32.7 & 23.3\end{array}$

$404142.864 .274 .9 \quad 86.8 \quad 88.0 \quad 86.6 \quad 78.7 \quad 71.961 .6 \quad 56.4 \quad 54.4 \quad 54.5$ $\begin{array}{lllllllllllllll}54.7 & 58.3 & 64.1 & 67.6 & 67.3 & 71.7 & 72.6 & 67.7 & 61.1 & 53.7 & 33.9 & 23.6\end{array}$

$484140.0 \quad 59.2 \quad 70.5 \quad 82.2 \quad 85.1 \quad 85.284 .3 \quad 80.9 \quad 71.968 .267 .967 .5$ $\begin{array}{lllllllllllll}65.9 & 67.2 & 71.0 & 72.6 & 67.5 & 65.8 & 63.0 & 54.2 & 48.1 & 40.5 & 25.8 & 18.1\end{array}$

$\begin{array}{llllllllllllll}5641 & 21.7 & 31.2 & 35.9 & 42.8 & 47.7 & 51.0 & 56.2 & 59.6 & 61.5 & 60.0 & 61.7 & 62.3\end{array}$ $\begin{array}{llllllllllllll}58.2 & 57.1 & 55.8 & 52.6 & 46.0 & 45.5 & 42.1 & 35.1 & 30.6 & 24.7 & 15.2 & 10.6\end{array}$

$84926.3 \quad 40.5 \quad 48.5 \quad 57.0 \quad 61.260 .2 \quad 58.1 \quad 54.6 \quad 50.6 \quad 46.6 \quad 45.2 \quad 45.0$ $\begin{array}{lllllllllllll}43.9 & 42.9 & 43.4 & 42.3 & 38.9 & 38.5 & 35.6 & 30.2 & 26.1 & 21.9 & 13.5 & 8.8\end{array}$

$\begin{array}{llllllllllllll}1649 & 38.3 & 58.3 & 72.2 & 87.7 & 91.4 & 89.9 & 84.2 & 77.9 & 67.2 & 62.2 & 62.6 & 62.0\end{array}$

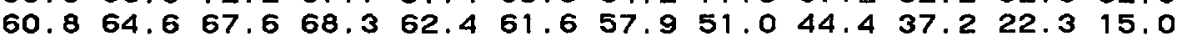

$244936.7 \quad 54.4 \quad 64.5 \quad 75.4 \quad 77.6 \quad 77.078 .7 \quad 75.8 \quad 69.4 \quad 65.0 \quad 67.4 \quad 67.0$ $\begin{array}{llllllllllll}66.9 & 69.9 & 73.8 & 76.1 & 71.1 & 69.4 & 67.0 & 59.4 & 52.6 & 43.9 & 27.9 & 17.8\end{array}$

$\begin{array}{lllllllllllll}3249 & 39.5 & 56.9 & 67.6 & 79.1 & 82.5 & 81.1 & 76.2 & 72.0 & 65.0 & 65.1 & 67.0 & 68.9\end{array}$

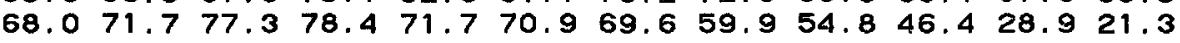

$404938.6 \quad 59.3 \quad 71.4 \quad 85.9 \quad 92.7 \quad 95.8 \quad 92.6 \quad 86.2 \quad 74.5 \quad 71.1 \quad 69.8 \quad 69.5$ $69.470 .375 .978 .973 .172 .0 \quad 68.0 \quad 61.0 \quad 53.9 \quad 45.3 \quad 28.2 \quad 18.5$

$\begin{array}{llllllllllllll}4849 & 31.8 & 49.5 & 60.8 & 78.5 & 84.5 & 85.1 & 79.1 & 75.2 & 67.4 & 60.3 & 58.3 & 58.0\end{array}$ $\begin{array}{llllllllllllll}57.4 & 56.4 & 58.1 & 57.6 & 51.4 & 50.5 & 48.1 & 41.5 & 35.7 & 30.4 & 18.8 & 13.0\end{array}$

$\begin{array}{lllllllllllllll}1657 & 18.6 & 27.6 & 32.3 & 39.0 & 42.5 & 43.5 & 44.5 & 45.4 & 44.4 & 44.2 & 44.7 & 44.5\end{array}$ $\begin{array}{lllllllllllllllll}43.0 & 43.3 & 41.6 & 41.5 & 37.6 & 36.5 & 33.7 & 28.2 & 24.0 & 19.6 & 12.0 & 7.1\end{array}$

$\begin{array}{llllllllllllllll}2457 & 19.0 & 28.5 & 33.9 & 42.1 & 47.7 & 51.2 & 56.1 & 60.8 & 63.0 & 62.2 & 65.1 & 64.8\end{array}$ $\begin{array}{llllllllllll}63.6 & 62.3 & 60.0 & 58.9 & 51.5 & 50.1 & 46.2 & 38.2 & 32.5 & 26.9 & 16.5 & 10.8\end{array}$

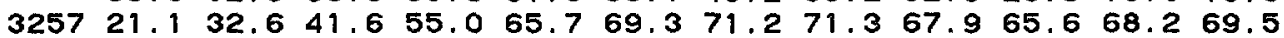
$\begin{array}{llllllllllllll}67.9 & 65.6 & 63.7 & 62.2 & 55.7 & 52.6 & 48.7 & 40.3 & 34.0 & 28.3 & 17.1 & 11.2\end{array}$

$405720.6 \quad 30.8 \quad 36.2 \quad 44.148 .1 \quad 51.8 \quad 56.6 \quad 61.6 \quad 63.6 \quad 63.1 \quad 63.8 \quad 65.0$ $\begin{array}{llllllllllll}62.0 & 60.2 & 57.8 & 55.6 & 49.6 & 48.9 & 44.8 & 37.3 & 31.7 & 26.1 & 15.6 & 10.0\end{array}$ 


\section{CYCLE 1 DATA}

DATASET 06, JULY 15, 1974

\section{Reactor Conditions}

Core Average Exposure, $1585 \mathrm{MWd} / \mathrm{t}$

Core Thermal Power, 3280 MWT

Dome Pressure, P, 1033 psia

Core Flow, 97.1 MIb/hr

Inlet Subcooling at P, 25.7 Btu/lb

Control Configuration

Legend: 48, Full Out; O, Full In.

$\begin{array}{rrrrrrrrrrrrrrr}48 & 48 & 48 & 48 & 48 & 48 & 48 & 48 & 48 & 48 & 48 & 48 & 48 & 48 & 48 \\ 48 & 48 & 48 & 48 & 48 & 32 & 48 & 40 & 48 & 32 & 48 & 48 & 48 & 48 & 48 \\ 48 & 48 & 48 & 48 & 48 & 48 & 48 & 48 & 48 & 48 & 48 & 48 & 48 & 48 & 48 \\ 48 & 48 & 48 & 40 & 48 & 32 & 48 & 20 & 48 & 32 & 48 & 40 & 48 & 48 & 48 \\ 48 & 48 & 48 & 48 & 48 & 48 & 48 & 48 & 48 & 48 & 48 & 48 & 48 & 48 & 48 \\ 48 & 32 & 48 & 32 & 48 & 8 & 48 & 36 & 48 & 8 & 48 & 32 & 48 & 32 & 48 \\ 48 & 48 & 48 & 48 & 48 & 48 & 48 & 48 & 48 & 48 & 48 & 48 & 48 & 48 & 48 \\ 48 & 40 & 48 & 20 & 48 & 36 & 48 & 10 & 48 & 36 & 48 & 20 & 48 & 40 & 48 \\ 48 & 48 & 48 & 48 & 48 & 48 & 48 & 48 & 48 & 48 & 48 & 48 & 48 & 48 & 48 \\ 48 & 32 & 48 & 32 & 48 & 8 & 48 & 36 & 48 & 8 & 48 & 32 & 48 & 32 & 48 \\ 48 & 48 & 48 & 48 & 48 & 48 & 48 & 48 & 48 & 48 & 48 & 48 & 48 & 48 & 48 \\ 48 & 48 & 48 & 40 & 48 & 32 & 48 & 20 & 48 & 32 & 48 & 40 & 48 & 48 & 48 \\ 48 & 48 & 48 & 48 & 48 & 48 & 48 & 48 & 48 & 48 & 48 & 48 & 48 & 48 & 48 \\ 48 & 48 & 48 & 48 & 48 & 32 & 48 & 40 & 48 & 32 & 48 & 48 & 48 & 48 & 48 \\ 48 & 48 & 48 & 48 & 48 & 48 & 48 & 48 & 48 & 48 & 48 & 48 & 48 & 48 & 48\end{array}$

Axial TIP Distribution,

No TIP data taken for this Data Set. 


\section{CYCLE 1 DATA}

DATASET 07, AUGUST 17, 1974

Reactor Conditions

Core Average Exposure, $2080 \mathrm{MWd} / \mathrm{t}$

Core Thermal Power, 3292 MWT

Dome Pressure, P, 1015 psia

Core Flow, 103.1 Mlb/hr

Inlet Subcooling at P, $23.8 \mathrm{Btu} / \mathrm{lb}$

Control Configuration

Legend: 48, Full Out; O, Full In.

$\begin{array}{rrrrrrrrrrrrrrr}48 & 48 & 48 & 48 & 48 & 48 & 48 & 48 & 48 & 48 & 48 & 48 & 48 & 48 & 48 \\ 48 & 48 & 48 & 48 & 42 & 48 & 26 & 48 & 26 & 48 & 42 & 48 & 48 & 48 & 48 \\ 48 & 48 & 48 & 48 & 48 & 48 & 48 & 48 & 48 & 48 & 48 & 48 & 48 & 48 & 48 \\ 48 & 48 & 34 & 48 & 30 & 48 & 8 & 48 & 8 & 48 & 30 & 48 & 34 & 48 & 48 \\ 48 & 48 & 48 & 44 & 48 & 42 & 48 & 48 & 48 & 42 & 48 & 44 & 48 & 48 & 48 \\ 48 & 48 & 32 & 48 & 8 & 48 & 28 & 48 & 28 & 48 & 8 & 48 & 32 & 48 & 48 \\ 48 & 48 & 48 & 44 & 48 & 40 & 48 & 44 & 48 & 40 & 48 & 44 & 48 & 48 & 48 \\ 48 & 48 & 14 & 48 & 32 & 48 & 6 & 48 & 6 & 48 & 32 & 48 & 14 & 48 & 48 \\ 48 & 48 & 48 & 44 & 48 & 40 & 48 & 44 & 48 & 40 & 48 & 44 & 48 & 48 & 48 \\ 48 & 48 & 32 & 48 & 8 & 48 & 28 & 48 & 28 & 48 & 8 & 48 & 32 & 48 & 48 \\ 48 & 48 & 48 & 44 & 48 & 42 & 48 & 48 & 48 & 42 & 48 & 44 & 48 & 48 & 48 \\ 48 & 48 & 34 & 48 & 30 & 48 & 8 & 48 & 8 & 48 & 30 & 48 & 34 & 48 & 48 \\ 48 & 48 & 48 & 48 & 48 & 48 & 48 & 48 & 48 & 48 & 48 & 48 & 48 & 48 & 48 \\ 48 & 48 & 48 & 48 & 42 & 48 & 26 & 48 & 26 & 48 & 42 & 48 & 48 & 48 & 48 \\ 48 & 48 & 48 & 48 & 48 & 48 & 48 & 48 & 48 & 48 & 48 & 48 & 48 & 48 & 48\end{array}$

Axial TIP Distribution,

No TIP data taken for this Data Set. 


\section{CYCLE 1 DATA}

\section{DATASET 08, SEPTEMBER 10, 1974}

\section{Reactor Conditions}

Core Average Exposure, $2555 \mathrm{MWd} / \mathrm{t}$

Core Thermal Power, 3265 MWT

Dome Pressure, P, 1022 psia

Core Flow, 101.2 Mib/hr

Inlet Subcooling at P, $24.4 \mathrm{Btu} / \mathrm{lb}$

Control Configuration

Legend: 48, Full Out; O, Full In.

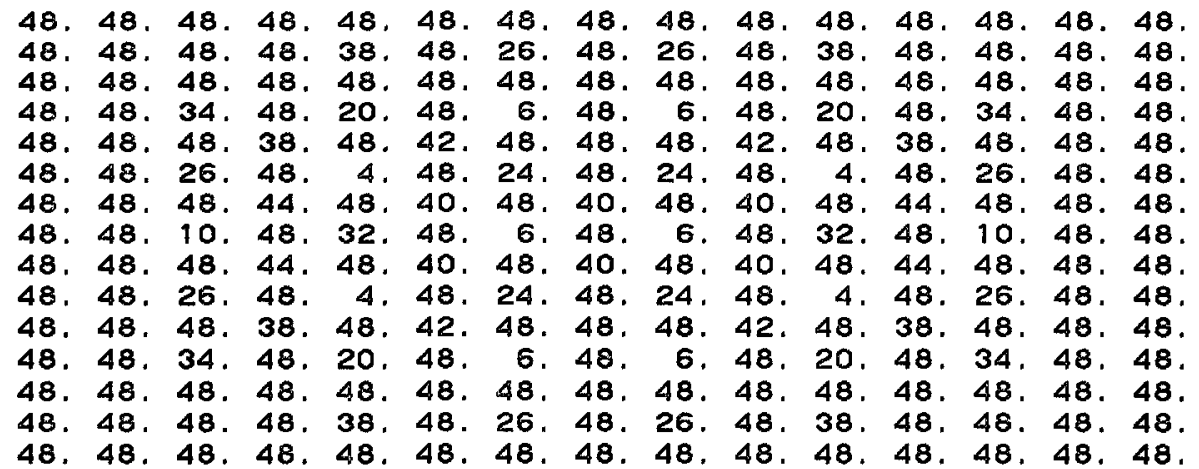

Axial TIP Distribution, Bottom to Top of Core

See Figure 22

$\begin{array}{lllllllllllllllllll}16 & 9 & 34.9 & 50.9 & 61.4 & 71.6 & 76.9 & 75.6 & 74.4 & 71.9 & 64.8 & 57.8 & 57.3 & 58.1\end{array}$ $\begin{array}{lllllllllllll}55.3 & 54.5 & 56.2 & 54.4 & 48.9 & 47.2 & 43.7 & 36.5 & 31.9 & 25.9 & 15.9 & 10.8\end{array}$

$24946.5 \quad 64.9 \quad 74.0 \quad 82.2 \quad 79.5 \quad 76.8 \quad 71.0 \quad 66.5 \quad 60.960 .4 \quad 67.1 \quad 70.7$ $\begin{array}{lllllllllllll}70.6 & 73.4 & 72.9 & 69.6 & 65.4 & 61.5 & 56.4 & 46.5 & 41.0 & 31.8 & 19.7 & 14.8\end{array}$

$32945.6 \quad 66.175 .7 \quad 84.9 \quad 82.7 \quad 76.2 \quad 70.0 \quad 66.961 .261 .165 .8 \quad 73.2$ $\begin{array}{llllllllllll}72.9 & 75.9 & 75.7 & 74.9 & 67.5 & 64.6 & 59.9 & 50.4 & 43.3 & 35.3 & 21.4 & 15.0\end{array}$

$\begin{array}{lllllllllllllllll}40 & 9 & 47.6 & 67.3 & 78.4 & 92.4 & 94.9 & 91.2 & 90.4 & 84.2 & 76.1 & 70.3 & 72.6 & 74.2\end{array}$

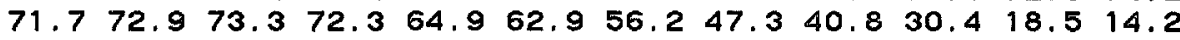

$\begin{array}{lllllllllllll}489 & 94.4 & 38.9 & 48.5 & 60.2 & 65.8 & 66.5 & 66.1 & 65.2 & 60.8 & 55.9 & 55.1 & 53.4\end{array}$ $\begin{array}{lllllllllllllll}53.5 & 51.6 & 50.5 & 49.5 & 44.9 & 42.8 & 39.0 & 33.2 & 28.0 & 23.2 & 15.1 & 9.3\end{array}$

$81734.350 .759 .171 .677 .077 .079 .979 .075 .571 .271 .3 \quad 69.4$

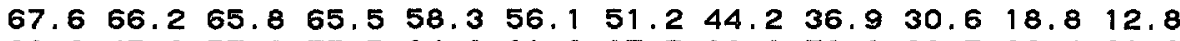

$161729.2 \quad 45.8 \quad 57.9 \quad 75.5 \quad 84.8 \quad 91.2 \quad 87.5 \quad 80.3 \quad 70.0 \quad 63.7 \quad 62.4 \quad 61.8$ $\begin{array}{lllllllllllll}62.9 & 66.9 & 72.9 & 74.6 & 69.2 & 66.2 & 62.0 & 54.2 & 47.0 & 38.6 & 23.5 & 17.1\end{array}$

$2417 \quad 40.8 \quad 62.7 \quad 80.8 \quad 94.2 \quad 96.4 \quad 88.2 \quad 83.2 \quad 70.5 \quad 62.5 \quad 55.9 \quad 57.0 \quad 56.9$ $\begin{array}{llllllllllllll}57.4 & 62.0 & 65.9 & 66.6 & 62.7 & 62.7 & 58.5 & 51.1 & 48.2 & 41.0 & 25.2 & 18.8\end{array}$

$\begin{array}{llllllllllllll}3217 & 53.2 & 75.5 & 87.7 & 93.6 & 90.6 & 83.1 & 75.6 & 65.9 & 57.5 & 53.1 & 54.2 & 56.1\end{array}$ $\begin{array}{llllllllllll}55.7 & 62.0 & 64.5 & 66.2 & 61.4 & 59.3 & 56.2 & 50.9 & 47.5 & 38.7 & 23.9 & 19.5\end{array}$

$401731.5 \quad 53.0 \quad 70.6 \quad 90.7 \quad 96.6 \quad 93.8 \quad 86.0 \quad 81.0 \quad 69.162 .460 .7 \quad 61.2$ $\begin{array}{llllllllllll}62.0 & 64.7 & 74.0 & 78.5 & 74.1 & 72.3 & 67.8 & 59.4 & 51.7 & 45.7 & 28.9 & 19.6\end{array}$

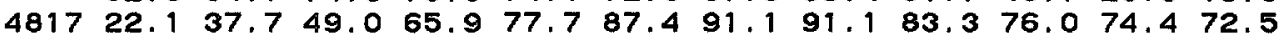
$\begin{array}{lllllllllllll}71.1 & 68.5 & 74.5 & 75.2 & 69.3 & 65.2 & 60.1 & 53.1 & 44.7 & 38.9 & 25.7 & 16.0\end{array}$

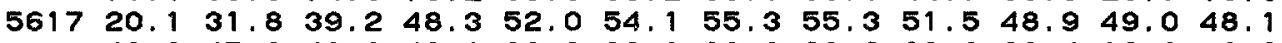
$\begin{array}{llllllllllllllll}48.0 & 45.0 & 43.9 & 43.9 & 38.8 & 38.1 & 33.3 & 28.6 & 23.8 & 20.4 & 12.3 & 8.0\end{array}$

$82552.0 \quad 76.3 \quad 86.8 \quad 98.6 \quad 95.1 \quad 88.7 \quad 79.6 \quad 75.7 \quad 69.8 \quad 68.3 \quad 67.8 \quad 73.0$ $\begin{array}{lllllllllllll}72.3 & 72.4 & 72.7 & 71.4 & 64.8 & 63.1 & 58.1 & 50.5 & 43.6 & 36.1 & 22.6 & 15.8\end{array}$

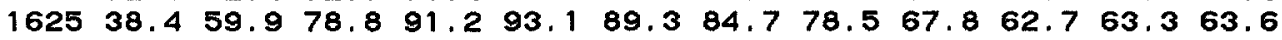

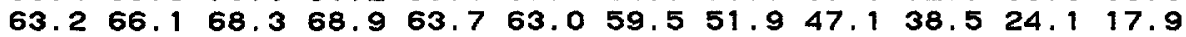


$\begin{array}{llllllllllllllllll}2425 & 32.2 & 49.3 & 66.4 & 85.3 & 93.9 & 90.6 & 87.2 & 76.5 & 67.6 & 63.1 & 66.3 & 71.5\end{array}$

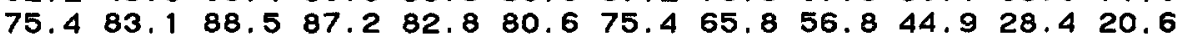
$\begin{array}{llllllllllllll}3225 & 35.5 & 53.6 & 70.6 & 89.6 & 97.2 & 92.2 & 85.0 & 74.4 & 65.3 & 60.7 & 64.0 & 69.2\end{array}$ $\begin{array}{llllllllllllll}70.6 & 80.1 & 85.1 & 83.8 & 78.7 & 77.3 & 71.8 & 62.3 & 54.9 & 44.2 & 27.1 & 21.5\end{array}$ $\begin{array}{llllllllllllll}4025 & 29.2 & 48.6 & 65.5 & 87.7 & 97.3 & 96.8 & 89.9 & 81.4 & 69.5 & 64.1 & 62.9 & 63.9\end{array}$ $\begin{array}{llllllllllll}63.1 & 64.9 & 69.9 & 73.0 & 68.3 & 66.6 & 63.5 & 57.3 & 50.2 & 45.3 & 29.6 & 20.8\end{array}$

$\begin{array}{lllllllllllllll}4825 & 31.5 & 54.8 & 72.0 & 88.7 & 95.6 & 92.9 & 85.1 & 77.8 & 69.4 & 64.0 & 54.6 & 69.8\end{array}$ $\begin{array}{llllllllllllll}70.3 & 70.9 & 75.9 & 77.6 & 70.0 & 68.1 & 63.9 & 57.0 & 49.6 & 42.3 & 27.3 & 18.3\end{array}$

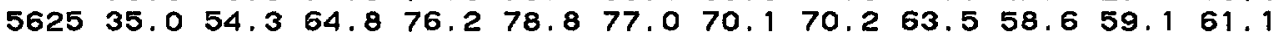
$\begin{array}{llllllllllll}59.7 & 57.2 & 56.6 & 58.5 & 52.8 & 50.4 & 46.9 & 41.1 & 33.8 & 28.6 & 18.2 & 12.0\end{array}$

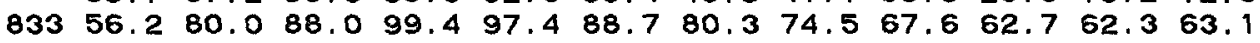
$\begin{array}{lllllllllllll}60.8 & 61.7 & 62.0 & 60.9 & 55.7 & 56.1 & 54.0 & 48.3 & 42.5 & 35.2 & 21.6 & 15.1\end{array}$

$\begin{array}{lllllllllllll}1633 & 37.1 & 60.2 & 76.1 & 88.9 & 92.0 & 88.4 & 86.0 & 80.3 & 74.6 & 68.4 & 69.4 & 69.6\end{array}$ $\begin{array}{llllllllllllll}66.6 & 69.6 & 73.3 & 73.3 & 68.8 & 67.4 & 64.4 & 55.6 & 48.5 & 37.8 & 23.8 & 17.9\end{array}$

$243326.7 \quad 43.9 \quad 59.0 \quad 80.0 \quad 89.6 \quad 90.1 \quad 86.0 \quad 78.3 \quad 68.6 \quad 63.1 \quad 63.4 \quad 63.1$ $\begin{array}{llllllllllll}63.8 & 66.1 & 69.5 & 72.3 & 68.2 & 67.5 & 64.5 & 58.0 & 52.3 & 45.2 & 28.9 & 20.4\end{array}$

$\begin{array}{llllllllllllll}3233 & 21.6 & 37.8 & 54.2 & 74.7 & 90.1 & 93.6 & 87.9 & 82.1 & 68.2 & 59.7 & 57.9 & 58.1\end{array}$

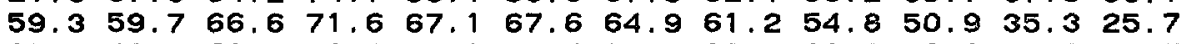

$\begin{array}{lllllllllllllllll}4033 & 25.0 & 42.4 & 59.1 & 79.8 & 93.1 & 95.4 & 91.7 & 89.1 & 83.0 & 76.6 & 74.0 & 72.7\end{array}$ $\begin{array}{llllllllllll}71.0 & 69.7 & 74.0 & 78.6 & 73.4 & 71.6 & 68.5 & 61.9 & 53.6 & 46.7 & 31.8 & 20.7\end{array}$

$\begin{array}{lllllllllllll}4833 & 34.7 & 58.0 & 74.7 & 90.0 & 93.9 & 90.4 & 82.5 & 78.8 & 68.1 & 61.5 & 62.3 & 63.4\end{array}$

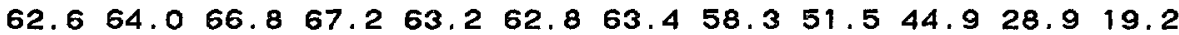

$\begin{array}{llllllllllllll}5633 & 44.0 & 63.0 & 71.6 & 81.8 & 83.6 & 75.8 & 70.8 & 67.4 & 62.9 & 57.2 & 55.8 & 57.1\end{array}$ $54.153 .051 .950 .6 \quad 45.7 \quad 44.441 .1 \quad 36.0 \quad 30.925 .6 \quad 16.011 .6$

$84144.564 .3 \quad 76.4 \quad 87.5 \quad 88.1 \quad 85.5 \quad 80.3 \quad 76.971 .3 \quad 67.4 \quad 71.6 \quad 74.6$ $\begin{array}{llllllllllll}72.0 & 72.3 & 75.2 & 74.4 & 66.5 & 64.0 & 58.7 & 50.2 & 42.3 & 34.3 & 20.2 & 14.3\end{array}$

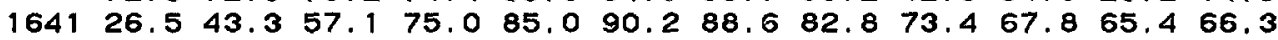
$\begin{array}{llllllllllllll}65.6 & 66.2 & 70.8 & 71.6 & 66.3 & 64.5 & 60.6 & 52.7 & 46.6 & 40.4 & 27.2 & 18.4\end{array}$

$\begin{array}{llllllllllllll}2441 & 31.6 & 50.8 & 66.6 & 83.8 & 85.9 & 82.5 & 76.7 & 67.3 & 56.9 & 52.9 & 54.8 & 58.2\end{array}$ $\begin{array}{llllllllllllll}60.3 & 67.1 & 73.0 & 74.7 & 66.9 & 68.1 & 64.8 & 57.5 & 49.5 & 41.7 & 26.4 & 18.9\end{array}$

$\begin{array}{lllllllllllllll}3241 & 44.2 & 65.3 & 77.7 & 89.5 & 90.6 & 85.1 & 76.1 & 68.1 & 57.5 & 54.8 & 56.5 & 61.6\end{array}$ $\begin{array}{lllllllllllll}65.3 & 72.0 & 77.8 & 80.7 & 76.7 & 75.9 & 70.7 & 63.2 & 54.3 & 46.1 & 29.0 & 20.8\end{array}$

$404128.2 \quad 48.266 .4 \quad 87.4 \quad 94.3 \quad 92.0 \quad 85.3 \quad 77.6 \quad 65.6 \quad 58.3 \quad 58.0 \quad 59.8$

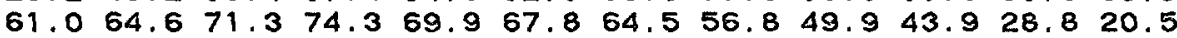

$\begin{array}{lllllllllllll}4841 & 29.7 & 46.3 & 58.9 & 75.7 & 87.4 & 89.9 & 87.2 & 82.4 & 72.3 & 70.4 & 72.9 & 75.2\end{array}$

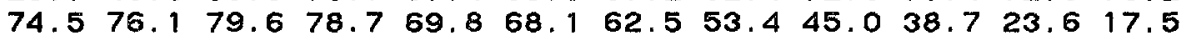

$\begin{array}{llllllllllllll}5641 & 38.3 & 54.8 & 62.4 & 72.6 & 72.6 & 71.3 & 68.8 & 64.8 & 59.2 & 55.3 & 55.7 & 54.5\end{array}$ $\begin{array}{llllllllllll}52.6 & 51.6 & 50.6 & 49.6 & 43.1 & 42.3 & 38.9 & 32.8 & 27.7 & 23.2 & 14.1 & 10.1\end{array}$

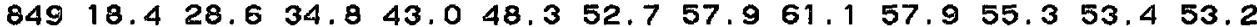
$\begin{array}{llllllllllllll}50.3 & 48.1 & 47.6 & 46.8 & 43.4 & 42.7 & 38.9 & 32.8 & 27.6 & 22.4 & 13.9 & 9.4\end{array}$

$\begin{array}{lllllllllllll}1649 & 36.4 & 55.8 & 67.8 & 81.4 & 88.2 & 86.5 & 82.8 & 75.8 & 65.8 & 62.2 & 62.4 & 63.3\end{array}$

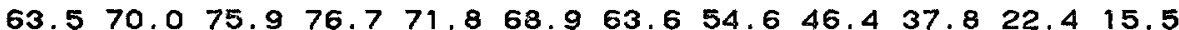

$2449 \quad 42.1 \quad 63.6 \quad 74.7 \quad 36.1 \quad 86.1 \quad 84.4 \quad 76.8 \quad 69.0 \quad 60.2 \quad 56.3 \quad 56.8 \quad 57.9$ $\begin{array}{lllllllllllll}58.9 & 61.6 & 66.6 & 67.9 & 61.7 & 62.1 & 58.5 & 51.2 & 47.4 & 40.2 & 25.2 & 18.3\end{array}$

$324946.266 .3 \quad 79.2 \quad 88.6 \quad 89.0 \quad 82.5 \quad 75.7 \quad 69.3 \quad 58.9 \quad 55.3 \quad 57.0 \quad 57.5$ $\begin{array}{lllllllllllll}58.4 & 61.1 & 54.5 & 64.4 & 59.1 & 58.3 & 56.2 & 51.2 & 46.9 & 41.1 & 25.6 & 18.9\end{array}$

$404939.3 \quad 61.5 \quad 74.8 \quad 89.0 \quad 91.8 \quad 90.1 \quad 83.0 \quad 77.4 \quad 68.4 \quad 61.4 \quad 62.6 \quad 63.6$ $\begin{array}{llllllllllllll}66.1 & 71.4 & 78.6 & 82.8 & 77.0 & 74.4 & 69.0 & 61.1 & 51.9 & 43.6 & 26.8 & 18.1\end{array}$

$\begin{array}{lllllllllllll}4849 & 27.4 & 42.3 & 52.0 & 64.8 & 72.4 & 75.9 & 77.4 & 77.9 & 73.2 & 67.4 & 66.0 & 64.3\end{array}$ $62.461 .963 .5 \quad 62.8 \quad 55.3 \quad 53.8 \quad 50.0 \quad 43.0 \quad 36.2 \quad 30.6 \quad 18.5 \quad 13.5$

$\begin{array}{llllllllllllll}1657 & 13.7 & 20.4 & 25.9 & 34.9 & 43.4 & 50.0 & 53.2 & 52.5 & 50.3 & 48.1 & 48.7 & 48.2\end{array}$ $\begin{array}{llllllllllllll}46.8 & 46.3 & 45.3 & 45.2 & 39.3 & 38.2 & 35.2 & 29.6 & 24.6 & 19.9 & 11.8 & 7.3\end{array}$

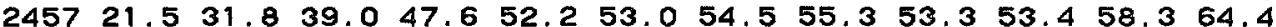
$\begin{array}{lllllllllllll}64.5 & 64.4 & 62.3 & 60.1 & 53.3 & 50.8 & 46.9 & 38.3 & 32.6 & 26.3 & 16.1 & 11.1\end{array}$

$325722.533 .9 \quad 39.948 .252 .653 .152 .853 .0 \quad 51.452 .960 .968 .8$

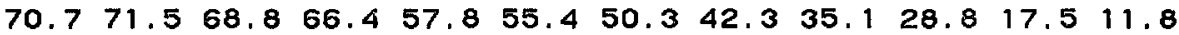

$405722.1 \quad 34.2 \quad 42.4 \quad 55.4 \quad 65.470 .972 .3 \quad 72.265 .461 .362 .0 \quad 61.9$ $\begin{array}{lllllllllllll}60.5 & 59.7 & 58.1 & 57.2 & 49.4 & 48.9 & 46.0 & 38.1 & 31.6 & 25.8 & 15.3 & 10.0\end{array}$ 


\section{CYCLE 1 DATA}

DATASET 09, OCTOBER 4, 1974

\section{Reactor Conditions}

Core Average Exposure, $2920 \mathrm{MWd} / \mathrm{t}$

Core Thermal Power, 2856 MWT

Dome Pressure, P, 1016 psia

Core Flow, $78.7 \mathrm{Mlb} / \mathrm{hr}$

Inlet Subcooling at P, $31.2 \mathrm{Btu} / \mathrm{lb}$

Control Configuration

Legend: 48, Full Out; O, Full In.

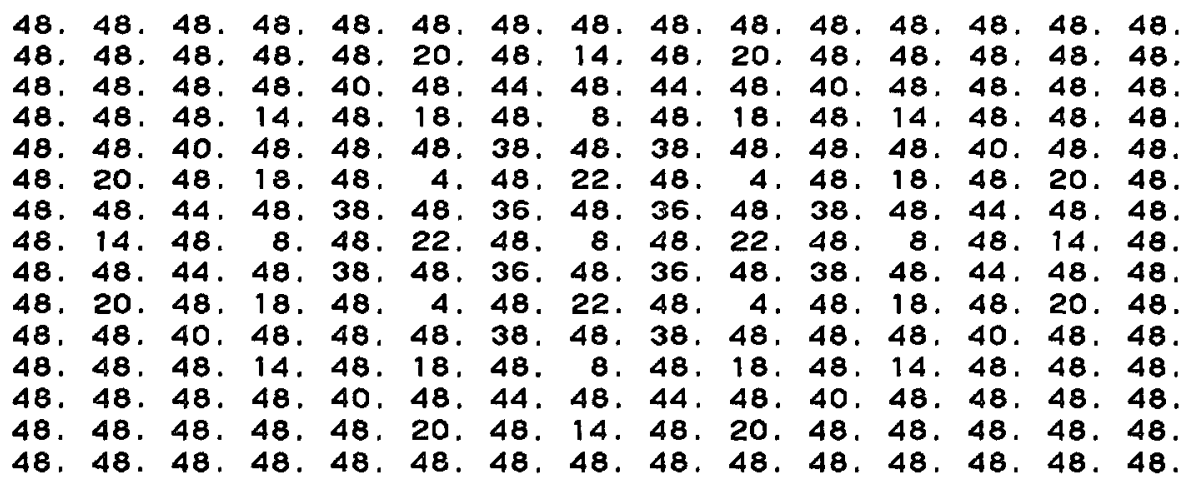

Axial TIP Distribution, Bottom to Top of Core

See Figure 22

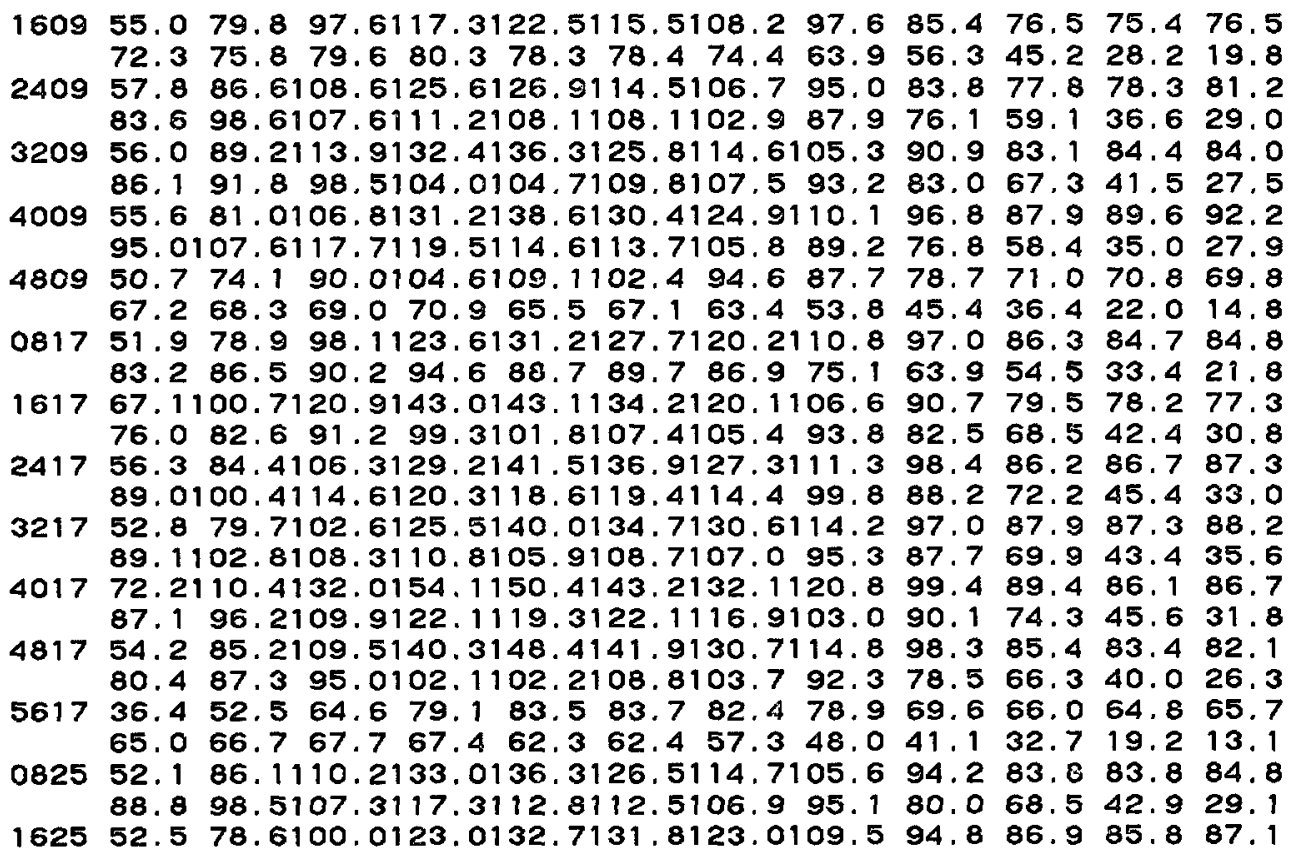


88.0 99.0111.9119.2118.8123.4116.2103.991.5 72.4 44.933.5 242544.566 .986 .7110 .6125 .6132 .8135 .9124 .3108 .3100 .9103 .1107 .1 110.6125 .6131 .4135 .0128 .3128 .3119 .5104 .491 .675 .648 .536 .6 322543.166 .188 .1111 .5126 .7135 .0136 .0123 .3107 .7100 .8101 .2105 .9 111.7129 .9140 .8140 .6131 .8130 .9123 .3108 .395 .977 .046 .237 .7 402549.077 .3100 .3125 .7141 .6148 .3139 .7123 .7103 .494 .091 .192 .1 93.0104 .2115 .9120 .0117 .0117 .5111 .099 .188 .075 .648 .533 .8

482552.487 .9113 .5144 .2149 .6139 .9130 .1111 .996 .485 .183 .280 .4 83.1 93.4109.6120.2118.4121.0117.1104.2 92.3 $77.6 \quad 46.731 .4$

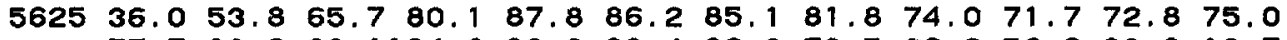

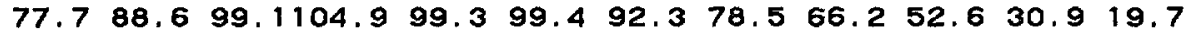

083358.191 .7114 .9134 .8134 .6123 .5113 .2102 .590 .081 .982 .282 .2 $82.090 .3 \quad 96.4103 .8106 .4109 .3104 .993 .4 \quad 79.564 .5 \quad 39.8 \quad 28.4$

163345.370 .690 .1114 .7125 .8129 .3123 .4109 .093 .085 .382 .884 .1 86.8 96.0105.4109.0105.6107.7105.1 96.4 87.9 71.0 45.0 32.7

243334.954 .972 .095 .8111 .2121 .2125 .3120 .0103 .495 .195 .6101 .6 105.8121 .4132 .8134 .9129 .2130 .2125 .5110 .696 .481 .250 .536 .5

323336.958 .076 .4101 .8117 .0129 .7133 .0123 .8109 .297 .899 .599 .6 102.1115 .4123 .7126 .3122 .5124 .2120 .5110 .599 .783 .052 .437 .5

403342.166 .165 .9113 .4133 .1137 .5136 .3123 .5109 .398 .296 .098 .2 102.1116 .2128 .3134 .5129 .6128 .7122 .6107 .594 .978 .748 .935 .3

483358.893 .6121 .2146 .9145 .9136 .3126 .7111 .394 .485 .487 .085 .8 86. $496.4102 .7109 .4108 .4110 .8107 .4 \quad 99.5 \quad 91.5 \quad 73.6 \quad 45.934 .0$

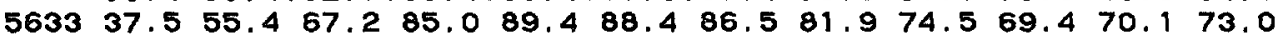

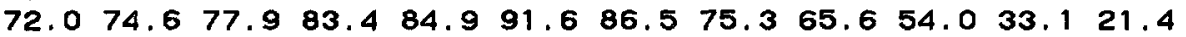

084147.271 .494 .7121 .1130 .9129 .4120 .3110 .197 .786 .985 .288 .2 88.198 .9112 .0116 .8109 .5114 .6106 .591 .978 .963 .938 .926 .0

164163.596 .5117 .8141 .0143 .9137 .7124 .4113 .195 .286 .283 .884 .1 85. $191.9107 .1120 .3116 .6121 .2117 .5104 .090 .8 \quad 77.948 .834 .5$

$244142.265 .5 \quad 84.7108 .5120 .3123 .7120 .3106 .2 \quad 91.182 .781 .984 .2$ 83.8 93.9100.9108.1101.9104.1 99.9 89.0 78.8 66.0 43.5 32.6

324140.063 .082 .7108 .7127 .1133 .1125 .8115 .098 .490 .892 .195 .1 99.7114 .2130 .5134 .4129 .3130 .5124 .7110 .494 .380 .949 .836 .9

404164.7102 .2123 .0146 .9148 .6143 .5128 .7116 .798 .788 .985 .785 .9 86.8 96.3107.6115.0113.4111.7109.1 96.7 85.2 69.7 48.6 36.0

484150.379 .9106 .0138 .3147 .2144 .5134 .3117 .3101 .291 .688 .586 .4 88.1 96.2110.3122.2117.4119.5119.1 98.6 85.3 70.443 .731 .0

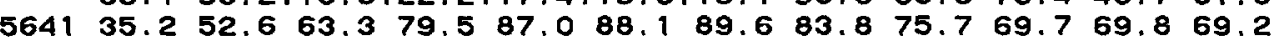
$\begin{array}{llllllllllll}72.2 & 79.7 & 84.0 & 87.2 & 81.9 & 81.3 & 75.1 & 63.2 & 55.1 & 45.5 & 28.1 & 19.1\end{array}$

$084950.6 \quad 69.984 .3 \quad 96.2100 .8 \quad 94.8 \quad 89.6 \quad 82.3 \quad 71.6 \quad 64.863 .763 .4$ $\begin{array}{llllllllllll}62.2 & 63.0 & 64.2 & 65.6 & 63.1 & 65.0 & 60.7 & 51.7 & 44.8 & 35.8 & 21.4 & 14.5\end{array}$

164954.581 .0105 .7128 .9131 .9128 .3117 .9106 .092 .183 .883 .382 .0 $81.491 .099 .6105 .1107 .0112 .5108 .094 .5 \quad 82.065 .939 .827 .3$

244952.0 82.9108.3128.6131.4124.2115.1 99.4 86.0 77.5 79.2 80.0 83.0 95.1108.2118.6117.2120.1115.0101.4 89.7 72.7 45.6 31.9

$324951.081 .8106 .9131 .5136 .5127 .2116 .7107 .892 .081 .7 \quad 78.882 .2$ $\begin{array}{llllllll}81.8 & 89.5 & 97.7102 .4100 .7103 .3102 .7 & 95.1 & 88.7 & 76.5 & 47.7 & 33.4\end{array}$

404952.681 .0106 .3133 .1143 .0140 .1126 .4114 .199 .290 .990 .992 .2 93.3107 .7122 .1132 .8130 .8133 .3127 .9112 .296 .479 .248 .433 .7

484945.771 .788 .6110 .9117 .6117 .5108 .5100 .187 .677 .073 .973 .9 $\begin{array}{llllllllllll}70.6 & 73.1 & 76.8 & 81.9 & 81.5 & 87.0 & 85.3 & 74.5 & 65.4 & 56.3 & 34.6 & 23.7\end{array}$

$165732.245 .656 .366 .972 .571 .372 .169 .6 \quad 64.6 \quad 60.060 .062 .1$ $\begin{array}{lllllllllllll}61.3 & 65.1 & 67.1 & 67.1 & 64.7 & 63.5 & 58.3 & 49.7 & 42.5 & 33.3 & 19.3 & 13.2\end{array}$

$\begin{array}{lllllllllllllll}2457 & 32.1 & 47.3 & 57.9 & 71.3 & 76.6 & 77.3 & 76.9 & 73.3 & 67.7 & 65.2 & 66.3 & 69.3\end{array}$ $\begin{array}{lllllllllllll}72.8 & 81.7 & 90.1 & 94.9 & 88.7 & 89.0 & 83.7 & 69.2 & 61.0 & 49.7 & 30.4 & 19.4\end{array}$

$325736.452 .263 .276 .960 .8 \quad 81.0 \quad 81.074 .7 \quad 72.566 .970 .173 .4$

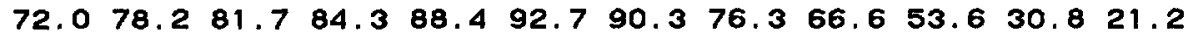

$405736.4 \quad 52.0 \quad 63.5 \quad 79.0 \quad 85.4 \quad 66.5 \quad 87.1 \quad 82.1 \quad 74.7 \quad 71.3 \quad 72: 0 \quad 74.4$ $\begin{array}{llllllllllllll}76.1 & 86.9 & 91.7 & 95.9 & 89.0 & 88.5 & 79.7 & 68.4 & 58.2 & 46.7 & 27.9 & 18.6\end{array}$ 


\section{CYCLE 1 DATA}

DATASET 10, NOVEMBER 21, 1974

\section{Reactor Conditions}

Core Average Exposure, $3542 \mathrm{MWd} / \mathrm{t}$

Core Thermal Power, 3271 MWT

Dome Pressure, P, 1035 psia

Core Flow, 103.8 Mlb/hr

Inlet Subcooling at P, 24.1 Btu/lb

Control Configuration

Legend: 48, Full Out; O, Full In.

\begin{tabular}{|c|c|c|c|c|c|c|c|c|c|c|c|c|c|c|}
\hline $\begin{array}{l}8 . \\
8 . \\
8 . \\
8 .\end{array}$ & $\begin{array}{l}3 \\
4 \\
1 \\
48 \\
30 \\
48 \\
48 \\
48 \\
48 \\
48\end{array}$ & $\begin{array}{l}48 . \\
48 .\end{array}$ & $\begin{array}{r}48 . \\
48 . \\
14 . \\
48 . \\
12 . \\
48 . \\
8 .\end{array}$ & $\begin{array}{l}4 \\
3 \\
4 \\
3 \\
4 \\
3 \\
4 \\
3 \\
4\end{array}$ & $\begin{array}{r}48 \\
12 \\
48 \\
6 \\
48 \\
12 \\
48 \\
6 \\
48\end{array}$ & $\begin{array}{l}4 \\
3 \\
4\end{array}$ & $\begin{array}{r}48 . \\
8 . \\
48 .\end{array}$ & & $\begin{array}{r}40 . \\
6 . \\
48 . \\
12 . \\
48 . \\
6 .\end{array}$ & & $\begin{array}{l}4 \\
1\end{array}$ & & 48. & 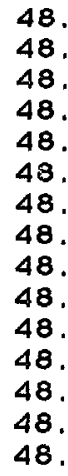 \\
\hline
\end{tabular}

Axial TIP Distribution, Bottom to Top of Core

See Figure 22

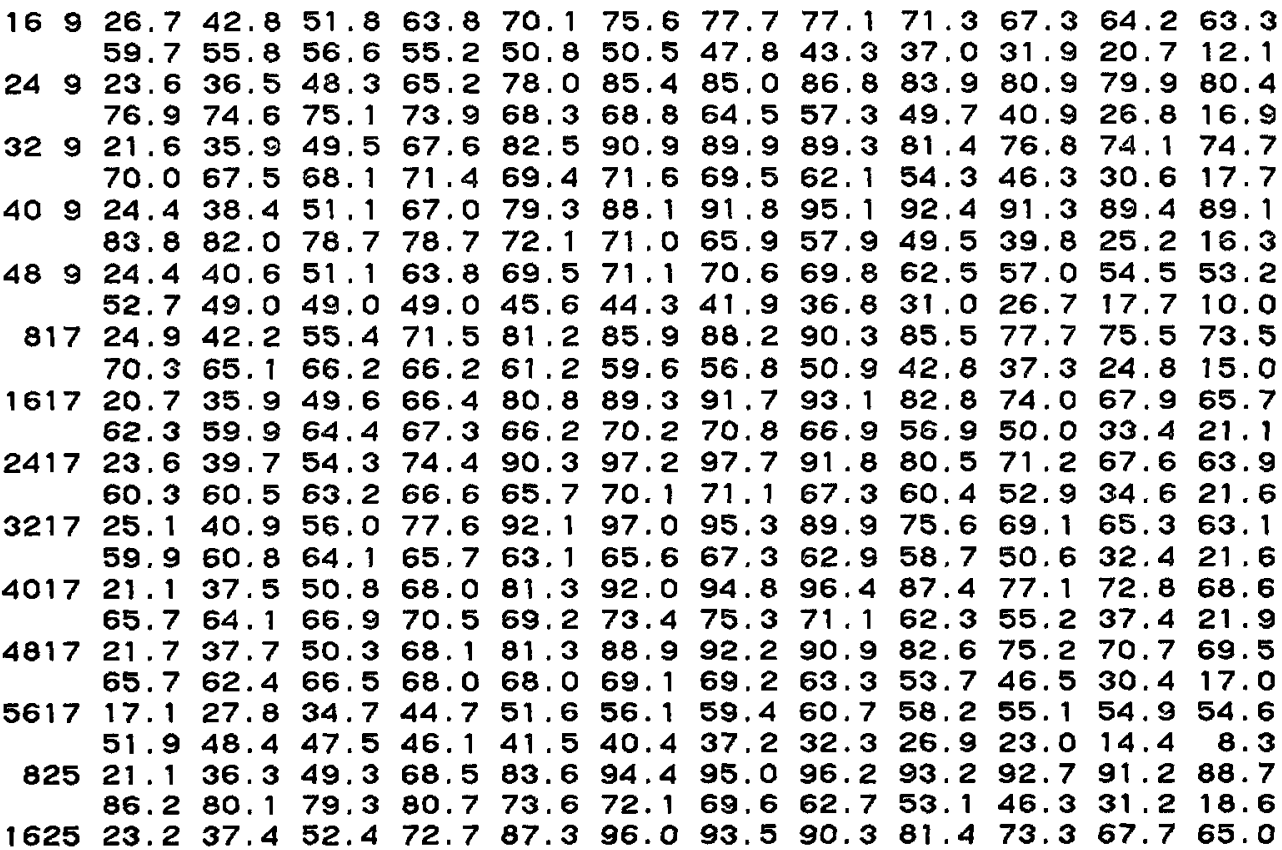


$\begin{array}{lllllllllllllll}62.3 & 62.4 & 65.4 & 68.9 & 67.0 & 72.5 & 75.1 & 70.1 & 62.6 & 55.1 & 35.5 & 22.4\end{array}$ $242522.937 .651 .268 .0 \quad 79.4 \quad 87.7 \quad 91.991 .6 \quad 82.4 \quad 74.671 .870 .6$ $\begin{array}{llllllllllll}68.0 & 70.5 & 74.8 & 76.6 & 75.4 & 77.8 & 78.2 & 72.8 & 66.7 & 59.1 & 39.2 & 24.8\end{array}$ $\begin{array}{lllllllllllll}3225 & 22.6 & 37.6 & 51.3 & 67.5 & 77.7 & 83.8 & 88.3 & 88.2 & 78.5 & 70.4 & 67.2 & 64.9\end{array}$ $\begin{array}{llllllllllll}62.7 & 65.0 & 69.4 & 72.6 & 71.7 & 78.2 & 80.5 & 74.8 & 68.1 & 58.2 & 37.9 & 24.3\end{array}$

$\begin{array}{llllllllllllll}4025 & 21.2 & 36.4 & 51.3 & 71.3 & 89.1 & 97.1 & 96.0 & 94.1 & 81.8 & 73.6 & 67.6 & 64.7\end{array}$ $\begin{array}{llllllllllll}62.3 & 61.8 & 65.5 & 69.4 & 67.4 & 69.6 & 71.1 & 67.2 & 61.7 & 57.7 & 38.9 & 24.6\end{array}$

$\begin{array}{llllllllllllllll}4825 & 18.7 & 33.2 & 47.5 & 66.2 & 85.7 & 97.1 & 97.1 & 96.8 & 84.9 & 77.6 & 72.8 & 71.0\end{array}$ $\begin{array}{llllllllllll}67.5 & 65.3 & 67.7 & 71.3 & 69.5 & 72.2 & 74.1 & 70.2 & 61.2 & 54.1 & 37.1 & 21.5\end{array}$

$\begin{array}{llllllllllllll}5625 & 15.1 & 25.4 & 33.1 & 44.6 & 52.7 & 58.4 & 64.7 & 68.7 & 70.5 & 73.2 & 76.4 & 76.6\end{array}$ $\begin{array}{lllllllllllll}73.4 & 69.0 & 67.9 & 66.6 & 61.4 & 59.6 & 56.2 & 49.1 & 40.5 & 35.2 & 22.4 & 13.1\end{array}$

$83322.437 .6 \quad 50.3 \quad 68.9 \quad 83.4 \quad 92.1 \quad 91.8 \quad 90.9 \quad 84.1 \quad 79.2 \quad 75.272 .9$

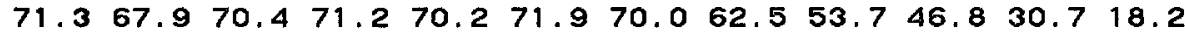

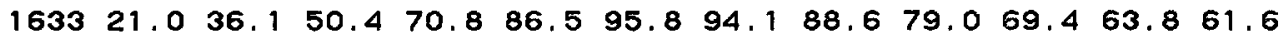
$\begin{array}{lllllllllllll}58.7 & 59.1 & 63.5 & 65.4 & 62.6 & 65.7 & 66.7 & 64.6 & 59.8 & 52.2 & 34.2 & 21.0\end{array}$

$243318.1 \quad 31.2 \quad 43.4 \quad 58.1 \quad 69.7 \quad 77.5 \quad 83.0 \quad 84.977 .6 \quad 70.5 \quad 65.6 \quad 65.0$

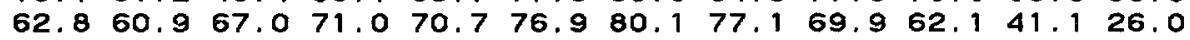

$\begin{array}{llllllllllllllll}3233 & 19.5 & 33.2 & 44.9 & 60.8 & 72.1 & 78.9 & 85.8 & 86.8 & 78.5 & 70.3 & 66.3 & 64.1\end{array}$

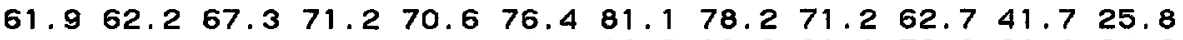

$4033 \quad 19.8 \quad 34.2 \quad 48.1 \quad 67.9 \quad 84.0 \quad 94.5 \quad 93.2 \quad 91.8 \quad 81.3 \quad 72.3 \quad 68.0 \quad 64.2$ $\begin{array}{lllllllllllll}61.5 & 59.3 & 64.1 & 67.8 & 67.1 & 73.2 & 76.9 & 73.7 & 65.1 & 58.6 & 40.1 & 23.7\end{array}$

$\begin{array}{lllllllllllllll}4833 & 20.8 & 35.8 & 50.6 & 70.2 & 88.3 & 96.0 & 95.5 & 92.6 & 83.0 & 75.3 & 72.5 & 69.5\end{array}$ $\begin{array}{llllllllllll}65.0 & 65.6 & 68.3 & 70.4 & 67.7 & 69.6 & 69.5 & 67.0 & 60.1 & 53.9 & 35.1 & 20.6\end{array}$

$\begin{array}{lllllllllllll}5633 & 16.2 & 26.5 & 36.0 & 47.2 & 57.1 & 62.3 & 65.1 & 66.6 & 62.6 & 60.0 & 59.7 & 60.3\end{array}$ $\begin{array}{lllllllllllll}57.7 & 54.9 & 54.6 & 56.1 & 52.2 & 56.2 & 55.0 & 49.5 & 41.5 & 35.4 & 23.4 & 13.8\end{array}$

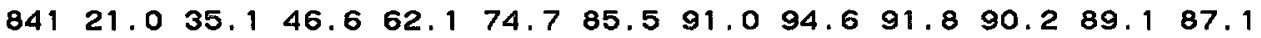
$\begin{array}{llllllllllll}83.1 & 78.2 & 79.4 & 79.4 & 74.1 & 71.4 & 67.3 & 60.2 & 50.9 & 43.9 & 27.8 & 16.9\end{array}$

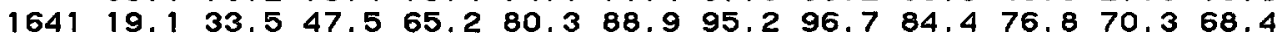
$\begin{array}{lllllllllllllll}65.7 & 62.2 & 66.6 & 69.1 & 68.4 & 71.9 & 74.6 & 71.7 & 62.7 & 55.7 & 38.8 & 23.1\end{array}$

$\begin{array}{lllllllllllll}2441 & 19.0 & 33.4 & 46.6 & 64.9 & 82.3 & 91.1 & 89.7 & 85.9 & 75.2 & 66.8 & 62.6 & 60.9\end{array}$ $\begin{array}{llllllllllll}58.1 & 57.1 & 62.8 & 66.4 & 62.1 & 65.4 & 66.6 & 62.8 & 57.7 & 52.6 & 36.2 & 22.1\end{array}$

$\begin{array}{lllllllllllllll}3241 & 20.4 & 35.5 & 50.1 & 67.8 & 83.7 & 92.8 & 91.9 & 87.8 & 78.4 & 69.5 & 65.8 & 63.2\end{array}$ $\begin{array}{llllllllllll}60.9 & 60.7 & 65.3 & 70.9 & 70.0 & 74.3 & 79.2 & 74.9 & 66.4 & 59.0 & 40.1 & 24.4\end{array}$

$\begin{array}{llllllllllllllll}4041 & 19.2 & 34.6 & 49.3 & 67.1 & 80.6 & 93.3 & 96.5 & 97.2 & 85.7 & 75.1 & 68.7 & 67.1\end{array}$ $\begin{array}{llllllllllll}65.6 & 64.6 & 69.1 & 73.4 & 72.0 & 73.1 & 73.8 & 70.3 & 62.8 & 58.0 & 41.4 & 25.4\end{array}$

$\begin{array}{lllllllllllllll}4841 & 19.6 & 33.7 & 46.9 & 63.3 & 78.0 & 91.5 & 97.5 & 99.4 & 89.8 & 83.3 & 79.8 & 76.6\end{array}$

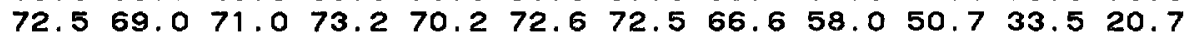

$\begin{array}{lllllllllllllll}5641 & 17.1 & 28.7 & 36.9 & 47.6 & 55.5 & 61.3 & 65.6 & 69.6 & 71.2 & 71.0 & 71.4 & 68.2\end{array}$

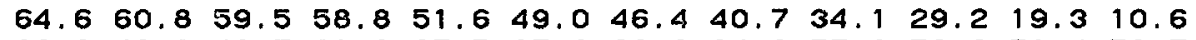

$84926.8 \quad 40.3 \quad 49.7 \quad 61.0 \quad 67.5 \quad 67.8 \quad 66.264 .0 \quad 57.3 \quad 52.6 \quad 51.450 .7$ $\begin{array}{llllllllllllll}47.8 & 45.9 & 44.9 & 44.7 & 42.4 & 42.4 & 40.0 & 35.4 & 29.7 & 24.9 & 15.5 & 9.1\end{array}$

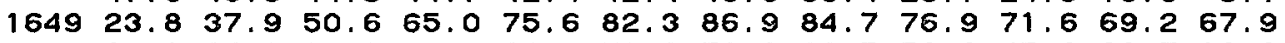
$\begin{array}{lllllllllllll}64.8 & 65.8 & 67.5 & 70.1 & 68.6 & 71.8 & 70.9 & 62.5 & 53.9 & 45.6 & 28.7 & 16.9\end{array}$

$244920.2 \quad 33.5 \quad 46.4 \quad 63.678 .5 \quad 88.0 \quad 88.1 \quad 85.4 \quad 77.4 \quad 71.068 .6 \quad 67.0$ $\begin{array}{llllllllllllll}65.8 & 64.5 & 67.6 & 70.4 & 67.9 & 71.4 & 72.8 & 67.5 & 60.3 & 51.0 & 32.6 & 20.8\end{array}$

$\begin{array}{lllllllllllllll}3249 & 19.1 & 33.8 & 47.1 & 65.7 & 82.5 & 92.2 & 95.0 & 92.7 & 81.1 & 73.2 & 69.1 & 68.3\end{array}$ $\begin{array}{lllllllllllll}66.6 & 64.5 & 68.1 & 69.6 & 66.6 & 68.0 & 69.9 & 66.7 & 61.1 & 54.2 & 36.2 & 22.8\end{array}$

$404920.3 \quad 33.5 \quad 45.7 \quad 62.4 \quad 76.9 \quad 86.9 \quad 91.8 \quad 92.5 \quad 85.6 \quad 82.2 \quad 78.8 \quad 75.9$ $\begin{array}{llllllllllll}74.0 & 72.1 & 75.4 & 78.6 & 76.3 & 79.5 & 80.1 & 74.0 & 64.3 & 55.2 & 36.1 & 20.9\end{array}$

$\begin{array}{lllllllllllllll}4849 & 20.6 & 35.7 & 47.6 & 61.3 & 72.3 & 76.4 & 76.2 & 77.0 & 70.6 & 63.6 & 60.3 & 57.5\end{array}$ $\begin{array}{lllllllllllll}55.2 & 51.7 & 53.7 & 55.0 & 55.3 & 56.4 & 56.8 & 51.5 & 44.0 & 38.6 & 25.9 & 15.5\end{array}$

$165715.924 .8 \quad 31.0 \quad 39.1 \quad 45.4 \quad 48.6 \quad 51.8 \quad 54.5 \quad 53.5 \quad 51.6 \quad 51.6 \quad 50.9$ $\begin{array}{llllllllllllll}49.8 & 47.4 & 47.0 & 46.0 & 41.6 & 41.2 & 38.6 & 32.8 & 27.7 & 23.2 & 14.6 & 8.2\end{array}$

$\begin{array}{lllllllllllllll}2457 & 14.8 & 23.4 & 29.9 & 39.3 & 46.8 & 52.4 & 57.8 & 62.0 & 65.3 & 68.2 & 69.8 & 68.9\end{array}$

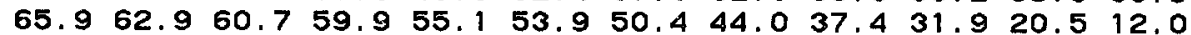

$\begin{array}{llllllllllllll}3257 & 16.1 & 25.3 & 33.0 & 43.4 & 51.8 & 56.2 & 58.8 & 61.6 & 60.6 & 56.5 & 58.4 & 58.6\end{array}$ $\begin{array}{llllllllllll}57.3 & 55.1 & 54.9 & 56.3 & 54.2 & 57.1 & 56.2 & 49.2 & 42.7 & 35.8 & 22.5 & 13.0\end{array}$

$\begin{array}{lllllllllllll}4057 & 17.2 & 27.5 & 35.8 & 45.3 & 54.2 & 59.2 & 65.9 & 70.8 & 72.0 & 72.8 & 74.8 & 73.0\end{array}$

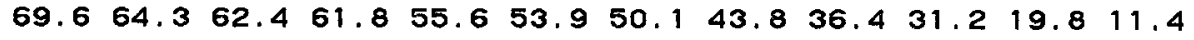




\section{CYCLE 1 DATA}

\section{DATASET 11, JANUARY 6, 1975}

\section{Reactor Conditions}

Core Average Exposure, $4364 \mathrm{MWd} / \mathrm{t}$

Core Thermal Power, 3280 MWT

Dome Pressure, P, 1027 psia

Core Flow, 104.1 Mlb/hr

Inlet Subcooling at P, $23.9 \mathrm{Btu} / \mathrm{lb}$

Control Configuration

Legend: 48, Full Out; O, Full In.

\begin{tabular}{|c|c|c|c|c|c|c|c|c|c|c|c|c|c|c|}
\hline 8. & $\begin{array}{l}48 . \\
48 . \\
48 . \\
48 \\
36 \\
48 \\
32 \\
48 \\
32 \\
48 \\
36 \\
48 . \\
48 \\
48 \\
48 .\end{array}$ & $\begin{array}{l}48 \\
48 \\
48 \\
48 \\
24 . \\
48 \\
10 . \\
48 \\
24 . \\
48 \\
48 \\
48 \\
48 \\
48\end{array}$ & $\begin{array}{r}48 . \\
48 . \\
36 . \\
48 . \\
8 . \\
48 . \\
6 . \\
48 . \\
6 . \\
48 . \\
8 . \\
48 . \\
36 . \\
48 . \\
48 .\end{array}$ & $\begin{array}{l}3 \\
4 \\
3 \\
4 \\
3 \\
4 \\
3 \\
4 \\
3\end{array}$ & $\begin{array}{r}16 \\
48 \\
6 \\
48 \\
10 \\
48 \\
10 \\
48 \\
6 \\
48 \\
16\end{array}$ & $\begin{array}{l}48 . \\
28 . \\
48 . \\
38 . \\
48 . \\
34 . \\
48 . \\
36 . \\
48 . \\
34 . \\
48 . \\
38 . \\
48 . \\
28\end{array}$ & $\begin{array}{r}48 . \\
48 . \\
6 . \\
48 . \\
16 . \\
48 . \\
12 . \\
48 . \\
12 . \\
48 . \\
16 . \\
48 . \\
6 . \\
48 . \\
48 .\end{array}$ & $\begin{array}{l}34 \\
48 \\
36 \\
48 \\
34 \\
48 \\
38 \\
48\end{array}$ & $\begin{array}{r}16 \\
48 \\
6 \\
48 \\
10 \\
48 \\
10 \\
48 \\
6 \\
48 \\
16\end{array}$ & & & & 48 & $\begin{array}{l}48 \\
3 E \\
48\end{array}$ \\
\hline
\end{tabular}

Axial TIP Distribution, Bottom to Top of Core

See Figure 22

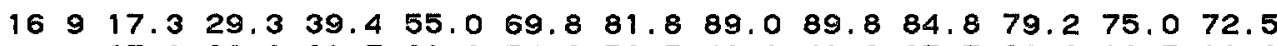
$\begin{array}{lllllllllllll}67.1 & 62.3 & 61.7 & 61.3 & 54.2 & 52.5 & 49.1 & 41.9 & 35.7 & 30.3 & 19.5 & 11.3\end{array}$

$\begin{array}{llllllllllllll}24 & 9 & 22.8 & 36.1 & 47.7 & 62.7 & 70.7 & 71.6 & 73.6 & 74.2 & 71.4 & 71.8 & 75.9 & 76.9\end{array}$

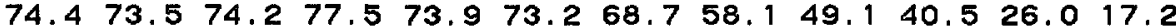

$\begin{array}{llllllllllllll}32 & 9 & 21.4 & 36.1 & 47.7 & 63.1 & 72.0 & 74.3 & 75.4 & 77.6 & 73.2 & 76.4 & 80.9 & 83.7\end{array}$ $\begin{array}{llllllllllll}82.1 & 79.2 & 78.9 & 78.4 & 71.5 & 69.7 & 64.5 & 56.5 & 49.4 & 43.0 & 28.8 & 17.0\end{array}$

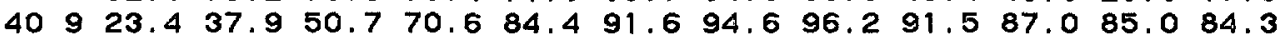
$\begin{array}{lllllllllllll}81.0 & 80.2 & 80.2 & 82.4 & 76.9 & 75.9 & 70.7 & 59.5 & 49.6 & 39.7 & 24.5 & 15.8\end{array}$

$\begin{array}{llllllllllllllll}48 & 9 & 16.6 & 28.7 & 38.1 & 51.5 & 63.6 & 75.0 & 79.6 & 82.6 & 78.1 & 73.0 & 68.9 & 66.5\end{array}$ $\begin{array}{lllllllllllll}62.4 & 57.7 & 57.3 & 54.8 & 48.3 & 46.2 & 42.8 & 36.7 & 30.0 & 25.3 & 16.9 & 9.0\end{array}$

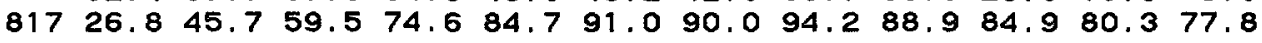
$\begin{array}{lllllllllllll}75.4 & 68.9 & 68.4 & 66.8 & 59.7 & 55.7 & 52.1 & 46.0 & 38.1 & 33.1 & 22.5 & 13.0\end{array}$

$161722.0 \quad 38.7 \quad 53.3 \quad 67.4 \quad 77.2 \quad 82.4 \quad 82.4 \quad 86.3 \quad 83.9 \quad 85.2 \quad 83.5 \quad 81.1$ $\begin{array}{llllllllllll}77.7 & 71.7 & 71.6 & 72.6 & 65.7 & 63.1 & 60.3 & 56.2 & 49.5 & 45.0 & 31.1 & 19.8\end{array}$

$241722.2 \quad 37.7 \quad 51.6 \quad 70.4 \quad 84.4 \quad 93.2 \quad 94.9 \quad 96.2 \quad 89.4 \quad 81.4 \quad 78.5 \quad 77.6$

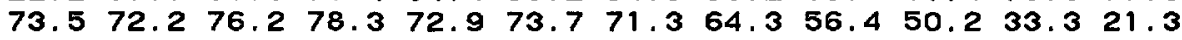

$\begin{array}{llllllllllllll}3217 & 24.4 & 40.1 & 54.3 & 73.6 & 88.1 & 95.5 & 96.3 & 93.8 & 84.7 & 77.5 & 76.2 & 75.2\end{array}$

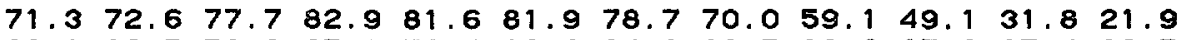

$401720.1 \quad 36.5 \quad 50.0 \quad 65.1 \quad 76.4 \quad 82.2 \quad 84.9 \quad 89.5 \quad 88.2 \quad 87.2 \quad 87.4 \quad 86.5$ $\begin{array}{llllllllllll}82.7 & 78.5 & 80.9 & 02.1 & 76.2 & 73.6 & 70.4 & 64.9 & 56.8 & 51.4 & 35.7 & 21.2\end{array}$

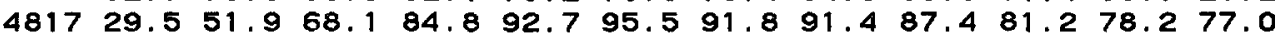

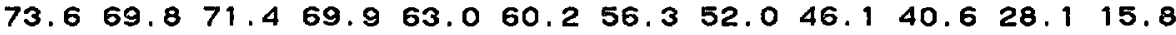

$\begin{array}{lllllllllllll}5617 & 13.0 & 21.7 & 28.4 & 37.4 & 46.9 & 56.2 & 63.9 & 67.8 & 64.8 & 62.1 & 61.4 & 59.5\end{array}$ $\begin{array}{lllllllllllllll}55.9 & 52.2 & 50.0 & 47.3 & 41.2 & 39.3 & 35.7 & 30.3 & 25.2 & 21.5 & 13.5 & 7.7\end{array}$

$82520.6 \quad 35.6 \quad 48.2 \quad 62.6 \quad 73.1 \quad 78.7 \quad 82.0 \quad 90.2 \quad 90.5 \quad 90.0 \quad 87.7 \quad 91.3$ $92.7 \quad 87.9 \quad 87.3 \quad 85.5 \quad 76.5 \quad 70.7 \quad 66.9 \quad 57.9 \quad 48.2 \quad 41.6 \quad 28.9 \quad 16.5$

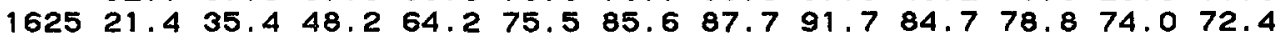


$\begin{array}{llllllllllllll}69.5 & 68.7 & 69.1 & 70.2 & 65.5 & 65.0 & 63.6 & 59.7 & 53.4 & 47.8 & 31.5 & 20.2\end{array}$

$\begin{array}{lllllllllllllll}2425 & 21.7 & 36.2 & 48.8 & 65.0 & 76.8 & 86.1 & 93.4 & 97.8 & 93.5 & 86.1 & 84.3 & 82.0\end{array}$ $\begin{array}{lllllllllllll}78.1 & 77.5 & 80.3 & 82.6 & 78.7 & 81.1 & 81.8 & 77.6 & 68.5 & 58.5 & 38.0 & 25.1\end{array}$

$\begin{array}{lllllllllllll}3225 & 22.4 & 37.4 & 50.4 & 67.3 & 79.1 & 87.9 & 92.9 & 97.6 & 91.3 & 83.5 & 80.5 & 77.6\end{array}$

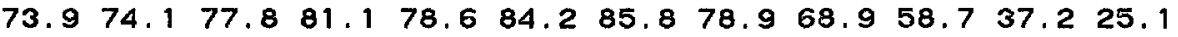

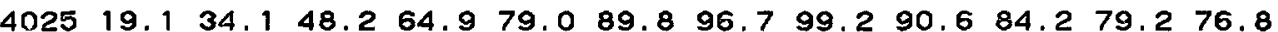

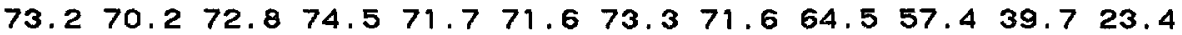

$\begin{array}{llllllllllllll}4825 & 16.8 & 30.3 & 42.2 & 55.8 & 68.2 & 73.7 & 75.4 & 76.5 & 72.0 & 68.1 & 67.4 & 71.7\end{array}$ $\begin{array}{lllllllllllll}73.8 & 74.0 & 76.6 & 76.2 & 70.0 & 67.4 & 64.6 & 60.1 & 52.4 & 47.9 & 34.2 & 19.2\end{array}$

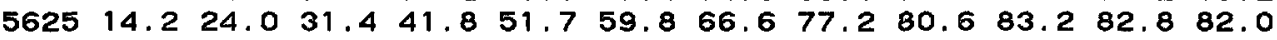
$\begin{array}{lllllllllllll}78.2 & 73.1 & 71.0 & 68.5 & 60.6 & 57.4 & 53.7 & 45.5 & 37.5 & 32.4 & 20.6 & 11.2\end{array}$

$83321.235 .647 .360 .968 .2 \quad 72.9 \quad 76.5 \quad 84.3 \quad 84.3 \quad 83.5 \quad 84.8 \quad 84.3$ $\begin{array}{lllllllllllll}79.2 & 74.4 & 74.4 & 71.8 & 63.9 & 63.0 & 60.3 & 55.1 & 47.8 & 41.6 & 27.7 & 16.2\end{array}$

$\begin{array}{lllllllllllll}1633 & 19.4 & 33.1 & 45.0 & 61.1 & 72.9 & 82.0 & 87.5 & 89.1 & 80.7 & 73.3 & 70.5 & 67.0\end{array}$ $\begin{array}{llllllllllllll}64.5 & 61.7 & 64.6 & 65.4 & 60.8 & 61.0 & 60.5 & 56.2 & 51.3 & 45.8 & 30.7 & 19.8\end{array}$

$\begin{array}{llllllllllllll}2433 & 18.5 & 32.2 & 44.8 & 60.9 & 74.3 & 85.9 & 92.6 & 96.4 & 89.2 & 82.8 & 77.5 & 74.4\end{array}$

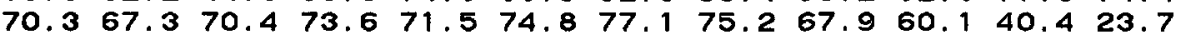

$\begin{array}{llllllllllllllll}3233 & 19.7 & 34.0 & 47.4 & 64.5 & 79.0 & 92.0 & 97.9 & 99.3 & 91.0 & 82.2 & 76.7 & 73.9\end{array}$ $\begin{array}{llllllllllll}68.8 & 68.3 & 71.3 & 75.7 & 75.7 & 82.6 & 85.2 & 81.8 & 71.6 & 62.4 & 40.7 & 26.2\end{array}$

$\begin{array}{llllllllllllll}4033 & 18.6 & 32.8 & 45.6 & 61.5 & 75.1 & 87.2 & 94.2 & 98.2 & 91.4 & 83.9 & 79.5 & 75.1\end{array}$ $\begin{array}{lllllllllllll}70.8 & 67.1 & 69.4 & 72.2 & 68.7 & 71.1 & 73.1 & 71.1 & 63.6 & 57.0 & 39.1 & 22.4\end{array}$

$483318.2 \quad 31.0 \quad 42.1 \quad 56.0 \quad 65.1 \quad 71.4 \quad 74.1 \quad 75.1 \quad 69.967 .165 .965 .0$ $\begin{array}{lllllllllllll}64.2 & 63.2 & 64.5 & 65.2 & 59.8 & 60.6 & 61.6 & 58.5 & 54.4 & 50.4 & 34.5 & 19.3\end{array}$

$\begin{array}{llllllllllllllll}5633 & 14.6 & 24.8 & 33.3 & 43.5 & 53.0 & 58.5 & 65.8 & 76.2 & 78.9 & 77.5 & 78.9 & 78.6\end{array}$ $\begin{array}{llllllllllllll}74.8 & 66.9 & 65.4 & 62.8 & 55.0 & 52.9 & 49.7 & 42.6 & 36.1 & 31.0 & 20.5 & 11.5\end{array}$

$84122.8 \quad 37.8 \quad 51.4 \quad 66.7 \quad 78.0 \quad 87.0 \quad 90.0 \quad 94.2 \quad 90.5 \quad 85.8 \quad 84.087 .2$ $\begin{array}{llllllllllllll}86.8 & 83.2 & 83.6 & 83.3 & 73.2 & 69.0 & 64.1 & 55.6 & 46.1 & 38.8 & 25.1 & 14.5\end{array}$

$\begin{array}{lllllllllllll}1641 & 19.2 & 33.3 & 47.2 & 63.3 & 75.2 & 85.7 & 91.8 & 94.5 & 88.6 & 82.1 & 78.3 & 77.5\end{array}$ $\begin{array}{lllllllllllll}75.0 & 70.5 & 72.6 & 72.8 & 67.1 & 65.5 & 64.2 & 60.9 & 54.4 & 49.8 & 34.6 & 21.0\end{array}$

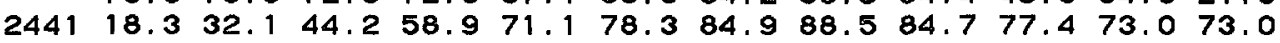
$\begin{array}{lllllllllllll}68.1 & 66.6 & 70.2 & 72.9 & 69.1 & 70.1 & 69.2 & 63.9 & 57.4 & 51.5 & 35.4 & 21.5\end{array}$

$\begin{array}{llllllllllllll}3241 & 19.2 & 33.3 & 46.2 & 61.0 & 72.4 & 79.3 & 85.3 & 90.8 & 88.7 & 81.6 & 77.1 & 75.8\end{array}$ $\begin{array}{llllllllllll}73.8 & 73.6 & 79.4 & 86.7 & 86.8 & 90.4 & 88.0 & 79.1 & 67.2 & 58.1 & 39.4 & 23.9\end{array}$

$4041 \quad 18.1 \quad 32.4 \quad 45.958 .8 \quad 74.0 \quad 84.0 \quad 91.0 \quad 95.5 \quad 89.2 \quad 83.2 \quad 80.1 \quad 78.1$ $75.672 .676 .077 .573 .272 .070 .2 \quad 65.6 \quad 57.954 .1 \quad 38.923 .4$

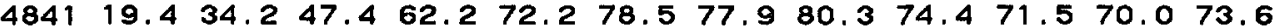
$\begin{array}{llllllllllll}74.0 & 73.2 & 74.9 & 74.5 & 66.4 & 63.8 & 61.1 & 55.4 & 49.2 & 44.4 & 30.7 & 18.5\end{array}$

$\begin{array}{lllllllllllllll}5641 & 15.9 & 26.8 & 34.6 & 46.3 & 58.0 & 70.2 & 79.8 & 84.3 & 80.8 & 75.4 & 71.2 & 68.4\end{array}$ $\begin{array}{llllllllllllll}66.0 & 59.9 & 59.0 & 56.5 & 49.4 & 47.7 & 43.9 & 37.7 & 31.2 & 26.7 & 17.8 & 10.2\end{array}$

$84928.7 \quad 44.956 .1 \quad 70.4 \quad 75.076 .5 \quad 75.2 \quad 72.7 \quad 67.3 \quad 60.7 \quad 58.6 \quad 56.0$ $\begin{array}{llllllllllll}52.3 & 49.5 & 48.4 & 47.3 & 41.3 & 40.3 & 38.1 & 32.4 & 27.1 & 22.9 & 14.4 & 8.3\end{array}$

$\begin{array}{lllllllllllllll}1649 & 23.1 & 37.4 & 50.0 & 66.0 & 77.6 & 87.5 & 93.9 & 97.0 & 97.5 & 93.5 & 94.4 & 93.0\end{array}$ $\begin{array}{llllllllllllll}88.3 & 84.9 & 84.2 & 83.6 & 76.4 & 73.0 & 67.3 & 59.4 & 49.4 & 40.7 & 25.7 & 15.8\end{array}$

$\begin{array}{lllllllllllll}2449 & 21.1 & 34.7 & 47.3 & 65.0 & 79.1 & 85.7 & 87.0 & 84.8 & 81.0 & 75.8 & 75.3 & 75.6\end{array}$ $\begin{array}{llllllllllllll}73.2 & 73.1 & 77.3 & 84.1 & 80.4 & 81.3 & 77.0 & 67.6 & 58.4 & 48.8 & 31.6 & 19.3\end{array}$

$324918.4 \quad 32.1 \quad 45.263 .7 \quad 79.490 .7$ 91.1 $93.3 \quad 84.7 \quad 78.875 .7 \quad 76.6$ $\begin{array}{llllllllllll}73.5 & 72.5 & 76.8 & 79.1 & 73.5 & 73.4 & 70.8 & 64.5 & 57.1 & 50.7 & 34.1 & 20.4\end{array}$

$\begin{array}{llllllllllllll}4049 & 20.6 & 34.4 & 47.2 & 63.3 & 74.9 & 79.9 & 84.2 & 88.8 & 87.9 & 89.9 & 91.7 & 89.6\end{array}$ $\begin{array}{lllllllllllll}86.3 & 83.9 & 87.8 & 91.8 & 88.1 & 87.7 & 83.4 & 73.3 & 61.9 & 51.9 & 33.7 & 20.2\end{array}$

$\begin{array}{lllllllllllll}4849 & 22.1 & 38.6 & 51.3 & 66.3 & 77.6 & 85.4 & 89.2 & 92.3 & 87.5 & 81.5 & 77.4 & 76.1\end{array}$ $\begin{array}{lllllllllllll}71.4 & 65.0 & 65.3 & 63.5 & 56.6 & 53.7 & 50.9 & 44.5 & 37.3 & 32.5 & 21.9 & 12.6\end{array}$

$\begin{array}{llllllllllllllll}1657 & 9.6 & 15.6 & 21.9 & 31.4 & 43.4 & 54.0 & 60.4 & 64.7 & 63.1 & 58.4 & 57.7 & 56.6\end{array}$ $\begin{array}{llllllllllllll}53.3 & 50.9 & 49.8 & 47.7 & 43.5 & 42.9 & 38.9 & 32.5 & 27.9 & 23.3 & 14.5 & 8.0\end{array}$

$2457 \quad 14.2 \quad 22.9 \quad 29.6 \quad 40.1 \quad 47.6 \quad 53.5 \quad 58.3 \quad 62.7 \quad 63.3 \quad 67.0 \quad 72.1 \quad 73.7$ $\begin{array}{lllllllllllll}70.9 & 67.5 & 66.5 & 65.9 & 58.4 & 56.4 & 52.9 & 44.7 & 37.2 & 31.3 & 20.3 & 11.6\end{array}$

$325715.525 .2 \quad 32.942 .8 \quad 50.2 \quad 55.5 \quad 59.8 \quad 62.6 \quad 63.066 .876 .178 .3$

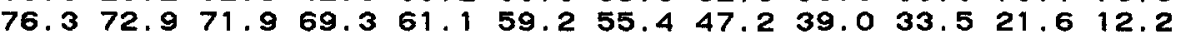

$405716.026 .7 \quad 36.451 .866 .877 .0 \quad 81.3 \quad 85.079 .374 .673 .271 .8$ $68.164 .762 .061 .255 .655 .451 .4 \quad 43.6 \quad 35.930 .6 \quad 19.211 .0$ 


\title{
CYCLE 1 DATA
}

\section{DATASET 12, FEBRUARY 3, 1975}

\section{Reactor Conditions}

Core Average Exposure, $4697 \mathrm{MWd} / \mathrm{t}$

Core Thermal Power, 3277 MWT

Dome Pressure, P, 1029 psia

Core Flow, 104.0 Mib/hr

inlet Subcooling at P, $23.9 \mathrm{Btu} / \mathrm{lb}$

Control Configuration

Legend: 48, Full Out; O, Full In.

\begin{abstract}
48, 48, 48, 48, 48, 48, 48, 48, 48, 48, 48, 48, 48, 48, 48,
48, 48, 48, 48, 48, 24, 48, 14, 48, 24, 48, 48, 48, 48, 48,

48, $48,48,48,36,48,38,48,38,48,36,48,48,48,48$,

48, 48. 48, 12. 48, 10, 48, 6, $48,10,48,12,48,48,48$.

48, 48, 36, $48,32,48,34,48,34,48,32,48,36,48,48$

48. 24. 48, 10. 48, 6, 48, 10, 48, 6. $48,10,48,24,48$.

48, 48, 38, 48, 34, 48, 34, 48, 34, 48, 34, 48, 38, 48, 48,

48. 14. 48. 6. $48,10,48,10,48,10,48,6.48,14,48$.

48, 48, 38, 48, 34, 48, 34, 48, 34, 48, 34, 48, 38, 48,48,

48. 24, 48, 10, 48, 6. $48,10,48,6,48,10,48,24,48$.

48, $48,36,48,32,48,34,48,34,48,32,48,36,48,48$

48, 48, 48, 12, 48, 10, 48, 6. $48,10,48,12,48,48,48$.

48, $48,48,48,36,48,38,48,38,48,36,48,48,48,48$.

48, 48, 48, 48, $48,24,48,14,48,24,48,48,48,48,48$.

48, $48,48,48,48,48,48,48,48,48,48,48,48,48,48$.
\end{abstract}

Axial TIP Distribution, Bottom to Top of Core

See Figure 22

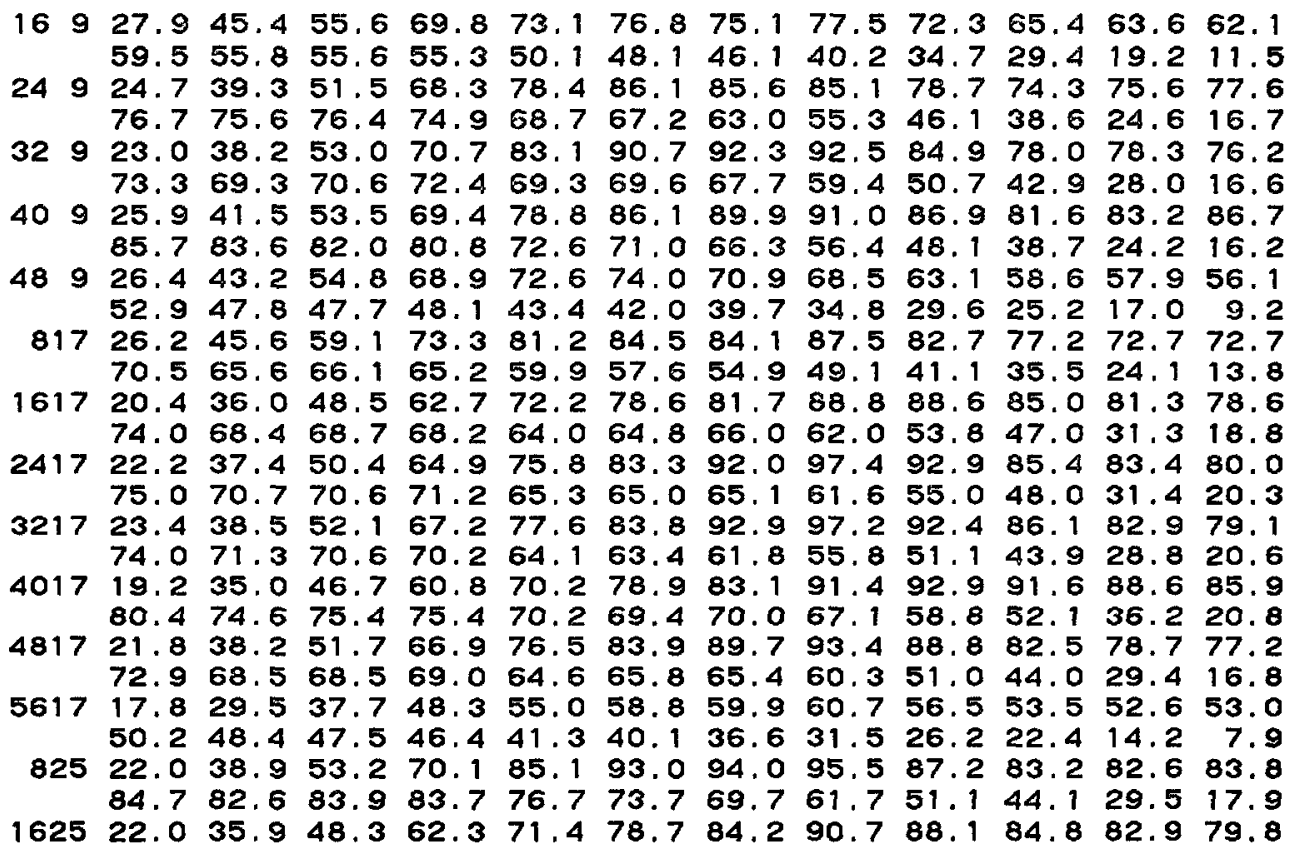


$\begin{array}{lllllllllllllll}74.1 & 71.0 & 71.7 & 70.7 & 66.0 & 67.7 & 68.2 & 63.4 & 56.4 & 48.8 & 31.8 & 20.5\end{array}$

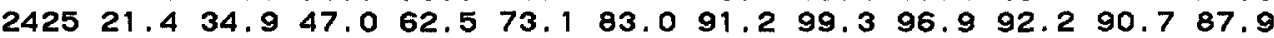
$\begin{array}{lllllllllllll}82.9 & 79.2 & 78.8 & 78.6 & 73.2 & 72.0 & 70.0 & 64.2 & 58.8 & 51.3 & 34.1 & 22.7\end{array}$

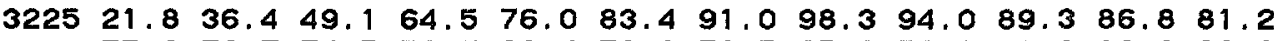
$\begin{array}{llllllllllll}75.2 & 72.7 & 74.5 & 73.7 & 68.4 & 70.3 & 70.5 & 65.6 & 59.2 & 51.3 & 32.9 & 22.9\end{array}$

$402518.3 \quad 32.7 \quad 45.2 \quad 60.4 \quad 71.2 \quad 81.0 \quad 87.1 \quad 96.3 \quad 93.8 \quad 89.5 \quad 85.2 \quad 81.1$ $\begin{array}{llllllllllllll}76.8 & 72.1 & 73.6 & 74.1 & 69.1 & 68.0 & 66.9 & 63.0 & 56.2 & 51.9 & 36.1 & 22.6\end{array}$

$\begin{array}{lllllllllllll}4825 & 17.7 & 32.6 & 47.1 & 65.0 & 80.3 & 92.6 & 93.9 & 97.2 & 88.8 & 84.5 & 82.4 & 78.4\end{array}$

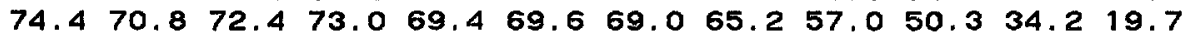

$\begin{array}{lllllllllllllll}5625 & 15.5 & 26.4 & 35.2 & 46.6 & 54.7 & 61.0 & 64.1 & 65.7 & 62.6 & 61.0 & 61.0 & 63.9\end{array}$

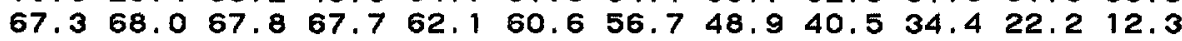

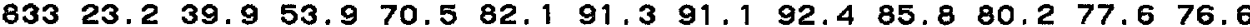
$\begin{array}{lllllllllllll}74.0 & 70.8 & 71.4 & 73.4 & 70.4 & 71.7 & 68.0 & 59.9 & 50.6 & 43.1 & 28.5 & 17.0\end{array}$

$\begin{array}{lllllllllllll}1633 & 19.7 & 34.0 & 46.2 & 61.6 & 70.3 & 78.3 & 85.7 & 91.0 & 87.4 & 82.1 & 78.2 & 74.3\end{array}$

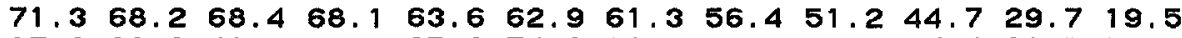

$243317.3 \quad 29.941 .4 \quad 56.0 \quad 65.974 .2 \quad 82.9 \quad 92.5 \quad 91.3 \quad 87.4 \quad 81.5 \quad 79.3$ $\begin{array}{llllllllllll}74.2 & 70.1 & 70.4 & 71.4 & 67.3 & 67.7 & 68.6 & 66.5 & 59.6 & 53.8 & 36.2 & 21.7\end{array}$

$\begin{array}{lllllllllllll}3233 & 18.5 & 32.1 & 44.1 & 59.8 & 71.0 & 81.0 & 90.0 & 96.9 & 91.8 & 87.6 & 83.4 & 79.0\end{array}$ $\begin{array}{llllllllllll}74.9 & 70.1 & 70.8 & 72.9 & 67.9 & 70.0 & 71.6 & 69.1 & 62.0 & 56.1 & 37.0 & 22.9\end{array}$

$403318.0 \quad 31.4 \quad 43.7 \quad 57.7 \quad 69.0 \quad 77.0 \quad 86.0 \quad 96.3 \quad 95.3 \quad 88.5 \quad 85.9 \quad 81.4$

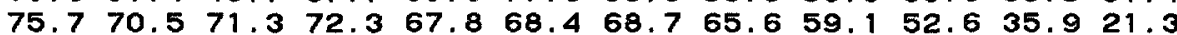

$\begin{array}{llllllllllllll}4833 & 20.4 & 35.3 & 49.4 & 67.9 & 82.5 & 92.5 & 96.1 & 96.5 & 91.0 & 83.2 & 80.8 & 78.0\end{array}$

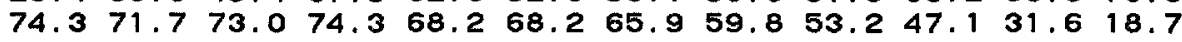

$\begin{array}{llllllllllllllll}5633 & 16.6 & 28.5 & 37.9 & 49.5 & 58.3 & 62.7 & 63.5 & 64.8 & 61.3 & 57.5 & 56.6 & 56.0\end{array}$ $\begin{array}{lllllllllllll}55.0 & 53.1 & 52.6 & 55.2 & 52.3 & 55.0 & 53.6 & 47.1 & 39.5 & 33.8 & 22.5 & 12.8\end{array}$

$\begin{array}{llllllllllllll}841 & 22.5 & 38.7 & 51.4 & 67.1 & 77.3 & 84.6 & 88.3 & 92.3 & 87.1 & 82.5 & 81.1 & 83.4\end{array}$ $\begin{array}{llllllllllll}83.2 & 80.2 & 82.3 & 82.2 & 75.7 & 71.7 & 67.7 & 59.5 & 50.2 & 41.8 & 26.8 & 15.9\end{array}$

$\begin{array}{llllllllllllll}1641 & 18.6 & 32.3 & 45.1 & 59.9 & 72.1 & 78.8 & 82.8 & 90.4 & 89.7 & 88.7 & 85.1 & 83.1\end{array}$

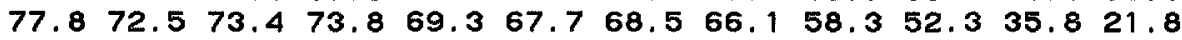

$\begin{array}{lllllllllllllllll}2441 & 17.8 & 31.1 & 43.2 & 56.4 & 66.3 & 75.7 & 82.5 & 89.8 & 87.9 & 82.4 & 78.8 & 75.7\end{array}$ $\begin{array}{lllllllllllll}70.6 & 66.9 & 67.8 & 68.1 & 61.5 & 62.3 & 61.1 & 57.1 & 51.4 & 46.9 & 32.3 & 19.9\end{array}$

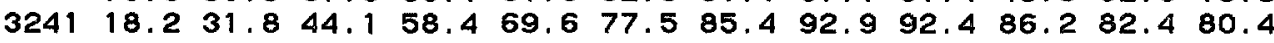

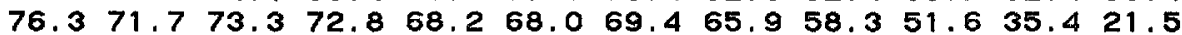

$4041 \quad 17.2 \quad 30.9 \quad 42.9 \quad 56.1 \quad 66.8 \quad 76.1 \quad 80.8 \quad 89.8 \quad 91.3 \quad 88.6 \quad 85.4 \quad 82.4$ $\begin{array}{lllllllllllll}79.3 & 74.9 & 76.6 & 76.8 & 72.0 & 70.6 & 68.8 & 63.9 & 58.1 & 52.6 & 37.3 & 22.3\end{array}$

$\begin{array}{llllllllllllll}4841 & 18.9 & 33.2 & 46.0 & 60.5 & 74.3 & 84.9 & 91.7 & 96.2 & 90.9 & 87.1 & 84.6 & 80.9\end{array}$ $\begin{array}{llllllllllll}77.8 & 73.0 & 74.3 & 75.0 & 68.0 & 67.4 & 67.4 & 62.7 & 54.0 & 47.3 & 31.8 & 19.8\end{array}$

$\begin{array}{lllllllllllll}5641 & 18.2 & 30.3 & 39.6 & 50.9 & 59.4 & 64.4 & 65.7 & 66.1 & 62.2 & 58.8 & 59.9 & 60.7\end{array}$ $\begin{array}{lllllllllllll}61.2 & 59.1 & 59.1 & 57.0 & 50.9 & 48.8 & 45.5 & 39.9 & 33.5 & 28.9 & 19.4 & 11.1\end{array}$

$\begin{array}{lllllllllllll}849 & 29.4 & 44.9 & 55.3 & 65.6 & 70.2 & 68.4 & 66.9 & 64.6 & 59.9 & 55.2 & 53.1 & 50.5\end{array}$ $\begin{array}{lllllllllllll}46.7 & 44.8 & 44.3 & 42.7 & 40.7 & 40.3 & 38.5 & 33.2 & 28.8 & 23.9 & 14.8 & 8.7\end{array}$

$\begin{array}{llllllllllllllll}1649 & 24.7 & 39.7 & 51.8 & 68.0 & 77.1 & 82.6 & 86.6 & 87.9 & 83.5 & 78.5 & 77.7 & 75.1\end{array}$

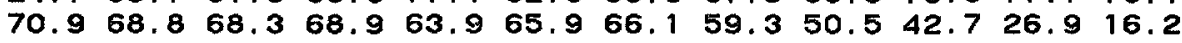

$\begin{array}{llllllllllllllll}2449 & 20.7 & 34.6 & 46.9 & 63.6 & 77.2 & 86.6 & 89.5 & 89.8 & 83.6 & 77.4 & 76.8 & 74.6\end{array}$ $\begin{array}{lllllllllllll}71.8 & 69.3 & 70.7 & 71.4 & 65.8 & 66.6 & 66.7 & 60.7 & 54.3 & 46.6 & 30.2 & 18.6\end{array}$

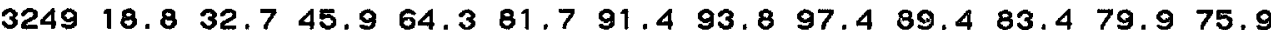

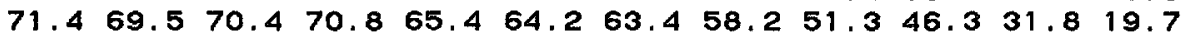

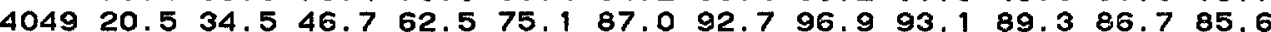
$\begin{array}{lllllllllllll}81.8 & 78.7 & 79.3 & 81.1 & 74.8 & 74.6 & 73.8 & 68.7 & 59.8 & 51.1 & 33.2 & 19.9\end{array}$

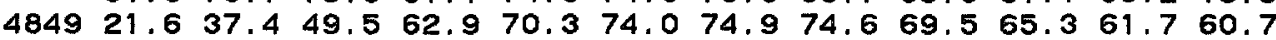

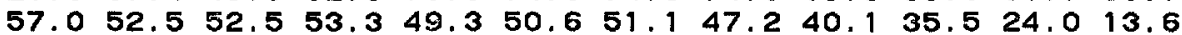

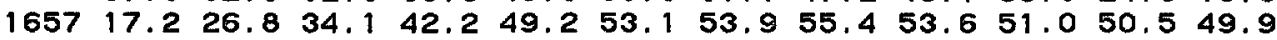
$\begin{array}{llllllllllllll}47.7 & 46.0 & 46.2 & 46.7 & 41.9 & 39.6 & 37.0 & 32.0 & 26.9 & 22.6 & 14.2 & 7.9\end{array}$

$245715.825 .1 \quad 32.942 .951 .355 .759 .0 \quad 60.458 .6 \quad 56.0 \quad 57.8 \quad 61.2$ $\begin{array}{lllllllllllll}62.2 & 61.7 & 62.8 & 62.2 & 55.2 & 54.5 & 51.3 & 43.8 & 36.4 & 30.7 & 20.1 & 11.1\end{array}$

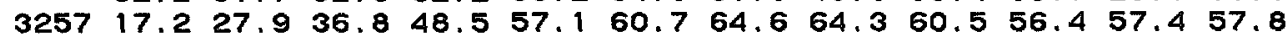
$\begin{array}{llllllllllllll}56.5 & 54.7 & 55.1 & 56.0 & 53.9 & 56.0 & 55.3 & 47.7 & 41.2 & 34.4 & 21.9 & 12.6\end{array}$

$4057 \quad 18.5 \quad 29.8 \quad 38.9 \quad 50.5 \quad 59.6 \quad 64.0 \quad 66.967 .0 \quad 63.6 \quad 59.6 \quad 61.3 \quad 63.3$ $\begin{array}{lllllllllllll}64.3 & 64.0 & 63.9 & 62.7 & 55.4 & 54.2 & 51.4 & 43.6 & 35.6 & 30.3 & 18.9 & 10.7\end{array}$ 


\section{CYCLE 1 DATA}

\section{DATASET 13, MARCH 13, 1975}

\section{Reactor Conditions}

Core Average Exposure, $5262 \mathrm{MWd} / \mathrm{t}$

Core Thermal Power, 3293 MWT

Dome Pressure, P, 1033 psia

Core Flow, 103.1 Mlb/hr

Inlet Subcooling at P, 24.2 Btu/lb

Control Configuration

Legend: 48, Full Out; O, Full In.

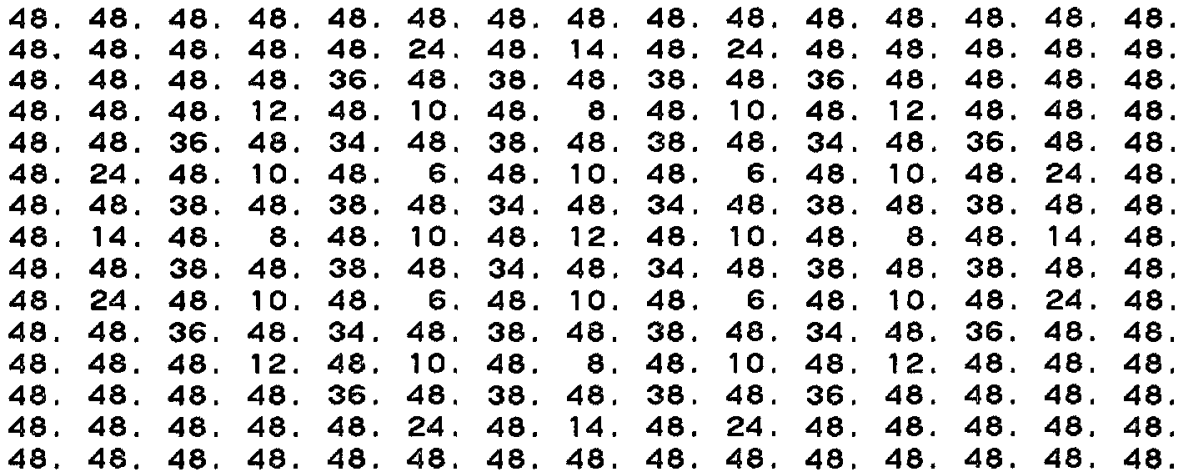

Axial TIP Distribution, Bottom To top of Core

See Figure 22

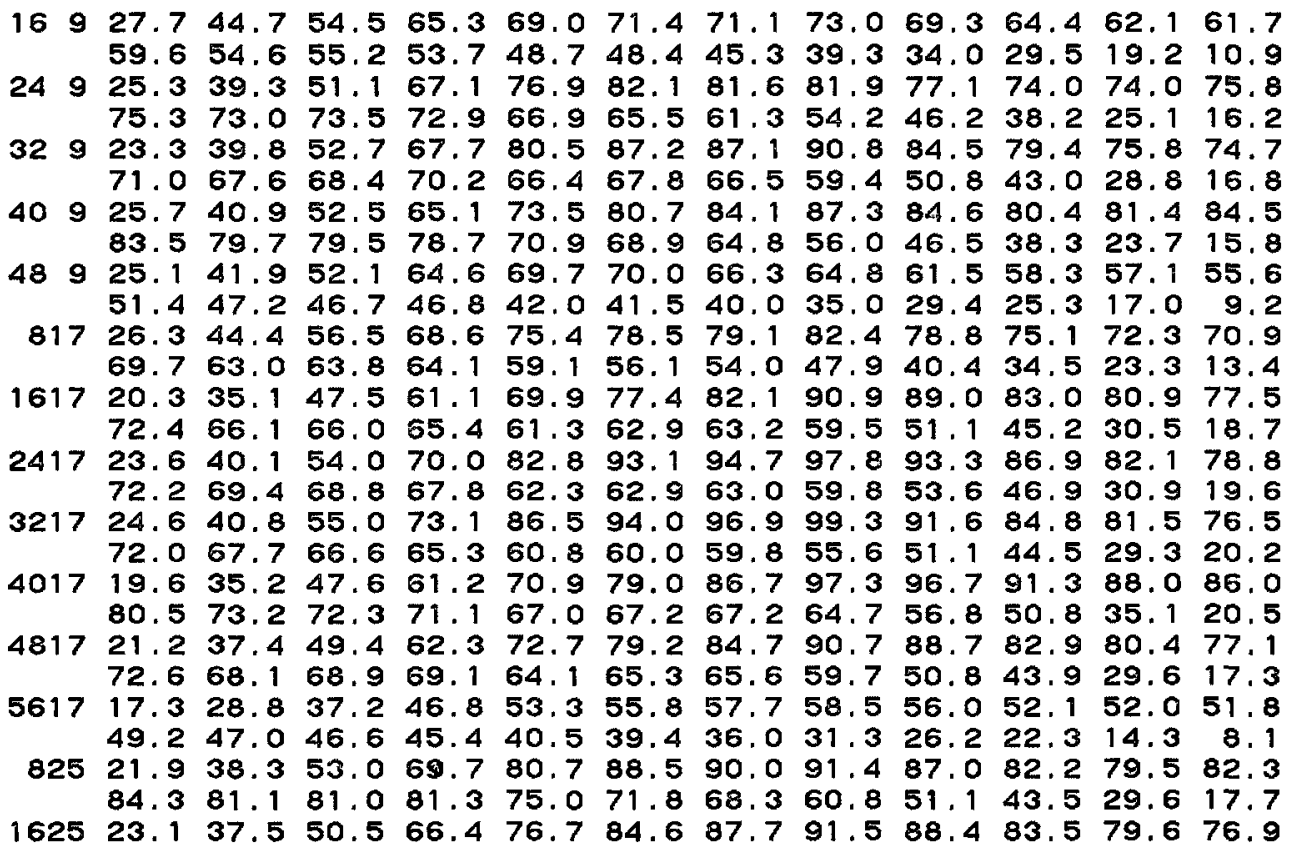


$\begin{array}{lllllllllllllll}71.2 & 68.3 & 67.3 & 67.0 & 62.3 & 64.2 & 64.9 & 61.3 & 55.0 & 47.6 & 31.3 & 19.6\end{array}$

$\begin{array}{lllllllllllll}2425 & 22.9 & 37.3 & 50.7 & 65.9 & 75.1 & 83.6 & 90.0 & 97.4 & 97.6 & 92.9 & 90.3 & 85.5\end{array}$ $80.976 .6 \quad 75.974 .3 \quad 68.7 \quad 68.6 \quad 66.8 \quad 60.7 \quad 55.1 \quad 48.5 \quad 32.4 \quad 21.9$

$322523.1 \quad 38.5 \quad 51.966 .0 \quad 75.3 \quad 82.6 \quad 90.3 \quad 95.8 \quad 93.9 \quad 88.4 \quad 86.6 \quad 82.6$

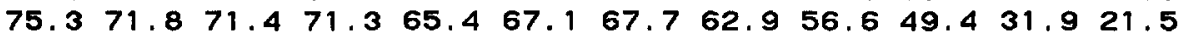

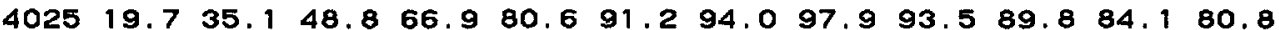
$\begin{array}{lllllllllllll}75.8 & 69.9 & 70.7 & 70.5 & 66.0 & 64.3 & 63.3 & 60.3 & 54.2 & 49.9 & 35.0 & 21.6\end{array}$

$\begin{array}{llllllllllllll}4825 & 18.4 & 33.7 & 47.5 & 64.4 & 79.6 & 89.1 & 90.4 & 93.8 & 90.5 & 83.5 & 78.7 & 77.6\end{array}$ $\begin{array}{lllllllllllll}73.7 & 68.9 & 70.6 & 71.3 & 68.0 & 66.5 & 67.8 & 64.3 & 56.1 & 49.3 & 33.5 & 19.2\end{array}$

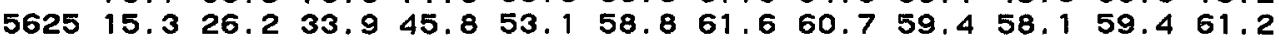
$\begin{array}{llllllllllllll}65.5 & 64.6 & 65.6 & 65.9 & 61.2 & 57.8 & 55.1 & 48.6 & 39.4 & 33.8 & 22.1 & 12.5\end{array}$

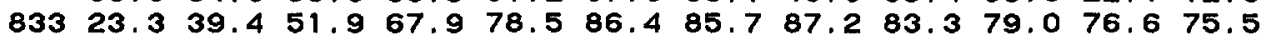
$\begin{array}{llllllllllllll}72.5 & 69.1 & 69.1 & 71.8 & 68.7 & 70.0 & 67.4 & 60.4 & 51.0 & 43.4 & 28.2 & 17.7\end{array}$

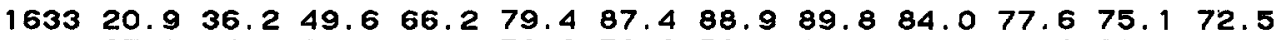
$\begin{array}{llllllllllllll}67.8 & 64.0 & 64.6 & 64.7 & 59.6 & 59.3 & 59.6 & 56.6 & 51.4 & 45.0 & 29.6 & 18.9\end{array}$

$243318.6 \quad 32.2 \quad 44.2 \quad 57.0 \quad 67.5 \quad 75.1 \quad 81.2 \quad 89.8 \quad 89.7 \quad 86.4 \quad 81.8 \quad 78.8$ $\begin{array}{llllllllllll}73.8 & 68.5 & 67.2 & 68.2 & 63.8 & 65.4 & 65.7 & 63.3 & 56.7 & 50.9 & 34.4 & 20.8\end{array}$

$\begin{array}{llllllllllllll}3233 & 21.7 & 36.1 & 47.8 & 62.0 & 71.6 & 79.8 & 88.9 & 94.1 & 92.2 & 87.2 & 84.5 & 79.6\end{array}$ $\begin{array}{llllllllllll}73.7 & 69.6 & 69.7 & 70.0 & 66.5 & 70.3 & 72.4 & 67.7 & 59.1 & 50.7 & 33.0 & 21.8\end{array}$

$\begin{array}{llllllllllllllll}4033 & 19.6 & 34.8 & 48.1 & 64.8 & 79.7 & 89.0 & 92.2 & 97.3 & 92.4 & 88.5 & 82.8 & 79.9\end{array}$

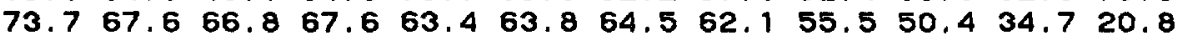

$\begin{array}{lllllllllllllll}4833 & 20.8 & 36.1 & 50.4 & 68.0 & 81.6 & 91.1 & 92.4 & 93.9 & 90.0 & 84.7 & 81.5 & 77.7\end{array}$ $\begin{array}{llllllllllll}73.7 & 70.7 & 70.2 & 71.7 & 66.6 & 66.5 & 65.6 & 61.7 & 55.0 & 48.4 & 32.3 & 18.8\end{array}$

$\begin{array}{llllllllllllll}5633 & 17.0 & 28.8 & 38.3 & 49.6 & 59.3 & 62.6 & 63.7 & 63.0 & 60.2 & 55.0 & 54.6 & 54.4\end{array}$

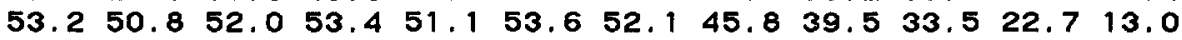

$\begin{array}{llllllllllllll}641 & 22.5 & 38.1 & 49.8 & 63.5 & 73.2 & 79.0 & 82.2 & 85.5 & 81.1 & 79.3 & 78.2 & 79.6\end{array}$

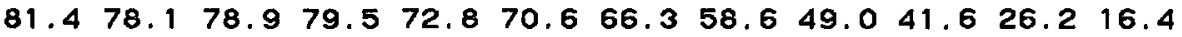

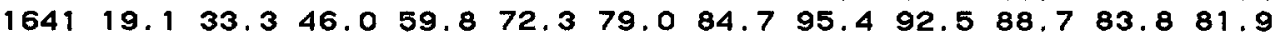

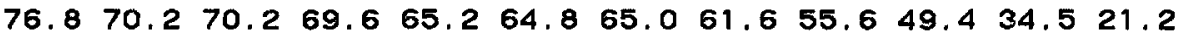

$2441 \quad 18.7 \quad 32.7 \quad 45.6 \quad 61.7 \quad 73.8 \quad 83.0 \quad 86.3 \quad 88.4 \quad 84.3 \quad 78.3 \quad 76.072 .0$ $\begin{array}{lllllllllllll}66.9 & 62.0 & 62.0 & 61.6 & 57.5 & 56.7 & 57.1 & 52.5 & 47.8 & 43.8 & 30.4 & 19.0\end{array}$

$\begin{array}{lllllllllllll}3241 & 20.3 & 35.6 & 49.5 & 65.6 & 79.1 & 89.5 & 92.1 & 95.4 & 90.5 & 85.4 & 81.5 & 78.0\end{array}$

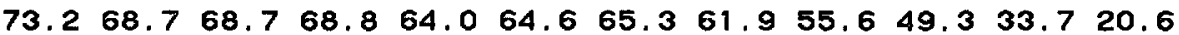

$404118.2 \quad 33.0 \quad 46.4 \quad 59.9 \quad 71.4 \quad 81.0 \quad 86.6 \quad 95.8 \quad 95.2 \quad 90.7 \quad 84.982 .6$ $\begin{array}{llllllllllll}78.0 & 72.3 & 72.4 & 73.5 & 67.8 & 67.0 & 64.6 & 60.9 & 54.3 & 50.1 & 35.2 & 21.5\end{array}$

$4841 \quad 18.932 .9 \quad 45.3 \quad 59.0 \quad 70.5 \quad 79.4 \quad 86.9 \quad 93.5 \quad 89.1 \quad 83.0 \quad 83.0 \quad 80.3$ $\begin{array}{lllllllllllll}77.1 & 71.3 & 72.3 & 70.6 & 65.2 & 65.9 & 64.1 & 59.5 & 51.7 & 45.2 & 30.5 & 18.3\end{array}$

$\begin{array}{lllllllllllll}5641 & 18.3 & 30.5 & 39.2 & 49.3 & 57.8 & 63.0 & 63.3 & 63.7 & 58.6 & 56.1 & 55.5 & 58.6\end{array}$ $\begin{array}{llllllllllllll}59.8 & 57.3 & 57.4 & 55.5 & 49.9 & 47.1 & 45.4 & 38.8 & 32.8 & 28.4 & 18.6 & 11.1\end{array}$

$\begin{array}{llllllllllllll}849 & 28.5 & 42.8 & 52.1 & 63.1 & 67.1 & 65.4 & 62.3 & 61.2 & 56.3 & 51.0 & 50.7 & 48.9\end{array}$ $\begin{array}{llllllllllll}46.2 & 44.2 & 43.2 & 42.6 & 39.7 & 39.2 & 37.3 & 32.3 & 27.8 & 23.1 & 14.6 & 8.4\end{array}$

$\begin{array}{lllllllllllllll}1649 & 24.3 & 38.9 & 51.4 & 64.1 & 72.5 & 77.1 & 81.8 & 85.9 & 81.1 & 77.9 & 77.2 & 75.4\end{array}$ $\begin{array}{llllllllllll}69.8 & 66.3 & 66.3 & 66.7 & 62.6 & 63.9 & 63.2 & 57.2 & 49.8 & 41.6 & 26.0 & 16.2\end{array}$

$\begin{array}{llllllllllllllll}2449 & 21.4 & 34.9 & 47.3 & 63.1 & 76.8 & 84.1 & 85.2 & 87.7 & 82.1 & 77.2 & 76.2 & 73.7\end{array}$ $\begin{array}{llllllllllll}69.6 & 68.0 & 67.7 & 68.2 & 63.4 & 64.0 & 63.1 & 59.2 & 52.6 & 45.5 & 29.7 & 18.3\end{array}$

$\begin{array}{llllllllllllll}3249 & 19.1 & 33.4 & 47.4 & 64.2 & 78.4 & 88.1 & 90.4 & 93.5 & 87.5 & 82.5 & 77.3 & 73.6\end{array}$ $\begin{array}{lllllllllllllll}69.5 & 65.5 & 67.4 & 67.1 & 61.8 & 62.0 & 61.7 & 58.5 & 52.8 & 46.7 & 31.7 & 19.5\end{array}$

$404920.4 \quad 34.7 \quad 46.8 \quad 61.6 \quad 71.3 \quad 82.9 \quad 90.2 \quad 94.9 \quad 91.3 \quad 88.0 \quad 86.4 \quad 83.9$ $\begin{array}{lllllllllllll}80.5 & 77.2 & 76.5 & 76.9 & 72.6 & 72.7 & 72.6 & 67.3 & 58.5 & 50.3 & 32.9 & 19.7\end{array}$

$484920.9 \quad 36.3 \quad 47.8 \quad 60.167 .5 \quad 70.3 \quad 68.969 .868 .064 .0 \quad 59.958 .9$ $\begin{array}{lllllllllllll}55.4 & 50.5 & 51.5 & 51.9 & 48.0 & 50.0 & 49.9 & 45.7 & 39.3 & 34.4 & 23.4 & 13.4\end{array}$

$\begin{array}{lllllllllllll}1657 & 16.6 & 26.0 & 33.5 & 41.8 & 47.8 & 50.7 & 52.5 & 53.4 & 52.1 & 49.4 & 48.7 & 50.0\end{array}$ $\begin{array}{llllllllllllll}48.0 & 45.9 & 45.3 & 45.0 & 40.4 & 38.7 & 36.4 & 31.8 & 26.2 & 22.0 & 14.0 & 7.9\end{array}$

$\begin{array}{llllllllllllll}2457 & 15.7 & 25.1 & 32.4 & 42.7 & 49.9 & 53.6 & 56.7 & 59.5 & 56.4 & 54.1 & 55.1 & 57.9\end{array}$ $\begin{array}{llllllllllll}59.4 & 59.1 & 58.0 & 58.2 & 52.3 & 51.7 & 48.9 & 41.9 & 35.4 & 30.6 & 19.7 & 11.1\end{array}$

$\begin{array}{lllllllllllll}3257 & 17.5 & 28.4 & 36.9 & 48.0 & 56.5 & 60.6 & 62.2 & 62.8 & 58.8 & 56.1 & 55.3 & 54.9\end{array}$ $\begin{array}{lllllllllllllll}53.2 & 51.3 & 52.7 & 52.6 & 50.9 & 53.9 & 53.6 & 46.5 & 39.7 & 34.2 & 21.4 & 12.6\end{array}$

$\begin{array}{lllllllllllll}4057 & 18.6 & 30.3 & 38.7 & 49.6 & 58.3 & 63.2 & 62.9 & 65.2 & 62.5 & 59.1 & 59.8 & 61.2\end{array}$

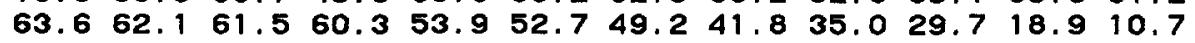




\section{CYCLE 1 DATA}

DATASET 14, APRIL 2, 1975

\section{Reactor Conditions}

Core Average Exposure, $5640 \mathrm{MWd} / \mathrm{t}$

Core Thermal Power, 3283 MWT

Dome Pressure, P, 1028 psia

Core Flow, 106.1 Mlb/hr

Inlet Subcooling at P, $23.4 \mathrm{Btu} / \mathrm{lb}$

Control Configuration

Legend: 48, Full Out; O, Full In.

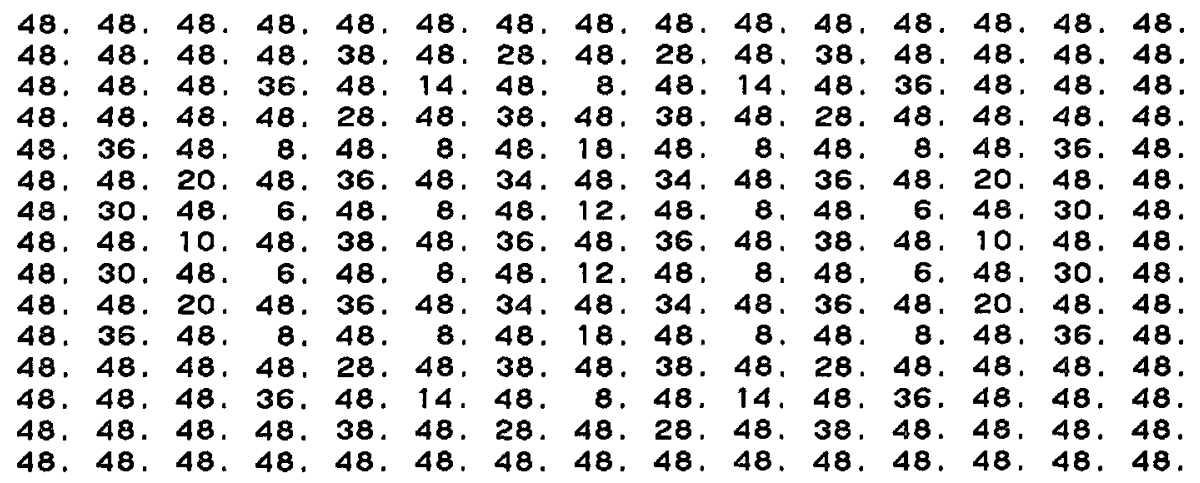

Axial TIP Distribution, Bottom To top of Core

See Figure 22

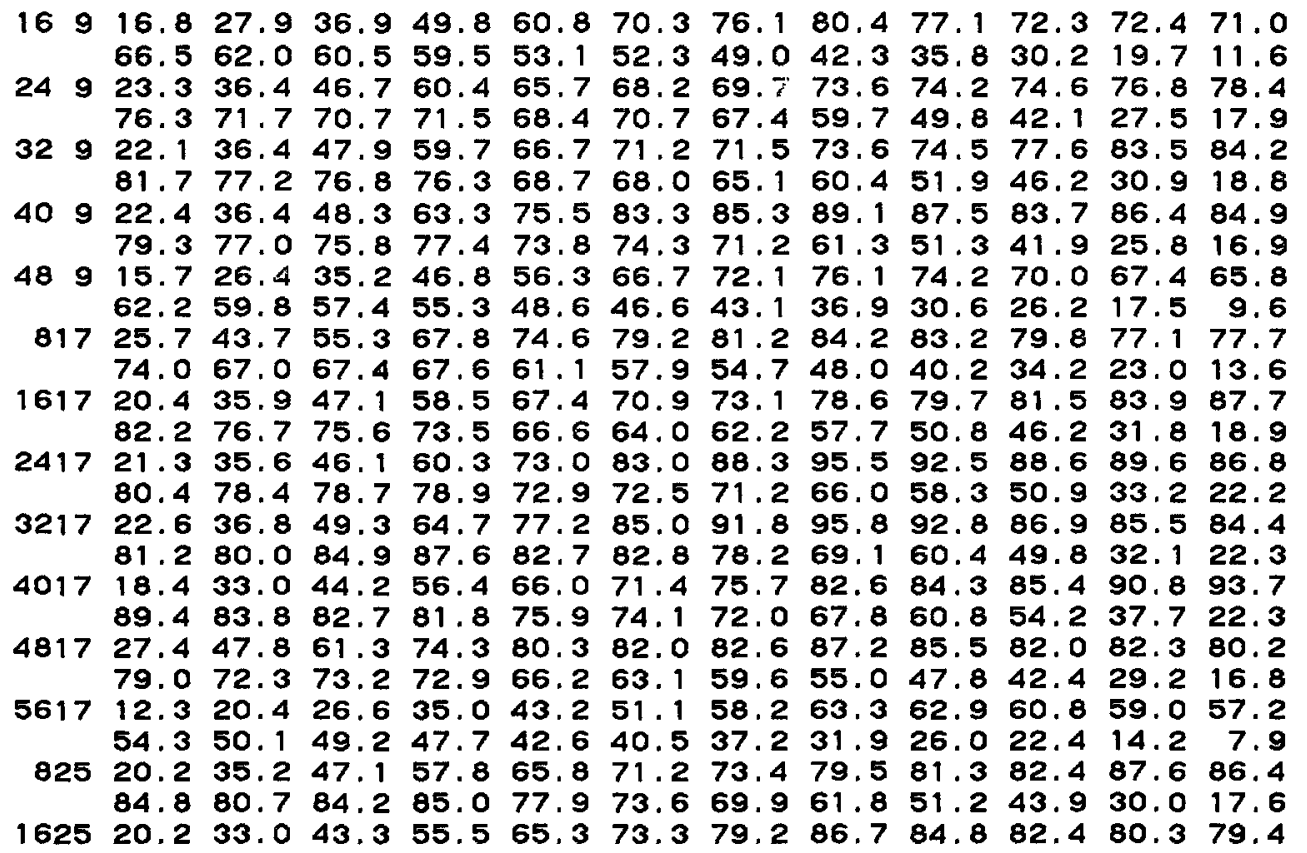


$\begin{array}{lllllllllllllll}75.1 & 70.9 & 69.9 & 70.7 & 64.9 & 64.9 & 63.2 & 57.6 & 52.9 & 47.7 & 31.8 & 20.4\end{array}$ $\begin{array}{lllllllllllll}2425 & 20.5 & 33.7 & 44.5 & 59.4 & 69.0 & 78.3 & 89.7 & 98.5 & 98.9 & 97.5 & 96.2 & 92.5\end{array}$ $\begin{array}{llllllllllll}87.5 & 83.9 & 83.6 & 81.6 & 75.1 & 75.0 & 73.0 & 67.9 & 61.5 & 53.6 & 35.7 & 23.4\end{array}$ $\begin{array}{lllllllllllllll}3225 & 21.4 & 36.0 & 47.9 & 61.6 & 72.0 & 81.7 & 92.0 & 98.7 & 98.9 & 96.3 & 94.5 & 89.6\end{array}$ $\begin{array}{lllllllllllll}84.4 & 80.1 & 81.2 & 82.1 & 76.8 & 79.5 & 80.1 & 72.4 & 63.5 & 55.1 & 35.6 & 23.6\end{array}$

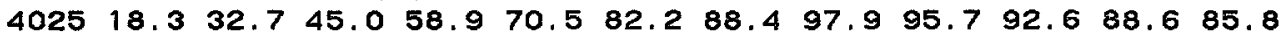

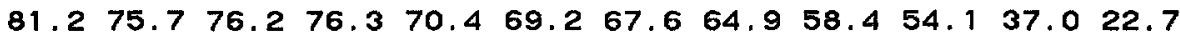

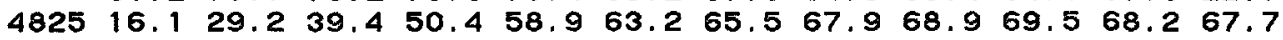
$\begin{array}{llllllllllll}66.9 & 66.9 & 72.0 & 75.8 & 71.5 & 70.8 & 70.0 & 64.4 & 56.4 & 51.3 & 36.1 & 20.7\end{array}$

$\begin{array}{llllllllllllll}5625 & 13.6 & 23.0 & 30.7 & 39.7 & 48.1 & 54.9 & 59.8 & 64.2 & 68.5 & 72.1 & 75.1 & 74.7\end{array}$ $\begin{array}{lllllllllllll}70.9 & 67.6 & 67.2 & 67.5 & 61.7 & 58.9 & 55.2 & 48.5 & 39.8 & 33.9 & 22.4 & 12.4\end{array}$

$\begin{array}{lllllllllllllll}833 & 21.2 & 35.5 & 45.3 & 56.5 & 60.9 & 64.6 & 65.7 & 71.3 & 77.3 & 79.5 & 82.7 & 83.0\end{array}$

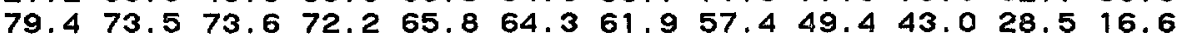

$\begin{array}{lllllllllllllll}1633 & 19.1 & 32.3 & 43.7 & 58.3 & 69.1 & 78.1 & 83.2 & 85.8 & 81.7 & 77.6 & 76.4 & 73.8\end{array}$ $68.064 .6 \quad 65.0 \quad 64.0 \quad 58.6 \quad 58.7 \quad 56.5 \quad 52.3 \quad 47.943 .3 \quad 28.8 \quad 19.1$

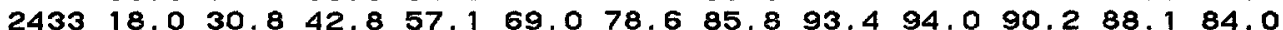
$79.272 .872 .271 .466 .6 \quad 67.266 .363 .658 .052 .8 \quad 36.522 .0$

$\begin{array}{llllllllllllll}3233 & 19.1 & 32.4 & 44.3 & 59.6 & 71.5 & 83.2 & 91.8 & 99.3 & 96.0 & 91.1 & 86.7 & 83.3\end{array}$ $\begin{array}{lllllllllllll}78.7 & 73.0 & 73.3 & 74.5 & 71.6 & 75.0 & 76.8 & 72.3 & 64.0 & 57.5 & 37.4 & 23.3\end{array}$

$403318.3 \quad 32.4 \quad 44.5 \quad 59.974 .0 \quad 84.2 \quad 90.6 \quad 98.0 \quad 94.790 .1 \quad 86.5 \quad 83.9$ $\begin{array}{llllllllllll}78.1 & 71.0 & 71.0 & 70.3 & 65.1 & 63.8 & 63.6 & 60.4 & 55.4 & 50.5 & 35.6 & 21.5\end{array}$

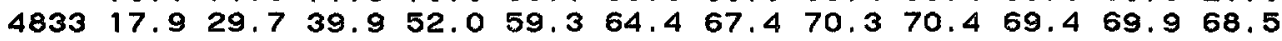
$\begin{array}{lllllllllllll}65.8 & 62.7 & 63.7 & 64.4 & 59.5 & 59.9 & 60.6 & 57.8 & 53.7 & 48.7 & 32.7 & 19.3\end{array}$

$\begin{array}{llllllllllllllll}5633 & 15.4 & 26.1 & 34.4 & 44.4 & 52.8 & 57.9 & 61.1 & 67.0 & 71.0 & 73.7 & 76.8 & 76.2\end{array}$ $\begin{array}{llllllllllllll}74.4 & 69.3 & 66.4 & 64.7 & 58.3 & 55.7 & 52.5 & 46.0 & 38.5 & 33.2 & 21.9 & 13.0\end{array}$

$84121.5 \quad 36.8 \quad 48.8 \quad 61.2 \quad 69.3 \quad 76.1 \quad 80.7 \quad 86.1 \quad 83.8 \quad 80.2 \quad 80.6 \quad 80.5$ $\begin{array}{lllllllllllllll}76.5 & 75.7 & 79.7 & 81.4 & 75.7 & 73.8 & 70.1 & 61.0 & 51.7 & 43.3 & 27.8 & 16.3\end{array}$

$\begin{array}{lllllllllllll}1641 & 18.2 & 31.5 & 43.6 & 56.1 & 67.5 & 76.5 & 84.0 & 89.8 & 87.8 & 85.7 & 84.4 & 83.8\end{array}$ $\begin{array}{llllllllllll}79.6 & 74.3 & 74.6 & 73.9 & 67.4 & 65.8 & 64.9 & 61.3 & 54.9 & 50.3 & 35.0 & 21.4\end{array}$

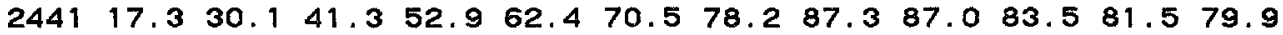

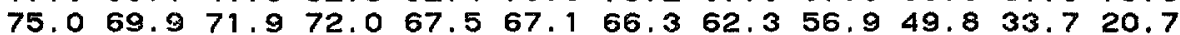

$\begin{array}{lllllllllllllll}3241 & 18.2 & 31.6 & 42.4 & 54.6 & 63.6 & 70.7 & 79.7 & 90.6 & 90.0 & 89.5 & 87.2 & 86.1\end{array}$ $82.679 .3 \quad 85.5 \quad 91.3 \quad 86.786 .283 .075 .1 \quad 64.3 \quad 54.9 \quad 36.922 .9$

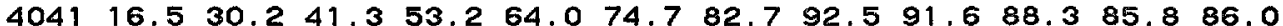
82.3 $77.7 \quad 78.277 .871 .6 \quad 69.4 \quad 68.965 .5 \quad 58.8 \quad 54.0 \quad 38.5 \quad 23.3$

$\begin{array}{lllllllllllllll}4841 & 19.2 & 33.8 & 45.3 & 56.6 & 64.8 & 69.2 & 71.5 & 77.4 & 75.7 & 72.7 & 73.4 & 73.0\end{array}$ $\begin{array}{lllllllllllll}70.2 & 69.3 & 74.3 & 75.6 & 69.4 & 66.8 & 64.4 & 58.7 & 51.2 & 46.3 & 31.6 & 18.5\end{array}$

$\begin{array}{llllllllllllllll}5641 & 15.9 & 26.8 & 35.6 & 46.5 & 57.1 & 67.4 & 72.9 & 77.4 & 74.5 & 71.6 & 68.0 & 66.7\end{array}$ $\begin{array}{lllllllllllll}63.4 & 58.7 & 57.4 & 56.3 & 50.6 & 47.8 & 45.3 & 38.5 & 32.5 & 27.9 & 18.8 & 10.8\end{array}$

$84927.7 \quad 43.6 \quad 53.5 \quad 64.1 \quad 67.7 \quad 67.8 \quad 67.967 .963 .3 \quad 58.2 \quad 58.1 \quad 56.4$ $\begin{array}{llllllllllllll}52.0 & 48.5 & 47.3 & 46.8 & 43.1 & 42.0 & 38.7 & 32.8 & 28.1 & 23.4 & 14.6 & 8.5\end{array}$

$\begin{array}{lllllllllllll}1649 & 22.1 & 35.2 & 45.2 & 59.5 & 67.5 & 77.0 & 85.1 & 90.5 & 89.2 & 93.3 & 97.1 & 97.0\end{array}$ $\begin{array}{lllllllllllll}92.8 & 87.4 & 85.6 & 84.5 & 77.0 & 74.4 & 68.8 & 60.4 & 51.4 & 42.2 & 26.7 & 16.6\end{array}$

$244920.5 \quad 33.6 \quad 44.9 \quad 58.7 \quad 70.8 \quad 78.5 \quad 81.8 \quad 85.6 \quad 82.5 \quad 81.3 \quad 81.8 \quad 81.0$ $\begin{array}{lllllllllllll}76.8 & 74.6 & 75.5 & 78.7 & 76.5 & 78.1 & 76.7 & 68.5 & 59.2 & 50.2 & 32.3 & 20.2\end{array}$

$\begin{array}{lllllllllllllll}3249 & 17.6 & 30.3 & 42.3 & 56.6 & 71.1 & 82.0 & 85.7 & 91.1 & 89.2 & 84.3 & 83.3 & 81.9\end{array}$ $\begin{array}{llllllllllll}77.8 & 74.9 & 76.9 & 76.6 & 72.3 & 72.1 & 71.5 & 66.6 & 59.6 & 52.7 & 35.7 & 21.0\end{array}$

$\begin{array}{lllllllllllllll}4049 & 19.8 & 33.1 & 43.7 & 56.6 & 66.3 & 71.6 & 75.8 & 81.8 & 83.2 & 88.2 & 93.5 & 95.0\end{array}$ $90.8 \quad 87.3 \quad 87.1 \quad 89.0 \quad 84.6 \quad 85.983 .9 \quad 75.7 \quad 64.1 \quad 54.6 \quad 35.0 \quad 21.4$

$\begin{array}{llllllllllllllll}4849 & 21.5 & 37.5 & 48.6 & 59.9 & 67.8 & 74.1 & 78.7 & 83.1 & 82.6 & 80.0 & 77.9 & 78.3\end{array}$ $\begin{array}{llllllllllll}72.8 & 66.6 & 66.1 & 65.5 & 59.5 & 56.1 & 52.5 & 46.5 & 39.2 & 34.4 & 23.2 & 13.8\end{array}$

$\begin{array}{lllllllllllllllll}1657 & 9.4 & 15.5 & 20.9 & 30.7 & 42.1 & 52.2 & 58.5 & 63.7 & 61.0 & 58.6 & 58.0 & 57.6\end{array}$ $\begin{array}{llllllllllll}54.0 & 50.4 & 49.2 & 48.2 & 42.9 & 42.6 & 39.2 & 32.6 & 27.9 & 23.3 & 14.8 & 8.0\end{array}$

$245714.923 .7 \quad 31.3 \quad 41.8 \quad 50.4 \quad 55.4 \quad 59.4 \quad 62.7 \quad 64.8 \quad 66.7 \quad 71.170 .5$ $66.163 .6 \quad 61.8 \quad 60.5 \quad 54.9 \quad 53.7 \quad 50.8 \quad 43.9 \quad 37.4 \quad 31.7 \quad 20.9 \quad 12.0$

$\begin{array}{lllllllllllllll}3257 & 16.6 & 26.9 & 34.7 & 44.9 & 52.4 & 56.0 & 60.4 & 63.4 & 64.4 & 66.0 & 72.0 & 74.8\end{array}$ $\begin{array}{llllllllllll}71.2 & 67.8 & 66.2 & 64.9 & 58.5 & 57.0 & 53.9 & 46.6 & 40.2 & 34.2 & 22.1 & 13.0\end{array}$

$4057 \quad 16.6 \quad 28.0 \quad 37.8 \quad 52.5 \quad 65.9 \quad 76.5 \quad 80.8 \quad 80.8 \quad 76.6 \quad 72.971 .070 .5$ $\begin{array}{llllllllllll}64.4 & 60.7 & 59.2 & 58.7 & 53.6 & 52.4 & 49.5 & 42.5 & 35.8 & 30.5 & 19.1 & 11.1\end{array}$ 


\title{
CYCLE 1 DATA
}

\section{DATASET 15, APRIL 24, 1975}

\section{Reactor Conditions}

Core Average Exposure, $6106 \mathrm{MWd} / \mathrm{t}$

Core Thermal Power, 3215 MWT

Dome Pressure, P, 1025 psia

Core Flow, 106.1 Mlb/hr

Inlet Subcooling at P, 22.9 Btu/lb

Control Configuration

Legend: 48, Full Out; O, Full In.

\begin{abstract}
48, 48, 48, 48, 48, 48, 48, 48, 48, 48, 48, 48, 48, 48, 48,
48, 48, 48, 48, 38, 48, 28, 48, 28, 48, 38, 48, 48, 48, 48.

48, $48,48,36,48,14,48$, 8. $48,14,48,36,48,48,48$.

48, $48,48,48,28,48,38,48,38,48,28,48,48,48,48$.

48. 36. $48,10,48,8,48,18,48,8,48,10,48,36,48$.

$48,48,20,48,36,48,34,48,34,48,36,48,20,48,48$.

48. 30, $48.6 .48,8,48,12,48,8,48,6,48,30,48$.

48, 48, 12, 48, 38, $48,36,48,36,48,38,48,12,48,48$,

$48,30,48,6,48,8,48,12,48,68,48,6,48,30,48$.

48, 48, 20, 48, 36, 48, 34, 48, 34, 48, 36, 48, 20, 48, 48.

48, 36, $48,10,48,8,48,18,48,8,48,10,48,36,48$.

48, 48, $48,48,28,48,38,48,38,48,28,48,48,48,48$.

48. 48, $48,36,48,14,48,8,48,14,48,36,48,48,48$.

48, $48,48,48,38,48,28,48,28,48,38,48,48,48,48$,

48, $48,48,48,48,48,48,48,48,48,48,48,48,48,48$,
\end{abstract}

Axial TIP Distribution, Bottom To top of Core

See Figure 22

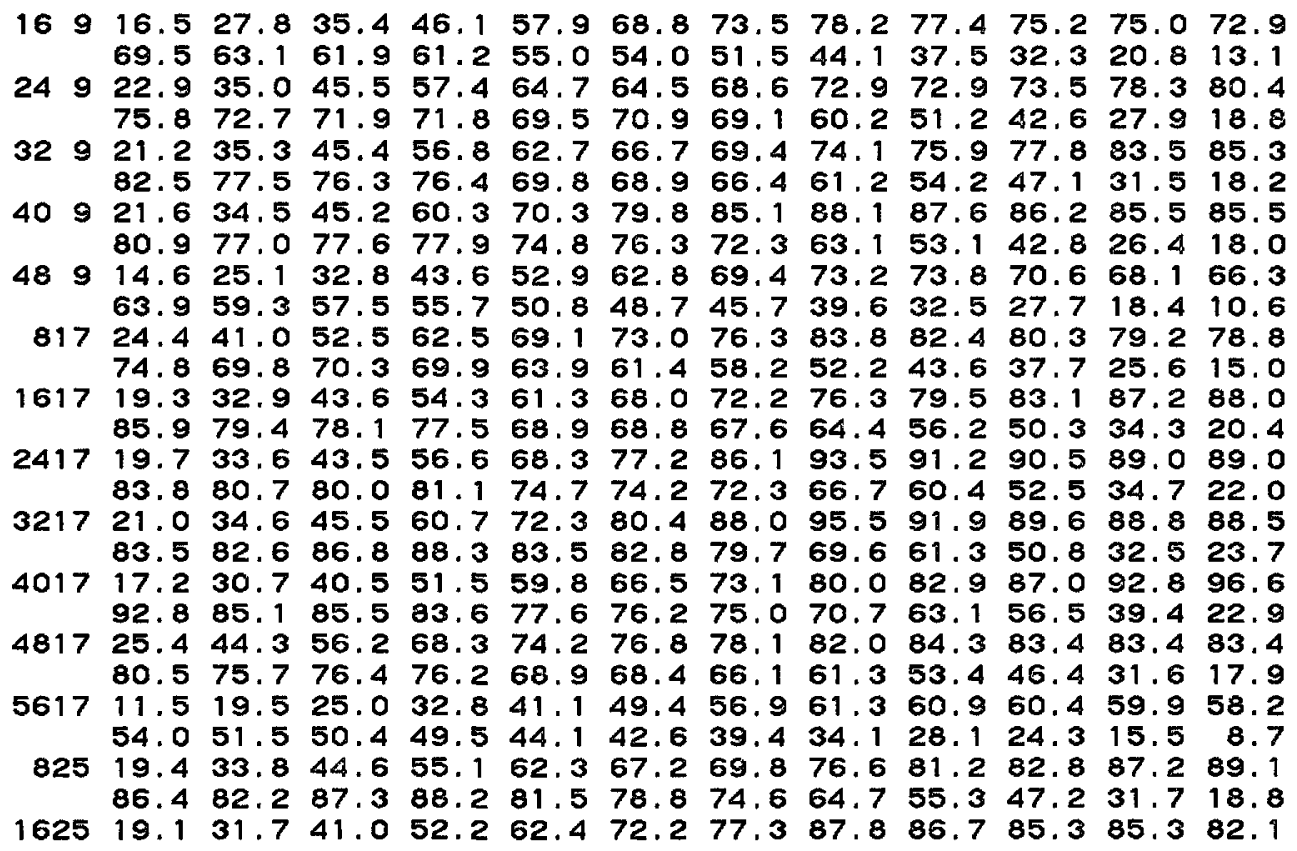


$\begin{array}{lllllllllllllllll}78.1 & 74.4 & 73.7 & 74.0 & 67.8 & 66.7 & 66.4 & 60.1 & 55.0 & 49.8 & 33.1 & 21.0\end{array}$ $242519.3 \quad 31.6 \quad 41.8 \quad 53.964 .4 \quad 74.1 \quad 85.8 \quad 97.6 \quad 99.3 \quad 98.2 \quad 99.0 \quad 97.3$ $90.686 .184 .4 \quad 83.076 .076 .273 .4 \quad 69.0 \quad 62.6 \quad 54.9 \quad 36.023 .7$

$\begin{array}{lllllllllllll}3225 & 20.2 & 33.3 & 44.2 & 56.3 & 66.6 & 76.5 & 87.5 & 96.1 & 97.5 & 97.5 & 95.9 & 93.8\end{array}$

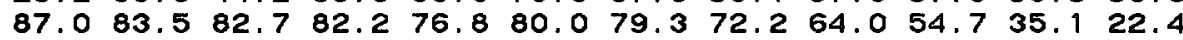

$\begin{array}{lllllllllllll}4025 & 17.1 & 30.4 & 41.6 & 55.4 & 65.2 & 76.2 & 85.8 & 95.1 & 95.4 & 94.9 & 92.3 & 91.3\end{array}$ $\begin{array}{llllllllllll}83.8 & 78.2 & 78.5 & 77.8 & 72.0 & 70.9 & 70.4 & 66.5 & 60.0 & 55.1 & 37.4 & 23.1\end{array}$

$\begin{array}{llllllllllllll}4825 & 15.3 & 27.2 & 36.9 & 47.2 & 54.0 & 59.4 & 62.5 & 67.7 & 69.1 & 68.5 & 68.4 & 68.8\end{array}$ $\begin{array}{llllllllllll}68.7 & 69.1 & 73.5 & 77.8 & 73.1 & 72.2 & 70.2 & 64.5 & 58.2 & 53.1 & 37.4 & 21.9\end{array}$

$\begin{array}{lllllllllllllllll}5625 & 12.9 & 22.3 & 29.6 & 38.8 & 45.4 & 51.9 & 57.9 & 62.7 & 66.9 & 70.1 & 75.0 & 75.0\end{array}$

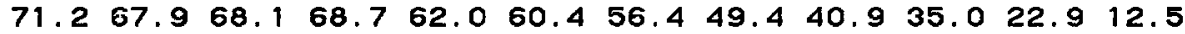

$83320.3 \quad 34.0 \quad 43.3 \quad 53.1 \quad 58.2 \quad 60.5 \quad 63.8 \quad 70.9 \quad 75.8 \quad 81.2 \quad 82.5 \quad 83.8$ $\begin{array}{llllllllllll}80.3 & 75.2 & 76.0 & 75.5 & 70.7 & 71.1 & 70.3 & 63.1 & 53.5 & 46.2 & 30.6 & 17.9\end{array}$

$\begin{array}{llllllllllllll}1633 & 18.0 & 30.5 & 40.0 & 53.0 & 64.6 & 74.0 & 80.4 & 84.3 & 81.5 & 77.7 & 77.5 & 74.7\end{array}$ $\begin{array}{llllllllllll}71.9 & 66.8 & 66.9 & 66.4 & 61.7 & 60.8 & 59.6 & 55.1 & 50.5 & 45.5 & 30.8 & 19.6\end{array}$

$2433 \quad 16.929 .9 \quad 40.2 \quad 52.8 \quad 64.0 \quad 74.4 \quad 84.5 \quad 93.2 \quad 93.8 \quad 92.1 \quad 91.6 \quad 88.6$ $81.875 .7 \quad 74.7 \quad 74.1 \quad 68.5 \quad 68.0 \quad 68.1 \quad 64.6 \quad 59.5 \quad 54.1 \quad 36.8 \quad 22.9$

$\begin{array}{llllllllllllll}3233 & 16.4 & 29.2 & 40.6 & 53.4 & 65.7 & 77.7 & 86.8 & 96.4 & 95.7 & 94.6 & 91.7 & 89.4\end{array}$ $83.176 .075 .475 .5 \quad 71.973 .975 .7 \quad 73.464 .0 \quad 57.6 \quad 40.2 \quad 24.0$

$\begin{array}{lllllllllllll}4033 & 17.2 & 30.6 & 41.7 & 55.9 & 67.5 & 79.9 & 87.6 & 94.2 & 94.0 & 91.8 & 91.2 & 87.6\end{array}$

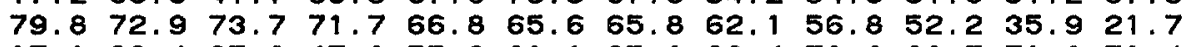

$\begin{array}{llllllllllllll}4833 & 17.1 & 28.4 & 37.3 & 47.9 & 55.6 & 61.1 & 65.3 & 69.4 & 70.6 & 69.7 & 71.8 & 71.4\end{array}$ $\begin{array}{llllllllllll}67.9 & 65.3 & 67.1 & 67.2 & 64.6 & 68.4 & 67.4 & 63.3 & 56.8 & 51.3 & 34.1 & 20.5\end{array}$

$\begin{array}{lllllllllllllll}5633 & 14.2 & 23.9 & 31.9 & 41.4 & 48.3 & 54.6 & 58.2 & 64.2 & 67.8 & 70.6 & 75.6 & 75.8\end{array}$ $\begin{array}{llllllllllll}73.6 & 68.3 & 66.8 & 64.8 & 58.5 & 57.8 & 54.9 & 46.7 & 40.1 & 34.2 & 22.6 & 12.9\end{array}$

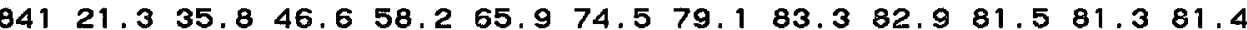
$\begin{array}{lllllllllllll}78.4 & 78.5 & 81.2 & 84.0 & 77.0 & 76.3 & 71.7 & 63.5 & 53.4 & 44.6 & 28.5 & 17.1\end{array}$

$164117.1 \quad 29.7 \quad 40.952 .4 \quad 62.9 \quad 71.4 \quad 79.6 \quad 87.8 \quad 88.6 \quad 87.5 \quad 86.486 .8$ $83.577 .277 .276 .871 .4 \quad 70.370 .6 \quad 68.0 \quad 59.954 .5 \quad 37.3 \quad 23.2$

$244116.428 .4 \quad 37.4 \quad 48.7 \quad 57.966 .7 \quad 74.5 \quad 84.9 \quad 85.0 \quad 84.0 \quad 83.0 \quad 81.3$ $\begin{array}{llllllllllllll}76.4 & 72.7 & 72.0 & 71.9 & 67.3 & 68.4 & 66.6 & 63.7 & 57.4 & 51.2 & 34.6 & 21.1\end{array}$

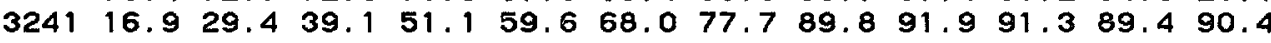
$\begin{array}{lllllllllllll}85.6 & 82.1 & 86.4 & 90.9 & 87.7 & 86.7 & 84.6 & 75.4 & 65.6 & 56.0 & 37.5 & 23.5\end{array}$

$\begin{array}{lllllllllllll}4041 & 15.8 & 27.9 & 38.4 & 48.4 & 59.2 & 69.9 & 79.4 & 88.2 & 92.8 & 91.7 & 91.3 & 89.2\end{array}$ $85.979 .979 .7 \quad 79.274 .072 .5 \quad 72.0 \quad 69.0 \quad 61.6 \quad 55.8 \quad 39.5 \quad 23.4$

$\begin{array}{lllllllllllllll}4841 & 18.4 & 31.5 & 41.3 & 51.6 & 58.2 & 63.3 & 67.1 & 71.3 & 72.6 & 72.9 & 72.8 & 72.7\end{array}$ $\begin{array}{llllllllllll}70.9 & 70.3 & 74.4 & 75.9 & 70.5 & 70.8 & 70.1 & 64.4 & 55.9 & 49.3 & 33.2 & 19.5\end{array}$

$\begin{array}{lllllllllllllll}5641 & 15.6 & 26.0 & 34.1 & 44.8 & 55.2 & 65.5 & 69.7 & 76.8 & 73.4 & 68.9 & 67.1 & 66.7\end{array}$ $\begin{array}{lllllllllllll}61.9 & 57.5 & 57.3 & 56.4 & 50.6 & 49.7 & 46.7 & 40.4 & 34.0 & 28.7 & 19.1 & 11.4\end{array}$

$84926.3 \quad 40.3 \quad 49.960 .6 \quad 63.8 \quad 65.5 \quad 65.7 \quad 67.8 \quad 63.0 \quad 58.6 \quad 58.8 \quad 56.7$ $\begin{array}{lllllllllllll}53.4 & 50.2 & 49.8 & 48.9 & 44.7 & 44.0 & 41.0 & 35.2 & 29.7 & 24.8 & 15.6 & 9.1\end{array}$

$164920.7 \quad 33.8 \quad 42.953 .962 .972 .2 \quad 79.4 \quad 89.0 \quad 90.5 \quad 92.7 \quad 98.4 \quad 98.2$

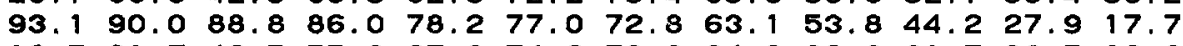

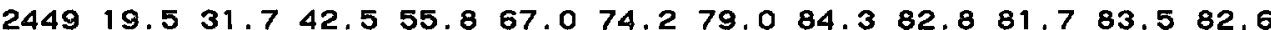
$78.976 .977 .579 .976 .979 .177 .670 .060 .5 \quad 51.4 \quad 33.321 .2$

$324916.6 \quad 29.0 \quad 39.4 \quad 53.2 \quad 65.9 \quad 75.5 \quad 81.4 \quad 87.7 \quad 87.5 \quad 85.984 .6 \quad 83.2$ $\begin{array}{llllllllllll}79.2 & 75.4 & 76.4 & 77.1 & 72.5 & 71.7 & 71.0 & 67.2 & 60.1 & 52.4 & 35.3 & 21.2\end{array}$

$404918.8 \quad 31.1 \quad 41.4 \quad 53.1 \quad 62.0 \quad 68.5 \quad 74.1 \quad 79.7 \quad 83.3 \quad 88.2 \quad 95.6 \quad 95.7$ $\begin{array}{llllllllllll}93.7 & 87.6 & 88.6 & 89.8 & 85.5 & 88.2 & 85.6 & 77.0 & 65.5 & 55.4 & 35.9 & 21.8\end{array}$

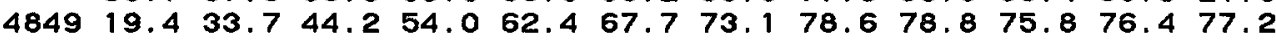
$\begin{array}{lllllllllllll}74.2 & 68.0 & 68.1 & 65.3 & 59.7 & 57.9 & 55.3 & 49.5 & 40.9 & 35.7 & 24.6 & 14.5\end{array}$

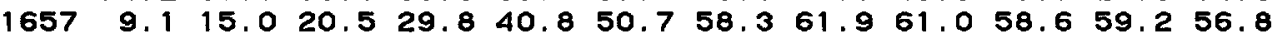
$\begin{array}{llllllllllll}53.4 & 51.2 & 50.6 & 48.7 & 43.6 & 42.9 & 39.8 & 34.7 & 28.9 & 23.9 & 15.2 & 8.4\end{array}$

$\begin{array}{lllllllllllllll}2457 & 14.5 & 23.4 & 30.5 & 40.9 & 49.7 & 54.0 & 58.3 & 61.2 & 63.0 & 65.7 & 70.2 & 69.1\end{array}$ $\begin{array}{llllllllllllll}65.6 & 61.8 & 60.8 & 59.9 & 54.8 & 54.3 & 51.6 & 44.7 & 38.0 & 32.7 & 21.4 & 12.1\end{array}$

$325716.2 \quad 26.1 \quad 34.0 \quad 44.451 .6 \quad 56.2 \quad 58.2 \quad 61.962 .6 \quad 66.3 \quad 71.173 .0$ $\begin{array}{llllllllllllll}70.7 & 67.5 & 64.8 & 64.9 & 57.7 & 56.7 & 54.6 & 47.4 & 40.5 & 34.7 & 22.4 & 13.3\end{array}$

$4057 \quad 16.4 \quad 27.3 \quad 37.4 \quad 51.6 \quad 64.9 \quad 73.0 \quad 79.2 \quad 81.3 \quad 77.972 .171 .1 \quad 69.6$ $64.860 .960 .859 .354 .054 .251 .344 .4 \quad 37.3 \quad 31.6 \quad 19.911 .7$ 


\section{CYCLE 1 DATA}

DATASET 16, MAY 13, 1975

\section{Reactor Conditions}

Core Average Exposure, $6470 \mathrm{MWd} / \mathrm{t}$

Core Thermal Power, 3172 MWT

Dome Pressure, P, 1025 psia

Core Flow, $105.4 \mathrm{Mlb} / \mathrm{hr}$

Inlet Subcooling at P, $22.8 \mathrm{Btu} / \mathrm{lb}$

Control Configuration

Legend: 48, Full Out; O, Full In.

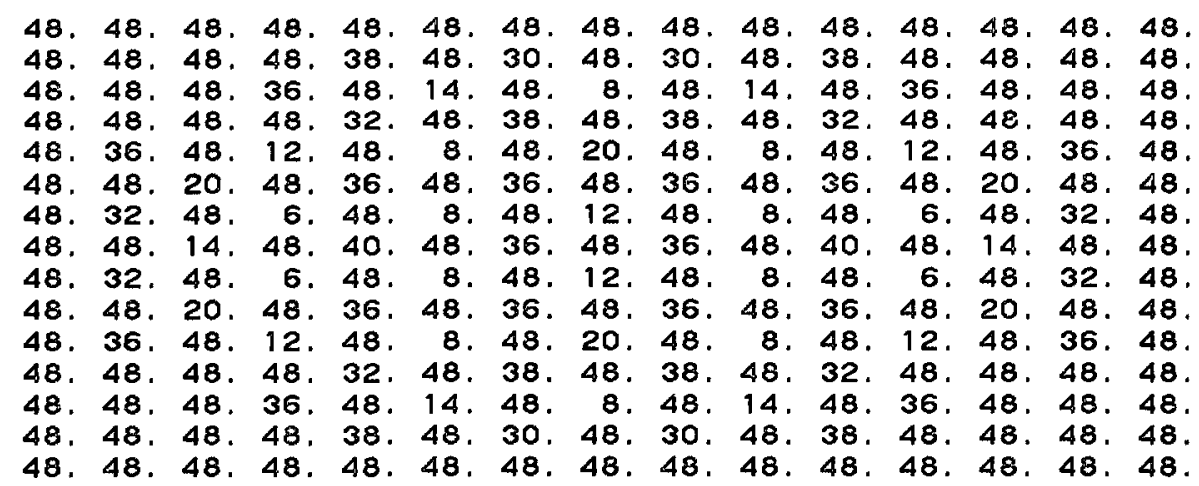

Axial TIP Distribution, Bottom To top of Core

See Figure 22

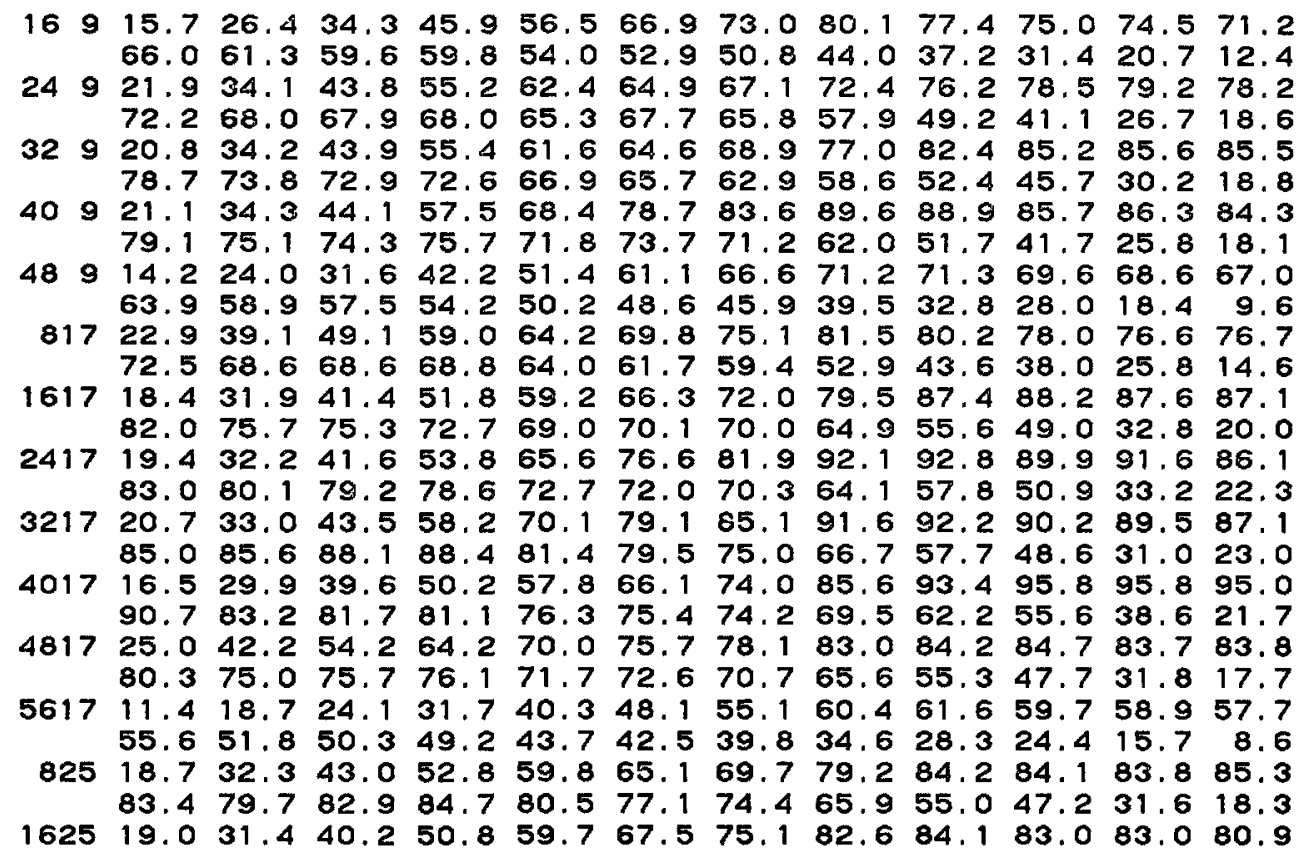


$76.472 .872 .072 .566 .766 .965 .960 .3 \quad 55.049 .2 \quad 32.620 .5$ $242519.2 \quad 31.641 .5 \quad 53.864 .7 \quad 77.1 \quad 86.7 \quad 96.8 \quad 96.995 .496 .093 .3$ 89.783 .982 .280 .174 .273 .071 .366 .160 .052 .434 .623 .2

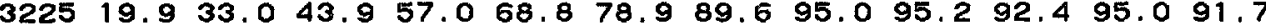

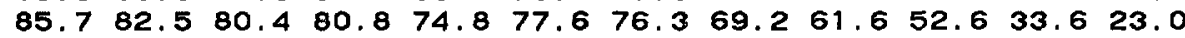

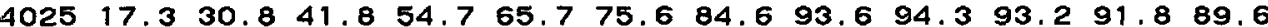
$\begin{array}{lllllllllllllll}84.8 & 78.8 & 78.4 & 77.3 & 72.3 & 69.8 & 69.6 & 66.0 & 59.9 & 54.8 & 37.3 & 22.1\end{array}$

$482515.0 \quad 27.0 \quad 36.445 .951 .857 .361 .466 .568 .768 .6 \quad 68.568 .1$ $68.468 .472 .677 .074 .074 .372 .466 .6 \quad 59.053 .136 .921 .0$

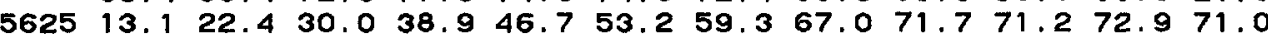

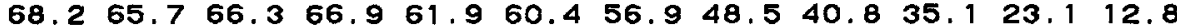

$83319.5 \quad 32.6 \quad 42.3 \quad 50.855 .8 \quad 61.1 \quad 65.4 \quad 75.379 .381 .480 .981 .8$ $\begin{array}{llllllllllllll}77.6 & 72.1 & 73.8 & 74.7 & 72.5 & 73.7 & 71.7 & 62.7 & 53.8 & 46.2 & 30.4 & 18.5\end{array}$

$163318.3 \quad 31.442 .3 \quad 56.6 \quad 66.972 .575 .278 .777 .075 .174 .573 .9$ $\begin{array}{llllllllllllllll}68.7 & 66.1 & 65.1 & 65.0 & 60.2 & 59.8 & 59.3 & 54.1 & 49.4 & 44.3 & 29.9 & 19.3\end{array}$

$243317.1 \quad 29.8 \quad 40.3 \quad 52.5 \quad 63.1 \quad 72.9 \quad 79.6 \quad 89.7 \quad 89.8$ 89.7 88.486 .8 $81.173 .7 \quad 73.272 .1 \quad 66.6 \quad 65.6 \quad 65.3 \quad 62.5 \quad 57.752 .3 \quad 35.921 .7$

$\begin{array}{llllllllllllllllllll}3233 & 17.8 & 31 & 3 & 41.8 & 54.3 & 66.5 & 77.7 & 87.2 & 94.6 & 94.9 & 92.3 & 90.9 & 88.4\end{array}$

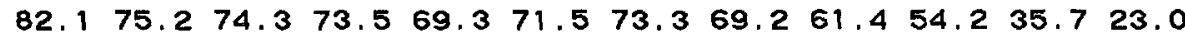

$403317.8 \quad 32.2 \quad 44.6 \quad 60.3 \quad 72.3 \quad 79.1 \quad 83.489 .492 .089 .288 .485 .3$

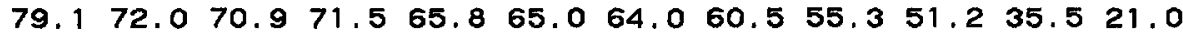

$483317.2 \quad 28.938 .349 .0 \quad 54.4 \quad 59.6 \quad 63.570 .7 \quad 70.169 .370 .769 .7$

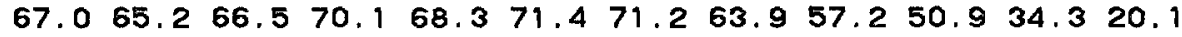

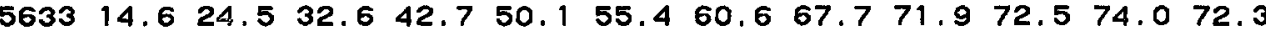
$\begin{array}{llllllllllllllll}68.9 & 64.2 & 64.0 & 63.5 & 57.6 & 55.9 & 53.9 & 47.5 & 39.5 & 34.3 & 22.6 & 13.0\end{array}$

$84120.6 \quad 34.6 \quad 43.955 .963 .7 \quad 69.9 \quad 76.6 \quad 81.7 \quad 80.5 \quad 80.079 .879 .5$

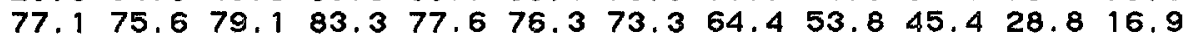

$164116.8 \quad 29.3 \quad 39.6 \quad 50.7 \quad 60.4 \quad 69.1 \quad 77.6 \quad 86.3 \quad 88.0 \quad 86.4 \quad 87.5 \quad 85.9$ 81.975 .776 .876 .272 .073 .474 .670 .461 .454 .437 .722 .9

$244115.9 \quad 27.6 \quad 37.1 \quad 48.0 \quad 57.8 \quad 68.7 \quad 77.2 \quad 84.3 \quad 83.7 \quad 81.482 .379 .5$

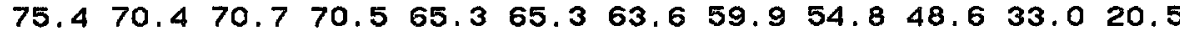

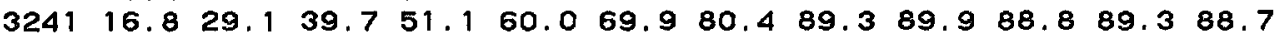

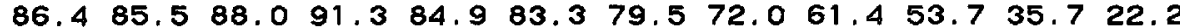

$4041 \quad 15.627 .4 \quad 36.7 \quad 47.7 \quad 57.2 \quad 68.5 \quad 77.986 .8 \quad 89.2 \quad 88.6 \quad 88.5 \quad 87.6$

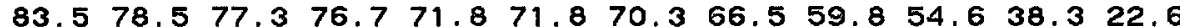

$484117.730 .439 .249 .0 \quad 55.361 .464 .968 .970 .771 .071 .671 .0$ $\begin{array}{lllllllllllllllll}69.6 & 68.0 & 72.1 & 75.2 & 72.0 & 73.9 & 74.5 & 66.7 & 57.3 & 49.6 & 33.1 & 19.3\end{array}$

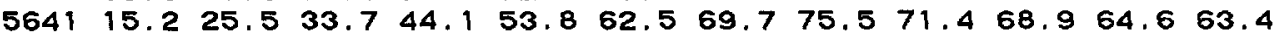

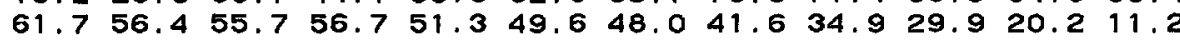

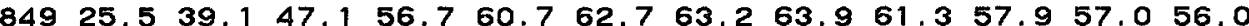
$52.150 .2 \quad 48.4 \quad 48.0 \quad 44.5 \quad 44.6 \quad 41.8 \quad 36.0 \quad 30.3 \quad 25.5 \quad 16.1 \quad 9.3$

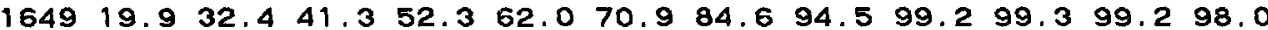

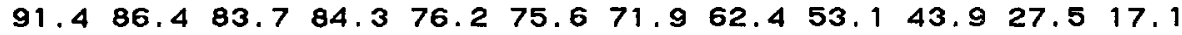

$244919.1 \quad 31.341 .5 \quad 54.764 .4 \quad 72.878 .8 \quad 84.6 \quad 83.6 \quad 83.8 \quad 84.083 .3$ $\begin{array}{lllllllllllllll}78.3 & 75.1 & 74.8 & 76.5 & 73.6 & 76.7 & 75.2 & 67.1 & 58.7 & 49.7 & 32.4 & 20.5\end{array}$

$324916.528 .0 \quad 38.7 \quad 51.7 \quad 64.1 \quad 74.2 \quad 79.7 \quad 87.2 \quad 87.3 \quad 86.0 \quad 34.983 .4$ $\begin{array}{lllllllllllllllllllllll}78.6 & 76.1 & 75.1 & 75.9 & 70.2 & 70.8 & 68.8 & 64.7 & 57.7 & 50.8 & 33.7 & 20.1\end{array}$

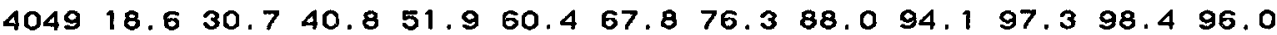
$91.0 \quad 85.784 .0 \quad 86.782 .685 .0 \quad 82.374 .6 \quad 63.5 \quad 54.4 \quad 35.021 .0$

$484919.0 \quad 33.0 \quad 43.451 .959 .2 \quad 67.871 .578 .678 .878 .477 .175 .2$ $\begin{array}{lllllllllllllll}70.4 & 65.1 & 64.9 & 64.4 & 59.3 & 56.9 & 55.3 & 48.8 & 41.1 & 35.6 & 23.7 & 13.8\end{array}$

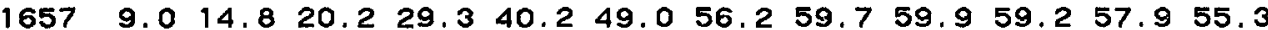
$\begin{array}{lllllllllllllll}53.6 & 49.5 & 48.6 & 48.1 & 43.9 & 42.4 & 40.2 & 34.3 & 28.8 & 23.5 & 15.3 & 8.5\end{array}$

$245714.623 .3 \quad 30.441 .450 .0 \quad 56.0 \quad 59.964 .767 .768 .670 .568 .3$ $\begin{array}{lllllllllllllll}62.3 & 58.7 & 57.8 & 56.7 & 51.5 & 52.3 & 49.0 & 42.9 & 36.9 & 31.1 & 20.4 & 12.1\end{array}$

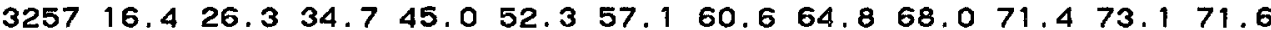
$67.663 .361 .660 .455 .454 .151 .1 \quad 45.6 \quad 39.0 \quad 33.7 \quad 21.512 .8$

$4057 \quad 16.327 .4 \quad 37.3 \quad 51.8 \quad 64.673 .876 .7 \quad 78.277 .173 .371 .3 \quad 68.4$ $\begin{array}{lllllllllllll}63.8 & 59.5 & 59.0 & 56.9 & 52.1 & 52.1 & 50.0 & 43.9 & 36.5 & 30.6 & 19.3 & 11.3\end{array}$ 
CYCLE 1 DATA

DATASET 17, JULY 25, 1975

\section{Reactor Conditions}

Core Average Exposure, $7000 \mathrm{MWd} / \mathrm{t}$

Core Thermal Power, 1649 MWT

Dome Pressure, P, 986 psia

Core Flow, $50.1 \mathrm{Mlb} / \mathrm{hr}$

Inlet Subcooling at P, 29.7 Btu/lb

Control Configuration

Legend: 48, Full Out; O, Full In.

$\begin{array}{rrrrrrrrrrrrrrr}48 & 48 & 48 & 48 & 48 & 48 & 48 & 48 & 48 & 48 & 48 & 48 & 48 & 48 & 48 \\ 48 & 48 & 48 & 48 & 48 & 12 & 48 & 12 & 48 & 12 & 48 & 48 & 48 & 48 & 48 \\ 48 & 48 & 48 & 48 & 40 & 48 & 44 & 48 & 44 & 48 & 40 & 48 & 48 & 48 & 48 \\ 48 & 48 & 48 & 8 & 48 & 8 & 48 & 4 & 48 & 8 & 48 & 8 & 48 & 48 & 48 \\ 48 & 48 & 40 & 48 & 44 & 48 & 40 & 48 & 40 & 48 & 44 & 48 & 40 & 48 & 48 \\ 48 & 12 & 48 & 8 & 48 & 0 & 48 & 4 & 48 & 0 & 48 & 8 & 48 & 12 & 48 \\ 48 & 48 & 44 & 48 & 40 & 48 & 40 & 48 & 40 & 48 & 40 & 48 & 44 & 48 & 48 \\ 48 & 12 & 48 & 4 & 48 & 4 & 48 & 6 & 48 & 4 & 48 & 4 & 48 & 12 & 48 \\ 48 & 48 & 44 & 48 & 40 & 48 & 40 & 48 & 40 & 48 & 40 & 48 & 44 & 48 & 48 \\ 48 & 12 & 48 & 8 & 48 & 0 & 48 & 4 & 48 & 0 & 48 & 8 & 48 & 12 & 48 \\ 48 & 48 & 40 & 48 & 44 & 48 & 40 & 48 & 40 & 48 & 44 & 48 & 40 & 48 & 48 \\ 48 & 48 & 48 & 8 & 48 & 8 & 48 & 4 & 48 & 8 & 48 & 8 & 48 & 48 & 48 \\ 48 & 48 & 48 & 48 & 40 & 48 & 44 & 48 & 44 & 48 & 40 & 48 & 48 & 48 & 48 \\ 48 & 48 & 48 & 48 & 48 & 12 & 48 & 12 & 48 & 12 & 48 & 48 & 48 & 48 & 48 \\ 48 & 48 & 48 & 48 & 48 & 48 & 48 & 48 & 48 & 48 & 48 & 48 & 48 & 48 & 48\end{array}$

Axial TIP Distribution,

No TIP data taken for this Data Set. 


\section{CYCLE 1 DATA}

DATASET 18, AUGUST 16, 1975

\section{Reactor Conditions}

Core Average Exposure, $7300 \mathrm{MWd} / \mathrm{t}$

Core Thermal Power, 1855 MWT

Dome Pressure, P, 995 psia

Core Flow, $39.9 \mathrm{MIb} / \mathrm{hr}$

Inlet Subcooling at P, $41.5 \mathrm{Btu} / \mathrm{lb}$

Control Configuration

Legend: 48, Full Out; O, Full In.

$\begin{array}{rrrrrrrrrrrrrrr}48 & 48 & 48 & 48 & 48 & 48 & 48 & 48 & 48 & 48 & 48 & 48 & 48 & 48 & 48 \\ 48 & 48 & 48 & 48 & 48 & 16 & 48 & 22 & 48 & 16 & 48 & 48 & 48 & 48 & 48 \\ 48 & 48 & 48 & 48 & 42 & 48 & 44 & 48 & 44 & 48 & 42 & 48 & 48 & 48 & 48 \\ 48 & 48 & 48 & 20 & 48 & 10 & 48 & 6 & 48 & 10 & 48 & 20 & 48 & 48 & 48 \\ 48 & 48 & 42 & 48 & 44 & 48 & 40 & 48 & 40 & 48 & 44 & 48 & 42 & 48 & 48 \\ 48 & 16 & 48 & 10 & 48 & 8 & 48 & 12 & 48 & 8 & 48 & 10 & 48 & 16 & 48 \\ 48 & 48 & 44 & 48 & 40 & 48 & 40 & 48 & 40 & 48 & 40 & 48 & 44 & 48 & 48 \\ 48 & 22 & 48 & 8 & 48 & 12 & 48 & 16 & 48 & 12 & 48 & 6 & 48 & 22 & 48 \\ 48 & 48 & 44 & 48 & 40 & 48 & 40 & 48 & 40 & 48 & 40 & 48 & 44 & 48 & 48 \\ 48 & 16 & 48 & 10 & 48 & 8 & 48 & 12 & 48 & 8 & 48 & 10 & 48 & 16 & 48 \\ 48 & 48 & 42 & 48 & 44 & 48 & 40 & 48 & 40 & 48 & 44 & 48 & 42 & 48 & 48 \\ 48 & 48 & 48 & 20 & 48 & 10 & 48 & 6 & 48 & 10 & 48 & 20 & 48 & 48 & 48 \\ 48 & 48 & 48 & 48 & 42 & 48 & 44 & 48 & 44 & 48 & 42 & 48 & 48 & 48 & 48 \\ 48 & 48 & 48 & 48 & 48 & 16 & 48 & 22 & 48 & 16 & 48 & 48 & 48 & 48 & 48 \\ 48 & 48 & 48 & 48 & 48 & 48 & 48 & 48 & 48 & 48 & 48 & 48 & 48 & 48 & 48\end{array}$

Axial TIP Distribution,

No TIP data taken for this Data Set. 


\section{CYCLE 1 DATA}

DATASET 19, SEPTEMBER 27, 1975

\section{Reactor Conditions}

Core Average Exposure, $7712 \mathrm{MWd} / \mathrm{t}$

Core Thermal Power, 1882 MWT

Dome Pressure, P, 1014 psia

Core Flow, $40.4 \mathrm{Mlb} / \mathrm{hr}$

Inlet Subcooling at P, $42.1 \mathrm{Btu} / \mathrm{lb}$

Control Configuration

Legend: 48, Full Out; O, Full In.

$\begin{array}{rrrrrrrrrrrrrrr}48 & 48 & 48 & 48 & 48 & 48 & 48 & 48 & 48 & 48 & 48 & 48 & 48 & 48 & 48 \\ 48 & 48 & 48 & 48 & 48 & 18 & 48 & 26 & 48 & 18 & 48 & 48 & 48 & 48 & 48 \\ 48 & 48 & 48 & 48 & 44 & 48 & 44 & 48 & 44 & 48 & 44 & 48 & 48 & 48 & 48 \\ 48 & 48 & 48 & 28 & 48 & 10 & 48 & 10 & 48 & 10 & 48 & 28 & 48 & 48 & 48 \\ 48 & 48 & 44 & 48 & 44 & 48 & 44 & 48 & 44 & 48 & 44 & 48 & 44 & 48 & 48 \\ 48 & 18 & 48 & 10 & 48 & 8 & 48 & 12 & 48 & 8 & 48 & 10 & 48 & 18 & 48 \\ 48 & 48 & 44 & 48 & 44 & 48 & 42 & 48 & 42 & 48 & 44 & 48 & 44 & 48 & 48 \\ 48 & 26 & 48 & 10 & 48 & 12 & 48 & 22 & 48 & 12 & 48 & 10 & 48 & 26 & 48 \\ 48 & 48 & 44 & 48 & 44 & 48 & 42 & 48 & 42 & 48 & 44 & 48 & 44 & 48 & 48 \\ 48 & 18 & 48 & 10 & 48 & 8 & 48 & 12 & 48 & 8 & 48 & 10 & 48 & 18 & 48 \\ 48 & 48 & 44 & 48 & 44 & 48 & 44 & 48 & 44 & 48 & 44 & 48 & 44 & 48 & 48 \\ 48 & 48 & 48 & 28 & 48 & 10 & 48 & 10 & 48 & 10 & 48 & 28 & 48 & 48 & 48 \\ 48 & 48 & 48 & 48 & 44 & 48 & 44 & 48 & 44 & 48 & 44 & 48 & 48 & 48 & 48 \\ 48 & 48 & 48 & 48 & 48 & 18 & 48 & 26 & 48 & 18 & 48 & 48 & 48 & 48 & 48 \\ 48 & 48 & 48 & 48 & 48 & 48 & 48 & 48 & 48 & 48 & 48 & 48 & 48 & 48 & 48\end{array}$

Axial TIP Distribution,

No TIP data taken for this Data Set. 


\title{
CYCLE 1 DATA
}

\section{DATASET 20, OCTOBER 31, 1975}

\section{Reactor Conditions}

Core Average Exposure, $8100 \mathrm{MWd} / \mathrm{t}$

Core Thermal Power, 1858 MWT

Dome Pressure, P, 1001 psia

Core Flow, $40.9 \mathrm{Mlb} / \mathrm{hr}$

Inlet Subcooling at P, $40.9 \mathrm{Btu} / \mathrm{lb}$

Control Configuration

\author{
Legend: 48, Full Out; O, Full In.
}

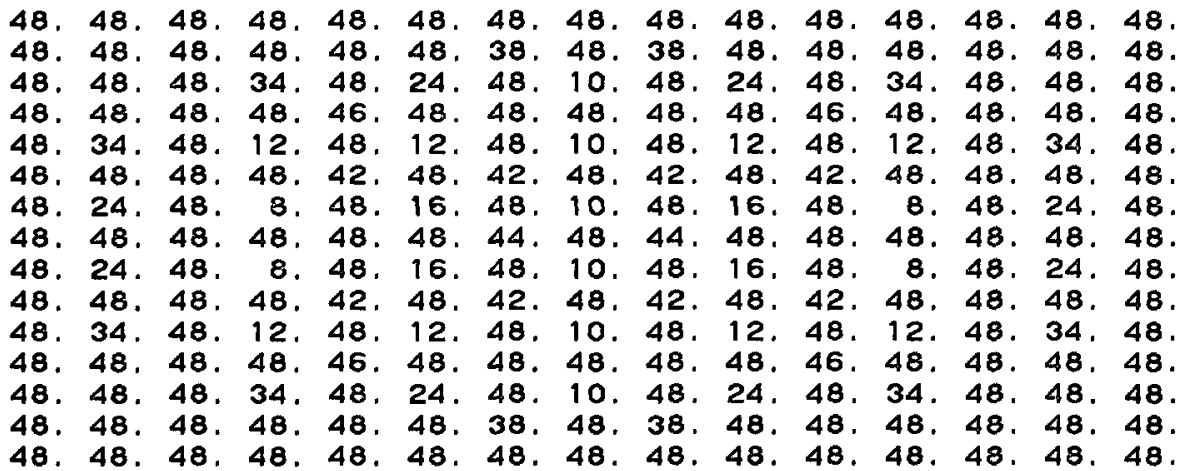

Axial TIP Distribution, Bottom To top of Core

See Figure 22

16949.470 .976 .377 .972 .569 .870 .071 .970 .469 .070 .068 .8 $\begin{array}{llllllllllllll}66.7 & 65.0 & 63.9 & 61.9 & 57.7 & 56.9 & 53.5 & 46.8 & 41.1 & 34.3 & 22.4 & 14.3\end{array}$

$24949.3 \quad 68.5 \quad 74.6 \quad 81.2 \quad 83.6 \quad 83.5 \quad 81.2 \quad 82.0 \quad 77.4 \quad 74.6 \quad 75.2 \quad 76.9$ $\begin{array}{llllllllllllll}76.0 & 75.5 & 74.3 & 72.9 & 68.1 & 67.3 & 63.3 & 56.1 & 49.8 & 42.4 & 27.7 & 21.7\end{array}$

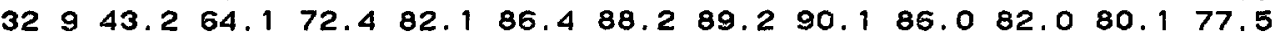

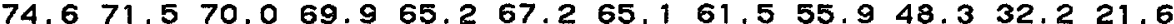

$\begin{array}{llllllllllllll}40 & 9 & 61.3 & 84.3 & 90.9 & 94.6 & 89.3 & 83.1 & 79.9 & 80.4 & 78.7 & 79.2 & 83.2 & 85.7\end{array}$ $\begin{array}{llllllllllllll}87.2 & 87.7 & 86.0 & 84.4 & 78.4 & 76.8 & 71.7 & 62.9 & 54.2 & 44.2 & 27.3 & 21.1\end{array}$

$\begin{array}{lllllllllllllll}48 & 9 & 27.5 & 41.8 & 48.6 & 54.0 & 56.3 & 57.5 & 61.2 & 67.3 & 68.9 & 68.7 & 68.8 & 67.5\end{array}$ $64.461 .958 .957 .453 .651 .248 .041 .9 \quad 35.930 .3 \quad 19.8 \quad 11.3$

$\begin{array}{lllllllllllll}817 & 38.8 & 59.2 & 66.5 & 70.4 & 70.7 & 68.2 & 68.9 & 76.2 & 78.2 & 78.2 & 78.7 & 77.3\end{array}$ $\begin{array}{llllllllllll}74.5 & 71.4 & 70.3 & 69.3 & 65.3 & 64.6 & 61.5 & 55.3 & 47.6 & 41.5 & 27.4 & 16.0\end{array}$

$161746.975 .0 \quad 86.4 \quad 92.5 \quad 90.2 \quad 84.4 \quad 78.7 \quad 79.7 \quad 77.4 \quad 77.478 .477 .4$ $\begin{array}{llllllllllll}75.4 & 71.1 & 69.9 & 69.5 & 67.9 & 70.4 & 71.2 & 66.1 & 59.3 & 52.6 & 35.2 & 22.5\end{array}$

$241760.9 \quad 89.7 \quad 95.4 \quad 98.6 \quad 90.3 \quad 84.078 .179 .3 \quad 76.976 .2 \quad 78.673 .3$ $\begin{array}{lllllllllllll}75.7 & 75.1 & 73.7 & 73.7 & 71.8 & 76.6 & 75.2 & 70.5 & 63.3 & 55.2 & 36.7 & 25.9\end{array}$

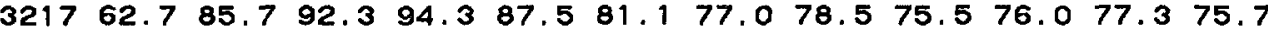
$72.170 .368 .968 .866 .6 \quad 69.4 \quad 70.166 .962 .7 \quad 52.8 \quad 34.8 \quad 26.8$

$401747.8 \quad 78.8 \quad 89.7 \quad 94.490 .6 \quad 85.981 .283 .580 .680 .781 .782 .6$ $\begin{array}{llllllllllll}80.9 & 78.8 & 78.7 & 79.0 & 77.3 & 79.8 & 80.4 & 74.7 & 67.3 & 59.4 & 40.3 & 24.7\end{array}$

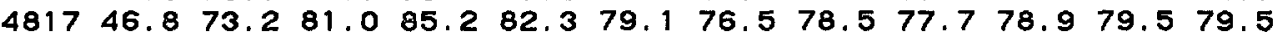
$\begin{array}{llllllllllll}76.4 & 73.3 & 73.2 & 73.0 & 69.0 & 72.4 & 71.4 & 67.0 & 59.2 & 51.5 & 34.1 & 20.3\end{array}$

$\begin{array}{llllllllllllll}5617 & 19.0 & 29.4 & 34.8 & 40.7 & 44.3 & 46.9 & 52.6 & 58.8 & 60.0 & 60.2 & 61.1 & 59.6\end{array}$ $\begin{array}{lllllllllllll}56.2 & 54.7 & 52.0 & 51.3 & 46.8 & 45.7 & 41.4 & 36.0 & 31.2 & 25.8 & 16.4 & 9.7\end{array}$

$\begin{array}{lllllllllllll}825 & 53.4 & 83.3 & 93.6 & 97.0 & 92.7 & 86.2 & 79.8 & 81.0 & 78.8 & 77.7 & 79.5 & 83.8\end{array}$ $\begin{array}{llllllllllllll}85.5 & 84.5 & 82.9 & 81.5 & 76.5 & 75.3 & 70.1 & 63.8 & 55.6 & 49.1 & 33.6 & 19.8\end{array}$

$\begin{array}{llllllllllllllll}1625 & 48.4 & 72.2 & 82.7 & 90.8 & 87.2 & 79.8 & 75.6 & 75.1 & 73.2 & 73.3 & 74.6 & 74.7\end{array}$ $\begin{array}{llllllllllll}72.2 & 70.7 & 69.2 & 70.7 & 69.4 & 72.4 & 71.9 & 68.1 & 64.0 & 56.5 & 37.5 & 24.6\end{array}$ 
$242544.7 \quad 68.8 \quad 83.4 \quad 94.9 \quad 94.1 \quad 88.8 \quad 84.4 \quad 81.6 \quad 79.6 \quad 79.7 \quad 82.3 \quad 82.7$

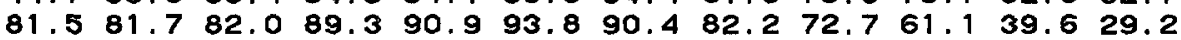

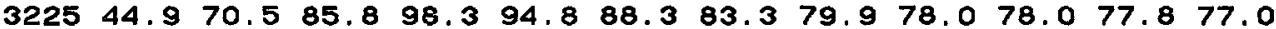

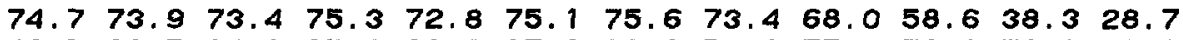

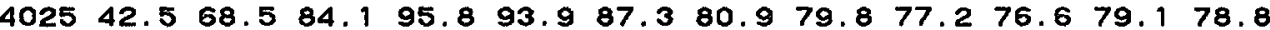
$\begin{array}{lllllllllllllll}76.5 & 75.1 & 78.4 & 84.3 & 85.3 & 88.7 & 86.6 & 80.0 & 71.5 & 63.4 & 42.0 & 27.7\end{array}$

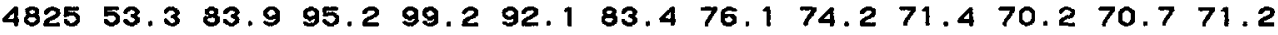
$\begin{array}{lllllllllllll}70.2 & 67.7 & 68.7 & 69.4 & 67.6 & 68.4 & 67.6 & 65.7 & 60.7 & 55.0 & 37.6 & 23.7\end{array}$

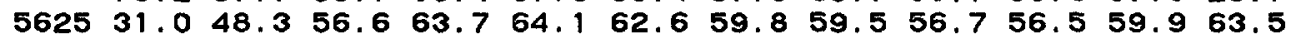
$\begin{array}{llllllllllllll}66.4 & 67.5 & 67.3 & 67.5 & 63.4 & 62.1 & 57.3 & 49.8 & 43.3 & 36.9 & 23.8 & 14.1\end{array}$

$\begin{array}{llllllllllllll}833 & 57.9 & 85.9 & 93.8 & 95.0 & 88.3 & 82.5 & 74.9 & 75.7 & 72.2 & 72.4 & 76.7 & 80.7\end{array}$ $\begin{array}{lllllllllllll}83.9 & 84.1 & 82.3 & 82.2 & 77.0 & 76.2 & 71.1 & 63.5 & 55.7 & 49.0 & 32.1 & 20.4\end{array}$

$163357.284 .0 \quad 89.493 .585 .379 .272 .070 .067 .067 .269 .069 .2$

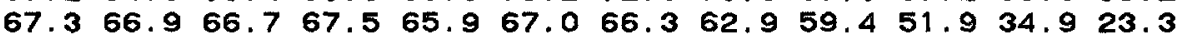

$243345.0 \quad 73.4 \quad 88.4 \quad 96.4 \quad 92.1 \quad 83.975 .7 \quad 75.3 \quad 72.8 \quad 73.674 .274 .2$ $\begin{array}{llllllllllll}72.8 & 72.4 & 74.1 & 79.5 & 82.2 & 85.4 & 83.6 & 77.9 & 69.7 & 62.5 & 42.1 & 26.1\end{array}$

$\begin{array}{lllllllllllllll}3233 & 45.3 & 73.7 & 89.6 & 99.3 & 95.3 & 88.1 & 81.6 & 79.5 & 75.9 & 75.3 & 76.1 & 75.4\end{array}$

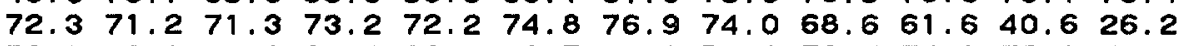

$\begin{array}{lllllllllllllll}4033 & 56.1 & 86.1 & 96.3 & 99.3 & 92.6 & 82.7 & 76.3 & 75.3 & 73.7 & 74.3 & 75.1 & 74.0\end{array}$

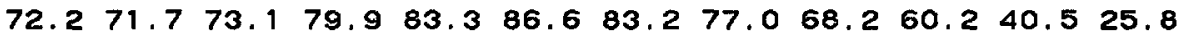

$\begin{array}{lllllllllllll}4833 & 63.1 & 91.5 & 97.3 & 98.3 & 90.0 & 82.0 & 77.5 & 76.8 & 75.1 & 75.1 & 76.9 & 77.8\end{array}$

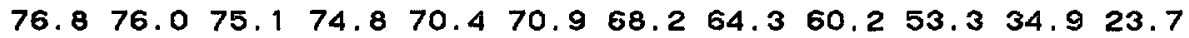

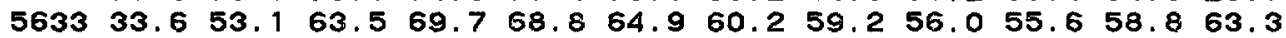
$\begin{array}{lllllllllllll}66.3 & 67.2 & 66.8 & 65.9 & 61.6 & 60.9 & 56.5 & 50.5 & 43.2 & 36.7 & 23.9 & 14.7\end{array}$

$84152.2 \quad 78.084 .689 .186 .283 .6 \quad 82.186 .384 .984 .786 .0 \quad 85.0$ $83.580 .5 \quad 79.979 .7 \quad 75.3 \quad 74.871 .6 \quad 64.7 \quad 56.4 \quad 47.9 \quad 30.5 \quad 18.7$

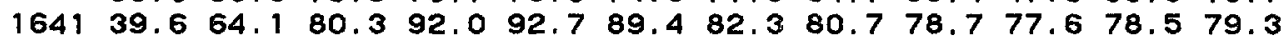
$\begin{array}{llllllllllll}77.8 & 74.1 & 73.6 & 74.7 & 73.6 & 76.7 & 78.4 & 74.4 & 66.3 & 59.7 & 41.2 & 25.2\end{array}$

$244137.8 \quad 61.5 \quad 75.0 \quad 85.6 \quad 85.4 \quad 79.5 \quad 73.5 \quad 72.2 \quad 69.5 \quad 67.5 \quad 68.3 \quad 67.3$ $\begin{array}{llllllllllll}66.0 & 63.6 & 64.2 & 66.0 & 65.8 & 70.2 & 72.2 & 69.1 & 62.4 & 55.2 & 37.0 & 23.1\end{array}$

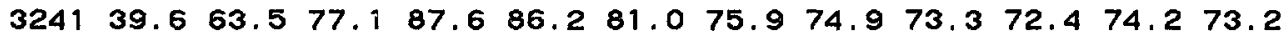

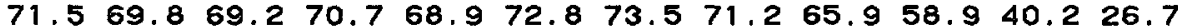

$404138.6 \quad 64.0 \quad 79.3 \quad 90.2 \quad 90.9 \quad 85.8 \quad 79.4 \quad 78.6 \quad 75.8 \quad 75.0 \quad 75.8 \quad 77.3$ $\begin{array}{llllllllllllll}75.7 & 74.1 & 74.1 & 76.3 & 75.8 & 81.2 & 82.5 & 77.9 & 69.9 & 62.5 & 42.7 & 26.9\end{array}$

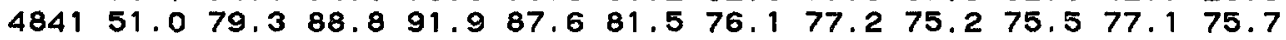

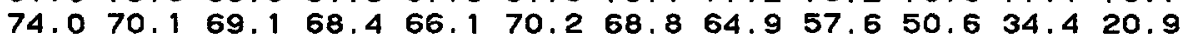

$564130.7 \quad 48.0 \quad 57.0 \quad 63.8 \quad 64.264 .5 \quad 64.8 \quad 67.6 \quad 67.6 \quad 65.1 \quad 64.862 .8$ $\begin{array}{lllllllllllll}60.8 & 58.8 & 56.5 & 56.3 & 52.2 & 51.0 & 47.1 & 41.5 & 35.7 & 30.4 & 20.3 & 12.1\end{array}$

$84938.954 .2 \quad 59.5 \quad 63.963 .4 \quad 61.6 \quad 61.962 .1 \quad 60.260 .160 .3 \quad 58.1$ $\begin{array}{lllllllllllll}55.5 & 53.3 & 51.9 & 51.1 & 48.9 & 48.6 & 45.0 & 39.4 & 33.9 & 27.9 & 17.5 & 10.8\end{array}$

$164951.6 \quad 76.584 .489 .485 .3 \quad 82.5 \quad 82.286 .388 .487 .0 \quad 90.0 \quad 89.9$ $\begin{array}{lllllllllllll}88.0 & 86.7 & 84.9 & 83.4 & 78.7 & 78.4 & 74.1 & 65.7 & 57.2 & 48.3 & 30.5 & 18.9\end{array}$

$244961.0 \quad 86.3 \quad 89.8 \quad 92.1 \quad 83.978 .073 .7 \quad 72.3 \quad 70.3 \quad 70.475 .2 \quad 78.5$

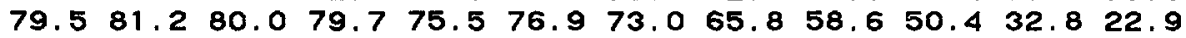

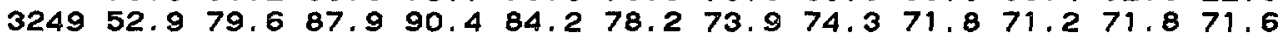
$\begin{array}{lllllllllllll}70.3 & 67.8 & 66.9 & 67.8 & 64.7 & 67.9 & 68.2 & 65.7 & 60.1 & 53.3 & 35.7 & 22.4\end{array}$

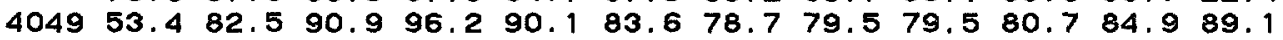
$\begin{array}{lllllllllllll}92.9 & 93.4 & 92.2 & 93.7 & 88.1 & 88.0 & 83.6 & 75.7 & 65.9 & 56.9 & 37.0 & 22.8\end{array}$

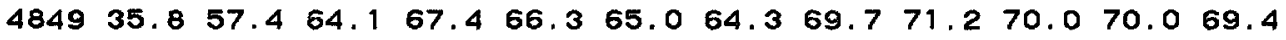
$\begin{array}{lllllllllllll}66.9 & 64.0 & 63.2 & 61.9 & 59.2 & 58.6 & 55.5 & 50.6 & 44.7 & 38.3 & 25.7 & 15.2\end{array}$

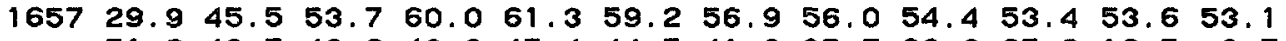
$\begin{array}{lllllllllllll}51.2 & 49.5 & 48.6 & 48.8 & 45.4 & 44.5 & 41.0 & 35.7 & 30.6 & 25.6 & 16.5 & 9.7\end{array}$

$245734.351 .862 .675 .4 \quad 81.0 \quad 81.6 \quad 79.1 \quad 77.3 \quad 71.4 \quad 66.4 \quad 64.2 \quad 62.3$

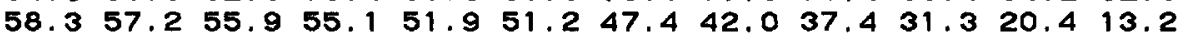

$325733.954 .1 \quad 66.078 .8 \quad 84.285 .4 \quad 83.2 \quad 81.3 \quad 73.967 .6 \quad 65.0 \quad 61.8$ $\begin{array}{llllllllllll}58.4 & 55.8 & 55.0 & 55.1 & 51.1 & 51.7 & 49.2 & 44.6 & 39.6 & 34.1 & 22.2 & 14.0\end{array}$

$405750.1 \quad 76.0 \quad 86.2 \quad 93.4 \quad 87.8 \quad 81.2 \quad 76.0 \quad 73.1 \quad 69.5 \quad 65.7 \quad 64.6 \quad 63.0$ $60.759 .5 \quad 57.757 .854 .0 \quad 53.4 \quad 49.6 \quad 43.5 \quad 37.7 \quad 31.7 \quad 20.2 \quad 12.2$ 


\section{CYCLE 1 DATA}

\section{DATASET 21, DECEMBER 24, 1975}

\section{Reactor Conditions}

Core Average Exposure, $8430 \mathrm{MWd} / \mathrm{t}$

Core Thermal Power, 3285 MWT

Dome Pressure, P, 1023 psia

Core Flow, 107.1 Mlb/hr

Inlet Subcooling at $\mathrm{P}, 22.0 \mathrm{Btu} / \mathrm{lb}$

Control Configuration

Legend: 48, Full Out; O, Full In.

$\begin{array}{lllllllllllllll}48 & 48 & 48 & 48 & 48 & 48 & 48 & 48 & 48 & 48 & 48 & 48 & 48 & 48 & 48 \\ 48 & 48 & 48 & 48 & 48 & 48 & 38 & 48 & 38 & 48 & 48 & 48 & 48 & 48 & 48 \\ 48 & 48 & 48 & 38 & 48 & 32 & 48 & 20 & 48 & 32 & 48 & 38 & 48 & 48 & 48 \\ 48 & 48 & 48 & 48 & 48 & 48 & 48 & 48 & 48 & 48 & 48 & 48 & 48 & 48 & 48 \\ 48 & 36 & 48 & 30 & 48 & 12 & 48 & 32 & 48 & 12 & 48 & 30 & 48 & 36 & 48 \\ 48 & 48 & 48 & 48 & 44 & 48 & 44 & 48 & 44 & 48 & 44 & 48 & 48 & 48 & 48 \\ 48 & 32 & 48 & 12 & 48 & 20 & 48 & 18 & 48 & 20 & 48 & 12 & 48 & 32 & 48 \\ 48 & 48 & 48 & 48 & 48 & 48 & 48 & 48 & 48 & 48 & 48 & 48 & 48 & 48 & 48 \\ 48 & 32 & 48 & 12 & 48 & 20 & 48 & 18 & 48 & 20 & 48 & 12 & 48 & 32 & 48 \\ 48 & 48 & 48 & 48 & 44 & 48 & 44 & 48 & 44 & 48 & 44 & 48 & 48 & 48 & 48 \\ 48 & 36 & 48 & 30 & 48 & 12 & 48 & 32 & 48 & 12 & 48 & 30 & 48 & 36 & 48 \\ 48 & 48 & 48 & 48 & 48 & 48 & 48 & 48 & 48 & 48 & 48 & 48 & 48 & 48 & 48 \\ 48 & 48 & 48 & 38 & 48 & 32 & 48 & 20 & 48 & 32 & 48 & 38 & 48 & 48 & 48 \\ 48 & 48 & 48 & 48 & 48 & 48 & 38 & 48 & 38 & 48 & 48 & 48 & 48 & 48 & 48 \\ 48 & 48 & 48 & 48 & 48 & 48 & 48 & 48 & 48 & 48 & 48 & 48 & 48 & 48 & 48\end{array}$

Axial TIP Distribution,

No TIP data taken for this Data Set. 


\section{CYCLE 1 DATA}

\section{DATASET 22, JANUARY 15, 1976}

\section{Reactor Conditions}

Core Average Exposure, $8766 \mathrm{MWd} / \mathrm{t}$

Core Thermal Power, 3292 MWT

Dome Pressure, P, 1024 psıa

Core Flow, $1012 \mathrm{Mlb} / \mathrm{hr}$

Inlet Subcoolıng at P, $246 \mathrm{Btu} / \mathrm{lb}$

Control Configuratıon

Legend 48, Full Out, O, Full In.

\begin{tabular}{|c|c|c|c|c|c|c|c|c|c|c|c|c|c|c|c|}
\hline 8. & $\begin{array}{l}48 \\
48 \\
48 \\
42 \\
48 \\
38 \\
38 \\
48 \\
38 \\
48 \\
42 \\
48 \\
48 \\
48\end{array}$ & $\begin{array}{l}48 \\
48 \\
48 \\
48 \\
48 \\
48 \\
48 \\
48 \\
48 \\
48 \\
48 \\
48 \\
48 \\
48\end{array}$ & $\begin{array}{l}48 . \\
42 . \\
48 . \\
38 . \\
48 . \\
20 . \\
48 . \\
20 . \\
48 . \\
38 . \\
48 . \\
42 .\end{array}$ & $\begin{array}{l}4 \\
4\end{array}$ & $\begin{array}{l}4 \\
3 \\
4 \\
1 \\
4 \\
3 \\
4 \\
3 \\
4 \\
1\end{array}$ & 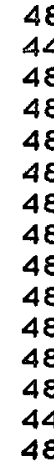 & & $\begin{array}{l}8 . \\
4 .\end{array}$ & $\begin{array}{l}4 . \\
8 . \\
8 . \\
8 . \\
8 . \\
8 . \\
8 . \\
8 . \\
8 . \\
8 .\end{array}$ & $\begin{array}{l}40 \\
34 \\
48 \\
18\end{array}$ & $\begin{array}{l}48 \\
48 \\
48 \\
48 \\
48 \\
48 \\
48 \\
48 \\
48 \\
48 \\
48\end{array}$ & $\begin{array}{l}8 \\
2\end{array}$ & $\begin{array}{l}8 . \\
8\end{array}$ & 4 & \\
\hline
\end{tabular}

Axıal TIP Distribution, Bottom To top of Core

See Figure 22

160969.3104 .8117 .5128 .7124 .8119 .5109 .2106 .2103 .199 .897 .793 .0 $\begin{array}{llllllllllll}88.1 & 81.6 & 80.3 & 76.2 & 67.2 & 65.8 & 62.0 & 53.8 & 46.5 & 39.6 & 26.5 & 16.8\end{array}$

240976.5112 .6131 .4141 .1141 .4138 .5139 .4140 .5132 .1125 .1120 .1113 .5

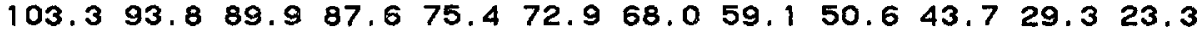

320970.3109 .8129 .9143 .5139 .3137 .5137 .4144 .7140 .5133 .9125 .9120 .4 $109.698 .994 .091 .078 .874 .4 \quad 70.1 \quad 60.6 \quad 52.0 \quad 45.3 \quad 30.521 .4$

400983.8118 .6125 .8135 .8134 .4133 .8135 .0135 .5128 .8119 .4118 .6113 .9 $\begin{array}{llllllllllll}103.7 & 96.0 & 92.5 & 88.2 & 77.8 & 74.1 & 70.1 & 59.9 & 51.6 & 43.6 & 28.4 & 22.9\end{array}$

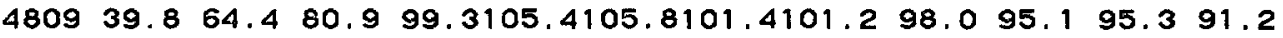
$85.7 \quad 79.6 \quad 76.5 \quad 73.8 \quad 66.0 \quad 64.2 \quad 59.2 \quad 51.6 \quad 43.3 \quad 37.3 \quad 24.7 \quad 13.3$

$081755.989 .1107 .3125 .2130 .8128 .7120 .3119 .3112 .8110 .4105 .6103,2$ $100.190 .3 \quad 89.6 \quad 86.8 \quad 77.3 \quad 74.1 \quad 71.6 \quad 62.7 \quad 53.6 \quad 47.8 \quad 32.6 \quad 19.0$

161772.6112 .6127 .0138 .2146 .3147 .8141 .9142 .5138 .7135 .1131 .4128 .0 $121.7109 .8108 .9106 .293 .388 .182 .071 .5 \quad 59.152 .6 \quad 36.423 .5$

241784.9121 .6130 .7135 .6129 .1124 .1122 .1126 .1123 .0117 .1116 .0114 .6 $109.1104 .6107 .4107 .596 .592 .685 .9 \quad 74.063 .154 .7 \quad 37.026 .7$

$321788.9122 .1134 .2146 .1145 .8140 .8138,0139.2137 .2131 .3130 .9129 .3$ $118.5110 .7106 .1102 .189 .785 .5 \quad 78.8 \quad 67.758 .5 \quad 50.0 \quad 33.129 .0$

401772.2112 .2125 .6132 .3130 .1126 .4123 .4125 .5122 .3117 .6115 .2114 .8 $109.4102 .2105 .1107 .297 .191 .384 .474 .6 \quad 61.655 .1 \quad 38.8 \quad 25.3$

481763.2100 .6117 .2130 .3138 .2143 .5143 .0142 .0137 .1129 .8128 .5126 .5 $117.2107 .0103 .6100 .387 .683 .1 \quad 77.368 .457 .1 \quad 50.6 \quad 35.421 .5$

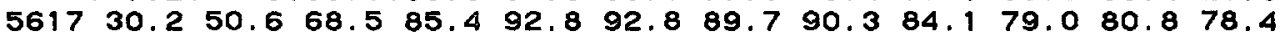

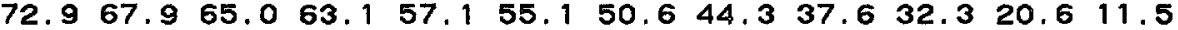

082567.7104 .8122 .4135 .5143 .4145 .2139 .7144 .6130 .9127 .3120 .3118 .8

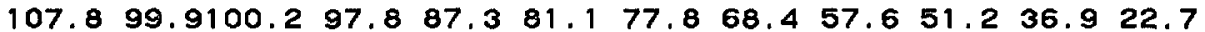

162580.9114 .6123 .5129 .5124 .4117 .0113 .1117 .0115 .9115 .0118 .7118 .4 $115.8114 .3115 .8115 .3102 .698 .0 \quad 92.679 .165 .856 .938 .126 .4$ 
$242587.4123,0131,1136.7132 .2128 .5129,1139.7142 .5139 .8142 .1141 .8$ $133.8125 .2121 .2118 .5104 .5 \quad 99.6 \quad 91.5 \quad 77.6 \quad 66.1 \quad 57.3 \quad 39.129 .4$ 322590.3128 .9137 .5142 .5136 .2128 .7122 .4123 .8122 .4120 .1123 .4123 .5 117.4114 .4117 .6118 .0103 .798 .989 .976 .665 .756 .237 .730 .2 402576.4117 .9129 .7134 .6130 .0125 .4124 .3136 .1135 .7140 .3139 .9139 .9 133.4123 .7122 .9122 .1109 .3101 .994 .181 .868 .660 .341 .327 .3

482571.4113 .8131 .1138 .3136 .1128 .7122 .6123 .9116 .5112 .5111 .7112 .2 $108.8106 .3111 .5113 .7103 .096 .690 .8 \quad 79.266 .5 \quad 57.840 .224 .8$

562541.866 .982 .798 .6106 .5113 .2109 .0111 .2100 .993 .489 .887 .5

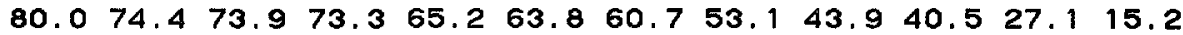

083371.7105 .3117 .4133 .0135 .8136 .4129 .3132 .9127 .3118 .2114 .7112 .7

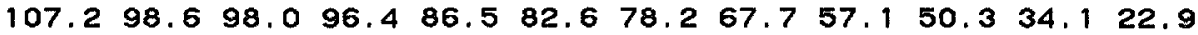

163373.1104 .6110 .6114 .9107 .8103 .398 .6103 .5101 .7100 .0103 .8104 .3 $102.5102 .7108 .4109 .396 .992 .4 \quad 86.174 .562 .453 .737 .926 .0$

243375.3112 .8126 .2131 .1124 .4119 .7116 .8128 .9134 .9134 .1137 .9136 .5 129.5118 .1116 .8114 .1100 .294 .8 89.0 77.365 .357 .640 .527 .2

323378.7116 .1124 .7131 .8124 .2116 .7111 .8115 .3113 .9114 .5117 .2117 .9 $112.2108 .5111 .6115 .5104 .599 .391 .7 \quad 78.766 .659 .041 .428 .4$

403377.5116 .9126 .0131 .1125 .1120 .7125 .9137 .5141 .9143 .7144 .6143 .0 $136.1124 .8123 .7121 .6107 .699 .9 \quad 92.3 \quad 79.066 .858 .240 .726 .5$

483376.8110 .6121 .6127 .3121 .9116 .0109 .8112 .5111 .7107 .9108 .6109 .4 108.3107 .8113 .8113 .2101 .696 .388 .675 .963 .454 .137 .025 .9

563346.274 .691 .4108 .4117 .4124 .6121 .5120 .3113 .3103 .899 .796 .4 $92.283 .8 \quad 81.6 \quad 77.268 .8 \quad 67.5 \quad 63.255 .4 \quad 46.9 \quad 41.3 \quad 28.4 \quad 16.5$

084174.1114 .1138 .3157 .6161 .4152 .6142 .4138 .2130 .2123 .3121 .0117 .5 $112.0101 .3101 .098 .588 .785 .180 .270 .860 .4 \quad 52.7 \quad 34.821 .9$

164170.2108 .0126 .1136 .4139 .4143 .0138 .6139 .5133 .4130 .0125 .1126 .6 $121.3112 .1112 .6109 .7100 .294 .987 .9 \quad 77.064 .457 .040 .826 .3$

244174.1112 .5123 .3129 .0123 .6114 .7108 .1110 .4108 .6105 .8105 .7105 .5

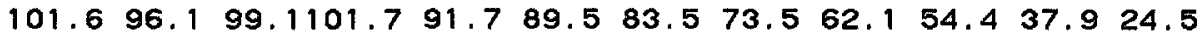

324178.5117 .0131 .0143 .9142 .8138 .7127 .7129 .9129 .8124 .6124 .7125 .7 $120.5111 .8109 .5107 .796 .690 .583 .3 \quad 73.461 .454 .739 .026 .6$

404171.4111 .5126 .5132 .3129 .6124 .8117 .9122 .3118 .5117 .6119 .9119 .4 $116.4109 .9112 .9117 .6107 .5102 .494 .882 .568 .660 .4 \quad 42.327 .1$

484172.7111 .1125 .3136 .5142 .2139 .9132 .4133 .0128 .3123 .4122 .0117 .9 $112.2101 .6100 .798 .787 .783 .978 .969 .459 .152 .5 \quad 36.523 .0$

564148.079 .1100 .4121 .6126 .7122 .6111 .9110 .9102 .8 95.6 89.9 88.7 $81.675 .373 .371 .6 \quad 63.261 .3 \quad 58.0 \quad 50.4 \quad 43.1 \quad 37.5 \quad 25.5 \quad 14.8$

084953.980 .7 92.0102.4102.5 $98.6 \quad 95.5 \quad 96.2 \quad 91.287 .2$ 86.2 84.6 $\begin{array}{llllllllllllll}78.5 & 74.2 & 72.1 & 69.7 & 62.8 & 61.7 & 56.3 & 48.8 & 41.7 & 34.5 & 22.3 & 12.5\end{array}$

$164978.2116 .7135 .0150 .0149,1141.0133 .2131 .2124 .2118 .5118 .4115 .8$

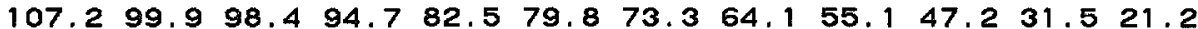

244982.9120 .1130 .3137 .0135 .2132 .5131 .0137 .3132 .3124 .5122 .6121 .4 $112.2101 .798 .996 .7 \quad 86.382 .8 \quad 77.566 .957 .049 .7 \quad 33.6 \quad 22.7$

324977.5114 .4129 .0138 .6136 .6132 .7133 .2144 .1143 .0137 .8136 .7133 .0 $122.2109 .4106 .0102 .089 .685 .580 .369 .859 .951 .7 \quad 35.423 .0$

404979.7119 .2131 .9141 .0138 .9139 .8142 .1144 .2139 .1134 .1130 .7128 .2 120.5110 .0109 .0107 .394 .888 .783 .073 .862 .853 .937 .224 .0

484954.589 .5109 .6125 .0128 .3123 .5114 .5114 .7109 .4104 .8103 .1102 .6 $97.388 .686 .8 \quad 84.976 .272 .068 .861 .5 \quad 51.345 .3 \quad 31.419 .6$

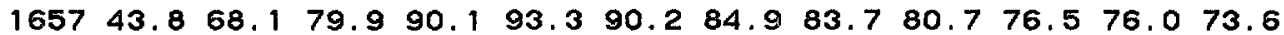

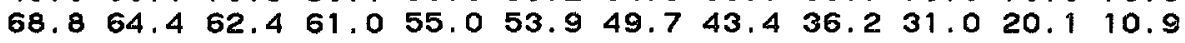

245759.395 .4117 .7133 .1131 .0122 .2111 .1106 .899 .491 .988 .184 .9

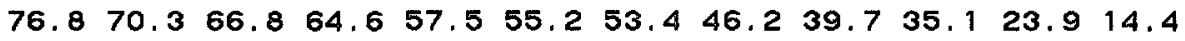

325766.2108 .6127 .8143 .8134 .6122 .2111 .6109 .2102 .794 .490 .8 86.6

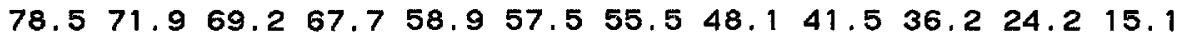

405773.0112 .6129 .2140 .1134 .0124 .6113 .2114 .7108 .0100 .997 .595 .3

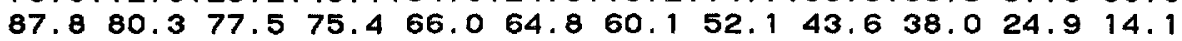




\section{CYCLE 1 DATA}

\section{DATASET 23, FEBRUARY 14, 1976}

\section{Reactor Conditions}

Core Average Exposure, $9295 \mathrm{MWd} / \mathrm{t}$

Core Thermal Power, 3255 MWT

Dome Pressure, P, 1025 psia

Core Flow, 106.7 MIb/hr

Inlet Subcooling at P, $22.1 \mathrm{Btu} / \mathrm{lb}$

Control Configuration

Legend: 48, Full Out; O, Full In.

$\begin{array}{lllllllllllllll}48 & 48 & 48 & 48 & 48 & 48 & 48 & 48 & 48 & 48 & 48 & 48 & 48 & 48 & 48 \\ 48 & 48 & 48 & 48 & 48 & 38 & 48 & 38 & 48 & 38 & 48 & 48 & 48 & 48 & 48 \\ 48 & 48 & 48 & 48 & 48 & 48 & 48 & 48 & 48 & 48 & 48 & 48 & 48 & 48 & 48 \\ 48 & 48 & 48 & 40 & 48 & 36 & 48 & 36 & 48 & 36 & 48 & 40 & 48 & 48 & 48 \\ 48 & 48 & 48 & 48 & 48 & 48 & 48 & 48 & 48 & 48 & 48 & 48 & 48 & 48 & 48 \\ 48 & 38 & 48 & 36 & 48 & 32 & 48 & 20 & 48 & 32 & 48 & 36 & 48 & 38 & 48 \\ 48 & 48 & 48 & 48 & 48 & 48 & 48 & 48 & 48 & 48 & 48 & 48 & 48 & 48 & 48 \\ 48 & 38 & 48 & 36 & 48 & 20 & 48 & 26 & 48 & 20 & 48 & 36 & 48 & 38 & 48 \\ 48 & 48 & 48 & 48 & 48 & 48 & 48 & 48 & 48 & 48 & 48 & 48 & 48 & 48 & 48 \\ 48 & 38 & 48 & 36 & 48 & 32 & 48 & 20 & 48 & 32 & 48 & 36 & 48 & 38 & 48 \\ 48 & 48 & 48 & 48 & 48 & 48 & 48 & 48 & 48 & 48 & 48 & 48 & 48 & 48 & 48 \\ 48 & 48 & 48 & 40 & 48 & 36 & 48 & 36 & 48 & 36 & 48 & 40 & 48 & 48 & 48 \\ 48 & 48 & 48 & 48 & 48 & 48 & 48 & 48 & 48 & 48 & 48 & 48 & 48 & 48 & 48 \\ 48 & 48 & 48 & 48 & 48 & 38 & 48 & 38 & 48 & 38 & 48 & 48 & 48 & 48 & 48 \\ 48 & 48 & 48 & 48 & 48 & 48 & 48 & 48 & 48 & 48 & 48 & 48 & 48 & 48 & 48\end{array}$

Axial TIP Distribution, Bottom To top of Core

See Figure 22

$16943.963 .368 .6 \quad 73.3 \quad 73.2 \quad 70.3 \quad 68.0 \quad 69.3 \quad 67.364 .863 .763 .0$ $\begin{array}{llllllllllll}58.8 & 53.3 & 51.4 & 50.5 & 43.7 & 41.4 & 39.5 & 34.9 & 30.2 & 26.6 & 17.4 & 10.7\end{array}$

$\begin{array}{llllllllllllllllll}24 & 9 & 45.2 & 62.8 & 70.7 & 79.5 & 84.0 & 88.7 & 88.7 & 93.1 & 88.3 & 80.6 & 78.9 & 74.5\end{array}$ $\begin{array}{lllllllllllll}67.7 & 61.1 & 58.3 & 55.5 & 48.6 & 46.8 & 43.6 & 37.4 & 32.8 & 28.4 & 19.1 & 14.9\end{array}$

$\begin{array}{lllllllllllllll}32 & 9 & 40.0 & 59.2 & 67.3 & 76.9 & 84.3 & 91.4 & 93.1 & 97.2 & 92.2 & 88.2 & 84.4 & 79.1\end{array}$ $\begin{array}{llllllllllll}70.6 & 64.3 & 60.8 & 58.7 & 50.8 & 48.2 & 44.6 & 38.9 & 33.2 & 29.8 & 20.2 & 13.4\end{array}$

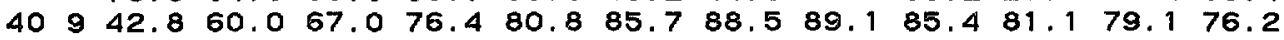
$\begin{array}{llllllllllllll}69.1 & 63.8 & 60.9 & 57.0 & 49.7 & 48.6 & 45.4 & 38.9 & 33.6 & 28.5 & 19.1 & 14.2\end{array}$

$48929.045 .252 .358 .261 .060 .960 .262 .061 .1 \quad 59.860 .157 .6$ $\begin{array}{llllllllllllll}55.2 & 50.7 & 49.3 & 46.9 & 42.8 & 40.6 & 38.2 & 33.1 & 28.4 & 24.2 & 16.0 & 8.6\end{array}$

$81737.5 \quad 57.1 \quad 64.5 \quad 70.7 \quad 72.974 .072 .074 .171 .970 .6 \quad 69.169 .1$ $\begin{array}{llllllllllllll}65.8 & 59.1 & 57.4 & 54.4 & 49.6 & 47.0 & 45.4 & 40.2 & 34.1 & 30.3 & 21.0 & 12.3\end{array}$

$161738.0 \quad 58.1 \quad 68.9 \quad 80.2 \quad 90.2 \quad 93.3 \quad 93.3 \quad 97.1 \quad 95.993 .0 \quad 92.490 .8$ $\begin{array}{lllllllllllll}85.0 & 75.0 & 72.8 & 69.0 & 59.9 & 54.5 & 50.1 & 44.1 & 36.6 & 32.2 & 22.9 & 14.7\end{array}$

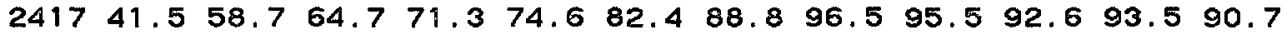
$\begin{array}{lllllllllllll}83.1 & 76.3 & 72.2 & 68.9 & 59.0 & 56.4 & 51.4 & 43.9 & 37.4 & 32.7 & 22.2 & 16.4\end{array}$

$\begin{array}{lllllllllllll}3217 & 42.0 & 57.7 & 62.9 & 69.6 & 73.4 & 78.9 & 86.6 & 93.5 & 94.5 & 91.5 & 92.1 & 88.4\end{array}$ $81.575 .371 .867 .257 .654 .850 .6 \quad 43.4 \quad 37.0 \quad 32.021 .6 \quad 17.2$

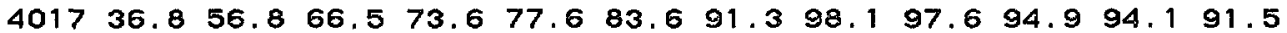

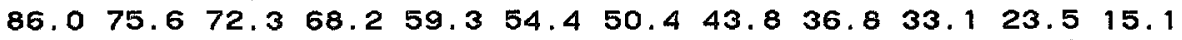

$481736.1 \quad 56.8 \quad 66.3 \quad 78.0 \quad 85.1 \quad 86.2 \quad 87.8 \quad 92.1 \quad 88.8 \quad 88.3 \quad 85.9$ 82.9 $\begin{array}{lllllllllllll}79.2 & 69.5 & 66.4 & 63.7 & 55.1 & 51.5 & 48.7 & 42.7 & 35.8 & 31.9 & 22.5 & 13.8\end{array}$

$\begin{array}{llllllllllllll}5617 & 23.0 & 35.8 & 42.7 & 48.6 & 51.9 & 52.3 & 51.4 & 53.5 & 51.8 & 51.8 & 51.8 & 49.9\end{array}$ $\begin{array}{llllllllllllll}46.5 & 43.8 & 41.8 & 40.2 & 35.9 & 35.0 & 32.8 & 28.2 & 24.2 & 20.5 & 13.3 & 7.4\end{array}$

$\begin{array}{lllllllllllll}825 & 35.0 & 52.5 & 61.8 & 72.4 & 82.6 & 90.6 & 91.6 & 96.7 & 94.1 & 87.9 & 86.3 & 83.8\end{array}$ $\begin{array}{llllllllllllll}76.9 & 67.5 & 65.0 & 62.4 & 54.5 & 49.8 & 46.6 & 41.3 & 35.2 & 31.7 & 22.6 & 14.1\end{array}$

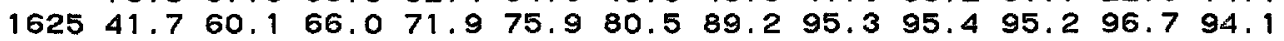

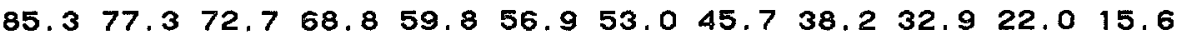


$\begin{array}{llllllllllllllllll}2425 & 43.1 & 61.3 & 66.1 & 71.1 & 70.7 & 71.3 & 76.5 & 86.7 & 91.6 & 94.6 & 98.5 & 98.0\end{array}$ $\begin{array}{lllllllllllll}92.7 & 87.0 & 82.5 & 78.1 & 68.3 & 63.7 & 58.0 & 48.9 & 41.8 & 36.2 & 25.1 & 18.3\end{array}$

$\begin{array}{lllllllllllllll}3225 & 44.1 & 62.4 & 66.7 & 70.1 & 70.3 & 68.9 & 70.7 & 74.9 & 77.4 & 80.8 & 85.8 & 87.1\end{array}$ $\begin{array}{llllllllllll}84.4 & 83.6 & 83.7 & 80.5 & 68.1 & 63.9 & 58.1 & 48.2 & 41.1 & 36.0 & 24.3 & 18.2\end{array}$

$\begin{array}{lllllllllllllll}4025 & 39.0 & 59.5 & 67.0 & 72.9 & 73.5 & 73.7 & 77.3 & 88.3 & 93.4 & 97.7 & 99.1 & 98.4\end{array}$ $\begin{array}{lllllllllllll}93.4 & 84.0 & 82.6 & 79.1 & 68.1 & 62.9 & 58.1 & 50.2 & 42.1 & 36.7 & 25.3 & 17.1\end{array}$

$\begin{array}{llllllllllllll}4825 & 37.3 & 58.9 & 68.5 & 75.6 & 82.7 & 88.7 & 93.2 & 99.4 & 98.2 & 95.3 & 91.9 & 89.5\end{array}$ $\begin{array}{lllllllllllll}84.4 & 75.4 & 71.6 & 67.7 & 60.1 & 55.4 & 52.2 & 45.8 & 38.3 & 34.1 & 23.9 & 14.9\end{array}$

$\begin{array}{llllllllllllll}5625 & 22.8 & 36.2 & 43.4 & 53.5 & 60.1 & 66.6 & 69.6 & 71.2 & 65.9 & 61.8 & 60.2 & 58.1\end{array}$ $\begin{array}{lllllllllllll}54.4 & 49.3 & 47.8 & 47.4 & 41.2 & 39.8 & 38.0 & 33.2 & 28.4 & 24.9 & 17.3 & 9.8\end{array}$

$\begin{array}{llllllllllllll}833 & 38.1 & 56.5 & 64.4 & 75.1 & 83.6 & 89.2 & 91.9 & 93.0 & 91.2 & 86.4 & 84.2 & 81.4\end{array}$ $\begin{array}{lllllllllllll}75.2 & 66.6 & 63.4 & 61.8 & 53.6 & 50.6 & 46.8 & 40.8 & 34.5 & 30.3 & 21.0 & 13.7\end{array}$

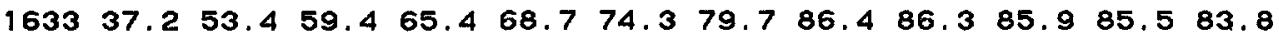
$\begin{array}{lllllllllllll}77.2 & 68.9 & 68.0 & 64.9 & 56.2 & 53.7 & 49.8 & 42.5 & 36.1 & 31.3 & 21.9 & 15.6\end{array}$

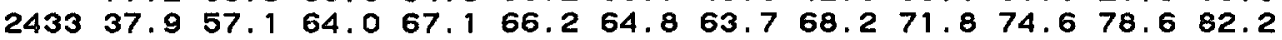
$\begin{array}{lllllllllllll}80.8 & 77.9 & 79.0 & 77.4 & 67.0 & 63.9 & 58.9 & 50.9 & 42.6 & 37.8 & 26.7 & 17.7\end{array}$

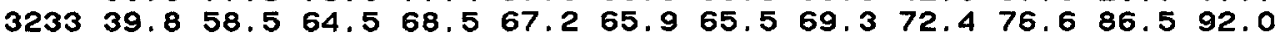
$\begin{array}{lllllllllllllll}90.8 & 85.1 & 83.7 & 80.4 & 68.2 & 64.6 & 58.2 & 50.2 & 42.4 & 37.0 & 26.5 & 17.6\end{array}$

$403340.0 \quad 61.1 \quad 68.1 \quad 71.2 \quad 71.5 \quad 71.7 \quad 75.1 \quad 81.0 \quad 84.5 \quad 84.5 \quad 86.7 \quad 86.6$ $\begin{array}{llllllllllllll}85.0 & 80.6 & 80.8 & 80.5 & 70.4 & 65.1 & 59.4 & 51.3 & 42.7 & 37.4 & 25.9 & 16.5\end{array}$

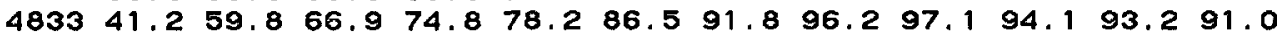
$84.874 .872 .1 \quad 67.7 \quad 57.954 .2 \quad 49.8 \quad 43.1 \quad 36.0 \quad 31.0 \quad 21.3 \quad 14.9$

$\begin{array}{llllllllllllllll}5633 & 25.5 & 41.4 & 51.4 & 61.0 & 69.8 & 76.3 & 78.2 & 81.7 & 75.5 & 71.9 & 69.2 & 66.2\end{array}$ $\begin{array}{lllllllllllll}61.7 & 54.4 & 53.0 & 50.3 & 43.5 & 41.7 & 39.3 & 34.3 & 28.8 & 25.8 & 17.7 & 10.1\end{array}$

$\begin{array}{lllllllllllllll}841 & 37.1 & 56.9 & 65.3 & 75.0 & 84.4 & 89.8 & 90.7 & 91.8 & 88.9 & 86.1 & 84.7 & 81.4\end{array}$

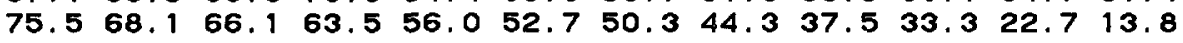

$\begin{array}{llllllllllllll}1641 & 36.5 & 56.8 & 66.9 & 73.5 & 79.7 & 85.4 & 91.0 & 98.3 & 97.7 & 96.7 & 95.2 & 93.7\end{array}$ $\begin{array}{llllllllllllll}87.5 & 78.5 & 75.1 & 70.9 & 62.8 & 57.9 & 53.9 & 47.4 & 39.4 & 34.7 & 25.1 & 16.9\end{array}$

$244136.956 .0 \quad 61.1 \quad 65.966 .6 \quad 67.6 \quad 69.9 \quad 77.1 \quad 82.5 \quad 84.0 \quad 86.0 \quad 84.6$ $\begin{array}{lllllllllllll}79.5 & 71.3 & 69.1 & 67.2 & 57.8 & 54.3 & 51.0 & 44.4 & 37.5 & 33.1 & 23.2 & 14.9\end{array}$

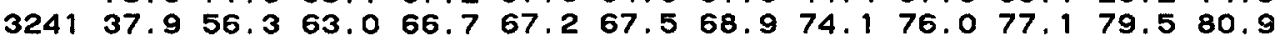
$\begin{array}{llllllllllllll}79.1 & 75.8 & 77.2 & 75.3 & 65.8 & 61.3 & 55.8 & 47.5 & 39.7 & 35.9 & 25.7 & 17.0\end{array}$

$404136.4 \quad 57.1 \quad 65.4 \quad 71.7 \quad 74.976 .7 \quad 79.7 \quad 90.7 \quad 95.6 \quad 97.8 \quad 99.298 .8$ $\begin{array}{lllllllllllll}93.5 & 83.5 & 79.4 & 75.6 & 65.7 & 60.4 & 55.8 & 48.0 & 39.9 & 35.3 & 25.0 & 16.7\end{array}$

$484138.0 \quad 58.4 \quad 66.8 \quad 74.2 \quad 79.3 \quad 82.9 \quad 88.3 \quad 93.2 \quad 90.5 \quad 89.4 \quad 87.6 \quad 84.3$ $\begin{array}{lllllllllllll}78.8 & 68.3 & 65.4 & 63.4 & 54.8 & 52.1 & 49.0 & 42.6 & 36.4 & 32.1 & 23.0 & 14.9\end{array}$

$\begin{array}{lllllllllllllll}5641 & 25.7 & 41.0 & 49.6 & 58.3 & 66.6 & 70.3 & 73.5 & 72.5 & 69.1 & 66.0 & 63.1 & 61.6\end{array}$ $\begin{array}{lllllllllllllll}56.2 & 51.1 & 49.2 & 47.4 & 41.9 & 40.2 & 38.0 & 32.9 & 27.9 & 24.9 & 17.1 & 9.6\end{array}$

$84931.845 .351 .0 \quad 56.857 .859 .8 \quad 59.0 \quad 61.0 \quad 59.458 .058 .255 .6$ $\begin{array}{lllllllllllll}51.6 & 48.2 & 46.8 & 45.4 & 41.5 & 40.2 & 37.3 & 31.7 & 26.8 & 22.8 & 14.8 & 8.1\end{array}$

$\begin{array}{lllllllllllllll}1649 & 43.1 & 62.9 & 71.4 & 82.4 & 88.1 & 87.4 & 86.9 & 86.1 & 84.5 & 81.3 & 81.5 & 79.7\end{array}$ $\begin{array}{llllllllllllll}74.6 & 67.9 & 65.2 & 62.0 & 53.7 & 50.9 & 47.3 & 41.5 & 35.2 & 31.0 & 20.7 & 13.1\end{array}$

$244945.0 \quad 63.969 .4 \quad 76.4 \quad 78.9 \quad 83.3 \quad 89.2 \quad 94.5 \quad 91.8 \quad 88.6 \quad 87.5 \quad 84.6$ $\begin{array}{lllllllllllll}77.5 & 70.5 & 66.7 & 64.6 & 55.5 & 52.8 & 48.8 & 42.1 & 35.8 & 31.0 & 21.2 & 14.9\end{array}$

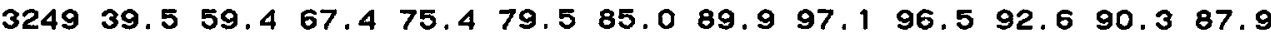
$\begin{array}{llllllllllllll}80.5 & 71.9 & 68.6 & 65.5 & 57.3 & 54.1 & 50.6 & 44.3 & 37.8 & 33.1 & 22.9 & 14.8\end{array}$

$404940.8 \quad 60.9 \quad 69.4 \quad 77.2 \quad 82.2 \quad 87.8 \quad 93.1 \quad 97.2 \quad 98.3 \quad 94.2 \quad 91.2 \quad 89.5$ $\begin{array}{lllllllllllll}84.4 & 75.5 & 72.0 & 69.6 & 60.0 & 55.9 & 52.8 & 46.4 & 38.8 & 34.3 & 23.7 & 15.3\end{array}$

$484929.347 .457 .365 .070 .973 .571 .973 .871 .6 \quad 69.969 .069 .6$ $\begin{array}{lllllllllllllll}65.1 & 59.0 & 57.1 & 54.7 & 49.2 & 46.8 & 44.1 & 38.9 & 33.1 & 29.6 & 20.8 & 11.7\end{array}$

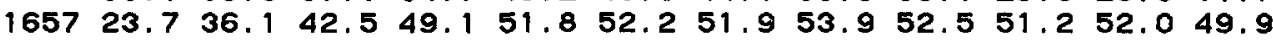
$\begin{array}{llllllllllllllll}46.4 & 42.6 & 42.0 & 40.7 & 36.5 & 35.1 & 32.8 & 28.7 & 24.4 & 20.9 & 13.6 & 7.2\end{array}$

$245725.1 \quad 39.4 \quad 47.958 .8 \quad 66.3 \quad 70.7 \quad 71.1 \quad 74.7 \quad 70.6 \quad 66.1 \quad 63.158 .9$ $\begin{array}{lllllllllllll}53.6 & 48.2 & 46.3 & 44.8 & 39.7 & 38.6 & 36.2 & 31.8 & 27.3 & 24.1 & 16.4 & 9.3\end{array}$

$325729.0 \quad 45.1 \quad 53.4 \quad 61.7 \quad 67.0 \quad 72.5 \quad 75.2 \quad 75.6 \quad 72.1 \quad 65.163 .260 .2$ $\begin{array}{llllllllllllll}55.1 & 49.9 & 47.7 & 45.3 & 40.5 & 39.4 & 37.5 & 32.9 & 28.5 & 24.9 & 16.5 & 10.1\end{array}$

405728.944 .352 .261 .067 .272 .176 .979 .576 .671 .670 .467 .2 $\begin{array}{llllllllllll}62.2 & 56.5 & 54.6 & 52.3 & 45.9 & 44.2 & 41.5 & 35.7 & 30.3 & 25.8 & 16.8 & 9.9\end{array}$ 


\section{CYCLE 1 DATA}

DATASET 24, MARCH 26, 1976

\section{Reactor Conditions}

Core Average Exposure, $10100 \mathrm{MWd} / \mathrm{t}$

Core Thermal Power, 3001 MWT

Dome Pressure, P, 1014 psia

Core Flow, $108.4 \mathrm{Mlb} / \mathrm{hr}$

Inlet Subcooling at P, 24.7 Btu/lb

Control Configuration

Legend: 48, Full Out; O, Full In.

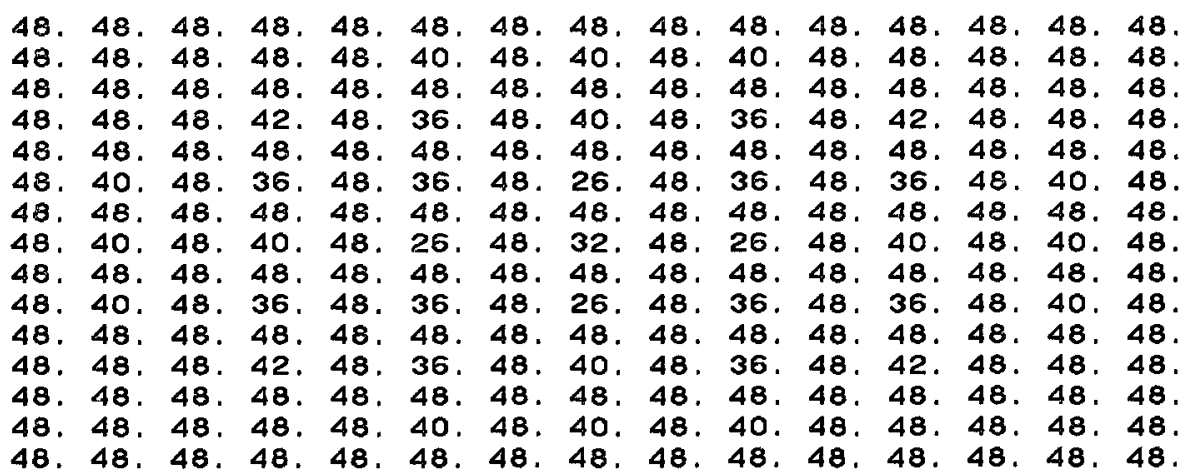

Axial TIP Distribution, Bottom To top of Core

See Figure 22

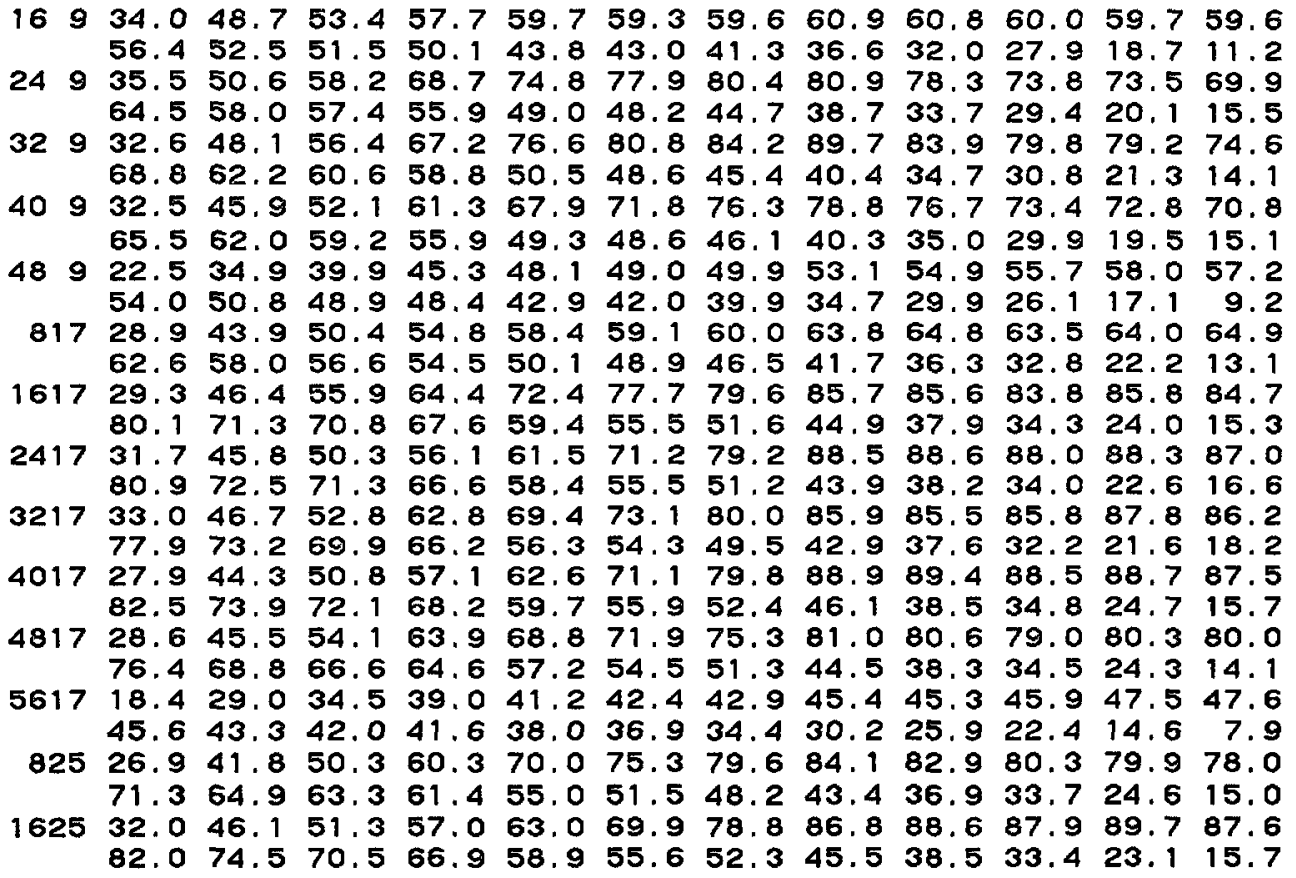


$242532.8 \quad 46.1 \quad 51.2 \quad 56.8 \quad 61.5 \quad 69.1 \quad 78.8 \quad 88.0 \quad 90.0 \quad 92.4 \quad 99.496 .9$ $\begin{array}{llllllllllll}92.2 & 85.4 & 79.7 & 75.2 & 65.2 & 61.8 & 56.3 & 47.1 & 40.5 & 35.2 & 24.1 & 18.2\end{array}$ $\begin{array}{lllllllllllll}3225 & 33.0 & 46.6 & 51.0 & 55.6 & 58.1 & 61.0 & 66.6 & 74.4 & 79.3 & 85.4 & 94.1 & 97.3\end{array}$ $\begin{array}{lllllllllllll}91.9 & 86.6 & 82.0 & 74.3 & 63.1 & 59.3 & 53.4 & 45.5 & 39.4 & 33.9 & 23.2 & 18.2\end{array}$ $\begin{array}{lllllllllllll}4025 & 30.1 & 46.0 & 52.4 & 57.8 & 62.8 & 70.0 & 78.8 & 88.3 & 91.4 & 93.9 & 96.7 & 96.4\end{array}$ $\begin{array}{lllllllllllll}91.7 & 83.4 & 81.4 & 77.3 & 66.9 & 62.8 & 57.9 & 50.1 & 42.2 & 37.3 & 25.5 & 16.8\end{array}$

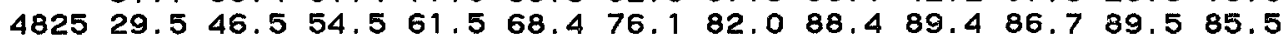
$\begin{array}{llllllllllllll}80.9 & 71.5 & 69.6 & 68.3 & 60.0 & 56.2 & 53.3 & 47.1 & 39.6 & 34.7 & 24.5 & 14.8\end{array}$

$\begin{array}{lllllllllllll}5625 & 19.5 & 31.0 & 38.4 & 47.3 & 54.1 & 58.2 & 59.8 & 61.6 & 59.0 & 57.2 & 56.3 & 54.3\end{array}$ $\begin{array}{lllllllllllll}51.0 & 48.4 & 48.5 & 46.7 & 42.8 & 40.9 & 39.7 & 34.5 & 29.5 & 26.7 & 18.0 & 9.9\end{array}$

$\begin{array}{llllllllllllll}833 & 30.1 & 45.6 & 54.5 & 65.7 & 74.2 & 79.8 & 80.4 & 84.2 & 80.2 & 78.1 & 77.9 & 76.9\end{array}$ $\begin{array}{lllllllllllll}72.0 & 65.2 & 63.4 & 61.2 & 54.1 & 51.2 & 48.2 & 42.3 & 35.8 & 32.1 & 22.1 & 14.1\end{array}$

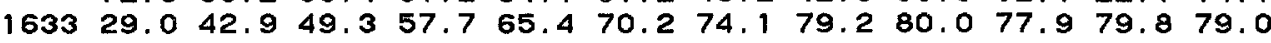
$\begin{array}{llllllllllllll}74.3 & 67.1 & 65.5 & 62.8 & 54.7 & 52.4 & 49.0 & 43.1 & 36.4 & 31.7 & 22.4 & 15.4\end{array}$

$243328.2 \quad 42.2 \quad 48.0 \quad 51.6 \quad 53.9 \quad 56.1 \quad 59.5 \quad 67.5 \quad 72.0 \quad 77.0 \quad 85.3 \quad 88.7$ $\begin{array}{llllllllllllll}87.2 & 80.8 & 76.5 & 71.4 & 61.6 & 57.8 & 53.6 & 46.2 & 39.3 & 34.7 & 24.7 & 16.1\end{array}$

$\begin{array}{lllllllllllll}3233 & 28.5 & 43.6 & 49.5 & 52.8 & 55.2 & 58.8 & 64.4 & 75.3 & 84.0 & 89.9 & 96.4 & 97.8\end{array}$

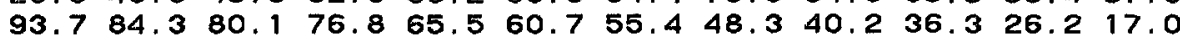

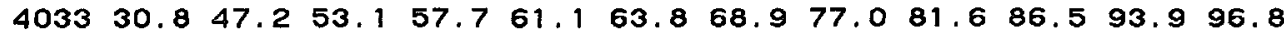
$\begin{array}{lllllllllllll}94.1 & 85.6 & 83.6 & 78.8 & 68.3 & 62.4 & 58.2 & 49.7 & 41.9 & 37.1 & 26.0 & 17.0\end{array}$

$\begin{array}{llllllllllllll}4833 & 33.1 & 48.8 & 57.5 & 67.8 & 75.1 & 79.5 & 81.4 & 88.2 & 87.2 & 86.6 & 85.5 & 85.7\end{array}$ $\begin{array}{lllllllllllll}79.5 & 72.8 & 69.3 & 66.7 & 57.7 & 54.2 & 50.6 & 43.5 & 37.2 & 32.3 & 22.1 & 15.3\end{array}$

$\begin{array}{lllllllllllllllll}5633 & 21.5 & 34.6 & 43.5 & 53.0 & 61.7 & 66.1 & 67.5 & 69.1 & 66.0 & 64.9 & 64.3 & 61.2\end{array}$ $\begin{array}{llllllllllllll}57.3 & 52.3 & 50.7 & 49.8 & 44.4 & 42.5 & 40.3 & 35.8 & 30.4 & 27.4 & 19.2 & 11.5\end{array}$

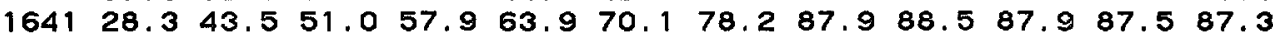
$\begin{array}{llllllllllllll}84.1 & 75.5 & 72.3 & 70.4 & 61.4 & 57.2 & 54.0 & 47.8 & 40.3 & 36.3 & 26.4 & 16.6\end{array}$

$\begin{array}{lllllllllllll}2441 & 28.3 & 43.6 & 49.1 & 53.8 & 58.0 & 64.7 & 71.8 & 79.4 & 81.6 & 81.8 & 83.4 & 82.9\end{array}$ $\begin{array}{llllllllllllll}77.3 & 70.3 & 67.7 & 64.9 & 55.3 & 52.9 & 49.8 & 43.4 & 37.5 & 33.0 & 23.4 & 15.5\end{array}$

$\begin{array}{llllllllllllll}3241 & 29.3 & 43.7 & 49.1 & 54.5 & 57.4 & 59.5 & 63.3 & 70.7 & 74.9 & 77.6 & 85.5 & 89.8\end{array}$ 86.9 $79.076 .872 .963 .3 \quad 59.1 \quad 53.4 \quad 46.3 \quad 39.5 \quad 35.325 .4 \quad 16.6$

$\begin{array}{lllllllllllllll}4041 & 27.9 & 43.4 & 50.6 & 55.3 & 61.1 & 69.7 & 78.0 & 87.7 & 91.1 & 91.7 & 92.7 & 93.5\end{array}$ $\begin{array}{llllllllllll}88.6 & 80.3 & 77.6 & 72.5 & 63.9 & 59.3 & 55.5 & 47.7 & 40.2 & 35.9 & 26.4 & 16.4\end{array}$

$\begin{array}{llllllllllllll}4841 & 29.8 & 45.9 & 52.5 & 58.4 & 63.9 & 69.2 & 75.5 & 82.4 & 83.2 & 82.3 & 82.9 & 80.5\end{array}$ $\begin{array}{lllllllllllll}75.0 & 67.1 & 65.6 & 62.9 & 55.2 & 52.7 & 49.8 & 44.1 & 38.1 & 34.8 & 24.2 & 15.1\end{array}$

$\begin{array}{lllllllllllllll}5641 & 21.3 & 34.1 & 41.8 & 50.6 & 57.1 & 61.0 & 62.4 & 64.4 & 62.0 & 60.6 & 59.6 & 58.2\end{array}$ $\begin{array}{lllllllllllll}54.6 & 49.9 & 48.8 & 47.8 & 42.5 & 41.5 & 39.1 & 34.9 & 30.2 & 26.9 & 18.7 & 10.5\end{array}$

$\begin{array}{llllllllllllllll}849 & 24.6 & 35.7 & 40.1 & 44.6 & 46.7 & 48.6 & 50.1 & 52.5 & 52.8 & 53.6 & 55.3 & 53.5\end{array}$ $\begin{array}{lllllllllllll}50.9 & 48.2 & 46.8 & 46.0 & 42.1 & 41.7 & 38.7 & 33.8 & 29.1 & 24.6 & 15.8 & 8.6\end{array}$

$164933.750 .558 .6 \quad 67.971 .7 \quad 73.075 .278 .677 .176 .476 .276 .0$ $\begin{array}{lllllllllllll}71.4 & 65.9 & 64.0 & 62.0 & 54.4 & 52.8 & 50.1 & 43.1 & 37.6 & 32.9 & 22.3 & 14.3\end{array}$

$244934.949 .8 \quad 56.0 \quad 62.2 \quad 66.7 \quad 72.9 \quad 79.9 \quad 84.8 \quad 84.8 \quad 81.3 \quad 83.0 \quad 80.4$ $\begin{array}{lllllllllllll}73.7 & 69.3 & 66.4 & 63.7 & 55.8 & 54.0 & 50.0 & 42.8 & 37.0 & 32.6 & 22.2 & 15.4\end{array}$

$324932.248 .4 \quad 56.5 \quad 66.6 \quad 76.0 \quad 80.1 \quad 83.0 \quad 88.6 \quad 88.2 \quad 84.3 \quad 84.0 \quad 82.8$ $\begin{array}{llllllllllllll}76.6 & 69.4 & 67.8 & 65.3 & 56.7 & 54.4 & 51.2 & 44.9 & 38.5 & 34.2 & 24.0 & 15.0\end{array}$

$404932.0 \quad 48.4 \quad 54.7 \quad 61.7 \quad 66.6 \quad 73.9 \quad 81.7 \quad 89.0 \quad 86.5 \quad 85.6 \quad 85.7 \quad 84.0$ $\begin{array}{llllllllllllll}80.4 & 73.1 & 70.9 & 68.5 & 59.9 & 56.8 & 54.3 & 47.5 & 40.6 & 35.9 & 25.1 & 15.7\end{array}$

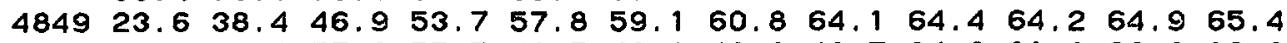
$\begin{array}{llllllllllllll}62.2 & 57.9 & 57.3 & 55.5 & 49.5 & 48.1 & 46.3 & 40.7 & 34.6 & 31.3 & 22.3 & 13.3\end{array}$

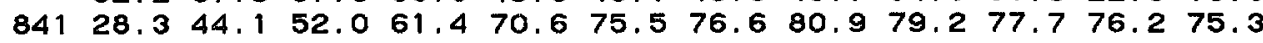
$\begin{array}{lllllllllllll}71.9 & 65.5 & 64.3 & 62.8 & 56.1 & 53.9 & 51.0 & 45.8 & 39.4 & 34.6 & 23.5 & 14.2\end{array}$

$\begin{array}{llllllllllllll}1657 & 19.4 & 29.8 & 35.2 & 40.0 & 42.7 & 43.0 & 44.5 & 46.1 & 46.8 & 46.7 & 48.6 & 48.5\end{array}$ $\begin{array}{lllllllllllllll}45.3 & 42.7 & 42.5 & 41.1 & 38.0 & 37.0 & 34.9 & 30.3 & 26.1 & 22.4 & 14.5 & 7.9\end{array}$

$245721.934 .6 \quad 43.1 \quad 53.8 \quad 61.3 \quad 64.1 \quad 64.4 \quad 64.7 \quad 61.6 \quad 59.3 \quad 58.955 .2$ $\begin{array}{lllllllllllllll}52.4 & 48.7 & 46.3 & 44.5 & 39.9 & 39.1 & 37.7 & 33.1 & 29.0 & 25.8 & 17.7 & 10.3\end{array}$

$\begin{array}{lllllllllllll}3257 & 25.3 & 39.5 & 47.1 & 56.3 & 61.9 & 64.0 & 65.2 & 66.4 & 63.5 & 59.3 & 57.7 & 56.2\end{array}$

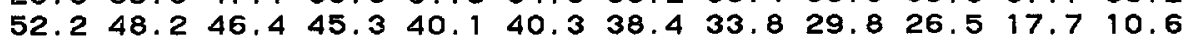

$\begin{array}{llllllllllllllll}4057 & 22.8 & 35.6 & 43.3 & 52.3 & 58.6 & 61.4 & 64.0 & 66.5 & 65.6 & 64.0 & 63.8 & 62.5\end{array}$

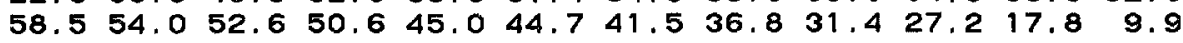




\section{CYCLE 2 DATA}

DATASET 25, JUNE 28, 1976

\section{Reactor Conditions}

Core Average Exposure, $8025 \mathrm{MWd} / \mathrm{t}$

Core Thermal Power, 2650 MWT

Dome Pressure, P, 1017 psia

Core Flow, $88.4 \mathrm{MIb} / \mathrm{hr}$

Inlet Subcooling at P, $24.7 \mathrm{Btu} / \mathrm{lb}$

Control Configuration

Legend: 48, Full Out; O, Full In.

\begin{tabular}{|c|c|c|c|c|c|c|c|c|c|c|c|c|c|}
\hline 48 & 48 & 48 & 48 & 48 & 48 & 48 & 48 & 48 & 48 & 48 & 48 & 48 & 48 \\
\hline 48 & 48 & 48 & 48 & 48 & 40 & 48 & 40 & 48 & 40 & 48 & 48 & 48 & 48 \\
\hline 48 & 48 & 48 & 48 & 20 & 48 & 12 & 48 & 12 & 48 & 20 & 48 & 48 & 48 \\
\hline 48 & 48 & 48 & 42 & 48 & 38 & 48 & 36 & 48 & 38 & 48 & 42 & 48 & 48 \\
\hline 48 & 48 & 20 & 48 & 10 & 48 & 8 & 48 & 8 & 48 & 10 & 48 & 20 & 48 \\
\hline 48 & 40 & 48 & 38 & 48 & 44 & 48 & 48 & 48 & 44 & 48 & 38 & 48 & 40 \\
\hline 48 & 48 & 12 & 48 & 8 & 48 & 6 & 48 & 6 & 48 & 8 & 48 & 12 & 48 \\
\hline 48 & 40 & 48 & 36 & 48 & 48 & 48 & 32 & 48 & 48 & 48 & 36 & 48 & 40 \\
\hline 48 & 48 & 12 & 48 & 8 & 48 & 6 & 48 & 6 & 48 & 8 & 48 & 12 & 48 \\
\hline 48 & 40 & 48 & 38 & 48 & 44 & 48 & 48 & 48 & 44 & 48 & 38 & 48 & 40 \\
\hline 48 & 48 & 20 & 48 & 10 & 48 & 8 & 48 & 8 & 48 & 10 & 48 & 20 & 48 \\
\hline 48 & 48 & 48 & 42 & 48 & 38 & 48 & 36 & 48 & 38 & 48 & 42 & 48 & 48 \\
\hline 48 & 48 & 48 & 48 & 20 & 48 & 12 & 48 & 12 & 48 & 20 & 48 & 48 & 48 \\
\hline 48 & 48 & 48 & 48 & 48 & 40 & 48 & 40 & 48 & 40 & 48 & 48 & 48 & 48 \\
\hline 48 & 48 & 48 & 48 & 48 & 48 & 48 & 48 & 48 & 48 & 48 & 18 & 48 & 48 \\
\hline
\end{tabular}

Axial TIP Distribution, Bottom To top of Core See Figure 22

160946.674 .190 .7106 .0109 .5107 .8104 .0104 .598 .194 .994 .397 .3 $98.799 .6108 .7113 .0106 .5108 .6107 .3 \quad 96.7 \quad 82.7 \quad 66.3 \quad 51.1 \quad 27.9$

240943.167 .185 .6108 .2123 .5127 .8125 .9131 .5121 .7112 .6109 .6108 .1 105.399 .8102 .2106 .3100 .6109 .0113 .3105 .994 .577 .760 .735 .8

$320944.570 .7 \quad 86.6109 .6128 .1135 .5136 .3136 .7130 .4126,0125.7122 .9$ 114.3108 .7113 .5114 .5110 .8119 .9122 .7113 .7103 .492 .161 .639 .5

400931.258 .180 .0101 .3122 .4127 .3127 .2131 .2131 .0120 .1117 .4117 .3 116.6115 .8125 .3132 .6132 .4128 .0129 .8124 .4106 .790 .169 .748 .1

480955.386 .9102 .1116 .2123 .4120 .6115 .0115 .1109 .3108 .3108 .8112 .9 $108.7106 .9110 .0112 .7106 .1106 .3 \quad 97.9 \quad 85.6 \quad 72.5 \quad 62.340 .923 .1$

081742.471 .590 .6105 .2115 .5114 .6111 .9111 .4108 .9106 .2105 .9107 .4 108.8108 .4118 .7125 .4120 .5122 .2118 .0109 .491 .975 .656 .933 .7

161745.570 .589 .7112 .5124 .4126 .0127 .8131 .3124 .8125 .1123 .4124 .2 124.1120 .8125 .6132 .9124 .9131 .1133 .1124 .6112 .391 .169 .243 .3

241747.569 .081 .395 .3107 .2122 .9128 .6130 .8125 .5122 .7126 .9122 .1 114.0110 .4109 .6110 .3108 .2111 .5117 .4113 .4110 .1101 .669 .051 .1

321749.969 .278 .690 .299 .3108 .3119 .9129 .1124 .1122 .5122 .4120 .0 111.3110 .4107 .9110 .3105 .8115 .1119 .2117 .0114 .3101 .567 .951 .0

401736.061 .276 .291 .6107 .6121 .0124 .6136 .1131 .4127 .0124 .1126 .0 122.8114 .0116 .7118 .8115 .5118 .1125 .4125 .8115 .299 .977 .848 .7

481748.181 .6101 .1126 .6136 .1142 .0145 .3143 .1135 .2130 .6132 .7132 .1 129.4131 .9138 .7144 .6135 .2138 .0134 .3122 .9107 .696 .367 .640 .2

$561745.771 .3 \quad 85.8 \quad 99.0106 .5104 .5102 .4101 .9 \quad 95.391 .491 .990 .1$

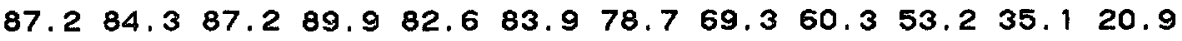

082540.266 .285 .2109 .7130 .3144 .8146 .2149 .2139 .3132 .0132 .6131 .2 123.4115 .7118 .3117 .5115 .2122 .5129 .1121 .8108 .298 .869 .442 .2

162542.864 .776 .691 .2105 .3115 .7125 .8133 .8134 .1130 .1131 .3130 .2 120.9115 .8116 .6118 .5112 .9118 .2120 .7116 .3108 .189 .870 .345 .5 
242559.489 .5104 .9114 .6117 .1114 .5113 .1115 .5111 .3110 .3111 .5109 .4 $\begin{array}{lllllllllllll}103.9 & 99.6 & 98.7 & 99.1 & 92.8 & 99.8100 .1 & 95.1 & 94.1 & 79.5 & 61.7 & 46.2\end{array}$

322573.2102 .5111 .4119 .6120 .0117 .3115 .4118 .3116 .0114 .6114 .6111 .4 $104.5104 .2101 .4100 .995 .299 .099 .5 \quad 94.994 .0 \quad 88.761 .248 .5$

402542.573 .895 .9110 .6117 .5117 .4115 .6121 .1116 .6111 .1111 .1110 .2 106.299 .6100 .9101 .197 .099 .9104 .2103 .2100 .592 .674 .748 .0

$482537.962 .378 .292 .5110 .9126 .4135 .814 i .0139 .8133 .5135 .8131 .6$ $125.3119 .2122 .0123 .3121 .0128 .3136,3131.8117 .894 .274 .642 .0$

562537.967 .991 .6121 .2141 .2146 .7140 .3145 .3131 .5126 .6121 .0118 .3 $114.5105 .9108 .3107 .6100 .5102 .5 \quad 98.8 \quad 91.978 .5 \quad 64.2 \quad 47.728 .9$

083343.269 .688 .6110 .0129 .2136 .0139 .1140 .1133 .6122 .6121 .8119 .1 113.5108 .9108 .4112 .0106 .5117 .2123 .1113 .8102 .393 .363 .240 .4

163339.860 .069 .460 .090 .4102 .8115 .3124 .2124 .3121 .5121 .0115 .3 107.7102 .7102 .0104 .198 .7105 .9109 .0107 .3102 .286 .668 .144 .6

243356.991 .1105 .7114 .8113 .7112 .0109 .2110 .0108 .2106 .0106 .9106 .1 $101.894 .993 .994 .5 \quad 88.9 \quad 92.593 .6 \quad 91.7 \quad 88.6 \quad 78.965 .641 .6$

323353.281 .590 .498 .2100 .5101 .3104 .6116 .6123 .1124 .4129 .3127 .9 121.5110 .5109 .7106 .898 .099 .6101 .397 .594 .993 .870 .245 .6

403357.393 .7110 .6120 .6127 .6127 .8124 .7130 .4126 .7124 .9125 .8125 .2 119.6111 .2109 .6109 .5103 .9105 .0107 .1108 .3105 .293 .977 .349 .3

483337.459 .874 .786 .799 .4114 .1126 .8137 .3132 .9132 .7133 .4125 .1 114.9111 .2109 .8110 .3107 .2115 .3124 .9122 .0112 .195 .976 .446 .2

563342.371 .893 .7119 .2140 .9140 .4133 .7133 .9125 .5115 .2109 .8108 .5

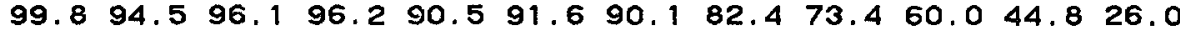

084145.973 .591 .4115 .6134 .0142 .1143 .4143 .1135 .9128 .8130 .5127 .4 126.8127 .2137 .7142 .9136 .8138 .9134 .2119 .9103 .290 .161 .437 .2

164149.170 .283 .7101 .6116 .7124 .2132 .8131 .5127 .0125 .2127 .9123 .2 117.3115 .7116 .7115 .6115 .4121 .4123 .4115 .9109 .987 .365 .949 .1

244146.877 .197 .0112 .7120 .5121 .3120 .9122 .6119 .0118 .7119 .1118 .1 111.6103 .6104 .4106 .0102 .2105 .6110 .1109 .3104 .890 .872 .246 .0

324161.194 .3108 .1116 .8118 .5116 .7113 .5118 .0119 .1115 .4117 .2116 .9 113.9105 .8105 .7106 .399 .8102 .9107 .9107 .1104 .8101 .575 .747 .7

404150.479 .597 .5114 .7121 .3123 .2125 .0127 .7122 .3119 .8118 .9116 .2 111.8109 .2106 .6109 .1103 .7112 .3119 .0114 .8107 .388 .568 .446 .4

484145.069 .686 .2104 .4119 .5128 .9135 .4136 .1131 .5127 .5124 .1123 .3 125.5128 .3137 .9141 .4139 .1142 .6138 .2127 .3114 .991 .168 .340 .5

564143.774 .198 .0123 .8146 .8156 .1152 .8152 .6144 .9128 .4126 .7122 .2 121.4114 .1116 .6117 .4109 .7110 .8106 .795 .880 .470 .448 .227 .1

084966.092 .6107 .6119 .8120 .9116 .6117 .5113 .2108 .5104 .8106 .9104 .6 $101.5104 .2105 .4103 .699 .398 .190 .5 \quad 76.6 \quad 64.8 \quad 49.5 \quad 34.0 \quad 22.4$

164957.684 .3103 .2118 .9123 .2123 .0117 .2117 .4110 .8109 .0109 .9111 .0 110.0120 .2128 .4131 .3127 .5128 .1123 .8108 .896 .876 .156 .840 .1

244945.065 .978 .495 .4110 .7118 .4127 .1127 .2124 .4120 .5119 .8118 .0 112.1112 .0115 .4118 .0115 .7124 .2125 .8117 .6106 .285 .464 .144 .7

324938.961 .973 .885 .997 .4110 .1122 .7132 .7133 .6129 .3127 .9126 .7 119.7112 .8116 .5118 .9115 .5127 .8137 .0137 .7124 .9116 .781 .650 .7

404953.175 .488 .9106 .9120 .1124 .7128 .8129 .4120 .5114 .9118 .7120 .0 114.9123 .2125 .2129 .7124 .9129 .7128 .4115 .7103 .1 80.1 59.5 44.1

484954.791 .1114 .9136 .3143 .9138 .7124 .6122 .4114 .1105 .7106 .7110 .6 110.1105 .5111 .7117 .1110 .8110 .5108 .198 .684 .371 .554 .132 .8

$165750.774 .987 .298 .3103 .7102 .997 .996 .590 .8 \quad 86.287 .285 .6$

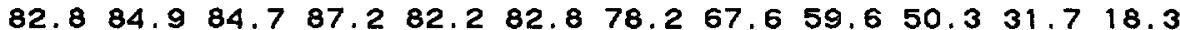

245750.778 .4100 .2127 .7139 .1138 .6137 .7134 .4123 .4117 .1115 .2112 .7 $104.4103 .0101 .1101 .496 .098 .495 .583 .273 .5 \quad 55.239 .523 .6$

325752.481 .5101 .1126 .9136 .0135 .7129 .7126 .0120 .1111 .9110 .1103 .1 $\begin{array}{llllllllllllll}97.8 & 93.7 & 92.5 & 95.3 & 89.8 & 93.7 & 91.2 & 83.0 & 73.6 & 64.7 & 41.5 & 26.5\end{array}$ 405752.080 .4101 .5129 .1142 .0143 .5139 .7139 .5130 .9126 .1126 .0123 .5 118.8117 .5118 .7119 .3112 .9113 .1108 .092 .479 .566 .941 .023 .9 


\section{CYCLE 2 DATA}

DATASET 26, JULY 14, 1976

\section{Reactor Conditions}

Core Average Exposure, $8264 \mathrm{MWd} / \mathrm{t}$

Core Thermal Power, 3262 MWT

Dome Pressure, P, 1013 psia

Core Flow, $101.2 \mathrm{MIb} / \mathrm{hr}$

Inlet Subcooling at P, $23.91 \mathrm{Btu} / \mathrm{lb}$

Control Configuration

Legend: 48, Full Out; O, Full In.

$\begin{array}{lllllllllllllll}48 & 48 & 48 & 48 & 48 & 48 & 48 & 48 & 48 & 48 & 48 & 48 & 48 & 48 & 48 \\ 48 & 48 & 48 & 48 & 48 & 48 & 34 & 48 & 34 & 48 & 48 & 48 & 48 & 48 & 48 \\ 48 & 48 & 48 & 38 & 48 & 38 & 48 & 42 & 48 & 38 & 48 & 38 & 48 & 48 & 48 \\ 48 & 48 & 48 & 48 & 22 & 48 & 12 & 48 & 12 & 48 & 22 & 48 & 48 & 48 & 48 \\ 48 & 38 & 48 & 38 & 48 & 38 & 48 & 40 & 48 & 38 & 48 & 38 & 48 & 38 & 48 \\ 48 & 48 & 22 & 48 & 10 & 48 & 10 & 48 & 10 & 48 & 10 & 48 & 22 & 48 & 48 \\ 48 & 40 & 48 & 40 & 48 & 48 & 48 & 44 & 48 & 48 & 48 & 40 & 48 & 40 & 48 \\ 48 & 48 & 22 & 48 & 14 & 48 & 14 & 48 & 14 & 48 & 14 & 48 & 22 & 48 & 48 \\ 48 & 40 & 48 & 40 & 48 & 48 & 48 & 44 & 48 & 48 & 48 & 40 & 48 & 40 & 48 \\ 48 & 48 & 22 & 48 & 10 & 48 & 10 & 48 & 10 & 48 & 10 & 48 & 22 & 48 & 48 \\ 48 & 38 & 48 & 38 & 48 & 38 & 48 & 40 & 48 & 38 & 48 & 38 & 48 & 38 & 48 \\ 48 & 48 & 48 & 48 & 22 & 48 & 12 & 48 & 12 & 48 & 22 & 48 & 48 & 48 & 48 \\ 48 & 48 & 48 & 38 & 48 & 38 & 48 & 42 & 48 & 38 & 48 & 38 & 48 & 48 & 48 \\ 48 & 48 & 48 & 48 & 48 & 48 & 34 & 48 & 34 & 48 & 48 & 48 & 48 & 48 & 48 \\ 48 & 48 & 48 & 48 & 48 & 48 & 48 & 48 & 48 & 48 & 48 & 48 & 48 & 48 & 48\end{array}$

Axial TIP Distribution, Bottom To top of Core See Figure 22

160941.767 .381 .996 .2104 .9112 .6111 .9115 .6113 .2106 .5107 .0106 .1

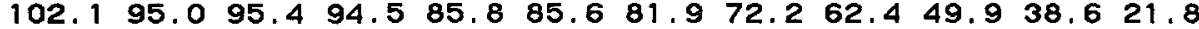
240939.863 .077 .191 .9104 .7117 .6126 .1140 .7138 .3130 .3125 .5124 .1 $115.2103 .4104 .4104 .895 .6 \quad 93.9 \quad 91.7 \quad 83.1 \quad 72.1 \quad 57.7 \quad 45.5 \quad 30.4$ 320943.366 .385 .6103 .9117 .1127 .1139 .2151 .7147 .4143 .8143 .7138 .6 128.4119 .1118 .4117 .1104 .9105 .2100 .689 .878 .068 .246 .531 .0

400938.469 .792 .9106 .4123 .7136 .5136 .7139 .7138 .7133 .5124 .6127 .9 123.8115 .0117 .3114 .0111 .4102 .199 .794 .280 .166 .451 .335 .4

480934.154 .866 .582 .897 .8110 .3115 .4120 .7115 .0112 .5115 .1112 .9 $106.9100 .9101 .098 .088 .586 .279 .5 \quad 68.7 \quad 58.549 .2 \quad 32.417 .8$

081734.357 .574 .489 .1104 .8116 .1118 .1126 .8121 .3117 .6119 .2118 .7 $114.6107 .1110 .8111 .9103 .199 .395 .8 \quad 86.674 .358 .844 .227 .4$

161736.555 .566 .081 .599 .2108 .3117 .6127 .4122 .8122 .8122 .2129 .0 $133.8133 .7138 .2139 .3127 .0125 .8120 .2105 .892 .372 .555 .0 \quad 36.8$

241736.354 .065 .781 .296 .0107 .0114 .4119 .1117 .9114 .9117 .7117 .2 109.3106.1105.1106.4103.4113.5116.2106.1 99.8 89.2 59.9 42.4

321739.658 .771 .988 .8100 .1106 .8114 .5115 .4114 .7110 .6113 .9109 .6 101.9100 .5101 .5102 .5102 .4114 .5116 .7109 .5102 .185 .658 .146 .1

401730.450 .161 .375 .391 .5102 .7111 .3123 .1122 .4119 .8122 .1123 .4 127.9125 .7125 .1128 .1120 .7121 .5120 .8110 .596 .881 .362 .439 .4

481748.176 .991 .6110 .3129 .1141 .4147 .7150 .5148 .3138 .5138 .7140 .5 135.1128 .6129 .4126 .7115 .5113 .2108 .296 .8 83.3 73.650 .931 .1

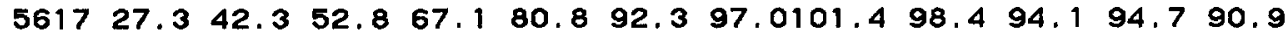

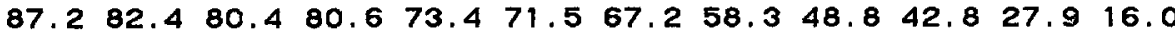

082534.255 .672 .693 .1112 .0123 .6126 .6131 .6124 .3123 .2126 .2128 .8 131.8130 .9135 .0135 .2124 .9122 .9118 .4105 .490 .480 .756 .235 .1

162537.557 .970 .985 .897 .9105 .8113 .3122 .0118 .9116 .9123 .1123 .1 117.4117 .3118 .1120 .4114 .2118 .6119 .3111 .399 .578 .660 .939 .4 
$\begin{array}{llllllllllllllll}2425 & 53.0 & 76.2 & 85.4 & 93.3 & 96.1 & 97.9 & 99.3 & 99.3 & 97.9 & 97.0 & 98.9 & 97.5\end{array}$ $95.093 .895 .499 .097 .3105 .6111 .8107 .299 .5 \quad 79.860 .6 \quad 43.6$

322550.777 .289 .5102 .7104 .1103 .8106 .2105 .3101 .3100 .6103 .7100 .6 $95.894 .697 .0101 .398 .4109 .2114 .5107 .8101 .5 \quad 89.860 .347 .8$

$402543.270 .1 \quad 82.8 \quad 92.498 .0100 .299 .5101 .7101 .798 .4101 .098 .8$

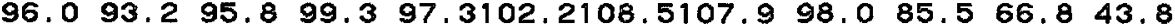

482533.855 .670 .788 .5104 .2114 .3116 .1125 .6121 .7120 .1122 .2128 .3 132.7135 .3141 .5140 .6130 .9129 .6125 .2112 .898 .077 .960 .036 .0

562531.154 .873 .997 .0117 .0125 .1121 .9128 .3119 .7112 .8111 .5111 .0 $111.0105 .4105 .8107 .997 .794 .0 \quad 90.4 \quad 79.4 \quad 65.7 \quad 53.0 \quad 39.722 .7$

083337.859 .976 .897 .6114 .1118 .7121 .7123 .7119 .5111 .8115 .9121 .2 126.0129 .4131 .5135 .9123 .2121 .6115 .1100 .985 .774 .550 .832 .0

163337.156 .567 .681 .896 .3103 .8106 .2109 .0103 .9105 .8106 .1106 .4 103.6100 .7106 .6113 .2109 .8116 .1116 .5106 .994 .576 .860 .138 .5

$243352.9 \quad 83.0 \quad 95.4104 .5105 .299 .797 .497 .0 \quad 95.491 .6 \quad 93.494 .0$ 92.386 .592 .598 .899 .7111 .7116 .9111 .8100 .163 .064 .840 .8

323350.982 .098 .0111 .1113 .1109 .6107 .7110 .9106 .8104 .5105 .8103 .8 102.197 .1100 .5105 .8108 .8118 .9123 .4118 .1107 .197 .770 .846 .0

403350.781 .397 .6104 .6108 .4108 .3105 .7110 .4109 .2108 .4109 .7109 .7 108.1104 .2106 .7113 .2116 .0123 .9125 .4119 .0106 .391 .271 .945 .8

483334.256 .771 .486 .6102 .3111 .2113 .9116 .3117 .3114 .7115 .9121 .7 126.7125 .1129 .6131 .8123 .5122 .0120 .0111 .092 .677 .160 .839 .1

563335.459 .878 .399 .0113 .9120 .8115 .9116 .9111 .2102 .9105 .3103 .2 $100.995 .899 .099 .6 \quad 90.588 .183 .373 .7 \quad 62.950 .0 \quad 38.421 .5$

084139.764 .079 .699 .2115 .5129 .1133 .5137 .5133 .9129 .0128 .4130 .1 132.9133 .2137 .3136 .2127 .3122 .8115 .5100 .083 .773 .349 .228 .6

164140.458 .270 .485 .6101 .4110 .6121 .9122 .6121 .8118 .7120 .5118 .4 113.8114 .5113 .8114 .8106 .9110 .2111 .8101 .992 .371 .954 .641 .6

244133.352 .364 .876 .287 .9100 .3104 .9111 .9109 .7109 .4110 .6107 .3 104.6100 .3102 .6104 .7101 .4107 .0113 .8111 .0101 .985 .467 .343 .0

$324135.5 \quad 56.6 \quad 69.5 \quad 82.7 \quad 95.5101 .1107 .7112 .8111 .2109 .9110 .3111 .8$ 107.6102 .0104 .2106 .2101 .2108 .0115 .1113 .1104 .897 .571 .846 .2

404136.254 .163 .476 .588 .8100 .8109 .3117 .7115 .9114 .0114 .9116 .3 112.7108 .4109 .7110 .3104 .0109 .4113 .5106 .698 .480 .061 .241 .5

484137.258 .368 .985 .7102 .5115 .6124 .3131 .2125 .3120 .6122 .8124 .6 128.6130 .5135 .5135 .7125 .8126 .5122 .1109 .494 .773 .957 .334 .4

564131.453 .871 .991 .5112 .6125 .9134 .7138 .5134 .7124 .0121 .7118 .8 118.9110 .3111 .4110 .5101 .497 .892 .8 80.7 68.458 .440 .122 .2

084952.775 .788 .9102 .5107 .8111 .9117 .0115 .4108 .1108 .1108 .6105 .2

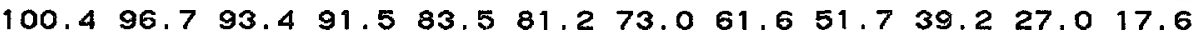

164948.069 .178 .492 .0106 .6115 .1118 .5118 .5113 .5112 .4116 .3116 .1 $113.4118 .7118 .0119 .4106 .8108,1102.8$ 89.7 76.559 .843 .730 .7

244943.162 .175 .0 93.2107.1115.4124.7128.3122.1117.2118.4114.5 109.6108 .0107 .1108 .7101 .3106 .5106 .996 .286 .267 .050 .837 .2

324937.462 .079 .599 .1113 .0119 .6124 .0129 .9128 .8125 .2123 .6122 .3 116.5108 .6109 .8110 .9106 .0111 .3119 .8113 .5100 .391 .965 .340 .6 404946.766 .8 80.0 96.6110.4118.9130.6129.2123.8116.7119.4121.7 118.4120 .2118 .6119 .5110 .2112 .1106 .794 .281 .963 .948 .035 .9

484941.765 .983 .498 .1111 .4120 .8121 .4126 .3120 .7116 .6113 .4111 .9 $109.8103 .0101 .8104 .096 .592 .790 .0 \quad 80.468 .1 \quad 55.943 .625 .7$

$\begin{array}{lllllllllllll}1657 & 49.1 & 71.5 & 83.8 & 95.5100 .0 & 97.8 & 96.6 & 97.3 & 92.5 & 88.8 & 87.9 & 85.7\end{array}$

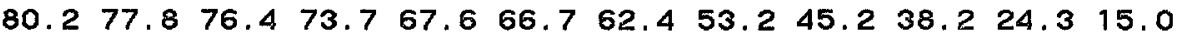

245750.674 .588 .8104 .9109 .8113 .8121 .8128 .9123 .9118 .1118 .7113 .4 $104.398 .496 .093 .184 .683 .4 \quad 78.3 \quad 66.3 \quad 57.2 \quad 42.6 \quad 31.0 \quad 18.6$

325746.670 .382 .995 .2102 .0107 .1113 .8122 .2120 .1113 .0117 .3110 .3 $99.393 .293 .3 \quad 89.9 \quad 81.8 \quad 80.2 \quad 77.1 \quad 67.9 \quad 57.6 \quad 49.7 \quad 32.3 \quad 20.4$

405762.233 .4109 .2130 .6130 .6135 .1129 .1133 .0131 .0124 .1121 .7118 .9 110.6106 .2105 .5103 .693 .491 .284 .672 .361 .350 .431 .619 .5 


\section{CYCLE 2 DATA}

\section{DATASET 27, SEPTEMBER 1, 1976}

\section{Reactor Conditions}

Core Average Exposure, $9035 \mathrm{MWd} / \mathrm{t}$

Core Thermal Power, 3276 MWT

Dome Pressure, P, 1027 psia

Core Flow, $104.5 \mathrm{MIb} / \mathrm{hr}$

Inlet Subcooling at P, $23.59 \mathrm{Btu} / \mathrm{lb}$

Control Configuration

Legend: 48, Full Out; O, Full In.

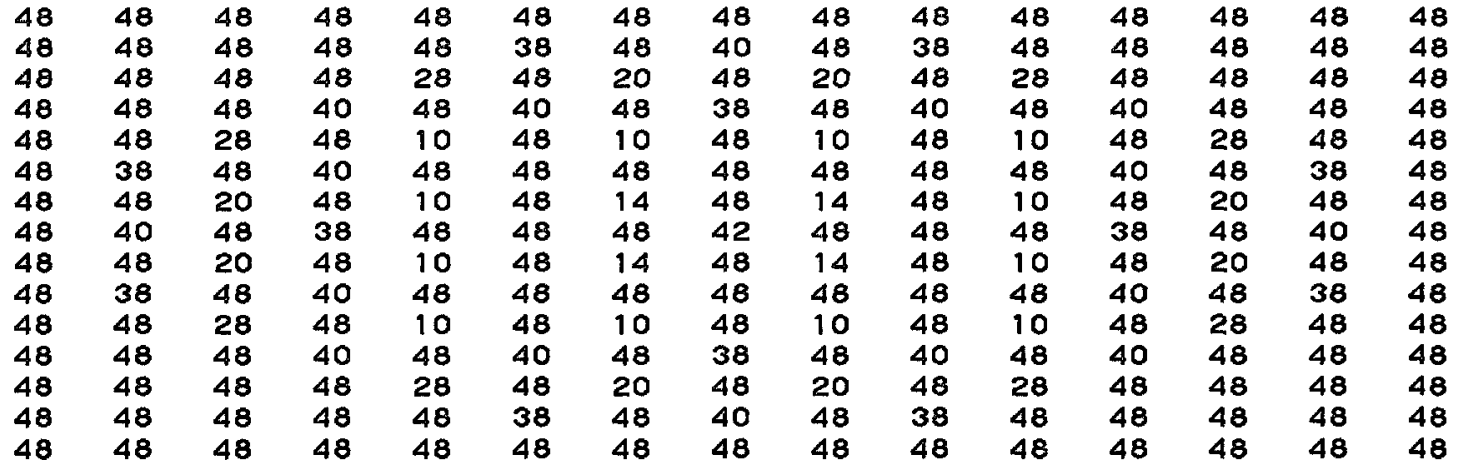

Axial TIP Distribution, Bottom To top of Core

See Figure 22

160938.362 .374 .689 .198 .198 .297 .2101 .5104 .5107 .5112 .8117 .0 $116.1105 .0104 .7104 .1 \quad 92.3 \quad 90.1 \quad 85.8 \quad 77.5 \quad 66.7 \quad 53.4 \quad 40.623 .2$ 240935.256 .169 .6 84.8100.2112.4111.7120.7117.1114.2112.4111.9 110.1109 .5115 .5118 .3108 .5106 .9102 .993 .578 .963 .549 .931 .2 320937.256 .870 .992 .0109 .0115 .8123 .9125 .3121 .3117 .3121 .4117 .0 117.0122 .2130 .5135 .3122 .2120 .0116 .099 .585 .675 .651 .135 .0 400926.046 .763 .476 .793 .8109 .1116 .0122 .1125 .8124 .6129 .7138 .6 140.0130 .3129 .4128 .8123 .4113 .8111 .7103 .086 .173 .655 .739 .0

480944.769 .582 .396 .698 .4100 .599 .9106 .7108 .4107 .3110 .9113 .0 $108.7101 .5100 .297 .5 \quad 88.686 .279 .5 \quad 69.2 \quad 58.350 .2 \quad 34.520 .3$

081735.258 .774 .788 .599 .1102 .4104 .9113 .4113 .2116 .9128 .4132 .8 128.8119 .7117 .6116 .6105 .8102 .798 .989 .575 .160 .845 .427 .6

161736.756 .169 .186 .4102 .4113 .9120 .3126 .8130 .4128 .0134 .6136 .3 129.1123 .2123 .4122 .9112 .0114 .3112 .7103 .993 .474 .256 .737 .6

241741.260 .172 .488 .9100 .5109 .2112 .4116 .8115 .7115 .1117 .4116 .6 113.4109 .2111 .2110 .9105 .2110 .1113 .8107 .798 .989 .560 .846 .3

321742.059 .067 .679 .290 .298 .4107 .6110 .4111 .3110 .0113 .5112 .2 105.9108 .1111 .1110 .8108 .5115 .7119 .2111 .3105 .191 .462 .045 .8

401735.054 .667 .982 .395 .8104 .3109 .5119 .1117 .7117 .7122 .2122 .3 116.4110 .6109 .4108 .7102 .3104 .9108 .7106 .297 .183 .066 .944 .1

481741.063 .075 .796 .3112 .3120 .5125 .4134 .0133 .1141 .2150 .7150 .8 144.6134 .0130 .9126 .4115 .2111 .4107 .7 96.9 84.976 .954 .835 .9

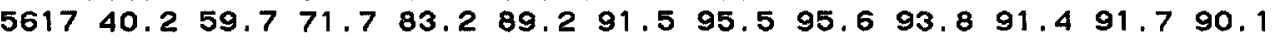
$\begin{array}{lllllllllllll}87.6 & 82.3 & 79.6 & 78.9 & 70.9 & 69.7 & 65.2 & 56.8 & 48.9 & 43.0 & 29.0 & 18.9\end{array}$

082533.152 .466 .882 .9102 .5118 .4124 .0134 .7131 .4131 .0132 .5131 .7 $130.5127 .4133 .1137 .0126 .3122 .9117 .2105 .590 .7 \quad 79.856 .934 .1$

162537.856 .068 .582 .095 .4103 .1110 .4119 .0117 .7118 .1123 .8122 .2 117.9115 .3115 .1114 .8108 .9114 .9115 .8108 .497 .878 .860 .841 .0 
$\begin{array}{llllllllllllllll}2425 & 59.4 & 83.1 & 39.8 & 96.2 & 97.7 & 96.0 & 95.6 & 97.0 & 94.4 & 94.8 & 97.7 & 97.2\end{array}$ $93.593 .197 .6101 .8104 .2116 .2120 .0109 .8101 .6 \quad 80.560 .544 .7$ 322563.488 .296 .0103 .9102 .3101 .2102 .7101 .2100 .899 .6102 .8101 .1 97.299 .2103 .8110 .5114 .3123 .6127 .3114 .4105 .292 .161 .751 .9

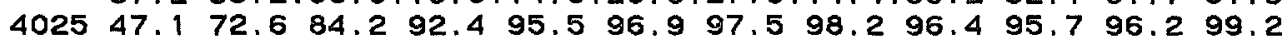

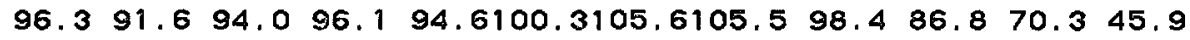
482533.953 .967 .384 .0100 .0110 .8118 .2125 .2126 .6127 .9128 .5130 .9 128.1128 .1134 .0135 .9127 .5127 .2123 .7111 .599 .179 .862 .741 .8

$562535.958 .878 .196 .5115 .8128,1130.6130 .5123 .6116 .2115 .1114 .8$ $110.9103 .0106 .7105 .097 .092 .8 \quad 89.3 \quad 79.765 .854 .241 .125 .8$

083335.956 .572 .592 .5109 .1116 .3122 .2126 .1118 .6115 .8117 .7119 .4 117.2120 .7128 .0132 .1122 .7122 .6116 .5100 .486 .875 .851 .933 .2

163333.650 .358 .970 .781 .092 .5100 .7110 .2106 .9104 .7105 .1105 .0 $100.797 .4100 .8105 .7101 .0105 .7107 .4102 .193 .7 \quad 77.960 .740 .1$

$243350.0 \quad 77.291 .297 .3 \quad 98.495 .991 .1 \quad 95.492 .191 .491 .492 .5$ 92.288 .492 .099 .7102 .9113 .8119 .4113 .6101 .385 .166 .641 .5

323347.674 .0 88.2 98.8105.8105.7103.5107.3104.5103.9105.9105.4 104.4100 .8104 .4112 .4114 .7126 .5131 .0122 .1109 .4102 .073 .548 .6

403349.276 .388 .695 .398 .6100 .2101 .4103 .8103 .6103 .0106 .5108 .7 105.0101 .2104 .0107 .8105 .1110 .4114 .4114 .7104 .890 .273 .750 .1

483332.651 .561 .474 .086 .398 .8108 .3113 .4116 .7113 .5116 .2116 .1 115.8114 .3121 .5126 .5120 .9119 .4117 .1107 .993 .580 .064 .643 .2

563336.761 .481 .7104 .6120 .9125 .7119 .8119 .8118 .2107 .4103 .1103 .1 $99.293 .098 .097 .0 \quad 90.590 .3 \quad 85.2 \quad 75.5 \quad 64.5 \quad 51.2 \quad 38.8 \quad 23.6$

084137.560 .172 .9 88.5106.6122.9129.9136.8139.3143.8150.2156.5 151.0139 .5139 .3138 .9124 .3120 .9115 .7100 .583 .772 .549 .230 .6

164142.261 .474 .491 .4105 .8110 .0119 .3120 .8120 .7119 .5123 .3120 .4 111.9112 .2109 .5108 .8102 .7108 .8108 .9100 .692 .674 .155 .844 .8

244148.875 .787 .696 .3101 .6100 .098 .8100 .1100 .0101 .0102 .5103 .6 99.595 .699 .4101 .8100 .6107 .7113 .5111 .4102 .785 .267 .843 .0

324153.8 83.3 94.5 99.0101.0102.6100.6102.4103.8104.0106.8109.9 108.8103 .0105 .6113 .0110 .1114 .9122 .3122 .4109 .8100 .875 .350 .2

404152.676 .587 .797 .6102 .0105 .3106 .9109 .2108 .1108 .4110 .9109 .7 103.1103 .1100 .8103 .699 .5106 .0110 .5104 .797 .579 .160 .941 .6

484136.557 .071 .990 .8104 .4116 .0122 .7127 .9130 .9132 .9142 .9144 .2 140.5133 .0134 .5134 .2122 .1122 .1119 .3107 .093 .173 .256 .335 .5

564136.460 .681 .1101 .1120 .1130 .4138 .4138 .7138 .5128 .1125 .8124 .8 $121.7114 .1112 .2110 .5100 .897 .593 .8 \quad 82.5 \quad 69.159 .4 \quad 40.424 .0$

084955.678 .391 .6105 .4108 .4109 .7113 .1114 .9114 .9113 .2116 .4113 .8 $\begin{array}{llllllllll}104.9104 .3100 .3 & 95.3 & 87.4 & 84.8 & 76.7 & 63.6 & 54.2 & 40.7 & 27.8 & 18.7\end{array}$

164945.565 .580 .295 .9105 .1107 .7113 .6120 .4119 .8122 .0132 .8132 .9 124.3120 .5120 .8117 .3107 .7106 .6102 .490 .378 .461 .345 .434 .3

244939.157 .7 70.3 89.1102.2107.1115.8119.4114.4118.1118.7119.8 $117.8124 .5130 .2131 .3120 .2121 .4115 .4103 .590 .570 .553 .3 \quad 39.8$

324932.651 .763 .774 .687 .9100 .2108 .9118 .3115 .8115 .1116 .5119 .2 121.2123 .4134 .3141 .1133 .6134 .5132 .7121 .8106 .096 .268 .543 .5

404942.561 .975 .795 .9107 .2114 .2119 .5122 .0122 .0130 .2135 .1139 .3 128.1124 .9121 .7121 .8112 .1112 .7109 .095 .084 .365 .049 .538 .0

484942.769 .689 .2106 .2119 .5115 .7116 .7121 .7117 .6114 .2114 .8116 .7 114.2103 .6103 .4103 .696 .693 .188 .881 .068 .457 .043 .727 .0

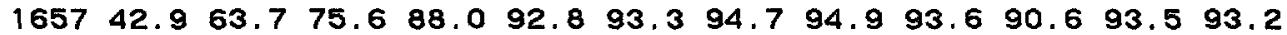

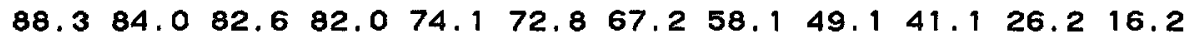

245744.870 .1 84.3106.8119.5126.0128.5126.4119.7113.8113.2109.7 $103.4102 .0100 .7 \quad 99.7 \quad 91.6 \quad 90.7 \quad 83.8 \quad 71.6 \quad 62.2 \quad 45.7 \quad 32.4 \quad 21.7$

325746.172 .389 .9111 .4123 .0125 .1122 .9118 .8110 .3104 .2102 .4100 .1

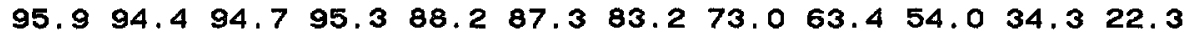

405743.968 .382 .9105 .1120 .5130 .1133 .2138 .2134 .0129 .2129 .9124 .2 119.4115 .4114 .4112 .4102 .7100 .693 .878 .765 .954 .934 .121 .0 


\section{CYCLE 2 DATA}

DATASET 28, OCTOBER 8, 1976

\section{Reactor Conditions}

Core Average Exposure, $9730 \mathrm{MWd} / \mathrm{t}$

Core Thermal Power, 3275 MWT

Dome Pressure, P, 1022 psia

Core Flow, $101.5 \mathrm{Mlb} / \mathrm{hr}$

Inlet Subcooling at $\mathrm{P}, 24.3 \mathrm{Btu} / \mathrm{lb}$

Control Configuration

Legend: 48, Full Out; O, Full In.

$\begin{array}{lllllllllllllll}48 & 48 & 48 & 48 & 48 & 48 & 48 & 48 & 48 & 48 & 48 & 48 & 48 & 48 & 48 \\ 48 & 48 & 48 & 48 & 48 & 38 & 48 & 40 & 48 & 38 & 48 & 48 & 48 & 48 & 48 \\ 48 & 48 & 48 & 48 & 28 & 48 & 18 & 48 & 18 & 48 & 28 & 48 & 48 & 48 & 48 \\ 48 & 48 & 48 & 40 & 48 & 40 & 48 & 38 & 48 & 40 & 48 & 40 & 48 & 48 & 48 \\ 48 & 48 & 28 & 48 & 10 & 48 & 14 & 48 & 14 & 48 & 10 & 48 & 28 & 48 & 48 \\ 48 & 38 & 48 & 40 & 48 & 48 & 48 & 48 & 48 & 48 & 48 & 40 & 48 & 38 & 48 \\ 48 & 48 & 18 & 48 & 14 & 48 & 18 & 48 & 18 & 48 & 14 & 48 & 18 & 48 & 48 \\ 48 & 40 & 48 & 38 & 48 & 48 & 48 & 42 & 48 & 48 & 48 & 38 & 48 & 40 & 48 \\ 48 & 48 & 18 & 48 & 14 & 48 & 18 & 48 & 18 & 48 & 14 & 48 & 18 & 48 & 48 \\ 48 & 38 & 48 & 40 & 48 & 48 & 48 & 48 & 48 & 48 & 48 & 40 & 48 & 38 & 48 \\ 48 & 48 & 28 & 48 & 10 & 48 & 14 & 48 & 14 & 48 & 10 & 48 & 28 & 48 & 48 \\ 48 & 48 & 48 & 40 & 48 & 40 & 48 & 38 & 48 & 40 & 48 & 40 & 48 & 48 & 48 \\ 48 & 48 & 48 & 48 & 28 & 48 & 18 & 48 & 18 & 48 & 28 & 48 & 48 & 48 & 48 \\ 48 & 48 & 48 & 48 & 48 & 38 & 48 & 40 & 48 & 38 & 48 & 48 & 48 & 48 & 48 \\ 48 & 48 & 48 & 48 & 48 & 48 & 48 & 48 & 48 & 48 & 48 & 48 & 48 & 48 & 48\end{array}$

Axial TIP Distribution, Bottom To top of Core See Figure 22

$160938.6 \quad 62.7 \quad 75.190 .797 .296 .394 .499 .6100 .6100 .5109 .8112 .2$ $108.5100 .299 .6 \quad 96.8 \quad 88.186 .2 \quad 82.974 .6 \quad 64.0 \quad 51.3 \quad 39.5 \quad 23.3$

240935.354 .068 .085 .596 .4106 .3113 .2117 .4111 .7106 .3106 .4106 .2 $100.597 .8103 .3108 .9103 .7102 .6 \quad 99.3 \quad 89.4 \quad 77.7 \quad 62.4 \quad 48.6 \quad 30.3$

320937.758 .070 .890 .2104 .6116 .4119 .9121 .3116 .4111 .5110 .5110 .0 108.2107 .5114 .5126 .2117 .4116 .9111 .697 .384 .472 .650 .135 .4

400926.748 .264 .077 .795 .5109 .0115 .2124 .7122 .9123 .2128 .3132 .7 130.9121 .8124 .2121 .8115 .8107 .9104 .799 .082 .669 .654 .038 .4

480952.779 .491 .3105 .0107 .8105 .0108 .9111 .8109 .5111 .6112 .5112 .2 $106.4102 .798 .8 \quad 95.9 \quad 86.9 \quad 82.5 \quad 75.9 \quad 65.0 \quad 55.3 \quad 45.2 \quad 28.6 \quad 20.1$

081735.660 .476 .088 .999 .5105 .1103 .1108 .4110 .1110 .8118 .8124 .5 $121.7111 .8111 .8110 .8102 .496 .894 .8 \quad 83.8 \quad 70.8 \quad 58.4 \quad 43.326 .0$

161734.552 .964 .081 .597 .5107 .5111 .5121 .8119 .3119 .4125 .5126 .4 122.0115 .8117 .3117 .4107 .4108 .3108 .699 .990 .370 .754 .136 .6

241737.154 .564 .779 .592 .499 .4105 .7111 .6108 .1108 .4113 .2110 .4 106.4105 .3108 .4112 .9114 .0122 .0122 .0108 .296 .588 .258 .643 .3

321737.953 .661 .170 .982 .590 .299 .6106 .1103 .7103 .6110 .5108 .0 104.7106 .9110 .8118 .7121 .9130 .0129 .9114 .6103 .8 86.9 58.5 47.7

401734.253 .464 .1 80.6 95.1102.9106.7114.9114.7113.4119.0119.3 $112.9108 .1108 .4108 .7102 .3107 .4109 .7102 .0 \quad 93.876 .259 .141 .1$

$481743.366 .782 .199,6114.6122,7129,0130.7133 .0136,7143,9140,5$ $134.7129 .2124 .1120 .5109 .1109 .0104 .090 .8 \quad 80.6 \quad 69.946 .7 \quad 35.0$

$\begin{array}{lllllllllllll}5617 & 45.1 & 67.1 & 77.8 & 88.6 & 95.4 & 95.1 & 95.9 & 94.6 & 93.5 & 89.9 & 89.9 & 87.5\end{array}$ $\begin{array}{lllllllllllll}82.9 & 78.9 & 76.6 & 74.4 & 67.5 & 66.1 & 61.4 & 53.3 & 45.9 & 38.5 & 24.1 & 17.6\end{array}$

$082532.651 .7 \quad 65.5 \quad 82.0100 .5113 .8122 .0130 .8127 .0121 .8122 .2122 .6$ 118.9113 .9118 .7123 .7118 .5118 .0114 .8101 .486 .776 .754 .832 .9

$162533.850 .7 \quad 62.075 .0 \quad 87.194 .5102 .4108 .8110 .0111 .5115 .9115 .9$ 112.0109 .9113 .3119 .5117 .4127 .6124 .1110 .295 .977 .959 .839 .9 
$2425 \quad 51.5 \quad 71.8 \quad 79.2 \quad 84.6 \quad 86.5 \quad 85.8 \quad 87.9 \quad 91.4 \quad 91.4 \quad 90.996 .398 .6$ 98. 1105.3115 .4123 .6123 .1128 .0124 .9109 .899 .177 .858 .744 .7 322554.575 .781 .588 .690 .989 .493 .094 .296 .496 .6101 .1100 .9 103.0114 .9123 .2134 .1129 .3135 .8131 .6113 .3101 .086 .457 .947 .1

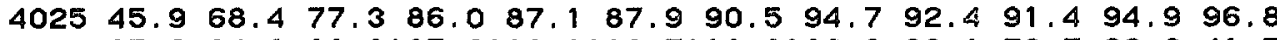
95.694 .399 .9107 .2110 .1118 .7119 .3108 .398 .179 .762 .241 .7

482534.753 .465 .483 .396 .5106 .5115 .0119 .3120 .5119 .1120 .8119 .5 $114.3116 .1123 .3129 .5123 .4125 .9120 .4105 .7 \quad 93.572 .655 .239 .7$

562540.569 .989 .8108 .9123 .5128 .3127 .1125 .4119 .0113 .0109 .5106 .1

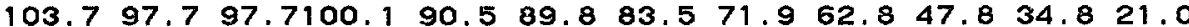

083335.456 .171 .088 .7105 .3110 .6115 .5117 .1115 .6109 .0108 .5108 .4 105.7105 .8111 .9120 .7114 .9117 .0112 .497 .483 .273 .450 .032 .5

$\begin{array}{lllllllllllll}1633 & 29.6 & 44.3 & 52.4 & 62.0 & 74.2 & 83.8 & 92.3 & 97.1 & 96.1 & 95.8 & 98.9 & 98.8\end{array}$ 96.495 .2101 .1108 .3108 .9118 .0118 .3106 .192 .675 .959 .938 .1

$\begin{array}{lllllllllllll}2433 & 42.9 & 66.7 & 76.2 & 83.7 & 85.3 & 85.3 & 81.9 & 88.2 & 86.5 & 88.2 & 91.2 & 95.7\end{array}$ $96.897 .6109 .2122 .7122 .9128 .1126 .0114 .0 \quad 98.5 \quad 80.663 .839 .2$

$323346.4 \quad 67.5 \quad 78.1 \quad 87.0 \quad 91.7 \quad 93.0 \quad 96.199 .3 \quad 99.3102 .1107 .2111 .3$ 111.4117 .9129 .4138 .5134 .5136 .3130 .7116 .3102 .991 .162 .749 .0

403348.470 .979 .587 .090 .992 .294 .6101 .4101 .9103 .7106 .9108 .4 107.6109 .5117 .5123 .9124 .3133 .1131 .2118 .6103 .984 .666 .146 .8

483333.650 .360 .072 .983 .993 .1100 .9107 .3106 .5107 .7109 .6106 .2 103.2103 .2113 .2118 .8117 .2119 .5118 .6103 .491 .673 .756 .942 .8

$563339.0 \quad 65.7 \quad 86.3107 .2122 .0124 .8120 .0118 .8108 .199 .897 .197 .8$

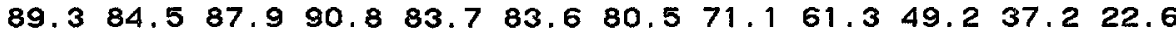

084137.559 .474 .490 .2106 .6119 .7124 .7131 .4130 .5134 .7143 .1145 .4 $138.0130 .6130 .5128 .4116 .4115 .1109 .993 .379 .869 .547 .3 \quad 30.3$

164139.155 .968 .285 .496 .3102 .1109 .5113 .3115 .3115 .3116 .7115 .5 109.2106 .8107 .9107 .5101 .0108 .9108 .698 .590 .971 .753 .842 .9

$244143.0 \quad 66.2 \quad 76.7 \quad 85.1 \quad 89.8 \quad 90.3 \quad 90.9 \quad 94.7 \quad 94.9 \quad 95.3 \quad 99.5102 .1$ 100.399 .6104 .0112 .9114 .5122 .9126 .6116 .7101 .383 .665 .139 .7

$324144.5 \quad 68.1 \quad 77.9 \quad 84.1 \quad 87.2 \quad 87.7 \quad 90.5 \quad 95.2 \quad 95.5 \quad 99.3101 .2104 .3$ 107.7106 .3112 .3121 .8124 .1132 .7134 .7122 .6104 .795 .970 .146 .0

$404146.568 .7 \quad 77.987 .993 .595 .598 .8102 .3102 .4102 .8107 .1105 .9$ 103.2101 .9103 .8109 .1104 .6111 .0113 .4103 .995 .876 .858 .740 .3

484135.755 .668 .986 .9102 .3113 .5117 .9124 .6124 .7126 .8135 .0137 .1 132.2127 .6126 .2128 .1119 .2119 .6116 .6103 .190 .271 .254 .233 .7

564137.466 .285 .4105 .6125 .0135 .4136 .5139 .7131 .9120 .0120 .9119 .1 $113.3103 .9103 .5102 .8 \quad 95.1 \quad 92.1 \quad 87.9 \quad 76.3 \quad 64.1 \quad 55.4 \quad 37.821 .1$

084957.080 .491 .8104 .4106 .5107 .8110 .6111 .4109 .4106 .8109 .9106 .4 $\begin{array}{lllllllllllll}99.7 & 98.1 & 94.5 & 89.8 & 83.2 & 78.8 & 70.1 & 60.1 & 51.0 & 39.1 & 26.9 & 18.1\end{array}$

164944.364 .876 .093 .2103 .0104 .0106 .3110 .8113 .0117 .0123 .9125 .5 118.1115 .3112 .6110 .9101 .8101 .897 .185 .575 .458 .643 .232 .9

244936.753 .464 .682 .595 .0101 .8106 .0109 .3107 .5109 .7110 .4112 .5 110.3109 .9116 .5122 .5115 .4117 .9114 .599 .087 .068 .451 .038 .3

$324929.947 .5 \quad 58.1 \quad 68.481 .2 \quad 92.1 \quad 98.4107 .8109 .1107 .8109 .3109 .3$ 110.5110 .6119 .1130 .3129 .0132 .6132 .3121 .9103 .793 .266 .839 .5

404941.360 .573 .191 .3106 .7113 .0115 .2117 .0120 .5123 .3130 .9129 .9 119.4116 .6116 .8114 .2106 .3107 .5102 .590 .8 80.3 62.447 .638 .6

484945.373 .394 .1110 .6124 .7119 .8115 .9117 .6115 .3112 .2109 .4111 .0 $\begin{array}{llllllllllll}105.1 & 99.9 & 98.5 & 97.7 & 90.2 & 87.8 & 83.7 & 76.1 & 64.4 & 53.7 & 42.4 & 26.2\end{array}$

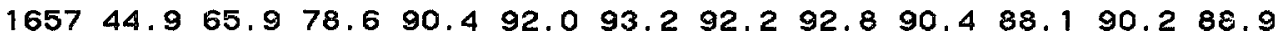

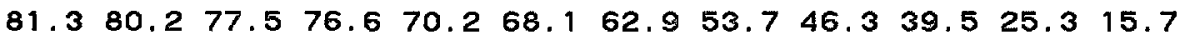

245747.671 .788 .0109 .2120 .8123 .7124 .4121 .2112 .0105 .7104 .1102 .5 $94.292 .991 .290 .683 .684 .380 .267 .858 .143 .0 \quad 31.519 .6$

325749.074 .691 .6115 .2122 .6119 .5116 .5113 .7108 .096 .895 .590 .7

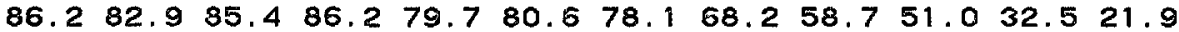

405746.171 .788 .3111 .1125 .4131 .1128 .4130 .9128 .9122 .4120 .2117 .1 112.0106 .9105 .2105 .094 .493 .487 .073 .061 .751 .132 .320 .0 
CYCLE 2 DATA

DATASET 29, OCTOBER 28, 1976

\title{
Reactor Conditions
}

Core Average Exposure, $10050 \mathrm{MWd} / \mathrm{t}$

Core Thermal Power, 3293 MWT

Dome Pressure, P, 1023 psia

Core Flow, $99.9 \mathrm{Mlb} / \mathrm{hr}$

Inlet Subcooling at $P, 24.8 \mathrm{Btu} / \mathrm{lb}$

Control Configuration

\author{
Legend: 48, Full Out; O, Full In.
}

$\begin{array}{lllllllllllllll}48 & 48 & 48 & 48 & 48 & 48 & 48 & 48 & 48 & 48 & 48 & 48 & 48 & 48 & 48 \\ 48 & 48 & 48 & 48 & 42 & 48 & 36 & 48 & 36 & 48 & 42 & 48 & 48 & 48 & 48 \\ 48 & 48 & 48 & 34 & 48 & 26 & 48 & 12 & 48 & 26 & 48 & 34 & 48 & 48 & 48 \\ 48 & 48 & 48 & 48 & 48 & 48 & 48 & 48 & 48 & 48 & 48 & 48 & 48 & 48 & 48 \\ 48 & 36 & 48 & 18 & 48 & 12 & 48 & 32 & 48 & 12 & 48 & 18 & 48 & 36 & 48 \\ 48 & 48 & 40 & 48 & 48 & 48 & 48 & 48 & 48 & 48 & 48 & 48 & 40 & 48 & 48 \\ 48 & 32 & 48 & 10 & 48 & 30 & 48 & 12 & 48 & 30 & 48 & 10 & 48 & 32 & 48 \\ 48 & 48 & 48 & 48 & 44 & 48 & 48 & 48 & 48 & 48 & 44 & 48 & 48 & 48 & 48 \\ 48 & 32 & 48 & 10 & 48 & 30 & 48 & 12 & 48 & 30 & 48 & 10 & 48 & 32 & 48 \\ 48 & 48 & 40 & 48 & 48 & 48 & 48 & 48 & 48 & 48 & 48 & 48 & 40 & 48 & 48 \\ 48 & 36 & 48 & 18 & 48 & 12 & 48 & 32 & 48 & 12 & 48 & 18 & 48 & 36 & 48 \\ 48 & 48 & 48 & 48 & 48 & 48 & 48 & 48 & 48 & 48 & 48 & 48 & 48 & 48 & 48 \\ 48 & 48 & 48 & 34 & 48 & 26 & 48 & 12 & 48 & 26 & 48 & 34 & 48 & 48 & 48 \\ 48 & 48 & 48 & 48 & 42 & 48 & 36 & 48 & 36 & 48 & 42 & 48 & 48 & 48 & 48 \\ 48 & 48 & 48 & 48 & 48 & 48 & 48 & 48 & 48 & 48 & 48 & 48 & 48 & 48 & 48\end{array}$

Axial TIP Distribution, Bottom To top of Core See Figure 22

160938.463 .180 .5100 .3110 .0112 .9116 .2121 .1121 .2116 .5115 .4113 .8 $109.299 .498 .096 .084 .784 .280 .4 \quad 72.062 .049 .6 \quad 38.121 .6$

240947.273 .084 .995 .2102 .4107 .0112 .2117 .4118 .7114 .1118 .3119 .9 117.2111 .8109 .8106 .696 .495 .391 .083 .072 .158 .345 .628 .4

$320951.474 .783 .290 .898 .4105 .3115 .4122 .2116 .8118,1119.1117 .0$ 112.1105 .9104 .0103 .897 .1101 .099 .589 .178 .468 .447 .032 .2

400937.165 .790 .6106 .8122 .2125 .3122 .5124 .4124 .9122 .5124 .0131 .2 132.8123 .0121 .7119 .3111 .4103 .799 .892 .477 .565 .651 .434 .6 480940.761 .772 .084 .992 .497 .2111 .4118 .9122 .8120 .3121 .0116 .8 $108.8103 .498 .597 .587 .6 \quad 83.6 \quad 76.6 \quad 65.6 \quad 55.945 .6 \quad 28.7 \quad 18.8$

081742.772 .391 .5105 .2118 .2122 .0127 .9136 .7130 .9124 .5121 .0121 .0 114.3103 .6103 .7105 .797 .193 .989 .980 .667 .555 .041 .225 .7 161772.1102 .0111 .8121 .5120 .8118 .7112 .7118 .7116 .2111 .1114 .4116 .7 $111.9111 .4114 .6123 .8117 .0117 .6111 .898 .784 .866 .851 .0 \quad 33.5$ 241780.3110 .2116 .9124 .2119 .5112 .3107 .3111 .2110 .1110 .4116 .7117 .8 110.9107 .5106 .1105 .9100 .2105 .8105 .995 .386 .278 .351 .840 .5

321782.8110 .6114 .1117 .4111 .2105 .4107 .7117 .1123 .8123 .0128 .4126 .4 $118.8115 .6114 .7113 .5105 .4108 .4105 .7 \quad 95.5 \quad 85.7 \quad 72.348 .841 .4$

401773.5108 .1114 .7121 .6121 .3116 .1110 .7113 .2110 .8109 .1113 .8113 .5 109.9104.1104.9104.5 99.9105.4106.5 95.8 85.8 67.8 53.3 36.8

481771.2103 .5115 .5127 .0129 .1131 .0129 .8129 .7127 .7119 .9118 .2117 .1 $110.4108 .9112 .2116 .2107 .9108 .6104 .788 .978 .767 .445 .3 \quad 33.7$

$561736.555 .064 .7 \quad 77.0 \quad 89.699 .2107 .6111 .0107 .3103 .198 .892 .0$ 83.7 $81.5 \quad 76.1 \quad 73.267 .265 .960 .5 \quad 51.8 \quad 45.9 \quad 38.3 \quad 23.6 \quad 16.8$

082544.268 .386 .0105 .0120 .3129 .5136 .6148 .6150 .5152 .2149 .4142 .3 135.5121 .5118 .7114 .5102 .9100 .997 .987 .976 .067 .949 .129 .7

162563.995 .0104 .9112 .2113 .2110 .6109 .8114 .3112 .5113 .7116 .0116 .8 112.7105 .3105 .1105 .398 .5100 .799 .892 .384 .367 .852 .934 .0 
242568.194 .0101 .0105 .7102 .597 .798 .5104 .8111 .0116 .7123 .6124 .8 119.4114 .4113 .9111 .9104 .0104 .6101 .990 .380 .865 .250 .237 .9 322575.5102 .3107 .3111 .3106 .6104 .4104 .0110 .0110 .5112 .4118 .1115 .0 110.9109 .1107 .9107 .7101 .5106 .9106 .596 .8 86.8 76.351 .540 .3 402564.293 .7102 .0106 .6105 .6103 .0101 .4105 .9110 .2115 .7119 .5118 .2 114.4107 .7108 .9110 .5102 .5102 .7100 .491 .280 .566 .452 .535 .8 $482555.282 .395 .6109 .6122 .2125 .1128,3130.6127 .7124 .5124 .5120 .9$ $113.1107 .9105 .6104 .695 .699 .099 .5 \quad 92.6 \quad 83.266 .049 .534 .8$

562546.375 .594 .6111 .8118 .5119 .1120 .9126 .2127 .7122 .9123 .8120 .9 $112.4103 .0101 .995 .1 \quad 36.4 \quad 83.1 \quad 78.1 \quad 67.7 \quad 56.8 \quad 44.3 \quad 32.6 \quad 19.0$

083363.394 .6106 .0119 .6123 .2122 .3122 .4132 .2135 .1132 .4131 .9131 .6 $122.9112 .9109 .8106 .7 \quad 96.994 .991 .3 \quad 81.4 \quad 69.461 .7 \quad 43.3 \quad 27.4$

163351.379 .693 .8103 .8106 .6104 .2102 .8106 .3104 .1103 .8103 .2102 .1 $99.691 .890 .4 \quad 93.0 \quad 84.8 \quad 86.7 \quad 88.8 \quad 84.078 .064 .8 \quad 51.2 \quad 33.1$

243358.188 .699 .9104 .8104 .8102 .7 97.6104.3109.5118.8124.0127.5 122.5114 .0114 .5113 .0104 .5104 .3103 .092 .981 .266 .953 .334 .3

323369.0101 .4109 .8113 .0110 .4106 .4104 .7110 .2110 .3112 .5117 .7119 .3 115.2106 .0106 .8107 .399 .7103 .2106 .0100 .089 .583 .259 .239 .5

403356.087 .1100 .0108 .8112 .7110 .7113 .5121 .5128 .1132 .4142 .5141 .0 137.6127 .7124 .9122 .6114 .3112 .8107 .094 .984 .868 .654 .240 .5

483367.696 .4107 .0115 .1114 .0112 .9111 .5114 .4115 .0112 .3114 .1110 .0

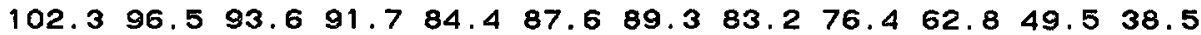

563347.576 .593 .3106 .2110 .7108 .9107 .2112 .3117 .0116 .0111 .8110 .1 101.292 .291 .188 .478 .277 .173 .064 .055 .044 .033 .319 .9

084150.178 .297 .6118 .1139 .0153 .1158 .8163 .0156 .2150 .5145 .0142 .6 $132.1119 .9119 .0118 .1106 .5102 .8 \quad 98.4 \quad 84.971 .562 .7 \quad 43.326 .7$

164175.1102 .7112 .0121 .0119 .9115 .4112 .6111 .1108 .9106 .2108 .4105 .7 99.2102 .1106 .7111 .1104 .2106 .3103 .090 .680 .364 .348 .839 .1

244163.294 .1104 .6110 .3108 .2103 .599 .2102 .2103 .8105 .5109 .2110 .6 108.0103 .3103 .4104 .999 .5103 .6107 .098 .186 .171 .956 .036 .0

324168.0100 .3109 .0112 .3109 .2104 .7102 .7113 .8120 .7127 .1130 .4131 .6 128.8120 .7121 .7120 .2111 .6110 .4107 .498 .585 .477 .958 .238 .1

404173.3104 .2111 .6118 .1116 .0110 .4109 .1108 .7109 .2107 .9111 .7110 .6 106.5103 .5104 .6105 .299 .9104 .7105 .295 .783 .968 .852 .837 .8

484151.878 .794 .0113 .9125 .1130 .4129 .5132 .1126 .2118 .6117 .9114 .4 112.1106 .9112 .0118 .6110 .3112 .0109 .497 .282 .466 .251 .932 .3

564140.367 .990 .1109 .9125 .6136 .0141 .9146 .3144 .1132 .4131 .3123 .6 $115.0103 .8101 .1101 .188 .885 .981 .5 \quad 72.259 .251 .435 .320 .6$

084962.488 .5100 .8113 .5116 .6117 .5118 .5119 .9115 .7111 .9109 .4105 .7

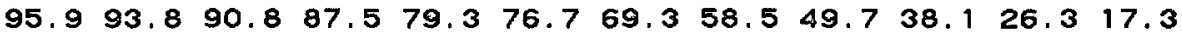

164976.0105 .7113 .6121 .1117 .8115 .1114 .6120 .3121 .9118 .5117 .4120 .3 112.1108 .5109 .3109 .8100 .199 .294 .982 .272 .355 .841 .830 .5

244978.3105 .4110 .8118 .7117 .0110 .0106 .9108 .7110 .8111 .2117 .9124 .3 120.0117 .9118 .3114 .6103 .0103 .1100 .087 .277 .461 .046 .035 .0

324966.798 .8110 .4111 .4108 .0106 .1103 .1110 .3108 .2110 .2114 .5115 .8 114.6107 .3108 .8110 .7103 .7107 .2111 .0103 .189 .983 .159 .936 .8

404984.5114 .3124 .5131 .7129 .4117 .8118 .9118 .6114 .8116 .3124 .6126 .1 118.1117 .9114 .6113 .2104 .6104 .297 .786 .576 .559 .444 .436 .3

484955.188 .6106 .2115 .0119 .1116 .6115 .2121 .2120 .1113 .6109 .5111 .0 $103.594 .995 .1 \quad 95.0 \quad 88.5 \quad 85.283 .4 \quad 74.963 .7 \quad 52.5 \quad 40.5 \quad 25.8$

165735.557 .875 .794 .4102 .1104 .6104 .6106 .7101 .594 .494 .991 .8

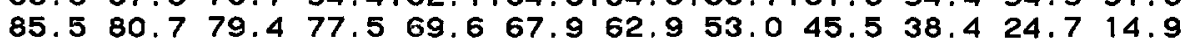

245754.582 .498 .2117 .9121 .4122 .4122 .2125 .0117 .5111 .7109 .9108 .2 $98.293 .791 .188 .980 .279 .374 .063 .755 .241 .5 \quad 30.519 .1$

325755.882 .295 .7110 .6113 .0114 .7119 .2121 .2110 .0101 .9101 .399 .5 89.9 $84.484 .483 .273 .874 .472 .5 \quad 63.0 \quad 54.948 .2 \quad 30.720 .4$

405754.187 .3112 .4140 .7143 .2139 .5137 .2138 .0128 .8123 .1121 .9121 .0 113.3106 .8104 .9104 .992 .089 .682 .869 .459 .549 .831 .218 .5 


\section{CYCLE 2 DATA}

DATASET 30, DECEMBER 16, 1976

\section{Reactor Conditions}

Core Average Exposure, $10730 \mathrm{MWd} / \mathrm{t}$

Core Thermal Power, 3256 MWT

Dome Pressure, P, 1021 psia

Core Flow, $102.3 \mathrm{Mlb} / \mathrm{hr}$

Inlet Subcooling at P, $23.7 \mathrm{Btu} / \mathrm{lb}$

Control Configuration

Legend: 48, Full Out; O, Full In.

$\begin{array}{lllllllllllllll}48 & 48 & 48 & 48 & 48 & 48 & 48 & 48 & 48 & 48 & 48 & 48 & 48 & 48 & 48 \\ 48 & 48 & 48 & 48 & 48 & 28 & 48 & 36 & 48 & 28 & 48 & 48 & 48 & 48 & 48 \\ 48 & 48 & 48 & 48 & 40 & 48 & 44 & 48 & 44 & 48 & 40 & 48 & 48 & 48 & 48 \\ 48 & 48 & 48 & 36 & 48 & 12 & 48 & 10 & 48 & 12 & 48 & 36 & 48 & 48 & 48 \\ 48 & 48 & 40 & 48 & 48 & 48 & 48 & 48 & 48 & 48 & 48 & 48 & 40 & 48 & 48 \\ 48 & 28 & 48 & 12 & 48 & 28 & 48 & 28 & 48 & 28 & 48 & 12 & 48 & 28 & 48 \\ 48 & 48 & 44 & 48 & 48 & 48 & 48 & 48 & 48 & 48 & 48 & 48 & 44 & 48 & 48 \\ 48 & 36 & 48 & 10 & 48 & 28 & 48 & 14 & 48 & 28 & 48 & 10 & 48 & 36 & 48 \\ 48 & 48 & 44 & 48 & 48 & 48 & 48 & 48 & 48 & 48 & 48 & 48 & 44 & 48 & 48 \\ 48 & 28 & 48 & 12 & 48 & 28 & 48 & 28 & 48 & 28 & 48 & 12 & 48 & 28 & 48 \\ 48 & 48 & 40 & 48 & 48 & 48 & 48 & 48 & 48 & 48 & 48 & 48 & 40 & 48 & 48 \\ 48 & 48 & 48 & 36 & 48 & 12 & 48 & 10 & 48 & 12 & 48 & 36 & 48 & 48 & 48 \\ 48 & 48 & 48 & 48 & 40 & 48 & 44 & 48 & 44 & 48 & 40 & 48 & 48 & 48 & 48 \\ 48 & 48 & 48 & 48 & 48 & 28 & 48 & 36 & 48 & 28 & 48 & 48 & 48 & 48 & 48 \\ 48 & 48 & 48 & 48 & 48 & 48 & 48 & 48 & 48 & 48 & 48 & 48 & 48 & 48 & 48\end{array}$

Axial TIP Distribution, Bottom To top of Core

See Figure 22

160949.580 .699 .7118 .1128 .1128 .8117 .9117 .1113 .2105 .6102 .7101 .6

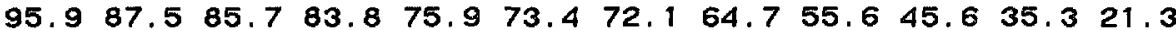
240949.381 .8101 .0114 .0117 .8116 .2109 .6112 .4110 .4112 .3115 .3115 .6 $109.4100 .1 \quad 98.0 \quad 96.0 \quad 86.9 \quad 85.2 \quad 82.5 \quad 75.4 \quad 64.6 \quad 53.1 \quad 42.2 \quad 26.4$

$320956.586,4104.0120 .1123 .8126 .3133 .2134 .7128 .1127 .3128 .2122 .4$ $114.9107 .0107 .5104 .1 \quad 92.5 \quad 92.1 \quad 87.8 \quad 77.2 \quad 68.161 .341 .6 \quad 28.1$

400934.962 .985 .199 .1116 .3124 .4118 .0119 .9120 .2118 .1118 .2123 .4 $119.6110 .3108 .4106 .3102 .692 .891 .185 .6 \quad 71.6 \quad 62.148 .6 \quad 34.6$

480965.597 .1109 .5123 .2122 .6119 .0116 .6116 .2111 .8107 .3105 .2104 .8

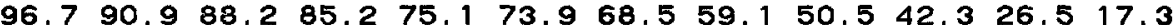

081745.975 .697 .4119 .3135 .4133 .5123 .8124 .4121 .0115 .2111 .4114 .1 $108.297 .998 .299 .289 .8 \quad 86.283 .3 \quad 74.4 \quad 61.5 \quad 51.2 \quad 38.924 .5$

161762.089 .7101 .7113 .8121 .8127 .3128 .6136 .3132 .7128 .9128 .0128 .0 123.4111 .9112 .0111 .8102 .5100 .997 .087 .275 .159 .245 .631 .0

241768.397 .1104 .5114 .0112 .4108 .9107 .7108 .7107 .1107 .8111 .6111 .4 $106.4100 .898 .696 .492 .096 .697 .688 .980 .4 \quad 70.649 .234 .7$

321770.395 .5103 .6109 .9107 .3101 .9102 .2104 .2102 .9104 .0107 .6106 .7

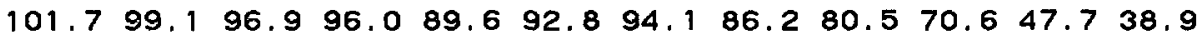

401761.792 .0102 .5113 .1114 .7114 .6113 .9115 .5112 .7110 .9114 .9114 .2 $109.3100 .299 .599 .791 .796 .898 .189 .980 .164 .5 \quad 51.2 \quad 33.6$

481759.288 .7104 .7125 .8138 .7144 .4148 .1150 .3141 .7132 .5131 .7127 .7 $119.0112 .1109 .0104 .496 .0 \quad 95.390 .8 \quad 80.4 \quad 71.862 .641 .930 .8$

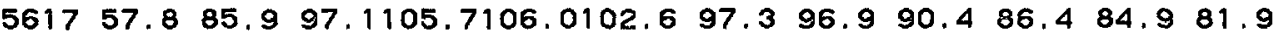
$\begin{array}{llllllllllll}75.0 & 71.8 & 69.9 & 66.6 & 60.7 & 59.6 & 55.8 & 47.6 & 41.7 & 35.7 & 22.5 & 16.4\end{array}$

082548.177 .499 .4114 .9123 .9123 .5120 .1124 .7120 .6124 .4128 .9131 .7 $126.3113 .8112 .4108 .399 .1 \quad 97.0 \quad 94.7 \quad 84.7 \quad 72.4 \quad 66.6 \quad 48.628 .0$

162563.592 .6102 .1109 .5108 .5105 .8106 .0109 .5108 .6110 .3116 .0115 .9 108.6105 .0101 .7101 .694 .599 .098 .688 .779 .263 .850 .035 .3 
$242563.6 \quad 88.595 .399 .596 .895 .192 .9101 .9104 .7113 .8125 .7131 .1$ $126.6123 .1120 .1116 .9104 .6103 .798 .1 \quad 86.075 .4 \quad 61.3 \quad 47.6 \quad 34.9$ 322568.894 .5100 .8106 .2102 .9100 .4102 .1106 .4111 .7120 .0132 .1136 .5 130.6130 .2125 .6124 .2110 .6107 .7102 .689 .478 .669 .245 .938 .0 402561.691 .398 .1105 .1101 .398 .297 .0101 .0103 .1108 .8119 .0123 .8 $119.3112 .6112 .7112 .1101 .7101 .0 \quad 97.1 \quad 87.1 \quad 76.964 .750 .2 \quad 33.3$

482558.090 .5109 .3123 .1123 .7121 .1119 .7120 .3113 .8113 .2114 .9114 .1 $105.5101 .1100 .398 .491 .297 .9 \quad 98.1 \quad 88.8 \quad 81.1 \quad 63.1 \quad 48.9 \quad 33.9$

562553.587 .4107 .3121 .9122 .8114 .6105 .7106 .0101 .7101 .9110 .2112 .0 $105.299 .498 .2 \quad 95.284 .1 \quad 80.4 \quad 75.4 \quad 66.3 \quad 56.6 \quad 43.9 \quad 32.1 \quad 18.7$

083353.687 .0104 .6122 .0128 .9133 .1133 .1134 .7131 .9122 .8123 .3122 .8 $114.6105 .4102 .6101 .292 .291 .4 \quad 86.677 .366 .760 .342 .027 .7$

$\begin{array}{lllllllllllllll}1633 & 58.8 & 84.9 & 94.1 & 99.4 & 99.7 & 97.3 & 95.4 & 98.2 & 97.3 & 98.4 & 98.7 & 98.6\end{array}$

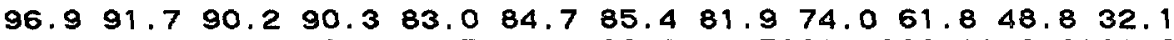

$243355.283 .6 \quad 94.5 \quad 99.799 .5 \quad 95.7 \quad 92.2 \quad 95.5101 .0108 .2119 .2126 .2$ $125.2118 .9117 .9116 .6105 .9103 .4100 .390 .077 .6 \quad 62.649 .732 .0$

323364.495 .2103 .8107 .1105 .1103 .1100 .9107 .3110 .0113 .3121 .7125 .4 123.4114 .9117 .6116 .1109 .0109 .5106 .896 .283 .076 .254 .935 .6

403365.694 .4102 .1109 .4108 .2105 .5105 .1111 .6116 .3122 .4136 .4139 .9 $135.8129 .8126 .5126 .2115 .4111 .4105 .5 \quad 92.481 .6 \quad 66.552 .538 .3$

$483358.888 .3102 .7114 .3117 .0115 .2111,5113.7108,8107.1107 .7106 .0$ $\begin{array}{llllllllllll}99.7 & 92.7 & 93.4 & 89.6 & 83.5 & 85.5 & 87.4 & 80.6 & 73.6 & 60.8 & 48.2 & 35.7\end{array}$

563350.484 .2103 .2117 .2117 .5118 .3112 .9113 .5111 .3104 .1100 .998 .1 $\begin{array}{llllllllllll}95.0 & 84.8 & 86.1 & 82.5 & 75.1 & 72.4 & 67.9 & 60.5 & 52.3 & 42.5 & 32.0 & 19.9\end{array}$

084151.280 .298 .9118 .6130 .8135 .5132 .0134 .1128 .9129 .0135 .1134 .8 $126.7116 .6113 .9111 .199 .8 \quad 97.9 \quad 92.8 \quad 80.2 \quad 69.1 \quad 61.041 .925 .9$

164172.598 .0108 .1117 .2118 .1116 .6116 .2115 .8113 .3111 .2113 .4109 .2

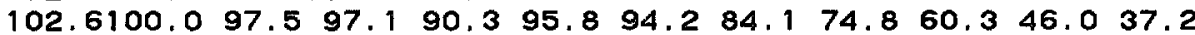

244159.990 .1100 .1106 .3105 .8101 .798 .399 .0103 .6110 .0121 .4128 .5 $125.7117 .7117 .8117 .7108 .1106 .1102 .292 .5 \quad 79.865 .951 .833 .7$

324161.691 .3102 .3106 .4104 .199 .197 .0102 .0107 .0114 .6126 .4134 .8 134.4124 .5124 .5122 .9111 .9107 .9104 .293 .680 .774 .554 .935 .8

404168.397 .6106 .9113 .1110 .1108 .7108 .6108 .6109 .5115 .4125 .4128 .1 122.1116 .1115 .5113 .5102 .2102 .397 .785 .676 .062 .347 .833 .1

484152.481 .097 .8116 .9127 .6128 .4128 .6130 .7121 .4117 .7119 .8115 .4 $108.7101 .5101 .8101 .496 .099 .398 .790 .680 .863 .4 \quad 48.6 \quad 31.7$

564148.181 .0100 .0118 .3123 .6120 .1112 .8109 .7107 .7108 .2112 .8116 .3 $110.4101 .5101 .3 \quad 97.287 .583 .780 .468 .958 .050 .935 .120 .1$

084972.398 .9110 .7121 .9121 .6119 .5115 .1114 .3107 .9103 .3101 .998 .5 $\begin{array}{llllllllllll}90.8 & 87.1 & 83.7 & 78.6 & 70.8 & 68.7 & 62.5 & 52.2 & 45.3 & 35.1 & 23.8 & 17.2\end{array}$

164962.485 .599 .2115 .9122 .3125 .7128 .4126 .9121 .6114 .8113 .9112 .0 $102.496 .997 .295 .586 .087 .784 .6 \quad 73.165 .350 .7 \quad 37.928 .9$

244961.289 .7105 .4118 .0118 .7112 .1106 .7108 .2105 .6101 .5104 .3102 .4

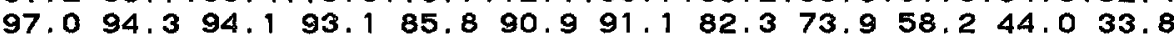

324953.286 .1103 .9112 .7114 .1114 .4112 .4113 .7110 .9108 .2108 .4108 .4

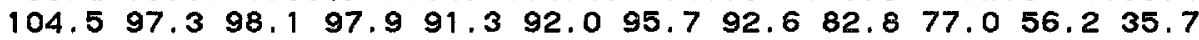

404963.790 .4103 .8120 .3125 .7126 .0121 .5121 .0115 .7107 .3109 .6106 .2 $99.493 .892 .190 .5 \quad 86.189 .090 .0 \quad 79.5 \quad 71.3 \quad 56.742 .6 \quad 33.5$

484958.494 .3115 .4127 .7133 .5133 .3125 .5125 .4117 .1109 .8104 .4103 .7

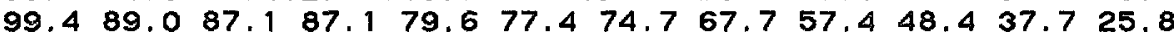

$\begin{array}{lllllllllll}1657 & 57.4 & 82.8 & 96.1104 .0102 .8 & 98.2 & 93.9 & 91.0 & 87.5 & 83.6 & 84.6 & 83.2\end{array}$

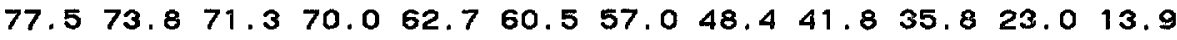

245762.792 .3104 .1116 .6116 .4107 .1104 .0100 .597 .398 .2104 .1105 .8

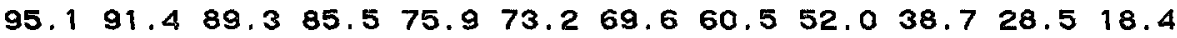

325763.393 .9105 .3118 .3117 .7115 .2112 .1111 .1104 .999 .398 .494 .6

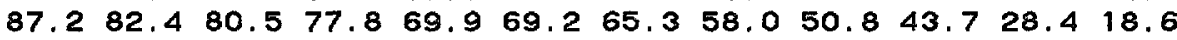

405759.991 .2106 .7121 .6118 .5111 .0108 .2108 .5106 .7107 .4117 .2118 .5 $109.3105 .7103 .299 .389 .185 .979 .366 .8 \quad 56.247 .829 .7 \quad 18.3$ 


\section{CYCLE 2 DATA}

\section{DATASET 31, DECEMBER 28, 1976}

\section{Reactor Conditions}

Core Average Exposure, $11030 \mathrm{MWd} / \mathrm{t}$

Core Thermal Power, 3285 MWT

Dome Pressure, P, 1018 psia

Core Flow, $106.5 \mathrm{Mlb} / \mathrm{hr}$

Inlet Subcooling at $\mathrm{P}, 23.1 \mathrm{Btu} / \mathrm{lb}$

Control Configuration

Legend: 48, Full Out; O, Full in.

$\begin{array}{lllllllllllllll}48 & 48 & 48 & 48 & 48 & 48 & 48 & 48 & 48 & 48 & 48 & 48 & 48 & 48 & 48 \\ 48 & 48 & 48 & 48 & 48 & 28 & 48 & 36 & 48 & 28 & 48 & 48 & 48 & 48 & 48 \\ 48 & 48 & 48 & 48 & 40 & 48 & 44 & 48 & 44 & 48 & 40 & 48 & 48 & 48 & 48 \\ 48 & 48 & 48 & 36 & 48 & 12 & 48 & 10 & 48 & 12 & 48 & 36 & 48 & 48 & 48 \\ 48 & 48 & 40 & 48 & 48 & 48 & 48 & 48 & 48 & 48 & 48 & 48 & 40 & 48 & 48 \\ 48 & 28 & 48 & 12 & 48 & 28 & 48 & 28 & 48 & 28 & 48 & 12 & 48 & 28 & 48 \\ 48 & 48 & 44 & 48 & 48 & 48 & 48 & 48 & 48 & 48 & 48 & 48 & 44 & 48 & 48 \\ 48 & 36 & 48 & 10 & 48 & 28 & 48 & 14 & 48 & 28 & 48 & 10 & 48 & 36 & 48 \\ 48 & 48 & 44 & 48 & 48 & 48 & 48 & 48 & 48 & 48 & 48 & 48 & 44 & 48 & 48 \\ 48 & 28 & 48 & 12 & 48 & 28 & 48 & 28 & 48 & 28 & 48 & 12 & 48 & 28 & 48 \\ 48 & 48 & 40 & 48 & 48 & 48 & 48 & 48 & 48 & 48 & 48 & 48 & 40 & 48 & 48 \\ 48 & 48 & 48 & 36 & 48 & 12 & 48 & 10 & 48 & 12 & 48 & 36 & 48 & 48 & 48 \\ 48 & 48 & 48 & 48 & 40 & 48 & 44 & 48 & 44 & 48 & 40 & 48 & 48 & 48 & 48 \\ 48 & 48 & 48 & 48 & 48 & 28 & 48 & 36 & 48 & 28 & 48 & 48 & 48 & 48 & 48 \\ 48 & 48 & 48 & 48 & 48 & 48 & 48 & 48 & 48 & 48 & 48 & 48 & 48 & 48 & 48\end{array}$

Axial TIP Distribution, Bottom To top of Core

See Figure 22

160949.482 .1100 .6119 .2126 .6123 .4117 .4116 .5112 .8106 .4102 .9102 .5

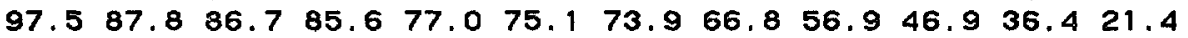
240949.581 .099 .2113 .6118 .1117 .2110 .0113 .3111 .3111 .8115 .1117 .7 $112.3101 .399 .4 \quad 97.3 \quad 87.986 .4 \quad 84.5 \quad 77.1 \quad 66.6 \quad 54.8 \quad 43.2 \quad 27.0$ 320955.786 .3104 .7118 .2122 .4127 .7130 .7132 .7127 .6126 .2126 .7124 .2 117.3108 .8107 .3106 .293 .393 .088 .678 .268 .561 .041 .928 .8 400934.562 .785 .298 .1116 .3122 .3119 .3117 .3117 .5116 .6116 .5122 .1 $122.0110 .8108 .3107 .7103 .694 .5 \quad 92.8 \quad 86.974 .163 .5 \quad 50.2 \quad 36.0$

480965.595 .2107 .3123 .3122 .7118 .1115 .4112 .5110 .6107 .9106 .3104 .6 $\begin{array}{llllllllllllll}97.0 & 91.6 & 90.1 & 86.7 & 76.8 & 75.5 & 69.5 & 59.6 & 51.7 & 42.9 & 27.2 & 17.7\end{array}$

081746.877 .998 .1118 .7134 .1135 .4126 .0128 .5123 .3117 .2112 .1116 .1 111.7101 .9101 .9101 .392 .989 .786 .276 .365 .653 .040 .224 .7

161762.691 .3103 .0115 .4119 .8127 .0129 .5139 .4135 .1132 .0132 .8132 .7 $124.0115 .3114 .3115 .3105 .1103 .999 .988 .0 \quad 77.1 \quad 60.6 \quad 46.8 \quad 32.2$

241766.996 .1106 .1112 .3112 .2108 .4110 .0111 .9109 .6108 .4112 .7114 .7 $108.6102 .6101 .3101 .895 .198 .9100 .991 .1 \quad 80.8 \quad 72.750 .135 .0$

321768.393 .7101 .5107 .9104 .7100 .9102 .2101 .6103 .2102 .3108 .9109 .1

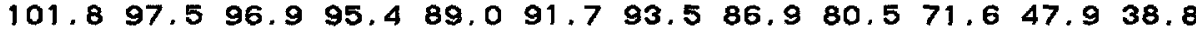

401759.989 .798 .6111 .0112 .6113 .1113 .6117 .4114 .4112 .3114 .2114 .9 109.1101 .2100 .399 .593 .296 .598 .389 .880 .465 .551 .234 .6

481758.487 .2101 .7122 .5135 .0142 .0147 .2146 .4140 .2133 .6133 .5128 .8 119.2112 .8111 .4106 .796 .796 .893 .682 .673 .364 .343 .0 30.8

$\begin{array}{lllllllllll}5617 & 58.5 & 84.8 & 95.7104 .8105 .0 & 97.7 & 97.6 & 95.3 & 90.5 & 84.3 & 85.1 & 82.6\end{array}$ $\begin{array}{llllllllllll}75.8 & 73.3 & 70.6 & 68.8 & 61.2 & 60.7 & 57.5 & 48.9 & 43.1 & 37.1 & 23.8 & 16.3\end{array}$

082548.678 .497 .7116 .0122 .9123 .5118 .0123 .0124 .7124 .0128 .8130 .8 $127.0116 .6112 .3109 .8100 .497 .996 .1 \quad 86.974 .067 .148 .829 .2$

162562.289 .498 .9107 .3107 .2105 .2105 .0109 .2110 .0111 .8114 .2116 .7 111.8104 .7104 .2104 .095 .899 .5100 .490 .980 .365 .751 .033 .3 
242560.786 .092 .297 .395 .994 .293 .4101 .7107 .2116 .5126 .5132 .6 $128.7124 .1122 .7117 .2106 .2104 .499 .1 \quad 86.7 \quad 77.361 .648 .034 .0$

322567.591 .996 .0104 .7102 .799 .9101 .7108 .0113 .3124 .6136 .0138 .7 131.7130 .7128 .3124 .9111 .2110 .1104 .390 .679 .369 .347 .437 .0

402560.288 .097 .4101 .5100 .198 .397 .6101 .9103 .5109 .1118 .4122 .6 $120.7115 .6114 .6112 .5103 .7103 .098 .5 \quad 89.3 \quad 79.265 .451 .434 .3$

482556.889 .7107 .1121 .1123 .0119 .9120 .4122 .2117 .8115 .7115 .0116 .0 $107.5102 .1100 .6100 .7 \quad 93.4 \quad 97.0 \quad 98.7 \quad 89.8 \quad 82.4 \quad 65.3 \quad 49.934 .6$

562554.589 .6111 .4123 .9120 .1111 .7104 .9108 .899 .3103 .8109 .1113 .4 $\begin{array}{llllllllllll}107.0 & 99.2 & 99.1 & 96.8 & 84.9 & 82.5 & 78.4 & 67.1 & 58.0 & 45.6 & 32.8 & 19.0\end{array}$

083350.982 .9106 .3121 .6127 .3134 .2131 .8135 .7130 .2125 .7123 .2123 .6 $115.7105 .8104 .9104 .393 .592 .0 \quad 90.0 \quad 78.367 .961 .4 \quad 42.927 .2$

$163357.0 \quad 82.592 .097 .398 .598 .197 .0100 .299 .498 .4101 .0100 .1$ $\begin{array}{lllllllllllll}96.8 & 92.2 & 90.8 & 91.1 & 83.9 & 86.3 & 86.4 & 81.5 & 74.9 & 62.3 & 49.1 & 31.7\end{array}$

243354.282 .993 .598 .198 .795 .693 .398 .6103 .1111 .0120 .0130 .3 128.9120 .3121 .1117 .3107 .7105 .3101 .590 .277 .763 .250 .232 .1

323362.190 .7100 .2103 .8103 .8101 .2102 .0107 .3110 .3114 .9121 .5127 .9 $123.7116 .2119 .6115 .9109 .4111 .2108 .896 .5 \quad 83.976 .654 .837 .7$

403362.889 .898 .8104 .0104 .7103 .9103 .4110 .1114 .0122 .5134 .0137 .9 134.8129 .2126 .9123 .1113 .6110 .3104 .492 .580 .965 .852 .037 .9

483356.488 .3101 .4112 .3115 .5114 .3113 .4114 .1111 .3110 .0110 .8106 .8

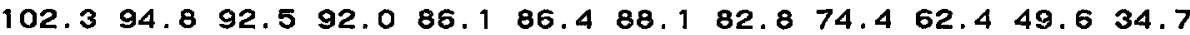

563351.386 .2104 .2119 .6120 .2117 .2112 .3115 .4109 .7108 .2101 .9101 .4 $\begin{array}{llllllllllllll}97.5 & 85.1 & 86.0 & 83.7 & 76.8 & 74.7 & 70.5 & 63.5 & 53.5 & 44.4 & 33.5 & 20.0\end{array}$

084150.979 .295 .9115 .6129 .2134 .5130 .5132 .0125 .5128 .3135 .0133 .1 $127.2116 .3116 .2111 .9100 .6 \quad 96.8 \quad 93.4 \quad 83.4 \quad 70.8 \quad 63.0 \quad 43.826 .7$

164168.494 .8104 .3115 .2117 .9114 .2114 .8117 .3113 .1111 .5112 .9110 .1 $104.598 .197 .896 .091 .395 .495 .4 \quad 85.977 .061 .4 \quad 47.3 \quad 36.9$

244158.787 .398 .0104 .7104 .8102 .197 .9102 .3104 .8111 .6123 .5127 .2 126.6118 .7119 .2118 .1108 .3107 .0103 .893 .880 .567 .453 .433 .7

324159.989 .099 .0104 .6103 .199 .898 .9104 .1108 .4117 .8128 .2136 .8 134.9126 .0125 .5125 .4112 .9109 .1106 .295 .281 .475 .456 .036 .5

404165.993 .4103 .4111 .0109 .6106 .8105 .5109 .2111 .2114 .2128 .7130 .8 $123.6118 .6115 .2116 .3104 .2103 .199 .588 .477 .563 .648 .5 \quad 32.8$

484152.980 .296 .0114 .2124 .7128 .7127 .3128 .0124 .2116 .2116 .8116 .6 111.0101 .5103 .2102 .795 .999 .8101 .592 .182 .366 .350 .132 .3

564148.482 .3101 .0120 .4124 .5119 .5111 .9108 .6107 .6109 .7115 .1117 .5 $115.0104 .8104 .3100 .089 .587 .882 .172 .158 .953 .3 \quad 36.821 .3$

084970.196 .2109 .8120 .0119 .0118 .2114 .1113 .8107 .6103 .2102 .399 .6

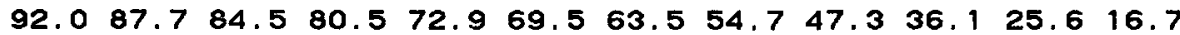

164959.685 .198 .5115 .9124 .3125 .4127 .8126 .5121 .9115 .4117 .1111 .5 $105.899 .397 .695 .8 \quad 88.388 .6 \quad 85.4 \quad 76.0 \quad 66.5 \quad 52.1 \quad 39.828 .8$

244958.387 .0103 .4117 .8117 .6113 .1111 .6111 .9106 .4102 .9105 .5104 .8 $99.494 .6 \quad 93.0 \quad 94.1 \quad 87.0 \quad 90.5 \quad 92.5 \quad 82.975 .460 .4 \quad 46.3 \quad 32.1$

324951.883 .9100 .7111 .4116 .3111 .5107 .6111 .9110 .7109 .0110 .4109 .0 $106.199 .899 .7100 .493 .394 .896 .694 .584 .7 \quad 79.158 .3 \quad 36.6$

404960.566 .3101 .3120 .2125 .1123 .2122 .9119 .2113 .4107 .5111 .5106 .4

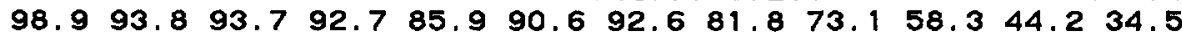

484959.194 .1114 .7127 .2130 .4130 .9122 .3123 .2117 .2110 .3105 .7104 .3

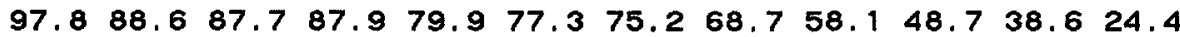

$165755.981 .6 \quad 94.2106 .3103 .499 .2 \quad 94.1 \quad 94.3 \quad 89.8 \quad 84.285 .084 .8$

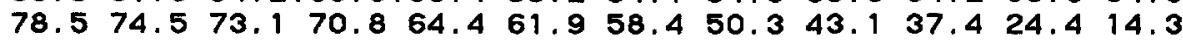

245761.792 .8105 .7118 .8114 .9107 .1102 .2100 .698 .397 .4105 .2106 .1

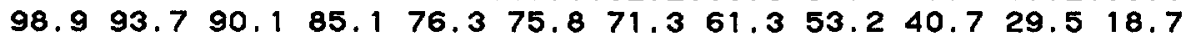

325762.595 .9106 .8118 .1116 .4116 .7112 .5114 .2105 .898 .999 .496 .3 $90.183 .8 \quad 81.178 .970 .970 .5 \quad 67.258 .7 \quad 51.6 \quad 44.6 \quad 29.1 \quad 18.1$

405757.688 .4105 .0116 .1116 .4111 .4109 .1108 .7108 .1108 .9118 .8119 .5 $113.0106 .8104 .4101 .690 .687 .781 .269 .1 \quad 59.549 .531 .918 .5$ 


\section{CYCLE 2 DATA}

DATASET 32, JANUARY 19, 1977

\section{Reactor Conditions}

Core Average Exposure, $11260 \mathrm{MWd} / \mathrm{t}$

Core Thermal Power, 3276 MWT

Dome Pressure, P, 1019 psia

Core Flow, 101.1 Mlb/hr

Inlet Subcooling at P, $24.4 \mathrm{Btu} / \mathrm{lb}$

Control Configuration

Legend: 48, Full Out; O, Full In.

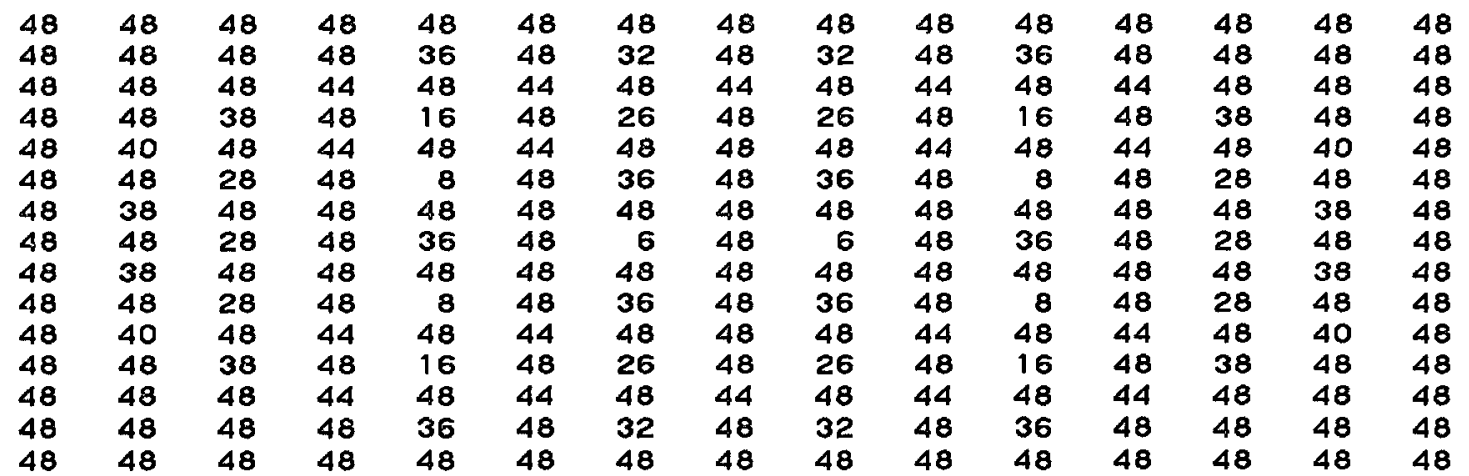

Axial TIP Distribution, Bottom To top of Core See Figure 22

160945.977 .8102 .0121 .2131 .7130 .6127 .6131 .1127 .4117 .2113 .9114 .1 106.396 .495 .294 .088 .084 .483 .074 .463 .452 .140 .623 .3 240957.495 .9116 .4133 .3134 .8130 .4126 .1132 .0139 .3136 .7136 .2136 .2 $128.5117 .7115 .9114 .2102 .6 \quad 99.4 \quad 94.7 \quad 83.1 \quad 71.1 \quad 57.4 \quad 45.729 .1$

320963.897 .0116 .1128 .9130 .3126 .2129 .2138 .6146 .7148 .5152 .1153 .2 $145.7134 .3133 .2129 .8113 .7108 .5101 .3 \quad 87.975 .265 .545 .0 \quad 31.5$

400942.979 .5111 .5129 .0143 .0145 .6138 .7146 .5146 .4137 .7136 .0136 .8 $130.9120 .8119 .3117 .0111 .4103 .4101 .6 \quad 94.3 \quad 79.0 \quad 67.5 \quad 52.9 \quad 36.5$

$480950.682 .6103 .4121 .3127 .5129 .0126 .9125,4120.4116 .8116 .0113 .3$ $105.8101 .3 \quad 98.3 \quad 94.4 \quad 86.0 \quad 84.2 \quad 79.1 \quad 66.8 \quad 58.1 \quad 48.3 \quad 30.520 .4$

081735.161 .583 .4109 .2137 .0150 .2146 .0150 .6146 .3136 .5133 .8134 .0 $126.9114 .5117 .0114 .1103 .298 .8 \quad 94.3 \quad 84.6 \quad 68.956 .8 \quad 42.8 \quad 26.4$

161758.492 .7112 .6128 .7133 .8132 .3128 .4132 .3128 .2125 .4126 .6124 .5 118.0114 .5116 .1122 .0114 .2115 .0110 .297 .384 .666 .151 .433 .8

241764.396 .9114 .8128 .4132 .1131 .3128 .6132 .4130 .3130 .9140 .6148 .6 143.4134 .7134 .2129 .6117 .2115 .4107 .694 .081 .273 .149 .337 .0

321776.6105 .2119 .7125 .6125 .2122 .8124 .5127 .8125 .9132 .3144 .2147 .4 138.4135 .5132 .4127 .2114 .1110 .8105 .091 .180 .668 .546 .239 .0

401756.491 .8110 .7125 .9128 .5125 .9124 .1126 .4122 .5122 .4124 .9125 .3 118.9112 .3111 .9115 .8109 .2112 .2108 .196 .285 .268 .553 .8 35.8

$481762.3100 .1123 .1145 .5155,5158.6155,7152,9148.2140 .8142 .4139 .4$ $130.0124 .3120 .3119 .2107 .0106 .2100 .788 .5 \quad 77.868 .045 .3 \quad 33.8$

561739.861 .679 .5103 .1115 .7121 .1120 .9117 .0111 .7102 .2101 .898 .6

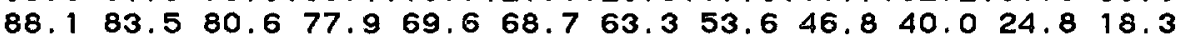

082549.177 .193 .7112 .1126 .8133 .3138 .3147 .1145 .6147 .6155 .2158 .7 $153.2139 .4132 .4129 .4114 .5107 .9104 .8 \quad 92.676 .768 .949 .2 \quad 30.2$

162571.6105 .1116 .8126 .6126 .6125 .5127 .7132 .2132 .2132 .6138 .6133 .9 127.9118 .2115 .5112 .8100 .098 .692 .984 .676 .363 .649 .833 .4 
242566.895 .4105 .0115 .5120 .1127 .5131 .8140 .3135 .5131 .5134 .2131 .0 $121.4113 .7112 .4108 .795 .494 .1 \quad 89.378 .871 .958 .7 \quad 46.0 \quad 32.9$

322576.3106 .2115 .8127 .2133 .3139 .5147 .2149 .8147 .5143 .6145 .0139 .2 129.4124 .3120 .3115 .2101 .899 .092 .480 .472 .764 .144 .035 .8

402565.598 .0110 .0117 .6119 .6120 .0120 .4125 .9123 .7119 .0120 .1117 .1 $110.8103 .9101 .0101 .0 \quad 90.589 .387 .181 .1 \quad 74.864 .450 .735 .8$

482574.5108 .5120 .4130 .9133 .7130 .8133 .3137 .1139 .3146 .8154 .4153 .1 146.0137 .6131 .7129 .1112 .8110 .0105 .990 .579 .261 .847 .935 .1

562556.895 .2117 .3137 .8149 .1150 .5146 .3140 .1134 .7128 .0125 .6125 .3 $118.4110 .3107 .6104 .5 \quad 91.6 \quad 86.8 \quad 81.4 \quad 70.460 .2 \quad 45.9 \quad 33.520 .6$

083353.380 .093 .3107 .2119 .5128 .0131 .9137 .7136 .9140 .9150 .3152 .6 $147.0132 .7130 .2126 .0111 .4107 .198 .4 \quad 85.071 .366 .4 \quad 44.029 .2$

163367.996 .6105 .8113 .5117 .8124 .4130 .6139 .5137 .4133 .2134 .6132 .8 $124.0113 .8110 .8108 .7 \quad 97.0 \quad 94.5 \quad 89.2 \quad 79.3 \quad 69.8 \quad 57.1 \quad 45.4 \quad 31.1$

243361.594 .0106 .1115 .3117 .7118 .1114 .6119 .6119 .3114 .9114 .7115 .1 $108.7100 .0 \quad 97.3 \quad 94.3 \quad 86.3 \quad 84.1 \quad 81.2 \quad 73.8 \quad 67.7 \quad 58.4 \quad 49.6 \quad 30.5$

323369.9102 .1113 .41205122 .7125 .0125 .1129 .0126 .9126 .8126 .3124 .1 117.5107 .4104 .5100 .590 .487 .383 .475 .369 .368 .951 .033 .4

403369.7100 .1111 .6118 .7125 .0133 .3143 .0152 .5149 .6145 .4147 .0144 .5 138.1126.4123.1117.8107.1102.1 96.3 85.4 75.8 63.0 49.9 36.1

483374.0103 .6112 .4120 .1120 .9122 .1124 .4129 .4131 .5139 .4149 .8150 .3 $141.3130 .9123 .9119 .4105 .9102 .8 \quad 96.5 \quad 84.973 .1 \quad 59.3 \quad 46.935 .9$

563355.291 .0111 .5131 .2138 .6139 .7135 .3135 .1129 .5122 .3119 .2116 .3 $112.2101 .2100 .098 .0 \quad 86.4 \quad 82.8 \quad 76.8 \quad 66.957 .145 .5 \quad 34.920 .6$

084155.690 .1109 .2132 .9148 .2155 .9151 .6153 .4150 .5148 .6156 .2153 .9 $146.0134 .1131 .4128 .7113 .9106 .3101 .5 \quad 86.5 \quad 73.464 .4 \quad 44.527 .3$

164169.9102 .6119 .7134 .4135 .4130 .6129 .4128 .4123 .5119 .8123 .4119 .9 $111.6107 .7106 .0102 .994 .3 \quad 95.5 \quad 91.0 \quad 83.977 .862 .6 \quad 47.5 \quad 39.0$

244154.486 .7106 .7122 .1129 .9135 .9138 .1144 .2146 .3144 .5146 .5143 .4 139.2127 .1126 .6125 .7113 .4109 .2104 .193 .980 .166 .752 .932 .6

324164.396 .6110 .6120 .2124 .1129 .1136 .4148 .3148 .5151 .0150 .8152 .6 148.5136 .8134 .7131 .3121 .5111 .0105 .292 .978 .671 .752 .835 .2

404158.288 .7106 .3120 .7124 .8127 .3125 .5129 .9128 .0123 .3126 .6123 .3 $116.6112 .1109 .3108 .297 .396 .493 .385 .377 .964 .650 .0 \quad 34.9$

484157.192 .1115 .0133 .1141 .9140 .0136 .7139 .3135 .4138 .6145 .2147 .1 140.7131 .2128 .9127 .5115 .1112 .9108 .794 .281 .866 .550 .631 .8

564149.786 .6116 .1142 .7162 .6162 .4153 .4151 .5143 .9133 .7131 .0128 .3 $121.0110 .5107 .0106 .394 .590 .083 .173 .860 .552 .3 \quad 36.820 .5$

084949.070 .485 .4107 .1122 .5131 .3135 .1135 .0126 .3120 .0119 .8114 .3 $104.9100 .3 \quad 96.3 \quad 92.2 \quad 82.4 \quad 79.2 \quad 72.4 \quad 60.8 \quad 52.8 \quad 40.3 \quad 27.5 \quad 19.6$

164970.6104 .7120 .7134 .1132 .9126 .8123 .8118 .6115 .6110 .8112 .2109 .5 $103.1100 .0102 .4106 .6102 .0102 .999 .3 \quad 86.5 \quad 74.259 .643 .832 .7$

244975.5108 .9124 .6141 .0137 .4129 .1121 .9124 .6126 .8126 .9135 .4142 .2 137.2133 .8132 .2128 .6114 .2109 .9102 .588 .977 .059 .844 .534 .9

324961.097 .3116 .3126 .8127 .1124 .4121 .2127 .9130 .3133 .4143 .1153 .1 $153.6145 .7145 .4140 .5127 .3120 .4113 .6103 .287 .679 .857 .3 \quad 36.1$

404975.9110 .8130 .1140 .6139 .8132 .8130 .6129 .9122 .8117 .2120 .2118 .3 109.1105.7108.4111.1104.6105.2100.2 87.3 76.5 60.6 45.2 36.8

484946.782 .6109 .7130 .6140 .9141 .3137 .6139 .8126 .9121 .1113 .7113 .6 $\begin{array}{llllllllllll}107.1 & 95.8 & 97.1 & 98.6 & 91.9 & 88.6 & 86.0 & 77.5 & 65.7 & 56.4 & 43.6 & 28.5\end{array}$

$165741.562 .874 .3 \quad 87.296 .0103 .4112 .7118 .4110 .2103 .7103 .399 .3$

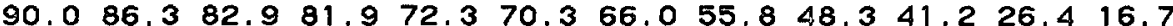
245773.4107 .1120 .9130 .2123 .1117 .7118 .2124 .6129 .6122 .3125 .0121 .8 $110.2103 .6100 .4 \quad 95.484 .781 .5 \quad 77.2 \quad 66.1 \quad 56.0 \quad 42.1 \quad 30.4 \quad 20.4$

325775.3109 .6123 .0132 .0123 .6115 .1111 .7117 .6120 .3116 .7118 .3116 .4 $106.698 .9 \quad 95.992 .981 .680 .075 .7 \quad 65.2 \quad 56.7 \quad 48.5 \quad 31.221 .0$

405765.8101 .0119 .4139 .6143 .3144 .9145 .3149 .9146 .2136 .9136 .9131 .1 121.8114 .3113 .8110 .298 .294 .886 .174 .162 .851 .633 .220 .6 


\section{CYCLE 2 DATA}

DATASET 33, JANUARY 26, 1977

\section{Reactor Conditions}

Core Average Exposure, $11420 \mathrm{MWd} / \mathrm{t}$

Core Thermal Power, 3273 MWT

Dome Pressure, $\mathrm{P}, 1019$ psia

Core Flow, 104.0 Mlb/hr

Inlet Subcooling at P, $23.6 \mathrm{Btu} / \mathrm{lb}$

Control Configuration

Legend: 48, Full Out; O, Full In.

$\begin{array}{rrrrrrrrrrrrrrr}48 & 48 & 48 & 48 & 48 & 48 & 48 & 48 & 48 & 48 & 48 & 48 & 48 & 48 & 48 \\ 48 & 48 & 48 & 48 & 36 & 48 & 32 & 48 & 32 & 48 & 36 & 48 & 48 & 48 & 48 \\ 48 & 48 & 48 & 44 & 48 & 44 & 48 & 44 & 48 & 44 & 48 & 44 & 48 & 48 & 48 \\ 48 & 48 & 38 & 48 & 16 & 48 & 26 & 48 & 26 & 48 & 16 & 48 & 38 & 48 & 48 \\ 48 & 40 & 48 & 44 & 48 & 44 & 48 & 48 & 48 & 44 & 48 & 44 & 48 & 40 & 48 \\ 48 & 48 & 28 & 48 & 8 & 48 & 36 & 48 & 36 & 48 & 8 & 48 & 28 & 48 & 48 \\ 48 & 38 & 48 & 48 & 48 & 48 & 48 & 48 & 48 & 48 & 48 & 48 & 48 & 38 & 48 \\ 48 & 48 & 28 & 48 & 36 & 48 & 6 & 48 & 6 & 48 & 36 & 48 & 28 & 48 & 48 \\ 48 & 38 & 48 & 48 & 48 & 48 & 48 & 48 & 48 & 48 & 48 & 48 & 48 & 38 & 48 \\ 48 & 48 & 28 & 48 & 8 & 48 & 36 & 48 & 36 & 48 & 8 & 48 & 28 & 48 & 48 \\ 48 & 40 & 48 & 44 & 48 & 44 & 48 & 48 & 48 & 44 & 48 & 44 & 48 & 40 & 48 \\ 48 & 48 & 38 & 48 & 16 & 48 & 26 & 48 & 26 & 48 & 16 & 48 & 38 & 48 & 48 \\ 48 & 48 & 48 & 44 & 48 & 44 & 48 & 44 & 48 & 44 & 48 & 44 & 48 & 48 & 48 \\ 48 & 48 & 48 & 48 & 36 & 48 & 32 & 48 & 32 & 48 & 36 & 48 & 48 & 48 & 48 \\ 48 & 48 & 48 & 48 & 48 & 48 & 48 & 48 & 48 & 48 & 48 & 48 & 48 & 48 & 48\end{array}$

Axial TIP Distribution, Bottom To top of Core See Figure 22

160944.778 .3102 .4118 .7129 .2130 .5128 .0129 .0126 .9117 .9115 .8114 .0

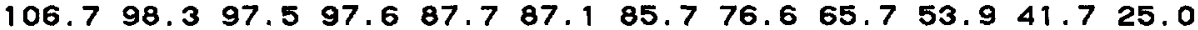

240956.894 .5115 .2130 .3133 .1126 .7127 .0136 .3141 .7140 .9139 .0139 .0 132.1119 .8118 .7116 .3104 .3101 .395 .785 .772 .959 .447 .029 .2

320961.396 .4115 .6132 .2127 .5131 .4130 .8141 .9146 .5145 .6151 .6151 .2 $147.0137,8137.0131 .0116 .7109 .6104 .090 .576 .968 .145 .931 .5$

400941.679 .6110 .7127 .7140 .1142 .5143 .9144 .6145 .1142 .2135 .3136 .7 133.7122 .3119 .1119 .8114 .5104 .7103 .396 .381 .769 .354 .238 .7

480948.380 .4100 .4119 .5124 .8126 .8124 .9125 .4122 .8118 .1116 .9113 .4 $107.4104 .099 .297 .288 .986 .480 .368 .859 .849 .5 \quad 31.120 .0$

081734.660 .981 .6106 .8132 .3144 .9146 .5149 .6139 .7136 .2134 .0134 .8 $129.0118 .0117 .5117 .5106 .2100 .996 .986 .7 \quad 71.7 \quad 58.744 .527 .5$

161756.889 .2107 .9128 .3131 .8130 .9128 .2133 .0128 .3124 .0124 .9126 .5 $121.2117 .5119 .7124 .3117 .7117 .7112 .5 \quad 99.8 \quad 86.767 .752 .834 .8$

241762.494 .7111 .5125 .9129 .6130 .7130 .1132 .6131 .7133 .9141 .9152 .3 145.3135 .7138 .1131 .8118 .5117 .9110 .796 .483 .773 .750 .637 .5

321774.3103 .5113 .4124 .7124 .9122 .7123 .7127 .1127 .5137 .2144 .1151 .2 144.3136 .6133 .7129 .4116 .1113 .3107 .193 .081 .570 .047 .240 .1

401755.090 .4106 .5121 .7126 .6124 .7123 .3125 .3125 .3121 .4123 .8125 .0 121.6113 .6113 .6118 .6111 .1114 .1109 .997 .386 .070 .354 .835 .4

481760.397 .3120 .3143 .3153 .7155 .4154 .0153 .9149 .5142 .3145 .3142 .8 131.8124 .9123 .6121 .7110 .1108 .1102 .891 .279 .669 .947 .035 .5

561738.659 .278 .299 .7113 .4117 .6118 .0115 .6110 .3104 .0103 .098 .2 $90.985 .6 \quad 81.6 \quad 79.3 \quad 71.5 \quad 68.5 \quad 64.4 \quad 54.8 \quad 48.7 \quad 40.8 \quad 25.9 \quad 18.4$

082548.175 .991 .9111 .0123 .8133 .9138 .8145 .5145 .5148 .9159 .2162 .8 153.4139 .8138 .4131 .5118 .5113 .0107 .194 .280 .872 .351 .731 .8

162568.4100 .2113 .0120 .3125 .8123 .7125 .1130 .8135 .9133 .1135 .6135 .2 $131.5120 .6117 .1114 .2100 .699 .494 .787 .378 .764 .951 .3 \quad 33.6$ 
242565.592 .7103 .2113 .2118 .9126 .3130 .5138 .3138 .8134 .4134 .9131 .7 $123.4116 .7115 .1110 .3 \quad 97.7 \quad 96.5 \quad 92.3 \quad 81.6 \quad 73.260 .147 .3 \quad 34.7$

322574.0102 .1111 .8123 .8130 .4136 .3144 .7152 .0150 .8142 .0143 .1140 .3 $130.0123 .8120 .9115 .3101 .9100 .294 .0 \quad 82.373 .165 .044 .835 .8$

402563.594 .5105 .9115 .9118 .4119 .5119 .5124 .1122 .1120 .6120 .1118 .5 $111.5105 .2103 .5100 .4 \quad 91.2 \quad 90.3 \quad 88.1 \quad 82.6 \quad 75.7 \quad 64.7 \quad 51.2 \quad 35.4$

482570.8102 .5114 .6125 .6128 .9128 .5132 .7135 .4140 .4143 .3154 .9154 .5 $146.8135 .5132 .5128 .7112 .9111 .9105 .1 \quad 92.5 \quad 82.0 \quad 63.7 \quad 49.2 \quad 34.9$

562556.394 .1118 .9135 .3144 .2148 .4139 .9138 .7136 .6127 .6125 .8124 .5 $117.7109 .6108 .2104 .5 \quad 93.6 \quad 88.1 \quad 83.4 \quad 70.5 \quad 61.8 \quad 48.0 \quad 34.8 \quad 19.4$

083352.378 .391 .8105 .3118 .1126 .0131 .4137 .8139 .3143 .4152 .4155 .8 149.1137 .3132 .2128 .2113 .8109 .4102 .186 .874 .266 .445 .829 .8

163365.294 .2103 .6112 .4116 .8123 .4132 .4140 .4139 .0136 .7136 .0135 .3 124.3116 .2113 .2110 .398 .796 .191 .681 .071 .358 .146 .432 .0

243358.891 .5104 .1111 .6115 .1114 .6114 .8118 .4117 .1116 .2117 .4116 .7 $108.9100 .798 .496 .8 \quad 87.8 \quad 85.682 .075 .7 \quad 68.959 .8 \quad 49.031 .3$

323366.998 .6109 .5117 .9120 .8122 .5122 .2127 .6127 .0127 .5128 .1125 .5 $118.1109 .2106 .6102 .5 \quad 92.0 \quad 88.8 \quad 84.1 \quad 77.0 \quad 70.8 \quad 69.6 \quad 52.6 \quad 34.4$

403366.596 .5106 .9115 .9123 .2130 .5138 .4150 .0150 .2146 .5147 .3143 .2 $138.5126 .5123 .4120 .2107 .6104 .497 .685 .976 .764 .250 .2 \quad 37.0$

483370.098 .5106 .4115 .9117 .9117 .0120 .2128 .5130 .5138 .7147 .2146 .6 $139.6130 .0124 .5120 .2105 .7104 .5 \quad 97.1 \quad 85.1 \quad 74.6 \quad 60.7 \quad 47.836 .2$

563353.289 .1110 .2126 .0134 .9136 .8133 .6135 .5128 .1121 .9118 .6118 .2 $110.5101 .8102 .1 \quad 99.0 \quad 86.6 \quad 83.5 \quad 78.2 \quad 67.7 \quad 59.246 .7 \quad 36.021 .6$

084154.087 .8107 .3130 .4144 .8151 .3150 .4153 .6149 .6149 .1157 .6158 .0 $148.9137 .2134 .1131 .4116 .3111 .8103 .5 \quad 90.075 .766 .546 .327 .7$

164165.295 .4113 .1129 .7131 .6127 .8126 .8127 .8124 .6120 .8124 .5121 .1 $114.4108 .9106 .7104 .7 \quad 96.5 \quad 97.2 \quad 94.5 \quad 86.0 \quad 79.6 \quad 64.7 \quad 49.6 \quad 38.5$

244151.883 .0102 .6116 .3125 .6130 .5134 .2144 .6144 .0142 .6143 .5145 .2 138.6128 .8127 .0125 .3112 .7109 .3104 .293 .480 .466 .552 .733 .9

324161.294 .1107 .4117 .0122 .4128 .1135 .1149 .6150 .8148 .6149 .9153 .3 149.0137 .1136 .8134 .9117 .7111 .6105 .493 .579 .472 .954 .135 .1

404155.688 .1103 .6117 .8124 .1124 .0126 .7129 .6126 .4124 .8126 .5125 .4 118.4112 .6110 .7110 .498 .797 .295 .587 .379 .565 .951 .834 .8

484155.489 .1110 .2129 .8138 .2137 .9136 .9136 .7135 .9138 .2145 .4146 .6 $140.1132 .8130 .7130 .6116 .7114 .4109 .297 .083 .465 .5 \quad 51.6 \quad 33.6$

$564148.784 .4109 .8138 .6159 .1157 .8153 .0147 .8142 .7132,4133,4128.3$ $120.7112 .7108 .3106 .5 \quad 95.7 \quad 91.6 \quad 86.3 \quad 74.2 \quad 62.0 \quad 54.2 \quad 37.4 \quad 21.4$

084945.366 .981 .9101 .4116 .4124 .9131 .7130 .5124 .7121 .0119 .1114 .0 $105.1100 .8 \quad 98.1 \quad 93.7 \quad 83.2 \quad 81.5 \quad 74.4 \quad 62.5 \quad 54.3 \quad 41.4 \quad 29.1 \quad 19.3$

164966.299 .5116 .0129 .7128 .8126 .4118 .3119 .2116 .0111 .8112 .3110 .4 103.4102 .0104 .2108 .1103 .6106 .2101 .889 .078 .161 .346 .032 .4

244968.7102 .2119 .9135 .0131 .5124 .3121 .3124 .7127 .2127 .8136 .2141 .5 137.8135 .5131 .6130 .1114 .3110 .5104 .990 .178 .761 .847 .233 .5

324958.593 .8110 .7123 .0122 .9120 .5120 .0127 .7130 .4132 .8143 .1155 .3 153.7145 .6144 .5141 .4126 .7121 .0115 .2104 .587 .279 .457 .436 .5

404971.3108 .5126 .0143 .2140 .3132 .1129 .7128 .9123 .1115 .3123 .1120 .6 111.5108 .8110 .8114 .6107 .6109 .8104 .190 .679 .261 .448 .136 .5

484946.279 .1107 .0128 .3141 .3141 .3137 .0138 .1125 .6121 .4114 .5111 .8 $107.298 .598 .0 \quad 99.292 .8 \quad 90.5 \quad 86.4 \quad 78.967 .3 \quad 55.9 \quad 44.0 \quad 28.6$

165739.660 .573 .184 .992 .1102 .0110 .6113 .9109 .4105 .0102 .699 .8 $\begin{array}{llllllllllll}91.4 & 87.3 & 84.4 & 82.0 & 73.2 & 71.8 & 67.3 & 57.9 & 49.5 & 42.7 & 27.4 & 16.9\end{array}$

245768.8102 .1117 .9129 .7127 .6120 .5116 .9124 .7125 .9123 .6124 .4121 .3 111.2104 .6102 .397 .986 .784 .179 .068 .358 .744 .232 .819 .3

325772.3105 .6119 .1129 .9126 .0117 .3111 .0118 .5119 .1117 .7116 .5116 .3 $108.8100 .197 .793 .982 .381 .876 .5 \quad 67.458 .149 .932 .820 .2$

405763.499 .8118 .9134 .9140 .3142 .4145 .2150 .8143 .3139 .2134 .6129 .6 121.8115 .2112 .4110 .898 .895 .389 .074 .063 .553 .033 .620 .3 


\section{CYCLE 2 DATA}

\section{DATASET 34, FEBRUARY 2, 1977}

\section{Reactor Conditions}

Core Average Exposure, $11570 \mathrm{MWd} / \mathrm{t}$

Core Thermal Power, 3260 MWT

Dome Pressure, $P, 1018$ psia

Core Flow, 106.0 Mlb/hr

Inlet Subcooling at $\mathrm{P}, 23.0 \mathrm{Btu} / \mathrm{lb}$

Control Configuration

Legend: 48, Full Out; O, Full In.

$\begin{array}{rrrrrrrrrrrrrrr}48 & 48 & 48 & 48 & 48 & 48 & 48 & 48 & 48 & 48 & 48 & 48 & 48 & 48 & 48 \\ 48 & 48 & 48 & 48 & 36 & 48 & 32 & 48 & 32 & 48 & 36 & 48 & 48 & 48 & 48 \\ 48 & 48 & 48 & 44 & 48 & 44 & 48 & 44 & 48 & 44 & 48 & 44 & 48 & 48 & 48 \\ 48 & 48 & 38 & 48 & 16 & 48 & 26 & 48 & 26 & 48 & 16 & 48 & 38 & 48 & 48 \\ 48 & 40 & 48 & 44 & 48 & 44 & 48 & 48 & 48 & 44 & 48 & 44 & 48 & 40 & 48 \\ 48 & 48 & 28 & 48 & 8 & 48 & 36 & 48 & 36 & 48 & 8 & 48 & 28 & 48 & 48 \\ 48 & 38 & 48 & 48 & 48 & 48 & 48 & 48 & 48 & 48 & 48 & 48 & 48 & 38 & 48 \\ 48 & 48 & 28 & 48 & 36 & 48 & 6 & 48 & 6 & 48 & 36 & 48 & 28 & 48 & 48 \\ 48 & 38 & 48 & 48 & 48 & 48 & 48 & 48 & 48 & 48 & 48 & 48 & 48 & 38 & 48 \\ 48 & 48 & 28 & 48 & 8 & 48 & 36 & 48 & 36 & 48 & 8 & 48 & 28 & 48 & 48 \\ 48 & 40 & 48 & 44 & 48 & 44 & 48 & 48 & 48 & 44 & 48 & 44 & 48 & 40 & 48 \\ 48 & 48 & 38 & 48 & 16 & 48 & 26 & 48 & 26 & 48 & 16 & 48 & 38 & 48 & 48 \\ 48 & 48 & 48 & 44 & 48 & 44 & 48 & 44 & 48 & 44 & 48 & 44 & 48 & 48 & 48 \\ 48 & 48 & 48 & 48 & 36 & 48 & 32 & 48 & 32 & 48 & 36 & 48 & 48 & 48 & 48 \\ 48 & 48 & 48 & 48 & 48 & 48 & 48 & 48 & 48 & 48 & 48 & 48 & 48 & 48 & 48\end{array}$

Axial TIP Distribution, Bottom To top of Core

See Figure 22

160944.577 .8100 .1116 .2126 .7127 .2124 .3130 .1122 .2118 .1114 .2113 .9 $106.599 .097 .395 .388 .987 .285 .6 \quad 77.6 \quad 66.6 \quad 54.2 \quad 42.024 .3$ 240957.092 .0114 .1129 .7131 .4128 .2126 .4135 .6137 .6138 .5139 .9137 .9 $131.6120 .4117 .5117 .5103 .4100 .3 \quad 96.486 .473 .7 \quad 59.8 \quad 47.0 \quad 30.1$

320961.595 .2112 .2127 .3129 .6125 .3130 .5139 .8145 .8150 .3153 .4152 .3 146.0137 .6135 .3132 .8115 .2112 .8103 .690 .677 .368 .046 .731 .3

400941.177 .6107 .8124 .3137 .9141 .6138 .8142 .8144 .5142 .2134 .9136 .6 133.2121 .5120 .6120 .2116 .7105 .1103 .695 .882 .269 .355 .039 .8

480948.178 .499 .3118 .2125 .1124 .8123 .5124 .9118 .8119 .7120 .1114 .2 $107.1104 .5101 .296 .388 .388 .681 .270 .060 .850 .3 \quad 32.021 .1$

081733.859 .681 .2103 .7125 .6142 .1144 .1147 .5139 .3138 .4132 .8132 .7 $130.5118 .1118 .1118 .7107 .4102 .398 .686 .8 \quad 72.6 \quad 60.445 .529 .7$

161755.389 .7106 .9123 .8132 .0129 .2125 .9130 .1127 .6122 .9127 .5125 .1 $122.1117 .2120 .0125 .7118 .5119 .1114 .3101 .387 .368 .652 .3 \quad 36.4$

241761.493 .3111 .6124 .3127 .9126 .9129 .0134 .6133 .7134 .6144 .0149 .7 144.8139 .1134 .5133 .0119 .5117 .8110 .995 .983 .774 .450 .538 .1

321773.6101 .4111 .3121 .8123 .4122 .2126 .4128 .8130 .2134 .0146 .1151 .9 142.3137 .8135 .0130 .5114 .9114 .5107 .793 .183 .170 .947 .939 .1

401754.887 .1103 .8119 .0122 .9122 .7121 .9124 .7123 .6120 .6126 .0125 .7 119.8113 .2113 .9116 .1110 .9113 .8108 .797 .086 .769 .954 .337 .0

481758.594 .3118 .4139 .0148 .8154 .2155 .3152 .5146 .6142 .8145 .3141 .2 133.3125 .5123 .6122 .4109 .8108 .9104 .191 .180 .970 .947 .635 .5

561738.158 .975 .095 .7112 .7113 .4117 .1113 .2110 .0104 .8100 .598 .4

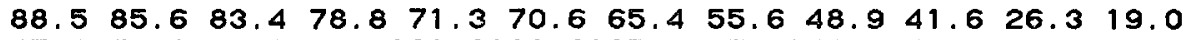

082547.874 .390 .6106 .5121 .0132 .6135 .5147 .1146 .9147 .6160 .0160 .7 $154.9142 .7138 .1131 .7119 .0114 .7108 .6 \quad 95.1 \quad 80.972 .152 .832 .6$

162568.399 .4110 .3121 .4122 .6124 .3128 .3135 .0130 .8134 .5138 .6139 .1 $130.5122 .2117 .8116 .8102 .4100 .596 .6 \quad 87.1 \quad 79.766 .2 \quad 51.3 \quad 34.7$ 
242563.390 .5100 .7111 .5116 .3125 .8132 .9138 .2136 .2134 .8135 .4133 .5 $124.1116 .6114 .3109 .8 \quad 97.1 \quad 96.2 \quad 92.1 \quad 81.4 \quad 73.2 \quad 60.2 \quad 47.4 \quad 34.7$

322572.4100 .9108 .8122 .5130 .6135 .8145 .6151 .5147 .6144 .6147 .0139 .7 $131.1124 .7121 .8117 .8103 .299 .7 \quad 95.3 \quad 82.3 \quad 73.3 \quad 65.5 \quad 45.136 .2$

402561.491 .8103 .9113 .0117 .1117 .0119 .8123 .2121 .6117 .0119 .8113 .9 $113.2104 .9104 .5101 .4 \quad 92.3 \quad 90.7 \quad 88.3 \quad 81.6 \quad 75.865 .1 \quad 52.3 \quad 35.7$

482569.2101 .1113 .0124 .1126 .3125 .9128 .7134 .4135 .8143 .7154 .2152 .3 144.7138 .2134 .1127 .8114 .9112 .3103 .992 .379 .564 .148 .936 .2

562554.991 .9113 .7132 .8141 .7143 .1139 .5140 .3131 .0128 .5127 .1125 .0 118.5110 .3109 .5106 .892 .589 .8 83.3 $73.461 .4 \quad 47.935 .719 .9$

083352.077 .090 .5100 .4114 .6127 .4130 .3136 .1135 .9144 .4150 .1153 .9 $150.1137 .4133 .7130 .1114 .2109 .2101 .8 \quad 88.674 .7 \quad 66.5 \quad 46.029 .5$

163363.492 .2101 .3110 .6116 .1121 .7131 .3141 .0140 .3134 .8137 .5134 .1 $128.4116 .9112 .8111 .198 .3 \quad 96.8 \quad 92.3 \quad 81.3 \quad 72.4 \quad 59.2 \quad 47.0 \quad 31.6$

243358.288 .1101 .4109 .1113 .6114 .5114 .1121 .2119 .9114 .7115 .1115 .3 110.8101 .6100 .097 .287 .686 .284 .175 .769 .860 .049 .131 .6

323365.597 .4107 .5114 .6117 .8120 .6122 .4128 .3127 .6126 .7129 .1127 .9 $119.3110 .7107 .2102 .992 .990 .184 .377 .472 .369 .953 .0 \quad 34.5$

403365.294 .3104 .6114 .6121 .1128 .4140 .5150 .8150 .1148 .0146 .0144 .1 $138.9129 .9124 .6121 .2109 .4104 .5 \quad 98.5 \quad 86.7 \quad 77.3 \quad 64.5 \quad 51.4 \quad 37.0$

483368.596 .2104 .0112 .9116 .8117 .9120 .9125 .8128 .5138 .3145 .7146 .5 $141.3129 .5125 .7120 .3107 .0102 .3 \quad 98.3 \quad 85.3 \quad 74.7 \quad 60.6 \quad 48.4 \quad 37.4$

$563353.988 .2108,9123.7133 .6133 .2133 .2136 .3130 .5122 .4119,1116.8$ $113.3104 .0103 .0100 .088 .585 .680 .769 .759 .248 .0 \quad 36.922 .2$

084153.885 .0105 .7127 .6142 .3147 .4148 .1153 .8147 .7149 .2157 .8159 .3 149.2138 .1136 .9131 .6117 .7113 .3104 .090 .677 .068 .446 .929 .3

164164.494 .9111 .9127 .4131 .4127 .4128 .3127 .3123 .5123 .8126 .2123 .1 $114.5110 .4109 .3107 .097 .898 .0 \quad 94.6 \quad 86.4 \quad 81.3 \quad 65.4 \quad 50.6 \quad 39.7$

244150.881 .5100 .1115 .1124 .1131 .1135 .4144 .7145 .5143 .5146 .2146 .1 $140.3129 .3129 .1126 .6112 .9109 .7106 .094 .681 .068 .153 .3 \quad 35.0$

324161.392 .6104 .9114 .7121 .5127 .0134 .0146 .4147 .4149 .1150 .3156 .0 151.0141 .1138 .2132 .5118 .3112 .6107 .094 .780 .573 .654 .935 .8

404154.984 .9102 .7115 .9120 .8123 .8128 .2130 .4129 .3127 .3127 .1125 .8 119.8114 .3111 .9111 .0100 .299 .096 .187 .280 .567 .252 .036 .9

484155.187 .8109 .0127 .4134 .4136 .6133 .1137 .4135 .8138 .4148 .0148 .1 143.8133 .1133 .7130 .2118 .3117 .5110 .699 .386 .267 .052 .632 .3

564148.283 .7110 .2137 .2155 .4159 .2152 .3153 .6145 .1133 .9132 .7130 .6 $123.8111 .6111 .1109 .097 .293 .987 .6 \quad 76.5 \quad 63.5 \quad 56.5 \quad 39.3 \quad 22.6$

084944.666 .180 .499 .3115 .6125 .5131 .4129 .8125 .4122 .0120 .9115 .4 $\begin{array}{llllllllllll}107.0103 .1 & 99.8 & 93.4 & 85.0 & 82.0 & 76.7 & 65.1 & 56.0 & 43.1 & 29.9 & 19.5\end{array}$

164964.397 .5113 .1125 .4126 .3122 .5118 .5121 .4115 .0111 .3112 .7111 .7 104.6102 .8104 .4109 .2104 .4108 .6105 .089 .379 .362 .047 .032 .8

244968.7101 .9118 .7134 .3132 .4125 .7123 .1127 .2127 .1120 .5138 .6144 .7 $140.5133 .8134 .2131 .4116 .4114 .6106 .593 .0 \quad 80.363 .248 .134 .6$

324958.290 .4109 .7120 .2124 .1119 .3119 .6126 .6130 .1133 .8143 .8155 .0 156.8147 .0144 .9143 .4128 .4121 .6116 .3105 .790 .580 .758 .536 .8

404971.4108 .7124 .1135 .4137 .7133 .3129 .5130 .8123 .5120 .1120 .7118 .4 111.4109 .3111 .8115 .2110 .2110 .3105 .690 .780 .064 .648 .738 .2

484945.578 .4105 .8124 .9137 .4139 .6138 .1137 .5128 .9120 .4114 .4115 .2 108.8100 .3101 .7100 .093 .691 .488 .681 .168 .958 .145 .128 .7

165738.959 .571 .483 .690 .7101 .5109 .3113 .9109 .8105 .3103 .2100 .3

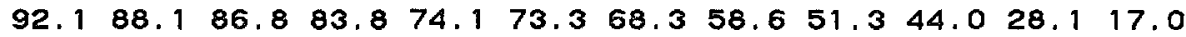

$245769.1102 .7117 .0127,7125.1119 .6119,1126,7128,7124,4123.8121 .8$ $113.5105 .4103 .198 .8 \quad 87.384 .980 .169 .5 \quad 59.745 .0 \quad 33.320 .3$ 325771.7106 .8119 .1129 .7123 .1114 .6110 .5117 .9121 .1116 .6120 .6116 .7 $109.0101 .998 .193 .784 .582 .6 \quad 78.0 \quad 67.4 \quad 58.951 .2 \quad 33.121 .0$

405765.0100 .1118 .2131 .7137 .7138 .9145 .3148 .6143 .0135 .9134 .8135 .2 122.9116 .8113 .7110 .998 .997 .489 .276 .064 .353 .933 .920 .5 


\section{CYCLE 2 DATA}

DATASET 35, FEBRUARY 23, 1977

\section{Reactor Conditions}

Core Average Exposure, $11910 \mathrm{MWd} / \mathrm{t}$

Core Thermal Power, 3258 MWT

Dome Pressure, P, 1026 psia

Core Flow, $105.1 \mathrm{Mlb} / \mathrm{hr}$

Inlet Subcooling at $\mathrm{P}, 23.4 \mathrm{Btu} / \mathrm{lb}$

Control Configuration

Legend: 48, Full Out; O, Full In.

$\begin{array}{lllllllllllllll}48 & 48 & 48 & 48 & 48 & 48 & 48 & 48 & 48 & 48 & 48 & 48 & 48 & 48 & 48 \\ 48 & 48 & 48 & 48 & 36 & 48 & 32 & 48 & 32 & 48 & 36 & 48 & 48 & 48 & 48 \\ 48 & 48 & 48 & 44 & 48 & 44 & 48 & 44 & 48 & 44 & 48 & 44 & 48 & 48 & 48 \\ 48 & 48 & 38 & 48 & 16 & 48 & 28 & 48 & 28 & 48 & 16 & 48 & 38 & 48 & 48 \\ 48 & 40 & 48 & 44 & 48 & 44 & 48 & 48 & 48 & 44 & 48 & 44 & 48 & 40 & 48 \\ 48 & 48 & 28 & 48 & 10 & 48 & 36 & 48 & 36 & 48 & 10 & 48 & 28 & 48 & 48 \\ 48 & 38 & 48 & 48 & 48 & 48 & 48 & 48 & 48 & 48 & 48 & 48 & 48 & 38 & 48 \\ 48 & 48 & 34 & 48 & 36 & 48 & 12 & 48 & 12 & 48 & 36 & 48 & 34 & 48 & 48 \\ 48 & 38 & 48 & 48 & 48 & 48 & 48 & 48 & 48 & 48 & 48 & 48 & 48 & 38 & 48 \\ 48 & 48 & 28 & 48 & 10 & 48 & 36 & 48 & 36 & 48 & 10 & 48 & 28 & 48 & 48 \\ 48 & 40 & 48 & 44 & 48 & 44 & 48 & 48 & 48 & 44 & 48 & 44 & 48 & 40 & 48 \\ 48 & 48 & 38 & 48 & 16 & 48 & 28 & 48 & 28 & 48 & 16 & 48 & 38 & 48 & 48 \\ 48 & 48 & 48 & 44 & 48 & 44 & 48 & 44 & 48 & 44 & 48 & 44 & 48 & 48 & 48 \\ 48 & 48 & 48 & 48 & 36 & 48 & 32 & 48 & 32 & 48 & 36 & 48 & 48 & 48 & 48 \\ 48 & 48 & 48 & 48 & 48 & 48 & 48 & 48 & 48 & 48 & 48 & 48 & 48 & 48 & 48\end{array}$

Axial TIP Distribution, Bottom To top of Core See Figure 22

160941.372 .491 .5104 .5113 .3116 .2118 .8118 .8116 .2112 .7109 .1108 .8 $\begin{array}{llllllllllll}102.0 & 93.5 & 93.9 & 93.5 & 87.1 & 86.5 & 83.9 & 76.0 & 66.4 & 53.7 & 41.9 & 24.4\end{array}$

240951.485 .6104 .4117 .1120 .4119 .0117 .5126 .0131 .2134 .1133 .7133 .0

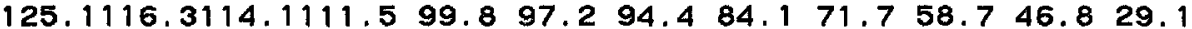

320957.286 .9102 .9117 .7115 .8120 .5121 .2131 .6136 .6141 .3147 .5144 .8 $140.3129 .4128 .5126 .1111 .0108 .2100 .988 .375 .466 .945 .3 \quad 31.3$

400937.672 .399 .3114 .3126 .7127 .4128 .4133 .5135 .6131 .0127 .1128 .7 127.4116 .8116 .5115 .3111 .5101 .9101 .995 .480 .669 .254 .738 .0

480943.872 .289 .9106 .6113 .0116 .1116 .6115 .6114 .4110 .3112 .6109 .1 $104.399 .0 \quad 98.6 \quad 97.7 \quad 86.986 .6 \quad 81.9 \quad 70.0 \quad 61.4 \quad 51.6 \quad 32.3 \quad 21.6$

081731.454 .074 .493 .4116 .6128 .6130 .8137 .8128 .1124 .7126 .6128 .9 122.4113 .3114 .2114 .0104 .7100 .697 .085 .471 .559 .045 .128 .0

161751.280 .397 .1114 .5117 .8118 .6118 .1122 .8117 .7118 .8121 .5120 .6 118.4112 .4117 .1121 .4115 .3115 .9112 .799 .686 .468 .851 .735 .3

241756.185 .4102 .8115 .7120 .4121 .9125 .0130 .0131 .2137 .5145 .8150 .3 $142.4133 .9129 .6128 .4116 .4114 .2106 .7 \quad 94.6 \quad 82.3 \quad 73.249 .7 \quad 36.7$

$321767.694 .1102,5114.3115 .3115 .9118,8128,5131,3136.3145 .4147 .6$ $138.2132 .5128 .1122 .4111 .7111 .2104 .4 \quad 91.8 \quad 81.070 .6 \quad 46.940 .9$ 401750.480 .396 .0109 .8116 .9115 .6113 .6120 .2119 .8116 .6121 .2120 .5 115.6109 .4110 .6113 .5108 .6111 .9108 .797 .886 .969 .954 .236 .6 481754.487 .5105 .5126 .5136 .8141 .7142 .9144 .7139 .4133 .8137 .7136 .1 $128.4122 .5121 .0118 .4109 .5109 .1104 .5 \quad 92.1 \quad 81.671 .748 .2 \quad 37.9$

561735.654 .369 .690 .1100 .8104 .8104 .3106 .9101 .598 .098 .294 .9

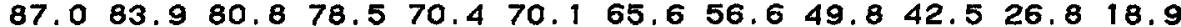
082544.468 .884 .1100 .2113 .0123 .9127 .5138 .2138 .3141 .0150 .8153 .0 144.8129 .9129 .2125 .5111 .4108 .6104 .991 .079 .270 .851 .132 .6 162562.792 .2102 .6112 .2115 .9119 .7121 .2128 .2129 .7127 .8130 .3129 .6 123.3115 .1111 .8111 .5100 .1101 .5100 .590 .281 .865 .852 .234 .5 
242559.384 .692 .5103 .9109 .4114 .7126 .5133 .5130 .4126 .4131 .2126 .5 $119.8113 .3112 .2109 .198 .799 .797 .3 \quad 86.178 .063 .049 .2 \quad 36.8$ $322567.193,6103,2114,3120,6127,2134,0140,6138,3137.4135 .3135 .1$ 126.7121 .1119 .2116 .3104 .3104 .2100 .588 .078 .568 .447 .239 .6 402557.987 .698 .6107 .5111 .1110 .9115 .3121 .7117 .5114 .2116 .5113 .9 $109.3102 .1101 .9100 .3 \quad 93.1 \quad 93.9 \quad 94.4 \quad 89.1 \quad 81.7 \quad 67.8 \quad 53.8 \quad 36.2$

482565.693 .9106 .1119 .0121 .2122 .7124 .7135 .5137 .7139 .6150 .7146 .6 $138.2130 .5128 .2123 .8112 .0109 .0104 .492 .8 \quad 81.964 .649 .435 .3$

562552.787 .4107 .5125 .5132 .4136 .1130 .9133 .3127 .6117 .7116 .5116 .9 $112.6107 .0103 .2102 .9 \quad 90.5 \quad 88.1 \quad 83.2 \quad 71.6 \quad 59.947 .8 \quad 35.220 .8$

083348.671 .383 .498 .6112 .2126 .3141 .5154 .0156 .5150 .5147 .7147 .6 $137.1125 .9121 .3119 .5106 .9103 .997 .1 \quad 84.6 \quad 72.7 \quad 64.1 \quad 44.530 .4$

163358.284 .793 .4101 .9110 .0118 .4129 .8137 .5136 .3132 .2130 .6127 .4 120.9109 .2108 .9105 .595 .194 .692 .580 .971 .758 .647 .631 .8

243353.681 .294 .2101 .1105 .6107 .3107 .8113 .9113 .5111 .3110 .1109 .2 $105.498 .598 .1 \quad 97.6 \quad 91.6 \quad 96.3 \quad 99.3 \quad 92.8 \quad 81.967 .0 \quad 53.7 \quad 34.2$

323361.589 .499 .5107 .5110 .2112 .2115 .8122 .3120 .4120 .4122 .7120 .1 115.8108 .1106 .0105 .698 .1100 .4104 .097 .286 .180 .558 .337 .9

403360.788 .097 .3107 .6115 .0125 .3133 .1145 .0142 .2142 .3142 .4138 .7 $132.1125 .3122 .3120 .2111 .6108 .1104 .3 \quad 94.183 .267 .453 .839 .2$

483362.988 .395 .5108 .3112 .7118 .6130 .8141 .4144 .3140 .2142 .7141 .4 131.4120 .8117 .6113 .7101 .999 .496 .284 .072 .960 .647 .935 .6

563351.384 .3101 .2116 .3123 .4128 .6129 .0132 .9125 .7119 .8114 .1112 .3 $104.998 .496 .4 \quad 94.8 \quad 84.8 \quad 81.3 \quad 77.1 \quad 67.958 .2 \quad 46.5 \quad 35.221 .9$

084150.079 .396 .9115 .7130 .6135 .6136 .8141 .0138 .5140 .9147 .0148 .6 $141.2132 .1126 .5125 .2113 .6110 .0103 .589 .7 \quad 75.767 .4 \quad 46.528 .5$

164159.788 .4103 .3119 .1121 .1119 .1120 .3120 .7120 .9117 .7121 .3117 .7 108.7105 .7106 .1104 .696 .099 .4101 .992 .084 .568 .351 .738 .2

244147.475 .492 .9106 .9115 .8123 .0131 .7141 .5138 .7137 .1142 .3141 .0 136.2125.4124.0123.9112.7110.4106.0 95.9 82.8 68.0 54.1 35.2

324156.684 .596 .8104 .7112 .8120 .8127 .9141 .9142 .7145 .0148 .3148 .8 144.9133 .1132 .2130 .2115 .6110 .9106 .094 .881 .274 .254 .636 .6

404152.079 .595 .5108 .6113 .4116 .3119 .9123 .1121 .1119 .3123 .6122 .1 113.6110 .7107 .9108 .099 .8101 .3100 .391 .184 .267 .052 .136 .5

484152.182 .8101 .9117 .3124 .6125 .5126 .7129 .4127 .6131 .9135 .3140 .4 $136.5128 .6126 .0125 .7114 .6114 .9108 .799 .8 \quad 85.868 .152 .732 .8$

564145.879 .8102 .4126 .0144 .0148 .6140 .9141 .3133 .7127 .0125 .2123 .3 $117.3107 .9106 .7104 .895 .492 .3 \quad 86.977 .063 .356 .739 .221 .5$

084940.559 .971 .787 .9102 .2113 .3118 .4120 .0116 .0113 .3114 .9109 .5 $102.6100 .5 \quad 96.5 \quad 92.0 \quad 83.8 \quad 82.1 \quad 75.1 \quad 64.5 \quad 55.9 \quad 43.0 \quad 29.5 \quad 19.6$

164958.787 .9104 .1116 .4115 .9112 .6111 .1113 .5109 .8106 .0108 .3109 .3 102.299 .5103 .4107 .5102 .7106 .9102 .089 .8 80.2 62.546 .334 .8

244966.197 .5109 .1121 .9121 .7117 .2113 .4121 .0124 .8131 .0138 .7141 .6 134.1129 .7129 .8124 .0111 .6109 .7103 .490 .078 .861 .046 .436 .5

324952.182 .9100 .1112 .7116 .1113 .2116 .1125 .6129 .1138 .0150 .4154 .0 148.7139 .0139 .8135 .9122 .1117 .0112 .2100 .686 .178 .956 .936 .6

404966.096 .4115 .5127 .5129 .0121 .7119 .6122 .4120 .1117 .3118 .6117 .5 108.1105 .0108 .6111 .6106 .1108 .1104 .591 .179 .163 .747 .938 .5

484941.871 .594 .7112 .4126 .1129 .6125 .9125 .0118 .4112 .9111 .8111 .0 $107.196 .297 .498 .5 \quad 91.2 \quad 90.3 \quad 87.8 \quad 80.469 .2 \quad 57.845 .4 \quad 27.5$

$165736.354 .664 .876 .3 \quad 82.691 .4101 .7106 .4102 .099 .196 .995 .1$

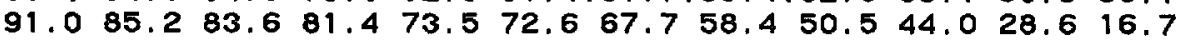

245765.697 .1107 .2115 .0114 .2108 .6109 .0116 .3117 .0116 .7116 .7116 .4 $108.0102 .5100 .2 \quad 95.8 \quad 85.6 \quad 83.4 \quad 79.5 \quad 67.958 .7 \quad 44.3 \quad 32.8 \quad 20.9$

325768.6100 .4111 .7119 .8112 .2105 .9103 .7111 .8114 .0111 .1113 .0113 .1

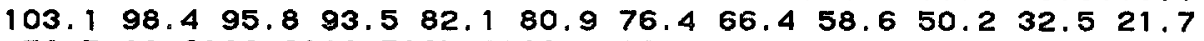

405759.590 .6106 .2122 .5127 .3129 .9132 .4139 .3135 .2127 .3131 .3127 .7 118.3114 .0110 .8108 .198 .796 .289 .977 .064 .354 .135 .120 .6 


\section{CYCLE 2 DATA}

DATASET 36, MARCH 11, 1977

\section{Reactor Conditions}

Core Average Exposure, $12190 \mathrm{MWd} / \mathrm{t}$

Core Thermal Power, 2858 MWT

Dome Pressure, $\mathrm{P}, 1026$ psia

Core Flow, $78.8 \mathrm{MIb} / \mathrm{hr}$

Inlet Subcooling at P, $29.8 \mathrm{Btu} / \mathrm{lb}$

Control Configuration

Legend: 48, Full Out; O, Full In.

\begin{tabular}{|c|c|c|c|c|c|c|c|c|c|c|c|c|c|c|}
\hline $\begin{array}{l}48 \\
48\end{array}$ & $\begin{array}{l}48 \\
48\end{array}$ & $\begin{array}{l}48 \\
48\end{array}$ & $\begin{array}{l}48 \\
48\end{array}$ & $\begin{array}{l}48 \\
48\end{array}$ & $\begin{array}{l}48 \\
38\end{array}$ & $\begin{array}{l}48 \\
48\end{array}$ & $\begin{array}{l}48 \\
40\end{array}$ & $\begin{array}{l}48 \\
48\end{array}$ & $\begin{array}{l}48 \\
38\end{array}$ & $\begin{array}{l}48 \\
48\end{array}$ & $\begin{array}{l}48 \\
48\end{array}$ & $\begin{array}{l}48 \\
48\end{array}$ & $\begin{array}{l}48 \\
48\end{array}$ & $\begin{array}{l}48 \\
48\end{array}$ \\
\hline $\begin{array}{l}48 \\
48\end{array}$ & $\begin{array}{l}48 \\
48\end{array}$ & $\begin{array}{l}48 \\
48\end{array}$ & $\begin{array}{l}48 \\
42\end{array}$ & $\begin{array}{l}32 \\
48\end{array}$ & $\begin{array}{l}48 \\
44\end{array}$ & $\begin{array}{l}34 \\
48\end{array}$ & $\begin{array}{l}48 \\
48\end{array}$ & $\begin{array}{l}34 \\
48\end{array}$ & $\begin{array}{l}48 \\
44\end{array}$ & $\begin{array}{l}32 \\
48\end{array}$ & $\begin{array}{l}48 \\
42\end{array}$ & $\begin{array}{l}48 \\
48\end{array}$ & & 10 \\
\hline 48 & 48 & 32 & 48 & 36 & 48 & 14 & 48 & 14 & 48 & 36 & 48 & 32 & 48 & 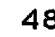 \\
\hline 48 & 38 & 48 & 44 & 48 & 48 & 48 & 48 & 48 & 48 & 48 & 44 & 48 & so & 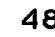 \\
\hline 48 & 48 & 34 & 48 & 14 & 48 & 24 & 48 & 24 & 48 & 14 & 48 & 34 & 48 & 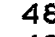 \\
\hline 48 & 40 & 48 & 48 & 48 & 48 & 48 & 48 & 48 & 48 & 48 & 48 & 48 & 40 & 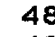 \\
\hline 48 & 48 & 34 & 48 & 14 & 48 & 24 & 48 & 24 & 48 & 14 & 48 & 34 & 48 & 4 \\
\hline 48 & 38 & 48 & 44 & 48 & 48 & 48 & 48 & 48 & 4 & 48 & 44 & 48 & 38 & \\
\hline 48 & 48 & 32 & 48 & 36 & 48 & 14 & 48 & 14 & 48 & 36 & 48 & 32 & 48 & 4 \\
\hline 48 & 48 & 48 & 42 & 48 & 44 & 48 & 48 & 48 & 44 & 48 & 42 & 48 & 48 & \\
\hline 48 & 48 & 48 & 48 & 32 & 48 & 34 & 48 & 32 & 48 & 32 & 48 & 48 & 48 & 4 \\
\hline 4 & 4 & 48 & 4 & 48 & 38 & 48 & 4 & 4 & 3 & 4 & 48 & 48 & 48 & \\
\hline & & 4 & 4 & 4 & & & & & & & & & & \\
\hline
\end{tabular}

Axial TIP Distribution, Bottom To top of Core See Figure 22

160954.886 .7100 .7108 .8110 .3105 .9102 .7107 .8111 .9109 .8111 .3106 .9

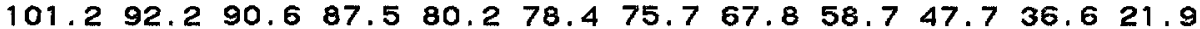
240949.075 .590 .2104 .2113 .4122 .7128 .7134 .9134 .9131 .1125 .8120 .6 $113.8102 .8102 .4100 .690 .287 .7 \quad 84.6 \quad 76.1 \quad 65.4 \quad 54.3 \quad 42.926 .2$ 320952.678 .592 .5109 .5121 .4127 .3135 .9145 .0139 .6138 .4133 .1127 .8 $122.0113 .9111 .0109 .398 .696 .090 .7 \quad 79.968 .860 .941 .629 .1$

400935.163 .381 .490 .8103 .4111 .7116 .8129 .0133 .8134 .5130 .5127 .6 $123.5112 .9111 .5109 .1104 .2 \quad 95.1 \quad 93.5 \quad 85.5 \quad 72.2 \quad 62.5 \quad 49.4 \quad 34.6$

480966.196 .8106 .3115 .3109 .9104 .5103 .8103 .5104 .5104 .0104 .7102 .2

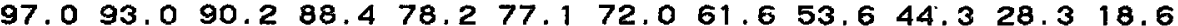

081750.385 .0102 .4111 .4117 .3112 .7105 .5111 .7117 .2116 .3117 .6116 .9 112.8104 .3105 .3101 .593 .490 .185 .475 .563 .053 .140 .625 .7

161753.280 .397 .2117 .7129 .1133 .4141 .4150 .6151 .7145 .8142 .5142 .9 134.3126 .0124 .1120 .5109 .0106 .7100 .086 .976 .059 .045 .333 .0

241759.388 .0103 .8117 .5119 .7120 .9124 .5126 .3125 .6120 .7121 .6117 .2 111.4105 .6103 .8105 .5100 .5104 .4102 .489 .778 .369 .647 .336 .4

321768.993 .1100 .6107 .8107 .2105 .3106 .4109 .2106 .9103 .8108 .0104 .6

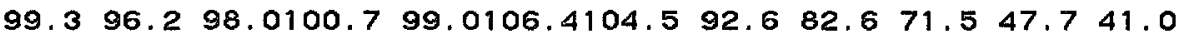

401750.281 .696 .7111 .4119 .8123 .4131 .7139 .8138 .4133 .3135 .3131 .6 $124.6113 .2112 .1109 .6101 .0100 .797 .186 .076 .161 .748 .7 \quad 34.2$

481759.489 .8105 .2122 .2125 .3125 .5129 .1136 .7139 .9134 .8138 .2136 .2 $126.2119 .9115 .2113 .1101 .499 .6 \quad 95.0 \quad 83.073 .463 .543 .3 \quad 32.7$

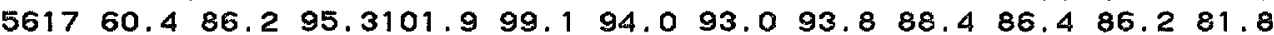

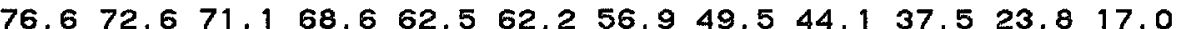

$082545.669 .1 \quad 83.997 .7110 .2122 .7135 .0146 .2145 .5141 .4139 .0132 .3$ $124.1114 .8113 .2107 .1 \quad 98.3 \quad 95.8 \quad 93.1 \quad 82.9 \quad 71.7 \quad 64.3 \quad 47.4 \quad 29.4$

162552.880 .696 .2108 .5112 .7112 .7117 .7123 .1123 .1120 .1122 .4119 .1 $111.9107 .2105 .2107 .4103 .3106 .5101 .8 \quad 89.979 .163 .649 .534 .9$ 
$242558.482 .4 \quad 89.494 .093 .692 .592 .794 .993 .995 .8103 .9110 .6$ $113.9117 .6118 .3117 .0108 .9108 .0103 .890 .5 \quad 81.364 .4 \quad 49.437 .6$

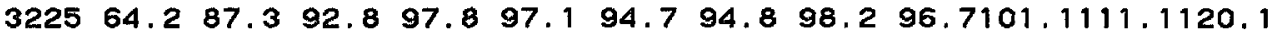
$124.8128 .2128 .7127 .5116 .7117 .3109 .094 .6 \quad 83.9 \quad 72.749 .840 .2$ 402556.584 .393 .9101 .8103 .0103 .6103 .4106 .2103 .5100 .7106 .4105 .7 103.9100 .7101 .1104 .6101 .5106 .7102 .893 .282 .067 .652 .535 .0

482555.185 .8102 .4114 .1121 .3124 .9134 .3146 .1148 .1141 .4139 .5138 .1 $126.2119 .2116 .5113 .4103 .7103 .5 \quad 99.287 .3 \quad 76.2 \quad 59.7 \quad 46.033 .7$

562557.792 .3109 .9127 .2131 .2132 .5126 .0126 .2119 .5113 .5111 .0108 .3 $100.994 .5 \quad 93.5 \quad 91.7 \quad 81.8 \quad 80.2 \quad 74.965 .0 \quad 55.6 \quad 43.6 \quad 32.1 \quad 19.6$

083350.476 .890 .7107 .5124 .8130 .8135 .0143 .8139 .2133 .7132 .4128 .5 120.0111 .3110 .3108 .096 .995 .190 .978 .268 .060 .641 .827 .9

163355.881 .290 .297 .399 .9100 .4103 .0106 .3103 .6100 .2102 .1101 .5

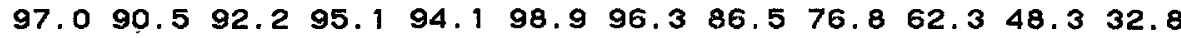

$243349.975 .186 .091 .791 .6 \quad 88.8 \quad 85.8 \quad 90.1 \quad 91.0 \quad 91.7100 .4107 .8$ 114.6116 .4119 .0119 .3111 .7111 .2107 .095 .481 .968 .253 .635 .6

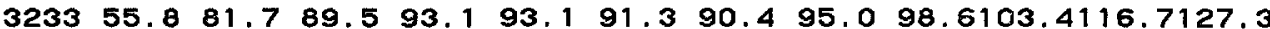
133.8134 .1137 .9136 .2123 .7119 .7112 .8100 .486 .580 .359 .539 .0

403357.382 .992 .698 .3100 .197 .997 .2103 .1102 .6105 .6109 .2112 .0 110.8110 .9110 .6115 .9112 .7117 .3113 .9100 .789 .572 .155 .940 .3

483360.386 .295 .3105 .3112 .1114 .2121 .5133 .9132 .5129 .4131 .3125 .6 $114.8108 .1107 .1104 .795 .595 .892 .782 .4 \quad 72.5 \quad 58.745 .935 .7$

563352.586 .6108 .1122 .3128 .9130 .3116 .2121 .3114 .2107 .3102 .8100 .5

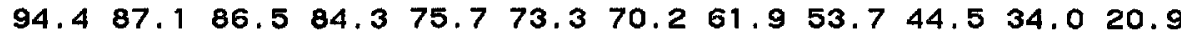

084151.079 .292 .9104 .6114 .1123 .7130 .1139 .2141 .7141 .6142 .2138 .2 $127.4118 .5116 .4114 .2102 .7 \quad 99.6 \quad 92.3 \quad 80.6 \quad 68.7 \quad 61.141 .8 \quad 26.9$

164156.782 .899 .4116 .6120 .9126 .6138 .0142 .6138 .1132 .8134 .3127 .9 120.1112 .8111 .2108 .999 .9100 .493 .983 .273 .258 .345 .035 .9

244152.281 .594 .5103 .0107 .8106 .0105 .0107 .3106 .4107 .0107 .1110 .6 109.6104 .2106 .9108 .4106 .8110 .7108 .899 .986 .271 .956 .736 .2

324153.079 .289 .893 .894 .293 .192 .697 .496 .598 .1102 .9106 .0 107.7105 .3107 .3111 .8109 .9113 .8112 .5101 .887 .780 .360 .839 .6

404159.988 .5102 .8112 .9117 .8122 .8130 .8135 .4132 .2125 .9128 .7125 .1 $119.1114 .2114 .4112 .4103 .7103 .199 .3 \quad 87.6 \quad 76.6 \quad 62.948 .3 \quad 32.8$

484151.283 .9102 .2118 .7124 .2125 .0127 .7136 .8140 .0137 .9136 .6133 .7 126.1117 .8117 .5114 .5105 .1103 .999 .8 88.3 76.061 .548 .629 .6

564149.083 .7104 .7118 .2127 .4132 .4129 .3133 .4124 .4117 .9114 .5111 .4 $107.595 .793 .8 \quad 90.8 \quad 84.079 .976 .7 \quad 68.2 \quad 57.2 \quad 50.6 \quad 36.121 .7$

084970.397 .0104 .8111 .7107 .6103 .1101 .8104 .7103 .7103 .0101 .8100 .3

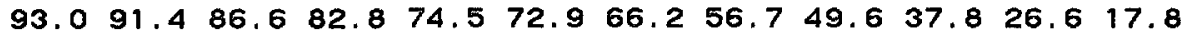

164959.986 .298 .2111 .6112 .6111 .8114 .2122 .0125 .3125 .1125 .8124 .2 $115.6110 .7107 .1104 .593 .294 .0 \quad 87.8 \quad 77.1 \quad 68.1 \quad 53.540 .7 \quad 30.9$

244960.387 .4103 .2118 .5121 .0121 .6128 .9138 .6138 .0134 .3130 .8127 .2 $119.7115 .6112 .2110 .699 .0 \quad 96.9 \quad 92.980 .7 \quad 71.855 .843 .0 \quad 33.7$

324954.985 .799 .3106 .4109 .2112 .2118 .0132 .2137 .1134 .2129 .8128 .7 123.0114 .4115 .4112 .9106 .1103 .0102 .794 .281 .374 .554 .936 .0 404963.594 .4109 .4121 .7126 .7125 .1130 .6136 .4134 .7129 .0133 .3127 .6 115.7110.9109.0104.9 94.3 95.5 89.5 $77.6 \quad 68.454 .741 .433 .5$

484959.498 .1120 .6131 .2129 .6124 .8113 .8112 .9110 .1103 .2100 .7101 .6

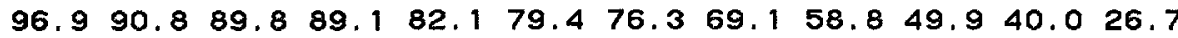

165758.284 .094 .6102 .8100 .196 .694 .794 .191 .088 .8 86.9 85.7

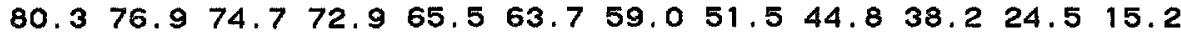

245766.899 .9111 .8126 .0130 .4126 .7124 .5124 .4115 .1108 .6106 .4103 .7

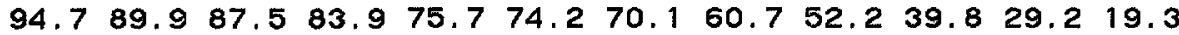

325771.2102 .7119 .5135 .5137 .7128 .5119 .0115 .7109 .9100 .298 .394 .4

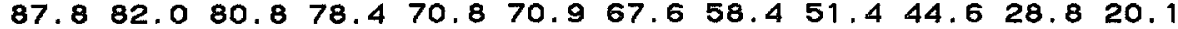
405763.194 .2108 .7125 .9130 .5131 .9130 .4131 .1127 .6120 .6120 .6114 .8 $106.8100 .398 .296 .988 .384 .477 .867 .057 .048 .5 \quad 30.818 .9$ 


\section{CYCLE 2 DATA}

DATASET 37, APRIL 3, 1977

\section{Reactor Conditions}

Core Average Exposure, $12530 \mathrm{MWd} / \mathrm{t}$

Core Thermal Power, 3247 MWT

Dome Pressure, P, 1023 psia

Core Flow, $104.2 \mathrm{Mlb} / \mathrm{hr}$

Inlet Subcooling at P, $23.5 \mathrm{Btu} / \mathrm{lb}$

Control Configuration

Legend: 48, Full Out; O, Full In.

$\begin{array}{lllllllllllllll}48 & 48 & 48 & 48 & 48 & 48 & 48 & 48 & 48 & 48 & 48 & 48 & 48 & 48 & 48 \\ 48 & 48 & 48 & 48 & 48 & 38 & 48 & 38 & 48 & 38 & 48 & 48 & 48 & 48 & 48 \\ 48 & 48 & 48 & 48 & 34 & 48 & 34 & 48 & 34 & 48 & 34 & 48 & 48 & 48 & 48 \\ 48 & 48 & 48 & 42 & 48 & 44 & 48 & 48 & 48 & 44 & 48 & 42 & 48 & 48 & 48 \\ 48 & 48 & 34 & 48 & 36 & 48 & 18 & 48 & 18 & 48 & 36 & 48 & 34 & 48 & 48 \\ 48 & 38 & 48 & 44 & 48 & 48 & 48 & 48 & 48 & 48 & 48 & 44 & 48 & 38 & 48 \\ 48 & 48 & 34 & 48 & 18 & 48 & 36 & 48 & 36 & 48 & 18 & 48 & 34 & 48 & 48 \\ 48 & 40 & 48 & 48 & 48 & 48 & 48 & 48 & 48 & 48 & 48 & 48 & 48 & 40 & 48 \\ 48 & 48 & 34 & 48 & 18 & 48 & 36 & 48 & 36 & 48 & 18 & 48 & 34 & 48 & 48 \\ 48 & 38 & 48 & 44 & 48 & 48 & 48 & 48 & 48 & 48 & 48 & 44 & 48 & 38 & 48 \\ 48 & 48 & 34 & 48 & 36 & 48 & 18 & 48 & 18 & 48 & 36 & 48 & 34 & 48 & 48 \\ 48 & 48 & 48 & 42 & 48 & 44 & 48 & 48 & 48 & 44 & 48 & 42 & 48 & 48 & 48 \\ 48 & 48 & 48 & 48 & 34 & 48 & 34 & 48 & 34 & 48 & 34 & 48 & 48 & 48 & 48 \\ 48 & 48 & 48 & 48 & 48 & 38 & 48 & 38 & 48 & 38 & 48 & 48 & 48 & 48 & 48 \\ 48 & 48 & 48 & 48 & 48 & 48 & 48 & 48 & 48 & 48 & 48 & 48 & 48 & 48 & 48\end{array}$

Axial TIP Distribution, Bottom To top of Core

See Figure 22

$160944.0 \quad 70.5 \quad 82.290 .194 .494 .297 .1104 .5104 .7104 .1101 .599 .1$

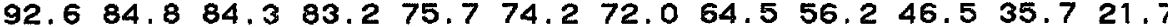

240938.559 .970 .682 .393 .8104 .4110 .6124 .1122 .7120 .8117 .0113 .7

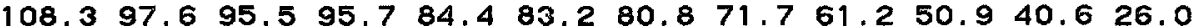
320942.061 .171 .283 .195 .1108 .0120 .0132 .5128 .0126 .9127 .5124 .3

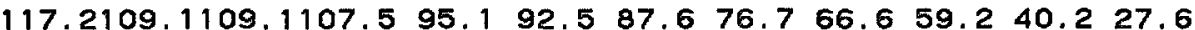

400928.751 .767 .976 .687 .999 .7107 .6122 .7127 .7124 .8119 .2120 .3 $116.4108 .8104 .9104 .0 \quad 98.389 .0 \quad 88.7 \quad 81.869 .4 \quad 59.9 \quad 47.433 .9$

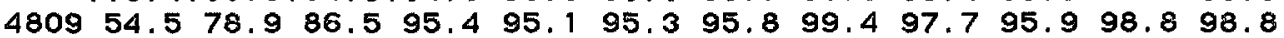
$\begin{array}{llllllllllll}91.8 & 88.2 & 89.2 & 83.3 & 76.4 & 74.8 & 70.1 & 60.6 & 52.7 & 44.1 & 28.0 & 18.8\end{array}$ $081740.6 \quad 69.083 .192 .296 .797 .698 .2110 .8113 .9113 .0111 .3108 .0$

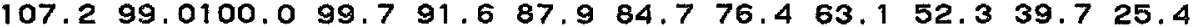

161743.765 .881 .5100 .3111 .4120 .1126 .3141 .9137 .5134 .5134 .5132 .6 $124.3118 .4117 .8115 .1104 .3100 .895 .0 \quad 83.271 .555 .743 .029 .7$

241748.372 .985 .699 .3104 .1108 .4112 .0118 .6115 .0111 .4114 .1112 .6 $107.8106 .1107 .8111 .1103 .4103 .096 .784 .7 \quad 72.764 .944 .432 .3$

$321757.3 \quad 78.486 .893 .995 .995 .398 .8103 .8103 .7103 .3101 .3102 .4$ 99. $1100.1106 .0111 .8102 .5103 .298 .3 \quad 85.0 \quad 76.565 .5 \quad 43.936 .9$

401741.868 .081 .997 .1104 .9114 .2122 .6129 .5129 .0125 .5127 .3126 .7 $118.6109 .9108 .6107 .299 .0 \quad 96.2 \quad 93.5 \quad 82.8 \quad 72.3 \quad 58.245 .8 \quad 31.2$

481748.573 .788 .8104 .7110 .2113 .7124 .5133 .6132 .2127 .5129 .6127 .0 $119.9113 .9110 .5106 .7 \quad 97.4 \quad 95.5 \quad 91.8 \quad 79.8 \quad 70.4 \quad 62.5 \quad 41.6 \quad 31.6$

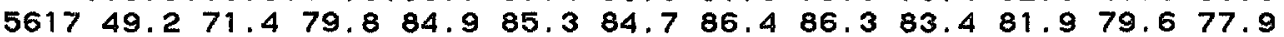
$\begin{array}{llllllllllllllll}73.4 & 69.0 & 67.0 & 66.1 & 61.8 & 60.0 & 56.2 & 49.3 & 43.7 & 37.0 & 23.7 & 16.7\end{array}$

082537.957 .069 .482 .192 .2105 .2118 .9130 .5135 .9129 .9128 .8126 .8 $121.9109 .6107 .1107 .996 .395 .2 \quad 91.0 \quad 31.070 .863 .945 .628 .3$

162544.467 .981 .293 .398 .3102 .9105 .3114 .0113 .6111 .3114 .6114 .6 108.3106 .0110 .3113 .6105 .9104 .698 .085 .672 .859 .245 .729 .9 
242552.674 .282 .290 .196 .5102 .7111 .6118 .9116 .6112 .4113 .8114 .0 109.1106 .4107 .2107 .696 .396 .290 .980 .170 .256 .543 .932 .3

322557.7 80.2 88.9 99.5106.5114.5122.4129.5127.5124.1124.5122.7 116.8113 .7112 .5112 .8100 .899 .593 .881 .470 .861 .942 .034 .4

402549.774 .585 .794 .397 .2100 .1102 .3106 .6105 .0102 .3102 .8102 .4 100.998 .6103 .8109 .0102 .1101 .097 .284 .774 .260 .348 .332 .3

482545.171 .4 85.8 99.6106.3113.5122.5137.0135.6130.3131.8130.8 $121.9115 .9114 .9113 .9101 .599 .495 .784 .4 \quad 72.957 .6 \quad 44.631 .3$

562548.979 .094 .2106 .0113 .1118 .0113 .2112 .5107 .1105 .4105 .1102 .5

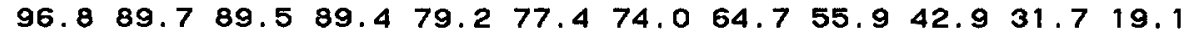

083341.662 .275 .390 .8104 .6111 .2119 .0127 .3126 .4123 .2123 .1122 .8 $114.4105 .9104 .8103 .1 \quad 94.2 \quad 91.5 \quad 86.2 \quad 75.0 \quad 63.8 \quad 57.7 \quad 40.526 .4$

$\begin{array}{lllllllllllll}1633 & 46.3 & 68.6 & 76.9 & 84.2 & 88.7 & 89.8 & 92.4 & 97.1 & 96.5 & 94.7 & 96.1 & 96.7\end{array}$ $92.291 .496 .4103 .0 \quad 95.5 \quad 95.990 .5 \quad 80.269 .2 \quad 56.1 \quad 44.429 .6$

243345.670 .681 .990 .798 .1105 .7110 .4119 .8119 .0114 .8114 .4114 .7 $111.2103 .6105 .0105 .3 \quad 97.0 \quad 94.6 \quad 90.8 \quad 81.3 \quad 69.7 \quad 56.5 \quad 44.7 \quad 30.0$

323351.576 .787 .597 .1106 .5115 .6126 .6135 .2134 .3133 .3133 .3132 .8 $127.5116 .5114 .5113 .0102 .498 .5 \quad 94.484 .072 .265 .648 .3 \quad 32.4$

403351.374 .884 .792 .695 .699 .3103 .7108 .1109 .7108 .9110 .4111 .3 110.3108 .2114 .9121 .8113 .5111 .2104 .591 .279 .563 .650 .435 .0

483350.173 .279 .089 .094 .5100 .5107 .8120 .8119 .3120 .4122 .4117 .7 111.6106 .4106 .9104 .894 .195 .189 .478 .668 .656 .243 .833 .6

563343.171 .789 .0101 .0109 .9112 .2108 .0110 .7108 .1101 .295 .794 .9

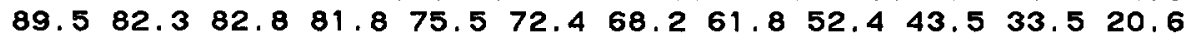

$084141.765 .677 .7 \quad 86.999 .6112 .0123 .8138 .8136 .2133 .3133 .9128 .9$ 123.0115 .5111 .8110 .598 .696 .090 .179 .667 .460 .641 .426 .2

164147.471 .085 .998 .4109 .3118 .8128 .2135 .3130 .9129 .0128 .3124 .2 $116.4110 .2110 .9109 .397 .396 .491 .979 .469 .5 \quad 55.442 .733 .3$

244145.370 .984 .194 .299 .3102 .8102 .5108 .7109 .7106 .8106 .8109 .8 107.9103 .4109 .6114 .4109 .9109 .5104 .192 .478 .365 .551 .233 .3

$324146.070 .8 \quad 80.786 .490 .391 .894 .5101 .6102 .3105 .4104 .7104 .7$ 105.4102 .6110 .3116 .1111 .4108 .4104 .993 .879 .271 .355 .236 .6

404151.177 .988 .4100 .1106 .4114 .6120 .9128 .3125 .0123 .3123 .0121 .6 $116.4110 .2111 .7112 .3101 .199 .2 \quad 93.6 \quad 82.0 \quad 71.6 \quad 57.945 .2 \quad 29.9$

$484142.5 \quad 69.286 .798 .8109 .1113 .8122 .1134 .8133 .3129 .6130 .3126 .4$ $120.8112 .8113 .1113 .4101 .8101 .498 .186 .7 \quad 73.6 \quad 60.4 \quad 47.629 .9$

564140.068 .985 .899 .0109 .8118 .4119 .0120 .9118 .6111 .7106 .1108 .5 $102.493 .0 \quad 92.9 \quad 88.9 \quad 84.1 \quad 79.7 \quad 75.8 \quad 67.5 \quad 57.5 \quad 50.7 \quad 36.920 .8$

$\begin{array}{llllllllllllll}0849 & 59.6 & 80.1 & 87.8 & 93.8 & 92.1 & 92.4 & 95.9 & 95.6 & 96.4 & 95.8 & 95.4 & 93.3\end{array}$

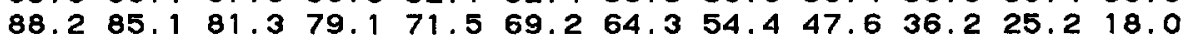

164948.570 .282 .095 .5100 .4103 .7109 .3117 .1118 .2114 .4117 .2116 .6 $107.2103 .6102 .499 .389 .589 .484 .674 .065 .251 .0 \quad 38.928 .2$

244949.071 .484 .798 .3104 .0107 .6116 .1129 .0129 .0123 .1123 .1120 .8 113.3110 .1111 .7107 .295 .492 .288 .976 .966 .752 .639 .932 .3

324945.470 .180 .287 .994 .399 .2104 .8122 .4124 .9126 .5123 .5125 .0 120.5113 .9113 .4113 .2105 .7102 .199 .291 .477 .570 .152 .833 .1

$404951.977 .890 .0104 .2110 .7116 .4124 .7133,1130.0120 .4121 .4116 .7$ 110.3103 .1101 .199 .890 .288 .784 .974 .064 .251 .239 .632 .3

484948.481 .198 .6107 .8116 .3111 .0103 .9106 .8103 .5100 .496 .098 .5 $93.186 .286 .585 .7 \quad 79.5 \quad 75.974 .5 \quad 68.6 \quad 57.949 .2 \quad 39.926 .0$

$\begin{array}{lllllllllllllll}1657 & 46.5 & 67.5 & 76.0 & 84.8 & 86.0 & 86.3 & 83.7 & 86.1 & 85.4 & 81.1 & 81.0 & 80.6\end{array}$ $\begin{array}{llllllllllllll}74.8 & 70.9 & 69.8 & 69.1 & 62.4 & 61.4 & 56.9 & 49.5 & 42.8 & 37.1 & 24.3 & 14.7\end{array}$

245756.079 .189 .5103 .5110 .5111 .6112 .6113 .3108 .4100 .7100 .898 .0

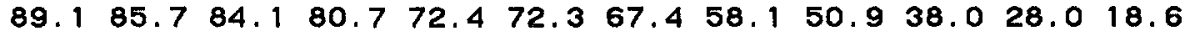

325755.8 81.4 92.2104.9109.0108.5109.0110.3104.8 96.6 95.9 91.9 $84.980 .0 \quad 77.7 \quad 77.2 \quad 69.269 .0 \quad 65.8 \quad 57.4 \quad 50.6 \quad 43.5 \quad 28.3 \quad 20.2$

405750.377 .289 .0103 .8113 .6117 .9121 .9122 .4121 .6111 .9111 .7110 .3 $101.197 .595 .692 .8 \quad 83.981 .9 \quad 78.1 \quad 65.7 \quad 55.8 \quad 46.9 \quad 30.3 \quad 18.0$ 